Francisco José Profito

\title{
On the Development of Advanced Techniques for Mixed-Elastohydrodynamic Lubrication Modelling of Journal and Sliding Bearing Systems
}


Francisco José Profito

\section{On the Development of Advanced Techniques for Mixed-Elastohydrodynamic Lubrication Modelling of Journal and Sliding Bearing Systems}


Francisco José Profito

\section{On the Development of Advanced Techniques for Mixed-Elastohydrodynamic Lubrication Modelling of Journal and Sliding Bearing Systems}

Thesis submitted for the Degree of Doctor of Philosophy of the University of São Paulo.

Department:

Mechanical Engineering

Supervisor:

Prof. Dr. Demetrio C. Zachariadis 
Este exemplar foi revisado e corrigido em relação à versão original, sob responsabilidade única do autor e com a anuência de seu orientador.

São Paulo, de de

Assinatura do autor:

Assinatura do orientador:

Profito, Francisco José

On the Development of Advanced Techniques for Mixed

Elastohydrodynamic Lubrication Modelling of Journal and Sliding Bearing Systems / F. J. Profito -- versão corr. -- São Paulo, 2015.

$236 \mathrm{p}$.

Tese (Doutorado) - Escola Politécnica da Universidade de São Paulo. Departamento de Engenharia Mecânica.

1.Lubrificação 2.Modelagem determinística do regime misto de lubrificação 3.Métodos particionados para interação fluido-estrutura 4.Método dos volumes finitos 5.Mancais de deslizamento I.Universidade de São Paulo. Escola Politécnica. Departamento de Engenharia Mecânica Il.t. 
To my family, and especially to my beloved wife Carla. 


\section{Acknowledgements}

Firstly, I would like to express my sincere gratitude to my supervisor Prof. Dr. Demetrio C. Zachariadis for the continuous support to my Ph.D. research, for his patience, motivation, immense encouragement, and most important, his friendship practically all along my study period at Escola Politécnica of the University of São Paulo (USP), starting early at the time of my undergraduate studies and maintained in the course of the MSc. and Ph.D. research projects. His mentorship helped me all the time of my research and of writing this thesis.

Besides my supervisor, I would like to thank the rest of my thesis committee: Dr. Daniele Dini (Imperial College London), Prof. Dr. Gustavo Buscaglia (ICMC-USP), Prof. Dr. Roberto Martins (POLI-USP), and Prof. Dr. Rodrigo Nicoletti (EESC-USP), for their insightful comments and encouragement, but also for the hard question which stimulated me to widen my research from various perspectives.

Special thanks go to Dr. Daniele Dini for hosting me in the Tribology Group at the Imperial College London throughout 2014. His intelligent suggestions, intensity, continuous enthusiasm and motivation made my research time in the laboratory even more fruitful and considerably helped the development of this thesis. I would like to 'salute' my then fellow labmates Carmine Putignano, Konstantinos Pagkalis (Kostas), Damon Lee, Alessio Natoli, Stefano Galvan, Alexis Wang, Nigel Marx, Debashis Puhan, Yuta Kanazawa, Francesco Erimani, Joanna Dawczyk, Artemis Kontou, and Nicola De Laurentis, for the stimulating (lunch) discussions and for all the enjoyable time we have had, which certainly contributed to making my time in the UK much more memorable.

My sincere thanks also go to Prof. Dr. Amilton Sinatora, who provided me with an opportunity to join his research group in the Laboratory of Surface Phenomena (LFS) at USP, and who gave access to the laboratory and research facilities. Without this precious support, it would not be possible to conduct this research. I am especially grateful to the friendly and cheerful group members who made the daily work even more enjoyable: Prof. Dr. Deniol Tanaka,

Prof. Dr. Izabel Machado, Prof. Dr. Rodrigo Stoeterau, Tiago Cousseau, Luiz Alberto Franco, Eduardo Tomanik (MAHLE Metal Leve S.A.), Eleir Bortoleto, Vanessa Seriacopi, Sebastián Ruiz, Gustavo Siebert, Rafael Obara, Marcos Zara, and Newton Fukumasu.

Furthermore, I would like to gratefully acknowledge the partial support of the ThyssenKrupp Metalúrgica Campo Limpo during the first period of the present research, as well as the São Paulo Research Foundation (FAPESP) via the R\&D Consortium 'TriboFlex' - PIT Project 2009/54891-8 and the Coordenation for the Improvement of Higher Education Personnel (CAPES) - Scholarship Proc. BEX 2025/13-2, both responsible for sponsoring the second phase of this Ph.D. research.

Finally, and most importantly, I would like to thank my parents Valdemar and Nadir Profito and my brother Flávio for supporting me spiritually and with unending encouragement throughout my life in general. Particularly, I would like to devote special thanks to my wife Carla. Her patient support, encouragement and unwavering love were undoubtedly determinant for the development of this thesis and upon which the past five years of my life were built. 


\section{Abstract}

The present thesis is focused on the development of a thorough mathematical modelling and computational solution framework aimed at the numerical simulation of journal and sliding bearing systems operating under a wide range of lubrication regimes (mixed, elastohydrodynamic and full film lubrication regimes) and working conditions (static, quasi-static and transient conditions). The fluid flow effects have been considered in terms of the Isothermal Generalized Equation of the Mechanics of the Viscous Thin Films (Reynolds equation), along with the massconserving $p-\theta$ Elrod-Adams cavitation model that accordingly ensures the so-called JFO complementary boundary conditions for fluid film rupture. The variation of the lubricant rheological properties due to the viscous-pressure (Barus and Roelands equations), viscous-shear-thinning (Eyring and Carreau-Yasuda equations) and density-pressure (Dowson-Higginson equation) relationships have also been taken into account in the overall modelling. Generic models have been derived for the aforementioned bearing components in order to enable their applications in general multibody dynamic systems (MDS), and by including the effects of angular misalignments, superficial geometric defects (form/waviness deviations, EHL deformations, etc.) and axial motion. The bearing flexibility (conformal EHL) has been incorporated by means of FEM model reduction (or condensation) techniques. The macroscopic influence of the mixedlubrication phenomena have been included into the modelling by the stochastic Patir and Cheng average flow model and the Greenwood-Williamson/Greenwood-Tripp formulations for rough contacts. Furthermore, a deterministic mixed-lubrication model with inter-asperity cavitation has also been proposed for full-scale simulations in the microscopic (roughness) level. According to the extensive mathematical modelling background established, three significant contributions have been accomplished. Firstly, a general numerical solution for the Reynolds lubrication equation with the mass-conserving $p-\theta$ cavitation model has been developed based on the hybridtype Element-Based Finite Volume Method (EbFVM). This new solution scheme allows solving lubrication problems with complex geometries to be discretized by unstructured grids. The numerical method was validated in agreement with several example cases from the literature, and further used in numerical experiments to explore its flexibility in coping with irregular meshes for reducing the number of nodes required in the solution of textured sliding bearings. Secondly, novel robust partitioned techniques, namely: Fixed Point Gauss-Seidel Method (PGMF), Point Gauss-Seidel Method with Aitken Acceleration (PGMA) and Interface Quasi-Newton Method with Inverse Jacobian from Least-Squares approximation (IQN-ILS), commonly adopted for solving fluid-structure interaction problems have been introduced in the context of tribological simulations, particularly for the coupled calculation of dynamic conformal EHL contacts. The performance of such partitioned methods was evaluated according to simulations of dynamically loaded connecting-rod big-end bearings of both heavy-duty and high-speed engines. Finally, the proposed deterministic mixed-lubrication modelling was applied to investigate the influence of the cylinder liner wear after a $100 \mathrm{~h}$ dynamometer engine test on the hydrodynamic pressure generation and friction of Twin-Land Oil Control Rings.

Keywords: Lubrication, Mass-conserving cavitation model, Conformal EHL, Deterministic mixed-lubrication modelling, Partitioned methods for fluid-structure interaction, Finite volume method, Journal bearings, Sliding bearings. 


\section{Resumo}

O presente trabalho é focado no desenvolvimento de uma modelagem matemática abrangente e um conjunto de ferramentas computacionais destinadas à simulação numérica de mancais hidrodinâmicos sujeitos a diferentes regimes de lubrificação (misto, elastohidrodinâmico e completamente hidrodinâmico) e condições operacionais (estático, quasi-estático e transiente). O escoamento do fluido lubrificante é descrito matematicamente através da Equação Generalizada da Mecânica dos Filmes Finos Viscosos (equação de Reynolds), juntamente com o modelo de cavitação $p-\theta$ proposto por Elrod-Adams que incorpora automaticamente as condições de contorno complementares de JFO para a completa conservação dos fluxos mássicos na presença de ruptura de filme lubricante (cavitação). As variações das propriedades reológicas do lubrificante devido aos efeitos de piezo-viscosidade (equação de Barus e Roelands), "shear-thinning" (equações de Eyring e Carreau-Yasuda) e piezo-densidade (equação de Dowson-Higginson) são também contempladas na modelagem. Modelos genéricos de mancais de deslizamento são propostos de forma a possibilitar suas aplicações em problemas de dinâmica multicorpos, assim como a consideração dos efeitos de desalinhamento angular, defeitos de superfícies (forma/ondulação, deformações EHL, etc.) e movimento axial. A flexibilidade dos mancais em situações envolvendo elastohidrodinâmica é incorporada por meio de técnicas de condensação de sistemas empregadas no MEF. A influência na escala macroscópica dos fenômenos de lubrificação mista é incluída na modelagem através das formulações estatísticas de Patir \& Cheng para a perturbação do escoamento devido à rugosidade das superfícies, e Greenwood-Williamson/Greenwood-Tripp para o contato rugoso. Adicionalmente, um modelo determinístico é também proposto no trabalho de forma a possibilitar simulações do regime misto de lubrificação diretamente na escala (microscópica) de rugosidade. A partir da extensa modelagem matemática desenvolvida, três principais contribuições são propostas. Inicialmente, uma formulação genérica para a solução da equação de Reynolds com o modelo de cavitação $p-\theta$ foi desenvolvida de acordo com o Método dos Volumes Finitos Baseado em Elementos (MVFbE). Este novo método permite a solução de problemas de lubrificação com geometrias complexas e discretizados por malhas nãoestruturadas. O método foi validado a partir da comparação com diversos exemplos da literatura, e posteriormente utilizado para a simulação de mancais texturizados. A segunda contribuição da pesquisa diz respeito à proposição de técnicas mais robustas e estáveis para a solução particionada do problema fluido-estrutura estabelecido em contatos EHL conformes. As técnicas de acoplamento apresentadas são: Método de Gauss-Seidel com Ponto Fixo (PGMF), Método de Gauss-Seidel com Aceleração de Aitken (PGMA) e Método de Quasi-Newton de Interface (IQN-ILS). O desempenho de tais métodos foi avaliado por meio de simulações dinâmicas de dois mancais de biela, o primeiro utilizado em motores de combustão interna (MCI) de alta velocidade (high-speed engine), e o segundo em motores sujeitos a elevado carregamento (heavy-duty diesel engine). Finalmente, a modelagem determinística proposta para o regime misto de lubrificação foi aplicada na investigação da influência do desgaste superficial de cilíndros de motores de combustão interna, obtidos após $100 \mathrm{~h}$ de teste em dinamômetro, na geração de sustentação hidrodinâmica e atrito em anéis de controle de óleo (TLOCRs).

Palavras-chave: Lubrificação, Modelo de cavitação com conservação de massa, Contato EHL conforme, Modelagem determinística do regime misto de lubrificação, Métodos particionados para interação fluido-estrutura, Método dos volumes finitos, Mancais de deslizamento. 


\section{Contents}

List of Figures $\quad$ ix

List of Tables $\quad$ xiii

Abbreviations $\quad$ Xv

Nomenclature $\quad$ xvi

Publications $\quad$ xxii

1 Introduction 1

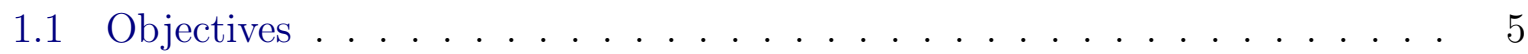

1.2 Original Contributions . . . . . . . . . . . . . . 5

1.3 Thesis Outline . . . . . . . . . . . . . . . . . 6

$\begin{array}{lll}\text { I } & \text { Mathematical Modelling } & 7\end{array}$

2 Hydrodynamic Lubrication (HL) Modelling $\quad 8$

2.1 Lubrication Theory (Reynolds Equation) . . . . . . . . . . . . . . . 8

2.2 Fluid Film Cavitation . . . . . . . . . . . . . . . . . . . . . . 12

2.2.1 Types of Fluid Film Cavitation . . . . . . . . . . . . . . . . . . . 12

2.2.2 Mathematical Modelling for Cavitation . . . . . . . . . . . . 13

2.2.3 Modified Reynolds Equation for Cavitation $(p-\theta$ Model $)$. . . . . 17

2.2.4 Modified Lubricant Flows and Shear Stresses $(p-\theta$ Model $)$. . . . 18

2.3 Lubricant Rheology . . . . . . . . . . . . . . . . . . . . . . . . 18

2.3.1 Viscosity-Pressure Correction . . . . . . . . . . . . . . . . . 19

2.3.2 Viscosity-Shear-Thinning Correction . . . . . . . . . . . . . . 19

2.3.3 Density-Pressure Correction . . . . . . . . . . . . . . . . 20

2.4 Journal Bearing Model . . . . . . . . . . . . . . . . . . . . . . . 20

2.4.1 Geometry of the Lubricant Film Thickness . . . . . . . . . . . . . . 21

2.4.2 Kinematic of the Contact Surfaces . . . . . . . . . . . . . . . 23

2.4 Reynolds Equation . . . . . . . . . . . . . . . . . . . 24

2.4.4 Lubricant Flows and Shear Stresses . . . . . . . . . . . . . . . 26

2.4.5 Operational Parameters . . . . . . . . . . . . . . 26

2.5 Sliding Bearing Model . . . . . . . . . . . . . . . . . . 26

2.5.1 Geometry of the Lubricant Film Thickness . . . . . . . . . . . . 26

2.5.2 Kinematic of the Contact Surfaces . . . . . . . . . . . . . . 27

2.5.3 Reynolds Equation . . . . . . . . . . . . . . . . . . . . 28 
2.5.4 Lubricant Flows and Shear Stresses . . . . . . . . . . . . . . . . . . 29

2.5.5 Operational Parameters . . . . . . . . . . . . . . . 30

3 Conformal Elastohydrodynamic Lubrication (EHL) Modelling 31

3.1 Types of EHL Regimes . . . . . . . . . . . . . . . . . . . . 31

3.2 Conformal EHL Model . . . . . . . . . . . . . . . . . . . . . . 32

3.2 .1 Full Structural Model . . . . . . . . . . . . . . . . . . . . . 32

3.2 .2 Reduced Structural Model . . . . . . . . . . . . . . . . . . . 33

3.3 Solution of the Reduced Structural FEM Model . . . . . . . . . . . . . . . 34

4 Mixed-Lubrication (ML) Modelling 36

4.1 Stochastic Mixed-Lubrication Modelling . . . . . . . . . . . . . . 36

4.1.1 Stochastic Asperity Contact Modelling . . . . . . . . . . . . . . . 36

4.1.2 Stochastic Hydrodynamic Modelling . . . . . . . . . . . . . . . . . 43

4.2 Deterministic Mixed-Lubrication Modelling . . . . . . . . . . . . . . . . 47

4.2.1 Deterministic Asperity Contact Model . . . . . . . . . . . . . . . . 48

4.2.2 Deterministic Hydrodynamic Model . . . . . . . . . . . . . . . . . . 49

5 Fully Coupled Mixed-Elastohydrodynamic Lubrication Modelling 50

5.1 Rigid Body Motion of the Bearing System . . . . . . . . . . . . . . 50

5.2 Fully Coupled System of Nonlinear Equations . . . . . . . . . . . . . . 51

\section{Numerical Solutions of the Mathematical Modelling 53}

6 Time Discretization of the Solver Operators $\quad 54$

6.1 Fourth-Order Accuracy BDF Scheme . . . . . . . . . . . . . . . . 54

6.2 Time Discretization of the Instantaneous Equilibrium Solver . . . . . . . . 55

6.2.1 Rigid Body Dynamics Solver . . . . . . . . . . . . . . 55

6.2 .2 Fluid Solver . . . . . . . . . . . . . . . . 56

6.2.3 Asperity Contact Solver . . . . . . . . . . . . . . 56

6.3 Time Discretization of the Rheological Solver . . . . . . . . . . . . 56

6.4 Time Discretization of the Structural Solver . . . . . . . . . . . . . . 57

7 General Finite Volume Method Solution of Reynolds Equation $\quad 58$

7.1 Fundamentals of the Element-Based Finite Volume Method (EbFVM) . . . 59

7.2 Temporal Discretization . . . . . . . . . . . . . . . . 61

7.3 Spatial Discretization . . . . . . . . . . . . . . . . . 61

7.3.1 Diffusivity Matrix (Poiseuille Term) . . . . . . . . . . . . 63

7.3.2 Convective Matrix (Couette Term) . . . . . . . . . . . . . . 63

7.3.3 Source Term Vectors . . . . . . . . . . . . . . . . . . . 63

7.4 Assembly of the Global Linear System of Equations . . . . . . . . . . . . . 64

7.5 Solution of the Linear System of Equations . . . . . . . . . . . . . . . 64

8 Discretization of the GW/GT Asperity Contact Models 66

9 Fluid-Structure Interaction (FSI) Solution of the Fully Coupled Mixed$\begin{array}{ll}\text { Elastohydrodynamic Lubrication Modelling } & \mathbf{6 7}\end{array}$

9.1 Solver Operators . . . . . . . . . . . . . . . . . . . . . . . 69

9.2 Interpolations on the Fluid-Structure Interface . . . . . . . . . . . . . . 69 
9.3 Fixed Point Gauss-Seidel Method (PGMF) . . . . . . . . . . . . . . . 70

9.4 Point Gauss-Seidel Method with Aitken Acceleration (PGMA) . . . . . . . 71

9.5 Interface Quasi-Newton Method (IQN-ILS) . . . . . . . . . . . . . . . 71

9.6 Algorithms for the EHL Solution of Dynamically Loaded Bearings . . . . . 75

\section{Application Cases}

10 Case I: Dynamically Loaded Connecting-Rod Big-End Bearing of a Heavy$\begin{array}{lr}\text { Duty Diesel Engine } & 78\end{array}$

10.1 Input Data . . . . . . . . . . . . . . . . . . . . . . . 78

10.2 Static Loading Conditions . . . . . . . . . . . . . . . . . . 81

10.2.1 Results for the High Load Condition . . . . . . . . . . . . . . 81

10.2.2 Results for the High Deformation Condition . . . . . . . . . . . . . 86

10.3 Dynamic Loading Conditions . . . . . . . . . . . . . . . . . 86

10.4 Performance of the Partitioned Algorithms . . . . . . . . . . . . . . . . 91

11 Case II: Dynamically Loaded Connecting-Rod Big-End Bearing of a $\begin{array}{lr}\text { High-Speed Engine } & 99\end{array}$

11.1 Input Data . . . . . . . . . . . . . . . . . . . . 100

11.2 Simulation results for the lubricant 'Oilo' . . . . . . . . . . . . . . . 103

11.3 Simulation results for the shear-thinning oils . . . . . . . . . . . . . . 113

11.4 Performance of the Partitioned Algorithms . . . . . . . . . . . . . . . . . . 114

12 Case III: Effect of Cylinder Liner Wear on the Mixed Lubrication Re$\begin{array}{lr}\text { gime of TLOCRs } & \mathbf{1 1 5}\end{array}$

12.1 Characterization of the Cylinder Liner Topographies . . . . . . . . . 116

12.2 Deterministic Simulations . . . . . . . . . . . . . . . 117

12.3 Global (Average) Results . . . . . . . . . . . . . . . . . . . . 118

12.4 Local Results . . . . . . . . . . . . . . . . . . . . . . . . . . 119

13 Case IV: Textured Sliding Bearing Simulation 124

14 Conclusions $\quad 129$

14.1 Future Works . . . . . . . . . . . . . . . . . . . 131

A Journal Bearing Model 133

A.1 Reference Frames and Coordinate Systems . . . . . . . . . . . . . 133

A.2 Transformation Matrices . . . . . . . . . . . . . . 135

A.3 Dimensionless Parameters . . . . . . . . . . . . . . . . . . 138

A.4 Derivation of the Lubricant Film Thickness and Kinematics . . . . . . . . 139

A.5 Operational Parameters . . . . . . . . . . . . . . . . . 143

$\begin{array}{lr}\text { B Sliding Bearing Model } & 147\end{array}$

B.1 Reference Frames and Coordinate Systems . . . . . . . . . . . . . . . . . 147

B.2 Dimensionless Parameters . . . . . . . . . . . . . . . . . . . 148

B.3 Operational Parameters . . . . . . . . . . . . . . . . 150 
C Conformal EHL Model $\quad 153$

C.1 Calculation of the Area Matrix . . . . . . . . . . . . . . . . 153

C.2 Substructure Generation into the Abaqus ${ }^{\circledR}$ Software . . . . . . . . . . . . 154

C.3 Dimensionless Parameters . . . . . . . . . . . . . . . . . 155

D Patir and Cheng's Model $\quad 156$

D.1 Contact and Contact Friction Factors . . . . . . . . . . . . . . . . 156

D.2 Flow Factors . . . . . . . . . . . . . . . . . . . . . . . . . 158

E GW/GT Models $\quad 164$

E.1 Dimensionless Parameters . . . . . . . . . . . . . . . . . . . . . . 164

E.2 Polynomial Fitting of the GW/GT Integrals . . . . . . . . . . . 165

F Numerical Solution of Reynolds Equation $\quad 167$

F.1 Mesh Elements for EbFVM Discretization . . . . . . . . . . . . . . . 167

F.2 Discretization of the Diffusion Term . . . . . . . . . . . . . . . . 172

F.3 Discretization of the Convective Term . . . . . . . . . . . . . . . . 173

F.4 Discretization of the Source Terms _ . . . . . . . . . . . . 176

F.5 Validation Cases . . . . . . . . . . . . . . . . . . . . . 177

G Algorithms $\quad 183$

$\begin{array}{ll}\text { Bibliography } & 191\end{array}$ 


\section{List of Figures}

1.1 Chart summarizing the overall mathematical modelling and solution techniques proposed in the present thesis. . . . . . . . . . . . 4

2.1 Coordinate system and kinematic variables for the General Equation of the Mechanics of Viscous Thin Films. . . . . . . . . . . . . . . . 9

2.2 Schematic illustration of the fluid film cavitation over a generic lubricated domain. . . . . . . . . . . . . . . . . . 14

2.3 Hydrodynamic pressure distribution for different cavitation models. . . . 15

2.4 Experimental photo illustrating the cavitation phenomenon in a piston ring contact of internal combustion engine. . . . . . . . . . . . . . 16

2.5 Main coordinate systems and kinematic variables of the journal bearing model. . . . . . . . . . . . . . . . . . 22

2.6 Illustration of the journal bearing contact interface surrounding the peri-

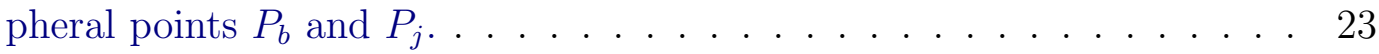

2.7 Main coordinate systems and kinematic variables of the sliding bearing model. . . . . . . . . . . . . . . . . . . . 28

3.1 Illustration of the FEM model reduction for conformal EHL systems. . . 33

4.1 Contact of an individual asperity modelled according to the Hertzian theory. . . . . . . . . . . . . . . . . . . . . . . 38

4.2 Contact between a rigid, smooth plane against a rough surface illustrating the main variables of the GW/GT models. . . . . . . . . . . . . . 39

4.3 Illustration of the misaligned contact between the asperities of two rough surfaces considered in the GT model. . . . . . . . . . . . . . . . . 40

4.4 Definition of the total film thickness for the Patir and Cheng's average flow model. . . . . . . . . . . . . . . . . . . . . . . . . 44

4.5 Schematic illustration of an asperity associated with the deterministic contact model. . . . . . . . . . . . . . . . . . . . . . . . 48

7.1 Geometric entities of the Element-Based Finite Volume Method (EbFVM). 60

10.1 Geometry and load conditions of the heavy-duty connecting-rod system. 79

10.2 Representative surface topography of the heavy-duty big-end bearing shells. . . . . . . . . . . . . . . . . . . 80

10.3 Performance of the partitioned methods for the high load condition

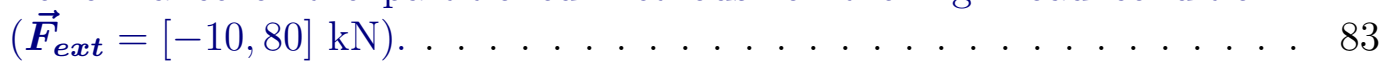

10.4 Hydrodynamic pressure distribution (top) and film fraction field (bottom) for the converged high load condition $\left(\overrightarrow{\boldsymbol{F}}_{\boldsymbol{e x t}}=[-10,80] \mathrm{kN}\right) . \quad . \quad . \quad 84$ 
10.5 Radial bearing deformation for the converged high load condition $\left(\overrightarrow{\boldsymbol{F}}_{\boldsymbol{e x t}}=\right.$

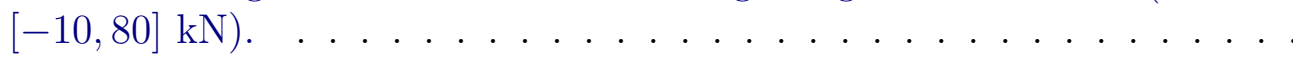

10.6 Performance of the partitioned methods for the high deformation condi-

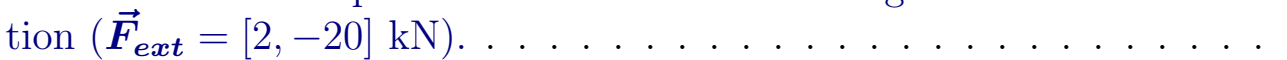

10.7 Hydrodynamic pressure distribution (top) and film fraction field (bot-
tom) for the converged high deformation condition $\left(\overrightarrow{\boldsymbol{F}}_{\boldsymbol{e x t}}=[2,-20] \mathrm{kN}\right)$

10.7 Hydrodynamic pressure distribution (top) and film fraction field (bot-
tom) for the converged high deformation condition $\left(\overrightarrow{\boldsymbol{F}}_{\boldsymbol{e x t}}=[2,-20] \mathrm{kN}\right)$.

10.8 Radial bearing deformation for the converged high deformation condition $\left(\overrightarrow{\boldsymbol{F}}_{\text {ext }}=[2,-20] \mathrm{kN}\right) . \ldots \ldots \ldots \ldots$. . . . . . . . . . . . . . 89

10.9 Scalar results of the dynamically loaded heavy-duty big-end bearing. . . 90

10.10 Field results of the heavy-duty big-end bearing $\left(C A=0^{\circ}\right.$ and $\left.C A=27^{\circ}\right) .93$

10.11 Field results of the heavy-duty big-end bearing $\left(C A=90^{\circ}\right.$ and $\left.C A=180^{\circ}\right) .94$

10.12 Field results of the heavy-duty big-end bearing $\left(C A=240^{\circ}\right.$ and $C A=$ $270^{\circ}$ ).

10.13 Field results of the heavy-duty big-end bearing $\left(C A=300^{\circ}\right.$ and $C A=$ $330^{\circ}$. . . . . . . . . . . . . . . . . . 96

10.14 Field results of the heavy-duty big-end bearing $\left(C A=360^{\circ}\right.$ and $C A=$ $\left.450^{\circ}\right) \ldots \ldots \ldots \ldots \ldots \ldots$

10.15 Field results of the heavy-duty big-end bearing $\left(C A=540^{\circ}\right.$ and $C A=$ $\left.630^{\circ}\right) \ldots \ldots \ldots \ldots \ldots$. . . . . . . . . . . . . . . . . . . . . . .

11.1 Illustration of the high-speed motorcycle engine. . . . . . . . . . . . . 99

11.2 Geometry and load conditions of the high-speed connecting-rod system. 100

11.3 Variation of the dynamic viscosity with shear rate for the different shearthinning oils investigated for friction reduction. . . . . . . . . . . . . 101

11.4 Representative surface topography of the high-speed big-end bearing shells.103

11.5 Scalar results of the dynamically loaded high-speed big-end bearing for the lubricant 'Oil0'.

11.6 Field results of the high-speed big-end bearing for lubricant 'Oil0' ( $C A=$ $0^{\circ}$ and $\left.C A=90^{\circ}\right) \ldots \ldots \ldots \ldots \ldots \ldots$

11.7 Field results of the high-speed big-end bearing for lubricant 'Oil0' $(C A=$ $180^{\circ}$ and $C A=270^{\circ}$ ). . . . . . . . . . . . . . . . 107

11.8 Field results of the high-speed big-end bearing for lubricant 'Oil0' $(C A=$ $330^{\circ}$ and $C A=351^{\circ}$ ).

11.9 Field results of the high-speed big-end bearing for lubricant 'Oil0' $(C A=$ $355^{\circ}$ and $\left.C A=357^{\circ}\right)$. . . . . . . . . . . . . . . . . 109

11.10 Field results of the high-speed big-end bearing for lubricant 'Oil0' $(C A=$ $360^{\circ}$ and $C A=390^{\circ}$ ). . . . . . . . . . . . . . . . . 110

11.11 Field results of the high-speed big-end bearing for lubricant 'Oil0' $(C A=$ $420^{\circ}$ and $C A=450^{\circ}$ ). . . . . . . . . . . . . . . .

11.12 Field results of the high-speed big-end bearing for lubricant 'Oil0' $(C A=$ $540^{\circ}$ and $C A=630^{\circ}$ ). . . . . . . . . . . . . . . . . . . 112

11.13 Scalar results for the dynamically loaded high-speed big-end bearing for the different shear-thinning oils.

12.1 Overall illustration of the TLOCR system. Adapted from [51] . . . . . . 115

12.2 Topography view at $109 \mathrm{~mm}$ from the TDC (approximately in the midstroke position). . . . . . . . . . . . . . . . 116

12.3 Splitting procedure adopted for the generation of the surface slices used in the deterministic simulations. 
12.4 Detailed topography view at mid-stroke, thrust-side (TS) position. . . . 119

12.5 Average results for the topographies measured at the Front position of the cylinder liner.

12.6 Average results for the topographies measured at the $T S$ position of the cylinder liner. . . . . . . . . . . . . . . . . . . . . . 122

12.7 Field results for the surface measured at the TS position (slice 7 and $\Lambda=1.25)$.

13.1 Schematic illustration of the textured sliding bearing and the general dimensions of a dimple cell. . . . . . . . . . . . . . . . . . . . . 124

13.2 Representative texture variants for the sliding bearing simulations and the associated hydrodynamic pressure results. . . . . . . . . . . 125

13.3 Detailed view of the hydrodynamic pressure distributions calculated in the regular and irregular meshes for the same texture variant. . . . . . . 127

A.1 Reference frames of the journal bearing model. . . . . . . . . . . . . 134

A.2 Main coordinate systems of the journal bearing model. . . . . . . . . . 135

A.3 Illustration of the journal bearing contact interface surrounding the points $P_{b}$ and $P_{j} \ldots \ldots \ldots \ldots \ldots$. . . . . . . . . . . . . . . . . . . . . .

B.1 Reference frames of the sliding bearing model. . . . . . . . . . . . . 147

B.2 Main coordinate systems of the sliding bearing model. . . . . . . . . . 148

C.1 Illustration of the element faces of the reduced model. . . . . . . . . . . 154

D.1 Contact factor for a Gaussian roughness height distribution. . . . . . . . 157

D.2 Contact friction factor for a Gaussian roughness height distribution. . . 158

D.3 Typical contact areas for longitudinally orientated, isotropic and transversally orientated surfaces. . . . . . . . . . . . . . . . . 159

D.4 Pressure flow factors for a Gaussian roughness height distribution. . . . 160

D.5 Shear flow factors for a Gaussian roughness height distribution. . . . . . . 161

D.6 Friction pressure flow factors for a Gaussian roughness height distribution.162

D.7 Friction shear flow factors for a Gaussian roughness height distribution. 163

E.1 Cumulative height distribution of a surface roughness profile before and after wear. . . . . . . . . . . . . . . 166

E.2 Curves of the integral functions of the GW/GT models. . . . . . . 166

F.1 Coordinate transformation for linear triangular element. . . . . . . . . 167

F.2 Coordinate transformation for bilinear quadrangular element. . . . . . . 170

F.3 Flow entities and illustration of the three typical flow configurations considered in the FWUS for a quadrangular element. . . . . . . . . . . . 174

F.4 Comparative results for the single parabolic slider case with densitypressure correction. . . . . . . . . . . . . . . . 178

F.5 Comparative results for the double parabolic slider case with densitypressure correction. . . . . . . . . . . . . . . . . . 179

F.6 Comparative results for the infinitely long journal bearing case with pressure-viscosity correction. . . . . . . . . . . . . . 180

F.7 Comparative results for the pure squeeze motion case between circular plates. . . . . . . . . . . . . . . . . . . . . 181 
F.8 Comparative results for the sliding bearing case with viscosity-pressure, viscosity-shear-thinning and density-pressure correction. . . . . . . . . . 182 


\section{List of Tables}

10.1 General data of the heavy-duty connecting-rod part. . . . . . . . . . 78

10.2 Main properties and operational conditions of the heavy-duty big-end bearing. . . . . . . . . . . . . . . . . . . 79

10.3 Rheological properties of the SAE $15 \mathrm{~W} 40$ engine oil at $95^{\circ} \mathrm{C}$ for the heavy-duty big-end bearing simulation. . . . . . . . . . . . . . 79

10.4 Contact and roughness parameters of the heavy-duty big-end bearing shells. . . . . . . . . . . . . . . . . . . 81

10.5 Numerical settings for the simulations of the heavy-duty big-end bearing. 82

10.6 Performance of the partitioned algorithms for the heavy-duty big-end bearing. . . . . . . . . . . . . . . . . . . . 91

11.1 General data of the high-speed connecting-rod part. . . . . . . . . . . 100

11.2 Main properties and operational conditions of the high-speed big-end bearing. . . . . . . . . . . . . . . . . . . . . 101

11.3 Rheological properties of the lubricants for the high-speed big-end bearing simulations. . . . . . . . . . . . . . . . . 102

11.4 Contact and roughness parameters of the high-speed big-end bearing shells. . . . . . . . . . . . . . . . . . . . . 102

11.5 Numerical settings for the static and dynamic simulations of the highspeed big-end bearing. . . . . . . . . . . . . . . . . . . . . . 104

11.6 Performance of the partitioned algorithms for the high-speed big-end bearing. . . . . . . . . . . . . . . . . 114

12.1 Roughness and contact parameters for the MS-Front region. . . . . . . . 117

13.1 Geometric and operational parameters for the textured sliding bearing simulations. . . . . . . . . . . . . . . . . . . . . 125

13.2 Performance of the EbFVM for the regular meshes . . . . . . . . . 126

13.3 Performance of the EbFVM for the irregular meshes . . . . . . . . . 126

D.1 Curve fitting coefficients of the pressure flow factors for a Gaussian roughness height distribution. . . . . . . . . . . . . . . 160

D.2 Curve fitting coefficients of the shear flow factors for a Gaussian roughness height distribution. . . . . . . . . . . . . . . 161

D.3 Curve fitting coefficients of the friction pressure flow factors for a Gaussian roughness height distribution. . . . . . . . . . . . . . . . 162

D.4 Curve fitting coefficients of the friction shear flow factors for a Gaussian roughness height distribution. . . . . . . . . . . . . . . 163

E.1 Coefficients of the polynomial fitting curves of the GW/GT integrals. . . 165 
F.1 General simulation parameters for the single parabolic slider case with density-pressure correction. . . . . . . . . . . . . . . . 178

F.2 General simulation parameters for the double parabolic slider case with density-pressure correction. . . . . . . . . . . . . . . . . 179

F.3 General simulation parameters for the infinitely long journal bearing case with pressure-viscosity correction. . . . . . . . . . . . . . 180

F.4 General simulation parameters for the pure squeeze motion case between circular plates. . . . . . . . . . . . . . . . . . 181

F.5 General simulation parameters for the sliding bearing case with viscositypressure, viscosity-shear-thinning and density-pressure correction. . . . . 182 


\section{Abbreviations}

$\begin{array}{ll}\text { BDF } & \text { Backward Differentiation Formula } \\ \text { CFD } & \text { Computational Fluid Dynamics } \\ \text { CV } & \text { Control Volume } \\ \text { DOFs } & \text { Degrees of Freedom } \\ \text { EbFVM } & \text { Element-Based Finite Volume Method } \\ \text { EHL } & \text { Elastohydrodynamic Lubrication } \\ \text { FDM } & \text { Finite Difference Method } \\ \text { FEM } & \text { Finite Element Method } \\ \text { FSI } & \text { Fluid-Structure Interaction } \\ \text { FVM } & \text { Finite Volume Method } \\ \text { FWUS } & \text { Flow Weighted Upwind Scheme } \\ \text { GW } & \text { Greenwood and Williamson } \\ \text { GT } & \text { Greenwood and Tripp } \\ \text { HDD } & \text { Heavy-Duty Diesel engine } \\ \text { HL } & \text { Hydrodynamic Lubrication } \\ \text { ICE } & \text { Internal Combustion Engine } \\ \text { IQN-ILS } & \text { Interface Quasi-Newton Method with Inverse Jacobian from a Least-Squares } \\ & \text { approximation } \\ \text { JFO } & \text { Jakobsson-Floberg-Olsson Cavitation Model } \\ \text { LCP } & \text { Linear Complementary Problem } \\ \text { MDS } & \text { Multibody Dinamic Systems } \\ \text { ML } & \text { Mixed Lubrication } \\ \text { MOFT } & \text { Minimum Oil Film Thickness } \\ \text { NR } & \text { Newton-Raphson Algorithm } \\ \text { ODE } & \text { Ordinary Differential Equation } \\ \text { PGMA } & \text { Point Gauss-Seidel Method with Aitken Acceleration } \\ \text { PGMF } & \text { Fixed Point Gauss-Seidel Method } \\ \text { PL } & \text { Power Loss } \\ \text { PP } & \text { Peak Pressure } \\ \text { SCV } & \text { Sub-Control Volume } \\ \text { SOR } & \text { Successive Over-Relaxation } \\ \text { TLOCR } & \text { Twin-Land Oil Control Ring } \\ \text { WRM } & \text { Weighted Residual Method } \\ & \end{array}$




\section{Nomenclature}

\section{Latin Symbols}

a Constant shear-thinning coefficient (Carreau-Yasuda equation)

$\vec{a} \quad$ Auxiliary vector of the translation squeeze term $\nabla H_{1}$ in the conservative form of Reynolds equation

$A_{A S P} \quad$ Total real contact area on the combined rough surface $\left[\mathrm{m}^{2}\right]$

$\bar{A}_{A S P} \quad$ Proportion of real contact area on the combined rough surface $[-]$

$A_{0} \quad$ Nominal (or apparent) contact area $\left[\mathrm{m}^{2}\right]$

$A_{i} \quad$ Coefficients of the 4th-order BDF scheme

$A_{r} \quad$ Rigid body angular misalignment of the journal (slider) relative to the bearing around the $\overrightarrow{\boldsymbol{I}}_{\boldsymbol{b}}\left(\overrightarrow{\boldsymbol{i}}_{\boldsymbol{b}}\right)$ axis $[\mathrm{rad}]$

$\dot{A}_{r} \quad$ Rigid body angular misalignment velocity of the journal (slider) relative to the bearing around the $\overrightarrow{\boldsymbol{I}}_{\boldsymbol{b}}\left(\overrightarrow{\boldsymbol{i}}_{\boldsymbol{b}}\right)$ axis $[\mathrm{rad} / \mathrm{s}]$

$\boldsymbol{A} \quad$ Total area matrix of the reduced bearing FEM model $\left[\mathrm{m}^{2}\right]$

$\overrightarrow{\boldsymbol{b}} \quad$ Auxiliary vector of the translation squeeze term $\nabla H_{2}$ in the conservative form of Reynolds equation

$B_{r} \quad$ Rigid body angular misalignment of the journal (slider) relative to the bearing around the $\overrightarrow{\boldsymbol{J}}_{\boldsymbol{b}}\left(\overrightarrow{\boldsymbol{k}}_{\boldsymbol{b}}\right)$ axis [rad]

$\dot{B}_{r} \quad$ Rigid body angular misalignment velocity of the journal (slider) relative to the bearing around the $\overrightarrow{\boldsymbol{J}}_{\boldsymbol{b}}\left(\overrightarrow{\boldsymbol{k}}_{\boldsymbol{b}}\right)$ axis $[\mathrm{rad} / \mathrm{s}]$

$\boldsymbol{B} \quad$ Damping matrix of the full bearing FEM model

$\boldsymbol{B}_{\boldsymbol{r}} \quad$ Damping matrix of the reduced bearing FEM model

$\boldsymbol{B}_{\boldsymbol{r l}} \quad$ Modified damping matrix of the reduced bearing FEM model

$\boldsymbol{B}_{r \boldsymbol{l}}^{\prime} \quad$ Modal damping matrix of the reduced bearing FEM model

$c \quad$ Clearance of the journal bearing system $[\mathrm{m}]$

$\overrightarrow{\boldsymbol{c}} \quad$ Auxiliary vector of the translation squeeze term $\nabla h$ in the conservative form of Reynolds equation

$\overrightarrow{\boldsymbol{c}}_{\boldsymbol{\delta}, \boldsymbol{\mu}, \boldsymbol{\rho}} \quad$ Coefficients of the linear approximations in the IQN-ILS coupling method $\overrightarrow{\boldsymbol{c}}_{e}^{n}$

$C A \quad$ Crank angle [ $\left.{ }^{\circ}\right]$

$C_{1} \quad$ Constant density coefficient (Dowson-Higginson equation), typically $C_{1}=$ $0.59 \mathrm{GPa}$

$C_{2} \quad$ Constant coefficient (Dowson-Higginson equation), typically $C_{2}=1.34$

$C_{b, j(s)} \quad$ Geometric centre of the bearing/journal (slider)

$\boldsymbol{C}_{e}^{n} \quad$ Convective matrix of the grid element $e$

$\overrightarrow{\boldsymbol{d}}_{e}^{n} \quad$ Diffusive fluxes through each SCV of the grid element $e$

$e \quad$ Journal eccentricity [m], or grid element on the hydrodynamic (fluid) mesh

$e_{E H L} \quad$ Relative error of the EHL solution

$E_{i} \quad$ Elastic modulus of the contact surfaces [Pa] 


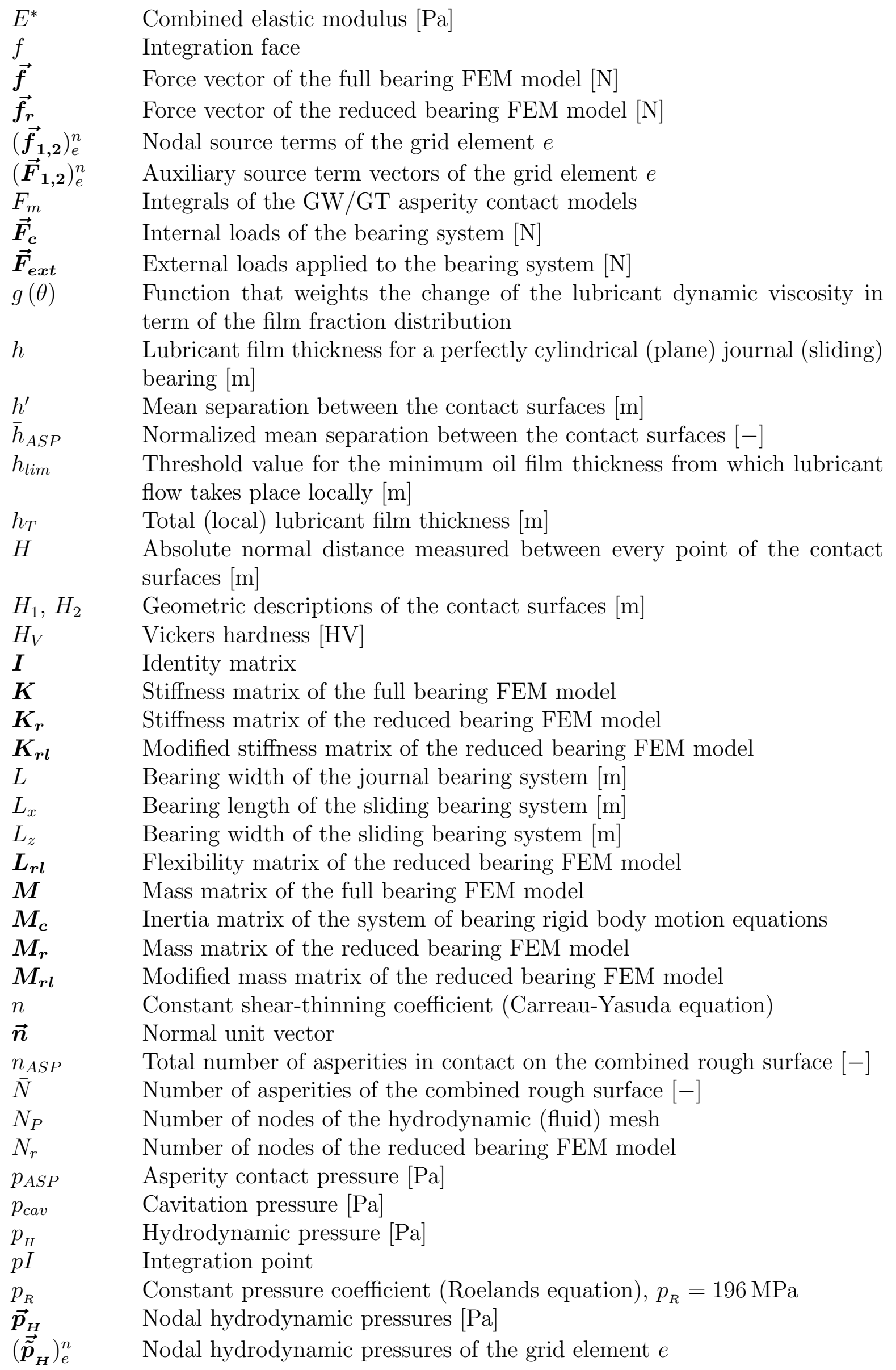


$P \quad$ Grid node on the hydrodynamic (fluid) mesh

$\overrightarrow{\boldsymbol{P}} \quad$ Modal force vector

$\boldsymbol{P}_{e}^{n} \quad$ Diffusivity matrix of the grid element $e$

$q_{H_{x}}, q_{H_{z}} \quad$ Lubricant flow in the $x$ - and $z$-directions $[\mathrm{kg} / \mathrm{s} \mathrm{m}]$

$\ddot{\overrightarrow{\boldsymbol{q}}}, \dot{\overrightarrow{\boldsymbol{q}}}, \overrightarrow{\boldsymbol{q}} \quad$ Acceleration, velocity and displacement vectors of the bearing rigid body motion $\left[\mathrm{m} / \mathrm{s}^{2}, \mathrm{~m} / \mathrm{s}, \mathrm{m}\right]$

$\left[\boldsymbol{Q}_{\boldsymbol{\delta}, \boldsymbol{\rho}, \boldsymbol{\mu}}\right] \quad$ Orthogonal matrices of the IQN-ILS coupling method

$r_{L D}$

$r_{L z x}$

$\overrightarrow{\boldsymbol{r}}_{\delta, \mu, \rho}$

$R_{b, j}$ Width to diameter ratio of the journal bearing system [-]

$\left[\boldsymbol{R}_{\boldsymbol{\delta}, \boldsymbol{\rho}, \boldsymbol{\mu}}\right] \quad$ Upper triangular matrices of the IQN-ILS coupling method

$\vec{s}$

$S_{N S}$

$S_{T}$

Width to length ratio of the sliding bearing system $[-]$

Residual vectors of the EHL coupling methods

Bearing/journal radius $[\mathrm{m}]$

Step of the Newton-Raphson algorithm

Normal squeeze source term in the conservative form of Reynolds equation

$S_{T S} \quad$ Translation squeeze source term in the conservative form of Reynolds equation

$t \quad$ Time $[\mathrm{s}]$

$\overline{\boldsymbol{T}}_{\boldsymbol{g l}} \quad$ (Semi)-dimensionless transformation matrix from global to local bearing coordinate systems

$\ddot{\overrightarrow{\boldsymbol{u}}}, \dot{\overrightarrow{\boldsymbol{u}}}, \overrightarrow{\boldsymbol{u}} \quad$ Acceleration, velocity and displacement vectors of the full bearing FEM model $\left[\mathrm{m} / \mathrm{s}^{2}, \mathrm{~m} / \mathrm{s}, \mathrm{m}\right]$

$\ddot{\overrightarrow{\boldsymbol{u}}}_{\boldsymbol{r}}, \dot{\overrightarrow{\boldsymbol{u}}}_{\boldsymbol{r}}, \overrightarrow{\boldsymbol{u}}_{\boldsymbol{r}} \quad$ Acceleration, velocity and displacement vectors of the reduced bearing FEM model $\left[\mathrm{m} / \mathrm{s}^{2}, \mathrm{~m} / \mathrm{s}, \mathrm{m}\right]$

$U_{1}, U_{2} \quad$ Velocities of the contact surfaces in the $x$-direction $[\mathrm{m} / \mathrm{s}]$

$U_{b, s} \quad$ Sliding velocity of the bearing/slider in the $\overrightarrow{\boldsymbol{i}}_{\boldsymbol{b}}$ direction $[\mathrm{m} / \mathrm{s}]$

$\overrightarrow{\boldsymbol{v}} \quad$ Average velocity vector in the conservative form of Reynolds equation $[\mathrm{m} / \mathrm{s}]$

$\overrightarrow{\boldsymbol{v}}_{\boldsymbol{r}} \quad$ Relative velocity vector in the conservative form of Reynolds equation $[\mathrm{m} / \mathrm{s}]$

$V_{1}, V_{2} \quad$ Velocities of the contact surfaces in the $y$-direction (normal or squeeze direction) $[\mathrm{m} / \mathrm{s}]$

$\left[\boldsymbol{V}_{\boldsymbol{\delta}, \boldsymbol{\rho}, \boldsymbol{\mu}}\right] \quad$ Auxiliary residual matrix of the IQN-ILS coupling method

$w_{s} \quad$ Contact interference $[\mathrm{m}]$

$W_{A S P} \quad$ Total contact load on the combined rough surface $[\mathrm{N}]$

$W_{1}, W_{2} \quad$ Velocities of the contact surfaces in the $z$-direction $[\mathrm{m} / \mathrm{s}]$

$W_{b, s} \quad$ Sliding velocity of the bearing/slider in the $\overrightarrow{\boldsymbol{k}}_{\boldsymbol{b}}$ direction $[\mathrm{m} / \mathrm{s}]$

$\left[\boldsymbol{W}_{\boldsymbol{\delta}, \boldsymbol{\rho}, \boldsymbol{\mu}}\right] \quad$ Auxiliary variable matrix of the IQN-ILS coupling method

$x_{b} \quad$ Bearing coordinate in the $x$-direction $[\mathrm{m}]$

$x_{i} \quad$ Local Cartesian coordinates $\left(i=1,2,3 \rightarrow x_{1}=x, x_{2}=y, x_{3}=z\right)[\mathrm{m}]$

$X_{r} \quad$ Rigid body displacement of the journal relative to the bearing in the $\overrightarrow{\boldsymbol{I}}_{\boldsymbol{b}}$ direction $[\mathrm{m}]$

$Y_{r} \quad$ Rigid body displacement of the journal (slider) relative to the bearing in the $\overrightarrow{\boldsymbol{J}}_{\boldsymbol{b}}\left(\overrightarrow{\boldsymbol{j}}_{\boldsymbol{b}}\right)$ direction $[\mathrm{m}]$

$z_{b} \quad$ Bearing coordinate in the $z$-direction $[\mathrm{m}]$

$Z \quad$ Exponential piezoviscous coefficient of Roelands equation, typically around 0.6 
$Z_{S} \quad$ Mean asperity height of the combined rough surface [m]

$\dot{\bar{Z}}_{C_{b, j}} \quad$ Axial velocity of the bearing/journal $[\mathrm{m} / \mathrm{s}]$

\section{Greek Symbols}

$\alpha \quad$ Pressure-viscosity coefficient of the lubricant $\left[\mathrm{m}^{2} / \mathrm{N}\right]$

$\alpha_{b} \quad$ Angular bearing rotation defined from the $\overrightarrow{\boldsymbol{J}}_{\boldsymbol{b}}$ axis [rad]

$\beta_{s} \quad$ Local asperity radius $[\mathrm{m}]$

$\beta_{S} \quad$ Mean asperity radius of the combined rough surface $[\mathrm{m}]$

$\gamma \quad$ Roughness correlation length [-]

$\dot{\gamma}_{H_{x}}, \dot{\gamma}_{H_{z}} \quad$ Lubricant shear rate in the $x$ - and $z$-directions $\left[\mathrm{s}^{-1}\right]$

$\dot{\gamma}_{e} \quad$ Equivalent shear rate $\left[\mathrm{s}^{-1}\right]$

$\Gamma^{c} \quad$ Convective matrix in the conservative form of Reynolds equation

$\Gamma^{c r} \quad$ Additional convective matrix in the conservative form of Reynolds equation

$\Gamma^{p} \quad$ Diffusivity matrix in the conservative form of Reynolds equation

$\delta \quad$ Combined geometric deviations around the bearing/journal (slider) cylindricities (flatnesses) [m]

$\overrightarrow{\boldsymbol{\delta}} \quad$ Nodal bearing displacements $[\mathrm{m}]$

$\ddot{\overrightarrow{\boldsymbol{\delta}}}, \dot{\overrightarrow{\boldsymbol{\delta}}}, \overrightarrow{\boldsymbol{\delta}} \quad$ Acceleration, velocity and displacement vectors of the reduced bearing FEM model $\left[\mathrm{m} / \mathrm{s}^{2}, \mathrm{~m} / \mathrm{s}, \mathrm{m}\right]$

$\Delta t \quad$ Time step $[\mathrm{s}]$

$\varepsilon \quad$ Journal eccentricity $[-]$

$\varepsilon_{E H L} \quad$ Maximum relative error of the EHL solution

$\varepsilon_{N R} \quad$ Maximum relative error of the Newton-Raphson algorithm

$\eta_{S} \quad$ Asperity density of the combined rough surface $\left[\mathrm{m}^{-2}\right]$

$\ddot{\vec{\eta}}, \dot{\vec{\eta}}, \overrightarrow{\boldsymbol{\eta}} \quad$ Modal acceleration, velocity and displacement vectors of the reduced bearing FEM model $\left[\mathrm{m} / \mathrm{s}^{2}, \mathrm{~m} / \mathrm{s}, \mathrm{m}\right]$

$\theta \quad$ Lubricant film fraction (cavitation) $[-]$

$\theta_{b} \quad$ Angular bearing coordinate defined from the $\overrightarrow{\boldsymbol{J}}_{\boldsymbol{b}_{\boldsymbol{r}}}$ axis [rad]

$\theta_{1}, \theta_{2} \quad$ Control parameters of the 4th-order BDF scheme

$(\overrightarrow{\tilde{\boldsymbol{\theta}}})_{e}^{n} \quad$ Nodal lubricant film fraction of the grid element $e$

$\lambda$

Characteristic relaxation time of the polymer in the Carreau-Yasuda equation $[\mathrm{s}]$

$\lambda^{\prime} \quad$ Step length of the Newton-Raphson algorithm

$\Lambda \quad$ Film thickness ratio, $\Lambda=\frac{h}{\sigma_{R}}[-]$

$\Lambda_{r l}^{*} \quad$ Truncated eigenvalue matrix

$\mu \quad$ Lubricant dynamic viscosity [Pas]

$\mu_{0} \quad$ Reference lubricant dynamic viscosity at given temperature and atmosphere pressure $[\mathrm{Pas}]$

$\mu_{P} \quad$ Lubricant dynamic viscosity at a given temperature, pressure and low shear rate $[\mathrm{Pas}]$

$\mu_{R} \quad$ Constant viscosity coefficient (Roelands equation), $\mu_{R}=6.31 \times 10^{-5} \mathrm{Pas}$

$\mu_{\infty_{P}} \quad$ Lubricant dynamic viscosity at infinite shear rate (Carreau-Yasuda equation) $[\mathrm{Pas}]$

$\mu_{B L} \quad$ Boundary coefficient of friction [-]

$\overrightarrow{\boldsymbol{\mu}} \quad$ Nodal lubricant dynamic viscosity [Pas]

$\nabla \quad$ Gradient operator 


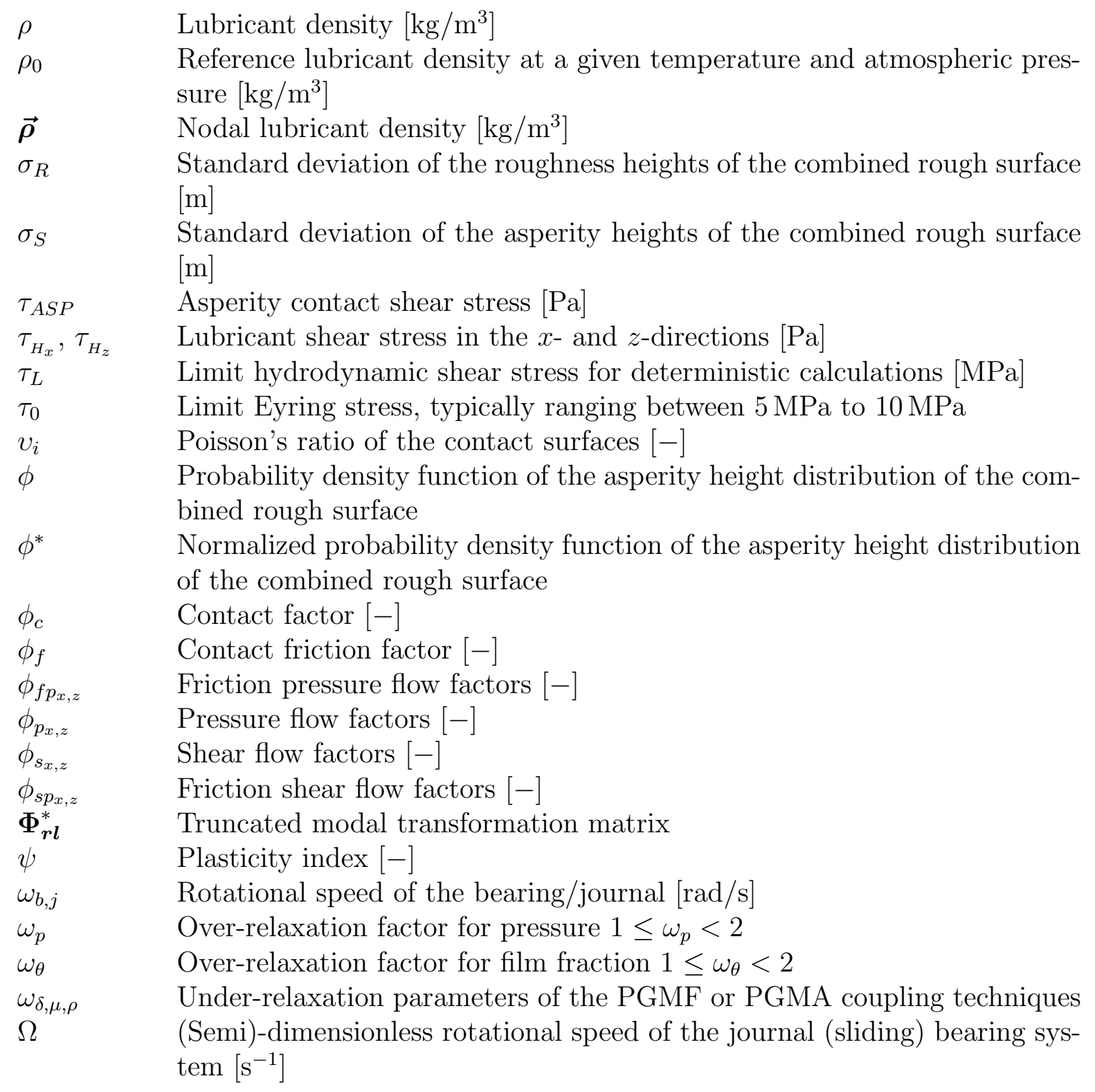

\section{Special Symbols}

e

$e^{-}$

$\mathrm{e}^{+}$

$\mathcal{C}_{\mathcal{H}}$

$\mathcal{D}$

$\mathcal{D}^{\prime}$

$\mathcal{D}^{0}$

$\mathcal{D}^{+}$

$\mathcal{D}_{\mathcal{H}}^{*}$

$\mathcal{D}_{\mathcal{H}}$

$\mathcal{E}$

$\mathcal{E}_{P}$

$\mathcal{F}$

$\mathcal{F}_{P}^{e}$
Solver operator of the asperity contact problem

Reformation cavitation boundary

Rupture cavitation boundary

Boundary that delimits each CV in the EbFVM

Solver operator of the dynamic equations

Jacobian matrix of $\mathcal{D}$

Cavitated regions

Pressured regions

Entire integration domain considered in the EbFVM

Sub-domain that encloses each CV in the EbFVM

Solver operator of the system "instantaneous equilibrium"

Set of grid elements that enclose each node $P$ in the EbFVM

Solver operator of the fluid problem

Set of faces inside each grid element $e$ that encloses node $P$ in the EbFVM 
$\mathcal{L} \quad$ Solver operator of the lubricant rheological corrections

$\mathcal{L}_{\mu} \quad$ Solver operator of the lubricant viscosity correction

$\mathcal{L}_{\rho} \quad$ Solver operator of the lubricant density correction

$\Re_{\boldsymbol{\delta}, \boldsymbol{\mu}, \boldsymbol{\rho}} \quad$ Residual operators of the IQN-ILS coupling method

$\Re_{\delta, \mu, \rho}^{\prime} \quad$ Jacobian matrices of $\Re_{\boldsymbol{\delta}, \boldsymbol{\mu}, \boldsymbol{\rho}}$

$\mathcal{S}$

Solver operator of the structural problem

\section{Subscripts and Superscripts}

$\begin{array}{ll}* & \text { Mean or expected quantities } \\ - & \text { Dimensionless quantities } \\ \cdot & \text { Time derivative } \\ \sim & \text { Intermediate or approximate solutions } \\ a & \text { Axial direction } \\ b & \text { Bearing } \\ j & \text { Journal } \\ k & \text { EHL coupling iteration } \\ l & \text { Local } \\ n & \text { Normal direction or time iteration } \\ N B & \text { Neighbouring nodes } \\ q & \text { Number of reused time steps } \\ r & \text { Relative motion, or iteration of the SOR algorithm } \\ s & \text { Slider } \\ t & \text { Tangential direction } \\ w & \text { Iteration of the Newton-Raphson algorithm }\end{array}$




\section{Publications}

\section{Journal Papers}

[1] PROfito, F.J., GiaCOPini, M., ZAChARIADIS, D.C., DINI, D. A General Finite Volume Method for the Solution of the Reynolds Lubrication Equation with a Mass-Conserving Cavitation Model. Tribology Letters 60, (2015).

[2] PROFITO, F.J., ZACHARIADIS, D.C. Partitioned Fluid-Structure Methods Applied to the Solution of Elastohydrodynamic Conformal Contacts. Tribology International 81, (2015), 321-332.

[3] PROFito, F.J., TOMANIK, E., ZACHARIADIS, D.C. Effect of Cylinder Liner Wear on the Mixed Lubrication Regime of TLOCRs. Tribology International 93, (2015), 723-732.

[4] DIMKOVsKI, Z., TOMANIK, E., PROFITO, F.J. Influence of Measurement and Filtering Type on Friction Predictions Between Cylinder Liner and Oil Control Ring. Tribology International, (2015). In Press (Available Online).

[5] BORTOLETO, E.M., ROVANI, A.C., SERIACOPI, V., PROFITO, F.J., ZACHARIADIS, D.C., MACHADO, I.F., SINATORA, A., SOUZA, R.M. Experimental and Numerical Analysis of Dry Contact in the Pin on Disc Test. Wear 301, (2013), $19-26$.

[6] TOMANIK, E., PROFITO, F.J., ZACHARIADIS, D.C. Modelling the Hydrodynamic Support of Cylinder Bore and Piston Rings with Laser Textured Surfaces. Tribology International 59, (2013), 90-96.

\section{Conference Papers}

[7] PROFITO, F.J., DINI, D., ZACHARIADIS, D.C. Partitioned Fluid-Structure Interaction Techniques Applied to the Conformal EHL Solution of Dynamically Loaded Connecting-Rod Bearings. In: 14th EDF/Pprime Workshop, Futuroscope - Influence of design and materials on journal and thrust bearing performance, October 8-9, 2015, Poitiers, France.

[8] Dimkovski, Z., TOMANIK, E., PROFITO, F.J. Influence of Measurement and Filtering Type on Friction Predictions Between Cylinder Liner and Oil Control Ring. In: 42nd Leeds-Lyon Symposium on Tribology - Surface and Interface Mysteries at Different Scales, 7-9 September 2015, Lyon, France. 
[9] MARX, N., PROFITO, F.J., DINI, D., SPIKES, H.A. Reducing Friction in Journal Bearing Systems: Polymer Shear-Thinning and Bearing Surface Texture. In: International Tribology Conference (ITC2015), 16-20 September 2015, Tokyo University of Science, Tokyo, Japan.

[10] PROFITO, F.J., DINI, D., ZACHARIADIS, D.C. A General Finite Volume Method Solution for the Reynolds Lubrication Equation with Mass-Conserving Cavitation Model. In: 70th STLE Annual Meeting 83 Exhibition, 17-21 May 2015, Omni Hotel, Dallas, Texas, USA.

[11] PROFITO, F.J., TOMANIK, E., ZACHARIADIS, D.C. Effect of Cylinder Liner Wear on the Mixed Lubrication Regime of TLOCRs. In: 41st Leeds-Lyon Symposium on Tribology - Integrated Tribology, 2-5 September 2014, Leeds, UK.

[12] BORTOLETO, E.M., ROVANI, A.C., SERIACOPI, V., PROFITO, F.J., ZACHARIADIS, D.C., MACHADO, I.F., SINATORA, A., SOUZA, R.M. Experimental and Numerical Analysis of Dry Contact in the Pin on Disc Test. In: 19th International Conference on Wear of Materials, 14-18 April 2013, Portland, Oregon, USA.

[13] TOMANIK, E., PROFITO, F.J., ZACHARIADIS, D.C. Modelling the Hydrodynamic Support of Cylinder Bore and Piston Rings with Laser Textured Surfaces. In: 38th Leeds-Lyon Symposium on Tribology - Energy and Health, 6-9 September 2011, Lyon, France.

[14] PROFito, F.J., ZACHARIADIS, D.C., TOMANIK, E., One Dimensional Mixed Lubrication Regime Model for Textured Piston Rings. In: COBEM 2011 - 21st Brazilian Congress of Mechanical Engineering, 2011, Natal, RN, Brazil.

\section{Other Papers}

[15] Profito, F.J., COUSSEAU, T., TOMAnIK, E. Folded Metal Effect on Lubricant Film Thickness and Friction Using a Mixed Lubrication Deterministic Model. SAE Technical Paper (2014), 2014-06-0302.

[16] PROFITO, F.J., TOMANIK, E., LASTRES, L., ZACHARIADIS, D. Effect of Lubricant Viscosity and Friction Modifier on Reciprocating Tests. SAE Technical Paper (2013), 2013-36-0155.

[17] PROFITO, F.J., TOMANIK, E., ZACHARIADIS, D.C. An Improved Surface Characterization of Textured Surfaces on Mixed Lubrication Regimes. SAE Technical Paper (2010), 2010-01-1525. 


\section{Chapter 1}

\section{Introduction}

Recently, the ever increasing market requirements, along with tighter Legislation toward the reduction of gas emissions, have pushed forward the research and development of more energetically efficient mechanical systems for a wide range of engineering applications. In most cases, such improvements have been achieved by increasing the specific power of the components, so that the same amount of energy can be produced more efficiently in smaller, compact systems (downsizing systems). The main consequences of these new design trends are the clearance reduction between the system parts, as well as the raising of the operational kinematic conditions and thermomechanical loads.

From the tribological point of view (friction, lubrication and wear), the combination of lower surface separations and higher load conditions contributes to intensifying the severity of lubricated contacts, leading the system to operate under mixed and/or elastohydrodynamic lubrication regimes. Under these conditions, the solid deformations induced by the fluid film pressures, along with the roughness pattern and distribution of the surface topographies, play an important role in the systems tribological performance. In this context, proper understanding of the physical phenomena taking place on such contact interfaces is extremely important for conceiving novel technologies aimed at reducing friction losses and fuel consumption in mechanical equipment, while keeping reliable control over superficial damage.

Particularly, for the tribosystems typically encountered in internal combustion engines, such as the piston and piston-rings cylinder liner interfaces, the connecting-rod big- and small-end bearings, main crankshaft bearings, the valves/valves seats, the camshaft contacts, etc., the utmost efficiency gains are mainly reached by improvements addressed to the contact surfaces and lubricant rheology. In the first case, modifications on the surface roughness (microscopic scale), material properties and coating technologies are the most common practices, while the engineering of advanced lubricant oils based mainly on friction and viscosity modifiers additives devised to reduce the dissipative forces under different lubrication conditions are the principal characteristics linked to the formulation of new multiviscous lubricants. Another possibility to achieve tribological enhancements deals with design changes in the macroscopic geometry of the contact surfaces (e.g. piston-rings and bearing profiles), or even in the overall system operational conditions (e.g. contact speeds, temperatures, loads, etc.).

The mathematical modelling and numerical simulations have proven to be important tools for the research and development of new components and technologies in the tribology field, especially due to its cheaper and faster approach in comparison with experimental procedures. In fact, parametric or optimization analysis are increasingly un- 
dertaken during the design process to select the most appropriate solutions in advance, thus contributing to reducing the number of laboratory tests. Furthermore, the use of computational simulations often allows identifying, and thus better understanding, specific physical phenomena that are not clearly detected from experiments. The knowledge of such hidden effects is sometimes determinant to provide meaningful technological outcomes.

In this scenario, the present thesis is devoted to developing advanced mathematical modelling and numerical simulation tools to predict the tribological performance of journal and sliding bearing systems operating under mixed-elastohydrodynamic lubrication regimes. Such advanced modelling approach aims to overcome some limitations of most commercial software, as well as to extend the state-of-the-art of some specific topics, including the simulation of lubrication systems with complex geometries to be discretized by irregular grids and the reliable partitioned solution of the fluid-structure interaction problem established in conformal EHL contacts. In addition, deterministic calculations for the mixed-lubrication conditions of Twin-Land Oil Control Rings (TLOCRs) contacts, which take into account the local, microscopic features of cylinder liner topographies of internal combustion engines, are also discussed throughout the work.

The simulation of lubrication problems with complex geometries is particularly important for unconventional applications involving textured surfaces, bearings with general grooves/holes patterns for lubricant feeding, or even in lubricated domains with special geometric features added to improve the overall system performance. The numerical calculation of the Reynolds lubrication equation together with the Jakobsson-Floberg-Olsson (JFO) complementary boundary conditions for cavitation $[87,88,115,160]$ is not straightforwardly accomplished in such domains, often discretized by unstructured meshes; such complementary conditions aim to ensure the whole mass conservation of the lubricant flow, which are in turn accounted for into the solution by means of the $p-\theta$ Elrod-Adams cavitation model $[77,78]$ that defines a modified diffusion-convection Reynolds equation. This solution on irregular grids traditionally uses Finite Element Method (FEM) schemes, which impose difficulties on the discretization process and on satisfying the fluid flow conservation on the moving cavitation boundaries, as well as in providing reliable convergence robustness [30, 32, 35, 82, 100, 128, 158, 162]. More recently, a novel FEM formulation that restates the lubrication modelling as a linear complementary problem (LCP) was proposed by [27, 93], and an efficient algorithm for the fluid pressure calculation was established by [217]. Concomitantly, the Finite Volume Method (FVM) has shown to be very useful for the solution of a wide range of lubrication problems, such as in textured surfaces [15] and piston-rings applications [16], lubrication systems with incompressible bulk flows [12] and discontinuous domains [11, 13, 18], as well as in thermohydrodynamic analysis $[74,75]$. The principal benefit of FVM techniques is their inherent conservative nature, which automatically guarantees the local and global flow conservation directly in the discrete formulation. Hence, the complementary JFO cavitation conditions, and its respective fluid transport conservation throughout the lubricated domain, particularly on the cavitation boundaries, can be more readily treated. The only drawback of usual FVM schemes, at least for lubrication applications, is the lack of standardized methodologies for the discretization on irregular grids. Indeed, some attempts have been made to deal with this issue [11, 13], but not for the combined solution of Reynolds equation with a mass-conserving cavitation model for liquid lubricants. In this context, a hybrid-type FVM formulation, namely Element-Based Finite Volume Method (EbFVM) [107, 108, 181, 189], which combines the geometric flexibility of the FEM, while preserving the whole fluid flow 
conservation aspect of FVM in the discrete domain, is explored in the present contribution for solving the complete isothermal lubrication problem on such unstructured meshes.

The solution of the fluid-structure interaction problem established between the hydrodynamic pressures and solid deformations is crucial for determining the tribological behaviour of EHL contacts. For conformal contacts encountered in journal and sliding bearing systems [36], the FSI coupling is commonly resolved by adopting either the less widespread mode-based approach [127], which computes surface displacements as a linear superposition of selected mode shapes obtained from the elastic modal solution of the bearing structure, or the node-based formulations, in which structural distortions are calculated directly from the bearing flexibility matrix. In the latter case, monolithic (or indirect) methods that solve all the problem equations (fluid flow, structural and load balance equations) simultaneously through a fully coupled Newton-Raphson algorithm based on the ready computation of the Jacobian matrix, are generally employed to accomplish the converged FSI results. In these situations, the Jacobian matrix is pre-defined analytically in terms of a Taylor series expansion of Reynolds equation $[34,90,95,98,122,123,140,147,153,154,157,220]$. Alternatively, the use of partitioned techniques prescribed by means of direct iterative schemes, wherein the fluid and structural problems are solved separately, i.e. there is no a priori explicit coupling between fluid and structure as posed by the Jacobian matrix in monolithic schemes, is rare in journal and sliding EHL bearing applications, especially due to convergence issues related to the large deformations involved [80, 132]. In this sense, in order to fill this gap and to provide a more robust and stable direct coupling method to cope with the solution of dynamically loaded conformal EHL systems, three partitioned techniques, Fixed Point Gauss-Seidel Method (PGMF), Point Gauss-Seidel Method with Aitken Acceleration (PGMA) and Interface Quasi-Newton Method with Inverse Jacobian from Least-Squares approximation (IQN-ILS), utilized in more complex FSI problems [64, 65, 211] will be here introduced in the context of tribological simulations. The essential advantage of partitioned schemes is the opportunity of using dedicate codes to resolve the fluid and solid equations separately as "black box" solvers.

Among other things, deterministic simulations are relevant for allowing evaluating the influence of different surface roughness patterns and any associated localized effects at the microscopic (roughness) scale on the mixed-lubrication regime of mechanical components operating under severe conditions. Particularly for piston-rings cylinder liner contacts, such detailed investigations permit to explore how diverse surface finishes affect the hydrodynamic loading capacity and the friction losses, as well as the intensity of the contact interactions at the asperity level over the engine cycle, which in turn can be used to identify critical situations prone to cause high wear and failure. For this application in particular, [51, 52, 136-138] conceived a full-scale deterministic model for predicting exclusively the fluid flow effects on Twin-Land Oil Control Rings (TLOCRs) interfaces under mixed-lubrication. An extension of such deterministic modelling approach that includes the simultaneous (local) asperity contact calculations and the fluid flow solution at the roughness scale with inter-asperity cavitation, is also addressed herein.

The aforementioned models and solution techniques have been incorporated into a general framework for journal and sliding bearing simulations developed in the course of this research. An overall picture of the mathematical modelling considered in such framework is shown in Fig. 1.1. The contents are displayed according to the tribological phenomena (hydrodynamic and contact mechanics) which are mathematically described at the different surface topography scales (form, waviness and roughness). The methods 
adopted for the model solutions are highlighted as well. The detailed presentation with the associated bibliographic review of the respective formulations and solution techniques are exposed in appropriate sections all over the thesis.

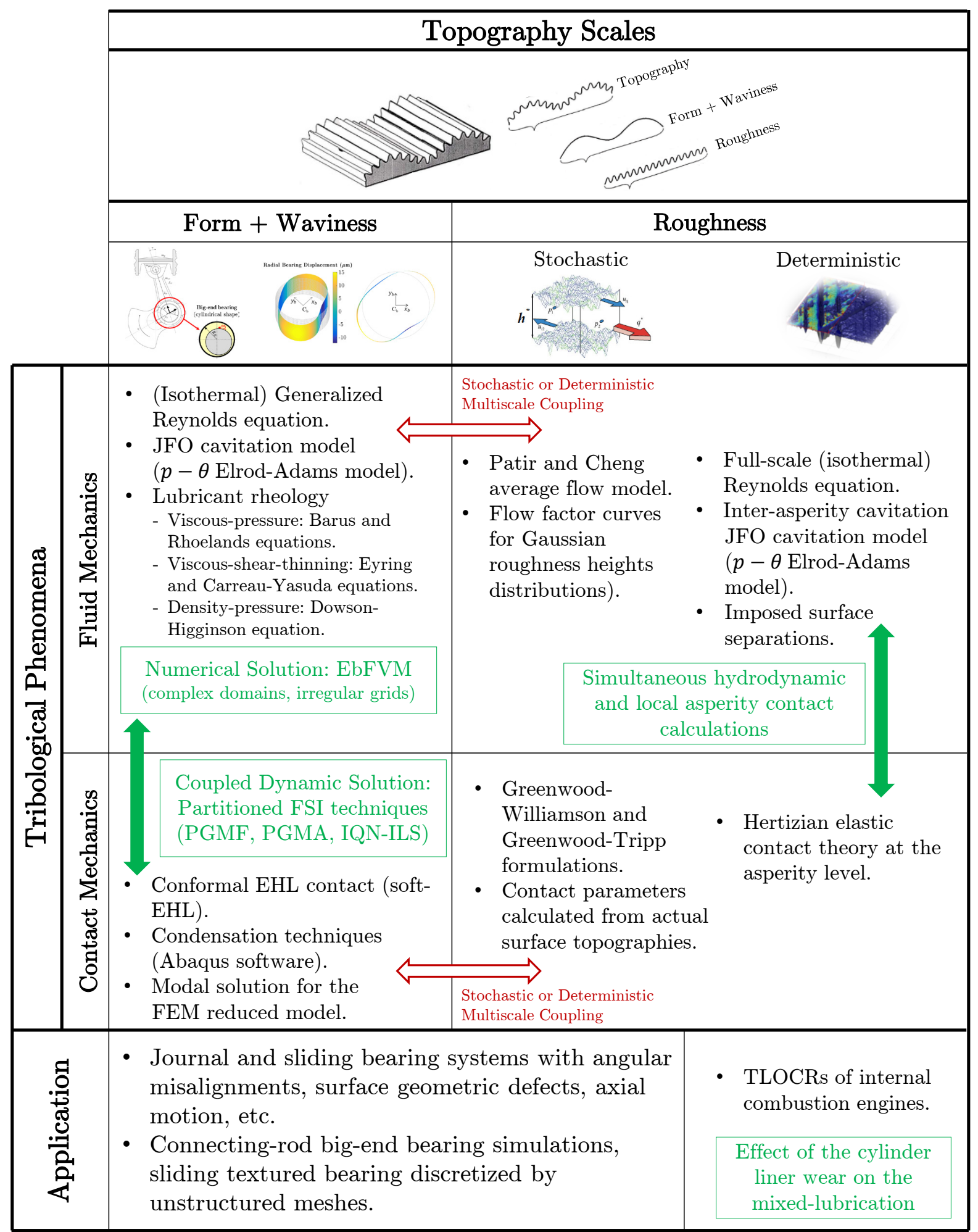

Figure 1.1: Chart summarizing the overall mathematical modelling and solution techniques proposed in the present thesis. The original contributions are highlighted in green. 


\section{$1.1 \quad$ Objectives}

The overall objectives of this thesis may be sorted into the following leading contents:

- Development of a comprehensive mathematical modelling, along with an entire numerical solution framework, aimed to computationally simulate the tribologial performance of bearing systems operating under mixed-elastohydrodynamic lubrication regimes subjected either to static or transient loading conditions. Such computational platform is intended to support general bearing design analysis and optimization processes.

- Numerical solution of the Reynolds lubrication equation with a mass-conserving cavitation model for unstructured (irregular) meshes. Such generality in terms of grid discretization allows the proper solution of lubrication problems in contacts characterized by complex geometries, e.g. generic-shaped textures, non-aligned grooves, etc.

- Partitioned solution of the transient EHL problem for conformal contacts, e.g. journal and sliding bearing systems, in which both the hydrodynamic (including the corrections of the lubricant rheological properties) and structural problems are solved separately as "black-box" solvers. The improvement of this solution approach in the context of tribological simulations is inspired by coupling techniques equally used in a wide range of fluid-structure interaction (FSI) applications.

\subsection{Original Contributions}

The main original contributions accomplished in the research, as already highlighted in Fig. 1.1, are outlined in the following topics:

- Development of a general Finite Volume Method (EbFVM) solution for the Reynolds lubrication equation with the mass-conserving $p-\theta$ Elrod-Adams cavitation model. This newly proposed method allows the complete static or transient solutions of the lubrication problem in applications involving complex geometries to be discretized by irregular meshes (Papers [1], [10]).

- Analysis of novel robust partitioned techniques, namely: Fixed Point Gauss-Seidel Method (PGMF), Point Gauss-Seidel Method with Aitken Acceleration (PGMA) and Interface Quasi-Newton Method with Inverse Jacobian from a Least-Squares approximation (IQN-ILS), for solving the full transient conformal EHL contact problem. Both the PGMA and IQN-ILS methods have proven to be the most effective and robust for simulating connecting-rod big-end bearings of internal combustion engines. Particularly, the IQN-ILS method presented the best performance in terms of computational efficiency for steady state conditions, while PGMA was the most appropriate under dynamic loading situations. (Papers [2], [7]).

- Application of the conceived solution framework to undertake deterministic simulations aimed to investigate the effect of cylinder liner wear on the mixed-lubrication regime of Twin-Land Oil Control Rings (TLOCRs) (Papers [4], [8], [3], [11], [15]). 


\subsection{Thesis Outline}

The present thesis is divided into three major parts. In Part I, all the mathematical models and formulations adopted throughout the work to describe the physical mechanisms ruling the different lubrication conditions that take place in sliding and journal bearing systems are exposed in detail. The presentation is separated according to the associated lubrication regimes considered in the modelling, namely: hydrodynamic lubrication (HL), conformal elastohydrodynamic lubrication (EHL) and mixed-lubrication (ML) regimes. The last section of Part I is particularly devoted to deriving and assembling the whole system of nonlinear equations that governs the full transient mixed-elastohydrodynamic lubrication problem for the aforementioned bearing systems.

Part II is dedicated to delineating all the numerical methods and techniques employed to solve the analytical modelling theories conceived in Part I. It corresponds to the core of the thesis wherein the main contributions of the work are presented with special attention. Initially, the numerical scheme used for the time discretization of the nonlinear equations is presented, along with the extrapolation expressions that provide good initial estimations for the solutions in next time steps. Later, a newly proposed hybrid-type finite volume method solution of the Reynolds equation with a mass-conserving cavitation model is developed for the general calculation of the hydrodynamic problem in complex geometries, followed by a brief section that outlines the discretization of the (stochastic) asperity contact models. Subsequently, Part II ends with a careful description of the different partitioned techniques devised for the complete solution of the conformal EHL problem under transient loading conditions.

Finally, Part III illustrates different case studies involving the simulation of actual lubrication systems that consider, to some extent, the mathematical models and numerical techniques covered in the thesis. The main intention of this part is to emphasize the effectiveness, efficiency and robustness of the entire solution framework developed, as well as the usefulness of the novel formulations here proposed, to predict the tribological behaviour of journal and sliding bearing components subjected to a wide range of operational conditions and lubrication regimes.

The thesis is brought to an end by a conclusion section which summarizes the final considerations and the suggestions for eventual future works and developments regarding the subjects contemplated in the research. 


\section{Part I}

\section{Mathematical Modelling}




\section{Chapter 2}

\section{Hydrodynamic Lubrication (HL) Modelling}

Hydrodynamic lubrication essentially consists of adding a liquid or gaseous lubricant fluid to the contact interface composed of two loaded surfaces moving relative to each other. The enforcement of the mass-conservation principle of the lubricant flow induced by both the relative motion and the local variations of the surfaces geometry yields the generation of a hydrodynamic pressure field that equilibrates the external loads acting on the surfaces. Such pressure generation also contributes to the building-up of a thin fluid film, which in turn allows the full separation of the contact surfaces and so the reduction of the frictional losses and interfacial damage [101, 199].

In this chapter, all the concepts regarding the mathematical modelling considered in the present work to describe the fluid flow effects that take place in the full-film hydrodynamic lubrication regime will be presented, beginning with a general overview of the lubrication theory, fluid film cavitation and lubricant rheology. Afterwards, the adjustments of such general concepts to particular applications involving journal bearings and sliding bearings will be explained in detail.

\subsection{Lubrication Theory (Reynolds Equation)}

The first mathematical model conceived specifically to describe the fluid flow effects in lubricated contacts was established by Osborne Reynolds in 1886 [183]. In this cornerstone work, Reynolds settled the fundamental assumptions of the modern lubrication theory, formally represented in terms of a partial differential equation (later on called Reynolds equation in his honour) that allows the calculation of the hydrodynamic pressures developed throughout the lubricated interface.

The lubrication theory originally proposed by Reynolds practically remained the same and with no conceptual change until 1962, when Duncan Dowson [70] published a set of more general equations which extended the lubrication theory by including the thermohydrodynamic effects and the variations of the lubricant properties, e.g. viscosity and density, across the film thickness. Afterwards, Berthe and Godet [26] generalized even further Dowson's model by taking into account the eventual existence of localized defects and/or geometric perturbations on both contact surfaces, thus providing the so-called Generalized Equations of the Mechanics of Viscous Thin Films [92]. The latter approach will be used in the present work as the mathematical basis for the development of the hydrodynamic modelling. 


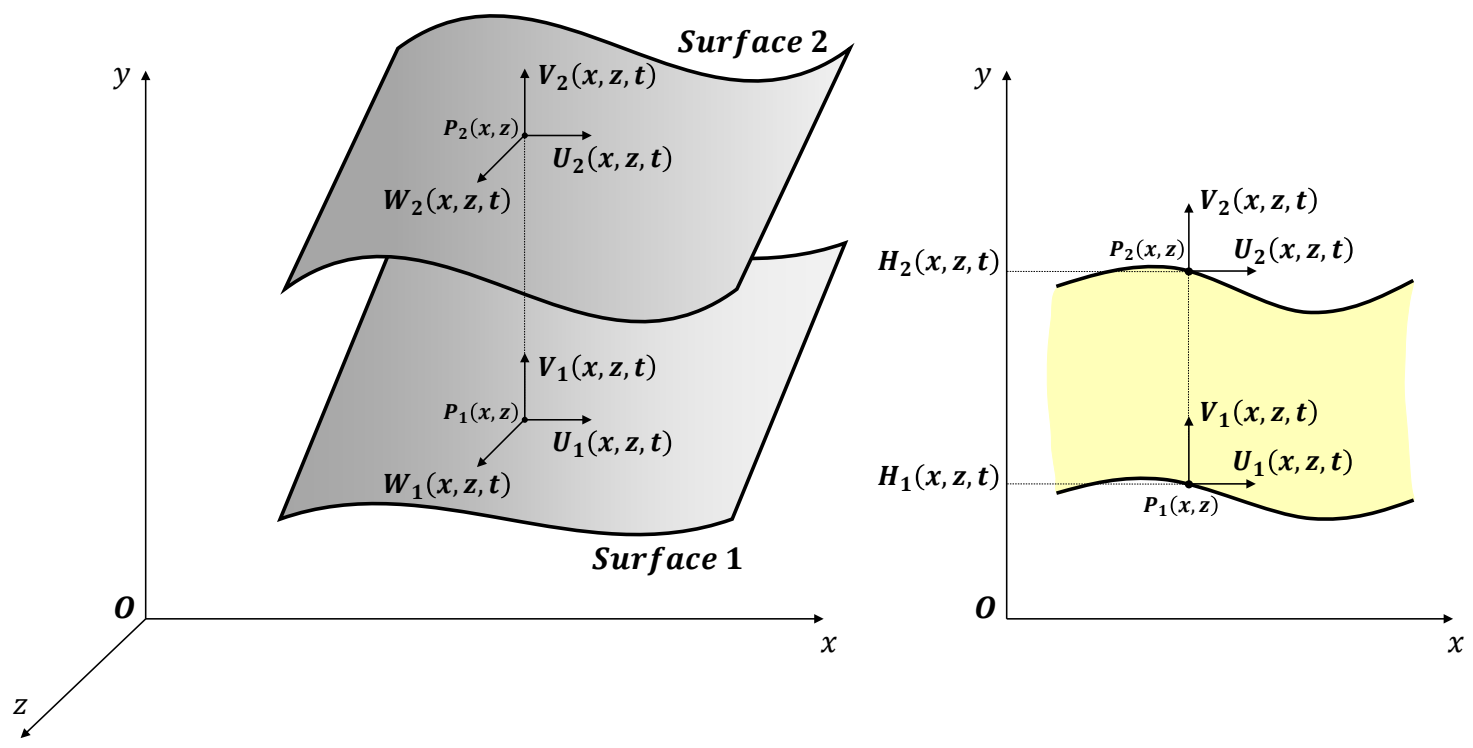

Figure 2.1: Left: General view illustrating the coordinate system and kinematic variables for the Equation of the Mechanics of Viscous Thin Films. Right: Detailed cross section showing the definition of the fluid film geometry.

The Generalized Equations of the Mechanics of Viscous Thin Films are derived from the Navier-Stokes equations by assuming the following fundamental assumptions of the lubrication theory [92]:

1) The fluid is assumed to be a continuum medium.

2) The lubricant fluid is Newtonian.

3) The dimensions across the film thickness $\mathcal{O}\left(10^{-6}\right)$ are much smaller when compared with other dimensions of the contact $\mathcal{O}\left(10^{-3}\right)$. This is the basic assumption of the lubrication theory, which mainly renders pressure gradient across the film thickness negligible.

4) The lubricant flow is assumed to be laminar so that the fluid inertia effects are negligible. This assumption is directly related to the thin film characteristic of lubricated contacts, which yields to flows with very small Reynolds numbers, i.e. the inertia effects are negligible in comparison with viscous behaviour.

5) The external mass/body forces are ignored.

6) The no-slip condition is considered between the fluid and the surfaces walls.

7) The contact surfaces are assumed to be perfectly smooth, i.e. without roughness; the effect of surface roughness on lubrication will be discussed later in Chapter 4 , which is especially devoted to mixed-lubrication modelling.

As the thermal effects have not been admitted in the present work, the lubricant flow will always be assumed isothermal and, as a consequence, any change in the lubricant rheology across the film thickness will be discarded. In such a way, the Isothermal Generalized Equations of the Mechanics of Viscous Thin Films, hereafter denominated simply 
as Reynolds Equation, can be expressed in the Cartesian coordinate system as follows $[92,171]$ :

$$
\begin{aligned}
& \underbrace{\frac{\partial}{\partial x}\left[\frac{\rho\left(H_{2}-H_{1}\right)^{3}}{12 \mu} \frac{\partial p_{H}}{\partial x}\right]+\frac{\partial}{\partial z}\left[\frac{\rho\left(H_{2}-H_{1}\right)^{3}}{12 \mu} \frac{\partial p_{H}}{\partial z}\right]}_{\text {Pressure-Flow (Poiseuille) Term }}= \\
& \underbrace{\frac{\partial}{\partial x}\left[\frac{\rho\left(U_{2}+U_{1}\right)}{2}\left(H_{2}-H_{1}\right)\right]+\frac{\partial}{\partial z}\left[\frac{\rho\left(W_{2}+W_{1}\right)}{2}\left(H_{2}-H_{1}\right)\right]}_{\text {Wedge-Flow (Couette) Term }}+ \\
& \underbrace{\rho\left[\left(U_{1} \frac{\partial H_{1}}{\partial x}-U_{2} \frac{\partial H_{2}}{\partial x}\right)+\left(W_{1} \frac{\partial H_{1}}{\partial z}-W_{2} \frac{\partial H_{2}}{\partial z}\right)\right]}_{\text {Normal-Squeeze Term }}+ \\
& \underbrace{\rho\left(V_{2}-V_{1}\right)}_{\text {Tocal-Expansion Term }}+\underbrace{\frac{\partial \rho}{\partial t}\left(H_{1}\right)}_{\left(H_{2}-H_{1}\right)}
\end{aligned}
$$

where (see Fig. 2.1)

$p_{H}$ is the hydrodynamic pressure $[\mathrm{Pa}]$;

$x_{i}$ are the local Cartesian coordinates $\left(i=1,2,3 \rightarrow x_{1}=x, x_{2}=y, x_{3}=z\right)[\mathrm{m}]$;

$t$ is the time $[\mathrm{s}]$;

$\mu$ is the lubricant dynamic viscosity [Pas];

$\rho$ is the lubricant density $\left[\mathrm{kg} / \mathrm{m}^{3}\right]$;

$\left(H_{1}, H_{2}\right)$ are the geometric descriptions of the contact surfaces [m];

$\left(U_{1}, U_{2}\right)$ are the velocities of the contact surfaces in the $x$-direction $[\mathrm{m} / \mathrm{s}]$;

$\left(W_{1}, W_{2}\right)$ are the velocities of the contact surfaces in the $z$-direction $[\mathrm{m} / \mathrm{s}]$;

$\left(V_{1}, V_{2}\right)$ are the velocities of the contact surfaces in the $y$-direction (normal or squeeze direction) $[\mathrm{m} / \mathrm{s}]$.

Alternatively, Eq. 2.1 is here rewritten in the conservative vector form as:

$$
\nabla \cdot\left(\boldsymbol{\Gamma}^{p} \nabla p_{H}\right)=\nabla \cdot\left(\boldsymbol{\Gamma}^{c} \overrightarrow{\boldsymbol{v}}\right)+\left[S_{T S}+S_{N S}\right]+S_{T} \frac{\partial \rho}{\partial t}
$$

where $\Gamma^{p}$ and $\Gamma^{c}$ are the diffusivity and convective matrices. Moreover, the scalars $S_{T S}$, $S_{N S}$, and $S_{T}$ correspond to the translation squeeze, normal squeeze and temporal expansion terms, respectively. Such quantities are defined as follows:

$$
\Gamma^{p}=\left[\begin{array}{cc}
\frac{\rho\left(H_{2}-H_{1}\right)^{3}}{12 \mu} & 0 \\
0 & \frac{\rho\left(H_{2}-H_{1}\right)^{3}}{12 \mu}
\end{array}\right]
$$




$$
\begin{aligned}
\Gamma^{\boldsymbol{c}} & =\left[\begin{array}{cc}
\rho\left(H_{2}-H_{1}\right) & 0 \\
0 & \rho\left(H_{2}-H_{1}\right)
\end{array}\right] \\
S_{T S} & =\rho\left(\overrightarrow{\boldsymbol{a}} \cdot \nabla H_{1}-\overrightarrow{\boldsymbol{b}} \cdot \nabla H_{2}\right) \\
S_{N S} & =\rho\left(V_{2}-V_{1}\right) \\
S_{T} & =\left(H_{2}-H_{1}\right) \\
\overrightarrow{\boldsymbol{v}} & =\left[\begin{array}{ll}
\frac{\left(U_{2}+U_{1}\right)}{2} & \frac{\left(W_{2}+W_{1}\right)}{2}
\end{array}\right]^{T} \\
\overrightarrow{\boldsymbol{a}} & =\left[\begin{array}{ll}
U_{1} & W_{1}
\end{array}\right]^{T} \\
\overrightarrow{\boldsymbol{b}} & =\left[\begin{array}{ll}
U_{2} & W_{2}
\end{array}\right]^{T}
\end{aligned}
$$

Each term of Eq. 2.1 is related to a different component of the lubricant flow developed through the contact interface. The physical meaning of each term can be interpreted as $[92,101]$ :

Pressure-Flow (Poiseuille) Term: lubricant flow induced by the pressure gradients.

Wedge-Flow (Couette) Term: lubricant flow induced by the relative motion of the contact surfaces (physical-wedge effect) and/or due to local variations in the lubricant density (density-wedge effect) and surfaces (vibrational) velocities (stretch-wedge effect).

Translation-Squeeze Term: lubricant flow induced by the fluid squeezing yielded by the relative translational motion of inclined contact surfaces.

Normal-Squeeze Term: lubricant flow induced by the fluid squeezing yielded by the normal relative motion of the contact surfaces.

Local-Expansion Term: lubricant flow induced by transient effects associated with the lubricant compressibility.

According to Eq. 2.1, it is possible to notice that the generation of hydrodynamic pressure is essentially dependent on the lubricant rheology $(\rho, \mu)$ as well as on the geometric $\left(H_{1}, H_{2}\right)$ and kinematic $\left(U_{1}, W_{1}, U_{2}, W_{2}\right)$ features of the contact surfaces. The latter two dependences are linked to peculiar characteristics of each lubrication system, so that Eq. 2.1 can be seen as general in the sense that it can be adjusted (or customized) for different applications, such as the journal and sliding bearing models later proposed in this thesis (see Sections 2.4 and 2.5, respectively).

Based on the same assumptions admitted for the development of the Generalized Equations of the Mechanics of Viscous Thin Films, expressions for the lubricant flows and shear stresses can be also obtained, which in turn are useful for the calculation of several operational parameters such as the frictional forces and lubricant leakage. Accordingly, the components of the lubricant flows in the $x$ - and $z$-directions can be expressed as $[92,101]$ :

$$
q_{H_{x}}=-\frac{\rho\left(H_{2}-H_{1}\right)^{3}}{12 \mu} \frac{\partial p_{H}}{\partial x}+\rho\left(H_{2}-H_{1}\right)\left(\frac{U_{1}+U_{2}}{2}\right)
$$




$$
q_{H z}=-\frac{\rho\left(H_{2}-H_{1}\right)^{3}}{12 \mu} \frac{\partial p_{H}}{\partial z}+\rho\left(H_{2}-H_{1}\right)\left(\frac{W_{1}+W_{2}}{2}\right)
$$

Similarly, the lubricant shear stresses can be written in terms of the shear rate components as:

$$
\begin{aligned}
& \tau_{H_{x}}=\mu \dot{\gamma}_{H_{x}} \quad \rightarrow \quad \dot{\gamma}_{H_{x}}=\frac{1}{2 \mu} \frac{\partial p_{H}}{\partial x}\left[2 y-\left(H_{2}+H_{1}\right)\right]+\left(\frac{U_{2}-U_{1}}{H_{2}-H_{1}}\right) \\
& \tau_{H_{z}}=\mu \dot{\gamma}_{H_{z}} \quad \rightarrow \quad \dot{\gamma}_{H_{z}}=\frac{1}{2 \mu} \frac{\partial p_{H}}{\partial z}\left[2 y-\left(H_{2}+H_{1}\right)\right]+\left(\frac{W_{2}-W_{1}}{H_{2}-H_{1}}\right)
\end{aligned}
$$

\section{$2.2 \quad$ Fluid Film Cavitation}

The hydrodynamic pressures calculated directly from the equations defined in the previous section are strongly dependent on the geometric and kinematic characteristics, along with the boundary conditions to which the lubrication system is subjected. Such predicted pressures can often reach negative values (relative pressures), which physically means that the fluid is undergoing considerable traction in some regions of the lubricated contact. However, in realistic lubrication environments featured by high shear stresses and viscous dissipation, the fluid capability to sustain high tensile stresses is unlikely. Consequently, when the hydrodynamic pressures fall below the saturation of a particular gas component dissolved in the lubricant (gaseous cavitation), or below the vapour pressure of the fluid at a given temperature (vapour cavitation), the lubricant film is broken and the resulting cavity is filled with a biphasic mixture of liquid and gases/vapours $[38,41,63,72,73]$. This phenomenon, usually denominated in the literature as lubrication cavitation or fluid film rupture, plays an important role in the lubrication system performance, and as such must be taken into account in the mathematical modelling of the problem. In the next sections, a brief description of the different types of cavitation that can occur in lubrication applications will be presented. Afterwards, the main mathematical models conceived to deal with such cavitation effects will be discussed, with greater emphasis on the mass-conserving cavitation model proposed by Jakobson-Floberg-Olsson (JFO) that will be adopted throughout the present work.

\subsubsection{Types of Fluid Film Cavitation}

The following three main types of fluid film cavitation can be identified in lubrication systems [38]:

Gaseous Cavitation: characterized by the presence of one or more species of gases dispersed in the biphasic mixture formed into the cavitated regions. In this case, the biphasic mixture (gas bubble) is developed essentially due to the diffusion of the gases previously dissolved in the liquid lubricant, which in turn were continuously detached from the lubricant as the fluid pressure reached values below the saturation pressure of each gas species. In some situations, gases from the atmospheric environment may also contribute to building-up the cavitation zone (air entrainment inlet suction $[10,89]$ ).

Pseudo-Cavitation: corresponds to a particular form of gaseous cavitation during which the biphasic mixture (gas bubble) expands due to its surrounding depressurization and without further gas mass diffusion from the liquid to the gas phase. In other words, the expansion occurs without the addition of mass within the cavitation zones. 
Vapour Cavitation: is the result of a thermodynamic non-equilibrium generated when film pressures fall below the vapour pressure of the liquid lubricant at a given temperature. This type of cavitation is prone to happen in systems operating under high transient conditions and tends to cause superficial damage on the contact surfaces due to the high frequencies in which the cavitation bubbles grow and collapse.

It is important to highlight at this point that the predominance of a specific type of cavitation depends on several factors such as whether the lubricant is a pure substance or a mixture of many others, or even if the lubrication condition is steady or transient. Those sort of characteristics determine if the gaseous portion of the biphasic mixture on the cavitation zones is composed either of gases/vapour or a mixture of both.

As most lubricants have significant amounts of dissolved gases in their composition at atmospheric pressure, the gaseous cavitation is predominant in lubrication systems operating under steady state or low transient conditions. In these cases, the gas bubbles are formed mainly by the gases detached from the liquid lubricant as the fluid pressures fall below the saturation pressure of the associated dissolved gases, which in turn are still higher than the vapour pressure of the lubricant. On the other hand, as already stated, vapour cavitation only prevails in systems subjected to high transients, where the relatively low rates of gaseous diffusion hinders the growth of gas bubbles.

As for stability, gaseous cavitation tends to be more stable than vapour cavitation, especially because of the inherent thermodynamic nature of the latter.

\subsubsection{Mathematical Modelling for Cavitation}

As stated above, since lubricants are unlikely to stand high tensile stresses, whenever fluid pressures reach values below the saturation pressure of the gases dissolved into the oil (gaseous cavitation), or below the vapour pressure of the fluid (vapour cavitation), the lubricant film is broken down. This fluid film rupture leads to the growth of unpressurised zones (cavitated zones) that are filled with a biphasic mixture of liquid (lubricant) and gases/vapour. According to Fig. 2.2, the cavitation boundaries that separate the pressured regions $\left(\mathcal{D}^{+}\right)$from the cavitated zones $\left(\mathcal{D}^{\mathbf{0}}\right)$ are discontinuities on the lubricated domain. Such boundaries can be of two types: (1) rupture boundary $\left(\mathfrak{C}^{+}\right)$, where cavitation initiates with fluid film rupture; and (2) reformation boundary $\left(\mathfrak{e}^{-}\right)$, where the lubricant film begins to be re-established due to the gradual rise in the hydrodynamic pressures.

As for the mathematical modelling of the cavitation phenomenon in lubrication problems, it is possible to sort three well-established formulations, namely: Gümbel (or halfSommerfeld) model, Swift-Steiber (or Reynolds) model and JFO model, which will be all described in detail in the next sections. The influence of each formulation on the location of the lubricant film rupture can be observed in Fig. 2.3.

\subsubsection{Gümbel's Model (half-Sommerfeld)}

Gümbel [94], in 1914, was the first researcher to address the issue of the lubricant film rupture in the calculation of hydrodynamic pressures. His approach, also denominated half-Sommerfeld assumption, is certainly the simplest formulation to deal with cavitation during the solution of Reynolds equation. The model only admits as valid the pressures whose values are equal to or greater than a given limit cavitation pressure $\left(p_{\text {cav }}\right)$. In this case, depending on the type of cavitation considered, $p_{\text {cav }}$ can correspond to the saturation pressure of the gases dissolved in the lubricant (gaseous cavitation), or the vapour pressure 
Reformation Boundary Rupture Boundary

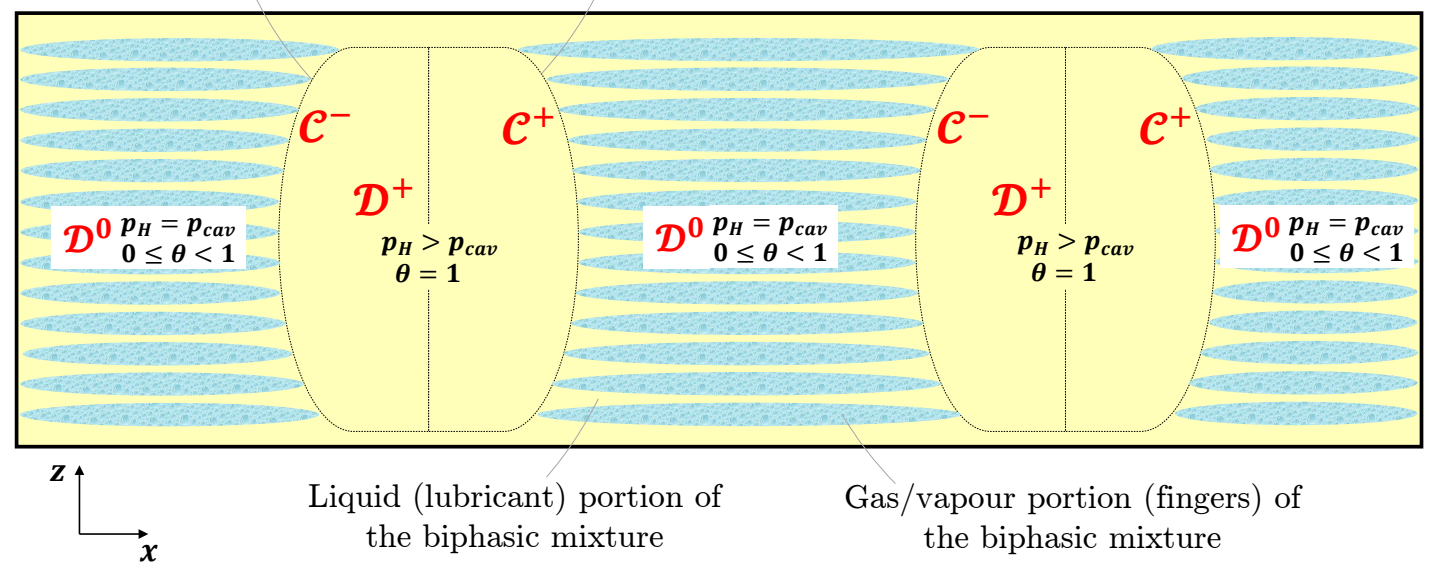

Figure 2.2: Schematic illustration of the fluid film cavitation over a generic lubricated domain. Notice that the pressured regions $\left(\mathcal{D}^{+}\right)$are separated by intermediate cavitation zones $\left(\mathcal{D}^{\mathbf{0}}\right)$ bounded by their respective reformation $\left(\mathrm{e}^{-}\right)$and rupture boundaries $\left(\mathrm{e}^{+}\right)$.

of the liquid lubricant (vapour cavitation). In contrast, all the calculated pressures with values below this threshold quantity are set equal to $p_{\text {cav }}$. The main limitation of the Gümbel's formulation is that the mass-conservation principle of the lubricant flow is not satisfied on the cavitation boundaries, since the cut-off like approach featured by $p_{H}<p_{c a v}$ breaks the original conservative nature of Reynolds equation.

\subsubsection{Swift-Steiber (or Reynolds) Model}

Swift [198] and Steiber [196] independently proposed an improvement in the Gümbel's model. In the new formulation, the authors assumed the pressure gradients as null on the cavitation boundaries, whereas within the cavitated zones the pressures are admitted constant and equal to the limit pressure $p_{\text {cav }}$. Such formulation is also known as Reynolds model for cavitation and can be stated mathematically as:

$$
\begin{cases}p_{H}=p_{c a v} & , \quad \text { within the cavitation regions }\left(\mathcal{D}^{\mathbf{0}}\right) \\ \frac{\partial p_{H}}{\partial \overrightarrow{\boldsymbol{n}}}=0 & , \quad \text { on the cavitation boundaries }\left(\mathbf{C}^{+} \text {and } \mathfrak{C}^{-}\right)\end{cases}
$$

According to [87], for moderate loaded journal bearings under steady-state conditions, the Swift-Steiber's model provides suitable results for design analysis purposes. On the other hand, [40, 41] pointed out that although the Swift-Steiber's conditions predict fairly well the cavitation boundaries for film rupture, the same conditions do not provide good predictions for the reformation boundaries. Similarly, for dynamically loaded bearings, the results were not satisfactory either [40, 41, 73]. The limitations of the Swift-Steiber's formulation are again related to the mass-conservation of the lubricant flow on the cavitation boundaries; particularly, such conservation is only ensured on the rupture boundaries but it is not enforced completely on the reformation ones [180].

\subsubsection{Jakobsson-Floberg-Olsson (JFO) Model}

Jakobsson, Floberg and Olsson (JFO) [87, 88, 115, 160] proposed a set of complementary boundary conditions that enforces the entire mass-conservation of the lubricant 


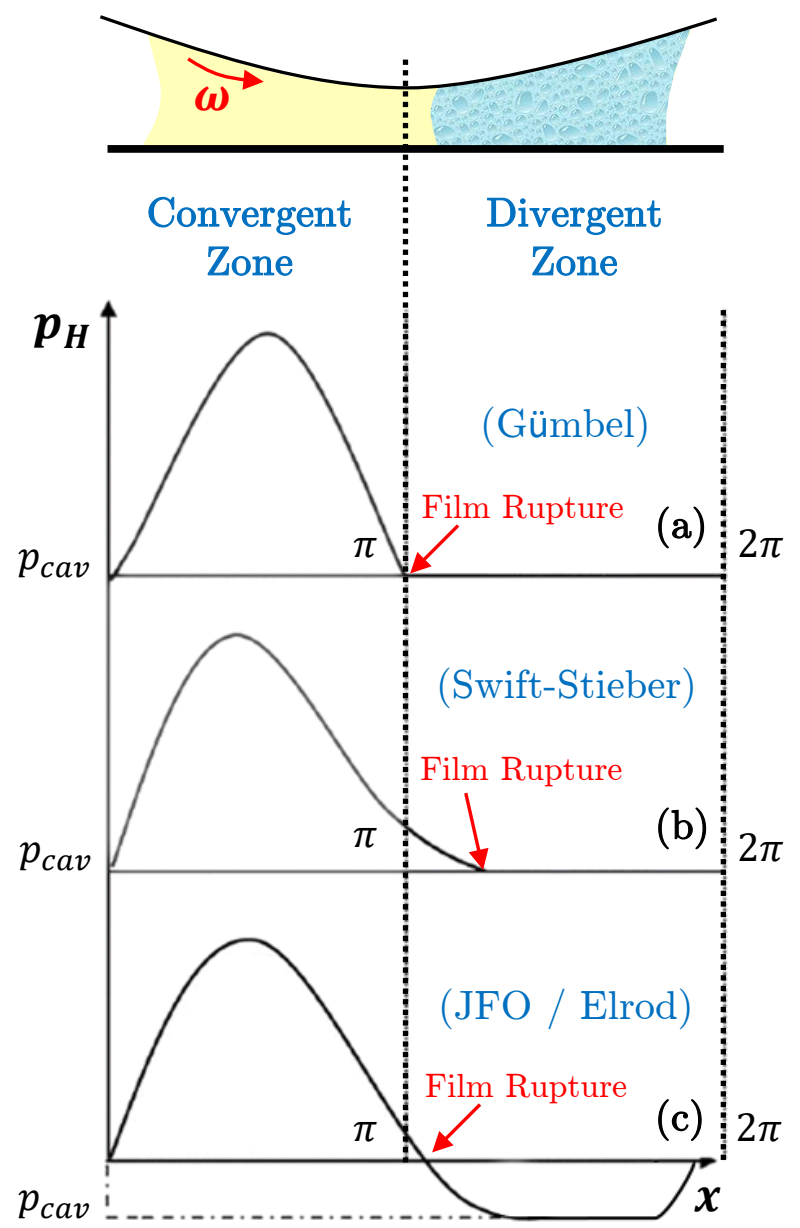

Figure 2.3: Hydrodynamic pressure distribution along the central circumferential direction of a cylindrical journal bearing obtained with different cavitation models. Notice the influence of the cavitation formulations on the position of the film rupture. Adapted from [38].

flow throughout the lubricated domain, including the rupture and reformation cavitation boundaries. The JFO's approach is probably the most widely accepted formulation for the cavitation modelling of lubrication systems. The main assumptions stated by the authors are listed as follows:

1) Within the cavitated regions, the fluid pressure in the biphasic mixture remains constant and equal to the limit pressure $\left(p_{\text {cav }}\right)$ associated with the type of cavitation considered (gaseous or vapour cavitation).

2) Within the cavitated regions, the liquid phase (lubricant) of the mixture is transported in-between gases/vapour fingers, which extend fully across the film thickness (see Fig. 2.2 and Fig. 2.4).

3) At the cavitation boundaries, the mass-conservation is enforced by imposing complementary boundary conditions that must be used concomitantly with the solution of the Reynolds equation. Such supplementary mass-conservation conditions can be posed mathematically as [92]:

$$
\rho h(\theta-1)\left(\frac{U_{n}}{2}-W_{n}\right)+\frac{\rho h^{3}}{12 \mu} \frac{\partial p_{H}}{\partial \overrightarrow{\boldsymbol{n}}}=0
$$




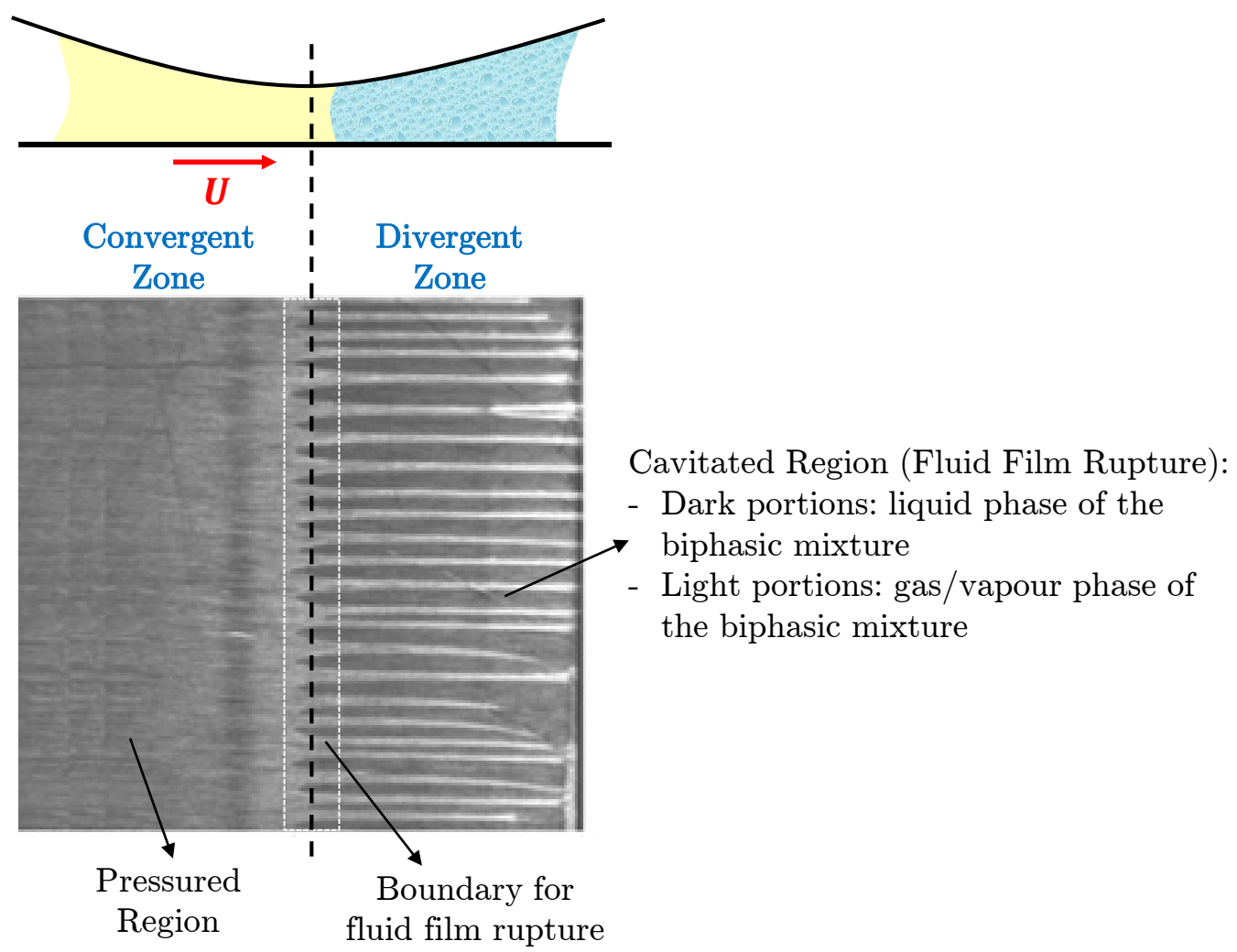

Figure 2.4: Experimental photo illustrating the cavitation phenomenon in a piston ring contact of internal combustion engine. Adapted from [66].

where $h$ is the film gap, $\theta$ the film fraction representing the proportion of lubricant contained in an infinitesimal volume of height $h$ (see detailed definition of $\theta$ in the next section) and $\overrightarrow{\boldsymbol{n}}$ the unit vector normal to the cavitation boundaries. $U_{n}$ and $W_{n}$ are the respective components of the sliding and moving boundary velocities upon the local normal direction $\overrightarrow{\boldsymbol{n}}$. Based on Eq. 2.7, when the fluid film is broken down, the negative pressure gradient in the vicinity of $\mathfrak{C}^{+}$shows that this must be zero and that $\theta$ is equal to one. Similarly, when the film is reformed, the surrounding pressure gradient is positive so that a discontinuity of $\theta$ as well as in the pressure gradient itself is expected.

Many other formulations based on different boundary conditions that do not consider the fully extended biphasic mixture across the film thickness have been proposed by several authors $[28,57,58,85-87,105,155]$. Such formulations are often denominated "flow separation conditions", and admit that the gas/vapour bubbles are initiated from a stationary surface and so expanded partially across the film thickness. However, the implementation of these alternative methodologies have serious limitations, especially in situations with moving cavitation boundaries, as those encountered in transient systems. In this manner, whenever the mass-conservation principle has to be totally satisfied over the lubricated domain, and regardless of the operational conditions of the system (either steady-state or transient), the additional assumptions established by JFO are the most consolidated and widespread ones in the literature for general lubrication applications [38].

The use of the JFO's complementary boundary conditions is not straightforward in practice since the cavitation boundaries are not known a priori (moving boundary prob- 
lem). In order to circumvent this issue, Elrod and Adams [77, 78] proposed some modifications in the Reynolds equation in order to automatically accomplish the JFO's conditions during the solution of the lubrication problem. In this case, a modified Reynolds equation is obtained that ensures the flow conservation on the entire domain, and particularly on the cavitation boundaries. This methodology is denominated in the literature as ElrodAdams cavitation model, or simply $p-\theta$ model for cativation. More details concerning the $p-\theta$ formulation will be described in the next section.

\subsubsection{Modified Reynolds Equation for Cavitation ( $p-\theta$ Model)}

The main idea of the $p-\theta$ Elrod-Adams formulation $[77,78]$ is based on the definition of a new flow parameter, namely lubricant film fraction $(\theta)$, which is aimed to account for the existence of the cavitation biphasic mixture directly into the Reynolds equation. Physically, the film fraction parameter can be interpreted as an auxiliary variable that represents the proportion of liquid (lubricant) at every point of the solution domain. Assuming that in the cavitation regions the mixture of liquid and gases/vapour behaves as a continuum medium and considering the rule of mixtures for density, the fluid density on the entire lubricated domain can be described in terms of $\theta(x, z, t)$ as:

$$
\rho \rightarrow \rho \theta \quad \text { with } \quad \begin{cases}0 \leq \theta<1 & , \quad \text { in the cavitated regions }\left(\mathcal{D}^{\mathbf{0}}\right) \\ \theta=1 \quad & \text { in the pressured regions }\left(\mathcal{D}^{+}\right)\end{cases}
$$

According to Eq. 2.8, the fluid density in the pressured regions $(\theta=1)$ remains the same as that of the liquid lubricant, i.e. $\rho$. On the other hand, in the cavitated zones $(0 \leq \theta<1)$ the density of the biphasic mixture is reduced to a fraction of $\rho$; this fraction is proportional to the amount of liquid (lubricant) in the mixture. For the particular case of $\theta=0$, the cavitated areas are filled only with gases/vapour. (In the latter situation, it was admitted implicitly that $\rho_{\text {gases/vapour }} \ll \rho_{\text {liquid }}$, so that $\frac{\rho_{\text {gases } / \text { vapour }}}{\rho_{\text {liquid }}} \approx 0$ and $\theta=0$ ).

By substituting the definition of Eq. 2.8 into the Eqs. 2.1-2.2, the following modified version of the Reynolds equation for the Elrod-Adams cavitation model ( $p-\theta$ model) is obtained:

$$
\nabla \cdot\left(\boldsymbol{\Gamma}^{p} \nabla p_{H}\right)=\nabla \cdot\left(\theta \boldsymbol{\Gamma}^{c} \overrightarrow{\boldsymbol{v}}\right)+\theta\left[S_{T S}+S_{N S}\right]+S_{T} \frac{\partial \theta}{\partial t}
$$

with the complementary conditions:

$$
\left(p_{H}-p_{\text {cav }}\right)(1-\theta)=0 \rightarrow \begin{cases}p_{H}>p_{\text {cav }} & \rightarrow \quad \theta=1 \\ p_{H}=p_{\text {cav }} & \rightarrow \quad 0 \leq \theta<1\end{cases}
$$

The matrix, vector and scalar terms in Eq. 2.9a are the same of those defined in Eqs. 2.3 , except for $S_{T}$ that is redefined as $S_{T} \equiv \rho\left(H_{2}-H_{1}\right)$. Moreover, the complementary conditions established in the above equation will be satisfied implicitly during the iterative numerical solution of the flow problem (see Section 7).

In consonance with the above defined equation, in the pressured regions $\left(\mathcal{D}^{+}\right)$, where the hydrodynamic pressures are higher than the limit cavitation pressure $\left(p_{H}>p_{\text {cav }}\right)$, the fluid film is completely developed $(\theta=1)$. On the other hand, in the cavitation zones the fluid pressures are kept constant $\left(p_{H}=p_{\text {cav }}\right)$ and the lubricant film is broken $(0 \leq \theta<1)$. Notice that the film fraction parameter $\theta$ does not appear in the Poiseuille term of the 
modified equation, since $\theta \neq 1$ only in the cavitation zones where no pressure gradients occur.

It is important to highlight that many other complex effects of temporal and spatial multiscale nature take place within the cavitation regions. Obviously, the JFO model and so the Elrod-Adams formulation are only a fair approximation of the real physical phenomena that simply ensure the global conservation of the lubricant flow on the contact interface. More advanced modelling aspects concerning the fluid flow behaviour involving cavitation effects can be found in reference [39].

\subsubsection{Modified Lubricant Flows and Shear Stresses ( $p-\theta$ Model)}

Analogously to the fluid density, the values of all lubricant properties can be assumed to vary depending on if the fluid is flowing within the pressured or cavitated regions. For the lubricant viscosity, such variation with respect to its nominal value can also be expressed as a function of the film fraction $\theta$ as:

$$
\mu \rightarrow \mu g(\theta)
$$

where $g(\theta)$ is a weighting function that determines how the lubricant dynamic viscosity changes in terms of the film fraction distribution. Notice again that it is not necessary to include such correction in the modified Reynolds equation, since the viscosity appears only on the Poiseuille term that is zero in the cavitation zones. However, such viscosity variation has to be considered in the calculations of the lubricant flows and shear stresses. Thus, the modified expressions for the components of lubricant flows are defined as:

$$
\begin{aligned}
& q_{H_{x}}=-\frac{\rho\left(H_{2}-H_{1}\right)^{3}}{12 \mu} \frac{\partial p_{H}}{\partial x}+\rho \theta\left(H_{2}-H_{1}\right)\left(\frac{U_{1}+U_{2}}{2}\right) \\
& q_{H_{z}}=-\frac{\rho\left(H_{2}-H_{1}\right)^{3}}{12 \mu} \frac{\partial p_{H}}{\partial z}+\rho \theta\left(H_{2}-H_{1}\right)\left(\frac{W_{1}+W_{2}}{2}\right)
\end{aligned}
$$

Similarly, the components of the modified shear stresses are:

$$
\begin{aligned}
& \tau_{H_{x}}=[\mu g(\theta)] \dot{\gamma}_{H_{x}} \quad \rightarrow \quad \dot{\gamma}_{H_{x}}=\frac{1}{2 \mu} \frac{\partial p_{H}}{\partial x}\left[2 y-\left(H_{2}+H_{1}\right)\right]+\left(\frac{U_{2}-U_{1}}{H_{2}-H_{1}}\right) \\
& \tau_{H_{z}}=[\mu g(\theta)] \dot{\gamma}_{H_{z}} \quad \rightarrow \quad \dot{\gamma}_{H_{z}}=\frac{1}{2 \mu} \frac{\partial p_{H}}{\partial z}\left[2 y-\left(H_{2}+H_{1}\right)\right]+\left(\frac{W_{2}-W_{1}}{H_{2}-H_{1}}\right)
\end{aligned}
$$

In this work, a linear correlation is assumed between the viscosity and the fluid film fraction within the cavitation zones, i.e. $g(\theta)=\theta[104,214]$.

\subsection{Lubricant Rheology}

The lubricant rheological properties $(\rho$ and $\mu$ ) are strongly affected by the temperature, pressure and shear rate conditions of the contact. As the thermal effects have been neglected in this work, only the isothermal density-pressure, viscosity-pressure and viscosityshear-thinning effects will be considered for the corrections of the lubricant properties. Furthermore, no changes in the lubricant rheology will be taken into account directly in the Reynolds equation, but only implicitly during the numerical solution of the problem. 


\subsubsection{Viscosity-Pressure Correction}

The classical equation utilized to correlate dynamic viscosity and fluid pressure in tribology applications is the well-known Barus equation, defined as [191]:

$$
\mu_{\text {Barus }}=\mu_{0} e^{\alpha\left(p_{H}\right)}
$$

where $\mu_{0}$ is the reference lubricant dynamic viscosity at a given temperature and atmosphere pressure, and $\alpha$ the pressure-viscosity coefficient of the lubricant. The coefficient $\alpha$ is a function of the molecular structure of the lubricant and its physical characteristics, generally varying in the ranges of $1.5 \times 10^{-8} \leq \alpha \leq 2.4 \times 10^{-8}\left[\mathrm{~m}^{2} / \mathrm{N}\right]$ for paraffinic oils and $2.5 \times 10^{-8} \leq \alpha \leq 3.5 \times 10^{-8}\left[\mathrm{~m}^{2} / \mathrm{N}\right]$ for aromatic oils [193]. As a first approximation of $\alpha$, the equation proposed by Wooster may be employed [193] (more accurate tabled values for $\alpha$ can be found in [101]):

$$
\alpha=\left[0.6+0.965 \log _{10}\left(1000 \mu_{0}\right)\right] \times 10^{-8}
$$

However, Barus equation provides suitable approximations only for moderate pressures not higher than $0.5 \mathrm{GPa}$. For more realistic applications involving a larger range of pressure magnitudes, the following (isothermal) Roelands equation can be used [191]:

$$
\mu_{\text {Roelands }}=\mu_{R} \mathrm{e}^{\left[\ln \left(\frac{\mu_{0}}{\mu_{R}}\right)\left(1+\frac{p_{H}}{p_{R}}\right)^{Z}\right]}
$$

where $\mu_{R}$ and $p_{R}$ are constants having the values of $6.31 \times 10^{-5} \mathrm{Pas}$ and $196 \mathrm{MPa}$, respectively. The exponent $Z$ is associated with the specific lubricant, typically around 0.6. Analogously to the pressure-viscosity coefficient of the Barus equation, $Z$ may be estimated as [193] (more accurate values can also be found in [101]):

$$
Z=\frac{\alpha p_{R}}{\ln \left(\frac{\mu_{0}}{\mu_{R}}\right)}
$$

In the above equation, $\alpha$ is the same pressure-viscosity coefficient of Barus equation (Eq. $2.13)$.

\subsubsection{Viscosity-Shear-Thinning Correction}

The Newtonian behaviour, i.e. the linear relationship between shear stress and shear rate of lubricant fluids, is usually valid under relatively low shear rate conditions; for pure mineral oils, for instance, such behaviour stands for shear rates up to $\sim 10^{6} \mathrm{~s}^{-1}$. However, under high shear rate situations not rarely found in lubricated contacts, this linear relationship is not valid anymore so that the lubricant starts to behave as a nonNewtonian fluid. The non-Newtonian characteristic typically observed in lubricant oils is denominated shear-thinning, which is associated with the "thinning" undergone by the fluid particles as the shear rate increases. For fluids with polymers dissolved in their base structure, the long polymeric molecules randomly oriented tend to be aligned by the high shear conditions, yielding the reduction of the lubricant bulk viscosity [193]. The multiviscous lubricants widely used in the lubrication of internal combustion engines are particularly susceptible to experience shear-thinning effects, especially due to the considerable amount of polymeric additives existent in their compositions. 
There are mainly two classes of methodologies to evaluate the lubricant viscosity variation caused by the non-Newtonian shear-thinning behaviour, which depend essentially on how the effect is investigated experimentally [191]. The first methodology is based on experimental results obtained from traction tests. In these cases, the so-called Eyring equation is usually adopted for the viscosity correction [191]:

$$
\mu_{\text {Eyring }}=\frac{\tau_{0}}{\dot{\gamma}_{e}} \sinh ^{-1}\left(\frac{\mu_{P} \dot{\gamma}_{e}}{\tau_{0}}\right)
$$

where $\mu_{P}$ is the lubricant dynamic viscosity at a given temperature, pressure and low shear rate, $\dot{\gamma}_{e}$ the equivalent operating shear rate and $\tau_{0}$ the Eyring stress. The latter is the limit shear stress from which significant shear-thinning occurs and typically has values between $5 \mathrm{MPa}$ to $10 \mathrm{MPa}$. The equivalent shear rate corresponds to the scalar magnitude of the second invariant of the shear rate tensor, which for fluid film lubrication assumes the following simplified expression [61]:

$$
\dot{\gamma}_{e}=\sqrt{\left(\dot{\gamma}_{H_{x}}\right)^{2}+\left(\dot{\gamma}_{H_{z}}\right)^{2}}
$$

The second approach commonly used to assess the lubricant shear-thinning effects relies on viscosity measurements obtained with precise viscometers. For these cases, the following power-law based Carreau-Yasuda equation commonly used to describe polymer shear-thinning fluids can be employed to correlate the viscosity changes [191]:

$$
\mu_{\text {Carreau-Yasuda }}=\mu_{\infty_{P}}+\left(\mu_{P}-\mu_{\infty_{P}}\right)\left[1+\left(\lambda \dot{\gamma}_{e}\right)^{a}\right]^{\frac{n-1}{a}}
$$

where $a$ and $n(n<1)$ are constants, $\lambda$ the characteristic relaxation time of the polymer, $\mu_{P}$ the low shear rate Newtonian viscosity at a given temperature and pressure, and $\mu_{\infty_{P}}$ is the lubricant viscosity at infinite shear rate.

\subsubsection{Density-Pressure Correction}

Regarding the relationship between lubricant density and pressure, the well-known Dowson-Higginson equation frequently used in calculations of elastohydrodynamic contacts will be here adopted [101]:

$$
\rho_{\text {Dowson-Higginson }}=\rho_{0}\left(\frac{C_{1}+C_{2} p_{H}}{C_{1}+p_{H}}\right)
$$

where $\rho_{0}$ is the reference lubricant density at atmospheric pressure and $C_{1}$ and $C_{2}$ are constants with typical values of $0.59 \mathrm{GPa}$ and 1.34 , respectively. Notice that in this case the fluid pressure has to be in GPa.

\subsection{Journal Bearing Model}

The journal bearing lubrication model proposed in this work will be presented in detail. For the sake of completeness, a generic model has been devised in order to allow its application in general multibody dynamic systems (MDS), such as the connectingrod bearings and piston-pins of internal combustion engines, as well as the floating ring bearings of high-speed turbocharges. Additionally, the effects of angular misalignments 
and the eventual existence of geometric defects on the contact surfaces are also taken into account in the model. The latter, in particular, are important for EHL problems in which the structural deformations have significant influence on the bearing performance. At this point, it is noteworthy the lack, if not the absence, of elucidative references in the literature covering all the aforementioned aspects in a unique model framework.

For the derivation of a general journal bearing model fitted to be used in MDS simulations, the definition of proper reference frames and coordinate systems is mandatory. Such definitions are presented in detail in Appendix A.1 and will be here suppressed for brevity. The main bearing coordinate systems are illustrated in Fig. 2.5.

\subsubsection{Geometry of the Lubricant Film Thickness}

In order to determine the lubricant film geometry for the current journal bearing model, the following further assumptions are admitted:

1) "Elliptization" of the journal cross sections: as can be observed in Fig. 2.5, whenever relative angular misalignments take place, the actual projections of the journal cross sections $(s)$ on the planes orthogonal to the bearing axial direction are ellipse-shaped curves. For angular misalignments of small magnitude, the influence of such "elliptization" on the lubricant film geometry will be neglected, so that the projected curves can be assumed as circles with the same journal radius [221]. However, despite the "elliptization" assumption, the equivalent eccentricities $\left(e^{\prime}\right)$ evaluated on the projected planes $(s)$ are still a function of the angular misalignments.

2) Curvature of the lubricant film: for $\left(\frac{c}{R}\right) \ll 1$ typically found in journal bearings, the curvature of the lubricant film can be neglected without affecting the overall system lubrication behaviour [60, 199]. In this context, the local Cartesian coordinate system $\boldsymbol{S}_{\mathcal{B}_{l}}$ can be used to describe the cylindrical-shaped bearing geometry, i.e. $\partial x_{b l} \approx R_{b} \theta_{b}$, hence allowing the development of the entire hydrodynamic modelling on an "unwrapped" rectangular domain.

The geometry of the lubricant film thickness is defined as the absolute normal distance measured between every point of the bearing surface $\left(P_{b}\right)$ and its respective point on the journal $\left(P_{j}\right)$, as shown in Fig. 2.6. Mathematically:

$$
H=\left(H_{2}-H_{1}\right)=\left(h+\delta_{b}-\delta_{j}\right)=\left(P_{j}-P_{b}\right) \cdot \vec{j}_{b_{l}}
$$

where $h$ is the lubricant film thickness for a perfectly cylindrical bearing and $\delta_{b, j}$ are the combined geometric deviations around the bearing/journal eccentricities; subscripts $b$ and $j$ denote bearing and journal, respectively. Such geometric deviations are caused by eventual superficial defects associated either with the form/waviness $\left(\delta_{b, j}^{G}\right)$ or roughness $\left(\delta_{b, j}^{R}\right)$ component scales of the surface topographies, or even to the solid deformations $\left(\delta_{b, j}^{D}\right)$ in EHL problems. Thus, the combined geometric deviations can be defined as:

$$
\delta_{b, j}=\left(\delta_{b, j}^{G}+\delta_{b, j}^{D}+\delta_{b, j}^{R}\right)
$$

After the evaluation of Eq. 2.21 in the local coordinate system $\boldsymbol{S}_{\mathcal{B}_{l}}$ and by assuming as negligible the terms of magnitude order $\mathcal{O}\left(\frac{c}{R}\right)^{n} \ll 1(n \geq 1)$, the geometry of the 


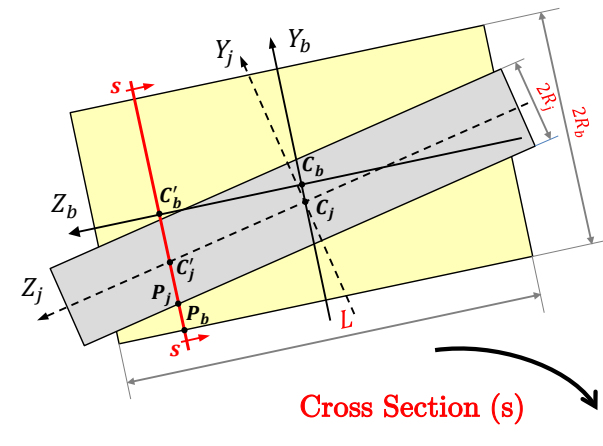

- - - Cylindrical geometry

Actual geometry with superficial defects

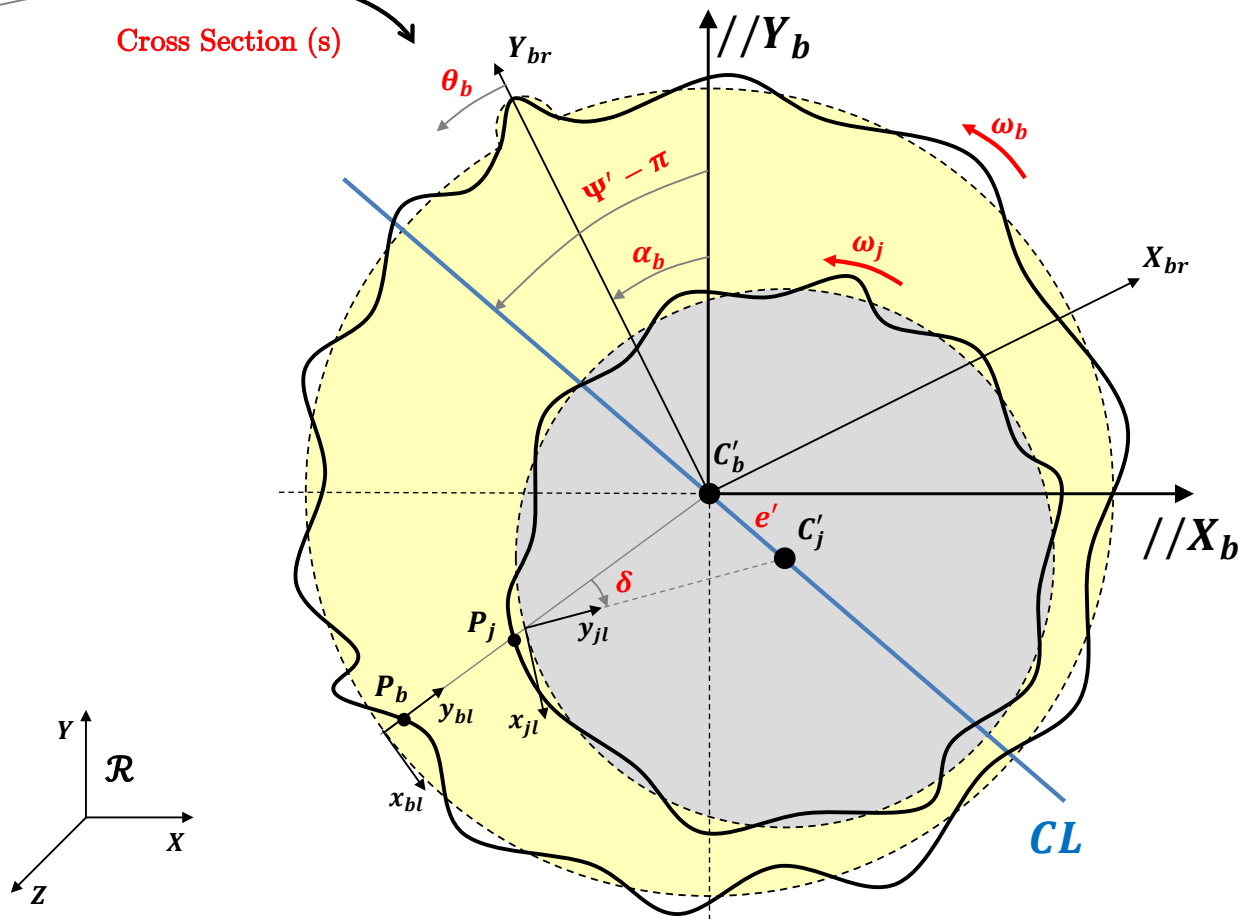

Figure 2.5: Main coordinate systems and kinematic variables of the journal bearing model.

lubricant film thickness for the current journal bearing model can be expressed in the dimensionless form as follows:

$$
\begin{aligned}
& \bar{H}_{2}\left(\theta_{b}, \bar{z}_{b}\right)=\underbrace{1-\left(\bar{Y}_{r}-\bar{A}_{r} \bar{z}_{b}\right) \cos \left(\theta_{b}+\alpha_{b}\right)+\left(\bar{X}_{r}+\bar{B}_{r} \bar{z}_{b}\right) \sin \left(\theta_{b}+\alpha_{b}\right)}_{\bar{h}}-\bar{\delta}_{j}^{n}\left(\theta_{b}, \bar{z}_{b}\right) \\
& \bar{H}_{1}\left(\theta_{b}, \bar{z}_{b}\right)=-\bar{\delta}_{b}^{n}\left(\theta_{b}, \bar{z}_{b}\right)
\end{aligned}
$$

where

$\left(\bar{X}_{r}, \bar{Y}_{r}\right)$ are the rigid body displacements of the journal relative to the bearing in the $\overrightarrow{\boldsymbol{I}}_{\boldsymbol{b}}$ and $\overrightarrow{\boldsymbol{J}}_{\boldsymbol{b}}$ directions, respectively, i.e. $\bar{X}_{r}=\left(\bar{X}_{j}-\bar{X}_{b}\right)$ and $\bar{Y}_{r}=\left(\bar{Y}_{j}-\bar{Y}_{b}\right)$;

$\left(\bar{A}_{r}, \bar{B}_{r}\right)$ are the rigid body angular misalignments of the journal relative to the bearing around the $\overrightarrow{\boldsymbol{I}}_{\boldsymbol{b}}$ and $\overrightarrow{\boldsymbol{J}}_{\boldsymbol{b}}$ axes, respectively, i.e. $\bar{A}_{r}=\left(\bar{A}_{j}-\bar{A}_{b}\right)$ and $\bar{B}_{r}=\left(\bar{B}_{j}-\bar{B}_{b}\right)$;

$\bar{\delta}_{b, j}^{n}$ are the normal components of the combined geometric deviations of the bearing/journal surfaces (combined geometric deviations at $P_{b, j}$ );

$\theta_{b}$ is the angular bearing coordinate defined as positive going counter-clockwise from the $\overrightarrow{\boldsymbol{J}}_{\boldsymbol{b}_{\boldsymbol{r}}}$ axis; 


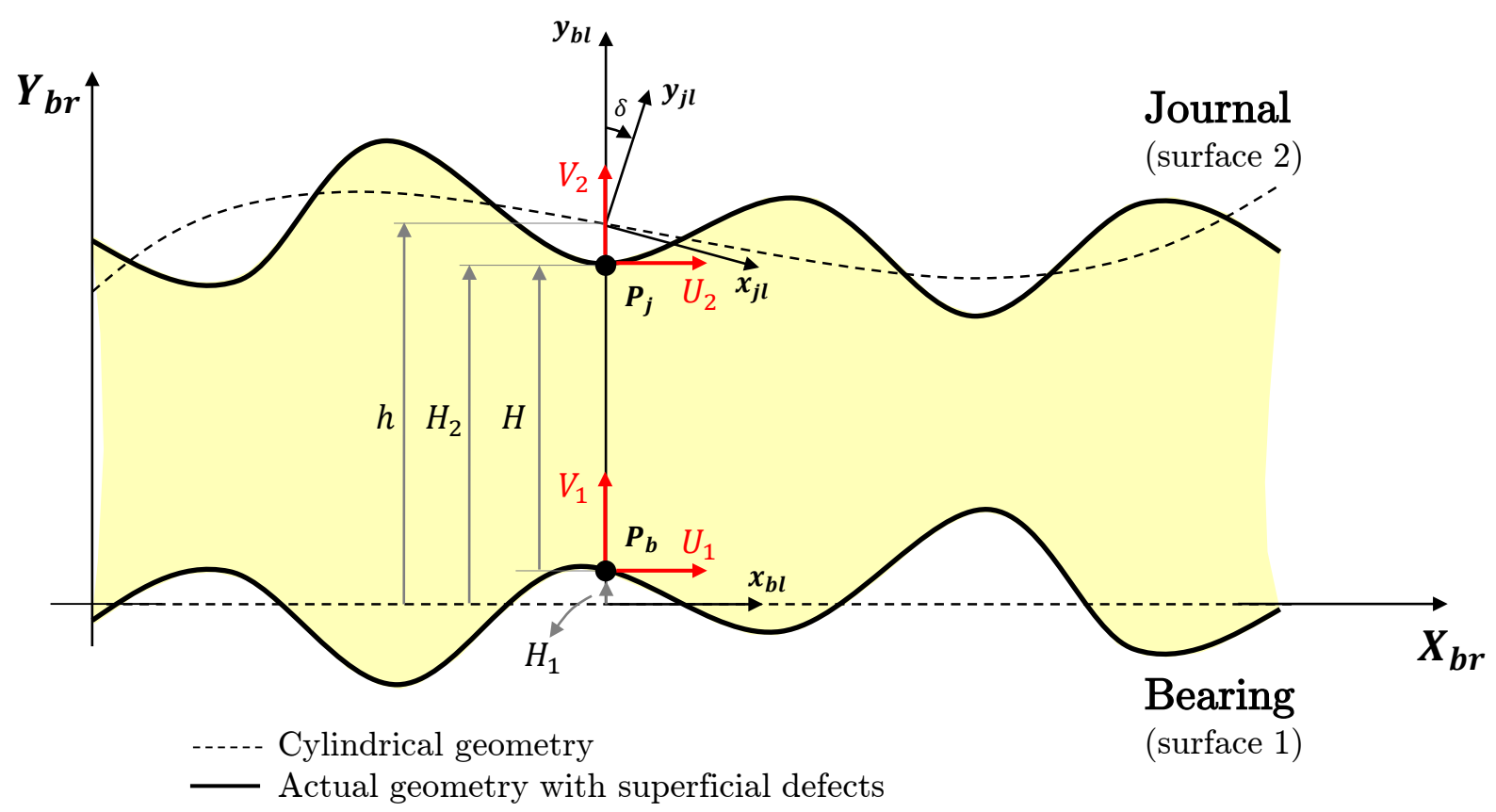

Figure 2.6: Illustration of the journal bearing contact interface surrounding the peripheral points $P_{b}$ and $P_{j}$.

$\bar{z}_{b}$ is the axial bearing coordinate (axial coordinate of $P_{b}$ );

$\alpha_{b}$ is the angular bearing rotation defined as positive going counter-clockwise from the $\overrightarrow{\boldsymbol{J}}_{\boldsymbol{b}}$ axis.

The over-bars in Eqs. 2.23 denote dimensionless quantities obtained according to the parameters listed in Appendix A.3. The derivation of Eqs. 2.23 from Eq. 2.21 is exposed in detail in Appendix A.4.

\subsubsection{Kinematic of the Contact Surfaces}

The kinematic description of the contact surfaces for the current journal bearing model consists in determining the absolute velocities of the points $P_{b}$ and $P_{j}$ in the local coordinate system $\mathcal{S}_{\mathcal{B}_{l}}$ where the lubricant film thickness is described. In the following analysis, such kinematic evaluations will be developed relative to the reference frame $\mathcal{R}$. Hence, in case of multibody dynamic applications where $\mathcal{R}$ can move with respect to other system components, a kinematic composition has to be considered to account for the frame relative motion. Thus, the absolute velocities of $P_{b}$ and $P_{j}$ relative to the frame $\mathcal{R}$ can be written as:

$$
{ }^{\mathcal{R}} \overrightarrow{\boldsymbol{V}}^{P_{b, j}}={ }^{\mathcal{B}_{r}, \mathcal{J}_{r}} \overrightarrow{\boldsymbol{V}}^{P_{b, j}}+{ }^{\mathcal{R}} \overrightarrow{\boldsymbol{V}}^{C_{b, j}}+{ }^{\mathcal{R}} \overrightarrow{\boldsymbol{\Omega}}^{\mathcal{B}_{r}, \mathcal{J}_{r}} \wedge\left(P_{b, j}-C_{b, j}\right)
$$

After the evaluation of Eq. 2.24 in the local coordinate system $\boldsymbol{S}_{\mathcal{B}_{l}}$ and by neglecting intermediate terms of magnitude order $\mathcal{O}\left(\frac{c}{R}\right)^{n} \ll 1, \mathcal{O}\left(\frac{c}{L}\right)^{n} \ll 1, \mathcal{O}\left(\frac{c}{L r_{L D}}\right)^{n} \ll 1(n \geq$ $1)^{1}$, the absolute velocities of the bearing/journal peripheral points $P_{b, j}$ (see Fig. 2.6) can

\footnotetext{
${ }^{1}$ The terms of order $\mathcal{O}\left(\frac{c}{L r_{L D}}\right)$ are associated with the "axial wedge effect", whose influence on the hydrodynamic pressure calculation is significant only for very short journal bearings [222]. This extreme bearing case will not be considered in the present work.
} 
be expressed in the dimensionless form as follows:

$$
\begin{aligned}
& \bar{U}_{1}=\left(2 \pi \bar{\omega}_{b}\right) \\
& \bar{V}_{1}=\left\{\left[\left(-\dot{\bar{Y}}_{C_{b}}+\dot{\bar{A}}_{b} \bar{z}_{b}\right) \cos \left(\theta_{b}+\alpha_{b}\right)+\left(\dot{\bar{X}}_{C_{b}}+\dot{\bar{B}}_{b} \bar{z}_{b}\right) \sin \left(\theta_{b}+\alpha_{b}\right)\right]+\right. \\
& \left.\left[2 \pi \bar{\omega}_{b} \bar{\delta}_{b}^{t}-\dot{\bar{\delta}}_{b}^{n}\right]\right\} \\
& \bar{W}_{1}=\left(\dot{\bar{Z}}_{C_{b}}\right) \\
& \bar{U}_{2}=\left(2 \pi \bar{\omega}_{j}\right) \\
& \bar{V}_{2}=\left\{\left[\left(-\dot{\bar{Y}}_{C_{j}}+\dot{\bar{A}}_{j} \bar{z}_{b}\right) \cos \left(\theta_{b}+\alpha_{b}\right)+\left(\dot{\bar{X}}_{C_{j}}+\dot{\bar{B}}_{j} \bar{z}_{b}\right) \sin \left(\theta_{b}+\alpha_{b}\right)\right]+\right. \\
& \left.\left[\left(2 \pi \bar{\omega}_{j} \bar{\delta}_{j}^{t}-\dot{\bar{\delta}}_{j}^{n}\right)+\left(2 \pi \bar{\omega}_{j} \frac{\partial \bar{h}}{\partial \theta_{b}}\right)\right]\right\} \\
& \bar{W}_{2}=\left(\dot{\bar{Z}}_{C_{j}}\right) \\
& \frac{\partial\left(\bar{H}_{2}-\bar{H}_{1}\right)}{\partial t}=\underbrace{\left(-\dot{\bar{Y}}_{r}+\dot{\bar{A}}_{r} \bar{z}_{b}\right) \cos \left(\theta_{b}+\alpha_{b}\right)+\left(\dot{\bar{X}}_{r}+\dot{\bar{B}}_{r} \bar{z}_{b}\right) \sin \left(\theta_{b}+\alpha_{b}\right)}_{\frac{\partial \bar{h}}{\partial t}}+ \\
& \left(\dot{\bar{\delta}}_{b}^{n}-\dot{\bar{\delta}}_{j}^{n}\right)
\end{aligned}
$$

where $\bar{\omega}_{b, j}$ and $\dot{\bar{Z}}_{C_{b, j}}$ are the rotational speeds and axial velocities of the bearing/journal components, respectively; once again, the over-dots in the equations designate time derivatives and the over-bars denote dimensionless quantities defined according to the parameters listed in Appendix A.3. The derivation of Eqs. 2.25 from Eq. 2.24 is presented in detail in Appendix A.4.

\subsubsection{Reynolds Equation}

In the following, the customized version of the Reynolds equation for the current journal bearing model will be developed according to the lubricant film thickness (Eqs. 2.23) and surfaces velocities (Eqs. 2.25) derived in the previous sections. In this fashion, it is convenient to write the expanded dimensionless form of the modified Reynolds equation (Eq. 2.9a) obtained from the parameters listed in Appendix A.3.

$$
\begin{aligned}
& \frac{\partial}{\partial \theta_{b}}\left[\frac{\bar{\rho}\left(\bar{H}_{2}-\bar{H}_{1}\right)^{3}}{12 \bar{\mu}} \frac{\partial \bar{p}_{H}}{\partial \theta_{b}}\right]+\left(\frac{1}{r_{L D}^{2}}\right) \frac{\partial}{\partial \bar{z}_{b}}\left[\frac{\bar{\rho}\left(\bar{H}_{2}-\bar{H}_{1}\right)^{3}}{12 \bar{\mu}} \frac{\partial \bar{p}_{H}}{\partial \bar{z}_{b}}\right]= \\
& \quad\left(\frac{1}{\Omega}\right)\left\{\frac{\partial}{\partial \theta_{b}}\left[\theta \bar{\rho}\left(\frac{\bar{U}_{2}+\bar{U}_{1}}{2}\right)\left(\bar{H}_{2}-\bar{H}_{1}\right)\right]+\frac{\partial}{\partial \bar{z}_{b}}\left[\theta \bar{\rho}\left(\frac{\bar{W}_{2}+\bar{W}_{1}}{2}\right)\left(\bar{H}_{2}-\bar{H}_{1}\right)\right]\right\}+ \\
& \quad\left(\frac{1}{\Omega}\right) \theta \bar{\rho}\left[\left(\bar{U}_{1} \frac{\partial \bar{H}_{1}}{\partial \theta_{b}}-\bar{U}_{2} \frac{\partial \bar{H}_{2}}{\partial \theta_{b}}\right)+\left(\bar{W}_{1} \frac{\partial \bar{H}_{1}}{\partial \bar{z}_{b}}-\bar{W}_{2} \frac{\partial \bar{H}_{2}}{\partial \bar{z}_{b}}\right)\right]+ \\
& \quad\left(\frac{1}{\Omega}\right) \bar{\rho}\left[\theta\left(\bar{V}_{2}-\bar{V}_{1}\right)+\left(\bar{H}_{2}-\bar{H}_{1}\right) \frac{\partial \theta}{\partial t}\right]
\end{aligned}
$$


Substituting Eqs. 2.25 into Eq. 2.26 and by rearranging the evolved terms, one obtains the full customized version of the modified Reynolds equation:

$$
\begin{aligned}
& \frac{\partial}{\partial \theta_{b}}\left[\frac{\bar{\rho}\left(\bar{H}_{2}-\bar{H}_{1}\right)^{3}}{12 \bar{\mu}} \frac{\partial \bar{p}_{H}}{\partial \theta_{b}}\right]+\left(\frac{1}{r_{L D}^{2}}\right) \frac{\partial}{\partial \bar{z}_{b}}\left[\frac{\bar{\rho}\left(\bar{H}_{2}-\bar{H}_{1}\right)^{3}}{12 \bar{\mu}} \frac{\partial \bar{p}_{H}}{\partial \bar{z}_{b}}\right]= \\
& \left(\frac{1}{\Omega}\right)\left\{(2 \pi) \frac{\partial}{\partial \theta_{b}}\left[\theta \bar{\rho}\left(\frac{\bar{\omega}_{j}+\bar{\omega}_{b}}{2}\right)\left(\bar{H}_{2}-\bar{H}_{1}\right)\right]+\frac{\partial}{\partial \bar{z}_{b}}\left[\theta \bar{\rho}\left(\frac{\dot{\bar{Z}}_{C_{j}}+\dot{\bar{Z}}_{C_{b}}}{2}\right)\left(\bar{H}_{2}-\bar{H}_{1}\right)\right]\right\}+ \\
& \left(\frac{1}{\Omega}\right) \theta \bar{\rho}\left\{(2 \pi)\left[\left(\bar{\omega}_{b} \frac{\partial \bar{H}_{1}}{\partial \theta_{b}}-\bar{\omega}_{j} \frac{\partial \bar{H}_{2}}{\partial \theta_{b}}+\bar{\omega}_{j} \frac{\partial \bar{h}}{\partial \theta_{b}}\right)\right]+\left[\left(\dot{\bar{Z}}_{C_{b}} \frac{\partial \bar{H}_{1}}{\partial \bar{z}_{b}}-\dot{\bar{Z}}_{C_{j}} \frac{\partial \bar{H}_{2}}{\partial \bar{z}_{b}}\right)\right]\right\}+ \\
& \left(\frac{1}{\Omega}\right) \theta \bar{\rho}\left[\frac{\partial\left(\bar{H}_{2}-\bar{H}_{1}\right)}{\partial t}+2 \pi\left(\bar{\omega}_{j} \bar{\delta}_{j}^{t}-\bar{\omega}_{b} \bar{\delta}_{b}^{t}\right)\right]+\left(\frac{1}{\Omega}\right) \bar{\rho}\left(\bar{H}_{2}-\bar{H}_{1}\right) \frac{\partial \theta}{\partial t}
\end{aligned}
$$

Finally, Eq. 2.27 can be rewritten in the conservative vector form as:

$$
\nabla \cdot\left(\overline{\boldsymbol{\Gamma}}^{p} \nabla \bar{p}_{H}\right)=\nabla \cdot\left(\theta \overline{\boldsymbol{\Gamma}}^{c} \overrightarrow{\boldsymbol{v}}\right)+\theta\left[\bar{S}_{T S}+\bar{S}_{N S}\right]+\bar{S}_{T} \frac{\partial \theta}{\partial t}
$$

with

$$
\begin{aligned}
& \overline{\boldsymbol{\Gamma}}^{\boldsymbol{p}}=\left[\begin{array}{cc}
\left(\frac{\bar{\rho}\left(\bar{H}_{2}-\bar{H}_{1}\right)^{3}}{12 \bar{\mu}}\right) & 0 \\
0 & \left(\frac{1}{r_{L D}^{2}}\right)\left(\frac{\bar{\rho}\left(\bar{H}_{2}-\bar{H}_{1}\right)^{3}}{12 \bar{\mu}}\right)
\end{array}\right] \\
& \overline{\boldsymbol{\Gamma}}^{c}=\left[\begin{array}{cc}
\left(\frac{2 \pi}{\Omega}\right) \bar{\rho}\left(\bar{H}_{2}-\bar{H}_{1}\right) & 0 \\
0 & \left(\frac{1}{\Omega}\right) \bar{\rho}\left(\bar{H}_{2}-\bar{H}_{1}\right)
\end{array}\right] \\
& \bar{S}_{T S}=\bar{\rho}\left(\overrightarrow{\overrightarrow{\boldsymbol{a}}} \cdot \nabla \bar{H}_{1}-\overrightarrow{\overrightarrow{\boldsymbol{b}}} \cdot \nabla \bar{H}_{2}+\overrightarrow{\overrightarrow{\boldsymbol{c}}} \cdot \nabla \bar{h}\right) \\
& \bar{S}_{N S}=\bar{\rho}\left(\frac{1}{\Omega}\right)\left[\frac{\partial\left(\bar{H}_{2}-\bar{H}_{1}\right)}{\partial t}+2 \pi\left(\bar{\omega}_{j} \bar{\delta}_{j}^{t}-\bar{\omega}_{b} \bar{\delta}_{b}^{t}\right)\right] \\
& \bar{S}_{T}=\bar{\rho}\left(\frac{1}{\Omega}\right)\left(\bar{H}_{2}-\bar{H}_{1}\right)
\end{aligned}
$$

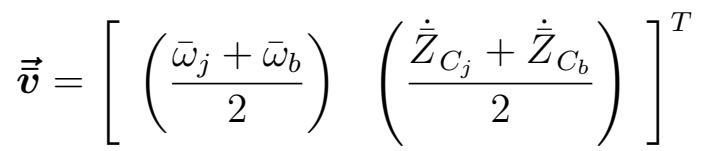

$$
\begin{aligned}
& \overrightarrow{\vec{a}}=\left[\begin{array}{ll}
\left(\frac{2 \pi}{\Omega}\right) \bar{\omega}_{b} & \left(\frac{1}{\Omega}\right) \dot{\bar{Z}}_{C_{b}}
\end{array}\right]^{T} \\
& \overrightarrow{\vec{b}}=\left[\left(\frac{2 \pi}{\Omega}\right) \bar{\omega}_{j} \quad\left(\frac{1}{\Omega}\right) \dot{\bar{Z}}_{C_{j}}\right]^{T} \\
& \overrightarrow{\overrightarrow{\boldsymbol{c}}}=\left[\left(\frac{2 \pi}{\Omega}\right) \bar{\omega}_{j} 0\right]^{T}
\end{aligned}
$$




\subsubsection{Lubricant Flows and Shear Stresses}

The expressions for the lubricant flows and shear stresses also have to be adapted in accordance with the geometric and kinematic features previously established for the current journal bearing model. Thus, the modified dimensionless components of the lubricant flows are:

$$
\begin{aligned}
& \bar{q}_{H_{\theta_{b}}}=-\frac{\bar{\rho}\left(\bar{H}_{2}-\bar{H}_{1}\right)^{3}}{12 \bar{\mu}} \frac{\partial \bar{p}_{H}}{\partial \theta_{b}}+\left(\frac{2 \pi}{\Omega}\right) \bar{\rho} \theta\left(\frac{\bar{\omega}_{j}+\bar{\omega}_{b}}{2}\right)\left(\bar{H}_{2}-\bar{H}_{1}\right) \\
& \bar{q}_{H_{z_{b}}}=-\left(\frac{1}{r_{L D}}\right) \frac{\bar{\rho}\left(\bar{H}_{2}-\bar{H}_{1}\right)^{3}}{12 \bar{\mu}} \frac{\partial \bar{p}_{H}}{\partial \bar{z}_{b}}+\left(\frac{r_{L D}}{\Omega}\right) \bar{\rho} \theta\left(\frac{\dot{\bar{Z}}_{C_{j}}+\dot{\bar{Z}}_{C_{b}}}{2}\right)\left(\bar{H}_{2}-\bar{H}_{1}\right)
\end{aligned}
$$

Analogously, the dimensionless components of the modified shear stresses acting on the bearing/journal surfaces are:

$$
\begin{aligned}
& \bar{\tau}_{H_{\theta_{b}}}^{b}=[\bar{\mu} g(\theta)] \dot{\bar{\gamma}}_{H_{\theta_{b}}}^{b} \rightarrow \dot{\bar{\gamma}}_{H_{\theta_{b}}}^{b}=-\frac{1}{2 \bar{\mu}} \frac{\partial \bar{p}_{H}}{\partial \theta_{b}}\left(\bar{H}_{2}-\bar{H}_{1}\right)+\left(\frac{2 \pi}{\Omega}\right)\left(\frac{\bar{\omega}_{j}-\bar{\omega}_{b}}{\bar{H}_{2}-\bar{H}_{1}}\right) \\
& \bar{\tau}_{H_{z_{b}}}^{b}=[\bar{\mu} g(\theta)] \dot{\bar{\gamma}}_{H_{z_{b}}}^{b} \rightarrow \dot{\bar{\gamma}}_{H_{z_{b}}}^{b}=-\frac{1}{2 \bar{\mu} r_{L D}} \frac{\partial \bar{p}_{H}}{\partial \bar{z}_{b}}\left(\bar{H}_{2}-\bar{H}_{1}\right)+\left(\frac{r_{L D}}{\Omega}\right)\left(\frac{\dot{\bar{Z}}_{C_{j}}-\dot{\bar{Z}}_{C_{b}}}{\bar{H}_{2}-\bar{H}_{1}}\right) \\
& \bar{\tau}_{H_{\theta_{b}}}^{j}=[\bar{\mu} g(\theta)] \dot{\bar{\gamma}}_{H_{\theta_{b}}}^{j} \rightarrow \dot{\bar{\gamma}}_{H_{\theta_{b}}}^{j}=\frac{1}{2 \bar{\mu}} \frac{\partial \bar{p}_{H}}{\partial \theta_{b}}\left(\bar{H}_{2}-\bar{H}_{1}\right)+\left(\frac{2 \pi}{\Omega}\right)\left(\frac{\bar{\omega}_{j}-\bar{\omega}_{b}}{\bar{H}_{2}-\bar{H}_{1}}\right) \\
& \bar{\tau}_{H_{z_{b}}}^{j}=[\bar{\mu} g(\theta)] \dot{\bar{\gamma}}_{H_{z_{b}}}^{j} \rightarrow \dot{\bar{\gamma}}_{H_{z_{b}}}^{j}=\frac{1}{2 \bar{\mu} r_{L D}} \frac{\partial \bar{p}_{H}}{\partial \bar{z}_{b}}\left(\bar{H}_{2}-\bar{H}_{1}\right)+\left(\frac{r_{L D}}{\Omega}\right)\left(\frac{\dot{\bar{Z}}_{C_{j}}-\dot{\bar{Z}}_{C_{b}}}{\bar{H}_{2}-\bar{H}_{1}}\right)
\end{aligned}
$$

\subsubsection{Operational Parameters}

Once the hydrodynamic pressure and fluid fraction (cavitation) fields are obtained from the solution of the Reynolds equation (Eq. 2.28), several operational parameters can be determined for the description of the overall system lubrication performance. For journal bearings, in particular, the definitions of some operational parameters are summarized in Appendix A.5.

\subsection{Sliding Bearing Model}

In this section, the sliding bearing lubrication model proposed in this work will be presented. Similarly to the journal bearing model of the previous section, a generic approach including angular misalignments and superficial defects is also considered for possible applications in multibody dynamic systems (MDS). The definitions of the model reference frames and coordinate systems are displayed in Appendix B.1 and will be here omitted for shortness. The main geometric characteristics and coordinate systems are illustrated in Fig. 2.7.

\subsubsection{Geometry of the Lubricant Film Thickness}

Analogously to the journal bearings, the geometry of the lubricant film thickness for the current sliding bearing model is defined as the normal absolute distance measured 
between every point of the bearing surface $\left(P_{b}\right)$ and its respective point on the slider $\left(P_{s}\right)$, as shown in Fig. 2.7. Mathematically:

$$
H=\left(H_{2}-H_{1}\right)=\left(h+\delta_{b}-\delta_{s}\right)=\left(P_{s}-P_{b}\right) \cdot \vec{j}_{\boldsymbol{b}}
$$

where $h$ is the lubricant film thickness for a perfectly plane bearing and $\delta_{b, s}$ are the combined geometric deviations around the bearing/slider surfaces; subscripts $b$ and $s$ denote bearing and slider, respectively. Such geometric deviations are related to eventual superficial defects associated either with the form/waviness $\left(\delta_{b, s}^{G}\right)$ or roughness $\left(\delta_{b, s}^{R}\right)$ components of the surface topographies, or even to the solid deformations $\left(\delta_{b, s}^{D}\right)$ in EHL problems. Thus, the combined geometric deviations can be defined as: $\delta_{b, s}=\left(\delta_{b, s}^{G}+\delta_{b, s}^{D}+\delta_{b, s}^{R}\right)$.

After the evaluation of Eq. 2.32 in the local coordinate system $\boldsymbol{S}_{\mathcal{B}}$ and by admitting as negligible the terms of magnitude order $\mathcal{O}\left(\frac{h_{0}}{L_{x}}\right)^{n} \ll 1$ and $\mathcal{O}\left(\frac{h_{0}}{L_{z}}\right)^{n} \ll 1(n \geq 1)$, as well as the small angular misalignments assumption, the geometry of the lubricant film thickness for the present bearing model can be written in the dimensionless form as follows:

$$
\begin{aligned}
\bar{H}_{2}\left(\bar{x}_{b}, \bar{z}_{b}\right) & =\underbrace{\bar{Y}_{r}+\bar{B}_{r}\left(\bar{x}_{b}-\bar{x}_{P}\right)-\bar{A}_{r}\left(\bar{z}_{b}-\bar{z}_{P}\right)}_{\bar{h}}-\bar{\delta}_{s}^{n}\left(\bar{x}_{b}, \bar{z}_{b}\right) \\
\bar{H}_{1}\left(\bar{x}_{b}, \bar{z}_{b}\right) & =-\bar{\delta}_{b}^{n}\left(\bar{x}_{b}, \bar{z}_{b}\right)
\end{aligned}
$$

where

$\bar{Y}_{r}$ is the rigid body displacement of the slider relative to the bearing in the $\overrightarrow{\boldsymbol{j}}_{\boldsymbol{b}}$ direction, i.e. $\bar{Y}_{r}=\left(\bar{Y}_{s}-\bar{Y}_{b}\right)$;

$\left(\bar{A}_{r}, \bar{B}_{r}\right)$ are the rigid body angular misalignments of the slider relative to the bearing around the $\overrightarrow{\boldsymbol{i}}_{\boldsymbol{b}}$ and $\overrightarrow{\boldsymbol{k}}_{\boldsymbol{b}}$ axes, respectively, i.e. $\bar{A}_{r}=\left(\bar{A}_{s}-\bar{A}_{b}\right)$ and $\bar{B}_{r}=\left(\bar{B}_{s}-\bar{B}_{b}\right)$; such misalignments are defined with respect to a fixed pole (pivot) located at the bearing point $\left(\bar{x}_{P}, \bar{z}_{P}\right)$;

$\bar{\delta}_{b, s}^{n}$ are the normal components of the combined geometric deviations of the bearing/slider surfaces (combined geometric deviations at $P_{b, s}$ );

$\left(\bar{x}_{b}, \bar{z}_{b}\right)$ are the bearing coordinates (coordinates of $\left.P_{b, s}\right)$;

The over-bars in Eqs. 2.33 denote dimensionless quantities defined according to the parameters listed in Appendix B.2.

\subsubsection{Kinematic of the Contact Surfaces}

The absolute velocities of the peripheral points $P_{b, s}$ (see Fig. 2.7) relative to the reference frame $\mathcal{R}$ can be expressed in the coordinate system $\boldsymbol{S}_{\mathcal{B}}$ as (dimensionless form):

$$
\begin{aligned}
\bar{U}_{1} & =\bar{U}_{b} \\
\bar{V}_{1} & =\dot{\bar{Y}}_{b}+\dot{\bar{B}}_{b}\left(\bar{x}_{b}-\bar{x}_{P}\right)-\dot{\bar{A}}_{b}\left(\bar{z}_{b}-\bar{z}_{P}\right)-\dot{\bar{\delta}}_{b}^{n} \\
\bar{W}_{1} & =\bar{W}_{b} \\
\bar{U}_{2} & =\bar{U}_{s}
\end{aligned}
$$



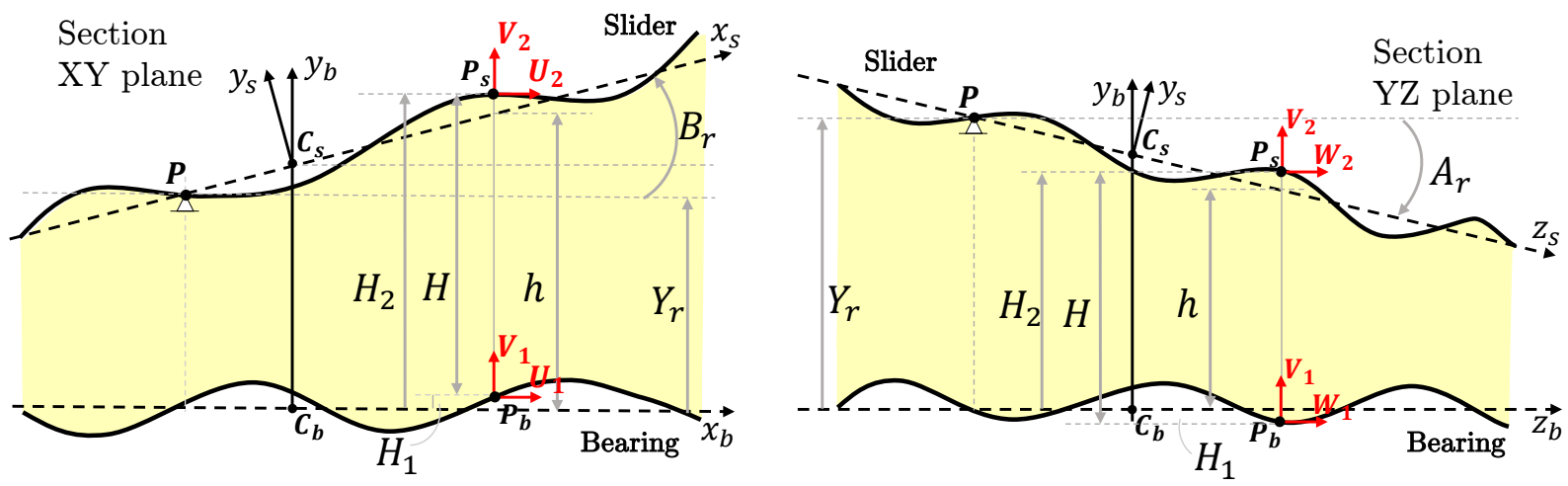

Figure 2.7: Main coordinate systems and kinematic variables of the sliding bearing model.

$$
\begin{aligned}
\bar{V}_{2} & =\dot{\bar{Y}}_{s}+\dot{\bar{B}}_{s}\left(\bar{x}_{b}-\bar{x}_{P}\right)-\dot{\bar{A}}_{s}\left(\bar{z}_{b}-\bar{z}_{P}\right)-\dot{\bar{\delta}}_{s}^{n} \\
\bar{W}_{2} & =\bar{W}_{s} \\
\frac{\partial\left(\bar{H}_{2}-\bar{H}_{1}\right)}{\partial t} & =\underbrace{\dot{\bar{Y}}_{r}+\dot{\bar{B}}_{r}\left(\bar{x}_{b}-\bar{x}_{P}\right)-\dot{\bar{A}}_{r}\left(\bar{z}_{b}-\bar{z}_{P}\right)}_{\frac{\partial \bar{h}}{\partial t}}+\left(\dot{\bar{\delta}}_{b}^{n}-\dot{\bar{\delta}}_{s}^{n}\right)
\end{aligned}
$$

where $\bar{U}_{b, s}$ and $\bar{W}_{b, s}$ are the bearing/slider velocities in the $\overrightarrow{\boldsymbol{i}}_{\boldsymbol{b}}$ and $\overrightarrow{\boldsymbol{k}}_{\boldsymbol{b}}$ directions (the sliding velocities are always assumed parallel to the coordinate directions of $\boldsymbol{S}_{\mathcal{B}}$ ); once again, the over-dots in Eqs. 2.34 designate time derivatives and the over-bars denote dimensionless quantities defined according to the parameters listed in Appendix B.2. In the same equations, the terms of magnitude order $\mathcal{O}\left(\frac{h_{0}}{L_{x}}\right)^{n} \ll 1$ and $\mathcal{O}\left(\frac{h_{0}}{L_{z}}\right)^{n} \ll 1$ $(n \geq 1)$, have been also neglected.

\subsubsection{Reynolds Equation}

In the following, the customized version of the Reynolds equation for current sliding bearings model will be developed according to the lubricant film thickness (Eqs. 2.33) and surfaces velocities (Eqs. 2.34) derived in the previous sections. Thus, the expanded dimensionless form of the modified Reynolds equation (Eq. 2.9a) obtained from the parameters listed in Appendix B.2 can be expressed as follows:

$$
\begin{aligned}
& \frac{\partial}{\partial \bar{x}_{b}}\left[\frac{\bar{\rho}\left(\bar{H}_{2}-\bar{H}_{1}\right)^{3}}{12 \bar{\mu}} \frac{\partial \bar{p}_{H}}{\partial \bar{x}_{b}}\right]+\left(\frac{1}{r_{L x z}^{2}}\right) \frac{\partial}{\partial \bar{z}_{b}}\left[\frac{\bar{\rho}\left(\bar{H}_{2}-\bar{H}_{1}\right)^{3}}{12 \bar{\mu}} \frac{\partial \bar{p}_{H}}{\partial \bar{z}_{b}}\right]= \\
& \left(\frac{1}{\Omega}\right)\left\{\frac{\partial}{\partial \bar{x}_{b}}\left[\theta \bar{\rho}\left(\frac{\bar{U}_{s}+\bar{U}_{b}}{2}\right)\left(\bar{H}_{2}-\bar{H}_{1}\right)\right]+\frac{\partial}{\partial \bar{z}_{b}}\left[\theta \bar{\rho}\left(\frac{\bar{W}_{s}+\bar{W}_{b}}{2}\right)\left(\bar{H}_{2}-\bar{H}_{1}\right)\right]\right\}+ \\
& \quad\left(\frac{1}{\Omega}\right) \theta \bar{\rho}\left[\left(\bar{U}_{b} \frac{\partial \bar{H}_{1}}{\partial \bar{x}_{b}}-\bar{U}_{s} \frac{\partial \bar{H}_{2}}{\partial \bar{x}_{b}}\right)+\left(\bar{W}_{b} \frac{\partial \bar{H}_{1}}{\partial \bar{z}_{b}}-\bar{W}_{s} \frac{\partial \bar{H}_{2}}{\partial \bar{z}_{b}}\right)\right]+ \\
& \quad\left(\frac{1}{\Omega}\right) \theta \bar{\rho} \frac{\partial\left(\bar{H}_{2}-\bar{H}_{1}\right)}{\partial t}+\left(\frac{1}{\Omega}\right) \bar{\rho}\left(\bar{H}_{2}-\bar{H}_{1}\right) \frac{\partial \theta}{\partial t}
\end{aligned}
$$

Accordingly, Eq. 2.35 can be rewritten in the conservative vector form as:

$$
\nabla \cdot\left(\overline{\boldsymbol{\Gamma}}^{p} \nabla \bar{p}_{H}\right)=\nabla \cdot\left(\theta \overline{\boldsymbol{\Gamma}}^{c} \overrightarrow{\overrightarrow{\boldsymbol{v}}}\right)+\theta\left[\bar{S}_{T S}+\bar{S}_{N S}\right]+\bar{S}_{T} \frac{\partial \theta}{\partial t}
$$


with

$$
\begin{aligned}
& \overline{\boldsymbol{\Gamma}}^{\boldsymbol{p}}=\left[\begin{array}{cc}
\left(\frac{\bar{\rho}\left(\bar{H}_{2}-\bar{H}_{1}\right)^{3}}{12 \bar{\mu}}\right) & 0 \\
0 & \left(\frac{1}{r_{L x z}^{2}}\right)\left(\frac{\bar{\rho}\left(\bar{H}_{2}-\bar{H}_{1}\right)^{3}}{12 \bar{\mu}}\right)
\end{array}\right] \\
& \overline{\boldsymbol{\Gamma}}^{c}=\left[\begin{array}{cc}
\left(\frac{1}{\Omega}\right) \bar{\rho}\left(\bar{H}_{2}-\bar{H}_{1}\right) & 0 \\
0 & \left(\frac{1}{\Omega}\right) \bar{\rho}\left(\bar{H}_{2}-\bar{H}_{1}\right)
\end{array}\right] \\
& \bar{S}_{T S}=\bar{\rho}\left(\overrightarrow{\vec{a}} \cdot \nabla \bar{H}_{1}-\overrightarrow{\vec{b}} \cdot \nabla \bar{H}_{2}\right) \\
& \bar{S}_{N S}=\bar{\rho}\left(\frac{1}{\Omega}\right) \frac{\partial\left(\bar{H}_{2}-\bar{H}_{1}\right)}{\partial t} \\
& \bar{S}_{T}=\bar{\rho}\left(\frac{1}{\Omega}\right)\left(\bar{H}_{2}-\bar{H}_{1}\right)
\end{aligned}
$$

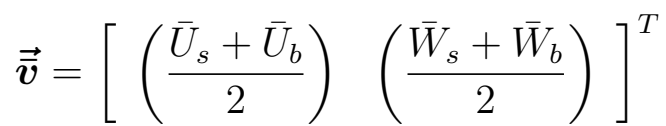

$$
\begin{aligned}
& \overrightarrow{\overrightarrow{\boldsymbol{a}}}=\left[\begin{array}{ll}
\left(\frac{1}{\Omega}\right) \bar{U}_{b} & \left(\frac{1}{\Omega}\right) \bar{W}_{b}
\end{array}\right]^{T}
\end{aligned}
$$

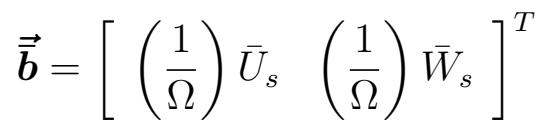

\subsubsection{Lubricant Flows and Shear Stresses}

The expressions for the lubricant flows and shear stresses also have to be adapted in accordance with the geometric and kinematic features of the current sliding bearing model. In this fashion, the modified dimensionless equations for the lubricant flows are:

$$
\begin{aligned}
& \bar{q}_{H_{x_{b}}}=-\frac{\bar{\rho}\left(\bar{H}_{2}-\bar{H}_{1}\right)^{3}}{12 \bar{\mu}} \frac{\partial \bar{p}_{H}}{\partial \bar{x}_{b}}+\left(\frac{1}{\Omega}\right) \bar{\rho} \theta\left(\frac{\bar{U}_{s}+\bar{U}_{b}}{2}\right)\left(\bar{H}_{2}-\bar{H}_{1}\right) \\
& \bar{q}_{H_{z_{b}}}=-\left(\frac{1}{r_{L x z}}\right) \frac{\bar{\rho}\left(\bar{H}_{2}-\bar{H}_{1}\right)^{3}}{12 \bar{\mu}} \frac{\partial \bar{p}_{H}}{\partial \bar{z}_{b}}+\left(\frac{r_{L x z}}{\Omega}\right) \bar{\rho} \theta\left(\frac{\bar{W}_{s}+\bar{W}_{b}}{2}\right)\left(\bar{H}_{2}-\bar{H}_{1}\right)
\end{aligned}
$$

Analogously, the dimensionless components of the modified shear stresses acting on the bearing/slider surfaces are:

$$
\begin{aligned}
& \bar{\tau}_{H_{x_{b}}}^{b}=[\bar{\mu} g(\theta)] \dot{\bar{\gamma}}_{H_{x_{b}}}^{b} \rightarrow \dot{\bar{\gamma}}_{H_{x_{b}}}^{b}=-\frac{1}{2 \bar{\mu}} \frac{\partial \bar{p}_{H}}{\partial \bar{x}_{b}}\left(\bar{H}_{2}-\bar{H}_{1}\right)+\left(\frac{1}{\Omega}\right)\left(\frac{\bar{U}_{s}-\bar{U}_{b}}{\bar{H}_{2}-\bar{H}_{1}}\right) \\
& \bar{\tau}_{H_{z_{b}}}^{b}=[\bar{\mu} g(\theta)] \dot{\bar{\gamma}}_{H_{z_{b}}}^{b} \rightarrow \dot{\bar{\gamma}}_{H_{z_{b}}}^{b}=-\frac{1}{2 \bar{\mu} r_{L x z}} \frac{\partial \bar{p}_{H}}{\partial \bar{z}_{b}}\left(\bar{H}_{2}-\bar{H}_{1}\right)+\left(\frac{r_{L x z}}{\Omega}\right)\left(\frac{\bar{W}_{s}-\bar{W}_{b}}{\bar{H}_{2}-\bar{H}_{1}}\right) \\
& \bar{\tau}_{H_{x_{b}}}^{s}=[\bar{\mu} g(\theta)] \dot{\bar{\gamma}}_{H_{x_{b}}}^{s} \rightarrow \dot{\bar{\gamma}}_{H_{x_{b}}}^{s}=\frac{1}{2 \bar{\mu}} \frac{\partial \bar{p}_{H}}{\partial \bar{x}_{b}}\left(\bar{H}_{2}-\bar{H}_{1}\right)+\left(\frac{1}{\Omega}\right)\left(\frac{\bar{U}_{s}-\bar{U}_{b}}{\bar{H}_{2}-\bar{H}_{1}}\right)
\end{aligned}
$$




$$
\bar{\tau}_{H_{z_{b}}}^{s}=[\bar{\mu} g(\theta)] \dot{\bar{\gamma}}_{H_{z_{b}}}^{s} \rightarrow \dot{\bar{\gamma}}_{H_{z_{b}}}^{s}=\frac{1}{2 \bar{\mu} r_{L x z}} \frac{\partial \bar{p}_{H}}{\partial \bar{z}_{b}}\left(\bar{H}_{2}-\bar{H}_{1}\right)+\left(\frac{r_{L x z}}{\Omega}\right)\left(\frac{\bar{W}_{s}-\bar{W}_{b}}{\bar{H}_{2}-\bar{H}_{1}}\right)
$$

\subsubsection{Operational Parameters}

Once the hydrodynamic pressure and fluid fraction (cavitation) fields are obtained from the solution of the Reynolds equation (Eq. 2.28), several operational parameters can be determined in order to describe the overall system lubrication performance. For sliding bearings, in particular, the definition of some operational parameters are summarized in Appendix B.3. 


\section{Chapter 3}

\section{Conformal Elastohydrodynamic Lubrication (EHL) Modelling}

The elastohydrodynamic lubrication (EHL) regime plays an important role in the tribological performance of lubrication systems. It is characterized by significant modifications in the fluid film geometry induced by the fluid-structure interaction (FSI) coupling established between the hydrodynamic pressures and the surfaces (elastic) deformations. Furthermore, the high pressures typically developed in EHL contacts also affect the lubricant rheology (piezoviscous effect), and consequently the calculations of the oil film thicknesses and the hydrodynamic friction losses. It is noteworthy that even under severe EHL conditions, the contact surfaces remain fully separated by a continuum lubricant film, i.e. no asperity contact occurs (the influence of eventual asperity interactions will be discussed later in Chapter 4). In this sense, the EHL regime can be seen as a limiting case of the full hydrodynamic regime.

\subsection{Types of EHL Regimes}

According to the geometry, load magnitude and mechanical properties of the contact surfaces, two subclasses of EHL regimes can be identified [101]:

Hard EHL: EHL regime typically found in lubricated non-conformal contacts, such as line and point contacts involving surfaces with high elastic modulus (metals). In these cases, the structural deformations occur manly due to the surfaces compression caused by the extreme hydrodynamic pressures ( 0.5-3.0 GPa) developed in the contact. Moreover, those extremely high pressures considerably affect the lubricant rheology, especially the piezo-viscosity relationship, and so must be taken into account for proper performance predictions. Examples of lubrication systems operating under hard EHL are: gear tooth flanks, rolling and roller bearings, cams, etc. More details about this type of EHL regime can be found in the elucidative reviews [71, 142, 190, 192, 230].

Soft EHL: EHL regime often developed in lubricated conformal contacts and/or in systems involving surfaces with relatively low elastic modulus, e.g. rubber. Particularly, even for conformal contacts with high elastic modulus and under moderate loads, significant solid deformations can still take place not due to the surfaces compression, but by the global flexibility of the entire structure. In these cases, since the load magnitudes, and therefore the hydrodynamic pressures, are lower, the changes in 
the lubricant rheology may eventually be neglected. Examples of lubrication systems operating under soft EHL are: elastomers, biomechanics devices, journal bearings, etc.

The EHL contacts established in journal and sliding bearing systems are essentially conformal. Thus, only the structural deformations yielded by the overall bearing compliance have to be considered in the calculations [36]. In the following sections, the mathematical formulations employed in the present work for the computation of the surfaces distortions in journal and sliding bearing applications will be described in detail.

\subsection{Conformal EHL Model}

The proper calculation of the surfaces elastic displacements is indispensable for accurate EHL predictions. As already stated, such displacements produce significant changes in the lubricant film thickness and hence in the overall system lubrication behaviour. The first publications dedicated to EHL analyses in journal bearings utilized simple techniques for solving the structural and hydrodynamic problems. In those cases, the structural deformations were calculated from analytical formulations based either on the plane stress or plane strain approximations, while the hydrodynamic pressures were assessed using the short-bearing theory $[79,80,91,167]$. Afterwards, 3D solid deformations were incorporated in the analyses concomitantly to the full solution of the Reynolds equation, which in turn enabled the evaluation of the significance of non-uniform bearing distortions, especially in applications of dynamically loaded connecting-rod big-end bearings of internal combustion engines [132, 157, 194].

In this context, the 3D solid modelling approach will be here adopted to deal with the bearing flexibility for the EHL calculations. In particular, for journal bearing cases characterized by the absence of a hollow pin-journal, the journal compliance tends to be considerably lower than the bearing structure one. Thus, the journal will be always assumed rigid and only the bearing deformations are considered in the simulations carried out later in this thesis.

\subsubsection{Full Structural Model}

The equations of motion for the structural problem will be described according to the classical mathematical theory of linear elasticity of solid mechanics. The main assumptions of the theory are summarized as follows [201]:

1) Solid is assumed as a continuum medium;

2) Lagrangian description of the motion with respect to a reference (undeformed) system configuration;

3) Solid displacements and deformations are assumed of small magnitudes;

4) Linear-elastic, homogeneous and isotropic material behaviour (generalized Hooke's Law).

The complete set of continuum equations that describe the general motion of elastic solid systems can be found in any specialized bibliography, e.g. [201], and so will be here suppressed for brevity. Furthermore, in order to make the overall modelling consistent, the structural displacements are evaluated with respect to the reference frame attached to the 


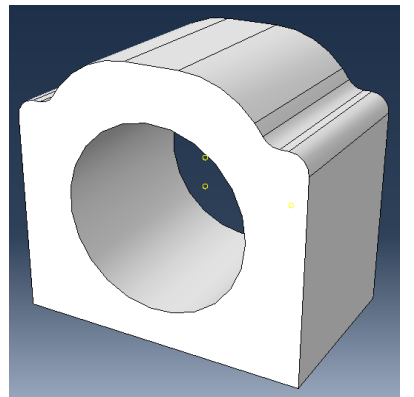

Continuum Model

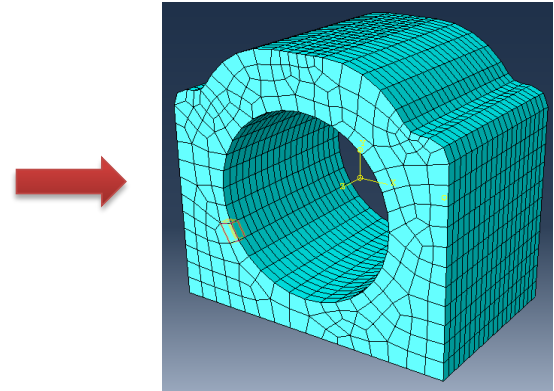

Full FEM Model

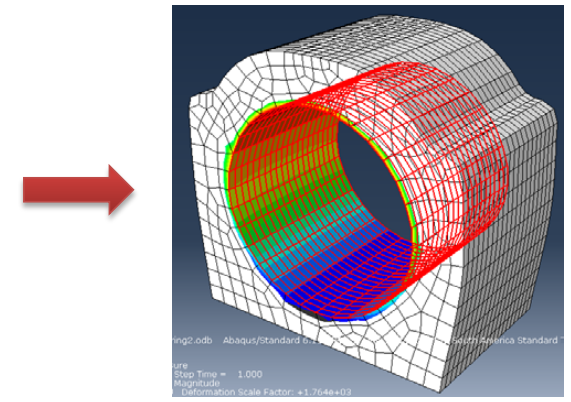

Reduced FEM Model

Figure 3.1: Illustration of the FEM model reduction for conformal EHL systems.

bearing structure $(\mathcal{R})$. Accordingly, the numerical solution of the solid problem is accomplished by solving the following system of ordinary differential equations (ODEs) obtained after the Finite Element Method (FEM) discretization of the continuum equations [19]:

$$
M \ddot{\vec{u}}+B \dot{\vec{u}}+K \vec{u}=\vec{f}
$$

where $\boldsymbol{M}, \boldsymbol{B}$ and $\boldsymbol{K}$ are the mass, damping and stiffness matrices of the full FEM model of the bearing structure, respectively; the system is assumed to have proportional equivalent viscous damping, i.e. $\boldsymbol{B}=\alpha \boldsymbol{M}+\beta \boldsymbol{K}$ where $\alpha$ and $\beta$ are real constant scalars. Vectors $\ddot{\overrightarrow{\boldsymbol{u}}}, \dot{\overrightarrow{\boldsymbol{u}}}$ and $\overrightarrow{\boldsymbol{u}}$ are the respective nodal accelerations, velocities and displacements, and $\overrightarrow{\boldsymbol{f}}$ the nodal force loads. These vector quantities embrace all the (3D) degrees of freedom (DOFs) of every node of the entire FEM model.

\subsubsection{Reduced Structural Model}

In EHL analyses, only the structural displacements of the contact surfaces are important for the calculations. In this fashion, it is convenient to use substructure (or superelement/condensation) techniques [232] to reduce the entire FEM model to an equivalent system that retains only the DOFs of the nodes placed on the bearing surfaces (see Fig. 3.1). Thus, the reduced form of Eq. 3.1 can be written as:

$$
M_{r} \ddot{\vec{u}}_{r}+B_{r} \dot{\vec{u}}_{r}+K_{r} \vec{u}_{r}=\vec{f}_{r}
$$

where subscript $r$ denotes quantities of the reduced system. Notice that unlike the sparse matrices associated with the entire FEM model, the matrices of the reduced system are fully populated due to the inherent whole coupling of the DOFs in substructure systems (super-elements). As for the boundary conditions that are eventually defined for the full FEM model, they are automatically enforced in the condensed model. In this work, the reduced matrices will be generated as a pre-processing procedure in the Abaqus ${ }^{\circledR}$ software [62], whose techniques for system reduction are based on the Guyan and modal condensation methods [59]. The Abaqus ${ }^{\circledR}$ [62] keywords and commands for substructure generation are listed in Appendix C.2.

The nodal load vector of the reduced model can be expressed in terms of the hydrodynamic pressure distribution acting over the entire bearing surface as (see details in Appendix C.1):

$$
\overrightarrow{f_{r}}=A \vec{p}_{H}
$$


where $\boldsymbol{A}$ is the total area matrix and $\overrightarrow{\boldsymbol{p}}_{\boldsymbol{H}}$ the vector of the nodal hydrodynamic pressures acting on the bearing surface. Substituting Eq. 3.3 into Eq. 3.2, one obtains:

$$
M_{r} \ddot{\vec{u}}_{r}+B_{r} \dot{\vec{u}}_{r}+K_{r} \vec{u}_{r}=A \vec{p}_{H}
$$

The kinematic quantities in Eq. 3.4 are originally calculated with respect to a global coordinate system established during the construction of the full FEM model. However, for the correction of the lubricant film thickness, such quantities have to be determined relative to the local bearing reference system where the fluid film geometry is described $\left(\boldsymbol{S}_{\mathcal{B}_{l}}\right.$ for journal bearings and $\boldsymbol{S}_{\mathcal{B}}$ for sliding bearings). Thus, a coordinate transformation has to be considered, yielding the following modified (semi)-dimensionless equation of the condensed model:

$$
\begin{aligned}
& M_{r l} \ddot{\overrightarrow{\tilde{\delta}}}+B_{r l} \dot{\overrightarrow{\hat{\delta}}}+K_{r l} \overrightarrow{\bar{\delta}}=A \vec{p}_{H} \\
& \overrightarrow{\bar{\delta}}=\bar{T}_{g l} \vec{u}_{r} \quad \dot{\overrightarrow{\hat{\delta}}}=\bar{T}_{g l} \dot{\vec{u}}_{r} \quad \ddot{\vec{\delta}}=\bar{T}_{g l} \ddot{\vec{u}}_{r} \\
& M_{r l}=M_{r} \overline{\boldsymbol{T}}_{\boldsymbol{g l}}^{T} \quad \boldsymbol{B}_{\boldsymbol{r l}}=\boldsymbol{B}_{\boldsymbol{r}} \overline{\boldsymbol{T}}_{\boldsymbol{g l}}^{T} \quad \boldsymbol{K}_{\boldsymbol{r l}}=\boldsymbol{K}_{\boldsymbol{r}} \overline{\boldsymbol{T}}_{\boldsymbol{g l}}^{T}
\end{aligned}
$$

In the above equations, $\overrightarrow{\vec{\delta}}, \dot{\overrightarrow{\boldsymbol{\delta}}}$ and $\ddot{\overrightarrow{\boldsymbol{\delta}}}$ are the dimensionless structural displacements, velocities and accelerations, respectively, and $\overline{\boldsymbol{T}}_{\boldsymbol{g} \boldsymbol{l}}$ is the (semi)-dimensionless transformation matrix from the global to the local bearing coordinate systems (see Appendix A.2 for details about the definition of $\left.\overline{\boldsymbol{T}}_{\boldsymbol{g l}}\right)$. Since $\overline{\boldsymbol{T}}_{\boldsymbol{g} \boldsymbol{l}}$ is constant, the modified matrices $\left(\boldsymbol{M}_{\boldsymbol{r} \boldsymbol{l}}\right.$, $\boldsymbol{B}_{\boldsymbol{r l}}$ and $\boldsymbol{K}_{\boldsymbol{r l}}$ ) can be calculated once in a pre-processing step of the simulation. Furthermore, the kinematic vectors in Eqs. 3.5 are sorted according to the local bearing directions (tangential, normal and axial) as:

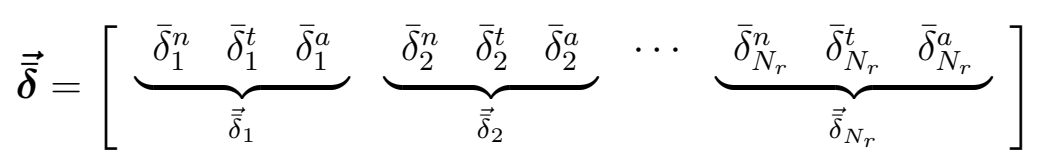

where $N_{r}$ is the number of nodes of the reduced model; the velocity and acceleration vectors are sorted in the same fashion.

\subsection{Solution of the Reduced Structural FEM Model}

The structural problem described mathematically by Eqs. 3.5 can be seen as a linear vibration system externally loaded by the hydrodynamic pressures developed on the bearing interface. In many applications, the solid boundary conditions are not clearly defined in the FEM model and so the structure is often treated as an unconstrained body (e.g. in connecting-rod bearings under dynamic conditions, or in many flexible MDS systems, the structural boundary conditions are not surely determined). In terms of solution, it poses some difficulties regarding the inherent singularity of the stiffness matrix for free-free systems. In order to circumvent this issue, a modal solution based on the suppression of the rigid body modes of the reduced model will be here adopted. Accordingly, in view of the expansion theorem established by the eigenvalue theory of vibration systems [148], the following linear transformation is used:

$$
\overrightarrow{\vec{\delta}}=\Phi_{r l}^{*} \vec{\eta} \quad \dot{\overrightarrow{\hat{\delta}}}=\Phi_{r l}^{*} \dot{\vec{\eta}} \quad \ddot{\vec{\delta}}=\Phi_{r l}^{*} \ddot{\vec{\eta}}
$$


where $\Phi_{r l}^{*}=\left[\begin{array}{llll}\varphi_{D}^{1} & \varphi_{D}^{2} & \varphi_{D}^{3} & \cdots\end{array}\right]$ is the truncated modal transformation matrix and $\overrightarrow{\boldsymbol{\eta}}, \dot{\overrightarrow{\boldsymbol{\eta}}}$ and $\ddot{\overrightarrow{\boldsymbol{\eta}}}$ the modal coordinates. The truncated matrix is constructed by considering only the deformation eigenvectors $\left(\varphi_{D}^{i}\right)$ of the reduced system, i.e. the eigenvectors associated with the rigid body motion (zero eigenvalues) are discarded from the analysis. Notice that similarly to the original modal matrix, $\boldsymbol{\Phi}_{r l}^{*}$ is defined as orthogonal with respect to the mass matrix, i.e. $\boldsymbol{\Phi}_{\boldsymbol{r l}}^{* \boldsymbol{T}} \boldsymbol{M}_{\boldsymbol{r l}} \boldsymbol{\Phi}_{\boldsymbol{r l}}^{*}=\boldsymbol{I}$ and $\boldsymbol{\Phi}_{\boldsymbol{r l}}^{* \boldsymbol{T}} \boldsymbol{K}_{\boldsymbol{r l}} \boldsymbol{\Phi}_{\boldsymbol{r l}}^{*}=\boldsymbol{\Lambda}_{\boldsymbol{r l}}^{*}$, with $\boldsymbol{\Lambda}_{\boldsymbol{r l}}^{*}=\operatorname{diag}\left(\begin{array}{cccc}\lambda_{D}^{1} & \lambda_{D}^{2} & \lambda_{D}^{3} & \cdots\end{array}\right)$ where $\lambda_{D}^{i}$ are the eigenvalues associated with $\boldsymbol{\varphi}_{\boldsymbol{D}}^{\boldsymbol{i}}$. By substituting Eq. 3.7 into Eq. 3.5a and assuming the matrices as symmetric and constant, one obtains:

$$
\ddot{\vec{\eta}}+B_{r l}^{\prime} \dot{\vec{\eta}}+\Lambda_{r l}^{*} \vec{\eta}=\vec{P}
$$

where $\boldsymbol{B}_{r l}^{\prime}=\boldsymbol{\Phi}_{r l}^{* \boldsymbol{T}} \boldsymbol{B}_{\boldsymbol{r l}} \boldsymbol{\Phi}_{\boldsymbol{r l}}^{*}$ and $\overrightarrow{\boldsymbol{P}}=\boldsymbol{\Phi}_{\boldsymbol{r l}}^{* \boldsymbol{T}} \boldsymbol{A} \vec{p}_{\boldsymbol{H}}$ are the respective modal damping matrix and modal force vector.

Finally, after the solution of Eq. 3.8 in the modal domain, the kinematics of the reduced system in the physical domain can be recovered straightforwardly by using the linear transformation defined in Eq. 3.7. For the particular case where the distributed structural inertia is neglected (quasi-static solutions), $\boldsymbol{M}_{\boldsymbol{r l}}=\mathbf{0}$ and $\boldsymbol{B}_{\boldsymbol{r l}}=\mathbf{0}$, so that the solid displacements can be calculated directly from the hydrodynamic pressure distribution as:

$$
\vec{\delta}=L_{r l} \vec{p}_{H}
$$

In the above equation $\boldsymbol{L}_{\boldsymbol{r l}}$ is the flexibility matrix of the reduced system, which for an unconstrained solid may be computed as [83, 215]:

$$
\boldsymbol{L}_{r l}=\left(\boldsymbol{K}_{r l}^{+}\right) \boldsymbol{A}=\left(\boldsymbol{\Phi}_{r l}^{*} \boldsymbol{\Lambda}_{r l}^{*-1} \boldsymbol{\Phi}_{r l}^{* T}\right) \boldsymbol{A}
$$

where superscript ' + ' denotes pseudo-inverse matrix. In fact, $\boldsymbol{L}_{\boldsymbol{r} \boldsymbol{l}}$ and $\boldsymbol{K}_{\boldsymbol{r} \boldsymbol{l}}$ are the pseudoinverse (also called the Moore-Penrose generalized inverses) of each other [83, 215]. 


\section{Chapter 4}

\section{Mixed-Lubrication (ML) Modelling}

The mixed-lubrication regime is characterized by lubricated interfaces where the fluid film geometry is not thick enough to completely separate the contact surfaces. Consequently, direct asperity interactions take place and the lubricant flow is also perturbed by the geometric irregularities of the roughness topographies. Such local features and interactions considerably affect the system lubrication performance, especially by increasing friction losses and superficial damage [101]. The mixed-lubrication regime can be seen as governed by a combination of the inherent effects that rule the boundary (at the asperity contact zones) and full-film hydrodynamic (on the non-contact regions) lubrication regimes. In terms of modelling, both the asperity contacts, as well as the perturbations in the lubricant flow, have to be taken into account for proper numerical predictions. In this context, two different spatial-scale models, namely stochastic and deterministic models, will be considered in this thesis for the mathematical description of the mixedlubrication conditions. Notice that the spatial-scale accounted in each model depends on which components of the surface topographies are used for the computation of the fluid

film geometry. In the stochastic approach, only the overall macroscopic geometry of the contact surfaces are admitted, while in the deterministic cases both the waviness and roughness microscopic components of the topographies are considered.

\subsection{Stochastic Mixed-Lubrication Modelling}

The stochastic description of the mixed-lubrication regime consists in representing the local, roughness scale effects in terms of statistical parameters incorporated in largescale models. In other words, only the overall influence of the local effects are taken into account in the hydrodynamic and asperity contact calculations. In the following sections, the stochastic models here adopted for the asperity contact interactions (GreenwoodWilliamson and Greenwood-Tripp models) and fluid flow perturbation in mixed-lubricated contact (Patir and Cheng formulation) will be exposed in detail.

\subsubsection{Stochastic Asperity Contact Modelling}

The direct contact between solid bodies is limited by the existence of microscopic irregularities on the surface topographies, i.e. surface roughness, which allow the actual interaction of only a small fraction of the nominal (or apparent) contact area. In this context, the real area of contact can be seen as the sum of all micro-areas produced at every discrete contact spot distributed above the surfaces reference planes [171]. 
Over the last fifty years, a substantial amount of stochastic, asperity-based contact models have been proposed. The pioneering work by Greenwood and Williamson (GW) [97] assumed the problem of a nominally flat and parallel rough surface in a statistical fashion by extending the elastic Hertzian theory for an individual asperity to an aggregation of asperities with Gaussian distribution heights. Later, Greenwood and Tripp (GT) [96] enhanced the former formulation by taking into account the contact between two rough surfaces and so the non-aligned interaction among the asperities. Afterwards, many other statistic-based models were proposed by relaxing some assumptions of the GW and GT formulations, but keeping similar trends regarding the linear proportionality between the real contact area and load $[46,99,156,161,179,216]$. Cheng, Etsion and Bogy (CEB) [50] proposed an elastic-plastic asperity model based on the volume conservation of an asperity control volume during plastic deformation. The incorporation of the transitional regime from elastic deformation to fully-plastic flow was achieved by Zhao, Maietta and Chang (ZMC), [224] who provided an elastic-elasto/plastic-fully plastic model that links the elastic and plastic material behaviours of the asperities using an analytical function. Lee and Ree (LE) [133] also proposed curve-fitted formulas obtained from a series of contact simulations correlating the effects of surface topography, material hardness and load on the deformational behaviour of rough surfaces.

More recently, advanced asperity contact models based on finite element analyses have been reported by Kogut and Etsion (KE) [124, 125] and Jackson and Green (JG) $[111,112]$. In these cases, appropriate empirical elasto-plastic correlations obtained from finite element results of a single asperity were extended to multi-asperity formulations. Furthermore, sophisticated models based on the fractal theory have also been suggested to account for the non-Euclidian, self-similar geometry of actual roughness topographies $[47,144,150]$, whose detailed discussion is out of the scope of the present work. A more comprehensive survey for asperity contact models can be found in references [1, 25, 139, 178].

In this thesis, the classical GW/GT models will be adopted for the asperity contact predictions under mixed-lubrication conditions. Despite their limitations, due to their simple computational implementations, the GW/GT models are the most utilized formulations for rough contact and have proven to be robust and efficient to provide satisfactory estimations for design purposes. In fact, most commercial software dedicated to the simulation of lubrication systems, e.g. AVL Excite [17] and Ricardo Software [184], adopt GW/GT models for asperity contact.

\subsubsection{Greenwood-Williamson Model}

The main assumptions of the asperity contact model initially proposed by Greenwood and Williamson are listed as follows [97]:

1) The original contact of two normally flat and parallel rough surfaces is idealized as the contact between a perfectly rigid, smooth plane against a combined rough topography, i.e. an equivalent roughness topography associated with the actual surfaces in contact.

2) The asperity heights of the rough surfaces are admitted randomly distributed and described according to their respective probability density functions.

3) The asperities of the rough surfaces are assumed spherical in shape and with constant mean radius. 


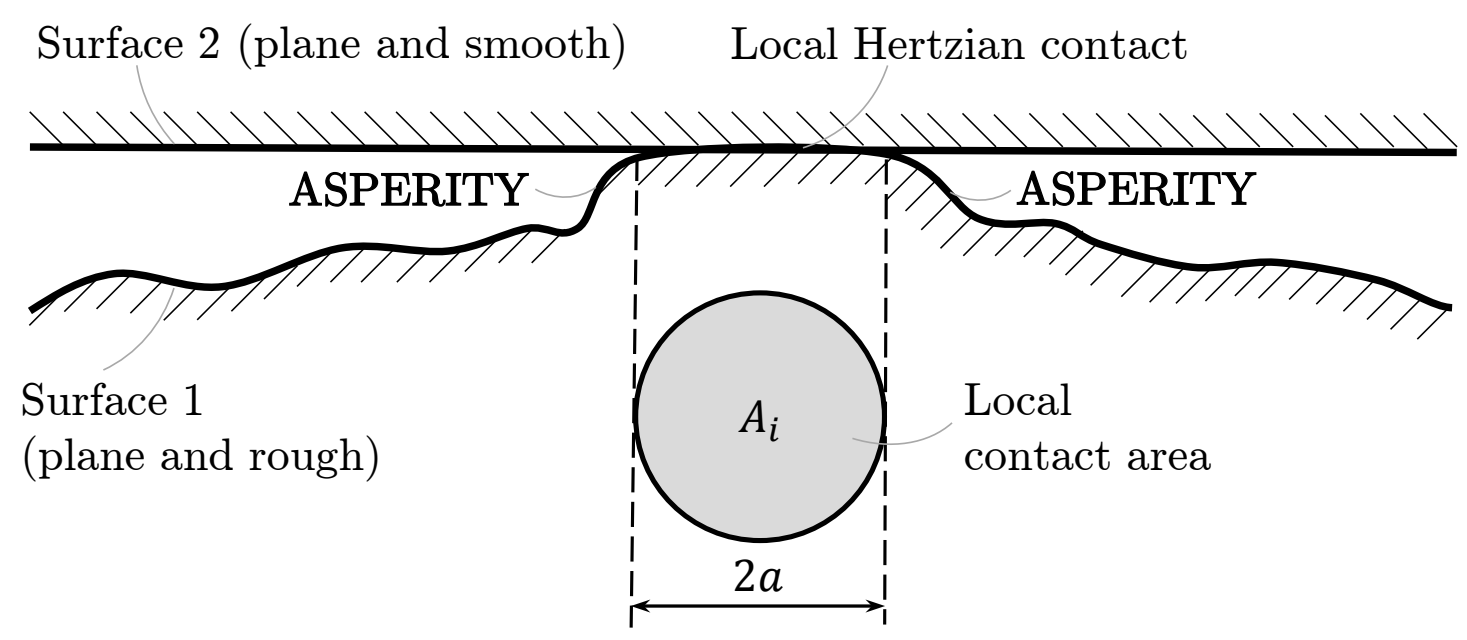

Figure 4.1: Contact of an individual asperity modelled according to the Hertzian theory.

4) The asperities deformations and displacements are assumed small and elastic, and so their influence on the deformation of the neighbours' asperities is neglected.

5) The contact of each asperity is modelled as a Hertzian contact between an elastic semi-sphere against a rigid plane (see Fig. 4.1).

It is noteworthy that the terms asperity and roughness height (or point) have different meanings in the GW/GT formulations. Roughness height is related to any measured point of the topography with no special significance, while an asperity itself corresponds to a particular roughness height whose magnitude is higher than its neighbours. Thus, it is intuitive that only the latter ones are relevant for the contact interactions.

Number of Asperities in Contact The probability of a given asperity of the combined rough surface to be in contact with the rigid plane corresponds to the likelihood of the respective asperity height to be greater than the mean surfaces separation $h^{\prime}=\left(h-Z_{S}\right)$ (see Fig. 4.2). Mathematically:

$$
\operatorname{prob}\left(z>h^{\prime}\right)=\int_{h^{\prime}}^{\infty} \phi(z) d z
$$

where $h^{\prime}$ is the mean surfaces separation determined with respect to the reference planes defined according to the asperity heights distribution (see Fig. 4.2). By assuming there exist $\bar{N}$ asperities on the rough surface, the total number of asperities in contact $\left(n_{A S P}\right)$ as a function of $h^{\prime}$ can be expressed as:

$$
n_{A S P}\left(h^{\prime}\right)=\bar{N} \int_{h^{\prime}}^{\infty} \phi(z) d z
$$

Real Contact Area As already stated, the real contact area is assumed as the sum of all micro-areas yielded by the local deformations at every asperity interaction. In agreement with the aforementioned assumption of the GW model concerning the use of the Hertzian theory for local contact evaluations, the total real contact area $\left(A_{A S P}\right)$ as a function of $h^{\prime}$ 


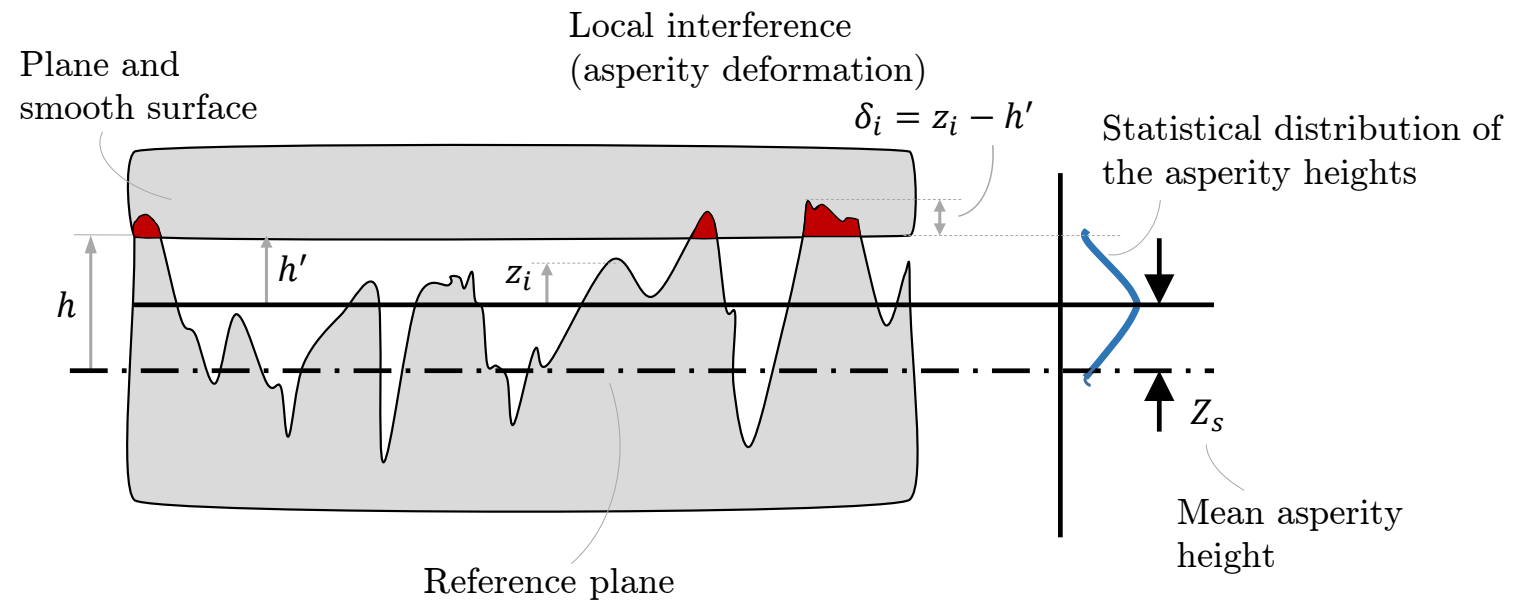

Figure 4.2: Contact between a rigid, smooth plane against a rough surface illustrating the main variables of the $G W / G T$ models.

can be defined as:

$$
A_{A S P}\left(h^{\prime}\right)=\sum_{i=1}^{n_{A S P}} \underbrace{\left[\pi \beta_{S}\left(z_{i}-h^{\prime}\right)\right]}_{\begin{array}{c}
\text { (micro-area" for each } \\
\text { asperity interaction } \\
\text { (Hertzian Theory) }
\end{array}}=\pi \bar{N} \beta_{S} \int_{h^{\prime}}^{\infty}\left[\left(z-h^{\prime}\right) \phi(z)\right] d z
$$

where $\beta_{S}$ is the mean asperity radius of the combined rough surface.

Total Contact Load Analogously to the real contact area, the total contact load can be seen as the sum of all micro-loads acting on every asperity interaction. Thus, by assuming again some definitions of the Hertzian theory, the total contact load $\left(W_{A S P}\right)$ as a function of $h^{\prime}$ can be written as:

$$
W_{A S P}\left(h^{\prime}\right)=\sum_{i=1}^{n_{A S P}} \underbrace{\left[\frac{4}{3} E^{*} \beta_{S}^{1 / 2}\left(z_{i}-h^{\prime}\right)^{3 / 2}\right]}_{\begin{array}{c}
\text { "micro-load" for each } \\
\text { asperity interaction } \\
\text { (Hertzian Theory) }
\end{array}}=\frac{4}{3} \bar{N} E^{*} \beta_{S}^{1 / 2} \int_{h^{\prime}}^{\infty}\left[\left(z-h^{\prime}\right)^{3 / 2} \phi(z)\right] d z
$$

In the above equation, $E^{*}$ is the combined elastic modulus defined as:

$$
\frac{1}{E^{*}}=\left(\frac{1-v_{1}^{2}}{E_{1}}+\frac{1-v_{2}^{2}}{E_{2}}\right)
$$

where $v_{i}$ and $E_{i}$ are the respective Poisson's ratio and the elastic modulus of each contact surface.

For generality, it is convenient to normalize Eqs. 4.2-4.4 by considering the following variables:

$$
\begin{aligned}
\eta_{S} & =\frac{\bar{N}}{A_{0}} \\
\bar{h}_{A S P} & =\frac{h^{\prime}}{\sigma_{S}}=\frac{h-Z_{S}}{\sigma_{S}}
\end{aligned}
$$




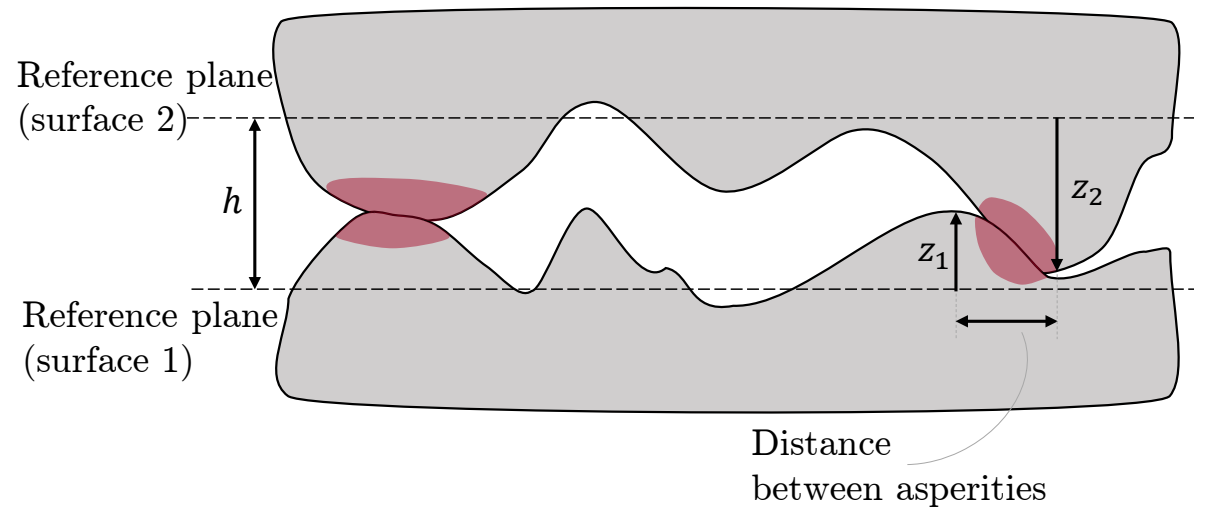

Figure 4.3: Illustration of the misaligned contact between the asperities of two rough surfaces considered in the GT model.

where

$A_{0}$ is the nominal (or apparent) contact area $\left[\mathrm{m}^{2}\right]$;

$\bar{h}_{A S P}$ is the normalized mean surfaces separation $[-]$;

$\eta_{S}$ is the asperity density of the combined rough surface $\left[\mathrm{m}^{-2}\right]$;

$Z_{S}$ is the mean asperity height of the combined rough surface $[\mathrm{m}]$;

$\sigma_{S}$ is the standard deviation of the asperity heights of the combined rough surface $[\mathrm{m}]$

Thus, the normalized version of Eqs. 4.2-4.4 can be rewritten as:

$$
\begin{aligned}
n_{A S P}^{(G W)}\left(\bar{h}_{A S P}\right) & =\eta_{S} A_{0} F_{0}\left(\bar{h}_{A S P}\right) \\
A_{A S P}^{(G W)}\left(\bar{h}_{A S P}\right) & =\pi\left(\eta_{S} \beta_{S} \sigma_{S}\right) A_{0} F_{1}\left(\bar{h}_{A S P}\right) \\
W_{A S P}^{(G W)}\left(\bar{h}_{A S P}\right) & =\frac{4}{3} E^{*}\left(\eta_{S} \beta_{S}^{1 / 2} \sigma_{S}^{3 / 2}\right) A_{0} F_{3 / 2}\left(\bar{h}_{A S P}\right) \\
F_{m}\left(\bar{h}_{A S P}\right) & =\int_{\bar{h}_{A S P}}^{\infty}\left[\left(s-\bar{h}_{A S P}\right)^{m} \phi^{*}(s)\right] d s
\end{aligned}
$$

where $\phi^{*}$ is the normalized probability density function of the asperity height distribution. Furthermore, it is still advantageous to represent the real contact as a proportion of the nominal area, as well as the total load in terms of the average contact pressure. Mathematically:

$$
\begin{aligned}
& \bar{A}_{A S P}^{(G W)}\left(\bar{h}_{A S P}\right)=\frac{A_{A S P}^{(G W)}}{A_{0}}=\pi\left(\eta_{S} \beta_{S} \sigma_{S}\right) F_{1}\left(\bar{h}_{A S P}\right) \\
& p_{A S P}^{(G W)}\left(\bar{h}_{A S P}\right)=\frac{W_{A S P}^{(G W)}}{A_{0}}=\frac{4}{3} E^{*}\left(\eta_{S} \beta_{S}^{1 / 2} \sigma_{S}^{3 / 2}\right) F_{3 / 2}\left(\bar{h}_{A S P}\right)
\end{aligned}
$$

According to Eqs. 4.7-4.8, all the results of the GW model clearly depend on the statistical distribution of the asperity heights. Such dependence is implicitly established by the integrals $F_{m}$, whose values are here determined numerically and fitted to polynomial 
curves; the coefficients of the polynomials for a Gaussian asperity height distribution can be found in Appendix E.2. Additionally, the asperity contact effects are also influenced by the mechanical properties of the surfaces materials $\left(v_{i}, E_{i}\right)$, as well as by four combined statistical parameters $\left(Z_{S}, \sigma_{S}, \beta_{S}, \eta_{S}\right)$ that describe the global features of the asperities. In general, those combined parameters relating both contact surfaces can be obtained from the following relationships [146]:

$$
\begin{aligned}
Z_{S} & =\sqrt{Z_{S_{1}}^{2}+Z_{S_{2}}^{2}} \\
\sigma_{S} & =\sqrt{\sigma_{S_{1}}^{2}+\sigma_{S_{2}}^{2}} \\
\frac{1}{\beta_{S}} & =\sqrt{\frac{1}{\beta_{S_{1}}^{2}}+\frac{1}{\beta_{S_{2}}^{2}}} \\
\eta_{S} & =\sqrt{\eta_{S_{1}}^{2}+\eta_{S_{2}}^{2}}
\end{aligned}
$$

where subscript $i$ denotes each contact surface.

\subsubsection{Greenwood-Tripp Model}

In the GW formulation, since the contact is idealized as occurring between a rigid, smooth plane against a combined rough surface, only the normal interactions at the asperities tips are considered. In reality, though, both surfaces are mutually rough and so the misaligned contact on the asperities shoulders (see Fig. 4.3) have to be taken into account to provide more accurate predictions. In order to overcome such drawback, Greenwood and Tripp (GT) [96] proposed a generalization of the GW model that admits the actual contact between two rough plane surfaces. In the new formulation, the surfaces were admitted as covered with paraboloidal asperities and the tangential forces locally generated at the non-aligned interactions are ignored due to the relatively small inclination of the asperities.

Accordingly, the expressions for the total number of asperities in contact, the proportion of real contact area and the contact pressure established by the GT model can be written in the normalized form as:

$$
\begin{aligned}
n_{A S P}^{(G T)}\left(\bar{h}_{A S P}\right) & =4 \pi\left(\eta_{S} \beta_{S} \sigma_{S}\right) \eta A_{0} F_{1}\left(\bar{h}_{A S P}\right) \\
\bar{A}_{A S P}^{(G T)}\left(\bar{h}_{A S P}\right) & =\pi^{2}\left(\eta_{S} \beta_{S} \sigma_{S}\right)^{2} F_{2}\left(\bar{h}_{A S P}\right) \\
p_{A S P}^{(G T)}\left(\bar{h}_{A S P}\right) & =\frac{16 \pi \sqrt{2}}{15} E^{*}\left(\eta_{S}^{2} \beta_{S}^{3 / 2} \sigma_{S}^{5 / 2}\right) F_{5 / 2}\left(\bar{h}_{A S P}\right) \\
F_{m}\left(\bar{h}_{A S P}\right) & =\int_{\bar{h}_{A S P}}^{\infty}\left[\left(s-\bar{h}_{A S P}\right)^{m} \phi^{*}(s)\right] d s
\end{aligned}
$$

The combined statistical parameters are the same as those defined in Eqs. 4.9.

\subsubsection{Asperity Contact Friction}

The friction forces produced by the asperity interactions will be here calculated based on the Coulomb-Amontons' Laws applied to dry solid contacts. The three laws are summarized below [193]: 
1) The friction force is independent of the surfaces velocities;

2) The friction force is directly proportional to the external loads;

3) The friction force is independent of the nominal contact area.

Therefore, the contact shear stresses can be defined mathematically as:

$$
\tau_{A S P}^{(G W, G T)}\left(\bar{h}_{A S P}\right)=\mu_{B L} p_{A S P}^{(G W, G T)}\left(\bar{h}_{A S P}\right)
$$

where $\mu_{B L}$ is the boundary coefficient of friction often obtained from experimental tests.

Once the contact shear stresses are determined, the associated friction forces can be easily calculated by numerical integration over the contact domain.

\subsubsection{Dimensionless Expressions}

According to the dimensionless parameters listed in Appendix E.1, the dimensionless form of the asperity contact pressures and shear stresses can be stated as:

$$
\begin{aligned}
\bar{p}_{A S P}^{(G W)}\left(\bar{h}_{A S P}\right) & =\bar{E}^{*}\left(\bar{\eta}_{S} \bar{\beta}_{S}^{1 / 2} \bar{\sigma}_{S}^{3 / 2}\right) F_{3 / 2}\left(\bar{h}_{A S P}\right) \\
\bar{p}_{A S P}^{(G T)}\left(\bar{h}_{A S P}\right) & =\bar{E}^{*}\left(\bar{\eta}_{S}^{2} \bar{\beta}_{S}^{3 / 2} \bar{\sigma}_{S}^{5 / 2}\right) F_{5 / 2}\left(\bar{h}_{A S P}\right) \\
\bar{\tau}_{A S P}^{(G W, G T)}\left(\bar{h}_{A S P}\right) & =\left(\bar{\mu}_{B L}\right) \bar{p}_{A S P}^{(G W, G T)}\left(\bar{h}_{A S P}\right)
\end{aligned}
$$

\subsubsection{Limit for Elastic Deformation}

Based on the classical theory of contact mechanics, a given asperity in contact begins to undergo plastic deformations whenever the magnitude of the mean contact pressure exceeds the yield strength of the softer surface material by $10 \%$ [117]. In this case, plastic deformation regions are initially verified underneath the interface and expand towards the surface as the contact load is increased. Conversely, the mean contact pressure also rises asymptotically until it reaches a maximum value of approximately three times the yield strength of the softer material (hardness), remaining unchanged even under further growth of the contact forces [109].

The occurrence of plastic deformations at the roughness/asperity scale depends on both the geometric features of the surface topographies, as well as on the constitutive properties of the contact materials. In order to assess the susceptibility of a given rough contact to experience plastic deformations, Greenwood and Williamson [97] proposed the following dimensionless quantity denominated plasticity index:

$$
\psi=\left(\frac{E^{*}}{H_{V}}\right)\left(\frac{\sigma_{S}}{\beta_{S}}\right)^{1 / 2}
$$

where $H_{V}$ is the Vickers hardness of the softer surface material.

For $\psi<0.6$ elastic deformations are predominant, while for $\psi>1$ most of the asperities in contact are plastically deformed. For metallic materials with usual engineering surface finishes, $\psi>1$ [109]. However, after the first running-in cycles under prevalent plastic contacts, the asperities are hardened and so the superficial yield strength is increased (shakedown mechanism). Therefore, the following asperity interactions tend to remain under the elastic regime, so that justifying the use of the GW/GT models (originally derived for elastic contacts) for rough contact in a wide range of engineering simulations. 


\subsubsection{Operational Parameters}

After the asperity contact pressure field is calculated from Eqs. 4.7, 4.8 and 4.10, several operational parameters can be determined for the description of the overall rough contact effects on the bearing systems. The definitions of some parameters are summarized in Appendices A.5 and B.3.

\subsubsection{Stochastic Hydrodynamic Modelling}

As already stated in the previous sections, full-film hydrodynamic lubrication is accurately modelled by the Reynolds equation that, for general purposes, is solved numerically in the discrete domain. However, when roughness scale is involved, many degrees of freedom are needed to properly capture the local geometric features of the interface, requiring much more computational efforts. Therefore, various methods have been proposed in the literature to avoid dense discretization grids for solving the fluid flow problem in mixedlubricated contacts.

The effect of surface roughness on lubrication was first reported by Tzeng and Saibel [206] for one-dimensional transversal roughness and followed by Christensen [54-56] and Chow and Cheng [53] for two-dimensional transverse and longitudinal topographies. All of those pioneering works were established within the framework of the stochastic process theory, which is based on the concept of viewing the film thickness as a stochastic process that results in a Reynolds type equation for the mean or expected pressure.

Patir and Cheng $[164,165]$ were the first to propose an average flow model for general roughness patterns by incorporating "flow factor" coefficients directly into a modified Reynolds equation that is solved in the smooth global domain. The flow factors are determined independently by solving the local deterministic flow problem for a specified roughness topography. Unlike the Christensen's methodology that particularly weights the film thickness oscillations according to a expectancy operator defined for a given roughness height distribution, the Patir and Cheng model is based on mean flow quantities obtained by solving Reynolds equation at the microscope scale for a representative bearing cell. The major drawback of this approach is a consequence of its heuristic derivation, which poses limitations on dealing with eventual cross-flow produced in case of surface roughness anisotropy, i.e. roughness directions are not identical to the coordinate axes. Such lack of generality was first highlighted by Elrod [76] and subsequently by many authors, including Tripp [205] who proposed a more complete tensor form of the averaged Reynolds equation using stochastic approach. In the latter case, the effects of roughness anisotropy on lubrication are accounted for in the off-diagonal terms of the diffusion and convective flow tensors; in particular, when the off-diagonal terms are negligible the Tripp model is essentially identical to that of Patir and Cheng.

Afterwards, the modelling of the fluid flow problem in the mixed-lubrication regime was addressed by Bayada [20, 22] and later by Jai [113] and Buscaglia [43-45] within the framework of the homogenization theory for spatially periodic roughness. This approach is based on the derivation of a homogenized Reynolds equation, defined at the macroscopic scale, which captures the overall effects of the surface roughness on the lubricant flow. Besides posing the average flow model on a more rigorous mathematical basis that overcomes the pure heuristic induction of the Patir and Cheng's model, one further feature of the homogenization technique is the definition of the local (or auxiliary) problem, which has to be solved over a periodic unit cell in order to compute the average flow tensors [169]. With this approach, statistical information related to the short length and time 


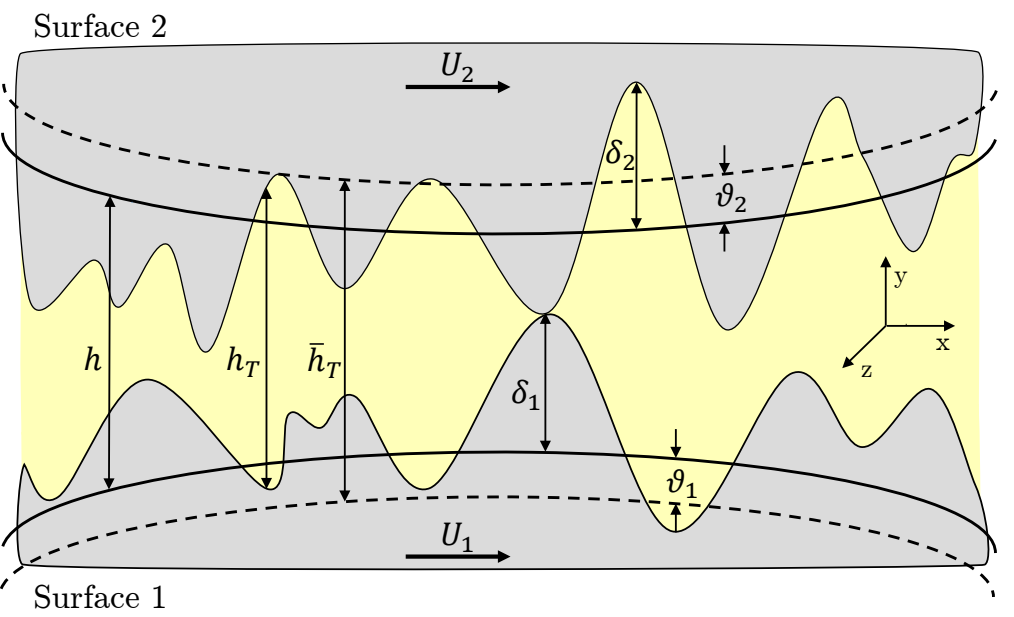

Figure 4.4: Definition of the total film thickness for the Patir and Cheng's average flow model.

scale effects is provided to the homogenized (averaged) Reynolds equation from the local problem solutions. Other more recent publications in which the homogenization method is used are references [4-9, 21, 23, 24, 114, 118, 186, 187].

In the mixed-lubrication models discussed above, the local roughness slope is always assumed small (Reynolds roughness), so that the flow equations at the microscopic scale are well described by the Reynolds equation, i.e. the local inertia effects can be neglected without significant loss of accuracy. Furthermore, the direct asperity contact under very small film thickness is generally not considered during the flow factors calculations, except in references [169, 187].

In this context, despite the wide variety of methodologies with different complexities existing in the literature to describe the fluid flow behaviour under mixed-lubrication conditions, the Patir and Cheng average approach, in particular, has proven effective in many applications and is thus used in the majority of theoretical models in tribology. Therefore, this flow average model will be adopted in the present work for modelling the mixed-lubrication regime.

\subsubsection{Patir and Cheng's Average Flow Model}

The average flow model proposed by Patir and Cheng is based on expressing the average lubricant flow quantities, e.g. the mean hydrodynamic pressure and nominal film thickness, in terms of flow factors obtained by numerically solving the Reynolds equation deterministically on a set of local, microscopic bearing cells. Thus, an average Reynolds equation governing the mean pressure at the global, macroscopic scale is derived in terms of those flow factor coefficients $[164,165]$.

Film Thickness and Roughness Functions According to Fig. 4.4, the local film thickness $\left(h_{T}\right)$ between two rough surfaces can be defined as:

$$
h_{T}=h+\underbrace{\delta_{2}^{R}+\delta_{1}^{R}}_{\delta_{R}}
$$

where $\delta_{i}^{R}$ is the roughness amplitude of the contact surfaces measured from their reference plane (attention to the orientation of the local coordinate systems) and $h$ the nominal film thickness that already includes eventual surface deformations in EHL contacts. 
Moreover, $\delta_{R}=\left(\delta_{2}^{R}+\delta_{1}^{R}\right)$ is the combined roughness with composite standard deviation $\sigma_{R}=\sqrt{\left(\sigma_{2}^{R}\right)^{2}+\left(\sigma_{1}^{R}\right)^{2}}$. It is also assumed that the roughness has small slopes so that Reynolds equation is locally applicable (Reynolds roughness). In addition, it is important to highlight that the Patir and Cheng's average flow model is independent of the roughness pattern in study and can be adopted with any type of isotropic topography.

The film thickness ratio $\Lambda=\frac{h}{\sigma_{R}}$ is defined to emphasize the relative influence of surface roughness on lubrication $[164,165]$. This influence is more significant for $\Lambda<3$, which is termed partial lubrication regime as a result of the occurrence of asperity interactions. In this regime, the total load and friction are shared by the combination of the hydrodynamic and rough contact effects. For $\Lambda \gg 3$, the film thickness tends to be sufficiently large so that the roughness effects are not important and the smooth film theory is satisfactorily accurate.

Average Reynolds Equation The Patir and Cheng's derivation of the average Reynolds equation consists in heuristically defining the expected unit lubricant flows described in terms of pressure $\phi_{p_{x, z}}$ and shear $\phi_{s_{x, z}}$ flow factors as [164]:

$$
\begin{aligned}
q_{H_{x}}^{*} & =-\phi_{p_{x}} \frac{\rho h^{3}}{12 \mu} \frac{\partial p_{H}^{*}}{\partial x}+\rho\left(\frac{U_{1}+U_{2}}{2}\right) h_{T}^{*}-\rho\left(\frac{U_{2}-U_{1}}{2}\right) \sigma_{R} \phi_{s_{x}} \\
q_{H_{z}}^{*} & =-\phi_{p_{z}} \frac{\rho h^{3}}{12 \mu} \frac{\partial p_{H}^{*}}{\partial z}+\rho\left(\frac{W_{1}+W_{2}}{2}\right) h_{T}^{*}-\rho\left(\frac{W_{2}-W_{1}}{2}\right) \sigma_{R} \phi_{s_{z}}
\end{aligned}
$$

where superscript ' $*$ ' denotes mean or expected quantities. In the above definition, the mean lubricant flows are assumed to be a combination of three terms:

$\mathbf{1}^{\text {st }}$ Term: Average flow due to the pressure gradients. In this case, the pressure flowfactors $\phi_{p_{x, z}}$ can be seen as a correction factor relating the mean Poiseuille flow in rough surfaces to that of smooth ones having the same nominal geometry.

$2^{\text {nd }}$ Term: Lubricant flow due to the movement of the contact surfaces (Couette flow).

$3^{\text {rd }}$ Term: Additional flow transport induced by the relative motion of the rough contact surfaces. Notice that this term is not originally present in the Reynolds equation for smooth surfaces, and so it can be interpreted physically as the combined perturbation effect yielded by the superficial roughness and sliding onto the fluid flow. For instance, considering a rough surface sliding against a stationary smooth one, the lubricant carried in the valleys of the moving rough topography contributes to the fluid flow transport through the contact gap $\left(\phi_{s_{x, z}}>0\right)$. Conversely, for a smooth surface sliding against a stationary rough one, the lubricant transport will be interfered by the fluid entrapment in the valleys of the rough surface $\left(\phi_{s_{x, z}}<0\right)$.

Finally, by performing a mean flow balance on a control volume with height $h_{T}$ and area big enough to include a large number of asperities (microscopic scale), but still small compared to the size of the global bearing system (macroscopic scale), the following average Reynolds equation with mass-conserving cavitation model ( $p-\theta$ model) for rough contacts is obtained:

$$
\nabla \cdot\left(\boldsymbol{\Gamma}^{\boldsymbol{p}^{*}} \nabla p_{H}^{*}\right)=\nabla \cdot\left(\theta^{*}\left[\boldsymbol{\Gamma}^{\boldsymbol{c}^{*}} \overrightarrow{\boldsymbol{v}}+\boldsymbol{\Gamma}^{\boldsymbol{c} \boldsymbol{r}^{*}} \overrightarrow{\boldsymbol{v}}_{\boldsymbol{r}}\right]\right)+\theta^{*}\left[S_{T S}^{*}+S_{N S}^{*}\right]+S_{T}^{*} \frac{\partial \theta^{*}}{\partial t}
$$


with the complementary conditions:

$$
\left(p_{H}^{*}-p_{\text {cav }}^{*}\right)\left(1-\theta^{*}\right)=0 \rightarrow \begin{cases}p_{H}^{*}>p_{c a v}^{*} & \rightarrow \quad \theta^{*}=1 \\ p_{H}^{*}=p_{c a v}^{*} & \rightarrow \quad 0 \leq \theta^{*}<1\end{cases}
$$

where the modified diffusive and convective matrices, as well as the modified translation and normal squeeze terms and the temporal expansion term are expressed as:

$$
\begin{aligned}
& \Gamma^{\boldsymbol{p}^{*}}=\left[\begin{array}{cc}
\frac{\rho\left(H_{2}-H_{1}\right)^{3}}{12 \mu} \phi_{p_{x}} & 0 \\
0 & \frac{\rho\left(H_{2}-H_{1}\right)^{3}}{12 \mu} \phi_{p_{z}}
\end{array}\right] \\
& \boldsymbol{\Gamma}^{\boldsymbol{c}^{*}}=\left[\begin{array}{cc}
\rho\left(H_{2}-H_{1}\right) \phi_{c} & 0 \\
0 & \rho\left(H_{2}-H_{1}\right) \phi_{c}
\end{array}\right] \\
& \boldsymbol{\Gamma}^{c \boldsymbol{r}^{*}}=\left[\begin{array}{cc}
-\rho \sigma_{R} \phi_{s_{x}} & 0 \\
0 & -\rho \sigma_{R} \phi_{s_{z}}
\end{array}\right] \\
& S_{T S}^{*}=\rho\left(\overrightarrow{\boldsymbol{a}} \cdot \nabla H_{1}-\overrightarrow{\boldsymbol{b}} \cdot \nabla H_{2}\right) \phi_{c} \\
& S_{N S}^{*}=\rho\left(V_{2}-V_{1}\right) \phi_{c} \\
& S_{T}^{*}=\rho\left(H_{2}-H_{1}\right) \phi_{c} \\
& \overrightarrow{\boldsymbol{v}}=\left[\begin{array}{ll}
\frac{\left(U_{2}+U_{1}\right)}{2} & \frac{\left(W_{2}+W_{1}\right)}{2}
\end{array}\right]^{T} \\
& \overrightarrow{\boldsymbol{v}}_{\boldsymbol{r}}=\left[\begin{array}{ll}
\frac{\left(U_{2}-U_{1}\right)}{2} & \frac{\left(W_{2}-W_{1}\right)}{2}
\end{array}\right]^{T} \\
& \overrightarrow{\boldsymbol{a}}=\left[\begin{array}{ll}
U_{1} & W_{1}
\end{array}\right]^{T} \\
& \overrightarrow{\boldsymbol{b}}=\left[\begin{array}{ll}
U_{2} & W_{2}
\end{array}\right]^{T}
\end{aligned}
$$

In the above equations, a new dimensionless coefficient denoted as contact factor $\left(\phi_{c}\right)$ is introduced to deal with the average or expected roughness values of $\delta_{R}, \delta_{1}^{R}$ and $\delta_{2}^{R}$. See details in Appendix D.1 on how $\phi_{c}$ contact factor can be estimated as a function of the surfaces nominal separation. Approximations for the pressure and shear flow factors for topographies with Gaussian roughness height distributions can be found in Appendix D.2.

Average Shear Stresses Similarly to the derivation of the average Reynolds equation, the average hydrodynamic shear stresses acting on each rough surface can be defined in terms of shear stress (or friction) flow factors $\phi_{f p_{x, z}}$ and $\phi_{f s_{x, z}}$ as [165]:

$$
\begin{aligned}
& \tau_{H_{x}}^{1^{*}}=\left[\mu g\left(\theta^{*}\right)\right] \dot{\gamma}_{H_{x}}^{1^{*}} \rightarrow \dot{\gamma}_{H_{x}}^{1^{*}}=-\frac{1}{2 \mu} \frac{\partial p_{H}^{*}}{\partial x}\left(H_{2}-H_{1}\right) \phi_{f p_{x}}+\left(\frac{U_{2}-U_{1}}{H_{2}-H_{1}}\right)\left(\phi_{f}-\phi_{f s_{x}}\right) \\
& \tau_{H_{x}}^{2^{*}}=\left[\mu g\left(\theta^{*}\right)\right] \dot{\gamma}_{H_{x}}^{2^{*}} \rightarrow \dot{\gamma}_{H_{x}}^{2^{*}}=\frac{1}{2 \mu} \frac{\partial p_{H}^{*}}{\partial x}\left(H_{2}-H_{1}\right) \phi_{f p_{x}}+\left(\frac{U_{2}-U_{1}}{H_{2}-H_{1}}\right)\left(\phi_{f}+\phi_{f s_{x}}\right) \\
& \tau_{H_{z}}^{1^{*}}=\left[\mu g\left(\theta^{*}\right)\right] \dot{\gamma}_{H_{z}}^{1^{*}} \rightarrow \dot{\gamma}_{H_{z}}^{1^{*}}=-\frac{1}{2 \mu} \frac{\partial p_{H}^{*}}{\partial z}\left(H_{2}-H_{1}\right) \phi_{f p_{z}}+\left(\frac{W_{2}-W_{1}}{H_{2}-H_{1}}\right)\left(\phi_{f}-\phi_{f s_{z}}\right)
\end{aligned}
$$




$$
\tau_{H_{z}}^{2^{*}}=\left[\mu g\left(\theta^{*}\right)\right] \dot{\gamma}_{H_{z}}^{2^{*}} \rightarrow \dot{\gamma}_{H_{z}}^{2^{*}}=\frac{1}{2 \mu} \frac{\partial p_{H}^{*}}{\partial z}\left(H_{2}-H_{1}\right) \phi_{f p_{z}}+\left(\frac{W_{2}-W_{1}}{H_{2}-H_{1}}\right)\left(\phi_{f}+\phi_{f s_{z}}\right)
$$

where $\phi_{f}$ is the friction contact factor whose definition can be seen in Appendix D.1. Approximations of the shear stress flow factors for surfaces with Gaussian roughness height distributions can be found in Appendix D.2.

Notice that the average Reynolds equation displayed in Eq. 4.16a and the average shear stresses of Eqs. 4.18 were expressed generically in a Cartesian coordinate system, but its particular usage for journal or sliding bearing applications follows the same procedures described in Sections 2.4 and 2.5, respectively. In other words, only the tensor terms of Eq. 4.17 have to be adjusted. Moreover, superscript ' $*$ ' will be hereafter suppressed for clearness whenever the average flow model is considered.

\subsection{Deterministic Mixed-Lubrication Modelling}

As seen in the previous sections, stochastic models use selected statistic quantities, e.g. asperity contact parameters and flow factor coefficients, to account for the surface roughness influence on the mixed-lubrication regime. In these cases, the hydrodynamic and rough contact pressures are calculated separately and then superimposed to balance the external applied loads. They are useful in capturing general features and handling problems with large solution domains. However, the use of such modelling approach is not precise enough for studies involving material and surface finishing investigations, since the microscopic geometric details of the roughness topographies are not included in the formulations.

Realistic deterministic models are indispensable for more in-depth simulation assessments of the boundary and mixed-lubrication regimes. They directly couple the tribosystem performance to the materials and surfaces design, and provide a reliable base for the development, refinement and verification of stochastic analytical models.

In the last few decades, fast advancing computational power allowed the digitalization of engineering topographies and the development of improved numerical techniques (e.g. multigrid methods and parallel computation) for the solution of the contact and lubrication problems. Therefore, increasingly greater attention has been devoted to deterministic mixed-lubrication simulations.

Early deterministic solutions of the full-film EHL regime under limited rough contact were undertaken for artificial roughness topographies with simple geometric irregularities, such as dimples and sinusoidal waviness [2, 48, 49, 130, 141]. Actual 2D roughness profiles were then employed by [131, 209] and later 3D measured topographies were used by $[219,226]$.

More recently, $\mathrm{Hu}$ and $\mathrm{Zhu}$ [106, 225, 227-231] proposed a fully coupled mixed-EHLlubrication model that assumes the lubricant film continuous in the non-contacting regions and the occurrence of asperity contact wherever the local fluid film is sufficiently thin. In this model, the film thickness is computed from the deformed average gap and the lubricant flows and asperity interactions are accounted for in a unified solution framework. With this formulation, 3D roughness surfaces can be used as input data to simulate the entire transitions from full-film and mixed-EHL regimes, along with the boundary lubrication under severe conditions. Since no averaging technique was adopted, the model only may be applied to small regions such as point contacts. 


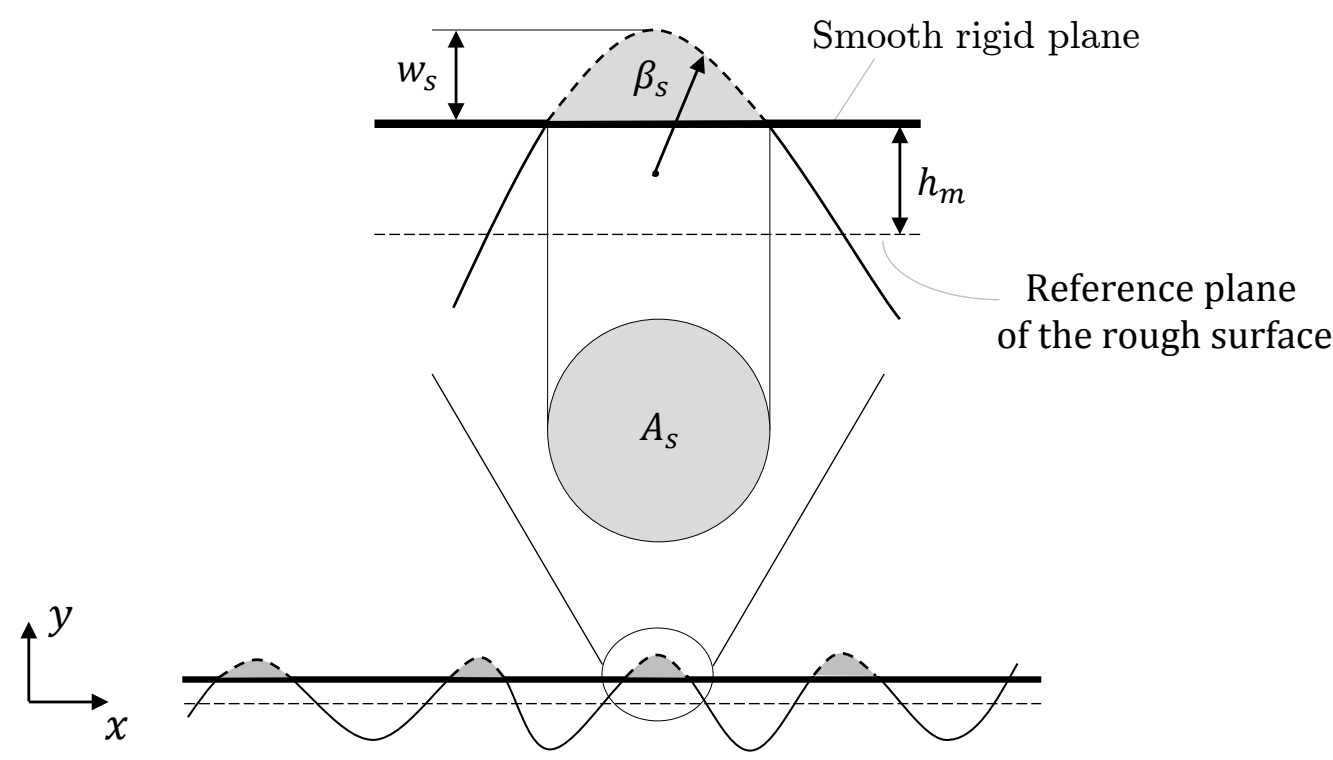

Figure 4.5: Schematic illustration of an asperity interaction and the respective quantities associated with the deterministic contact model.

Li and Chen [51, 52, 136-138] proposed a deterministic mixed-lubrication model for the simulation of Twin Land Oil Controls Rings (TLOCRs) sliding against cylinder liner topographies of internal combustion engines. The model is based on the calculation of the oil transport and the hydrodynamic pressure generation on the contact between a parallel, flat rigid plane and a rough surface under a reference sliding speed and constant mean separations. Thus, a correlation method was introduced to estimate the hydrodynamic carrying capacity and the associated viscous friction forces for different operating velocities and fluid film thicknesses. However, the local asperity interactions have been neglected in the formulation.

The deterministic mixed-lubrication regime model proposed in the present work consists in an extension of the $\mathrm{Li}$ and Chen's approach. The novelty is based on the further calculation of the local asperity contact interactions concomitantly to the hydrodynamic solutions. More details about the proposed model are exposed in the following sections. The results from the herein devised deterministic model have been published by the author in references [67, 68, 172, 175, 176].

\subsubsection{Deterministic Asperity Contact Model}

The pressure that arises when an individual asperity is brought into contact with a smooth, rigid plane is calculated by assuming an elastic-perfectly plastic model, whose pressures in the elastic regime are computed based on the classical Hertz formulas for spherical-shaped bodies and the plastic deformations are limited by surface hardness of the softer material. Thus, the real contact area $\left(A_{s}\right)$ and the mean contact pressure $\left(p_{A S P_{s}}\right)$ for each asperity interaction can be expressed as [117]:

$$
\begin{aligned}
& A_{s}=\pi \beta_{s} w_{s}
\end{aligned}
$$

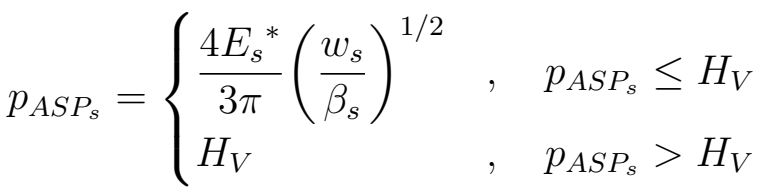


where $w_{s}$ is the contact interference (see Fig. 4.5), $\beta_{s}$ the local asperity radius, $E_{s}{ }^{*}$ the combined elastic modulus of the asperity and $H_{V}$ the hardness of the softer material. $\beta_{s}$ is determined locally by approximating the geometry of each asperity to a spherical-shape fitted surface.

For a given mean separation $\left(h_{m}\right)$ between the rough surface and the rigid plane, the integration of Reynolds equation and the contact calculations are simultaneously performed. At the contact spots, no hydrodynamic pressure is admitted (boundary contact), and a threshold value of $h_{\text {lim }}=1 \mathrm{~nm}$ is adopted for the minimum film thickness to avoid singularities during the solution of Reynolds equation. In other words, wherever $0<h_{T}<h_{\text {lim }}$, the local gap is fixed to $h_{\text {lim }}$, i.e. $h_{T} \rightarrow h_{l i m}$. Additionally, for every point with $h_{T}<0$, contact interference takes place $\left(w_{s}>0\right)$ and so only boundary contact occurs.

It is worth noticing that the threshold of $h_{\text {lim }}=1 \mathrm{~nm}$ was adopted based on the smaller gap from which hydrodynamic flow (either pressure or shear flow) is possible, below which continuum fluid flow would become impossible. According to reference [225], the magnitude of $\sim 1 \mathrm{~nm}$ for the cut-off film thickness value is a good estimate that is in accordance with the average molecular sizes of most mineral and synthetic oils (assuming the smallest possible lubricant molecule width/thickness layer). Further references [207, 223] also admit the same magnitude for the smaller gap from which continuum mechanics can be used to describe the lubricant flow. The influence of different threshold values on the overall results is difficult to speculate. Although such evaluation is interesting and worth to be undertaken in future contributions, this is out of the scope of the present work.

Furthermore, for very small film thickness and high shear rate conditions, especially those observed at the contact spots, liquid lubricants might undergo shear-thinning effects (see Section 2.3.2) and generally a limiting shear stress behaviour. In order to account for such situation in the present deterministic modelling, the calculated local hydrodynamic shear stresses are constrained to a limit value $\tau_{L}$ and kept constant in case of further shear rate increase [192]; the value of $\tau_{L}=1 \mathrm{MPa}$ is here adopted according to [116]. Finally, it is worth remarking that the local elastohydrodynamic (micro-EHL) and rheological coupled effects are not considered in the proposed deterministic model.

\subsubsection{Deterministic Hydrodynamic Model}

The deterministic fluid flow problem is modelled according to the modified Reynolds equation with mass-conserving cavitation model described in Section 2. In this case, as the full-scale representation of the surface roughness is considered entirely in the calculation of the lubricant film thickness, the inter-asperity cavitation effects are also contemplated in the solution. 


\section{Chapter 5}

\section{Fully Coupled \\ Mixed-Elastohydrodynamic Lubrication Modelling}

In this section, the full system of nonlinear equations that governs the fully coupled dynamic EHL problems considered in this work will be presented. The explanation begins with the derivation of the rigid body dynamic equations of motion, followed by a section that summarizes all equations that must be simultaneously computed for the entire problem solution.

\subsection{Rigid Body Motion of the Bearing System}

In order to derive the dynamic equations that describe the rigid body motion of the journal/slider relative to the bearing surface, the following kinematic assumptions are admitted:

1) Only the relative rigid body displacements/misalignments of small magnitude are assumed in the modelling. In other words, the eventual gross motion of the system is ignored from the dynamic equations, whose effects may be ultimately considered as (external) inertial loads.

2) Transient operational conditions are not accounted for in the modelling, i.e. the system rotational/sliding speeds are known a priori as input variables.

3) The bearing (e.g. bearing shell in case of connecting-rod systems) is assumed fixed in the structure, i.e. the bearing relative displacements/misalignments are assumed null.

By applying Newton's second law of motion, the rigid body dynamic equations can be expressed in the bearing coordinate system $\left(\boldsymbol{S}_{\mathcal{B}}\right)$ as:

$$
M_{c} \ddot{\overrightarrow{\boldsymbol{q}}}(t)+\overrightarrow{\boldsymbol{F}}_{\boldsymbol{c}}(\overrightarrow{\boldsymbol{q}}, \dot{\overrightarrow{\boldsymbol{q}}}, t)=\overrightarrow{\boldsymbol{F}}_{\text {ext }}(t)
$$

where $\boldsymbol{M}_{\boldsymbol{c}}$ is the inertia matrix, $\overrightarrow{\boldsymbol{F}}_{\boldsymbol{c}}$ the internal load vector associated with the hydrodynamic and asperity contact effects and $\overrightarrow{\boldsymbol{F}}_{\text {ext }}$ the vector of the external loads applied to the system. Furthermore, $\overrightarrow{\boldsymbol{q}}, \dot{\overrightarrow{\boldsymbol{q}}}$ and $\ddot{\overrightarrow{\boldsymbol{q}}}$ are the bearing rigid body displacement, velocity and 
acceleration vectors, respectively. The particular definition of such quantities for journal and sliding bearing systems are displayed as follows:

- Journal Bearings

$$
\begin{gathered}
\boldsymbol{M}_{\boldsymbol{c}}=\left[\begin{array}{cccc}
m_{J} & 0 & 0 & 0 \\
0 & m_{J} & 0 & 0 \\
0 & 0 & I_{x} & 0 \\
0 & 0 & 0 & I_{y}
\end{array}\right] \quad \overrightarrow{\boldsymbol{q}}=\left[\begin{array}{c}
X_{r} \\
Y_{r} \\
A_{r} \\
B_{r}
\end{array}\right] \quad \dot{\vec{q}}=\left[\begin{array}{c}
\dot{X}_{r} \\
\dot{Y}_{r} \\
\dot{A}_{r} \\
\dot{B}_{r}
\end{array}\right] \quad \ddot{\vec{q}}=\left[\begin{array}{c}
\ddot{X}_{r} \\
\ddot{Y}_{r} \\
\ddot{A}_{r} \\
\ddot{B}_{r}
\end{array}\right] \\
\overrightarrow{\boldsymbol{F}}_{\boldsymbol{c}}=\left[\begin{array}{c}
W_{H}^{x}+W_{A S P}^{x}+F_{A S P}^{x} \\
W_{H}^{y}+W_{A S P}^{y}+F_{A S P}^{y} \\
M_{H}^{x}+M_{A S P}^{x}+T_{A S P}^{x} \\
M_{H}^{y}+M_{A S P}^{y}+T_{A S P}^{y}
\end{array}\right] \quad \overrightarrow{\boldsymbol{F}}_{\text {ext }}=\left[\begin{array}{c}
F_{e x t}^{x} \\
F_{e x t}^{y} \\
M_{e x t}^{x} \\
M_{e x t}^{y}
\end{array}\right]
\end{gathered}
$$

where $m_{J}$ is the equivalent journal mass and $I_{x}$ and $I_{y}$ the journal moments of inertia in the $\left(\overrightarrow{\boldsymbol{I}}_{\boldsymbol{b}}\right)$ and $\left(\overrightarrow{\boldsymbol{J}}_{\boldsymbol{b}}\right)$ directions. Moreover, $W$ and $M$ denote the resulting loads and moments, and $F$ and $T$ the associated friction forces and torques. Notice that $F_{A S P}^{x}$ and $F_{A S P}^{y}$ should be considered in the simulation of transients involving rough contact, which will not be performed in this work.

\section{- Sliding Bearings}

$$
\begin{gathered}
\boldsymbol{M}_{\boldsymbol{c}}=\left[\begin{array}{ccc}
m_{J} & 0 & 0 \\
0 & I_{x} & 0 \\
0 & 0 & I_{z}
\end{array}\right] \quad \overrightarrow{\boldsymbol{q}}=\left[\begin{array}{c}
Y_{r} \\
A_{r} \\
B_{r}
\end{array}\right] \quad \dot{\overrightarrow{\boldsymbol{q}}}=\left[\begin{array}{c}
\dot{Y}_{r} \\
\dot{A}_{r} \\
\dot{B}_{r}
\end{array}\right] \quad \ddot{\vec{q}}=\left[\begin{array}{c}
\ddot{Y}_{r} \\
\ddot{A}_{r} \\
\ddot{B}_{r}
\end{array}\right] \\
\overrightarrow{\boldsymbol{F}}_{\boldsymbol{c}}=\left[\begin{array}{c}
W_{H}^{y}+W_{A S P}^{y} \\
M_{H}^{x}+M_{A S P}^{x} \\
M_{H}^{z}+M_{A S P}^{z}
\end{array}\right] \quad \overrightarrow{\boldsymbol{F}}_{\text {ext }}=\left[\begin{array}{c}
F_{e x t}^{y} \\
M_{e x t}^{x} \\
M_{e x t}^{z}
\end{array}\right]
\end{gathered}
$$

where the inertia and load quantities have the same physical meaning of those previously defined for journal bearings.

\subsection{Fully Coupled System of Nonlinear Equations}

Next, the full set of nonlinear equations that describe the tribological behaviour of dynamically loaded journal or sliding bearing systems operating under the mixedelastohydrodynamic lubrication regimes is summarized in terms of solver operators. Each operator is associated with a particular physical phenomenon that is mathematically modelled from the formulations discussed in the previous sections.

$$
\left.\begin{array}{c}
{[\overrightarrow{\boldsymbol{q}}, \dot{\overrightarrow{\boldsymbol{q}}}]=\mathcal{D}\left(\overrightarrow{\boldsymbol{F}}_{\text {ext }}, t\right)} \\
{\left[p_{H}, \theta, \dot{\gamma}\right]=\mathcal{F}(\overrightarrow{\boldsymbol{q}}, \dot{\overrightarrow{\boldsymbol{q}}}, \delta, \dot{\delta}, \rho, \mu, t)} \\
{\left[p_{A S P}\right]=\mathcal{C}(\overrightarrow{\boldsymbol{q}}, \delta)}
\end{array}\right\} \rightarrow\left[\overrightarrow{\boldsymbol{q}}, \dot{\overrightarrow{\boldsymbol{q}}}, p_{H}, \theta, \dot{\gamma}, p_{A S P}\right]=\mathcal{E}\left(\delta, \dot{\delta}, \rho, \mu, \overrightarrow{\boldsymbol{F}}_{\text {ext }}, t\right)
$$




$$
\begin{gathered}
\left.\begin{array}{c}
{[\rho]=\mathcal{L}_{\boldsymbol{\rho}}\left(p_{H}\right)} \\
{[\mu]=\mathcal{L}_{\boldsymbol{\mu}}\left(p_{H}, \dot{\gamma}\right)}
\end{array}\right\} \rightarrow[\rho, \mu]=\mathcal{L}\left(p_{H}, \dot{\gamma}\right) \\
{[\delta, \dot{\delta}]=\boldsymbol{S}\left(p_{H}\right)}
\end{gathered}
$$

The above expressions are disposed in terms of operators that embrace all the equations associated with a particular solver. Operator $\mathcal{E}$ represents the full solution of the system "instantaneous equilibrium" for the external load condition $\overrightarrow{\boldsymbol{F}}_{\boldsymbol{e x t}}(t)$. This solver is composed of a combination of three intermediate solvers, namely: rigid body dynamics solver $(\mathcal{D})$ established by Eq. 5.1, fluid solver $(\mathcal{F})$ associated with the Reynolds equation (Eq. 2.28 or Eq. 2.36), and asperity contact solver (e) for the GW/GT models (Eqs. 4.7 or Eqs. 4.10). In order to resolve $\mathcal{E}$, the rheological $(\rho, \mu)$ and structural $(\delta, \dot{\delta})$ variables are kept fixed, so that only the instantaneous rigid body kinematics $(\overrightarrow{\boldsymbol{q}}, \dot{\overrightarrow{\boldsymbol{q}}})$ and the related hydrodynamic pressure $\left(p_{H}\right)$, film fraction $(\theta)$ and shear rate $(\dot{\gamma})$ fields are determined by solving the nonlinear equations outlined in Eq. 5.1. Afterwards, from the existing fluid film results, both the lubricant rheology and structural displacements are updated according to operators $\mathcal{L}$ and $\mathcal{S}$, respectively; the rheological operator $(\mathcal{L})$ is formed by the assemblage of two individual rheological solvers linked to the density-pressure $\left(\mathcal{L}_{\boldsymbol{\rho}}\right)$ and viscosity-pressure-shear-thinning relationships $\left(\mathcal{L}_{\boldsymbol{\mu}}\right)$. More details concerning the overall coupled solution including all the above defined operators will be described in Chapter 9 . 


\section{Part II}

\section{Numerical Solutions of the Mathematical Modelling}




\section{Chapter 6}

\section{Time Discretization of the Solver Operators}

The time discretization of the solver operators that embrace all the equations of the fully coupled EHL modelling conceived in this thesis will be presented in the following sections. In particular, the time discretization of the dynamic equations that govern the rigid body and structural deformation motions will be exposed in detail. An advanced fourth-order accuracy version of the so-called Backward Difference Formulas (BDF) proposed by [69] will be here adopted. The scheme is fully implicit and is based on parameters that control the stability and dissipativity properties of the temporal solution. Its convergence characteristics have been demonstrated to be considerably stable for nonlinear dynamic applications where the responses are controlled by a relatively small number of low frequency modes, as those encountered in the EHL problems considered in the present contribution. Furthermore, due to the multistep aspect of the algorithm, it is also possible to determine more accurate extrapolation formulas for the initial guesses to be used in the beginning of each time step. To the author's knowledge, that is the first time that such a high-order scheme has been employed in the context of dynamic EHL solutions.

\subsection{Fourth-Order Accuracy BDF Scheme}

The general four-step scheme proposed by [69] for solving semi-discrete dynamic equations is defined for a generic kinematic variable $y$ as:

$$
\begin{aligned}
\dot{y}^{n} \Delta t= & A_{1}\left(\theta_{1}, \theta_{2}\right) y^{n}+A_{2}\left(\theta_{1}, \theta_{2}\right) y^{n-1}+A_{3}\left(\theta_{1}, \theta_{2}\right) y^{n-2}+ \\
& A_{4}\left(\theta_{1}, \theta_{2}\right) y^{n-3}+A_{5}\left(\theta_{1}, \theta_{2}\right) y^{n-4}
\end{aligned}
$$

where

$$
\begin{aligned}
& A_{1}\left(\theta_{1}, \theta_{2}\right)=-\frac{1}{3} \theta_{1} \theta_{2}-\frac{1}{4} \theta_{2}+\frac{25}{12} \\
& A_{2}\left(\theta_{1}, \theta_{2}\right)=\theta_{1} \theta_{2}+\theta_{2}-4 \\
& A_{3}\left(\theta_{1}, \theta_{2}\right)=-\theta_{1} \theta_{2}-\frac{3}{2} \theta_{2}+3 \\
& A_{4}\left(\theta_{1}, \theta_{2}\right)=\frac{1}{3} \theta_{1} \theta_{2}+\theta_{2}-\frac{4}{3} \\
& A_{5}\left(\theta_{1}, \theta_{2}\right)=\frac{1}{4}-\frac{1}{4} \theta_{2}
\end{aligned}
$$


In the above equations, $\Delta t$ is the time step and superscript $n$ the time iteration, i.e. $t^{n}=n \Delta t$. Parameters $\theta_{1}$ and $\theta_{2}$ are real numbers that rule the stability and dissipative properties of the discretization scheme. For unconditional stability, such parameters must obey the following relation:

$$
\left\{\begin{array}{l}
\left(\theta_{1}+\frac{3}{2}\right) \theta_{2} \leq 4 \\
\left(\theta_{1}-\frac{1}{2}\right) \theta_{2}<5
\end{array}\right.
$$

Throughout this thesis, the values of $\left(\theta_{1}, \theta_{2}\right)=\left(2, \frac{4}{5}\right)$ are employed for ensuring unconditional stability and suitable numerical dissipation regardless of the time step considered; a sensitivity analysis for the appropriateness of $\left(\theta_{1}, \theta_{2}\right)$ for a wide range of nonlinear dynamic problems can be found in [69]. In the next developments, the pair $\left(\theta_{1}, \theta_{2}\right)$ will be suppressed from the coefficients $A_{i}\left(\theta_{1}, \theta_{2}\right)$ for compactness.

\subsection{Time Discretization of the Instantaneous Equi- librium Solver}

By enforcing operator $\mathcal{E}$ (Eq. 5.4a) at time step $n$, one obtains:

$$
\left[\overrightarrow{\boldsymbol{q}}^{n}, \dot{\boldsymbol{q}}^{n}, p_{H}{ }^{n}, \theta^{n}, \dot{\gamma}^{n}, p_{A S P}{ }^{n}\right]=\mathcal{E}\left(\delta^{n}, \dot{\delta}^{n}, \rho^{n}, \mu^{n}, \overrightarrow{\boldsymbol{F}}_{\boldsymbol{e x t}}{ }^{n}, t^{n}\right)
$$

The time discretization of each sub-solver that compound $\mathcal{E}$ (see Section 5.2) are shown in the next sections.

\subsubsection{Rigid Body Dynamics Solver}

By imposing the dynamic rigid body solver (Eq. 5.1) at time step $n$, one has:

$$
\boldsymbol{M} \ddot{\overrightarrow{\boldsymbol{q}}}^{n}+\overrightarrow{\boldsymbol{F}}_{\boldsymbol{c}}\left(\overrightarrow{\boldsymbol{q}}^{n}, \dot{\overrightarrow{\boldsymbol{q}}}^{n}, t^{n}\right)=\overrightarrow{\boldsymbol{F}}_{\boldsymbol{e x t}}\left(t^{n}\right)
$$

The discrete rigid body velocity $\left(\dot{\overrightarrow{\boldsymbol{q}}}^{n}\right)$ and acceleration $\left(\ddot{\overrightarrow{\boldsymbol{q}}}^{n}\right)$ can be written in agreement with the generic expression defined in Eq. 6.1 by using the respective quantities calculated in the previous time steps. Accordingly:

$$
\begin{aligned}
\dot{\overrightarrow{\boldsymbol{q}}}^{n} & =\frac{A_{1}}{\Delta t} \overrightarrow{\boldsymbol{q}}^{n}+\frac{A_{2}}{\Delta t} \overrightarrow{\boldsymbol{q}}^{n-1}+\frac{A_{3}}{\Delta t} \overrightarrow{\boldsymbol{q}}^{n-2}+\frac{A_{4}}{\Delta t} \overrightarrow{\boldsymbol{q}}^{n-3}+\frac{A_{5}}{\Delta t} \overrightarrow{\boldsymbol{q}}^{n-4} \\
\ddot{\overrightarrow{\boldsymbol{q}}}^{n} & =\frac{A_{1}}{\Delta t} \dot{\overrightarrow{\boldsymbol{q}}}^{n}+\frac{A_{2}}{\Delta t} \dot{\overrightarrow{\boldsymbol{q}}}^{n-1}+\frac{A_{3}}{\Delta t} \dot{\overrightarrow{\boldsymbol{q}}}^{n-2}+\frac{A_{4}}{\Delta t} \dot{\overrightarrow{\boldsymbol{q}}}^{n-3}+\frac{A_{5}}{\Delta t} \dot{\overrightarrow{\boldsymbol{q}}}^{n-4}
\end{aligned}
$$

In this fashion, at each time step $n$, the only unknown variables in Eq. 6.5 are the rigid body displacements $\overrightarrow{\boldsymbol{q}}^{n}$. Thus, Eq. 6.5 can be rearranged and displayed as:

$$
\mathcal{D}\left(\overrightarrow{\boldsymbol{q}}^{n}\right)=\boldsymbol{M} \ddot{\overrightarrow{\boldsymbol{q}}}^{n}+\overrightarrow{\boldsymbol{F}}_{\boldsymbol{c}}\left(\overrightarrow{\boldsymbol{q}}^{n}, \dot{\overrightarrow{\boldsymbol{q}}}^{n}, t^{n}\right)-\overrightarrow{\boldsymbol{F}}_{\boldsymbol{e x t}}\left(t^{n}\right)=\overrightarrow{\mathbf{0}}
$$

Operator $\mathcal{D}$ represents the set of nonlinear equations that has to be solved at each time step to determine the "instantaneous equilibrium" of the bearing system. This set of equations is solved by using the advanced Newton-Raphson method with Armijo's line 
search technique to improve the solution step size at each iteration [119]. Accordingly, the Newton sequence derived from the local linear model of $\mathcal{D}\left(\overrightarrow{\boldsymbol{q}}^{n}\right)$ is

$$
\overrightarrow{\boldsymbol{q}}^{n, w}=\overrightarrow{\boldsymbol{q}}^{n, w-1}+\lambda^{\prime w} \overrightarrow{\boldsymbol{s}}^{w} \quad \mathcal{D}^{\prime}\left(\overrightarrow{\boldsymbol{q}}^{n, w-1}\right) \overrightarrow{\boldsymbol{s}}^{w}=-\mathcal{D}\left(\overrightarrow{\boldsymbol{q}}^{n, w-1}\right)
$$

where $\mathcal{D}^{\prime}$ is the Jacobian matrix, $\overrightarrow{\boldsymbol{s}}^{w}$ the Newton step and $\lambda^{\prime w}$ the step length. For each Newton iteration $w$, the Jacobian matrix is approximated by finite differences, while $\lambda^{\prime w}$ is selected to guarantee the decrease of $\|\mathcal{D}\|$ in accordance with the Armijo's line search method. The iterative process terminates whenever the norm of $\mathcal{D}$ is less than a relative error tolerance $\varepsilon_{N R}$ that depends on the magnitude of the external loads, i.e. $\left\|\mathcal{D}\left(\overrightarrow{\boldsymbol{q}}^{n, w}\right)\right\| \leq \varepsilon_{N R}\left\|\overrightarrow{\boldsymbol{F}}_{\text {ext }}\left(t^{n}\right)\right\|$.

The initial iterate $(w=0)$ for a given time step $n$ can be estimated from the previous converged solutions by extrapolating the generic expression of the BDF scheme (Eq. 6.1) as:

$$
\overrightarrow{\boldsymbol{q}}^{n, 0}=-\frac{1}{A_{1}}\left(A_{2} \overrightarrow{\boldsymbol{q}}^{n-1}+A_{3} \overrightarrow{\boldsymbol{q}}^{n-2}+A_{4} \overrightarrow{\boldsymbol{q}}^{n-3}+A_{5} \overrightarrow{\boldsymbol{q}}^{n-4}-\Delta t \dot{\overrightarrow{\boldsymbol{q}}}^{n-1}\right)
$$

As a high-order time discretization scheme is being considered, the above initial iterate tends to provide favourable starting guesses which contribute to the speeding-up of the iterative solution.

\subsubsection{Fluid Solver}

By enforcing the hydrodynamic solver (Eq. 5.4a) at time step $n$, one has:

$$
\left[p_{H}{ }^{n}, \theta^{n}, \dot{\gamma}^{n}\right]=\mathcal{F}\left(\overrightarrow{\boldsymbol{q}}^{n}, \dot{\overrightarrow{\boldsymbol{q}}}^{n}, \delta^{n}, \dot{\delta}^{n}, \rho^{n}, \mu^{n}, t^{n}\right)
$$

The hydrodynamic solver essentially comprises the solution of the modified Reynolds equation (Eq. 2.28 or Eq. 2.36), which is numerically calculated in this thesis by using a special finite volume method (FVM) scheme described in Chapter 7.

\subsubsection{Asperity Contact Solver}

By imposing the asperity contact solver (Eq. 5.4a) at time step $n$, one has (the spatial discretization of the asperity contact equations are described in Chapter 8):

$$
\left[p_{A S P}{ }^{n}\right]=\mathcal{C}\left(\overrightarrow{\boldsymbol{q}}^{n}, \delta^{n}\right)
$$

\subsection{Time Discretization of the Rheological Solver}

By enforcing the rheological solver (Eq. 5.4b) at time step $n$, one has:

$$
\left[\rho^{n}, \mu^{n}\right]=\mathcal{L}\left(p_{H}^{n}, \dot{\gamma}^{n}\right)
$$

As the equations for the lubricant rheology are not time dependent (see Section 2.3), no time discretization procedure has to be applied to the individual solvers that compound $\mathcal{L}$. Thus, the rheological corrections are evaluated only for the local values of the hydrodynamic pressure and shear rate fields. Accordingly,

$$
\left[\rho^{n}\right]=\mathcal{L}_{\boldsymbol{\rho}}\left(p_{H}{ }^{n}\right)
$$




$$
\left[\mu^{n}\right]=\mathcal{L}_{\boldsymbol{\mu}}\left(p_{H}^{n}, \dot{\gamma}^{n}\right)
$$

\subsection{Time Discretization of the Structural Solver}

By enforcing the structural solver (Eq. 5.4c) at time step $n$, one obtains:

$$
\left[\delta^{n}, \dot{\delta}^{n}\right]=\mathcal{S}\left(p_{H}{ }^{n}\right)
$$

or, more specifically, by imposing the structural modal solution (Eq. 3.8) at the same time step, one has:

$$
\ddot{\overrightarrow{\boldsymbol{\eta}}}^{n}+\boldsymbol{B}_{\boldsymbol{r l}}^{\prime} \dot{\overrightarrow{\boldsymbol{\eta}}}^{n}+\Lambda_{\boldsymbol{r l}}^{*} \overrightarrow{\boldsymbol{\eta}}^{n}=\overrightarrow{\boldsymbol{P}}^{n}
$$

Similarly to the rigid body kinematics, the discrete structural velocities $\left(\dot{\overrightarrow{\boldsymbol{\eta}}}^{n}\right)$ and accelerations $\left(\ddot{\overrightarrow{\boldsymbol{\eta}}}^{n}\right)$ can be written according to the generic expression defined in Eq. 6.1 as follows:

$$
\begin{aligned}
& \dot{\overrightarrow{\boldsymbol{\eta}}}^{n}=\frac{A_{1}}{\Delta t} \overrightarrow{\boldsymbol{\eta}}^{n}+\frac{A_{2}}{\Delta t} \overrightarrow{\boldsymbol{\eta}}^{n-1}+\frac{A_{3}}{\Delta t} \overrightarrow{\boldsymbol{\eta}}^{n-2}+\frac{A_{4}}{\Delta t} \overrightarrow{\boldsymbol{\eta}}^{n-3}+\frac{A_{5}}{\Delta t} \overrightarrow{\boldsymbol{\eta}}^{n-4} \\
& \ddot{\overrightarrow{\boldsymbol{\eta}}}^{n}=\frac{A_{1}}{\Delta t} \dot{\overrightarrow{\boldsymbol{\eta}}}^{n}+\frac{A_{2}}{\Delta t} \dot{\overrightarrow{\boldsymbol{\eta}}}^{n-1}+\frac{A_{3}}{\Delta t} \dot{\overrightarrow{\boldsymbol{\eta}}}^{n-2}+\frac{A_{4}}{\Delta t} \dot{\overrightarrow{\boldsymbol{\eta}}}^{n-3}+\frac{A_{5}}{\Delta t} \dot{\overrightarrow{\boldsymbol{\eta}}}^{n-4}
\end{aligned}
$$

By substituting Eqs. 6.16 into Eq. 6.15 and considering that the latter is a set of linear equations, it is possible to deduce an expression for the direct solution of $\overrightarrow{\boldsymbol{\eta}}^{n}$ as:

$$
\begin{aligned}
\overrightarrow{\boldsymbol{\eta}}^{n}= & {\left[\boldsymbol{\Lambda}_{\boldsymbol{r l}}^{*}+\frac{A_{1}}{\Delta t} \boldsymbol{B}_{\boldsymbol{r l}}^{\prime}+\frac{A_{1}{ }^{2}}{\Delta t^{2}} \boldsymbol{I}\right]^{-1}\left\{\overrightarrow{\boldsymbol{P}}^{n}-\left[\frac{A_{2}}{\Delta t} \dot{\overrightarrow{\boldsymbol{\eta}}}^{n-1}+\frac{A_{3}}{\Delta t} \dot{\overrightarrow{\boldsymbol{\eta}}}^{n-2}+\frac{A_{4}}{\Delta t} \dot{\overrightarrow{\boldsymbol{\eta}}}^{n-3}+\frac{A_{5}}{\Delta t} \dot{\overrightarrow{\boldsymbol{\eta}}}^{n-4}\right]\right.} \\
& -\boldsymbol{B}_{\boldsymbol{r l}}^{\prime}\left[\frac{A_{2}}{\Delta t} \overrightarrow{\boldsymbol{\eta}}^{n-1}+\frac{A_{3}}{\Delta t} \overrightarrow{\boldsymbol{\eta}}^{n-2}+\frac{A_{4}}{\Delta t} \overrightarrow{\boldsymbol{\eta}}^{n-3}+\frac{A_{5}}{\Delta t} \overrightarrow{\boldsymbol{\eta}}^{n-4}\right] \\
& \left.-\left[\frac{A_{1} A_{2}}{\Delta t^{2}} \overrightarrow{\boldsymbol{\eta}}^{n-1}+\frac{A_{1} A_{3}}{\Delta t^{2}} \overrightarrow{\boldsymbol{\eta}}^{n-2}+\frac{A_{1} A_{4}}{\Delta t^{2}} \overrightarrow{\boldsymbol{\eta}}^{n-3}+\frac{A_{1} A_{5}}{\Delta t^{2}} \overrightarrow{\boldsymbol{\eta}}^{n-4}\right]\right\}
\end{aligned}
$$

In the particular case where the distributed structural inertia is neglected (quasi-static solutions), Eq. 6.17 is reduced to the following expression:

$$
\overrightarrow{\boldsymbol{\eta}}^{n}=\left(\boldsymbol{\Lambda}_{\boldsymbol{r l}}^{*-1}\right) \overrightarrow{\boldsymbol{P}}^{n}
$$

As already stated in Section 3.3, Eq. 6.18 can be written directly in physical coordinates as (Eq. 3.9):

$$
\overrightarrow{\boldsymbol{\delta}}^{n}=\boldsymbol{L}_{\boldsymbol{r l}} \overrightarrow{\boldsymbol{p}}_{\boldsymbol{H}}{ }^{n}
$$

Although Eq. 6.17 is a set of linear equations that does not require an initial iterate for its solution, it is quite useful for the convergence of the whole EHL problem to provide good estimations for the structural displacements in the beginning of each time step. These estimations can be obtained analogously to the extrapolation process used for the rigid body displacements (Eq. 6.9), so:

$$
\overrightarrow{\boldsymbol{\eta}}^{n, 0}=-\frac{1}{A_{1}}\left(A_{2} \overrightarrow{\boldsymbol{\eta}}^{n-1}+A_{3} \overrightarrow{\boldsymbol{\eta}}^{n-2}+A_{4} \overrightarrow{\boldsymbol{\eta}}^{n-3}+A_{5} \overrightarrow{\boldsymbol{\eta}}^{n-4}-\Delta t \dot{\overrightarrow{\boldsymbol{\eta}}}^{n-1}\right)
$$




\section{Chapter 7}

\section{General Finite Volume Method Solution of Reynolds Equation}

The exact close-form solution of the Reynolds lubrication equation exists only for very particular problems involving simple contact geometries, boundaries conditions and isothermal flows. Examples of such peculiar applications comprise short and long journal bearing components, as well as special formulations conceived for sliding and pure-squeeze bearings. However, when the evaluation of more complex, realistic lubrication systems is desired, approximate solutions based on numerical techniques have to be considered. The numerical solution of Reynolds equation is traditionally performed by adopting the Finite Difference Method (FDM). The predominance of such discretization scheme is due to its relative simplicity in terms of formulation, along with the geometrically uncomplicated domains usually found in conventional lubrication systems, which may well be discretized using structured meshes. In this method, the partial derivatives of the equation are approximated by finite difference formulas obtained from the truncation of the high-order terms of Taylor expansions.

In contrast, for applications involving more complex geometries, where unstructured (or irregular) grids are necessary to accurately discretize the solution domain, the FDM has limited applicability. In the presence of irregular geometries and meshes, the Finite Element Method (FEM) is the most widespread method employed for the numerical solution of the Reynolds equation [30, 32, 35, 82, 100, 128, 158, 162], especially due to its great flexibility to deal with distorted elements (interpolation and shape functions). However, when mass conservation is contemplated in the fluid film cavitation modelling by the imposition of the JFO conditions (see Section 2.2.2.3), the solution of the modified diffusion-convection Reynolds equation (see $p-\theta$ Elrod-Adams model in Section 2.2.3) is not straightforwardly accomplished with the FEM formulation. Essentially, the main difficulties arise in the discretization of the convective term of the modified equation, as well as in the enforcement of the flow conservation on the cavitation boundaries throughout the lubricated contact. Recently, a new FEM formulation based on the mathematical derivation of a linear complementary problem (LCP) was proposed for solving the fluid film lubrication with mass-conserving cavitation model [27, 93]. Moreover, [217] have developed an efficient algorithm for fluid pressure calculation defined according to a reformulation of the complementary constraints imposed by the JFO conditions.

Alternatively, the Finite Volume Method (FVM), widely utilized in Computational Fluid Dynamic (CFD) simulations, has been proven to be very effective for solving lubrication problem, especially in thermohydrodynamic analysis [74, 75], lubrication with 
incompressible bulk flows [12], discontinuous domains [11, 13, 18] and textured surfaces [15], as well as in piston-ring applications [16]. The main advantage of the FVM is its conservative characteristic, which in turn enables to impose local and global flow conservation in the discretized formulation. This intrinsic conservative feature automatically enforces the complementary JFO conditions and allows a straightforward upwind-based treatment for the convective term of the modified Reynolds equation, hence facilitating the entire discretization procedure. The only disadvantage of conventional FVM schemes is the absence of a standardized strategy for the discretization of unstructured meshes, which is a prerogative of the FEM.

In this context, the present contribution proposes a general framework for the solution of Reynolds equation, together with the $p-\theta$ mass-conserving cavitation model, for lubrication problems with complex geometries. This scheme is based on a hybrid-type formulation, hereafter denominated the Element-Based Finite Volume Method (EbFVM), which combines the geometric flexibility of the FEM to deal with unstructured grids while preserving the local and global fluid flow conservation aspect of the FVM throughout the discretized domain [181, 189]. Essentially, the EbFVM is a FVM scheme that employs the concept of elements and their interpolation and shape functions adopted in the FEM. However, differently from the classical FEM methodologies, local and global conservation of the transport properties are exactly enforced in the EbFVM, since the construction of the discretized equations follows the FVM principles; this eliminates the potential issues arising from the use of the FEM mathematical foundation to derive the weak form of the formulation. In other words, in the EbFVM the discretized equations represent the physical balances over control volumes that are built as dual entities around all vertices of the primal grid, with contributions of neighbouring elements. The motivation for the use of the EbFVM in lubrication applications is based on its flexibility, generality and suitability for computational implementations. The results presented in the following sections have been published by the author in references [173, 174].

\subsection{Fundamentals of the Element-Based Finite Volume Method (EbFVM)}

Similarly to any numerical method used to approximate the solution of partial differential equations, the initial step of the EbFVM consists in the geometric discretization of the continuum domain in smaller, simple-shaped subregions denominated elements $(e)$, which are inter-connected by nodes $(P)$ that coincide with the vertices of the elements. The collection of these geometric entities is here denominated geometric grid and for the discretization of the Reynolds equation, it is composed of either triangular or quadrangular elements. In Fig. 7.1 a schematic illustration of the domain discretization and the geometric entities involved is shown.

In contrast to the classical finite volume method commonly used for structured grids, where elements and control volumes are coincident ("cell-centre" formulation) and the transport equations are integrated directly using the geometric grid, in the EbFVM scheme the elements are used as an auxiliary entity from which the control volumes are constructed ("cell-vertex" formulation). In this case, the control volumes constitute a secondary or computational grid, where the local conservation of the physical fluxes balance is effectively enforced. As depicted in Fig. 7.1, a control volume $(C V)$ is associated with a given node $(P)$ of the geometric grid and is composed of portions of the neighbouring elements. Those 


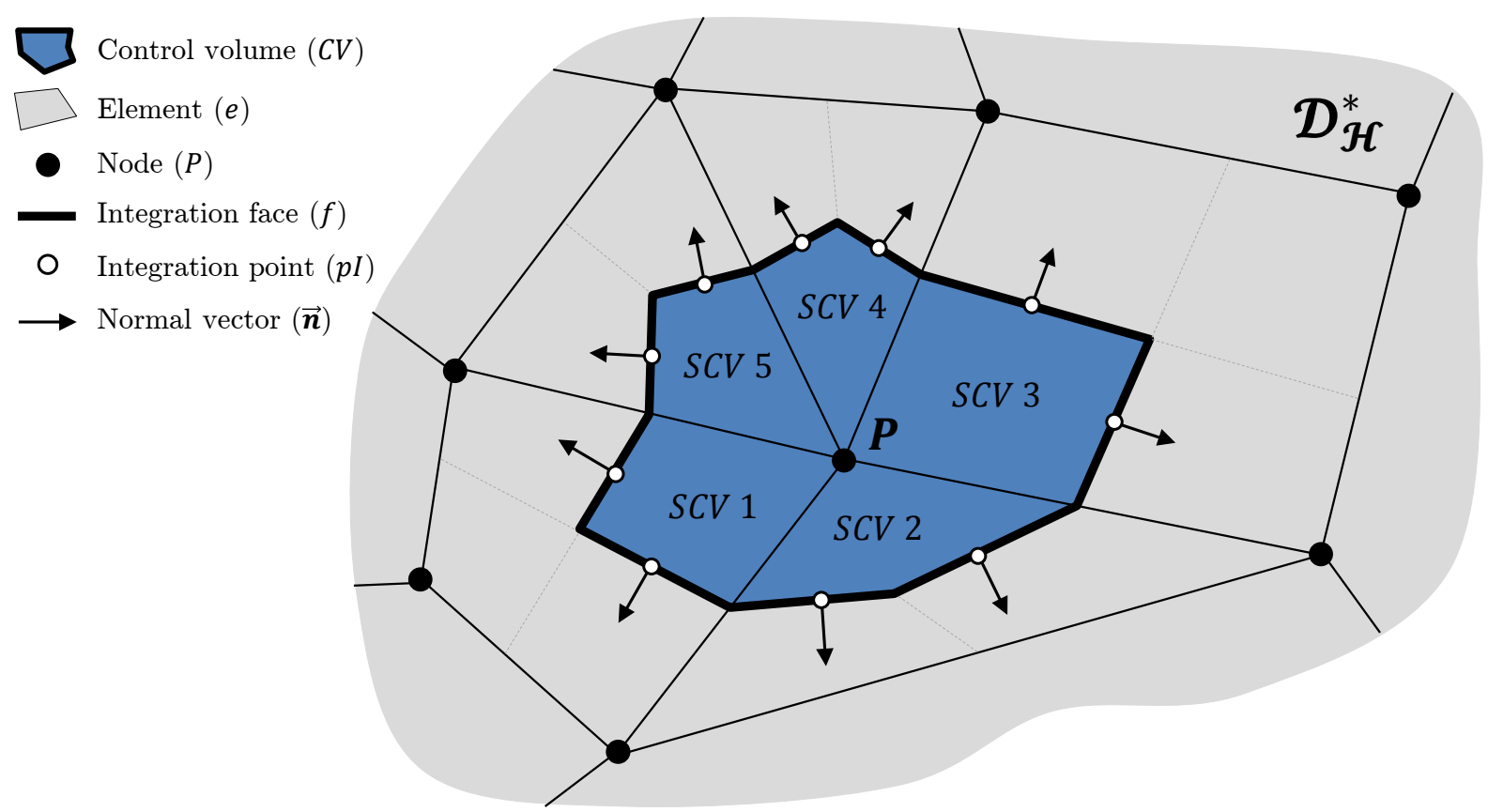

Figure 7.1: Geometric entities of the Element-Based Finite Volume Method (EbFVM).

portions are designated as sub-control volumes $(S C V)$ and result from a subdivision of the elements. For a centroid subdivision (or "median rule"), the division lines are obtained by joining the centroid of the element to the midpoints of the element edges. Those lines are denoted faces $(f)$ and are the basic geometric entity from which the fluxes are locally evaluated in the discretized equations. In particular, by assuming the "midpoint rule" to approximate the fluxes over the $C V$ faces, the midpoint of each face (where the fluxes are effectively calculated) and its respective normal vector are defined, respectively, as integration point $(p I)$ and normal vector $(\overrightarrow{\boldsymbol{n}})$. Following this strategy, each control volume associated with a given node $P$ may be seen as a sum of the nearest sub-control volumes of the elements that share $\mathrm{P}$, and the fluxes at one specific integration point can be calculated using data from the element in which the integration point is placed [107, 108].

As already stated, due to the "cell-vertex" nature of the EbFVM, the unknowns of the problem are approximated at the nodes of the geometric grid. Furthermore, in order to deal with the distorted elements of irregular grids, as well as local variations in the flow and transport properties, pre-defined families of interpolation and shape functions can be employed. These functions are based on the definition of parametric elements that are described with respect to a local (transformed) coordinate system. Thus, all the required calculations previously undertaken in the physical domain can be performed more easily in the transformed domain. The reader should refer to Appendix F.1 for details about the coordinate transformation and the associated shape functions of triangular and quadrangular elements.

Once the integration points are identified within each element, the fluxes of the transport properties through the faces of the sub-control volumes can be calculated according to the geometric and general parameters associated with the element nodes. This procedure allows elements, and hence the geometric grid, to be treated as the major entity over which all the calculations are performed independently. Subsequently, the conservation equation for every control volume can be determined from the fluxes contributions coming from its respective communal surrounding sub-control volumes. Thus, after an element-by-element 
assembly of the global system of equations (analogous to the assembly procedure in the FEM), the total physical balance of the conservation equation are automatically satisfied by the inherent connectivity of the elements.

In the next sections, the temporal and spatial discretization of each term of the modified Reynolds equation (Eq. 2.9a) derived in Section 2.2.3 will be presented in detail. The same discretization procedures are valid for the customized equations obtained for the journal (Eq. 2.28) and sliding (Eq. 2.36) bearing models. Further explanations concerning the EbFVM scheme for a generic conservation equation can be found in [107, 181, 189].

\subsection{Temporal Discretization}

The temporal approximation of Eq. 2.9a can be established by integrating all terms of the equation in a time interval $\Delta t$, as follows:

$$
\begin{aligned}
\int_{t}^{t+\Delta t}\left[\nabla \cdot\left(\boldsymbol{\Gamma}^{p} \nabla p_{H}\right)\right] d t= & \int_{t}^{t+\Delta t}\left[\nabla \cdot\left(\theta \boldsymbol{\Gamma}^{c} \overrightarrow{\boldsymbol{v}}\right)\right] d t+\int_{t}^{t+\Delta t}\left[\theta\left(S_{T S}+S_{N S}\right)\right] d t+ \\
& \int_{t}^{t+\Delta t}\left[S_{T} \frac{\partial \theta}{\partial t}\right] d t
\end{aligned}
$$

Assuming an implicit first-order time discretization scheme (backward Euler method), the integrands of Eq. 7.1 can be approximated to their values at the end of the time interval. The advantage of such formulation is its unconditional stability, so that the numerical accuracy of the solution is mainly controlled by the size of the time step $\Delta t$. Thus, by performing the temporal discretization using $t=n \Delta t$, where $n$ is the $\mathrm{n}$-th time instant, Eq. 7.1 can be rewritten as:

$$
\left[\nabla \cdot\left(\boldsymbol{\Gamma}^{p} \nabla p_{H}\right)\right]^{n}=\left[\nabla \cdot\left(\theta \boldsymbol{\Gamma}^{c} \overrightarrow{\boldsymbol{v}}\right)\right]^{n}+\left[\theta\left(S_{T S}+S_{N S}\right)\right]^{n}+S_{T}^{n}\left(\frac{\theta^{n}-\theta^{n-1}}{\Delta t}\right)
$$

\subsection{Spatial Discretization}

For the spatial discretization of Eq. 7.2 at each time instant $t=n \Delta t$, it is convenient to consider its integral formulation (or weak formulation), which may be obtained by adopting the Weighted Residual Method (WRM). The reason for using this integral approach is to mathematically ensure the minimization of the approximation errors throughout the solution domain [182]. In this case, Eq. 7.2 is expressed as follows:

$$
\begin{gathered}
\iint_{\mathcal{D}_{\mathcal{H}}^{*}} \mathcal{W}\left\{\left[\nabla \cdot\left(\tilde{\boldsymbol{\Gamma}}^{p} \nabla \tilde{p}_{H}\right)^{n}\right]-\left[\nabla \cdot\left(\tilde{\theta} \tilde{\boldsymbol{\Gamma}}^{c} \overrightarrow{\tilde{\boldsymbol{v}}}\right)^{n}\right]-\left[\tilde{\theta}\left(\tilde{S}_{T S}+\tilde{S}_{N S}\right)\right]^{n}-\right. \\
\left.\tilde{S}_{T}^{n}\left(\frac{\tilde{\theta}^{n}-\tilde{\theta}^{n-1}}{\Delta t}\right)\right\} d \mathcal{D}_{\mathcal{H}}^{*}=0
\end{gathered}
$$

where $\mathcal{W}$ corresponds to the weighting function that weights the discretization errors over the entire integration domain $\left(\mathcal{D}_{\mathcal{H}}^{*}\right)$, and superscript ' $\sim$ ' denotes the approximated solutions of the transport properties and unknown variables. Particularly for finite volume 
methods, $\mathcal{W}$ is assumed unitary within every control volume and null outside (sub-domain method) [135]. Thus, Eq. 7.3 can be restated for each control volume as:

$$
\begin{aligned}
\iint_{\mathcal{D}_{\mathcal{H}}}\left[\nabla \cdot\left(\tilde{\boldsymbol{\Gamma}}^{p} \nabla \tilde{p}_{H}\right)^{n}\right] d \mathcal{D}_{\mathcal{H}}= & \iint_{\mathcal{D}_{\mathcal{H}}}\left[\nabla \cdot\left(\tilde{\theta} \tilde{\boldsymbol{\Gamma}}^{c} \overrightarrow{\tilde{\boldsymbol{v}}}\right)^{n}\right] d \mathcal{D}_{\mathcal{H}}+\iint_{\mathcal{D}_{\mathcal{H}}}\left[\tilde{\theta}\left(\tilde{S}_{T S}+\tilde{S}_{N S}\right)\right]^{n} d \mathcal{D}_{\mathcal{H}}+ \\
& \iint_{\mathcal{D}_{\mathcal{H}}}\left[\tilde{S}_{T}^{n}\left(\frac{\tilde{\theta}^{n}-\tilde{\theta}^{n-1}}{\Delta t}\right)\right] d \mathcal{D}_{\mathcal{H}}
\end{aligned}
$$

where $\mathcal{D}_{\mathcal{H}}$ is the sub-domain enclosed by each control volume centred at every node $P$.

By applying the divergence (or Gauss-Ostrogradsky's) theorem to the first two integrals of Eq. 7.4, one obtains:

$$
\begin{aligned}
\oint_{\mathcal{C}_{\mathcal{H}}}\left[\left(\tilde{\boldsymbol{\Gamma}}^{p} \nabla \tilde{p}_{H}\right)^{n} \cdot \overrightarrow{\boldsymbol{n}}\right] d \mathfrak{C}_{\mathcal{H}}= & \oint_{\mathcal{C}_{\mathcal{H}}}\left[\left(\tilde{\theta} \tilde{\boldsymbol{\Gamma}}^{c} \overrightarrow{\tilde{\boldsymbol{v}}}\right)^{n} \cdot \overrightarrow{\boldsymbol{n}}\right] d \mathcal{C}_{\mathcal{H}}+\iint_{\mathcal{D}_{\mathcal{H}}}\left[\tilde{\theta}\left(\tilde{S}_{T S}+\tilde{S}_{N S}\right)\right]^{n} d \mathcal{D}_{\mathcal{H}}+ \\
& \iint_{\mathcal{D}_{\mathcal{H}}}\left[\tilde{S}_{T}^{n}\left(\frac{\tilde{\theta}^{n}-\tilde{\theta}^{n-1}}{\Delta t}\right)\right] d \mathcal{D}_{\mathcal{H}}
\end{aligned}
$$

where $\boldsymbol{C}_{\mathcal{H}}$ is the boundary that delimits each control volume and $\overrightarrow{\boldsymbol{n}}$ the unit vector normal to that boundary (see Fig. 7.1).

Finally, recalling that the fluxes over the $C V$ faces are approximated by the "midpoint rule" and by assuming a second-order approximation scheme for the area integrals (in which the integral is evaluated as the product between the integrand function at the $C V$ centre and the $C V$ area [84]), Eq. 7.5 can be expressed in the discretized form as follows:

$$
\begin{aligned}
& \sum_{e \in \mathcal{E}_{P}} \sum_{f \in \mathcal{F}_{P}^{e}}\left[\left(\tilde{\boldsymbol{\Gamma}}^{p} \nabla \tilde{p}_{H}\right)^{n} \cdot \overrightarrow{\boldsymbol{n}}^{*}\right]_{f}^{e}=\sum_{e \in \mathcal{E}_{P}} \sum_{f \in \mathcal{F}_{P}^{e}}\left[\left(\tilde{\theta} \tilde{\boldsymbol{\Gamma}}^{c} \overrightarrow{\tilde{\boldsymbol{v}}}\right)^{n} \cdot \overrightarrow{\boldsymbol{n}}^{*}\right]_{f}^{e}+ \\
& {\left[\tilde{\theta}\left(\tilde{S}_{T S}+\tilde{S}_{N S}+\frac{\tilde{S}_{T}}{\Delta t}\right)\right]_{P}^{n} \underbrace{\sum_{e \in \mathcal{E}_{P}}\left[\Delta \mathcal{D}_{\mathcal{H}_{s}^{e}}^{e}\right]}_{\Delta \mathcal{D}_{\mathcal{H}_{P}}}-} \\
& {\left[\tilde{\theta}^{n-1}\left(\frac{\tilde{S}_{T}}{\Delta t}\right)^{n}\right]_{P} \underbrace{\sum_{e \in \mathcal{E}_{P}}\left[\Delta \mathcal{D}_{\mathcal{H}_{s}^{e}}^{e}\right]}_{\Delta \mathcal{D}_{\mathcal{H}_{P}}}}
\end{aligned}
$$

where $\overrightarrow{\boldsymbol{n}}_{f}^{* e}=\overrightarrow{\boldsymbol{n}}_{f}^{e} \Delta \boldsymbol{\mathcal { C }}_{\mathcal{H}_{f}^{e}}$ is the normal face vector that retains, simultaneously, the normal orientation $\left(\overrightarrow{\boldsymbol{n}}_{f}^{e}\right)$ and the length $\left(\Delta \boldsymbol{\mathcal { C }}_{\mathcal{H}_{f}^{e}}^{e}\right)$ of each delimiting face associated with a given control volume; $\Delta \mathcal{D}_{\mathcal{H}_{s}^{e}}^{e}$ is the area of the s-th $S C V$ that compound the control volume and $\mathcal{D}_{\mathcal{H}_{P}}$ is the full area of the respective $C V$ (see Appendix F.1 for details about the computation of $\overrightarrow{\boldsymbol{n}}_{f}^{* e}$ for triangular and quadrangular elements). Moreover, in Eq. $7.6 \mathcal{E}_{P}$ denotes the set of elements that contribute to the formation of the $C V$ around node $P$ and $\mathcal{F}_{P}^{e}$ the set of faces inside the corresponding element $e$ that encloses $P$.

The above discrete equation is valid for every control volume constructed surrounding each node $P$ of the geometry grid. The inner summations in the first two terms of the equation respectively correspond to the diffusive and convective fluxes through each subcontrol volume, while the outer summations represent the entire flux balances over the 
associated $C V$. The last two components of the equation are the source terms evaluated explicitly at node $P$.

As already pointed out, the assembly of the global system of equations is performed in an element-by-element fashion, similar to the procedure employed in the FEM. Furthermore, all local computations are carried out at element level using the transformed domain approach (parametric elements); this allows the elements to be treated independently, no matter how distorted they are with respect to the global coordinate system. Such procedure renders the definition of element matrices and vectors constituting each term of Eq. 7.6 straightforward, analogously to the stiffness and mass matrices and load vectors used in FEM formulations. Next, those definitions will be exposed in detail by adopting index notation for compactness.

\subsubsection{Diffusivity Matrix (Poiseuille Term)}

The total diffusive flux (first term of Eq. 7.6) across the sub-control volumes of a given element $e$ can be represented as:

$$
\overrightarrow{\boldsymbol{d}}_{e}^{n}=\boldsymbol{P}_{e}^{n}\left(\overrightarrow{\tilde{\boldsymbol{p}}}_{\boldsymbol{H}}\right)_{e}^{n}
$$

where the components of vector $\overrightarrow{\boldsymbol{d}}_{e}^{n}$ correspond to the diffusive fluxes through each subcontrol volume, $\left(\overrightarrow{\tilde{\boldsymbol{p}}}_{\boldsymbol{H}}\right)_{e}^{n}$ is the vector of the nodal pressures and $\boldsymbol{P}_{e}^{n}$ is the diffusivity matrix of the element. See Appendix F.2 for details about the derivation of $\boldsymbol{P}_{e}^{n}$.

\subsubsection{Convective Matrix (Couette Term)}

Similar to the diffusion term, the total convective flux (second term of Eq. 7.6) flowing through the sub-control volumes of a given element $e$ can be expressed as:

$$
\overrightarrow{\boldsymbol{c}}_{e}^{n}=\boldsymbol{C}_{e}^{n}(\overrightarrow{\tilde{\boldsymbol{\theta}}})_{e}^{n}
$$

where the entries of vector $\overrightarrow{\boldsymbol{c}}_{e}^{n}$ are the convective fluxes through each sub-control volume, $(\overrightarrow{\tilde{\boldsymbol{\theta}}})_{e}^{n}$ the vector of the nodal lubricant film fraction and $\boldsymbol{C}_{e}^{n}$ is the convective matrix of the element. See Appendix F.3 for details about the derivation of $\boldsymbol{C}_{e}^{n}$.

\subsubsection{Source Term Vectors}

The source terms (third and fourth terms of Eq. 7.6) associated with the sub-control volumes of a given element $e$ can be written as follows:

$$
\left(\overrightarrow{\boldsymbol{f}}_{\mathbf{1}}\right)_{e}^{n}=\left(\overrightarrow{\boldsymbol{F}}_{\mathbf{1}}\right)_{e}^{n} \cdot(\overrightarrow{\tilde{\boldsymbol{\theta}}})_{e}^{n} \quad\left(\overrightarrow{\boldsymbol{f}}_{\mathbf{2}}\right)_{e}^{n}=\left(\overrightarrow{\boldsymbol{F}}_{\mathbf{2}}\right)_{e}^{n} \cdot(\overrightarrow{\tilde{\boldsymbol{\theta}}})_{e}^{n-1}
$$

where the members of vectors $\left(\overrightarrow{\boldsymbol{f}}_{\mathbf{1}}\right)_{e}^{n}$ and $\left(\overrightarrow{\boldsymbol{f}}_{\mathbf{2}}\right)_{e}^{n}$ are the source terms of each element node, while $\left(\overrightarrow{\boldsymbol{F}}_{\mathbf{1}}\right)_{e}^{n}$ and $\left(\overrightarrow{\boldsymbol{F}}_{\mathbf{2}}\right)_{e}^{n}$ are auxiliary element vectors (see Appendix F.4 for more details about their derivations). Additionally, $(\overrightarrow{\tilde{\boldsymbol{\theta}}})_{e}^{n}$ and $(\overrightarrow{\tilde{\boldsymbol{\theta}}})_{e}^{n-1}$ are vectors of the nodal lubricant film fraction at time instants $n$ and $(n-1)$, respectively. In the above equations, the operator '.' denotes scalar product. 


\subsection{Assembly of the Global Linear System of Equa- tions}

Subsequently to the calculation of the diffusivity and convective matrices, as well as the source term vectors for all the elements of the geometric grid, the discrete equation associated with every control volume are assembled in a global system of linear equations. The rows of the resulting linear system correspond to the entire flux balance over each control volume constructed around every node $P$ of the grid. This can be mathematically represented as:

$$
\left(a_{P}^{n}\right)\left(\tilde{p}_{H}\right)_{P}^{n}+\sum_{N B}\left[\left(a_{N B}^{n}\right)\left(\tilde{p}_{H}\right)_{N B}^{n}\right]=\left(b_{P}^{n}\right)(\tilde{\theta})_{P}^{n}+\sum_{N B}\left[\left(b_{N B}^{n}\right)(\tilde{\theta})_{N B}^{n}\right]+B_{P}^{n}
$$

where $N B$ denotes the neighbouring nodes around $P$ and the coefficients $a_{i}^{n}$ and $b_{i}^{n}$ are the entries of the linear system of equations computed during the assembly operation.

\subsection{Solution of the Linear System of Equations}

For each time instant $n$, the global system of equations defined in the previous section (Eq. 7.10), embraces two unknown variables, namely the hydrodynamic pressure $\left(\tilde{p}_{H}\right)$, and the lubricant film fraction $(\tilde{\theta})$ fields. Thus, a specific numerical procedure has to be considered for the simultaneous solution of $\tilde{p}_{H}$ and $\tilde{\theta}$. In the present contribution, these simultaneous calculations are accomplished by adopting a generalization of the algorithm proposed by [15], which was originally conceived for applications with structured grids. In this sense, the generalization here devised consists in an extension of the original algorithm for lubrication problems to be discretized by irregular meshes.

The main idea of the algorithm is based on the iterative solution of a linear system of equations according to the well-known Gauss-Seidel method with Successive OverRelaxation (SOR). In this case, by assuming that $a_{i}^{n}, b_{i}^{n}$ and $B_{i}^{n}$ are known a priori, the nodal values of $\left(\tilde{p}_{H}\right)_{P}^{n}$ and $(\tilde{\theta})_{P}^{n}$ can be computed iteratively, as follows:

$$
\begin{gathered}
{\left[\left(\tilde{p}_{H}\right)_{P}^{n},(\tilde{\theta})_{P}^{n}\right]=\lim _{r \rightarrow \infty}\left[\left(\tilde{p}_{H}\right)_{P}^{n, r},(\tilde{\theta})_{P}^{n, r}\right] \text { with }\left[\left(\tilde{p}_{H}\right)_{P}^{n, 0},(\tilde{\theta})_{P}^{n, 0}\right]=\left[\left(\tilde{p}_{H}\right)_{P}^{n-1},(\tilde{\theta})_{P}^{n-1}\right]} \\
\left(\tilde{p}_{H}\right)_{P}^{n, r}=\omega_{p}\left(\tilde{P}_{H}\right)_{P}^{n, r}+\left(1-\omega_{p}\right)\left(\tilde{p}_{H}\right)_{P}^{n, r-1} \\
(\tilde{\theta})_{P}^{n, r}=\omega_{\theta}(\tilde{\Theta})_{P}^{n, r}+\left(1-\omega_{\theta}\right)(\tilde{\theta})_{P}^{n, r-1}
\end{gathered}
$$

where $r$ is the r-th iteration of the SOR method, $\omega_{p}$ and $\omega_{\theta}$ the respective relaxation factors, and $\left(\tilde{P}_{H}\right)_{P}^{n, r}$ and $(\tilde{\Theta})_{P}^{n, r}$ the intermediate nodal values of $\tilde{p}_{H}$ and $\tilde{\theta}$.

The intermediate quantities $\left(\tilde{P}_{H}\right)_{P}^{n, r}$ and $(\tilde{\Theta})_{P}^{n, r}$ are approximated from Eq. 7.10 by isolating the respective nodal values and admitting the others fixed to the values calculated in the previous iteration, which mathematically corresponds to:

$$
\begin{aligned}
& \tilde{P}_{P}^{n, r}=\frac{1}{a_{P}^{n}}\left\{-\sum_{N B}\left[\left(a_{N B}^{n}\right)\left(\tilde{p}_{H}\right)_{N B}^{n, r-1}\right]+\left(b_{P}^{n}\right)(\tilde{\theta})_{P}^{n, r-1}+\sum_{N B}\left[\left(b_{N B}^{n}\right)(\tilde{\theta})_{N B}^{n, r-1}\right]+B_{P}^{n}\right\} \\
& \tilde{\Theta}_{P}^{n, r}=\frac{1}{b_{P}^{n}}\left\{\left(a_{P}^{n}\right)\left(\tilde{p}_{H}\right)_{P}^{n, r-1}+\sum_{N B}\left[\left(a_{N B}^{n}\right)\left(\tilde{p}_{H}\right)_{N B}^{n, r-1}\right]-\sum_{N B}\left[\left(b_{N B}^{n}\right)(\tilde{\theta})_{N B}^{n, r-1}\right]-B_{P}^{n}\right\}
\end{aligned}
$$


Furthermore, the complementary conditions related to the $p-\theta$ cavitation model displayed in Eq. 2.9b must be satisfied during the iterative procedure. In the discrete domain this reads:

$$
\left\{\begin{array} { l } 
{ ( \tilde { p } _ { H } ) _ { P } ^ { n , r } > p _ { \text { cav } } } \\
{ 0 \leq ( \tilde { \theta } ) _ { P } ^ { n , r } \leq 1 }
\end{array} \quad \Rightarrow \left\{\begin{array}{lll}
\left(\tilde{p}_{H}\right)_{P}^{n, r} \geq p_{\text {cav }} & \rightarrow & (\tilde{\theta})_{P}^{n, r}=1 \\
(\tilde{\theta})_{P}^{n, r}<1 & \rightarrow & \left(\tilde{p}_{H}\right)_{P}^{n, r}=p_{\text {cav }}
\end{array}\right.\right.
$$

The discrete complementary conditions of Eq. 7.13 enforce the cavitation regions and boundaries to be positioned always at the nodes of the geometric grid. Moreover, as the discretization method is inherently conservative in the discrete domain (finite volume method), the local conservation of the lubricant flow is ensured locally for all grid nodes (control volumes), including those laying on the cavitation boundaries; this automatically satisfies the mass-conservation conditions imposed by the JFO cavitation model. The extended SOR algorithm proposed in this contribution is shown as a pseudo-code in Algorithm 8. 


\section{Chapter 8}

\section{Discretization of the GW/GT Asperity Contact Models}

Once the stochastic asperity contact models considered in this thesis are not formulated in terms of differential or integral equations, no special discretization techniques are required for their numerical solutions. Hence, the equations of the GW/GT models (Eqs. 4.12) can be discretized directly at every grid node $P$ of the primary mesh [171] as:

$$
\begin{aligned}
& \bar{p}_{A S P}^{(G W)_{P}}= \begin{cases}\bar{E}_{P}^{* n}\left(\bar{\eta}_{S} \bar{\beta}_{S}^{1 / 2} \bar{\sigma}_{S}^{3 / 2}\right)_{P}^{n} F_{\frac{3}{2}}\left(\bar{h}_{A S P P}\right) & , \quad \bar{h}_{A S P P}^{n}>3 \\
1 & , \quad \bar{h}_{A S P P} \leq 3 \text { and } \bar{p}_{A S P}^{(G W)_{n}} \leq H_{V P}^{n} \\
H_{V P}^{n} & , \quad \bar{h}_{A S P P}^{n} \leq 3 \text { and } \bar{p}_{A S P}^{(G W)_{n}}>H_{V P}^{n}\end{cases}
\end{aligned}
$$

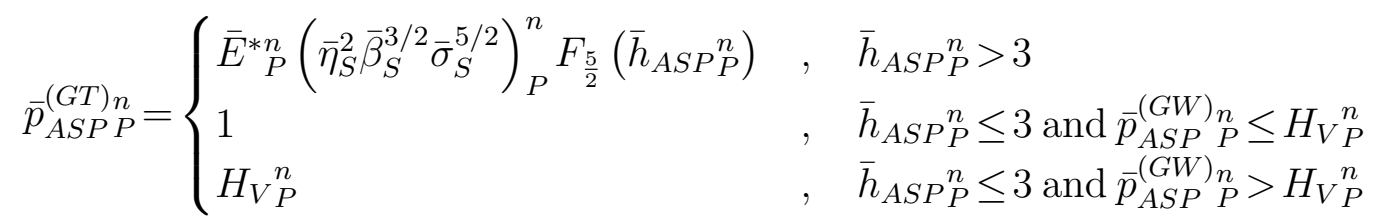

$$
\begin{aligned}
& \bar{\tau}_{A S P}^{(G W, G T)_{P}} \stackrel{P}{ }=\bar{\mu}_{B L P}{ }_{P}^{n}\left(\bar{p}_{A S P}^{(G W, G T)_{n}}\right)
\end{aligned}
$$

where superscript $n$ denotes the time step. 


\section{Chapter 9}

\section{Fluid-Structure Interaction (FSI) Solution of the Fully Coupled Mixed-Elastohydrodynamic Lubrication Modelling}

The solution of the fully coupled dynamic EHL problem summarized in Section 5.2 may be accomplished by taking one of the following approaches:

Full dynamic analysis: More complex approach where both the structural dynamics as well as the large rigid body motions of the (multibody) system are solved simultaneously in the same solution framework. In this case, the internal link forces acting on the joint components are not known a priori, and so the bearings tribological behaviour is determined in accordance with the general external loads applied to the entire (multibody) system. Such solution approach has been used by [122] for the transient analysis of a connecting-rod big-end bearing.

Partial dynamic analysis: Only the structural bearing dynamics (distributed inertia) are directly considered in the solution. In this approach, the link forces acting on the bearings are known a priori, whose values correspond to the reaction forces determined from previously (multibody) dynamic simulations of the entire system with perfectly rigid joints. The eventual inertia effects associated with the large rigid body motions are implicitly accounted into the reaction forces. This partial dynamic analysis has been employed by [31, 34] for connecting-rod bearings and by [159] for a simpler stationary bearing application.

Quasi-static analysis: The same as in the partial dynamic analysis described above, but neglects the structural distributed inertia effects. This is the most widespread approach reported in the literature for the solution of dynamically loaded EHL problems in conformal contacts [82, 95, 98, 120, 132, 147, 157, 194, 195]. Due to its wellestablished use in the specialized literature, the quasi-static analysis will be adopted for all EHL solutions throughout this thesis.

Regardless of the approach chosen for the solution of the dynamic EHL problem, the fluid-structure interaction (FSI) coupling inherent in EHL contacts has to be solved accordingly. For conformal contacts often encountered in journal and sliding bearing applications, such FSI calculations are traditionally addressed by using either the nodal or 
modal approach [36]. The modal (or mode-based) approach was introduced by [127] and is less widespread in the literature. According to this method, the nodal displacements are computed by adopting a linear combination of particular mode shapes determined from the linear elastic solution of the bearing structure. On the other hand, the nodal (or node-based) methodology is most predominant in publications involving journal bearing EHL problems. In the latter case, the nodal displacements are determined directly from the linear matrix-vector relationship between the nodal load vector and the compliance matrix of the elastic structure (quasi-static analysis). The computation of the compliance matrix is usually carried out by applying some model reduction (or condensation) technique to the complete FEM bearing model (see Section 3.2). Thus, only the degrees of freedom of the nodes placed on the internal bearing surface are retained for the EHL solutions.

Two subclasses of methods, namely indirect or direct methods, can be identified for the node-based approach. The nodal indirect (or monolithic) methods often employ sophisticated implicit Newton-Raphson schemes, where the solution step of each Newton iteration is computed by solving a system of residual equations defined both in terms of the applied external loads and a Taylor series expansion of Reynolds equation, which thus allows the ready evaluation of the Jacobian matrix [34, 90, 95, 98, 122, 123, 140, 147, 153, 154, 157, 220]. In other words, in the indirect methods all the equations involved in the EHL modelling are solved simultaneously. In contrast, the nodal direct (or partitioned) methods are established by means of direct iterative schemes [80, 132], in which the hydrodynamic and structural problems are solved separately. In that case, a coupling algorithm is required to incorporate the interaction between the fluid and structural solvers. The numerical convergence of the direct schemes commonly used to solve flexible bearing systems is often difficult, especially when large deformations take place, hence justifying the predominance of the indirect methods for EHL solutions of highly loaded bearings.

In this scenario, three partitioned methods [64, 65], namely: Fixed Point Gauss-Seidel Method (PGMF), Point Gauss-Seidel Method with Aitken Acceleration (PGMA) and Interface Quasi-Newton Method with Inverse Jacobian from a Least-Squares approximation (IQN-ILS), are analysed in this thesis. The aim of such techniques is to provide more robust and stable direct (nodal) solutions particularly applied to conformal EHL contacts under different operational conditions. These partitioned methods are used in a wide range of applications of FSI problems, such as in the analysis of flutter in wings of aircrafts and blades of turbo-machines, in the complex fluid-structure interaction of life-saving equipment such as parachutes and air bags, and even in the investigation of blood flow through arteries and heart valves [64]. The main advantage of the partitioned approach is the possibility of using optimized codes to solve hydrodynamic equations and structural equations separately. In other words, each component of the EHL problem can be solved with different techniques specialized for each type of equations. This flexibility is not verified in the implicit solutions where all equations are generally solved in the same solution framework. With these advantages in mind, another goal of this contribution is to introduce the aforementioned partitioned coupling techniques in the context of tribological simulations.

In the following sections, the partitioned methods considered in the present work will be described in detail. Although only conformal contacts are investigated, the methods may well have their application extended to EHL non-conformal line and point contact applications. For more in-depth explanations concerning the convergence of such partitioned techniques, the comprehensive references $[64,65,211]$ are strongly recommended. 
The results presented in this chapter have been published by the author in references $[170,177]$.

\subsection{Solver Operators}

For the development of the partitioned techniques, it is convenient to rewrite the time discretized version of the solver operators defined in Section 5.2 as:

$$
\begin{aligned}
{\left[\overrightarrow{\boldsymbol{q}}^{n}, \dot{\overrightarrow{\boldsymbol{q}}}^{n}, p_{H}{ }^{n}, \theta^{n}, \dot{\gamma}^{n}, p_{A S P}{ }^{n}\right] } & =\mathcal{E}\left(\delta^{n}, \dot{\delta}^{n}, \rho^{n}, \mu^{n}, \overrightarrow{\boldsymbol{F}}_{\boldsymbol{e x t}}{ }^{n}, t^{n}\right) \\
{\left[\delta^{n}, \dot{\delta}^{n}\right] } & =\mathcal{S}\left(p_{H}{ }^{n}\right) \\
{\left[\rho^{n}\right] } & =\mathcal{L}_{\boldsymbol{\rho}}\left(p_{H}{ }^{n}\right) \\
{\left[\mu^{n}\right] } & =\mathcal{L}_{\boldsymbol{\mu}}\left(p_{H}{ }^{n}, \dot{\gamma}^{n}\right)
\end{aligned}
$$

As the adopted time discretization scheme is fully implicit, the rigid body and structural velocities can be fully determined in terms of the associated quantities calculated in the previous time steps (see Section 6.1). Hence, the $\dot{\overrightarrow{\boldsymbol{q}}}^{n}$ and $\dot{\delta}^{n}$ variables may be omitted from the above equations without loss of generality. Furthermore, Eqs. 9.1 can be redefined by retaining explicitly only the variables directly involved in the coupled EHL solution. Accordingly:

$$
\begin{aligned}
{\left[\overrightarrow{\boldsymbol{q}}^{n}, p_{H}{ }^{n}, \dot{\gamma}^{n}\right] } & =\mathcal{E}\left(\delta^{n}, \rho^{n}, \mu^{n}, \overrightarrow{\boldsymbol{F}}_{\boldsymbol{e x t}}{ }^{n}\right) \\
{\left[\delta^{n}\right] } & =\mathcal{S}\left(p_{H}{ }^{n}\right) \\
{\left[\rho^{n}\right] } & =\mathcal{L}_{\boldsymbol{\rho}}\left(p_{H}{ }^{n}\right) \\
{\left[\mu^{n}\right] } & =\mathcal{L}_{\boldsymbol{\mu}}\left(p_{H}{ }^{n}, \dot{\gamma}^{n}\right)
\end{aligned}
$$

Notice that each solver operator displayed in Eqs. 9.1-9.2 represent a set of calculation procedures for the mathematical models that compound that solver. With respect to the notation for the following developments, superscript $n$ will be replaced by $k$ to indicate the coupling iteration within time step $t^{n}$. In addition, all the coupled field variables $\left(p_{H}\right.$, $\dot{\gamma}, \delta, \rho, \mu)$ will be denoted in terms of vectors $\left(\overrightarrow{\boldsymbol{p}}_{\boldsymbol{H}}, \overrightarrow{\dot{\gamma}}, \overrightarrow{\boldsymbol{\delta}}, \overrightarrow{\boldsymbol{\rho}}, \overrightarrow{\boldsymbol{\mu}}\right)$ representing their respective nodal values on the hydrodynamic mesh.

\subsection{Interpolations on the Fluid-Structure Interface}

Hydrodynamic and structural meshes may have different grid size requirements. Frequently, the hydrodynamic mesh tends to be finer then the structural grid in order to capture specific geometric features and their influence on the system hydrodynamic behaviour. Moreover, a finer hydrodynamic grid also provides a more accurate evaluation of the bearing performance parameters. In these situations, the structural displacements and hydrodynamic pressures have to be transferred from one mesh to another, and vice-versa.

The simplest approach to accomplish the information transfer between different meshes, adopted in the present thesis, is the so-called nearest-neighbour interpolation. The nearestneighbour interpolation approach does not require the knowledge of the connectivity between the grid points. Instead, the results at each node on a secondary mesh (target mesh) are obtained by projecting it onto a primary mesh (source mesh), followed 
by an interpolation using the values of the nearest (neighbour) source nodes. Such mesh interpolation methodology is designated as projection method [81]. In the current work, linear interpolation functions are used to approximate the nodal bearing displacements originally evaluated on the structural mesh in the fluid mesh. As for the determination of the nearest nodes, a simpler algorithm based on the nodes coordinates is employed. Furthermore, as only small structural displacements are admitted, any effect related to the movement of the fluid grid is neglected.

\subsection{Fixed Point Gauss-Seidel Method (PGMF)}

The Fixed Point Gauss-Seidel Method (PGMF) is the simplest iterative technique employed for the direct solution of EHL conformal contacts. In this method, at each coupling iteration $k$, the vectors of the structural displacements and rheological properties are updated according to the residual vectors $\left(\overrightarrow{\boldsymbol{r}}_{\boldsymbol{\delta}, \boldsymbol{\rho}, \boldsymbol{\mu}}{ }^{k}\right)$ computed in the previous iteration:

$$
\begin{aligned}
\overrightarrow{\boldsymbol{\delta}}^{k} & =\overrightarrow{\boldsymbol{\delta}}^{k-1}+\omega_{\delta} \overrightarrow{\boldsymbol{r}}_{\boldsymbol{\delta}}{ }^{k} \\
\overrightarrow{\boldsymbol{\rho}}^{k} & =\overrightarrow{\boldsymbol{\rho}}^{k-1}+\omega_{\rho} \overrightarrow{\boldsymbol{r}}_{\boldsymbol{\rho}}{ }^{k} \\
\overrightarrow{\boldsymbol{\mu}}^{k} & =\overrightarrow{\boldsymbol{\mu}}^{k-1}+\omega_{\mu} \overrightarrow{\boldsymbol{r}}_{\boldsymbol{\mu}}{ }^{k}
\end{aligned}
$$

The fixed under-relaxation parameters $\omega_{\delta, \rho, \mu}$, with $0<\omega_{\delta, \rho, \mu} \leq 1$, are introduced in order to accommodate abrupt changes in the variables that might cause difficulties in the numerical solution convergence. The optimum values of $\omega_{\delta, \rho, \mu}$ are problem dependent and have to be determined empirically for each particular simulation case. The computation of the residual vectors is based on the intermediate solutions obtained in the previous iteration. Mathematically:

$$
\begin{array}{ll}
\overrightarrow{\boldsymbol{r}}_{\boldsymbol{\delta}}^{k}=\overrightarrow{\tilde{\boldsymbol{\delta}}}^{k}-\overrightarrow{\boldsymbol{\delta}}^{k-1} & \overrightarrow{\tilde{\boldsymbol{\delta}}}^{k}=\mathcal{S} \circ \mathcal{E}\left(\overrightarrow{\boldsymbol{\delta}}^{k-1}, \overrightarrow{\boldsymbol{\rho}}^{k-1}, \overrightarrow{\boldsymbol{\mu}}^{k-1}, \overrightarrow{\boldsymbol{q}}^{k}\right) \\
\overrightarrow{\boldsymbol{r}}_{\boldsymbol{\rho}}{ }^{k}=\overrightarrow{\tilde{\boldsymbol{\rho}}}^{k}-\overrightarrow{\boldsymbol{\rho}}^{k-1} & \overrightarrow{\tilde{\boldsymbol{\rho}}}^{k}=\mathcal{L}_{\boldsymbol{\rho}} \circ \mathcal{E}\left(\overrightarrow{\boldsymbol{\delta}}^{k-1}, \overrightarrow{\boldsymbol{\rho}}^{k-1}, \overrightarrow{\boldsymbol{\mu}}^{k-1}, \overrightarrow{\boldsymbol{q}}^{k}\right) \\
\overrightarrow{\boldsymbol{r}}_{\boldsymbol{\mu}}{ }^{k}=\overrightarrow{\tilde{\boldsymbol{\mu}}}^{k}-\overrightarrow{\boldsymbol{\mu}}^{k-1} & \overrightarrow{\tilde{\boldsymbol{\mu}}}^{k}=\mathcal{L}_{\boldsymbol{\mu}} \circ \mathcal{E}\left(\overrightarrow{\boldsymbol{\delta}}^{k-1}, \overrightarrow{\boldsymbol{\rho}}^{k-1}, \overrightarrow{\boldsymbol{\mu}}^{k-1}, \overrightarrow{\boldsymbol{q}}^{k}\right)
\end{array}
$$

where the over-tilde represents intermediate solutions and the notation ' $\circ$ ' indicates function composition, in which the results from the $\mathcal{E}$ solver are given as input to the other operators. Notice that $\mathcal{E}$ is shown in terms of $\overrightarrow{\boldsymbol{q}}^{k}$, the converged rigid body position obtained by solving the "instantaneous equilibrium" equations (Eq. 6.4) for $\overrightarrow{\boldsymbol{F}}_{\boldsymbol{e x t}}$ and with the rheological properties and structural displacements evaluated in the previous iteration $(k-1)$. The solution of the equilibrium equations also provides the values of the hydrodynamic pressures $\left(\overrightarrow{\boldsymbol{p}}_{\boldsymbol{H}}{ }^{k}\right)$ and shear rates $\left(\overrightarrow{\dot{\gamma}}^{k}\right)$ needed to compute the intermediate structural displacements $\left(\overrightarrow{\tilde{\boldsymbol{\delta}}}^{k}\right)$, lubricant density $\left(\overrightarrow{\tilde{\boldsymbol{\rho}}}^{k}\right)$ and viscosity $\left(\overrightarrow{\tilde{\boldsymbol{\mu}}}^{k}\right)$. The pseudo-code displayed in Algorithm 1 summarizes the overall EHL solution with PGMF method. 


\subsection{Point Gauss-Seidel Method with Aitken Acceler- ation (PGMA)}

The convergence of the PGMF method can be improved by introducing the so-called Aitken relaxation scheme [110, 126, 151, 152]. This technique is based on "dynamically" varying the scalar under-relaxation parameters $\omega_{\delta, \rho, \mu}$ (which were previously assumed constant in the fixed-point method) within a time step. In this case, the Gauss-Seidel iteration can be rewritten as follows:

$$
\begin{aligned}
\overrightarrow{\boldsymbol{\delta}}^{k} & =\overrightarrow{\boldsymbol{\delta}}^{k-1}+\omega_{\delta}^{k} \overrightarrow{\boldsymbol{r}}_{\boldsymbol{\delta}}{ }^{k} \\
\overrightarrow{\boldsymbol{\rho}}^{k} & =\overrightarrow{\boldsymbol{\rho}}^{k-1}+\omega_{\rho}^{k} \overrightarrow{\boldsymbol{r}}_{\boldsymbol{\rho}}{ }^{k} \\
\overrightarrow{\boldsymbol{\mu}}^{k} & =\overrightarrow{\boldsymbol{\mu}}^{k-1}+\omega_{\mu}^{k} \overrightarrow{\boldsymbol{r}}_{\boldsymbol{\mu}}{ }^{k}
\end{aligned}
$$

Notice that iteration index $k$ also appears in the relaxation parameters, since their values are allowed to change in every coupling iteration. The computation of the residual vectors remains the same as those defined for the PGMF method (Eqs. 9.4). As for the values of the relaxation factors, they are updated according to the residues calculated in the previous iteration as:

$$
\omega_{\delta, \rho, \mu}^{k}=\omega_{\delta, \rho, \mu}^{k-1} \frac{\left(\overrightarrow{\boldsymbol{r}}_{\boldsymbol{\delta}, \boldsymbol{\rho}, \boldsymbol{\mu}}{ }^{k-1}\right)^{T} \cdot\left(\overrightarrow{\boldsymbol{r}}_{\boldsymbol{\delta}, \boldsymbol{\rho}, \boldsymbol{\mu}}{ }^{k-1}-\overrightarrow{\boldsymbol{r}}_{\boldsymbol{\delta}, \boldsymbol{\rho}, \boldsymbol{\mu}}{ }^{k}\right)}{\left(\overrightarrow{\boldsymbol{r}}_{\boldsymbol{\delta}, \boldsymbol{\rho}, \boldsymbol{\mu}}{ }^{k}-\overrightarrow{\boldsymbol{r}}_{\boldsymbol{\delta}, \boldsymbol{\rho}, \boldsymbol{\mu}}{ }^{k-1}\right)^{T} \cdot\left(\overrightarrow{\boldsymbol{r}}_{\boldsymbol{\delta}, \boldsymbol{\rho}, \boldsymbol{\mu}}{ }^{k}-\overrightarrow{\boldsymbol{r}}_{\boldsymbol{\delta}, \boldsymbol{\rho}, \boldsymbol{\mu}}{ }^{k-1}\right)}
$$

where superscript $T$ denotes transpose vector and the symbol '.' means dot product. In order to ensure that $0<\omega_{\delta, \rho, \mu} \leq 1$ (under-relaxation) and to avoid either negative or very small values, the following restrictions are imposed on the magnitude of the relaxation parameters:

$$
\omega_{\delta, \rho, \mu}^{k} \leftarrow \min \left[\max \left(\omega_{\delta, \rho, \mu}^{k}, \omega_{\min }\right), \omega_{\max }\right]
$$

The limit values of $\omega_{\min }=0.001$ and $\omega_{\max }=1$ are adopted throughout the thesis. Moreover, the first relaxation in a time step is executed with the relaxation factor from the end of the previous time step. The pseudo-code shown in Algorithm 2 summarizes the overall EHL solution with PGMA method.

\subsection{Interface Quasi-Newton Method (IQN-ILS)}

The Gauss-Seidel based methods previously described are prone to numerical instabilities or low convergence rates, especially when large elastic deformations occur [147]. In this section, a method expected to be more robust is proposed, in which the inverse of the Jacobian matrices of the complete nonlinear system of equations are successively approximated during the iterative procedure. The present derivation evolves from the method detailed in [64].

Initially, the full EHL problem (Eqs. 9.2) is reformulated as a set of nonlinear equations, as follows:

$$
\begin{array}{r}
\mathcal{E}\left(\vec{\delta}, \vec{\rho}, \vec{\mu}, \vec{F}_{\text {ext }}\right)-\left[\overrightarrow{\boldsymbol{q}}, \vec{p}_{H}, \overrightarrow{\dot{\gamma}}\right]=\overrightarrow{0} \\
\mathcal{S}\left(\vec{p}_{H}\right)-\vec{\delta}=\overrightarrow{0}
\end{array}
$$




$$
\begin{aligned}
\mathcal{L}_{\rho}\left(\vec{p}_{H}\right)-\vec{\rho} & =\overrightarrow{0} \\
\mathcal{L}_{\mu}\left(\vec{p}_{H}, \vec{\gamma}\right)-\vec{\mu} & =\overrightarrow{0}
\end{aligned}
$$

By considering the same function composition used in Eqs. 9.4, where the outputs of the $\mathcal{E}$ solver are given as input to the other operators, the above equations can be restated as:

$$
\begin{array}{r}
\mathcal{S} \circ \mathcal{E}(\vec{\delta}, \vec{\rho}, \vec{\mu}, \vec{q})-\vec{\delta}=\overrightarrow{0} \\
\mathcal{L}_{\rho} \circ \mathcal{E}(\vec{\delta}, \vec{\rho}, \vec{\mu}, \vec{q})-\vec{\rho}=\overrightarrow{0} \\
\mathcal{L}_{\mu} \circ \mathcal{E}(\vec{\delta}, \vec{\rho}, \vec{\mu}, \vec{q})-\vec{\mu}=\overrightarrow{0}
\end{array}
$$

Furthermore, the introduction of the residual operators

$$
\begin{aligned}
\Re_{\delta}(\vec{\delta}) & =\mathcal{S} \circ \mathcal{E}(\vec{\delta}, \vec{\rho}, \vec{\mu}, \vec{q})-\vec{\delta}=\overrightarrow{0} \\
\Re_{\rho}(\vec{\rho}) & =\mathcal{L}_{\rho} \circ \mathcal{E}(\vec{\delta}, \vec{\rho}, \vec{\mu}, \vec{q})-\vec{\rho}=\overrightarrow{0} \\
\Re_{\mu}(\vec{\mu}) & =\mathcal{L}_{\mu} \circ \mathcal{E}(\vec{\delta}, \vec{\rho}, \vec{\mu}, \vec{q})-\vec{\mu}=\overrightarrow{0}
\end{aligned}
$$

yields compact equations for three unknown variables, namely the structural displacements $(\overrightarrow{\boldsymbol{\delta}})$ and the lubricant density $(\overrightarrow{\boldsymbol{\rho}})$ and viscosity $(\overrightarrow{\boldsymbol{\mu}})$; such unknown quantities, in turn, are implicitly dependent on the hydrodynamic pressure $\left(\overrightarrow{\boldsymbol{p}}_{\boldsymbol{H}}\right)$ and shear rate $(\overrightarrow{\dot{\gamma}})$ fields.

The above defined residuals can be solved by using the well-known Newton-Raphson iterations:

$$
\begin{array}{ll}
\overrightarrow{\boldsymbol{\delta}}^{k}=\overrightarrow{\boldsymbol{\delta}}^{k-1}+\Delta \overrightarrow{\boldsymbol{\delta}}^{k} & {\left[\Re_{\boldsymbol{\delta}}^{k-1}\right] \Delta \overrightarrow{\boldsymbol{\delta}}^{k}=-\overrightarrow{\boldsymbol{r}}_{\boldsymbol{\delta}}{ }^{k}} \\
\overrightarrow{\boldsymbol{\rho}}^{k}=\overrightarrow{\boldsymbol{\rho}}^{k-1}+\Delta \overrightarrow{\boldsymbol{\rho}}^{k} & {\left[\Re_{\boldsymbol{\rho}}^{k-1}\right] \Delta \overrightarrow{\boldsymbol{\rho}}^{k}=-\overrightarrow{\boldsymbol{r}}_{\boldsymbol{\rho}}{ }^{k}} \\
\overrightarrow{\boldsymbol{\mu}}^{k}=\overrightarrow{\boldsymbol{\mu}}^{k-1}+\Delta \overrightarrow{\boldsymbol{\mu}}^{k} & {\left[\Re_{\boldsymbol{\mu}}{ }^{k-1}\right] \Delta \overrightarrow{\boldsymbol{\mu}}^{k}=-\overrightarrow{\boldsymbol{r}}_{\boldsymbol{\mu}}{ }^{k}}
\end{array}
$$

with residuals computed as:

$$
\begin{array}{rlrl}
\overrightarrow{\boldsymbol{r}}_{\boldsymbol{\delta}}{ }^{k} & =\Re_{\boldsymbol{\delta}}\left(\overrightarrow{\boldsymbol{\delta}}^{k-1}\right)=\overrightarrow{\tilde{\boldsymbol{\delta}}}^{k}-\overrightarrow{\boldsymbol{\delta}}^{k-1} & & \overrightarrow{\tilde{\boldsymbol{\delta}}}^{k}=\mathcal{S} \circ \mathcal{E}\left(\overrightarrow{\boldsymbol{\delta}}^{k-1}, \overrightarrow{\boldsymbol{\rho}}^{k-1}, \overrightarrow{\boldsymbol{\mu}}^{k-1}, \overrightarrow{\boldsymbol{q}}^{k}\right) \\
\overrightarrow{\boldsymbol{r}}_{\boldsymbol{\rho}}{ }^{k}=\Re_{\boldsymbol{\rho}}\left(\overrightarrow{\boldsymbol{\rho}}^{k-1}\right)=\overrightarrow{\tilde{\boldsymbol{\rho}}}^{k}-\overrightarrow{\boldsymbol{\rho}}^{k-1} & \overrightarrow{\tilde{\boldsymbol{\rho}}}^{k}=\mathcal{L}_{\boldsymbol{\rho}} \circ \mathcal{E}\left(\overrightarrow{\boldsymbol{\delta}}^{k-1}, \overrightarrow{\boldsymbol{\rho}}^{k-1}, \overrightarrow{\boldsymbol{\mu}}^{k-1}, \overrightarrow{\boldsymbol{q}}^{k}\right) \\
\overrightarrow{\boldsymbol{r}}_{\boldsymbol{\mu}}{ }^{k}=\Re_{\boldsymbol{\mu}}\left(\overrightarrow{\boldsymbol{\mu}}^{k-1}\right)=\overrightarrow{\tilde{\boldsymbol{\mu}}}^{k}-\overrightarrow{\boldsymbol{\mu}}^{k-1} & \overrightarrow{\tilde{\boldsymbol{\mu}}}^{k}=\mathcal{L}_{\boldsymbol{\mu}} \circ \mathcal{E}\left(\overrightarrow{\boldsymbol{\delta}}^{k-1}, \overrightarrow{\boldsymbol{\rho}}^{k-1}, \overrightarrow{\boldsymbol{\mu}}^{k-1}, \overrightarrow{\boldsymbol{q}}^{k}\right)
\end{array}
$$

In the above expressions, $\left[\Re_{\boldsymbol{\delta}, \boldsymbol{\rho}, \boldsymbol{\mu}}^{\prime}{ }^{k-1}\right]$ are the Jacobian matrices of $\Re_{\boldsymbol{\delta}, \boldsymbol{\rho}, \boldsymbol{\mu}}$ evaluated at $\overrightarrow{\boldsymbol{\delta}}^{k-1}, \overrightarrow{\boldsymbol{\rho}}^{k-1}$ and $\overrightarrow{\boldsymbol{\mu}}^{k-1}$, respectively, all at time level $n$. Again, notice that $\boldsymbol{E}$ is shown in terms of $\overrightarrow{\boldsymbol{q}}^{k}$, the converged rigid body position obtained by solving the "instantaneous equilibrium" equations (Eq. 6.4) for $\overrightarrow{\boldsymbol{F}}_{\text {ext }}$ and with the rheological properties and structural displacements evaluated in the previous iteration $(k-1)$.

In partitioned solutions, the exact Jacobian matrix is unknown a priori since all the operators involved are assumed "black-box" solvers. Although the number of degrees of 
freedom on the contact interface is smaller than in the entire bearing structure (reduction technique, see Section 3.2), Jacobian matrices $\Re_{\boldsymbol{\delta}, \boldsymbol{\rho}, \boldsymbol{\mu}}^{\prime}$ are normally dense. Consequently, the solution of the linear systems in Eqs. 9.11 at each iteration would still represent a drawback in terms of computational efforts, particularly in problems with a large number of retained nodes on the bearing surfaces.

An alternative to the inexistence of the exact Jacobian matrix is the use of some approximation technique. Quasi-Newton iterations could be used in such cases, but the direct solution of the linear systems would still have to be computed. However, one notices that the approximation of the Jacobian matrix can in fact be suppressed, in the sense that it is more advantageous to approximate the inverse of the Jacobian directly. In this case, the quasi-Newton iterations can be rewritten as:

$$
\begin{aligned}
\overrightarrow{\boldsymbol{\delta}}^{k} & =\overrightarrow{\boldsymbol{\delta}}^{k-1}+\left[{\widehat{\Re_{\boldsymbol{\delta}}^{\prime k-1}}}^{-1}\left(-\overrightarrow{\boldsymbol{r}}_{\boldsymbol{\delta}}{ }^{k}\right)\right. \\
\overrightarrow{\boldsymbol{\rho}}^{k} & =\overrightarrow{\boldsymbol{\rho}}^{k-1}+\left[\widehat{\Re_{\boldsymbol{\rho}}^{k-1}}\right]^{-1}\left(-\overrightarrow{\boldsymbol{r}}_{\boldsymbol{\rho}}{ }^{k}\right) \\
\overrightarrow{\boldsymbol{\mu}}^{k} & =\overrightarrow{\boldsymbol{\mu}}^{k-1}+\left[\widehat{\Re_{\boldsymbol{\mu}}^{k-1}}\right]^{-1}\left(-\overrightarrow{\boldsymbol{r}}_{\boldsymbol{\mu}}{ }^{k}\right)
\end{aligned}
$$

where $\left[\Re_{\boldsymbol{\delta}, \boldsymbol{\rho}, \boldsymbol{\mu}}^{{ }^{k-1}}\right]^{-1}$ indicates the approximation for the inverse of the Jacobian matrices. Additionally, it can also be observed in Eqs. 9.13 that such approximation does not need to be performed explicitly; only a procedure to calculate the matrix-vector products $\left[\Re_{\boldsymbol{\delta}, \boldsymbol{\rho}, \boldsymbol{\mu}}^{k-1}\right]^{-1}\left(\overrightarrow{\boldsymbol{r}}_{\boldsymbol{\delta}, \boldsymbol{\rho}, \boldsymbol{\mu}}{ }^{k}\right)$ is sufficient.

The main purpose of the coupling method presented in this section is essentially to conceive an ingenious and efficient approximation solution for the matrix-vector products of Eqs. 9.13. This intent is accomplished with a formulation that progressively reconstructs and improves the solution of the matrix-vector products by using the results calculated in the previous quasi-Newton iterations. These pieces of information are consecutively stored as the columns in the following auxiliary matrices:

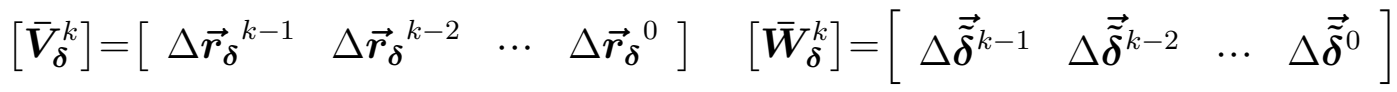

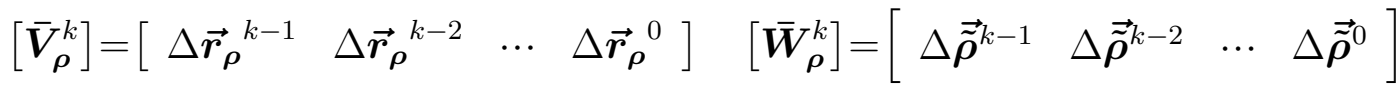

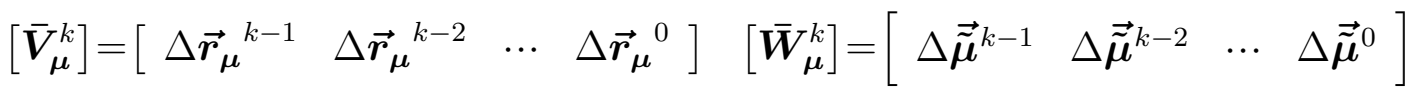

The information from previous time steps can be reused due to the similarity among their results. The matrices $\overline{\boldsymbol{V}}_{\boldsymbol{\delta}, \boldsymbol{\rho}, \boldsymbol{\mu}}{ }^{k}$ and $\overline{\boldsymbol{W}}_{\boldsymbol{\delta}, \boldsymbol{\rho}, \boldsymbol{\mu}}{ }^{k}$ are then combined with those from $q$ previous time steps (if, of course, at least $q$ time steps have already been computed), providing the following full auxiliary matrices (attention to the different superscripts):

$$
\begin{aligned}
& {\left[\boldsymbol{V}_{\boldsymbol{\delta}}^{k}\right]=\left[\begin{array}{llll}
\overline{\boldsymbol{V}}_{\boldsymbol{\delta}}^{k} & \boldsymbol{V}_{\boldsymbol{\delta}}^{n-1} & \cdots & \boldsymbol{V}_{\boldsymbol{\delta}}^{n-q}
\end{array}\right] \quad\left[\begin{array}{llll}
\boldsymbol{W}_{\boldsymbol{\delta}}^{k}
\end{array}\right]=\left[\begin{array}{llll}
\overline{\boldsymbol{W}}_{\boldsymbol{\delta}}^{k} & \boldsymbol{W}_{\boldsymbol{\delta}}^{n-1} & \cdots & \boldsymbol{W}_{\boldsymbol{\delta}}^{n-q}
\end{array}\right]} \\
& {\left[\begin{array}{llll}
\boldsymbol{V}_{\boldsymbol{\rho}}^{k}
\end{array}\right]=\left[\begin{array}{llll}
\overline{\boldsymbol{V}}_{\boldsymbol{\rho}}^{k} & \boldsymbol{V}_{\boldsymbol{\rho}}^{n-1} & \ldots & \boldsymbol{V}_{\boldsymbol{\rho}}^{n-q}
\end{array}\right] \quad\left[\begin{array}{llll}
\boldsymbol{W}_{\boldsymbol{\rho}}{ }^{n}
\end{array}\right]=\left[\begin{array}{llll}
\overline{\boldsymbol{W}}_{\boldsymbol{\rho}}^{k} & \boldsymbol{W}_{\boldsymbol{\rho}}^{n-1} & \ldots & \boldsymbol{W}_{\boldsymbol{\rho}}^{n-q}
\end{array}\right]} \\
& {\left[\begin{array}{llll}
\boldsymbol{V}_{\boldsymbol{\mu}}{ }^{k}
\end{array}\right]=\left[\begin{array}{llll}
\overline{\boldsymbol{V}}_{\boldsymbol{\mu}}^{k} & \boldsymbol{V}_{\boldsymbol{\mu}}{ }^{n-1} & \ldots & \boldsymbol{V}_{\boldsymbol{\mu}}{ }^{n-q}
\end{array}\right] \quad\left[\begin{array}{llll}
\boldsymbol{W}_{\boldsymbol{\mu}}{ }^{k}
\end{array}\right]=\left[\begin{array}{llll}
\overline{\boldsymbol{W}}_{\boldsymbol{\mu}}^{k} & \boldsymbol{W}_{\boldsymbol{\mu}}{ }^{n-1} & \cdots & \boldsymbol{W}_{\boldsymbol{\mu}}{ }^{n-q}
\end{array}\right]}
\end{aligned}
$$

The consideration of information from previous time steps substantially accelerates the convergence of the coupling iterations. However, if results from too many times steps 
are reused, the convergence can decay as the information from the time step $(n-q)$ might no longer be relevant in time step $n$. The optimum value of $q$ is problem-dependent but the convergence does not vary significantly near the optimum; the value of $q=5$ has shown to be effective for the EHL solutions presented later in this thesis. Notice that as the matrices $\left[\boldsymbol{V}_{\boldsymbol{\delta}, \boldsymbol{\rho}, \boldsymbol{\mu}}{ }^{k}\right]$ and $\left[\boldsymbol{W}_{\boldsymbol{\delta}, \boldsymbol{\rho}, \boldsymbol{\mu}}{ }^{k}\right]$ contain at least one column, simple relaxation has to be performed only in the beginning of the first time step if information from the previous time steps is reused $(q>0)$; if reuse is not considered $(q=0)$, such relaxation has to be done in the beginning of every time step.

In Eqs. 9.14, the differences among the vectors from the coupling iterations $k$ and $(k-1)$ are defined as (see Eqs. 9.12):

$$
\begin{array}{rlrl}
\Delta \overrightarrow{\boldsymbol{r}}_{\boldsymbol{\delta}}{ }^{k-1} & =\overrightarrow{\boldsymbol{r}}_{\boldsymbol{\delta}}{ }^{k}-\overrightarrow{\boldsymbol{r}}_{\boldsymbol{\delta}}^{k-1} & \Delta \overrightarrow{\tilde{\boldsymbol{\delta}}}^{k-1} & =\overrightarrow{\tilde{\boldsymbol{\delta}}}^{k}-\overrightarrow{\tilde{\boldsymbol{\delta}}}^{k-1} \\
\Delta \overrightarrow{\boldsymbol{r}}_{\boldsymbol{\rho}}{ }^{k-1} & =\overrightarrow{\boldsymbol{r}}_{\boldsymbol{\rho}}{ }^{k}-\overrightarrow{\boldsymbol{r}}_{\boldsymbol{\rho}}^{k-1} & \Delta \overrightarrow{\tilde{\boldsymbol{\rho}}}^{k-1}=\overrightarrow{\tilde{\boldsymbol{\rho}}}^{k}-\overrightarrow{\tilde{\boldsymbol{\rho}}}^{k-1} \\
\Delta \overrightarrow{\boldsymbol{r}}_{\boldsymbol{\mu}}{ }^{k-1} & =\overrightarrow{\boldsymbol{r}}_{\boldsymbol{\mu}}{ }^{k}-\overrightarrow{\boldsymbol{r}}_{\boldsymbol{\mu}}{ }^{k-1} & \Delta \overrightarrow{\tilde{\boldsymbol{\mu}}}^{k-1} & =\overrightarrow{\tilde{\boldsymbol{\mu}}}^{k}-\overrightarrow{\tilde{\boldsymbol{\mu}}}^{k-1}
\end{array}
$$

Moreover, the difference between the desired (converged) and current residuals, i.e. $\Delta \overrightarrow{\boldsymbol{r}}_{\boldsymbol{\delta}, \boldsymbol{\rho}, \boldsymbol{\mu}}=\overrightarrow{\mathbf{0}}-\overrightarrow{\boldsymbol{r}}_{\boldsymbol{\delta}, \boldsymbol{\rho}, \boldsymbol{\mu}}{ }^{k}$, can be approximated as a linear combination of the previous differences:

$$
\begin{array}{lll}
\Delta \overrightarrow{\boldsymbol{r}}_{\boldsymbol{\delta}} \approx\left[\boldsymbol{V}_{\boldsymbol{\delta}}{ }^{k}\right] \overrightarrow{\boldsymbol{c}}_{\boldsymbol{\delta}}{ }^{k} & \rightarrow & {\left[\boldsymbol{V}_{\boldsymbol{\delta}}{ }^{k}\right] \overrightarrow{\boldsymbol{c}}_{\boldsymbol{\delta}}{ }^{k}=-\overrightarrow{\boldsymbol{r}}_{\boldsymbol{\delta}}{ }^{k}} \\
\Delta \overrightarrow{\boldsymbol{r}}_{\boldsymbol{\rho}} \approx\left[\boldsymbol{V}_{\boldsymbol{\rho}}{ }^{k}\right] \overrightarrow{\boldsymbol{c}}_{\boldsymbol{\rho}}{ }^{k} & \rightarrow & {\left[\boldsymbol{V}_{\boldsymbol{\rho}}{ }^{k}\right] \overrightarrow{\boldsymbol{c}}_{\boldsymbol{\rho}}{ }^{k}=-\overrightarrow{\boldsymbol{r}}_{\boldsymbol{\rho}}{ }^{k}} \\
\Delta \overrightarrow{\boldsymbol{r}}_{\boldsymbol{\mu}} \approx\left[\boldsymbol{V}_{\boldsymbol{\mu}}{ }^{k}\right] \overrightarrow{\boldsymbol{c}}_{\boldsymbol{\mu}}{ }^{k} & \rightarrow & {\left[\boldsymbol{V}_{\boldsymbol{\mu}}{ }^{k}\right] \overrightarrow{\boldsymbol{c}}_{\boldsymbol{\mu}}{ }^{k}=-\overrightarrow{\boldsymbol{r}}_{\boldsymbol{\mu}}{ }^{k}}
\end{array}
$$

where $\overrightarrow{\boldsymbol{c}}_{\boldsymbol{\delta}, \boldsymbol{\rho}, \boldsymbol{\mu}}{ }^{k}$ are vectors with the coefficients of the linear approximation. As the number of columns of matrices $\left[\boldsymbol{V}_{\boldsymbol{\delta}, \boldsymbol{\rho}, \boldsymbol{\mu}}{ }^{k}\right]$ is $k \leq N_{P}$, where $N_{P}$ is the number of nodes of the hydrodynamic mesh, the system is overdetermined for $\overrightarrow{\boldsymbol{c}}_{\boldsymbol{\delta}, \boldsymbol{\rho}, \boldsymbol{\mu}}{ }^{k}$ and hence has to be calculated in least-squares sense. The solution of the posed least-squares problem can be accomplished by using the simplest normal decomposition of $\left[\boldsymbol{V}_{\boldsymbol{\delta}, \boldsymbol{\rho}, \boldsymbol{\mu}}{ }^{k}\right]$. However, for cases involving a high number of iterations, a more stable economy-size QR-decomposition method is recommended. Thus, for instance, by adopting the Householder transformation [64], such matrices are decomposed in $\left[\boldsymbol{V}_{\boldsymbol{\delta}, \boldsymbol{\rho}, \boldsymbol{\mu}}{ }^{k}\right]=\left[\boldsymbol{Q}_{\boldsymbol{\delta}, \boldsymbol{\rho}, \boldsymbol{\mu}}{ }^{k}\right]\left[\boldsymbol{R}_{\boldsymbol{\delta}, \boldsymbol{\rho}, \boldsymbol{\mu}}{ }^{k}\right]$, with $\left[\boldsymbol{Q}_{\boldsymbol{\delta}, \boldsymbol{\rho}, \boldsymbol{\mu}}{ }^{k}\right]$ being orthogonal matrices and $\left[\boldsymbol{R}_{\boldsymbol{\delta}, \boldsymbol{\rho}, \boldsymbol{\mu}}{ }^{k}\right]$ upper triangular matrices. In this manner, Eqs. 9.17 may be calculated for the coefficients vectors by solving the triangular systems $\left[\boldsymbol{R}_{\boldsymbol{\delta}, \boldsymbol{\rho}, \boldsymbol{\mu}}{ }^{k}\right] \overrightarrow{\boldsymbol{c}}_{\boldsymbol{\delta}, \boldsymbol{\rho}, \boldsymbol{\mu}}{ }^{k}=\left[\boldsymbol{Q}_{\boldsymbol{\delta}, \boldsymbol{\rho}, \boldsymbol{\mu}}{ }^{k}\right]^{T}\left(-\overrightarrow{\boldsymbol{r}}_{\boldsymbol{\delta}, \boldsymbol{\rho}, \boldsymbol{\mu}}{ }^{k}\right)$.

Analogously, the differences of the intermediate variables that correspond to $\Delta \overrightarrow{\boldsymbol{r}}_{\boldsymbol{\delta}, \boldsymbol{\rho}, \boldsymbol{\mu}}$ can be also approximated as a linear combination giving:

$$
\begin{aligned}
\Delta \overrightarrow{\tilde{\boldsymbol{\delta}}} & \approx\left[\boldsymbol{W}_{\boldsymbol{\delta}}{ }^{k}\right] \overrightarrow{\boldsymbol{c}}_{\boldsymbol{\delta}}{ }^{k} \\
\Delta \overrightarrow{\tilde{\boldsymbol{\rho}}} & \approx\left[\boldsymbol{W}_{\boldsymbol{\rho}}{ }^{k}\right] \overrightarrow{\boldsymbol{c}}_{\boldsymbol{\rho}}{ }^{k} \\
\Delta \overrightarrow{\tilde{\boldsymbol{\mu}}} & \approx\left[\boldsymbol{W}_{\boldsymbol{\mu}}{ }^{k}\right] \overrightarrow{\boldsymbol{c}}_{\boldsymbol{\mu}}{ }^{k}
\end{aligned}
$$

From Eqs. 9.12, the following combination of approximations can be established:

$$
\begin{aligned}
& \Delta \overrightarrow{\boldsymbol{r}}_{\boldsymbol{\delta}} \approx \Delta \overrightarrow{\tilde{\boldsymbol{\delta}}}-\Delta \overrightarrow{\boldsymbol{\delta}} \\
& \Delta \overrightarrow{\boldsymbol{r}}_{\boldsymbol{\rho}} \approx \Delta \overrightarrow{\tilde{\boldsymbol{\rho}}}-\Delta \overrightarrow{\boldsymbol{\rho}}
\end{aligned}
$$




$$
\Delta \overrightarrow{\boldsymbol{r}}_{\boldsymbol{\mu}} \approx \Delta \overrightarrow{\tilde{\mu}}-\Delta \vec{\mu}
$$

Substituting Eqs. 9.18 into Eqs. 9.19 and assuming again that $\Delta \overrightarrow{\boldsymbol{r}}_{\boldsymbol{\delta}, \boldsymbol{\rho}, \boldsymbol{\mu}}=\overrightarrow{\mathbf{0}}-\overrightarrow{\boldsymbol{r}}_{\boldsymbol{\delta}, \boldsymbol{\rho}, \boldsymbol{\mu}}{ }^{k}$, one has:

$$
\begin{aligned}
\Delta \overrightarrow{\boldsymbol{\delta}} & =\left[\boldsymbol{W}_{\boldsymbol{\delta}}{ }^{k}\right] \overrightarrow{\boldsymbol{c}}_{\boldsymbol{\delta}}{ }^{k}+\Delta \overrightarrow{\boldsymbol{r}}_{\boldsymbol{\delta}}{ }^{k} \\
\Delta \overrightarrow{\boldsymbol{\rho}} & =\left[\boldsymbol{W}_{\boldsymbol{\rho}}{ }^{k}\right] \overrightarrow{\boldsymbol{c}}_{\boldsymbol{\rho}}{ }^{k}+\Delta \overrightarrow{\boldsymbol{r}}_{\boldsymbol{\rho}}{ }^{k} \\
\Delta \overrightarrow{\boldsymbol{\mu}} & =\left[\boldsymbol{W}_{\boldsymbol{\mu}}{ }^{k}\right] \overrightarrow{\boldsymbol{c}}_{\boldsymbol{\mu}}{ }^{k}+\Delta \overrightarrow{\boldsymbol{r}}_{\boldsymbol{\mu}}{ }^{k}
\end{aligned}
$$

Finally, since $\overrightarrow{\boldsymbol{c}}_{\boldsymbol{\delta}, \boldsymbol{\rho}, \boldsymbol{\mu}}{ }^{k}$ are functions of $\overrightarrow{\boldsymbol{r}} \boldsymbol{\delta}, \boldsymbol{\rho}, \boldsymbol{\mu}^{k}$ (see Eqs. 9.17), Eqs. 9.20 can be interpreted as an approximation of the products between the inverse of the Jacobian matrices and the associated residual vectors (see Eqs. 9.13). Mathematically:

$$
\begin{aligned}
& \Delta \overrightarrow{\boldsymbol{\delta}}=\left[{\widehat{\Re_{\boldsymbol{\delta}}^{k-1}}}^{-1}\left(-\overrightarrow{\boldsymbol{r}}_{\boldsymbol{\delta}}^{k}\right)=\left[\boldsymbol{W}_{\boldsymbol{\delta}}^{k}\right] \overrightarrow{\boldsymbol{c}}_{\boldsymbol{\delta}}^{k}+\overrightarrow{\boldsymbol{r}}_{\boldsymbol{\delta}}^{k} \equiv \Delta \overrightarrow{\boldsymbol{\delta}}^{k}\right. \\
& \Delta \overrightarrow{\boldsymbol{\rho}}=\left[{\widehat{\Re_{\boldsymbol{\rho}}{ }^{k-1}}}^{-1}\left(-\overrightarrow{\boldsymbol{r}}_{\boldsymbol{\rho}}{ }^{k}\right)=\left[\boldsymbol{W}_{\boldsymbol{\rho}}{ }^{k}\right] \overrightarrow{\boldsymbol{c}}_{\boldsymbol{\rho}}{ }^{k}+\overrightarrow{\boldsymbol{r}}_{\boldsymbol{\rho}}{ }^{k} \equiv \Delta \overrightarrow{\boldsymbol{\rho}}^{k}\right. \\
& \Delta \overrightarrow{\boldsymbol{\mu}}=\left[{\widehat{\Re_{\boldsymbol{\mu}}^{\prime k-1}}}^{-1}\left(-\overrightarrow{\boldsymbol{r}}_{\boldsymbol{\mu}}{ }^{k}\right)=\left[\boldsymbol{W}_{\boldsymbol{\mu}}{ }^{k}\right] \overrightarrow{\boldsymbol{c}}_{\boldsymbol{\mu}}{ }^{k}+\overrightarrow{\boldsymbol{r}}_{\boldsymbol{\mu}}{ }^{k} \equiv \Delta \overrightarrow{\boldsymbol{\mu}}^{k}\right.
\end{aligned}
$$

The pseudo-code exposed in Algorithm 3 summarizes the overall EHL solution with IQN-ILS method.

\subsection{Algorithms for the EHL Solution of Dynamically Loaded Bearings}

The implementation details of the partitioned coupling techniques described in the above sections are summarized in Appendix G. In general, the iterative process established by each method within a given time step (Eqs. 9.3, Eqs. 9.5 or Eqs. 9.13) has to be computed until a convergence criterion is reached. The criterion adopted in this thesis is based on the definition of a relative error $\left(e_{E H L}{ }^{k}\right)$ that embraces all the solution variables, so that the calculation process is halted whenever the combined error is less than a limit value $\left(\varepsilon_{E H L}\right)$. Mathematically:

$$
\left[\overrightarrow{\boldsymbol{p}}_{\boldsymbol{H}}^{k}, \overrightarrow{\boldsymbol{\delta}}^{k}, \overrightarrow{\boldsymbol{\rho}}^{k}, \overrightarrow{\boldsymbol{\mu}}^{k}\right] \rightarrow\left[\overrightarrow{\boldsymbol{p}}_{\boldsymbol{H}}, \overrightarrow{\boldsymbol{\delta}}, \overrightarrow{\boldsymbol{\rho}}, \overrightarrow{\boldsymbol{\mu}}\right]^{n} \quad \text { if } \quad e_{E H L}{ }^{k} \leq \varepsilon_{E H L}
$$

with

$$
\begin{aligned}
e_{E H L}{ }^{k}= & \left(\frac{\left\|\overrightarrow{\boldsymbol{p}}_{\boldsymbol{H}}{ }^{k}-\overrightarrow{\boldsymbol{p}}_{\boldsymbol{H}}{ }^{k-1}\right\|}{\left\|\overrightarrow{\boldsymbol{p}}_{\boldsymbol{H}}{ }^{k}\right\|}\right)+\left(\frac{\left\|\overrightarrow{\boldsymbol{\delta}}^{k}-\overrightarrow{\boldsymbol{\delta}}^{k-1}\right\|}{\left\|\overrightarrow{\boldsymbol{\delta}}^{k}\right\|}\right)+ \\
& \left(\frac{\left\|\overrightarrow{\boldsymbol{\rho}}^{k}-\overrightarrow{\boldsymbol{\rho}}^{k-1}\right\|}{\left\|\overrightarrow{\boldsymbol{\rho}}^{k}\right\|}\right)+\left(\frac{\left\|\overrightarrow{\boldsymbol{\mu}}^{k}-\overrightarrow{\boldsymbol{\mu}}^{k-1}\right\|}{\left\|\overrightarrow{\boldsymbol{\mu}}^{k}\right\|}\right)
\end{aligned}
$$

For the calculation of the residual vectors at each coupling iteration (Eqs. 9.4 and 9.12 ), the bearing "instantaneous equilibrium" position $\overrightarrow{\boldsymbol{q}}^{k}$ has to be determined for the 
current external load $\overrightarrow{\boldsymbol{F}}_{\text {ext }}{ }^{n}$ and the rheological properties and structural displacements obtained in the previous iteration. This calculation is undertaken by solving operator $\mathcal{E}$, which in turn also provides the updated hydrodynamic pressures $\left(\overrightarrow{\boldsymbol{p}}_{\boldsymbol{H}}{ }^{k}\right)$ and shear rates $\left(\overrightarrow{\dot{\gamma}}^{k}\right)$ values needed for the next computations of the coupled variables $\left(\overrightarrow{\boldsymbol{\delta}}^{k}, \overrightarrow{\boldsymbol{\rho}}^{k}, \overrightarrow{\boldsymbol{\mu}}^{k}\right)$. This also explains the reason why $\overrightarrow{\boldsymbol{q}}^{k}$ appears within $\mathcal{E}$ in the function compositions defined for the residual equations. Furthermore, all coupling algorithms begin each time step with suitable extrapolations for both structural displacements (Eq. 6.20) and rigid body positions (Eq. 6.9); all the other coupled variables are not extrapolated at all, thus only their values at the previous time step are used to initiate the EHL iterations.

Alternative "hybrid" coupling procedures are also proposed in Algorithms 4-5. In these cases, the coupled structural and rheological solutions are each calculated using a different partitioned technique. For instance, the structural displacements can be iterated with the IQN-ILS method, while the PGMA formulation can be used for the rheological corrections, and vice-versa. 


\section{Part III}

\section{Application Cases}




\section{Chapter 10}

\section{Case I: Dynamically Loaded Connecting-Rod Big-End Bearing of a Heavy-Duty Diesel Engine}

The present case study aims to explore the use of the solution framework developed in this thesis for the simulation of connecting-rod bearings operating under severe tribological conditions. In this manner, the big-end bearing of a heavy-duty diesel (HDD) engine subjected to extremely high combustion loads is considered for analysis. Furthermore, the numerical simulations of such bearing application is prone to present convergence issues due to the high concentrated fluid pressures and large solid deformations involved, thus providing appropriate challenges for the evaluation of the robustness of the new partitioned coupling techniques contemplated in this work.

\subsection{Input Data}

The main data of the connecting-rod part, as well as the general properties and operational conditions associated with the big-end bearing are listed in Tables 10.1-10.2, respectively. The bearing bore and the conrod cap are assumed to have the same material properties of the entire rod structure. Any design details and shape variations provided by the system assemblage have been ignored in the analysis.

Moreover, Table 10.3 displays the rheological properties of the SAE $15 \mathrm{~W} 40$ engine oil at the operational temperature of $95^{\circ} \mathrm{C}$. Notice that the viscosity correction due to lubricant shear-thinning effect has not been considered at this point.

Table 10.1: General data of the heavy-duty connecting-rod part.

\begin{tabular}{lcc}
\hline Parameter & Value & Unit \\
\hline Material & Steel & - \\
Young modulus & 210 & $\mathrm{GPa}$ \\
Poisson's ratio & 0.3 & - \\
Density & 7800 & $\mathrm{~kg} / \mathrm{m}^{3}$ \\
Rod length & 192 & $\mathrm{~mm}$ \\
\hline
\end{tabular}

Similarly to the connecting-rod of the high-speed engine that will be studied in Chapter 11, the 3D FEM model of the current conrod part was condensed to the set of nodes loc- 


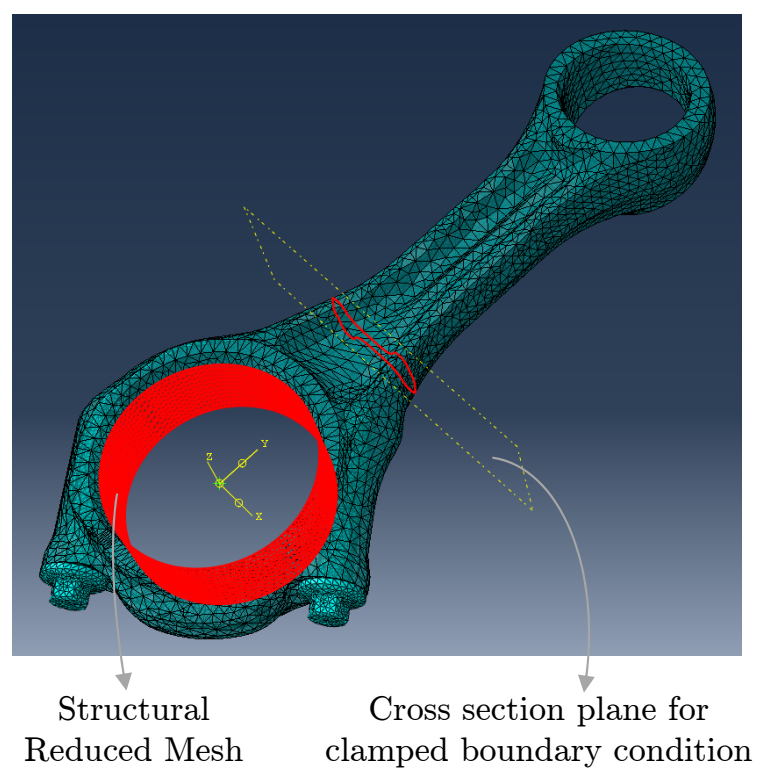

(a) 3D FEM model
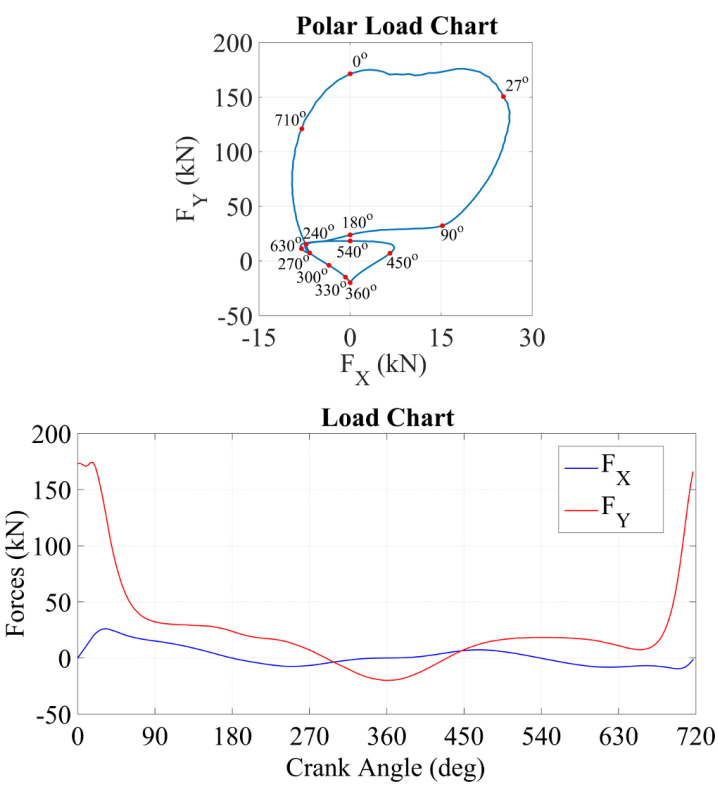

(b) Load chart of the big-end bearing

Figure 10.1: Geometry and load conditions of the heavy-duty connecting-rod system.

Table 10.2: Main properties and operational conditions of the heavy-duty big-end bearing.

\begin{tabular}{lcc}
\hline Parameter & Value & Unit \\
\hline Radius & 36.50 & $\mathrm{~mm}$ \\
Width & 30.14 & $\mathrm{~mm}$ \\
Radial clearance & 37.50 & $\mu \mathrm{m}$ \\
Ambient pressure (bearing sides) & $10^{5}$ & $\mathrm{~Pa}$ \\
Cavitation pressure & 0 & $\mathrm{~Pa}$ \\
Engine speed & 3000 & $\mathrm{RPM}$ \\
Supply hole & No & - \\
\hline
\end{tabular}

Table 10.3: Rheological properties of the SAE $15 \mathrm{~W} 40$ engine oil at $95^{\circ} \mathrm{C}$ for the heavy-duty big-end bearing simulation.

\begin{tabular}{|c|c|c|}
\hline Parameter & Value & Unit \\
\hline \multicolumn{3}{|c|}{ Reference values } \\
\hline$\eta_{0}$ & 13.55 & $\mathrm{mPas}$ \\
\hline$\rho_{0}$ & 850 & $\mathrm{~kg} / \mathrm{m}^{3}$ \\
\hline \multicolumn{3}{|c|}{ Piezo-viscosity effect (Roelands equation) ${ }^{*}$} \\
\hline$\alpha$ & $1.10 \times 10^{-8}$ & $\mathrm{~Pa}^{-1}$ \\
\hline \multicolumn{3}{|c|}{ Piezo-density effect (Dowson-Higgison equation) } \\
\hline$C_{1}$ & $5.9 \times 10^{8}$ & $\mathrm{~Pa}$ \\
\hline$C_{2}$ & 1.34 & - \\
\hline
\end{tabular}




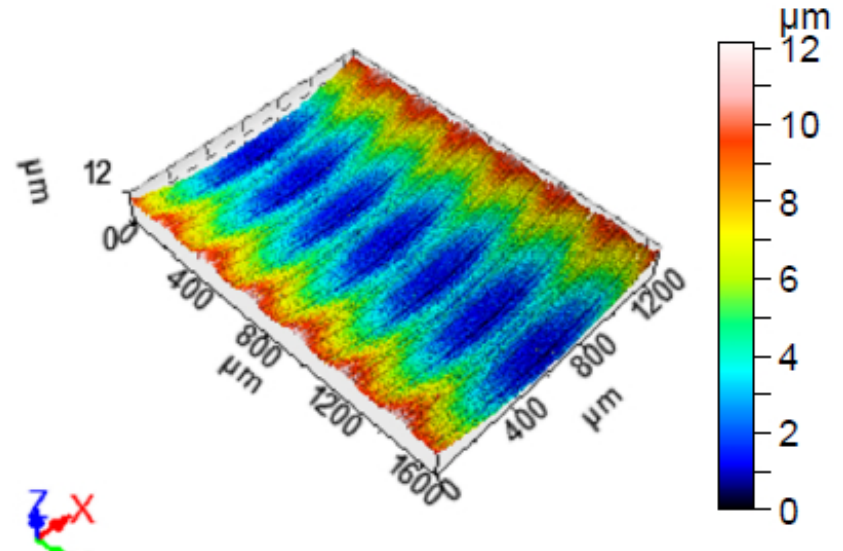

(a) Original topography as measured

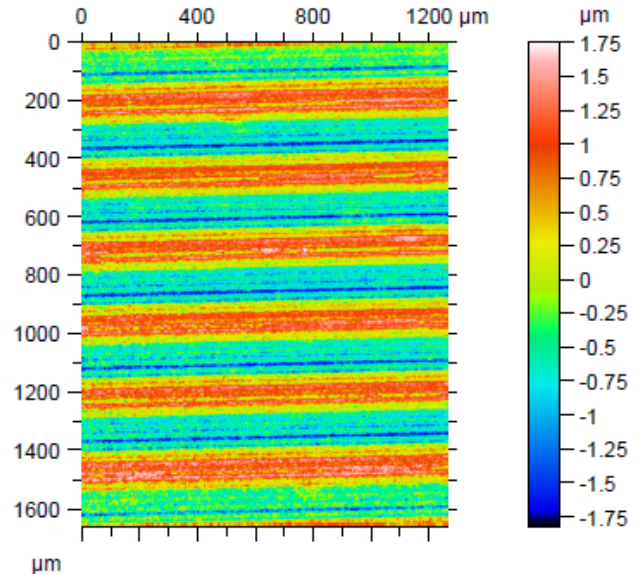

(b) Roughness topography after form and waviness removal (cut-off of $0.8 \mathrm{~mm}$ )

Figure 10.2: Representative surface topography of the heavy-duty big-end bearing shells.

ated on the big-end bearing surface by using the Abaqus ${ }^{\circledR}$ software [62]. In this case, however, a different approach widely adopted in the literature $[3,32,34,120,147,163$, $168,195,197,220]$ was chosen for the boundary condition of the solid structure. Now, the free-free condition that will be used in the high-speed big-end bearing case (Chapter 11) was substituted by the clamped condition imposed on an orthogonal cross section plane situated above the big-end bore, as illustrated in Fig. 10.1a. This condition automatically restrains the free-free rigid body motion of the structure, and is here considered to evaluate the appropriateness of the FSI partitioned techniques for different structural boundary conditions. In particular, the corresponding clamped constraint tend to yield large, unrealistic bending deformations in the rod, which in turn have to be removed to properly account for the local influence of the superficial distortions on the lubricant film thickness. The usual approach for that, here assumed, consists in subtracting the mean bending displacement of the structure from the respective bearing distortions [168]. With respect to the journal flexibility, though, it was assumed rigid due to the absence of a hollow pin-journal linking the conrod to the crankshaft. Furthermore, the external load forces depicted in Fig. 10.1b, which is represented with respect to the bearing coordinate systems illustrated in Fig. 10.1a, were extracted from earlier multibody dynamic simulations undertaken for the full (rigid) connecting-rod mechanism.

As for the inputs for the statistical mixed-lubrication models (GW/GT models for asperity contact and Patir and Cheng formulation for the average flow model), Table 10.4 collects the parameters associated with surface topographies measured from bearing shells employed in a similar diesel engine application (see Fig. 10.2). Such estimations have been here admitted due to the lack of roughness measurements of bearing shells actually used in the connecting-rod system under analysis. Notice that the shell topography in consideration is clearly designed with a circumferential micro-groove pattern $(250 \mu \mathrm{m}$ pitch), whose influence on the lubricant flow under eventual mixed-lubrication conditions is considered through the Patir and Cheng's approach for longitudinally oriented surface roughness, i.e. $\gamma \rightarrow \infty$ (see Appendix D.2); similar micro-grooved bearing topography under mixed-lubrication was investigated by [14]. Moreover, the steel journal surface has been assumed smooth due to its noticeable lower roughness compared to the bearing one. 
Table 10.4: Contact and roughness parameters of the heavy-duty big-end bearing shells.

\begin{tabular}{lcc}
\hline Parameter & Value & Unit \\
\hline Bearing material $^{*}$ & AlSnSi alloy & - \\
Overlay material $^{*}$ & PbSnCu alloy & - \\
$E$ (bearing material) $^{*}$ & 75 & $\mathrm{GPa}$ \\
$v$ (bearing material) $^{*}$ & 0.34 & - \\
$H_{V}$ (overlay material) $^{*}$ & 18 & $\mathrm{HV}$ \\
\hline$S a$ & 0.65 & $\mu \mathrm{m}$ \\
$S q$ & 0.77 & $\mu \mathrm{m}$ \\
$\gamma$ & 9 & - \\
$Z_{S}$ & 0.9 & $\mu \mathrm{m}$ \\
$\sigma_{S}$ & 0.49 & $\mu \mathrm{m}$ \\
$\beta_{S}$ & 26.17 & $\mu \mathrm{m}$ \\
$\eta_{S}$ & $2.68 \times 10^{9}$ & $\mathrm{~m}^{-2}$ \\
$\mu_{B L}$ & 0.12 & - \\
\hline${ }^{*}$ See $[14,208]$ & &
\end{tabular}

Finally, all the numerical settings, tolerance errors and specific parameter controls related to the different algorithms involved in the computational simulations of the static and dynamic EHL problems presented in the next sections are listed in Table 10.5.

\subsection{Static Loading Conditions}

Initially, the performance of the partitioned coupling methods will be evaluated for the current big-end bearing case under static loading conditions. In this case, the following two "instantaneous" critical situations were simulated individually by ignoring any transient effect: (1) high load condition with $\overrightarrow{\boldsymbol{F}}_{\text {ext }}=[-10,80] \mathrm{kN}$ pointing to the stiffer direction of the connecting-rod shank, and (2) high deformation condition with $\overrightarrow{\boldsymbol{F}}_{\boldsymbol{e x t}}=[2,-20] \mathrm{kN}$ aligned to the more compliant direction of the lower bearing shell. The results presented in the following sections have been published by the author in reference [177]. The performance of the partitioned techniques is assessed in terms of the total number of iterations needed for the final convergence of the static problem, including the intermediate load steps. The computational time of each simulation, as well as the percentage of the relative variation of the elapsed time with respect to the IQN-ILS method, are also presented as an overall evaluation of the coupling procedures.

\subsubsection{Results for the High Load Condition}

The converged static solution for the high load condition with $\overrightarrow{\boldsymbol{F}}_{\boldsymbol{e x t}}=[-10,80] \mathrm{kN}$ is summarized in Figs. 10.4-10.5, and the performance of each partitioned method is listed in table of Fig. 10.3. In Fig. 10.4, the hydrodynamic pressure distribution and the film fraction field representing the cavitation zones are illustrated for both rigid and flexible bearing cases. As can be observed, the slight increase in the area loaded by hydrodynamic pressures due to the structural deformations was enough to considerably reduce the peak pressure from $226 \mathrm{MPa}$ to $112 \mathrm{MPa}$. In contrast, the influence of the bearing distortions on 
Table 10.5: Numerical settings for the simulations of the heavy-duty big-end bearing.

\begin{tabular}{|c|c|}
\hline Parameter & Value \\
\hline \multicolumn{2}{|c|}{ Meshes } \\
\hline $\begin{array}{l}\text { Hydrodynamic } \\
\text { Structural (reduced model) }\end{array}$ & $\begin{array}{c}3600(120 \times 30) \\
984\end{array}$ \\
\hline \multicolumn{2}{|c|}{ Solver: Linear System } \\
\hline $\begin{array}{l}\text { Type } \\
\text { Relaxation (pressure) factor } \\
\text { Relaxation (film fraction) factor } \\
\text { Max. iterations } \\
\text { Tolerance error }\end{array}$ & $\begin{array}{c}\text { SOR } \\
1.8 \\
1 \\
20000 \\
10^{-6}\end{array}$ \\
\hline \multicolumn{2}{|c|}{ Solver: Rheology } \\
\hline $\begin{array}{l}\text { Type } \\
\text { Initial relaxation factor } \\
\text { Maximum iterations } \\
\text { Tolerance error } \\
\end{array}$ & $\begin{array}{c}\text { PGMA } \\
0.001 \\
30 \\
10^{-2} \\
\end{array}$ \\
\hline \multicolumn{2}{|c|}{ Solver: $E H L$} \\
\hline $\begin{array}{l}\text { Type } \\
\text { Initial relaxation factor } \\
\text { Maximum iterations } \\
\text { Tolerance error }\end{array}$ & $\begin{array}{c}\text { PGMF, PGMA, IQN-ILS } \\
0.001 \\
30 \\
10^{-2}\end{array}$ \\
\hline \multicolumn{2}{|c|}{ Solver: Static } \\
\hline $\begin{array}{l}\text { Type } \\
\text { Finite difference step } \\
\text { Maximum function evaluation } \\
\text { Maximum iterations } \\
\text { Tolerance for variables } \\
\text { Tolerance for function }\end{array}$ & $\begin{array}{l}\text { fsolveNR } \\
10^{-4} \\
30 \\
40 \\
10^{-4} \\
10^{-3} \\
\end{array}$ \\
\hline \multicolumn{2}{|c|}{ Solver: Dynamic } \\
\hline $\begin{array}{l}\text { Type } \\
\text { BDF order } \\
\theta_{1} \\
\theta_{2} \\
\text { Time step } \\
\text { Initial time } \\
\text { Maximum sub-steps } \\
\text { Maximum history }\end{array}$ & $\begin{array}{c}\text { BDF } \\
4 \\
2 \\
0.8 \\
\left.3^{\circ} \text { (crank angles }\right) \\
0.005 \mathrm{~s} \\
5 \\
5\end{array}$ \\
\hline
\end{tabular}




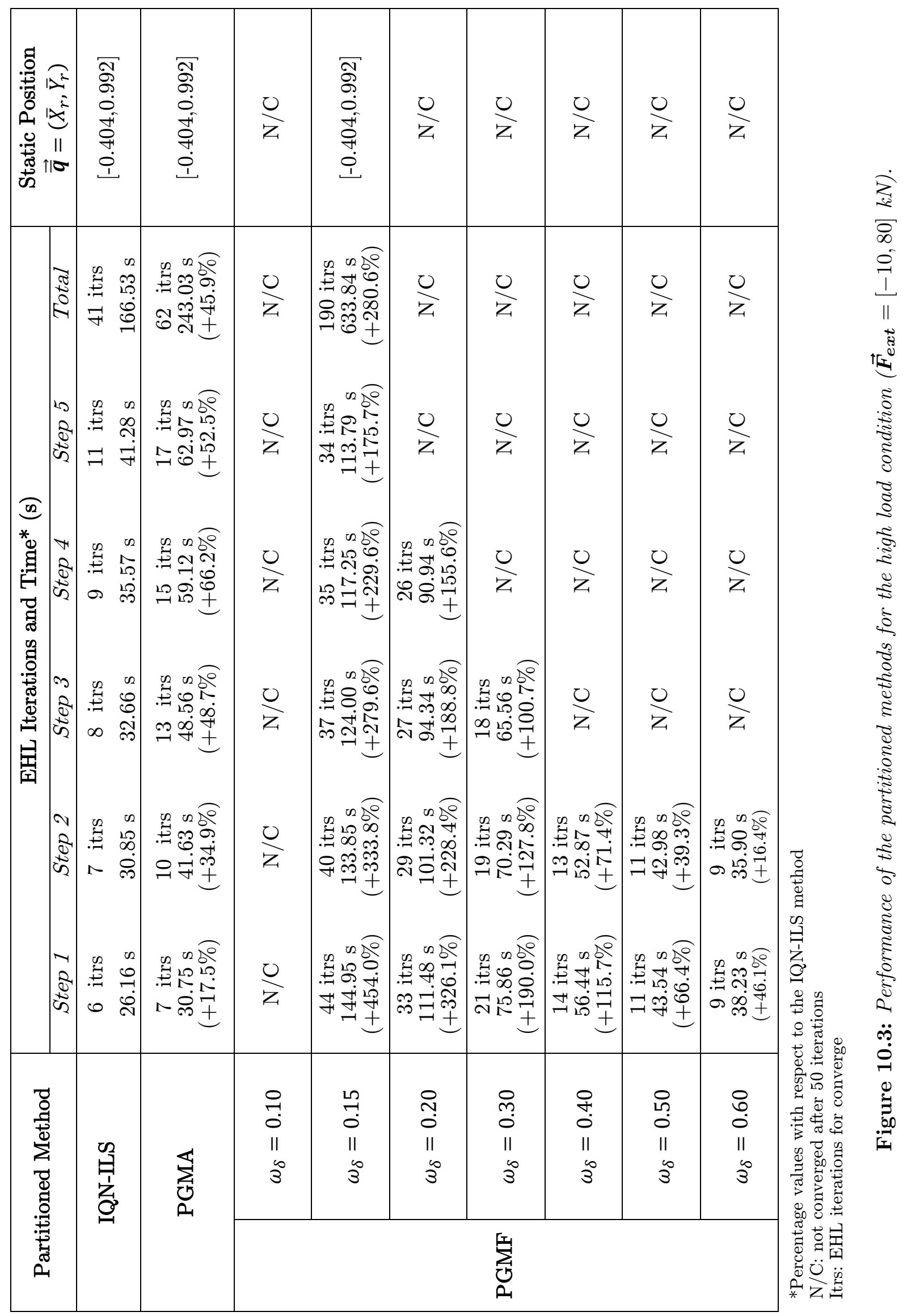



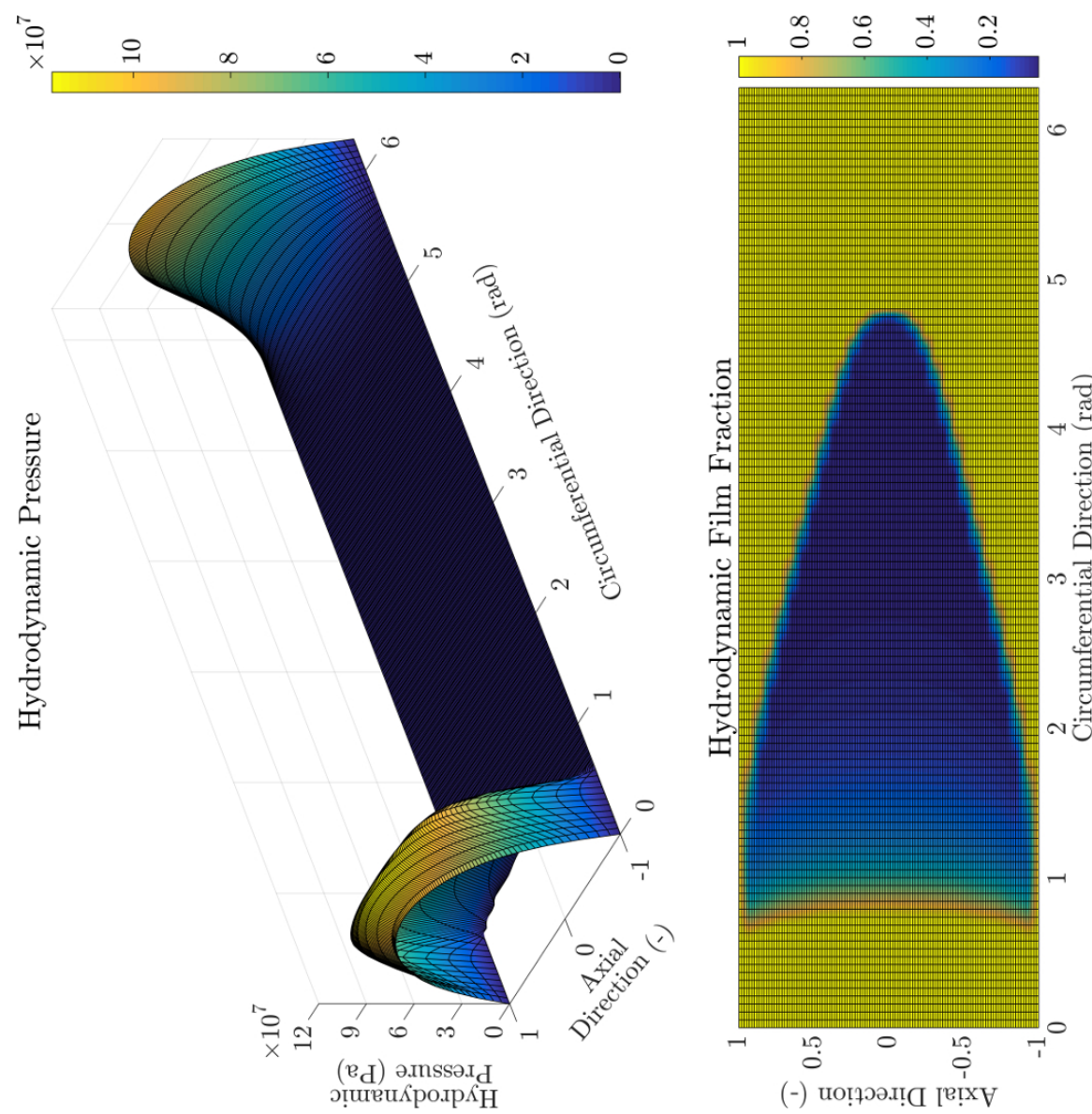

(5)
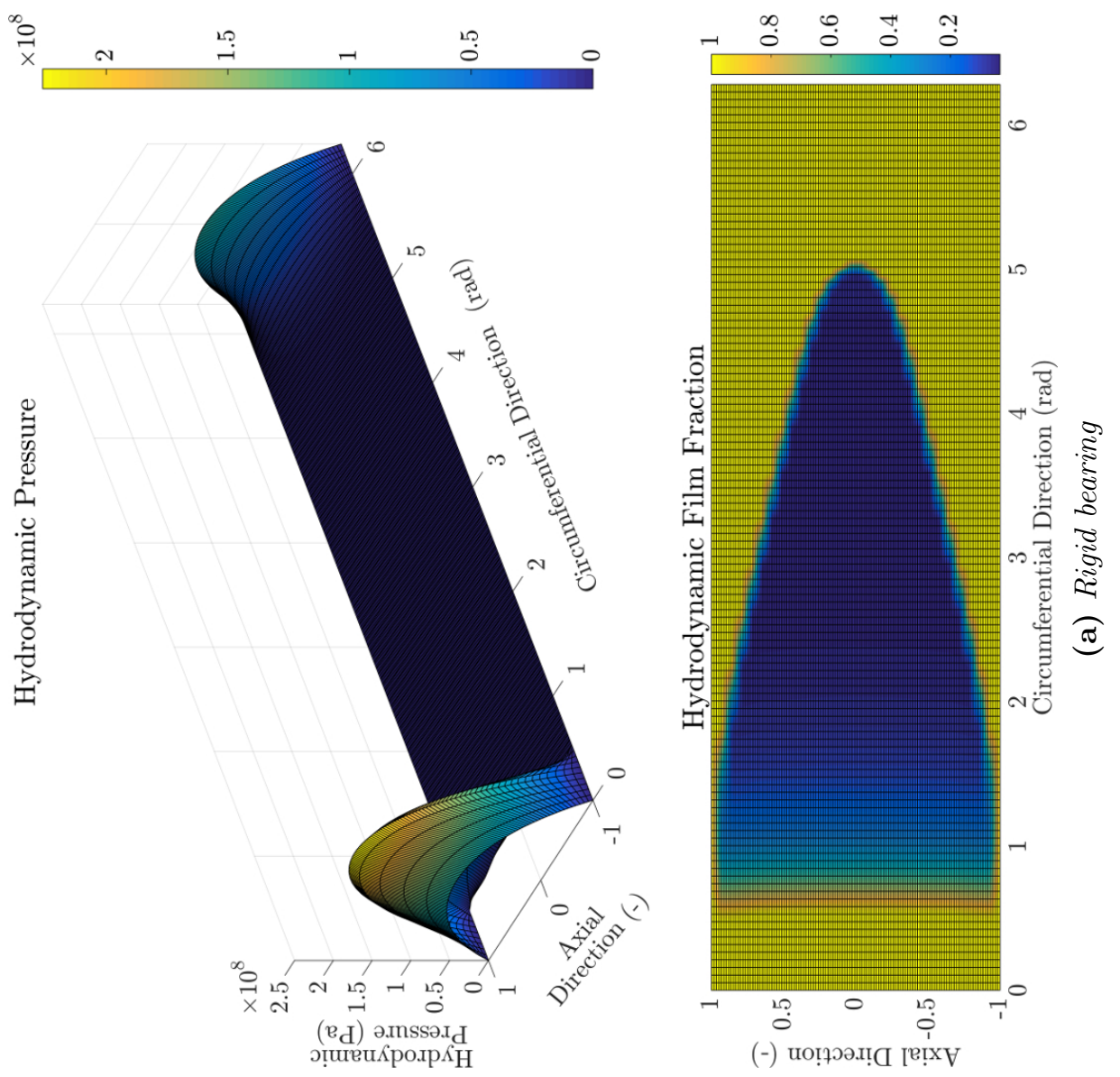

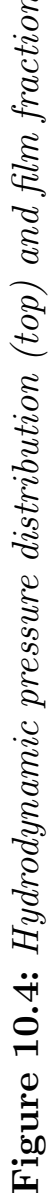



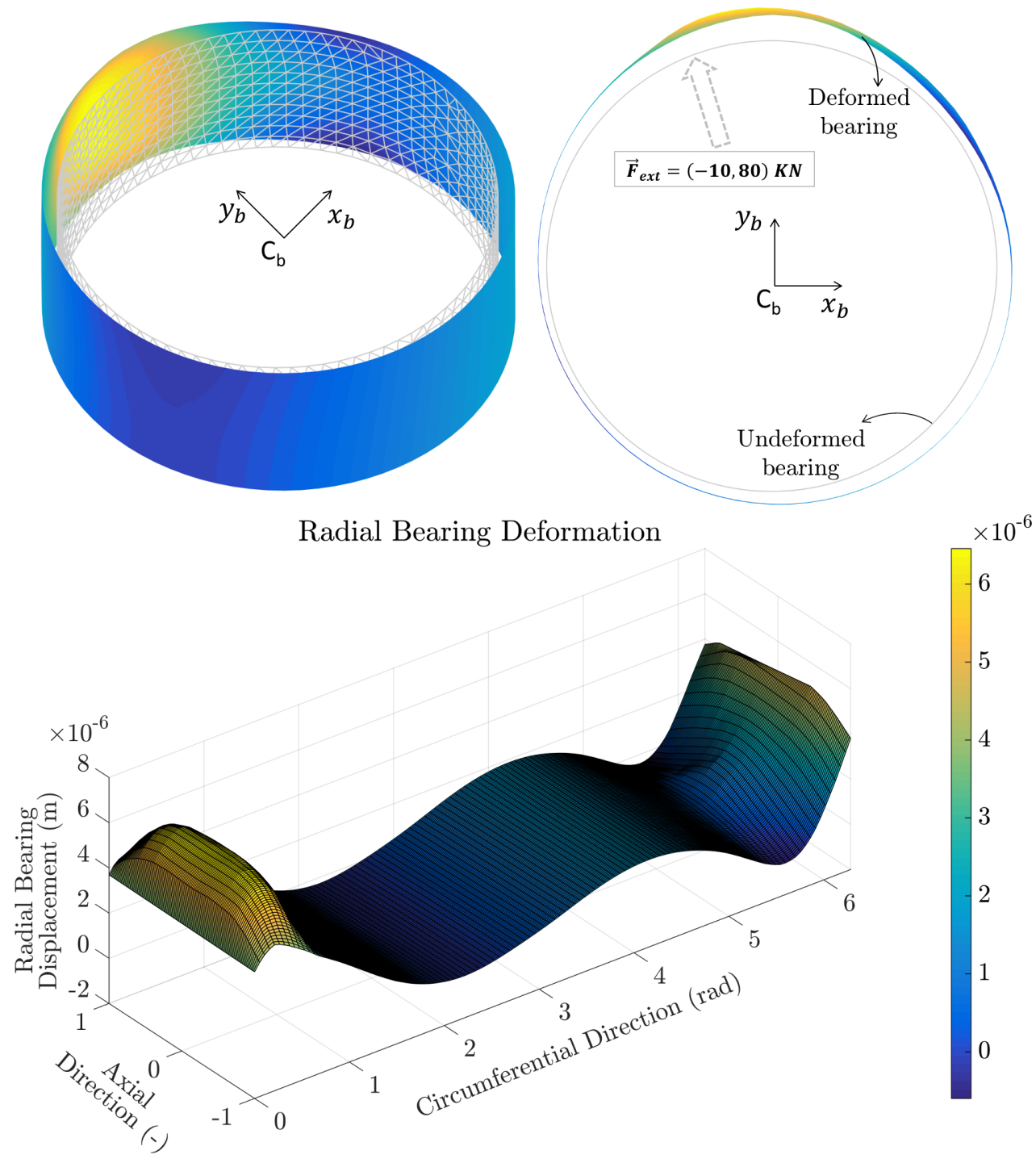

Figure 10.5: Radial bearing deformation for the converged high load condition $\left(\overrightarrow{\boldsymbol{F}}_{\text {ext }}=[-10,80]\right.$ $k N)$.

the cavitation zones was practically inexistent, and consisted only of a small change noticed in the curvature of the upstream cavitation boundary. In Fig. 10.5, the structural elastic displacements induced by the hydrodynamic pressures are depicted. As expected, the maximum distortions occurred near the regions of high pressures, located approximately between $340^{\circ}$ and $20^{\circ}$ along the bearing circumferential direction. As will be shown in the next section, the magnitude of the displacements in this high load condition is much smaller than that obtained for the high deformation case, once the bearing structure is less flexible in the direction of the conrod shank in which the respective load is applied. As for the performance of the partitioned coupling methods in the convergence of the static EHL problem, the Interface Quasi-Newton Method (IQN-ILS) was shown to be the best technique both in terms of number of iterations and computational time. In this case, the complete convergence, including the sequence of all load steps, was reached after 41 iterations (166.53 s). On the other hand, the Fixed Point Gauss-Seidel Method (PGMF) presented the worst performance, in which the whole convergence was obtained only for a (fixed) relaxation factor of $\omega_{E H L}=0.15$ and after 190 iterations (663.84 s); other values for the relaxation factor were tested, but no complete convergence was possible up to 
the last load step (see table in Fig. 10.3). The Point Gauss-Seidel Method with Aitken Acceleration (PGMA) exhibited an intermediate efficiency, with the convergence being achieved after 62 iterations (243.03 s). The table in Fig. 10.3 also shows the converged static position $\overrightarrow{\overrightarrow{\boldsymbol{q}}}=\left(\bar{X}_{r}, \bar{Y}_{r}\right)$ of the system for each partitioned method; as can be observed, the converged results were identical, concluding that the problem converged to the same solution for all methods.

\subsubsection{Results for the High Deformation Condition}

The converged results for the high deformation condition with $\overrightarrow{\boldsymbol{F}}_{\boldsymbol{e x t}}=[2,-20] \mathrm{kN}$ are illustrated in Figs. 10.7-10.8. As can be seen in Fig. 10.7, the local bearing deformations in this case yielded to a higher variation in the hydrodynamic pressure field, resulting in a bimodal pressure distribution typically found in big-end bearing simulations [34, 95, 129, 157]. Such higher perturbations in the pressure field also increased the area of hydrodynamic load capacity, and hence clearly reduced the peak pressure and the area of the cavitation zones as well. In Fig. 10.8, it is possible to notice the large structural distortions induced by the hydrodynamic pressures, especially those caused by the higher flexibility of the connecting-rod bearing along the direction in which the present load is applied. Regarding the convergence of the EHL solution, again the Interface Quasi-Newton Method (IQN-ILS) was the best, converging after 51 iterations $(212.53 \mathrm{~s}$ ). Similarly, the Fixed Point Gauss-Seidel Method (PGMF) showed the worst performance, converging completely only for the (fixed) relaxation factor $\omega_{E H L}=0.15$ and after 173 iterations (608.49 s). The Point Gauss-Seidel Method with Aitken Acceleration (PGMA) also presented an intermediate efficiency, obtained after 117 iterations (427.95 s). The converged static position $\overrightarrow{\overrightarrow{\boldsymbol{q}}}=\left(\bar{X}_{r}, \bar{Y}_{r}\right)$ of the system were the same for all partitioned methods as well (see table in Fig. 10.6).

\subsection{Dynamic Loading Conditions}

The overall scalar results of the conrod big-end bearing considered in the present analysis for the dynamic loading conditions depicted in Fig. 10.1b are summarized in Fig. 10.9. The plots illustrate the variation of the most important operational variables of the system over a full engine cycle. The results for both rigid (blue lines) and flexible (red lines) bearing cases are shown. As can be seen in Fig. 10.9e, the journal eccentricity considerably increased when the bearing flexibility was taken into account in comparison to the rigid condition; this situation can also be observed in Fig. 10.9c, where the journal trajectory for the EHL case clearly extrapolated the unit eccentricity circle. This situation typically occurs in conrod applications and is explained by the significant solid deformations that allow the larger rigid body displacements of the journal within the bearing bore. In particular, two important localized differences can be further highlighted with respect to the bearing eccentricity: $(1)$ at $C A \approx 360^{\circ}$, which corresponds to the situation of maximum structural distortions (see Fig. 10.14a) induced by the alignment of the external loads to the more compliant direction of the lower bearing shell, the journal rigid body displacements were significantly higher for the flexible bearing case in comparison with the rigid one; and $(2)$ at $C A \approx 450^{\circ}$, where the magnitude of the external loads is relatively small, the rapid eccentricity drop observed for the rigid bearing was attenuated when the system flexibility is considered. 


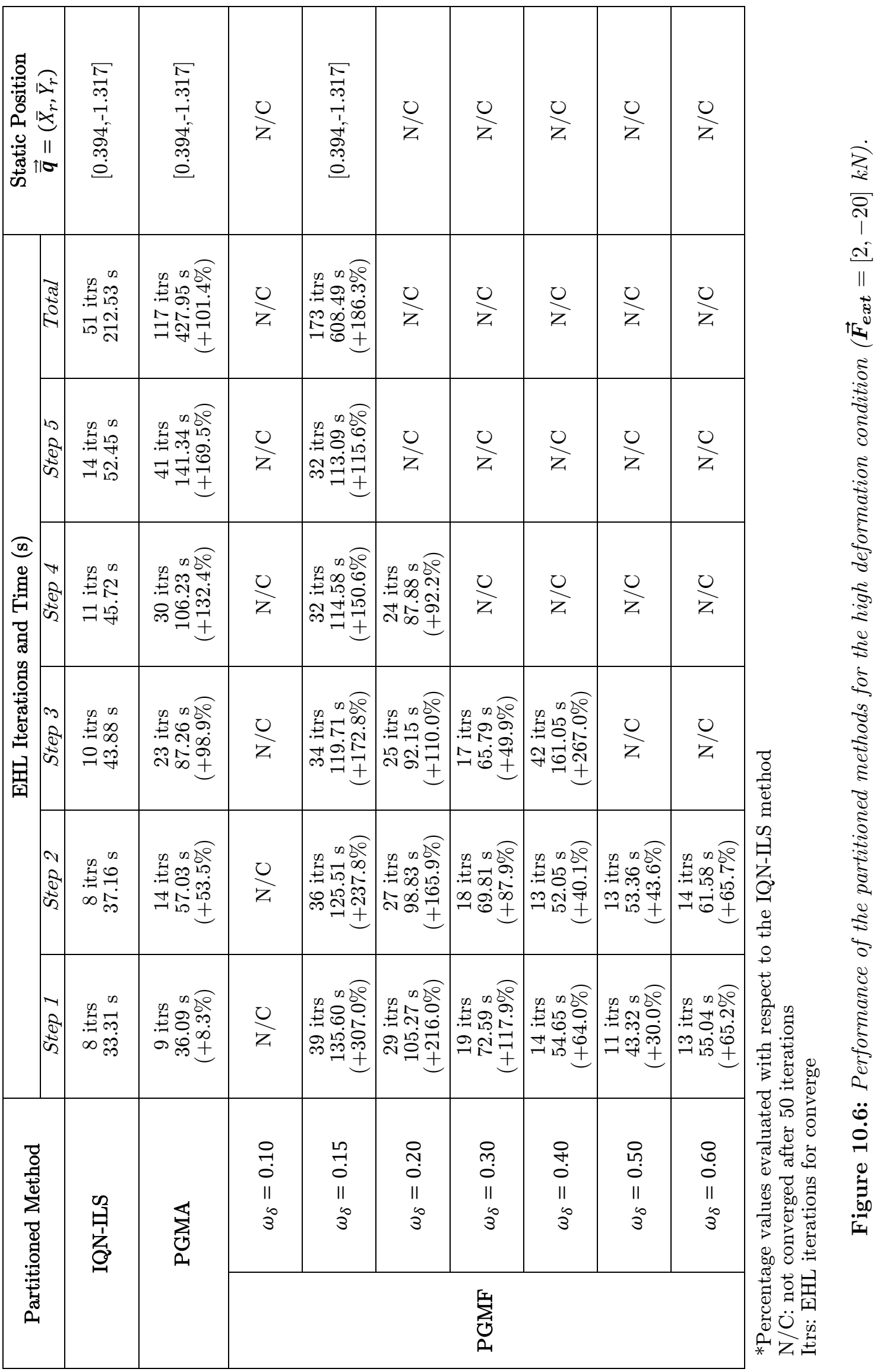




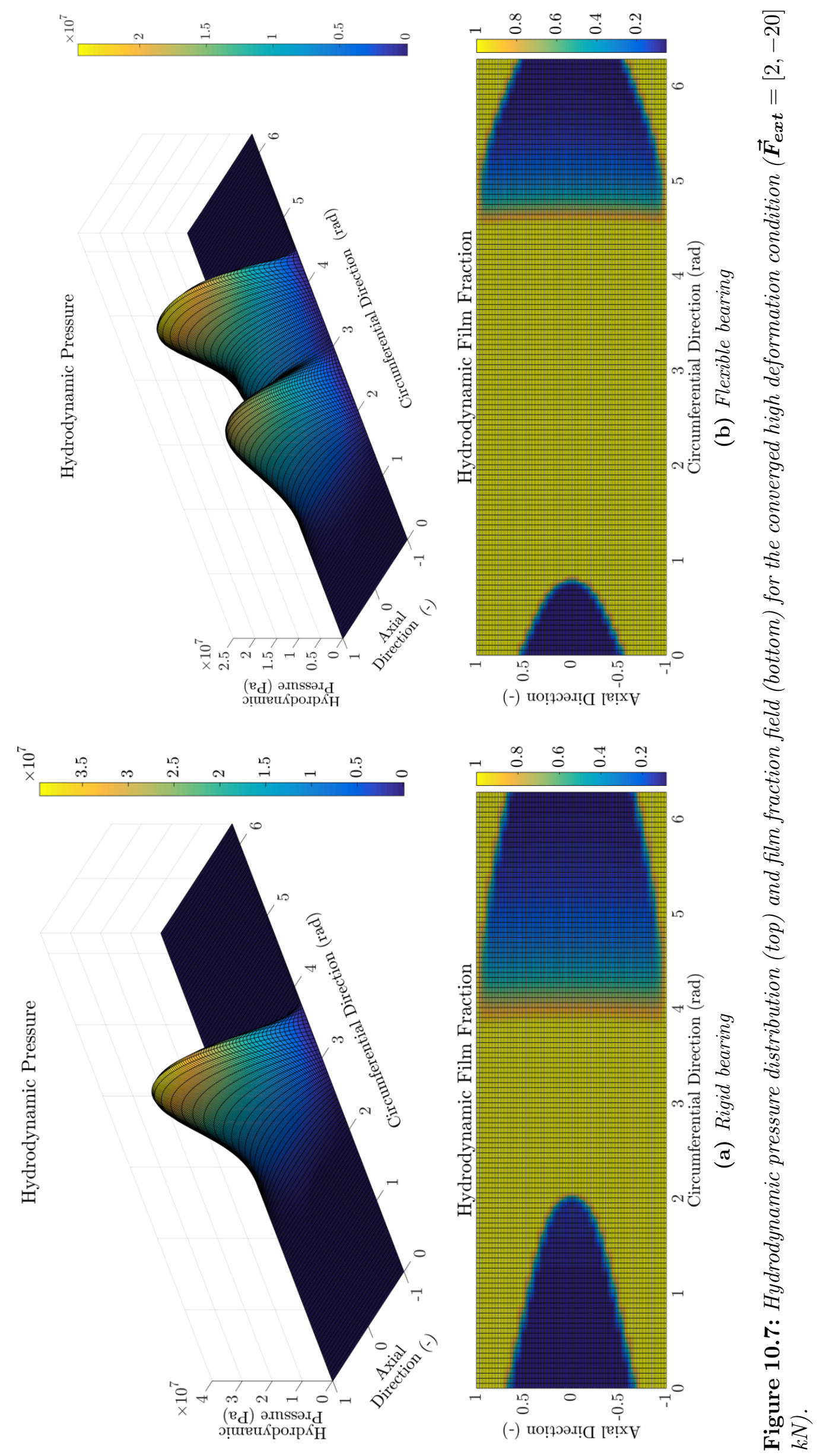



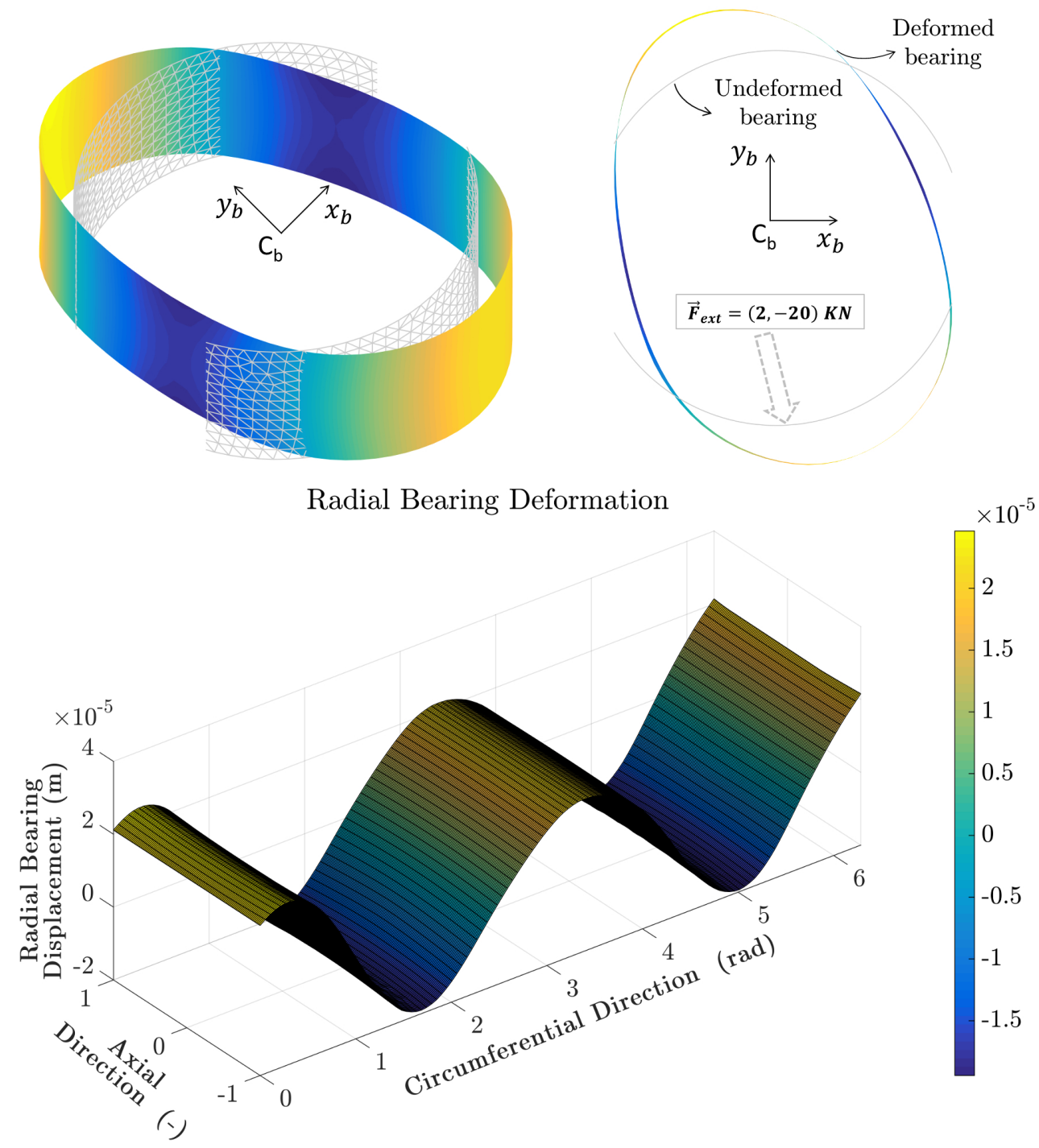

Figure 10.8: Radial bearing deformation for the converged high deformation condition $\left(\overrightarrow{\boldsymbol{F}}_{\mathbf{e x t}}=\right.$ $[2,-20] k N)$.

The general behaviour of the hydrodynamic peak pressures (PP) and the minimum oil film thicknesses (MOFT) were essentially the same, but the magnitudes of both tended to be lower when the bearing flexibility was taken into account. For the peak pressures (see Fig. 10.9d), very significant reductions from almost $1 \mathrm{GPa}$ to $300 \mathrm{MPa}$ were observed during combustion for $0^{\circ}<C A<45^{\circ}$ and $675^{\circ}<C A<720^{\circ}$. This decrease in PP is associated with the beneficial "spreading" of the hydrodynamic pressures over the deformed bearing surface (see Fig. 10.4b), i.e. the bearing distortions are prone to "accommodate" the fluid pressures throughout the contact (increase of the contact wet area), thus attenuating eventual localized "spikes" in the pressure distribution. Regarding the minimum oil film thicknesses (see Fig. 10.9b), a distinguished effect can also be visualized for the EHL case during combustion, where the MOFT reached very small values $(\approx 1.5 \mu \mathrm{m})$ that led the system to operate under mixed-EHL conditions (see Fig. 10.10b). Such critical lubrication situation occurred only locally at the bearing sides and was caused by deformations of the contact geometry surrounding those regions; this effect was not observed for the rigid bearing case, and even for the flexible high-speed big-end bearing to be investigated in Chapter 11. It is noteworthy that proper predictions of the mixed-EHL regime, along 


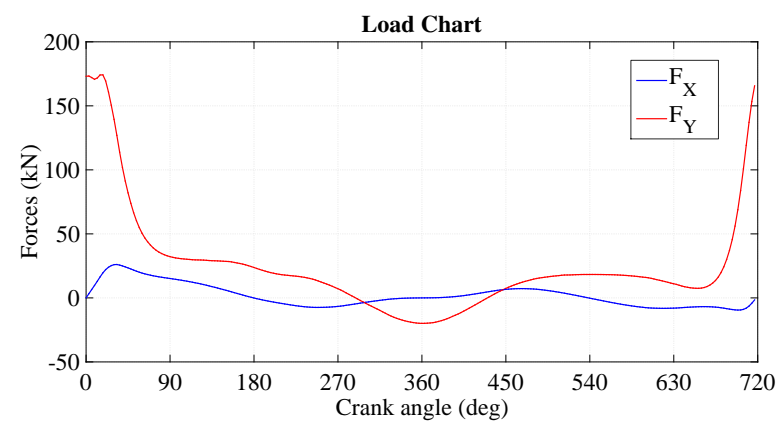

(a) External load forces

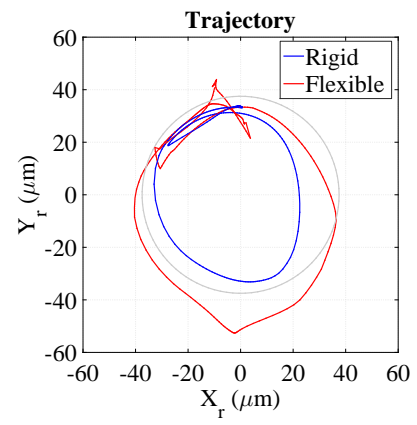

(c) Journal trajectory

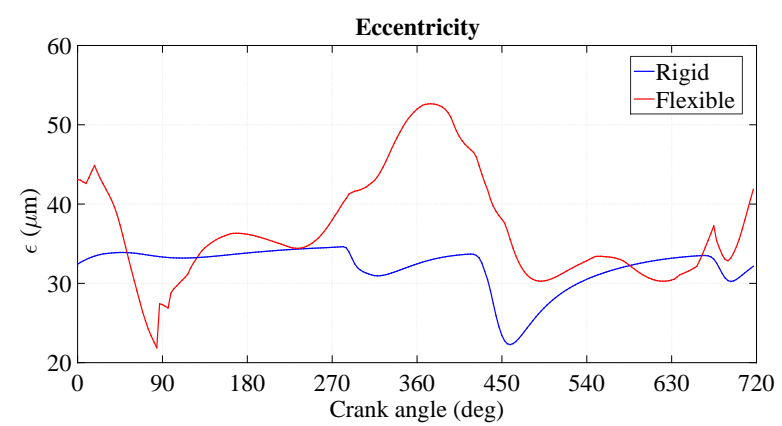

(e) Journal eccentricity

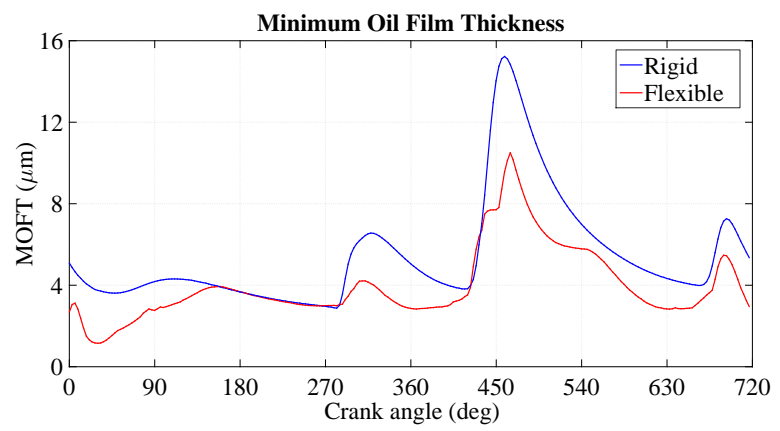

(b) Minimum oil film thickness

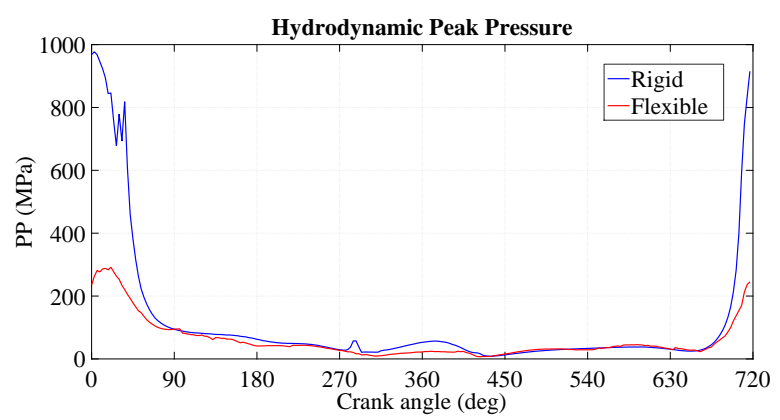

(d) Hydrodynamic peak pressure

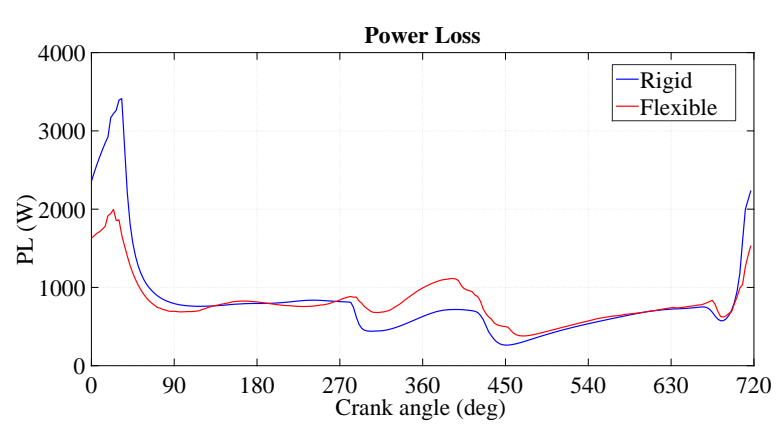

(f) Power loss

Figure 10.9: Scalar results of the dynamically loaded heavy-duty big-end bearing.

with the identification of the regions and positions at which asperity contact takes place, are crucial for designing reliable bearing components for applications with high loading conditions and abrupt transients. Finally, the curves shown in Fig. 10.9f indicate that the changes in the power friction losses were not so pronounced for the analyzed cases. Once again, the major differences were detected during combustion and around $C A=360^{\circ}$. In the former situation, the friction losses were reduced for the flexible bearing due to the lower lubricant viscosities produced by the moderate influence of the piezoviscous effect (see results for PP in Fig. 10.9d) in comparison with the rigid case. In contrast, for the crank angle positions near $360^{\circ}$, the smaller film thicknesses obtained for the deformable bearing (see results for the MOFT in Fig. 10.9b) contributed to raising the overall viscous dissipations.

The surface plots depicted in Figs. 10.10-10.15 illustrate the hydrodynamic pressure, hydrodynamic film fraction (cavitation), asperity contact pressure and bearing displacement fields for 8 crank angle positions covering the whole engine cycle (only results for the flexible bearing case are shown). The intention is to provide a global picture on how 
Table 10.6: Performance of the partitioned algorithms for the heavy-duty big-end bearing.

\begin{tabular}{lcc}
\hline Partitioned Method & EHL Iterations* & Time Elapsed* \\
\hline PGMF & N/C & N $/ \mathrm{C}$ \\
PGMA & $6(5)$ & $26.28 \mathrm{~s}(16.57 \mathrm{~s})$ \\
IQN-ILS & $8(6)$ & $37.74 \mathrm{~s}(23.82 \mathrm{~s})$ \\
\hline
\end{tabular}

${ }^{*}$ Values between brackets correspond to the statistical medians

$\mathrm{N} / \mathrm{C}$ : not converged

such field variables change along the engine cycle. In this fashion, as discussed in Section 10.2 for the static loading simulations, one can emphasize the EHL effects under the high load conditions around $C A=27^{\circ}$, where the structural distortions were mainly verified close to the regions of high pressure concentrated on the top bearing shell. In this case, however, despite the high intensity of the external loads, the magnitudes of the displacements were smaller than those obtained for the high deformation situations in the vicinity of $C A=360^{\circ}$. As already pointed out, such discrepancy is due to the less flexibility of the bearing structure along the direction of the conrod shank in which the high loads are applied. Furthermore, for the same high deformation conditions, the local bearing displacements also yielded significant perturbations in the hydrodynamic pressure fields, which in turn generated the well-known bimodal pressure distributions.

The connecting-rod system in study also showed an interesting effect regarding the collapse of the cavitation zones under situations of abrupt changes in the orientation of the external loads $[29,40,41,162]$. This phenomenon was first observed for the crank angle interval $240^{\circ}<C A<330^{\circ}$, as can be seen by following the evolution of the film fraction field in Figs. 10.10-10.15. Similar trends were also encountered for the stroke ranges $350^{\circ}<C A<450^{\circ}$ and $620^{\circ}<C A<720^{\circ}$. Under these conditions, the fast change in the journal movement, along with the relative high structural deformations and the associated lubricant squeeze effect, yielded the generation of a large depressurization zone in the contact that in turn contributed to hindering the local convergence ratio of the EHL solution.

\subsection{Performance of the Partitioned Algorithms}

The overall performance of the partitioned coupling algorithms for the present big-end bearing case under dynamic loading conditions is summarized in Table 10.6. The simple average and the statistical median of the number of EHL iterations and the associated elapsed time for the solution convergence are listed in the table. Both performance parameters are determined from their respective values required for the convergence of each crank angle of the entire dynamic solution. All simulations were carried out in a computer with processor Intel Core i7-3630 CPU $2.40 \mathrm{GHz}$ and $8 \mathrm{~GB}$ memory. According to the average values, the PGMA method has shown to be more efficient, stable and robust then the IQN-ILS method, in contrast to the static simulations described in Section 10.2. The main reason for such a difference is that the high transients experienced by the bearing tended to weaken the robustness of the IQN-ILS method, especially due to the accentuated squeeze film effects induced both by the rapid changes in the journal rigid body movement and the "vibrational" velocity of the bearing structure. Such effects added further challenges to the convergence of the EHL problem that were overcome more effi- 
ciently by the PGMA technique. The performances of the PGMF method were extremely poor, showing no convergence (within the maximum number of iterations allowed) for practically all time steps. Therefore, one can conclude that the proposed PGMA method is preferable and recommended for partitioned EHL solutions of bearings operating under severe lubrication conditions. 

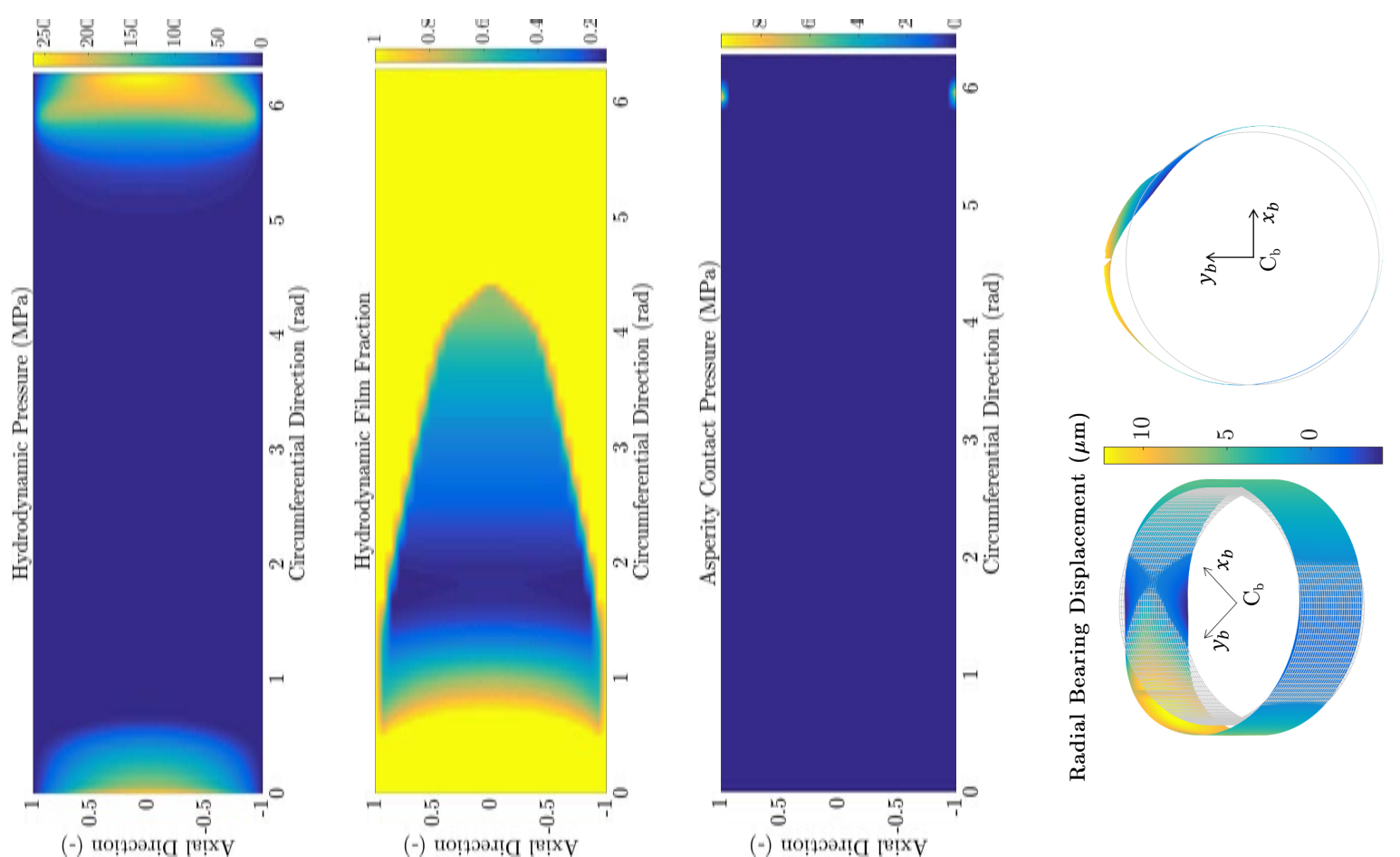

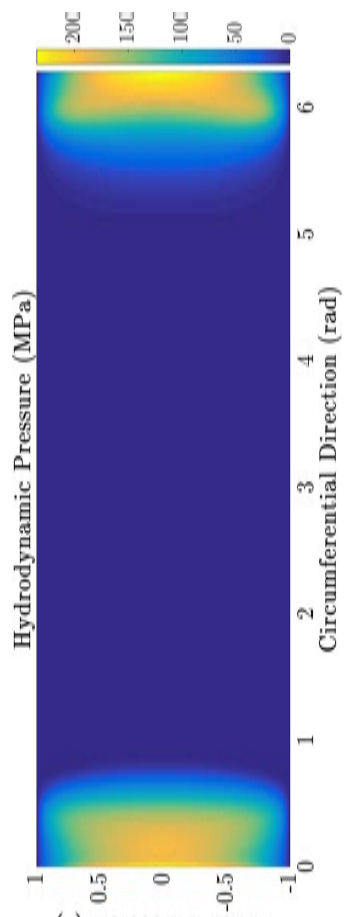

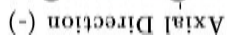

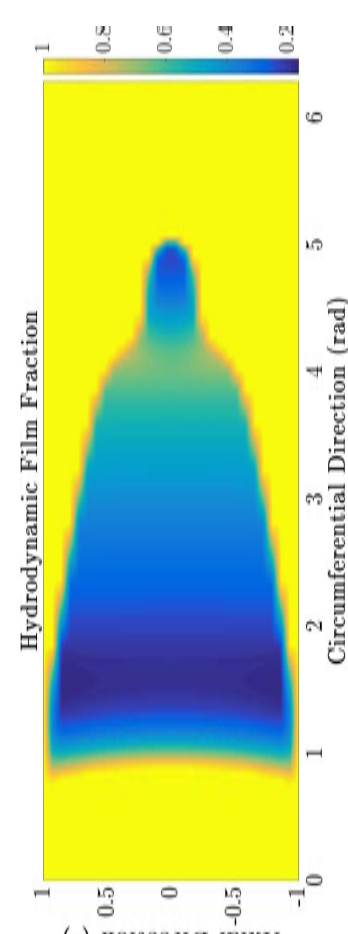

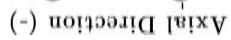

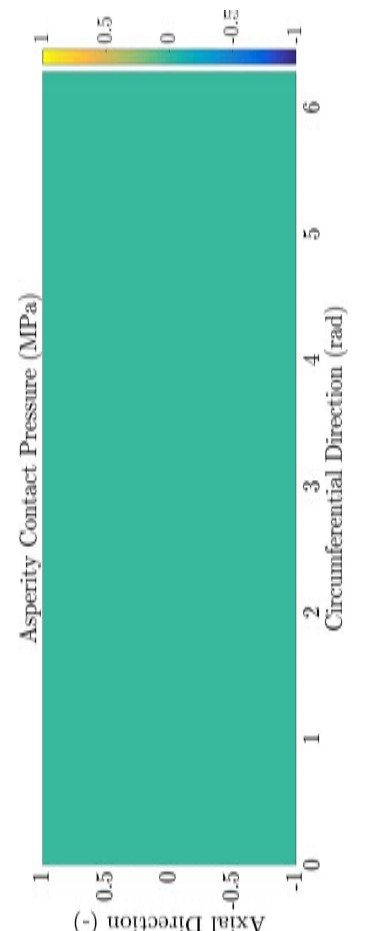

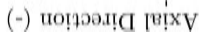

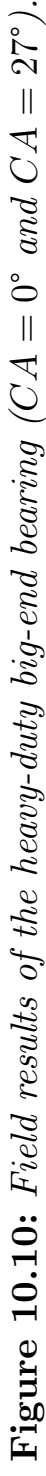

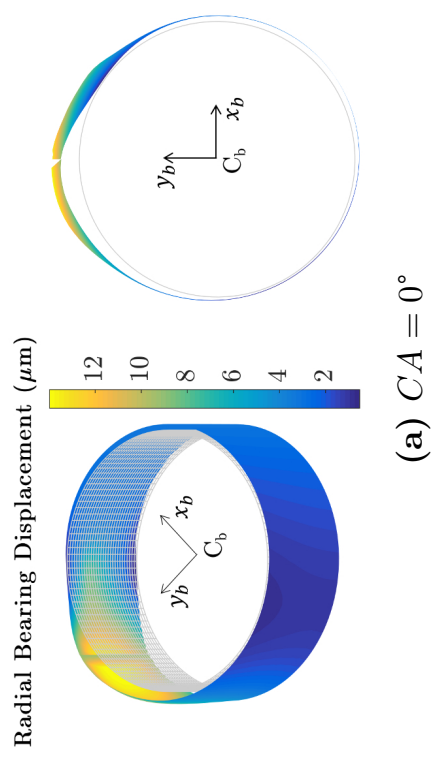




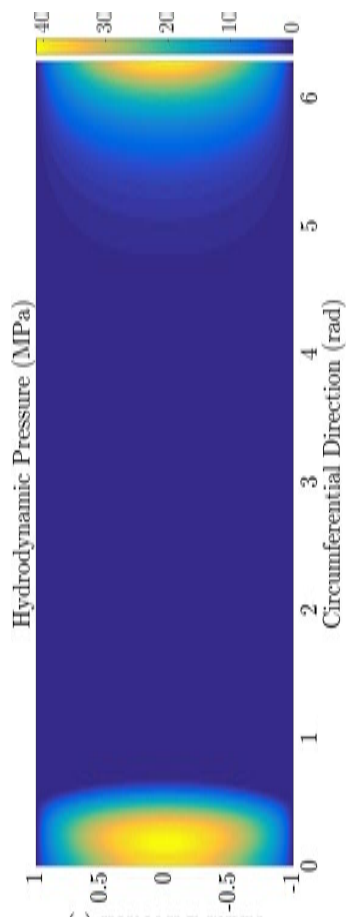

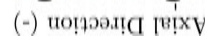

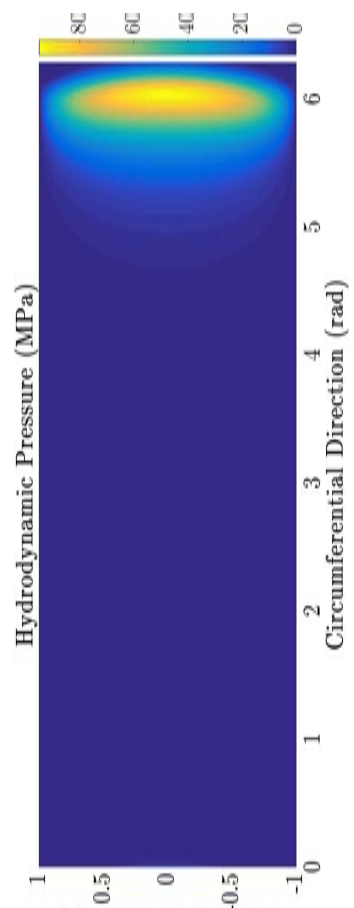

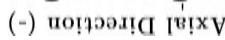

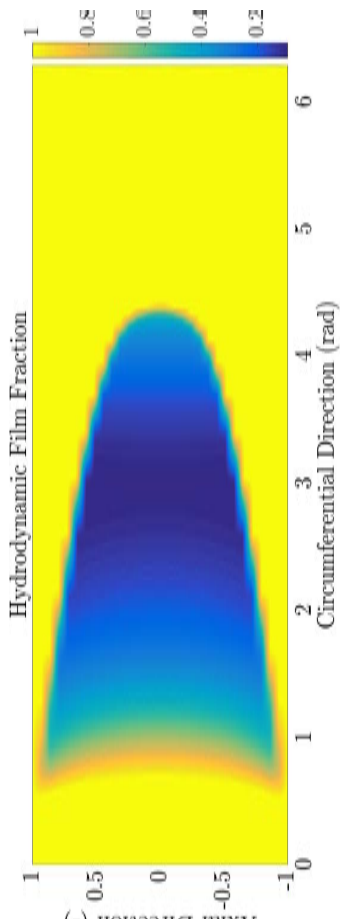

(-) นопрอง!ด [B!x

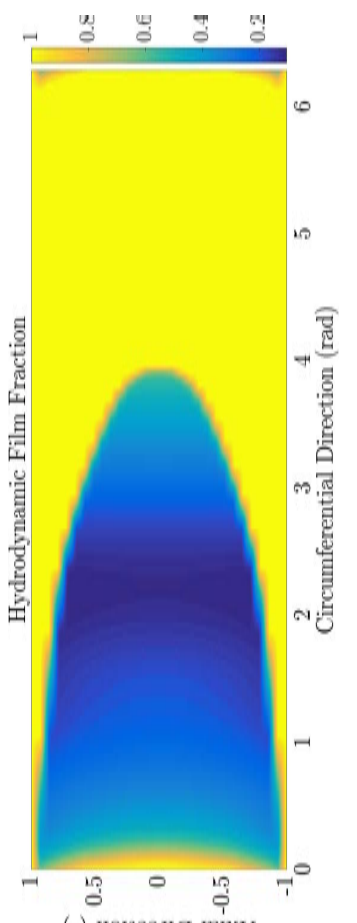

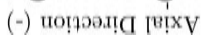
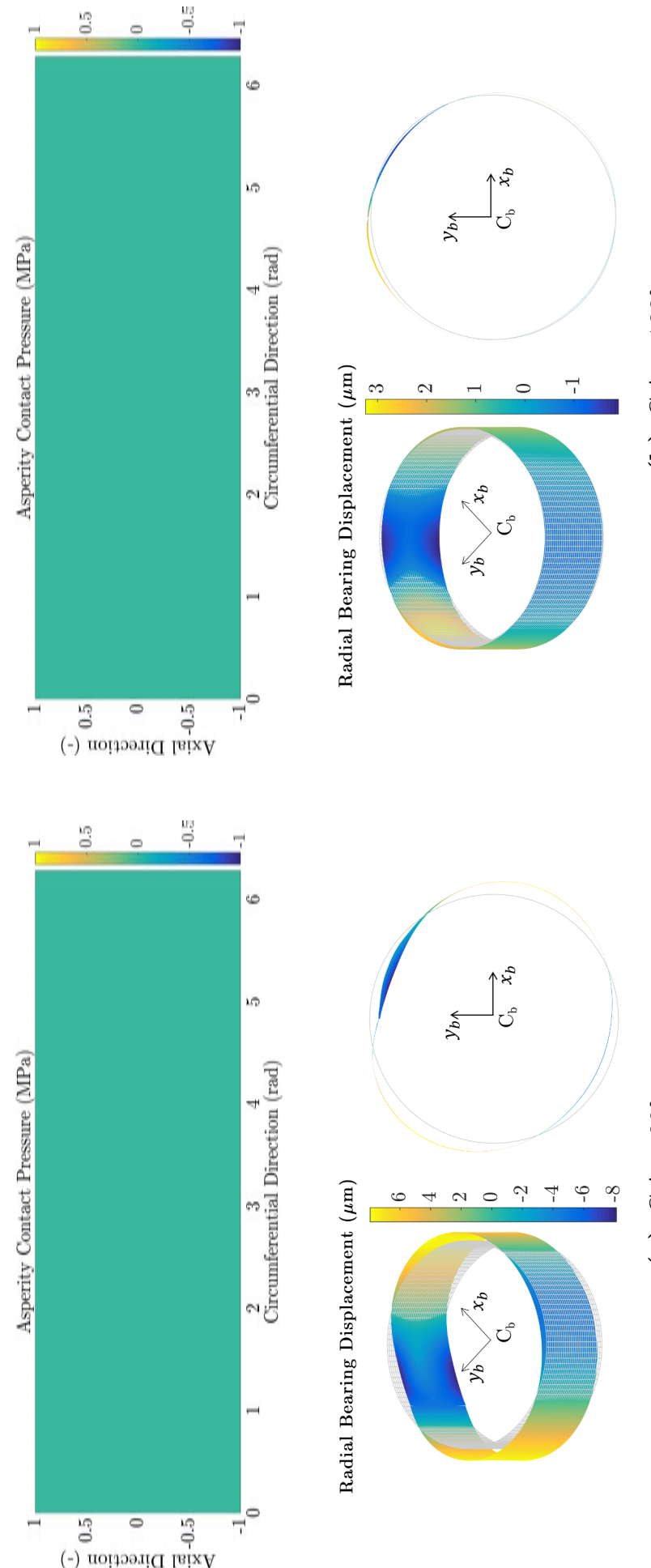

2

8

11

$\circlearrowright \quad \ddot{ت}$ 


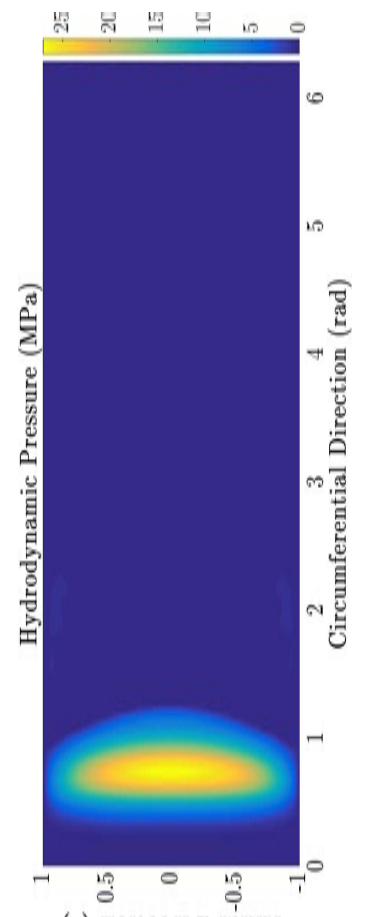

(-) uompos!C ['घ!xV

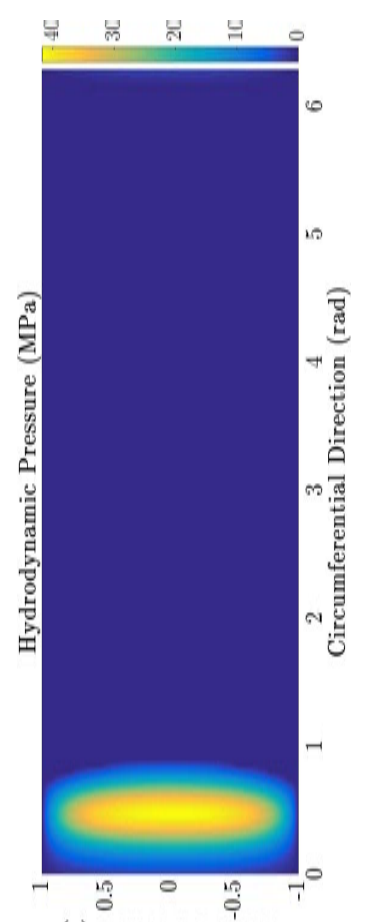

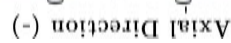
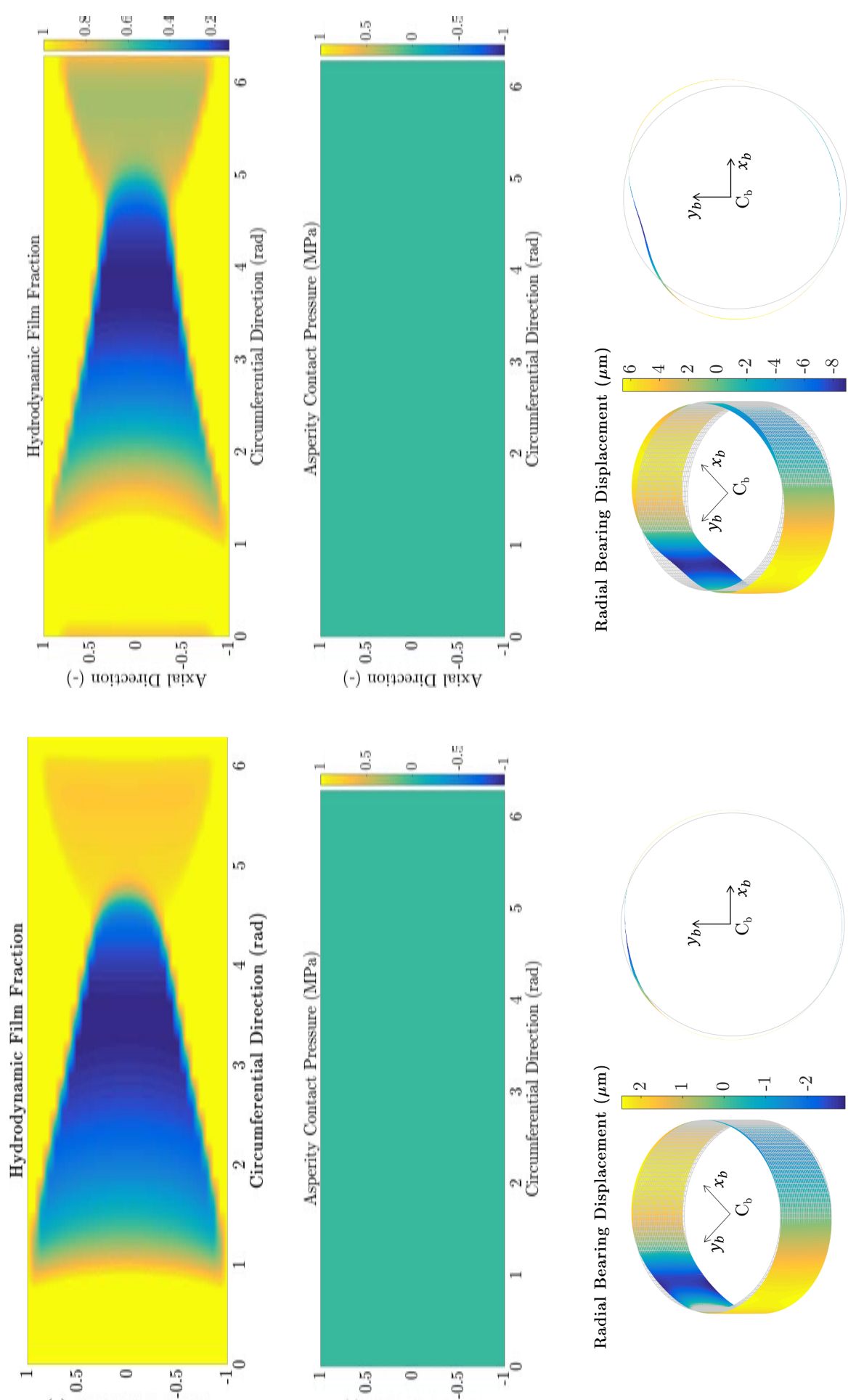

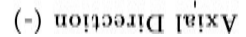

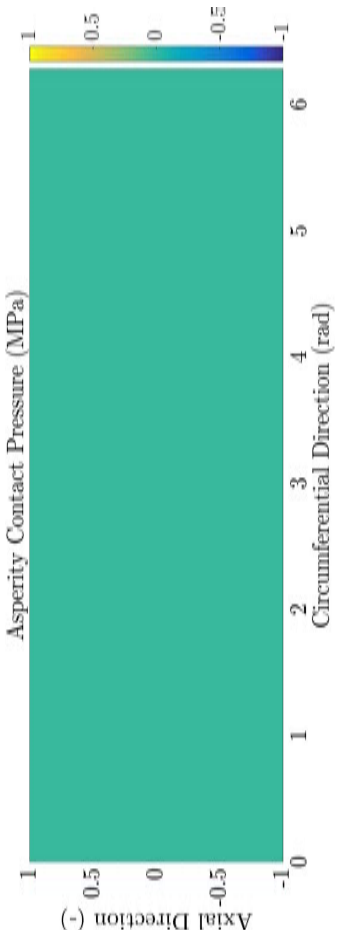

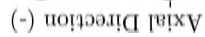
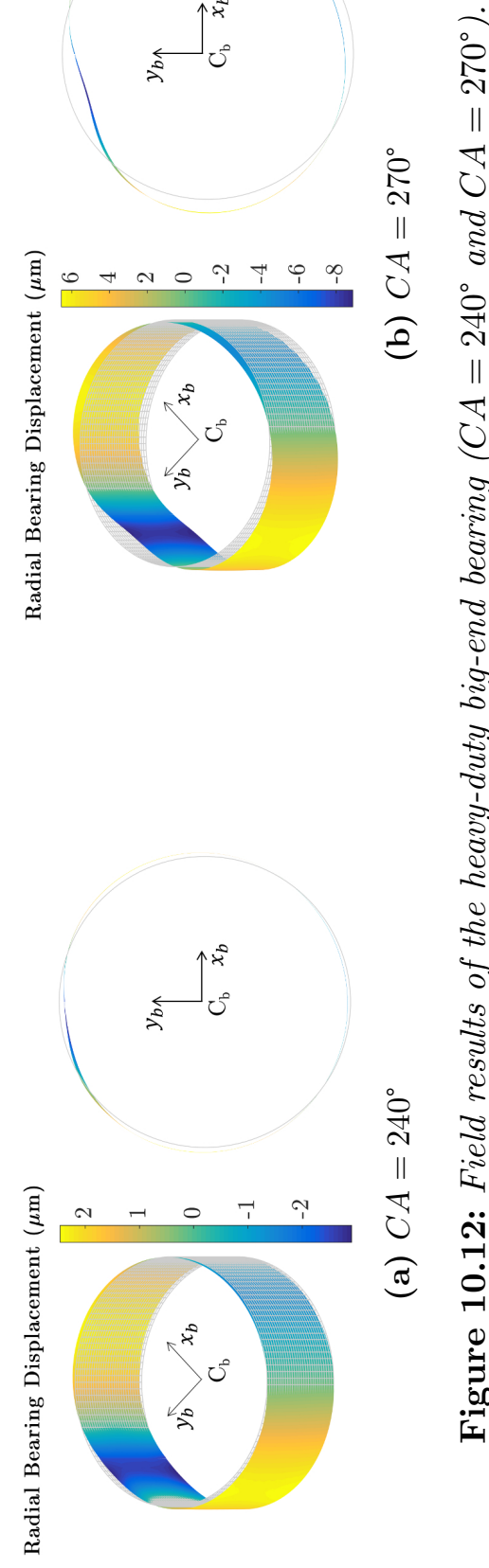

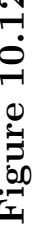




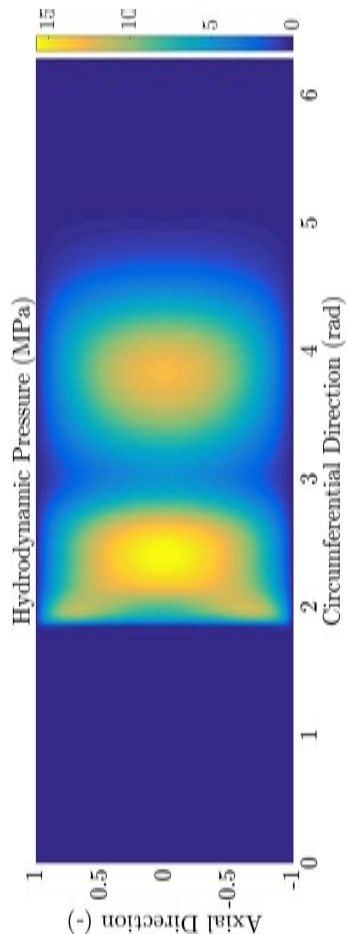

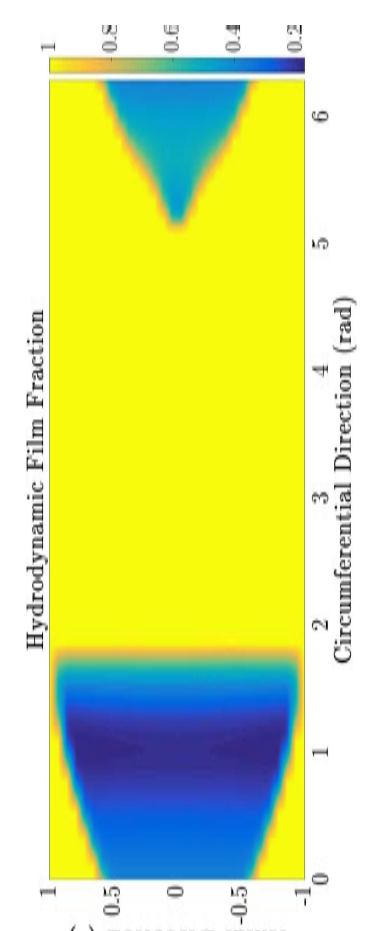

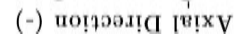
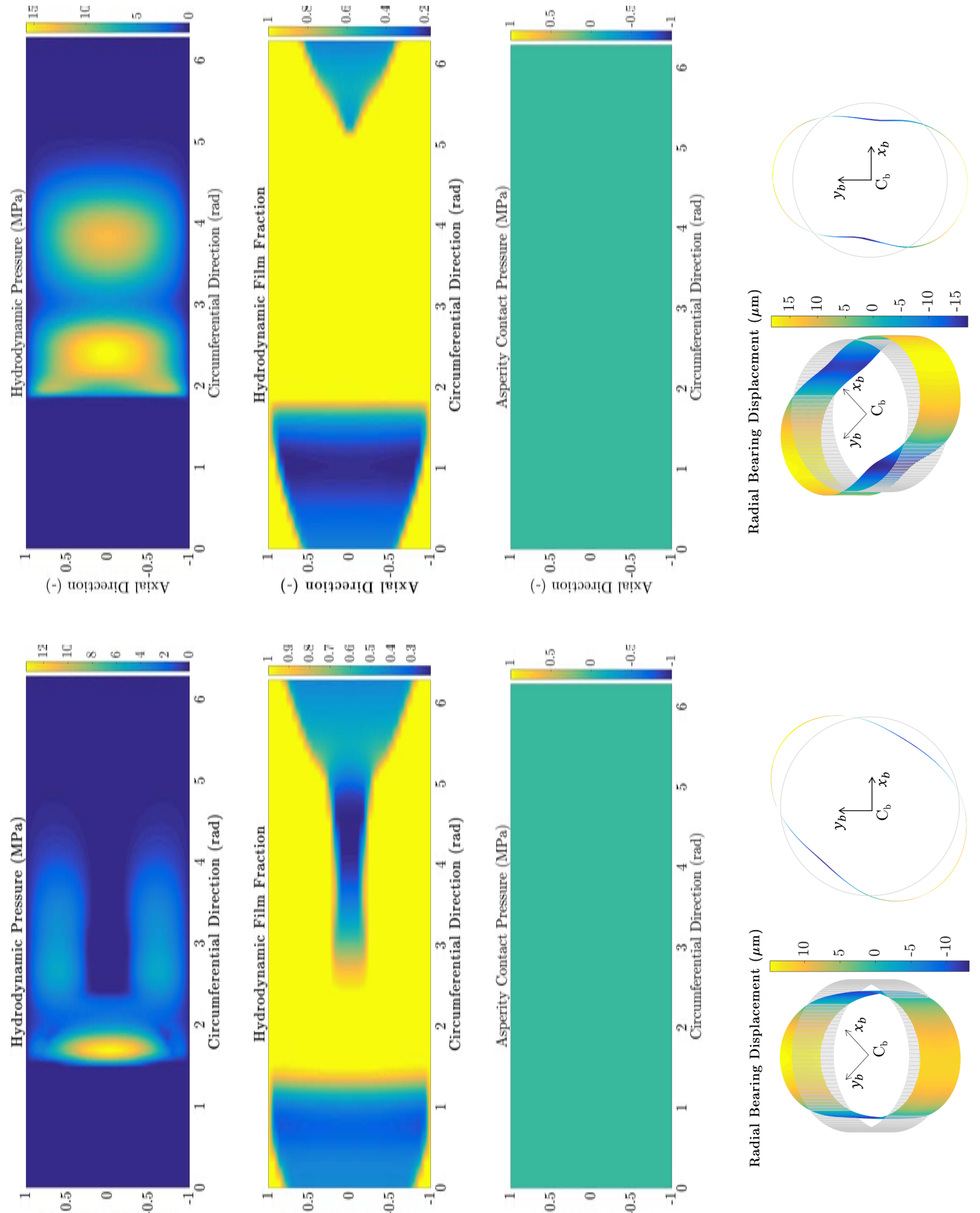

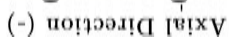

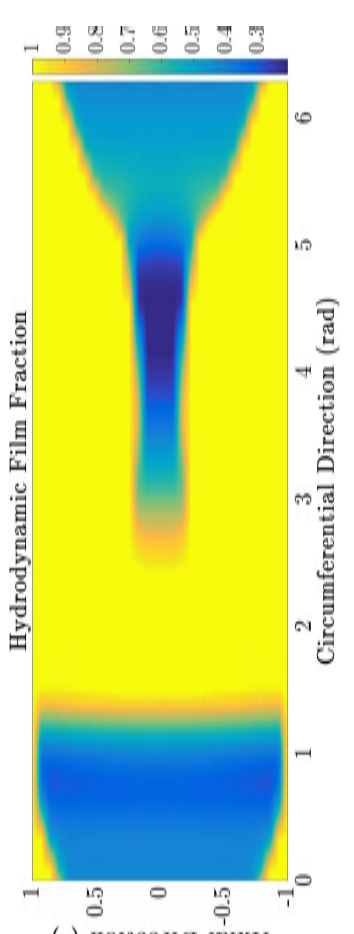

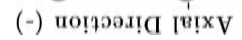

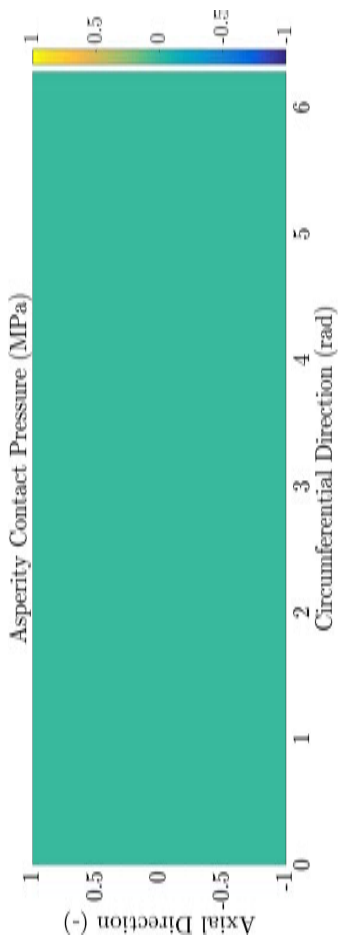

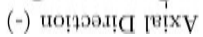

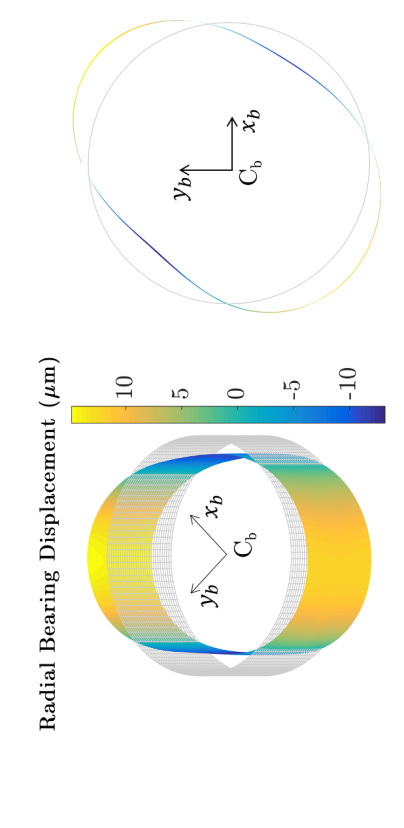

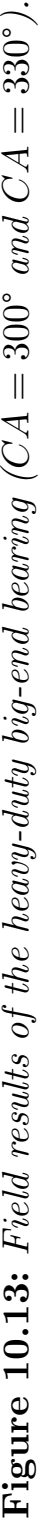



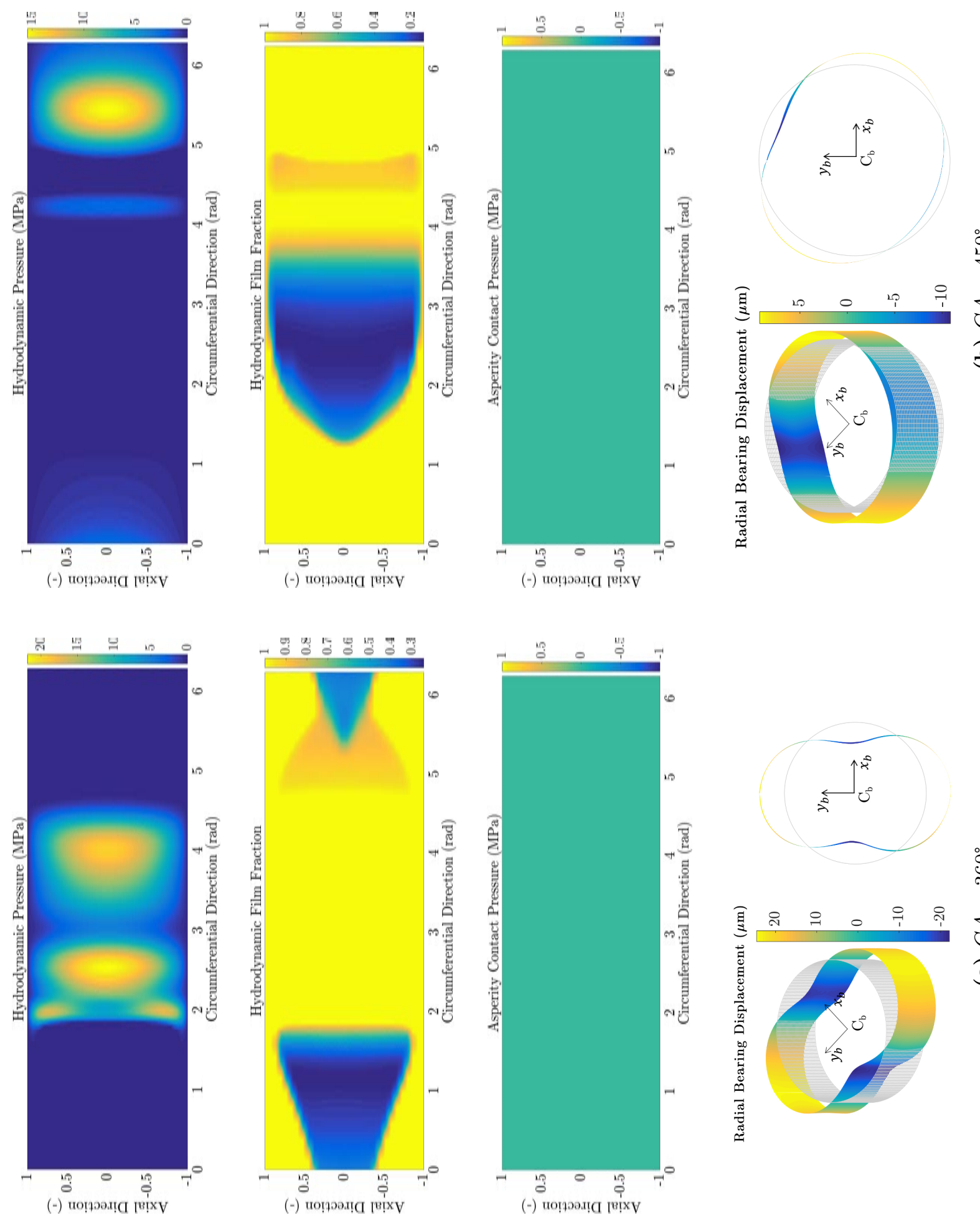

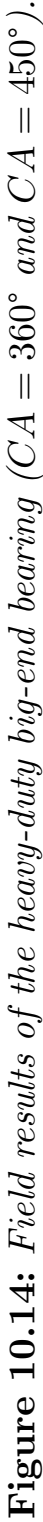




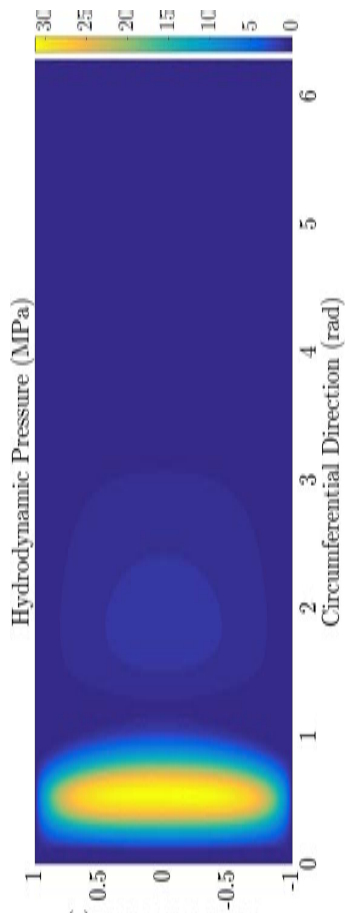

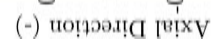

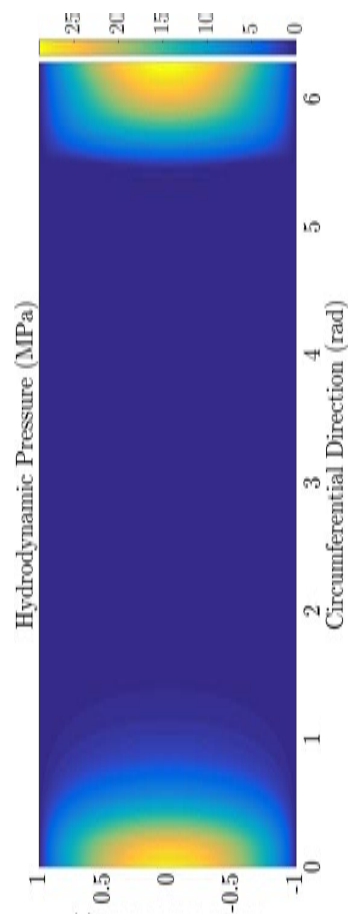

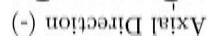
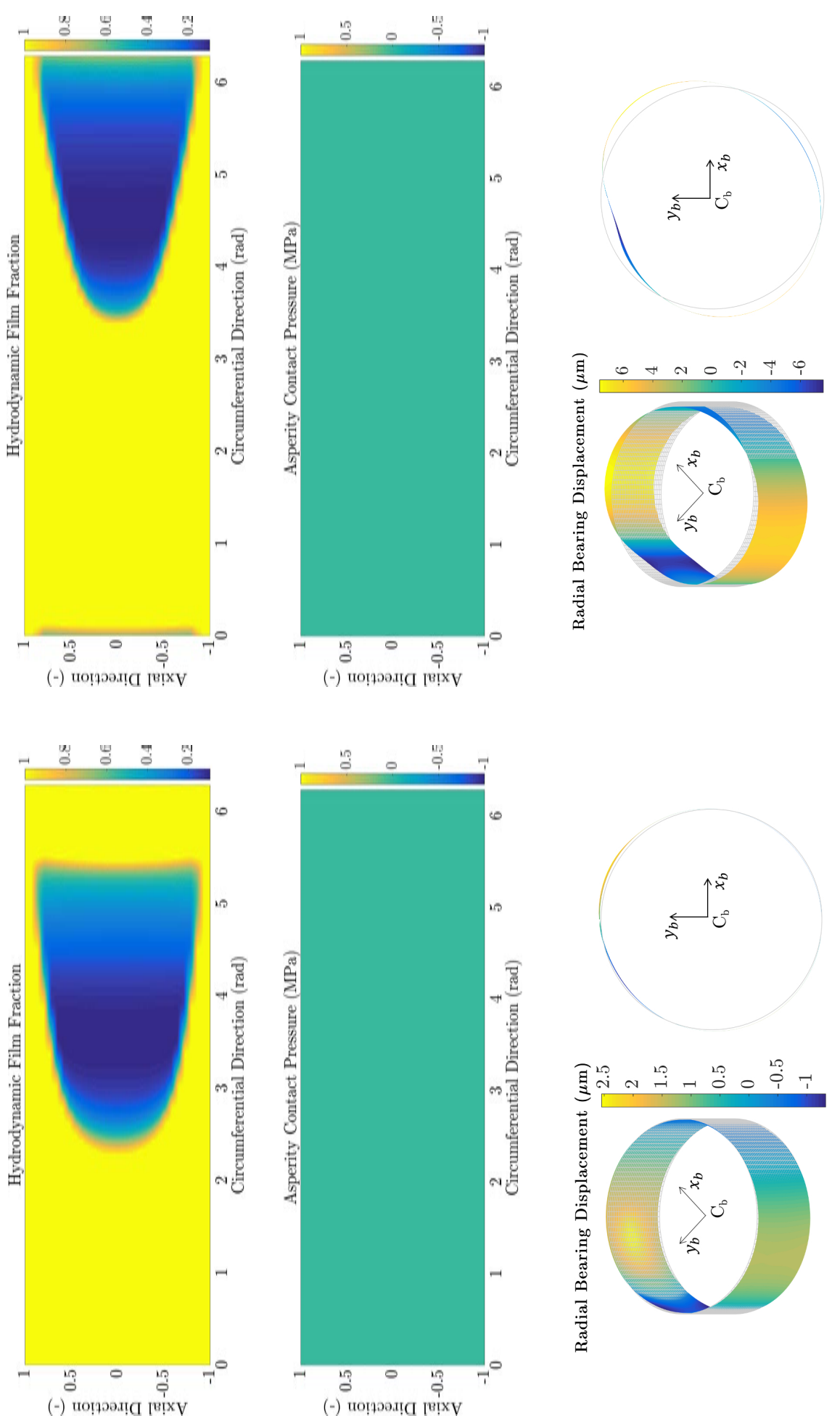

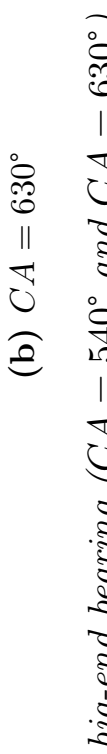

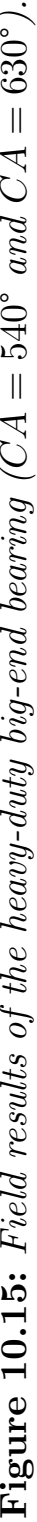




\section{Chapter 11}

\section{Case II: Dynamically Loaded Connecting-Rod Big-End Bearing of a High-Speed Engine}

In this second application case, a set of dynamic simulations undertaken for the connecting-rod big-end bearing of a high-speed motorcycle engine will be presented. Figure 11.1 illustrates an assemblage of the main engine parts, with particular emphasis on the connecting-rod system. This analysis is to evaluate the suitability and robustness of the new EHL coupling techniques described in Chapter 9, as well as the effectiveness of the high-order time discretization scheme introduced in Chapter 6, on the simulation of actual journal bearings under dynamic loading conditions. Furthermore, the influence of different shear-thinning oils on the friction reduction of the bearing system is evaluated as well to provide further information about their potential applicability to real engine components. Some of the results presented in this case study have been published by the author in reference [170].

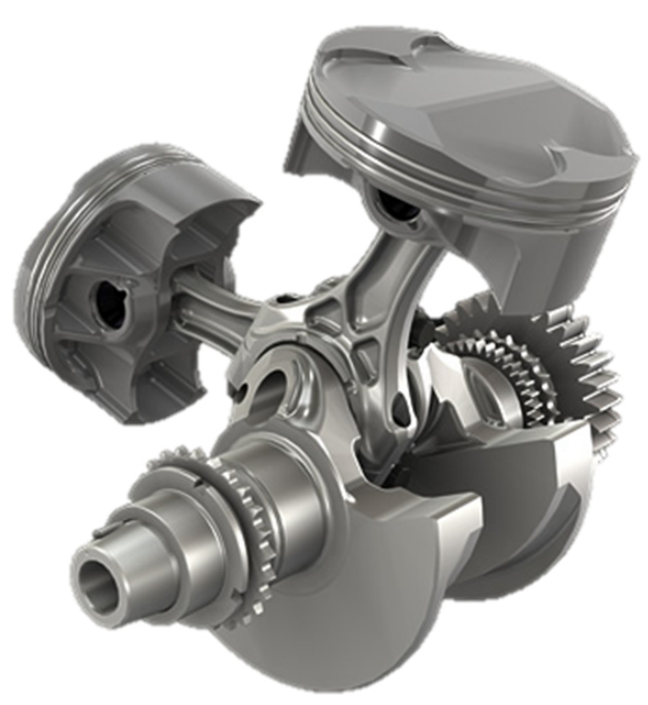

(a) Assembly of the main engine parts.

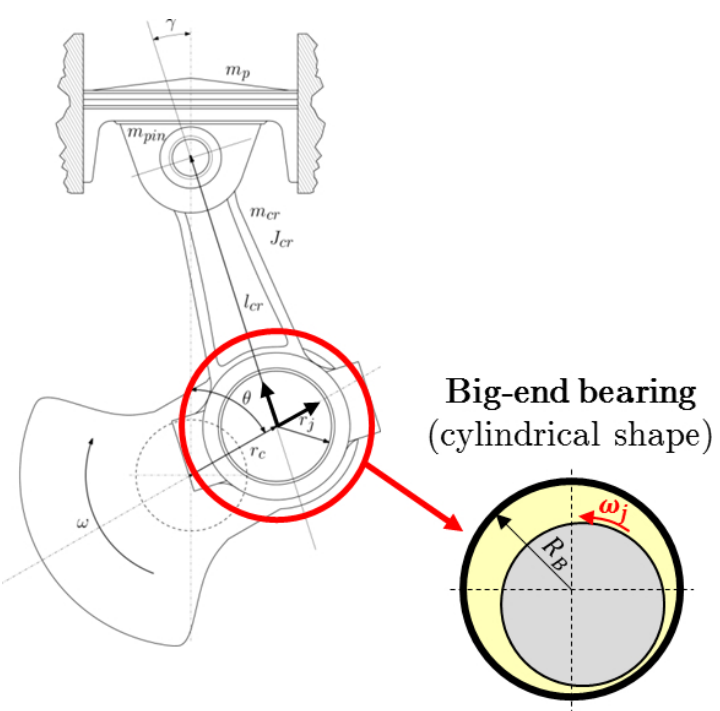

(b) Details of the components and references of the connecting-rod system.

Figure 11.1: Illustration of the high-speed motorcycle engine. 


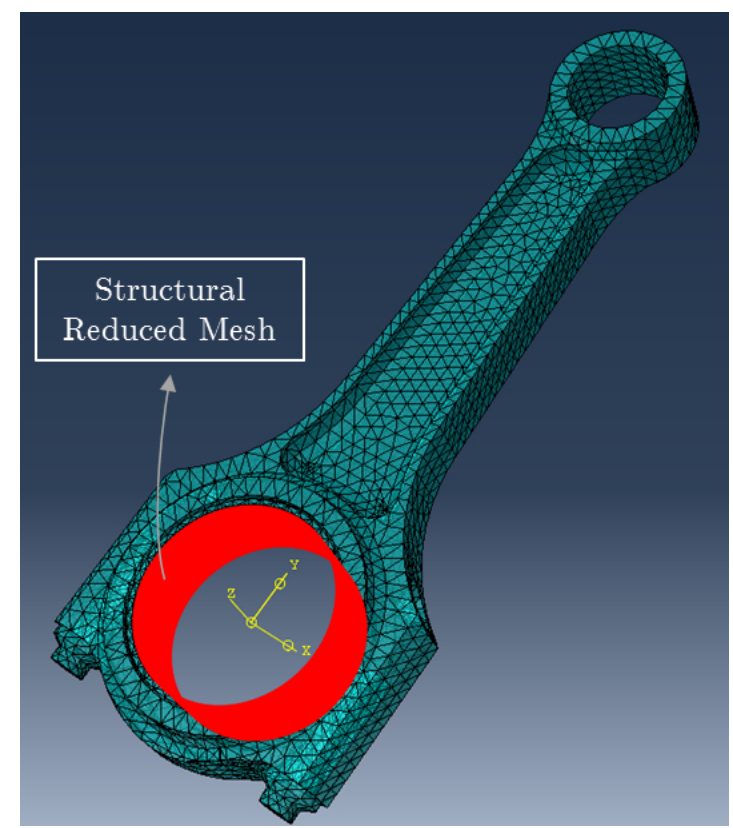

(a) 3D FEM model
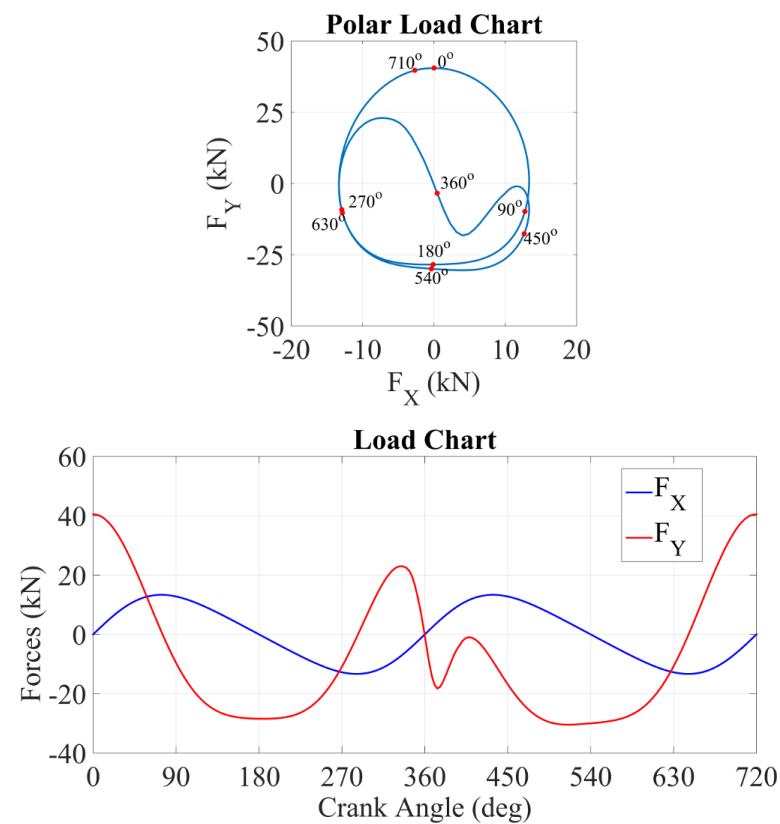

(b) Load chart of the big-end bearing

Figure 11.2: Geometry and load conditions of the high-speed connecting-rod system.

\subsection{Input Data}

The general input data of the connecting-rod in study are listed in Table 11.1. Similarly, Table 11.2 displays the principal properties and boundary conditions of the big-end bearing considered for the simulations.

Table 11.1: General data of the high-speed connecting-rod part.

\begin{tabular}{lcc}
\hline Parameter & Value & Unit \\
\hline Material & Steel & - \\
Young modulus & 210 & $\mathrm{GPa}$ \\
Poisson's ratio & 0.3 & - \\
Density & 8050 & $\mathrm{~kg} / \mathrm{m}^{3}$ \\
Rod length & 150 & $\mathrm{~mm}$ \\
\hline
\end{tabular}

The entire 3D FEM model of the connecting-rod part created in the Abaqus ${ }^{\circledR}$ software [62] is depicted in Fig. 11.2a. The bearing surface is highlighted to illustrate the region from which the full FEM system is reduced, i.e. only the degrees of freedom of the nodes located on this surface are retained in the reduced FEM model and effectively used for EHL calculations (see Section 3.2.2 for further explanations about FEM model reduction). Moreover, the system was admitted with no physical constraint, i.e. free-free boundary condition, so that the procedure described in Section 3.3 had to be applied to the reduced matrices to prevent the singularities associated with the rigid body modes. The effects of the distributed inertia were neglected in the analysis (quasi-static solution approach). The external load forces acting on the big-end bearing, which are represented with respect to the bearing coordinate systems illustrated in Fig. 11.2a, are depicted in the plots of Fig. 11.2b. Such loads correspond to the reaction forces obtained from multibody dynamic 
simulations of the whole connecting-rod system, assuming the respective bearings as ideal rigid joints. Furthermore, by considering the absence of a hollow pin-journal, the journal flexibility tends to be considerably lower than the bearing structure one. Thus, the journal is admitted rigid and only the bearing deformations are considered in the analysis.

Table 11.2: Main properties and operational conditions of the high-speed big-end bearing.

\begin{tabular}{lcc}
\hline Parameter & Value & Unit \\
\hline Radius & 21.5 & $\mathrm{~mm}$ \\
Width & 20.0 & $\mathrm{~mm}$ \\
Radial clearance & 50.0 & $\mu \mathrm{m}$ \\
Ambient pressure (bearing sides) & $10^{5}$ & $\mathrm{~Pa}$ \\
Cavitation pressure & 0 & $\mathrm{~Pa}$ \\
Engine speed & 9000 & $\mathrm{RPM}$ \\
Supply hole & $\mathrm{No}$ & - \\
\hline
\end{tabular}

Table 11.3, summarizes the rheological properties of each lubricant here investigated for potential reduction in the bearing friction losses. Oil0 is the standard lubricant used in the specified engine, and will thus be assumed as a reference for comparisons. On the other hand, OilA, OilB and OilC are newly formulated shear-thinning oils with different polymer concentrations [145]. Originally, nearly all engine lubricants contain viscosity index improvers (VIIs) aimed to attenuate the temperature dependence of the blend. These polymers may undergo both temporary and permanent shear-thinning at high shear stresses [145]. Furthermore, it is now commonly recognised that the temporary shear-thinning effect yields to local temporary viscosity loss, hence contributing to reducing the hydrodynamic friction at high sliding velocities, as those encountered in the particular highspeed engine studied in this section. Figure 11.3 illustrates the variation of the dynamic viscosity with shear rate for the three shear-thinning oils considered.

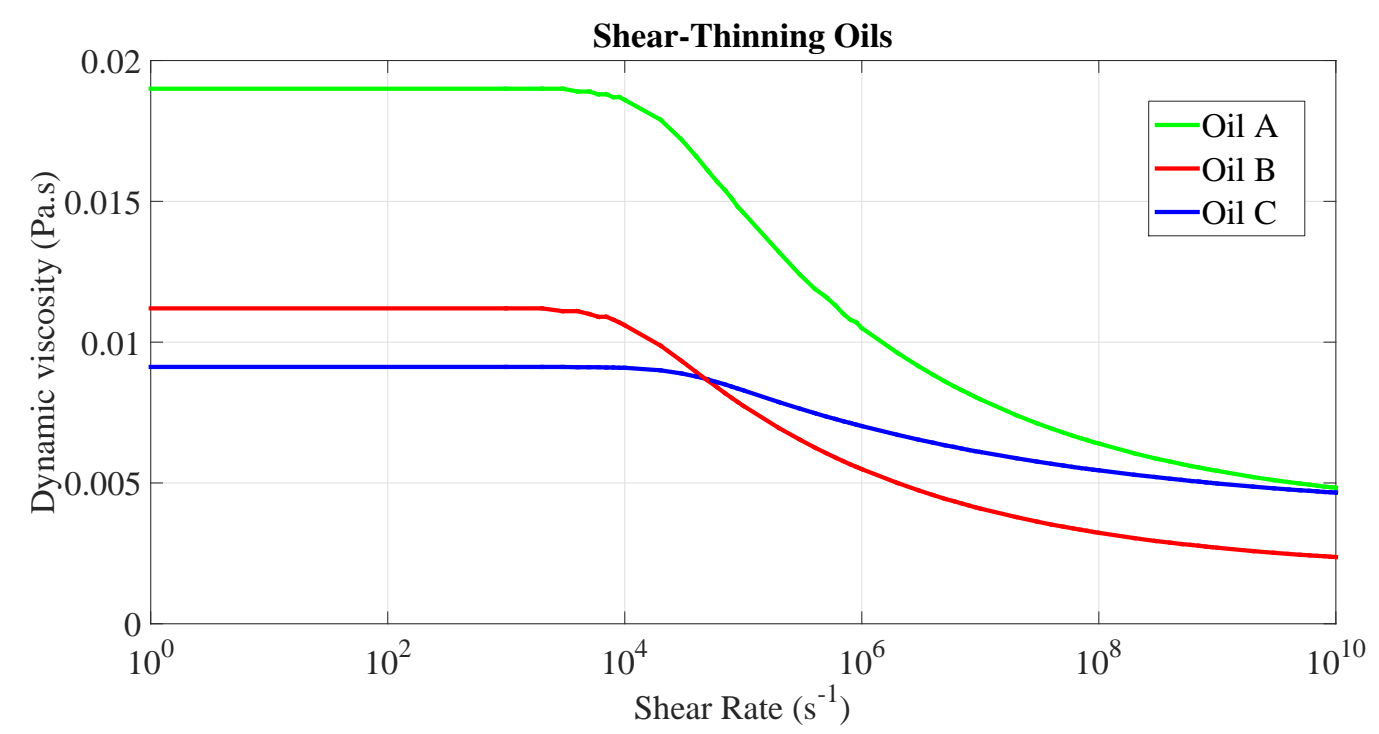

Figure 11.3: Variation of the dynamic viscosity with shear rate for the different shear-thinning oils investigated for friction reduction.

As for the inputs of the statistical mixed-lubrication models (GW/GT models for asperity contact and Patir and Cheng formulation for the average fluid flow), Table 11.4 
Table 11.3: Rheological properties of the lubricants for the high-speed big-end bearing simulations.

\begin{tabular}{|c|c|c|c|c|c|}
\hline \multirow[t]{2}{*}{ Parameter } & \multicolumn{4}{|c|}{ Value } & \multirow[t]{2}{*}{ Unit } \\
\hline & $\begin{array}{c}\text { OIL } 0 \\
\text { (Standard) }\end{array}$ & $\begin{array}{c}\text { OIL A } \\
(-)\end{array}$ & $\begin{array}{c}\text { OIL B } \\
(-)\end{array}$ & $\begin{array}{c}\text { OIL C } \\
(-)\end{array}$ & \\
\hline \multicolumn{6}{|c|}{ Piezo-viscosity effect (Roelands equation) ${ }^{*}$} \\
\hline$\alpha$ & \multicolumn{4}{|c|}{$1.10 \times 10^{-8}$} & $\mathrm{~Pa}^{-1}$ \\
\hline \multicolumn{6}{|c|}{ Shear-thinning effect (Carreau-Yasuda equation) ${ }^{* *}$} \\
\hline$\eta_{0}$ & $1.90 \times 10^{-2}$ & $1.90 \times 10^{-2}$ & $1.12 \times 10^{-2}$ & $9.12 \times 10^{-3}$ & Pas \\
\hline$\eta_{\infty}$ & - & $3.87 \times 10^{-3}$ & $1.85 \times 10^{-3}$ & $3.87 \times 10^{-3}$ & Pas \\
\hline$A$ & - & $5.00 \times 10^{-5}$ & $9.00 \times 10^{-5}$ & $3.00 \times 10^{-5}$ & - \\
\hline$n$ & - & 0.79 & 0.79 & 0.85 & - \\
\hline$a$ & - & 1.9 & 1.9 & 2 & - \\
\hline \multicolumn{6}{|c|}{ Piezo-density effect (Dowson-Higgison equation) ${ }^{*}$} \\
\hline$\rho_{0}$ & \multicolumn{4}{|c|}{850} & $\mathrm{~kg} / \mathrm{m}^{3}$ \\
\hline$C_{1}$ & \multicolumn{4}{|c|}{$5.90 \times 10^{8}$} & $\mathrm{~Pa}$ \\
\hline$C_{2}$ & \multicolumn{4}{|c|}{1.34} & - \\
\hline
\end{tabular}

shows the contact and roughness parameters obtained for the surface topography depicted in Fig. 11.4, which was measured from a bearing shell used in a similar high-speed engine. Notice that the surface roughness is assumed practically isotropic so that $\gamma \rightarrow 0$ (see Appendix D.2). Table 11.5 lists all the numerical settings and specific parameter controls related to the different algorithms used for the simulations presented in the next sections.

Table 11.4: Contact and roughness parameters of the high-speed big-end bearing shells.

\begin{tabular}{lcc}
\hline Parameter & Value & Unit \\
\hline Bearing material $^{*}$ & AlSnSi alloy & - \\
Overlay material $^{*}$ & PbSnCu alloy & - \\
$E$ (bearing material) $^{*}$ & 75 & $\mathrm{GPa}$ \\
$v$ (bearing material) $^{*}$ & 0.34 & - \\
$H_{V}$ (overlay material) $^{*}$ & 18 & $\mathrm{HV}$ \\
\hline$S a$ & 0.24 & $\mu \mathrm{m}$ \\
$S q$ & 0.32 & $\mu \mathrm{m}$ \\
$\gamma$ & 0.1 & - \\
$Z_{S}$ & 0.28 & $\mu \mathrm{m}$ \\
$\sigma_{S}$ & 0.23 & $\mu \mathrm{m}$ \\
$\beta_{S}$ & 61.42 & $\mu \mathrm{m}$ \\
$\eta_{S}$ & $6.50 \times 10^{9}$ & $\mathrm{~m}^{-2}$ \\
$\mu_{B L}$ & 0.12 & - \\
\hline${ }^{*}$ See $[14,208]$ & &
\end{tabular}




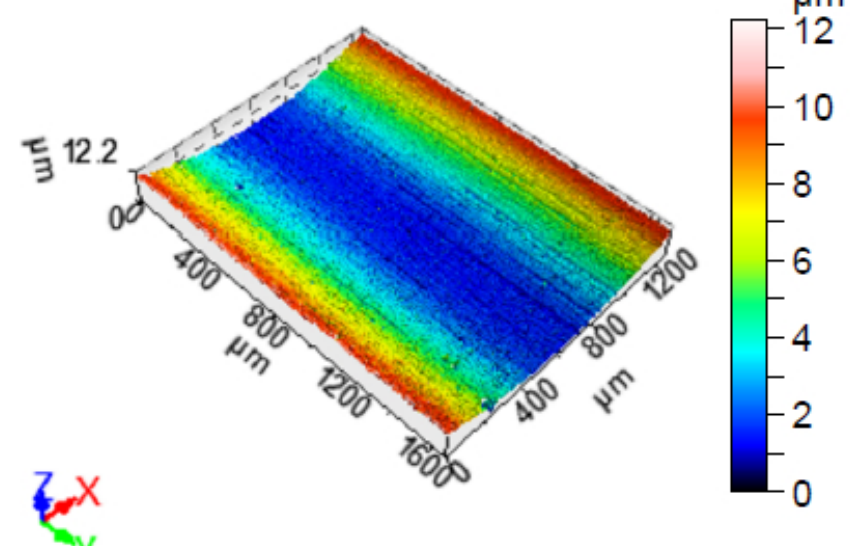

(a) Original topography as measured.

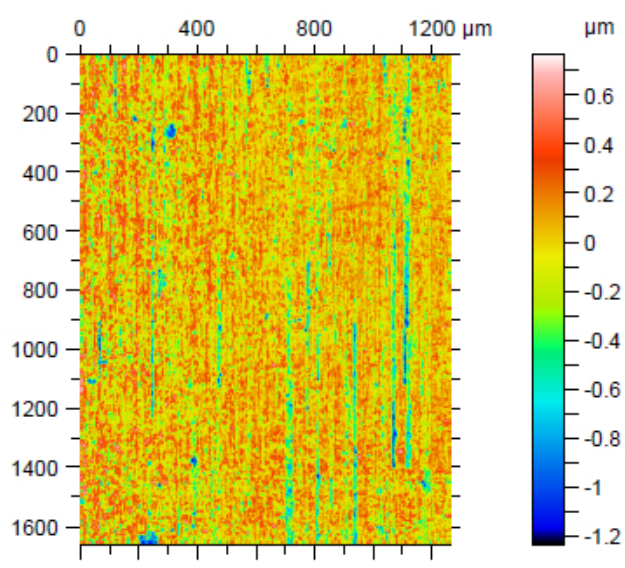

(b) Roughness topography after form and waviness removal (cut-off of $0.8 \mathrm{~mm}$ ).

Figure 11.4: Representative surface topography of the high-speed big-end bearing shells.

\subsection{Simulation results for the lubricant 'Oilo'}

The overall scalar results of the conrod big-end bearing considered in the present analysis for lubricant Oil0 are summarized in Fig. 11.5. The curves depict the evolution of the main operational lubrication variables over a full engine cycle subjected to the conditions described in Section 11.1. The results for both rigid (blue lines) and flexible (red lines) bearing cases are shown in the figure. The general behaviour of the system is similar to that verified in Section 10.3 for the heavy-duty big-end bearing. In this fashion, as expected for the EHL case, the journal orbit deviated around the unit eccentricity circle due to the large solid deformations of the bearing bore. Furthermore, the maximum structural displacements also occurred under equivalent situations in which the external loads are aligned to the more compliant direction of the lower shell $\left(C A \approx 180^{\circ}\right.$ and $C A \approx 540^{\circ}$.

The variation tendencies of the minimum oil film thickness (MOFT) and the hydrodynamic peak pressures (PP) showed to be similar along the complete engine cycle, except for the magnitudes of PP that were clearly lower for the flexible bearing case. In particular, significant decrease of PP is observed during combustion, which can be explained by the "spreading" effect of the fluid pressure over the distorted bearing surface. Analogously, distinguishable reduction in the hydrodynamic peak pressures also took place around the positions of larger structural deformations $\left(C A \approx 180^{\circ}\right.$ and $\left.C A \approx 540^{\circ}\right)$, which equally contributed to the "accommodation" of the lubricant pressure distributions on the interface. Regarding the MOFT, slightly lower values were obtained under EHL conditions, but in contrast to the heavy-duty diesel engine analysis they were not small enough to produce asperity contact interactions. Likewise, the main discrepancies in the power friction losses (PL) were noticed during combustion and for the situations of large structural displacements. In the former condition, the differences in the viscous dissipation are linked to the varying intensity of the piezoviscous effect, while in the latter situation the deviations are associated with the combined drop in the hydrodynamic pressures and film thickness for the flexible bearing case.

The surface plots illustrated in Figs. 11.6-11.12 aim to outline the progression in time of the hydrodynamic pressure, hydrodynamic film fraction (cavitation), asperity contact 
Table 11.5: Numerical settings for the static and dynamic simulations of the high-speed big-end bearing.

\begin{tabular}{|c|c|}
\hline Parameter & Value \\
\hline \multicolumn{2}{|c|}{ Meshes } \\
\hline Hydrodynamic & $2400(120 \times 20)$ \\
\hline Structural (reduced model) & 2520 \\
\hline \multicolumn{2}{|c|}{ Solver: Linear System } \\
\hline Type & SOR \\
\hline Relaxation (pressure) factor & 1.8 \\
\hline Relaxation (film fraction) factor & 1 \\
\hline Max. iterations & 20000 \\
\hline Tolerance error & $10^{-6}$ \\
\hline \multicolumn{2}{|c|}{ Solver: Rheology } \\
\hline Type & PGMA \\
\hline Initial relaxation factor & 0.001 \\
\hline Maximum iterations & 30 \\
\hline Tolerance error & $10^{-2}$ \\
\hline \multicolumn{2}{|c|}{ Solver: EHL } \\
\hline Type & PGMF, PGMA, IQN-ILS \\
\hline Initial relaxation factor & 0.001 \\
\hline Maximum iterations & 30 \\
\hline Tolerance error & $10^{-2}$ \\
\hline \multicolumn{2}{|c|}{ Solver: Static } \\
\hline Type & fsolveNR \\
\hline Finite difference step & $10^{-4}$ \\
\hline Maximum function evaluation & 30 \\
\hline Maximum iterations & 40 \\
\hline Tolerance for variables & $10^{-4}$ \\
\hline Tolerance for function & $10^{-3}$ \\
\hline \multicolumn{2}{|c|}{ Solver: Dynamic } \\
\hline Type & $\mathrm{BDF}$ \\
\hline BDF order & 4 \\
\hline$\theta_{1}$ & 2 \\
\hline$\theta_{2}$ & 0.8 \\
\hline Time step & $3^{\circ}$ (crank angles) \\
\hline Initial time & $0.0012 \mathrm{~s}$ \\
\hline Maximum sub-steps & 5 \\
\hline Maximum history & 5 \\
\hline
\end{tabular}




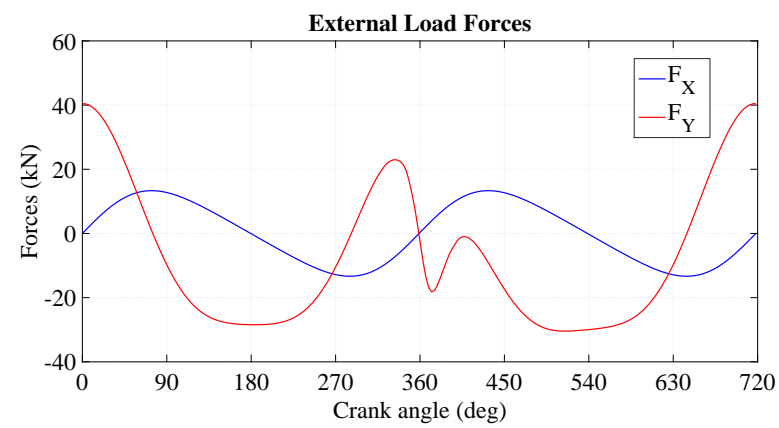

(a) External load forces

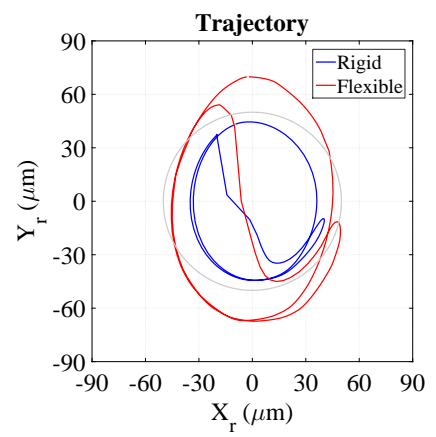

(c) Journal trajectory

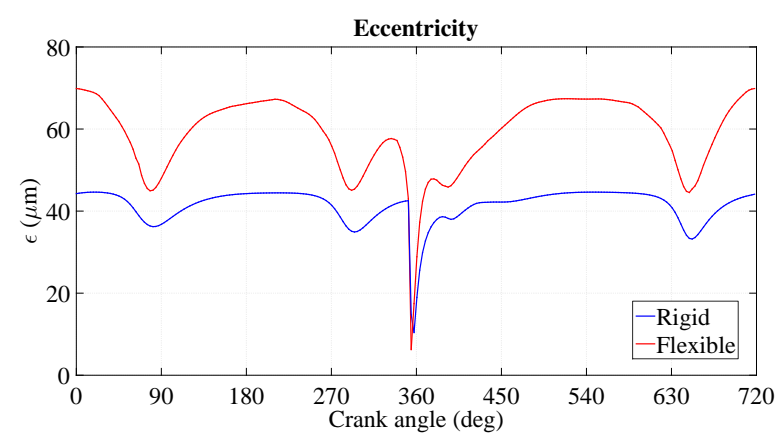

(e) Journal eccentricity

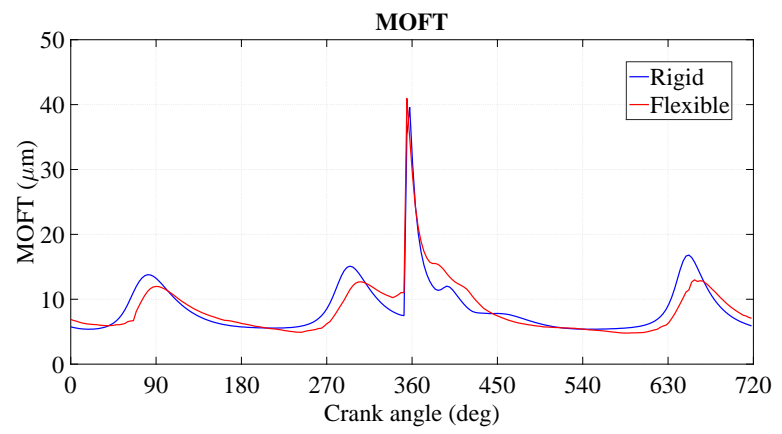

(b) Minimum oil film thickness

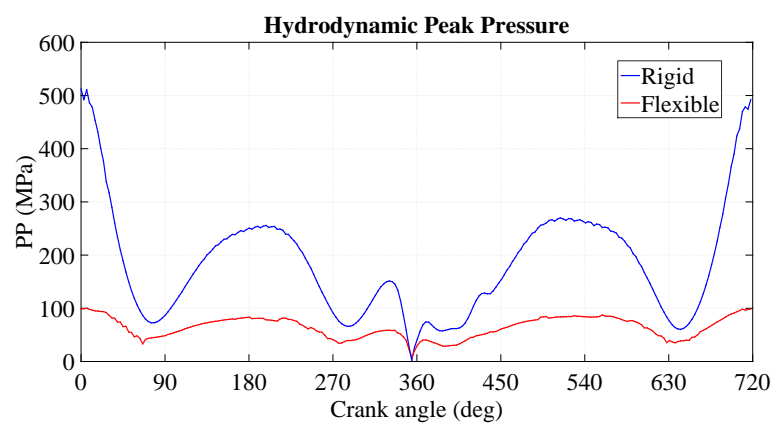

(d) Hydrodynamic peak pressure

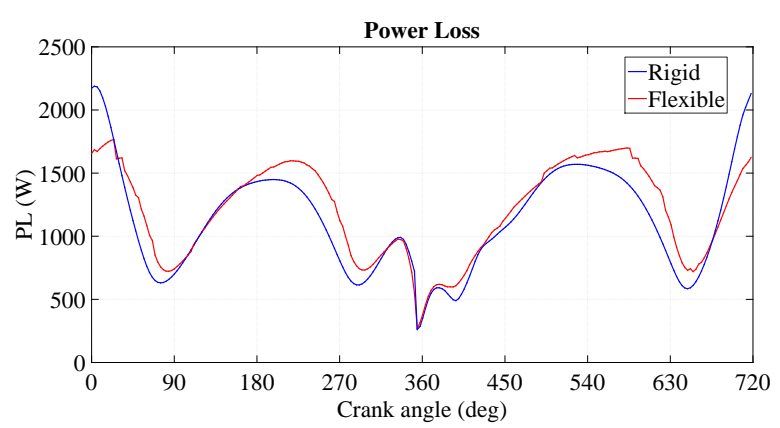

(f) Power loss

Figure 11.5: Scalar results of the dynamically loaded high-speed big-end bearing for the lubricant 'Oilo'.

pressure and bearing displacement fields over the entire engine cycle. Equivalently to the heavy-duty diesel engine case, for the situations of maximum structural deformations $\left(C A \approx 180^{\circ}\right.$ and $C A \approx 540^{\circ}$ ), the local geometric perturbations on the bearing surface yielded the hydrodynamic pressure distributions to the same bimodal shape pattern. Furthermore, a collapse of the cavitation zones comparable with that previously described in Section 10.3 was detected as well, but now only for the crank angle interval $330^{\circ}<C A<390^{\circ}$, as can be visualized by following the evolution of the film fraction field through Figs. 11.8-11.10. Physically, such sudden disruption onto the cavitation regions is closely related to the abrupt change of orientation and magnitude of the external loads acting on the big-end bearing [29, 40,41, 162]. This can be verified in the load chart of Fig. 11.2b, with special attention to the almost null loading situation experienced by the system at $C A \approx 360^{\circ}$. This instantaneous "unloading" condition explains the "spike" in the eccentricity and MOFT curves of Fig. 11.5. Furthermore, this extremely rapid transient behaviour configured the only bottleneck for the convergence of the EHL problem. 


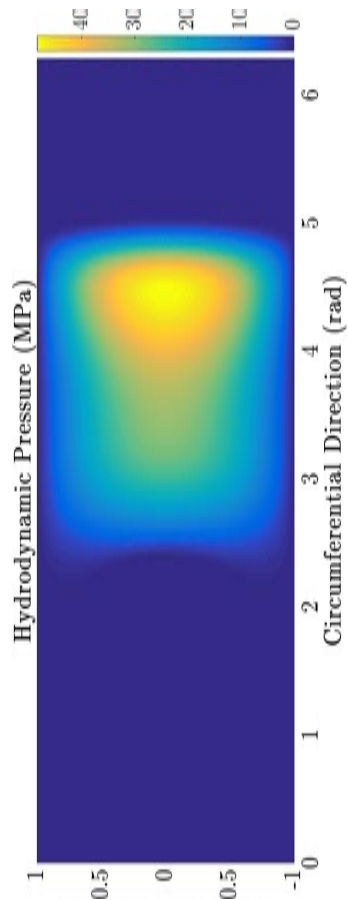

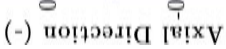

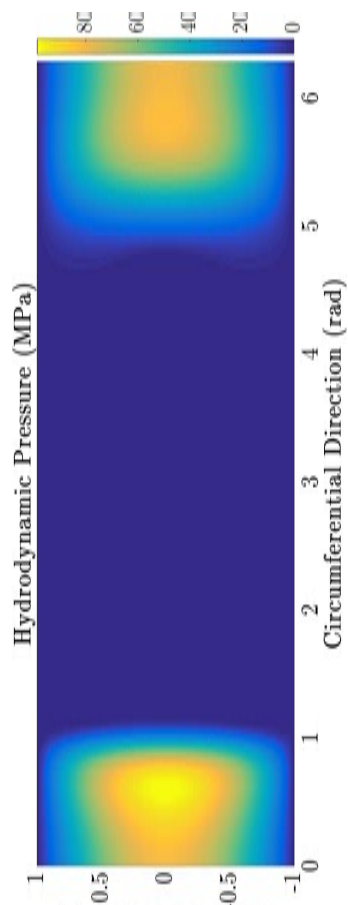

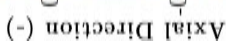

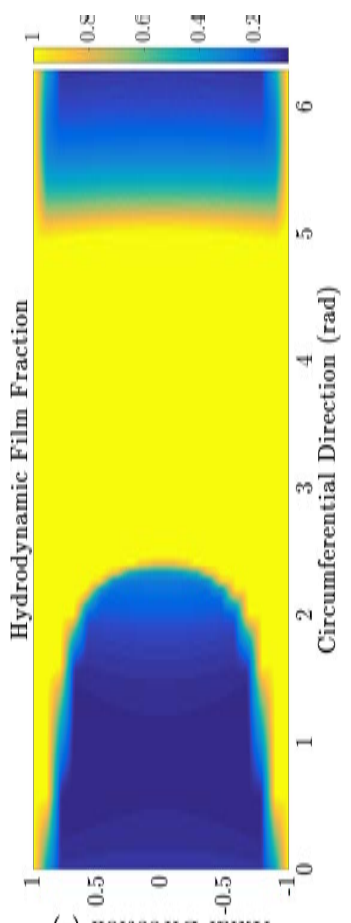

(-) попрал! [в!xy

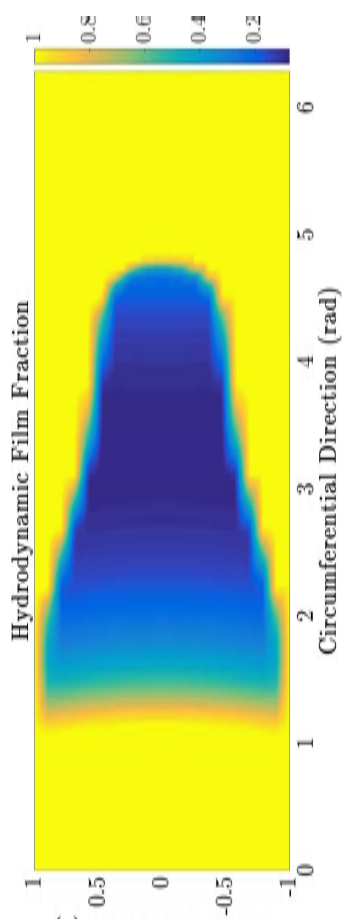

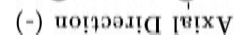

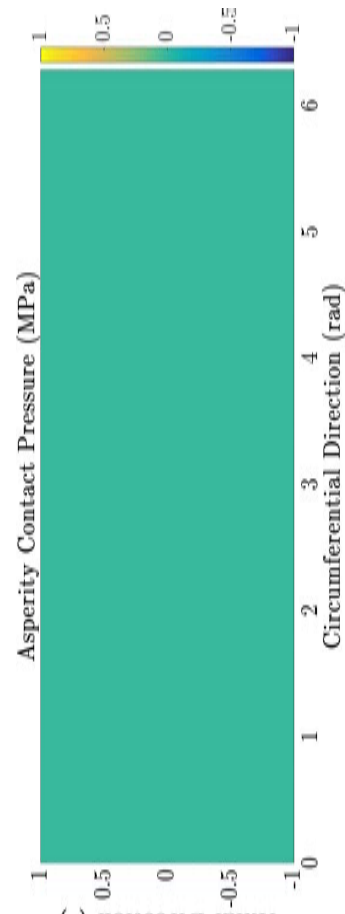

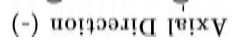

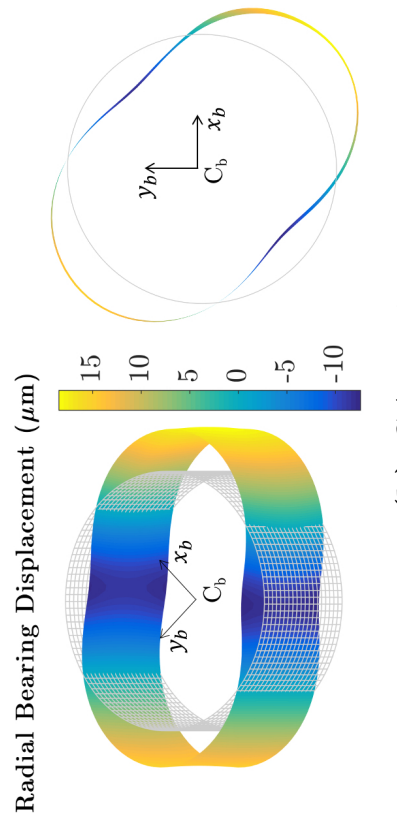



$8:$

U

อ :

है
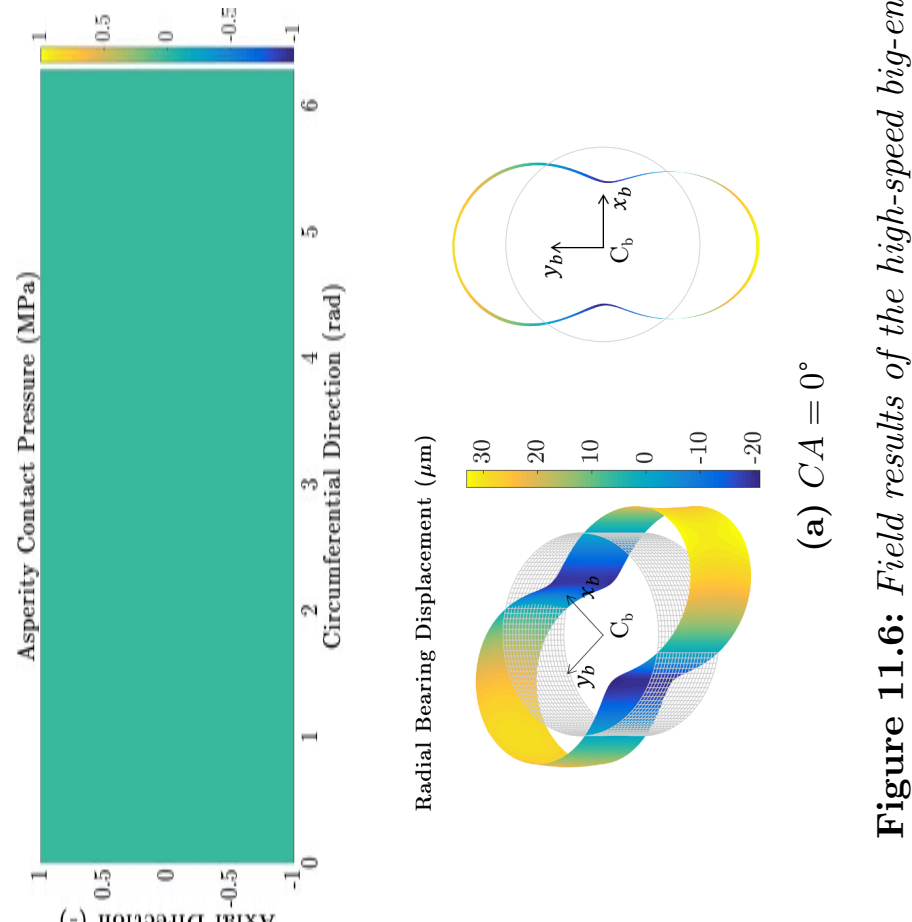


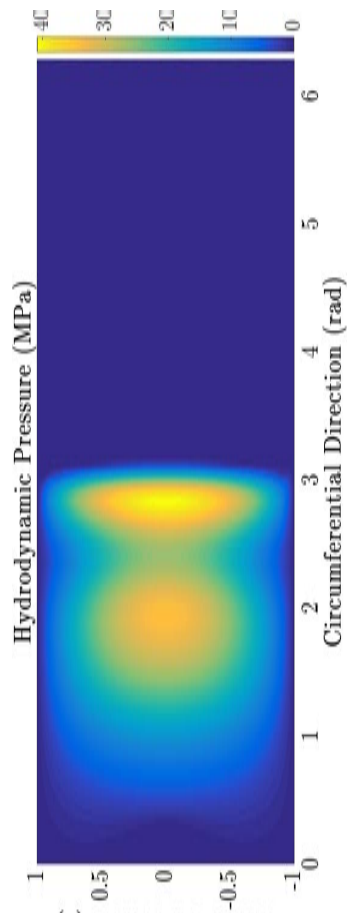

(-) uo!qpaม! [в!xV

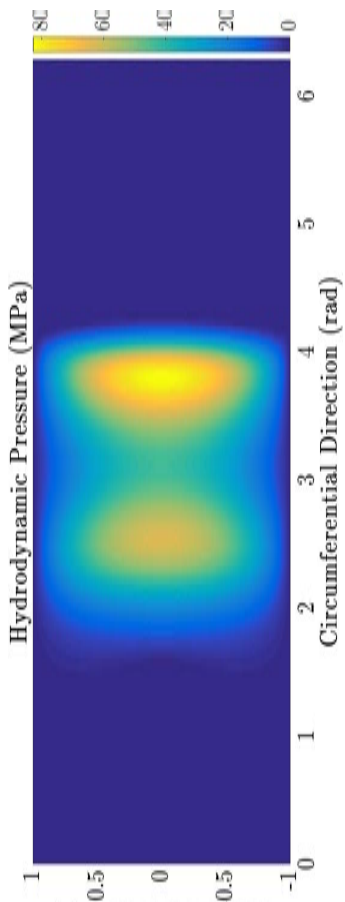

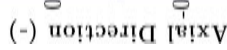

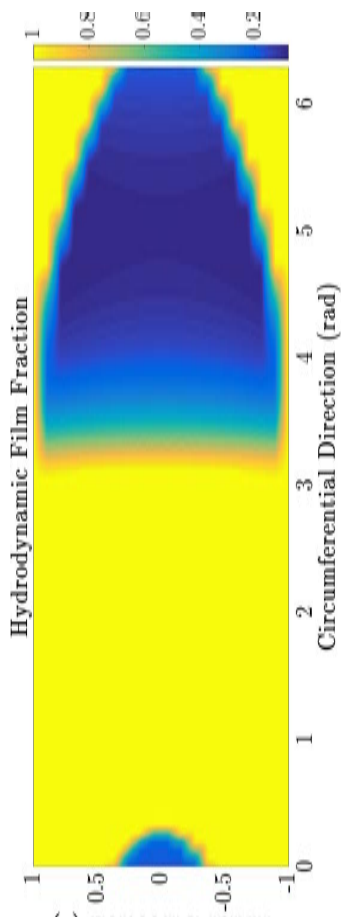

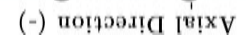

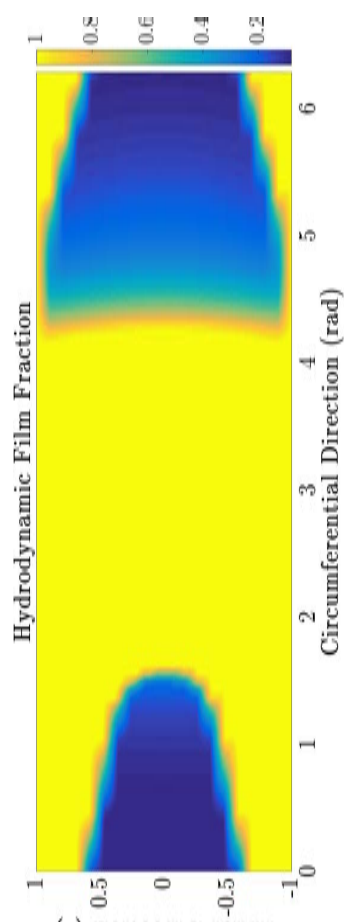

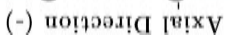

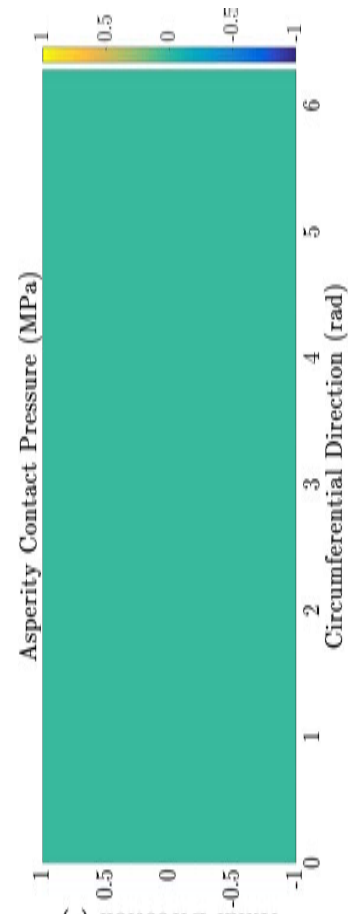

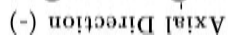

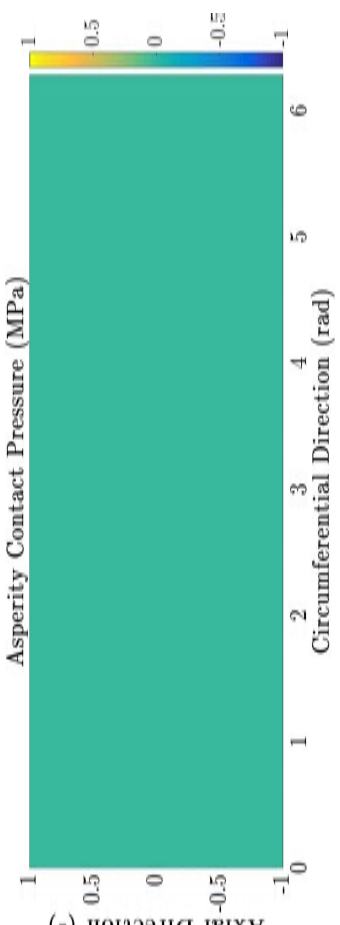

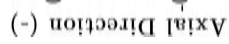

2

용

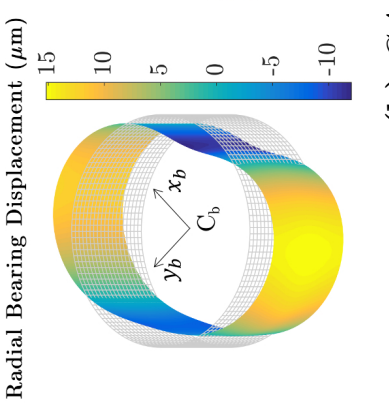

\| $\|$

ठ ठ

$\stackrel{0}{0}$

है

$\frac{\sqrt[3]{3}}{3}$

\&ิ

ปั

ชี่

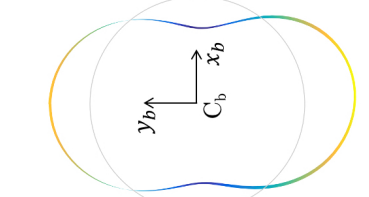

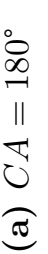

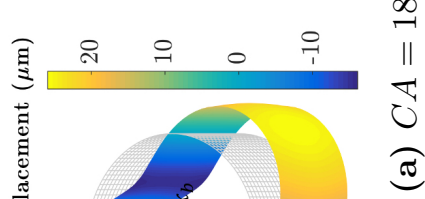

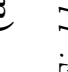




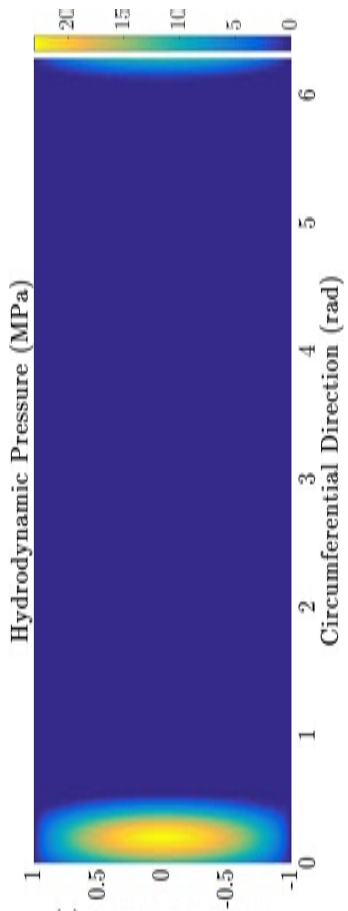

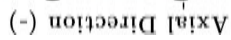

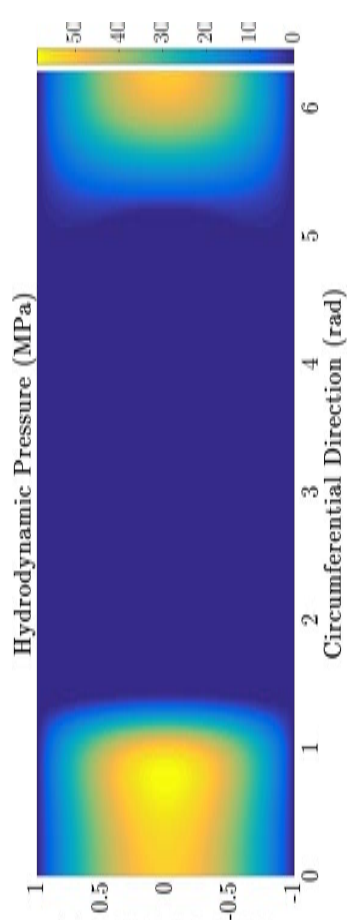

(-) प0!790x!ด [घ!xy

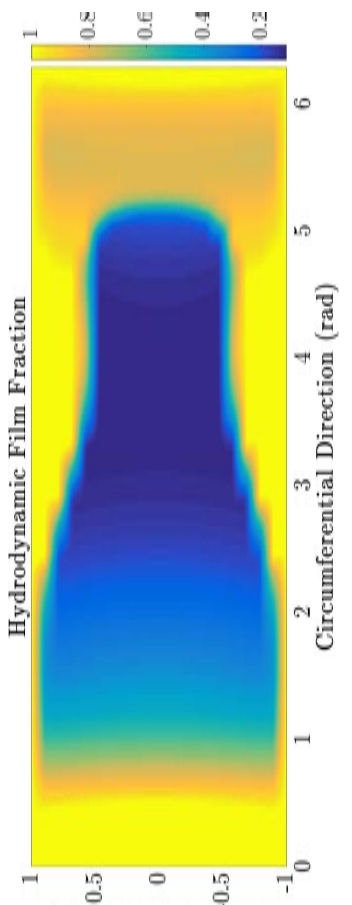

(-) хопюอง!ด [в!x

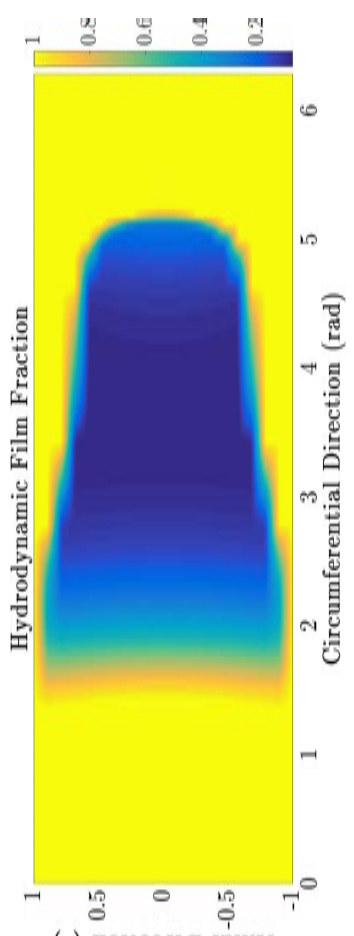

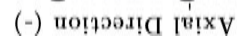

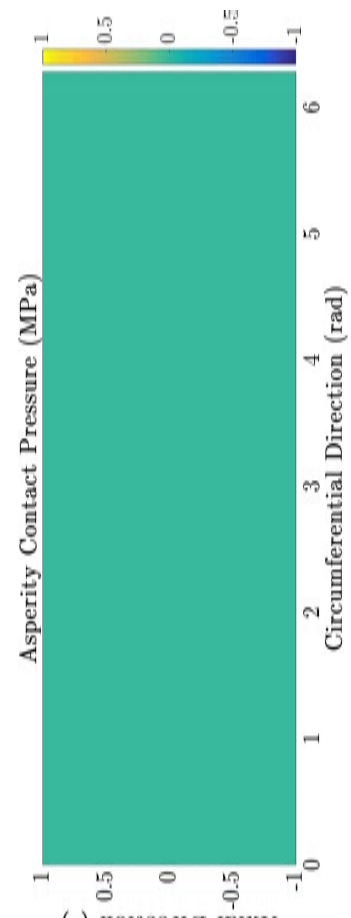

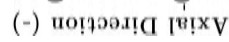

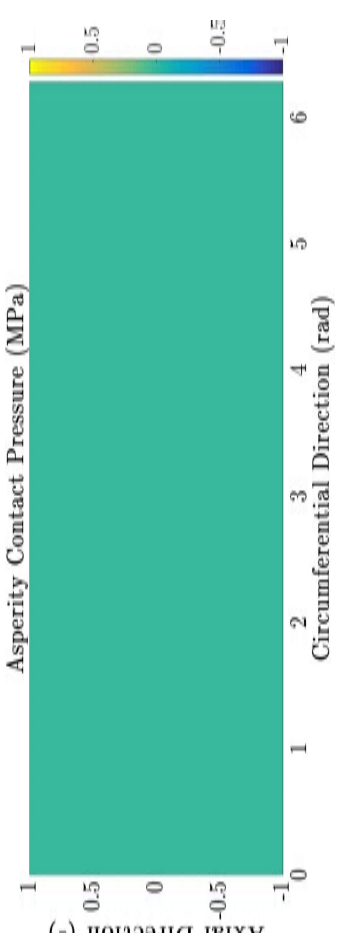

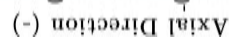

2

ำ ค

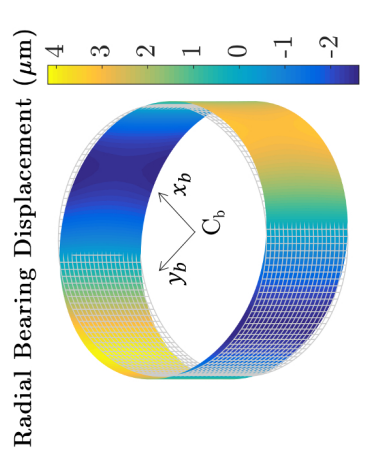

3

ช

$\circlearrowright$

$\stackrel{\overbrace{}}{\overparen{0}}$

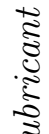

s

हี

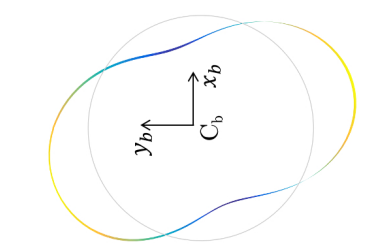

ฮ্ৰ

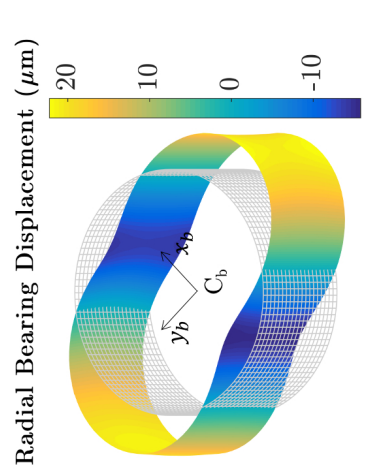

(2) 


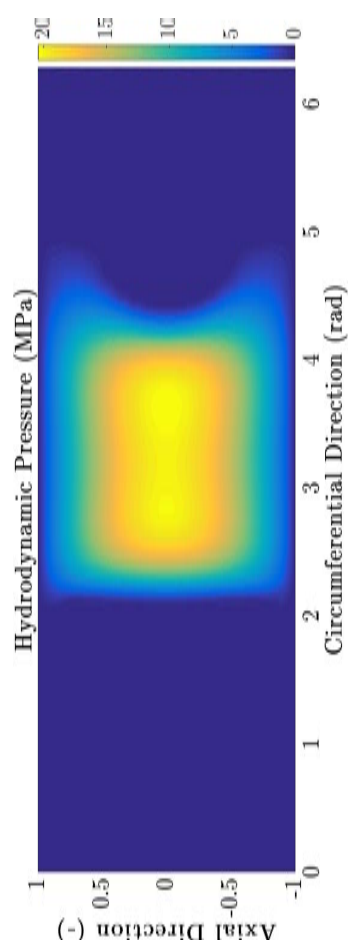

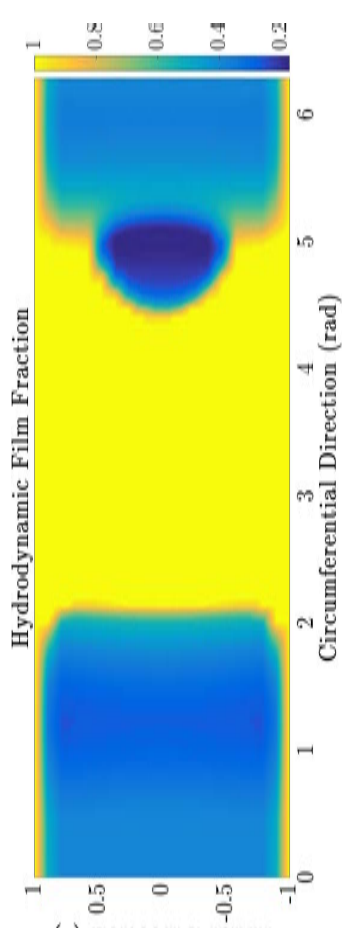

(-) попюอง!ด [в!x

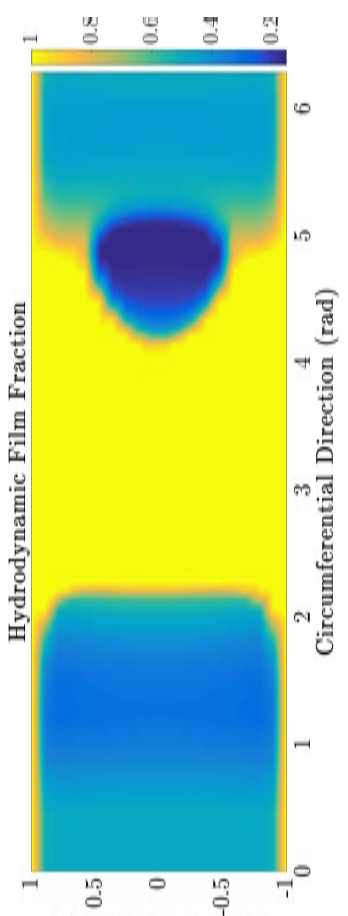

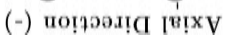

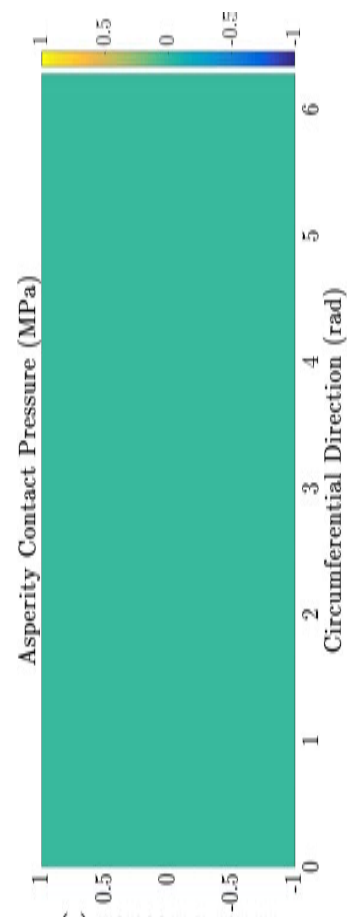

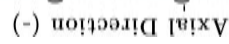

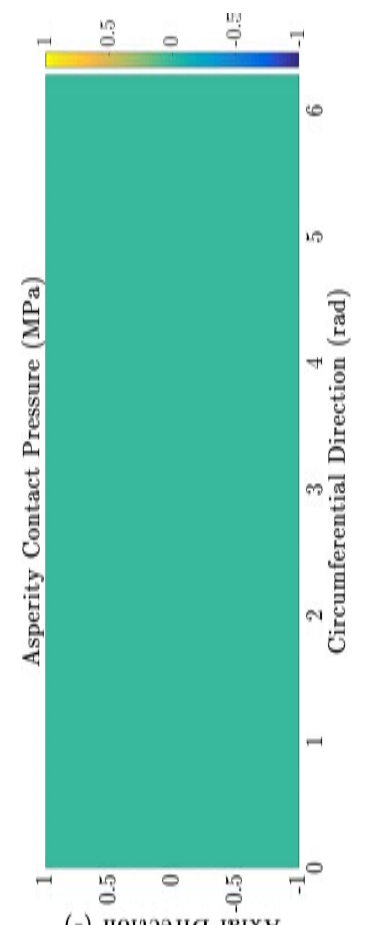

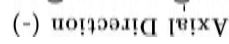

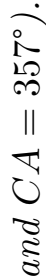

ก is

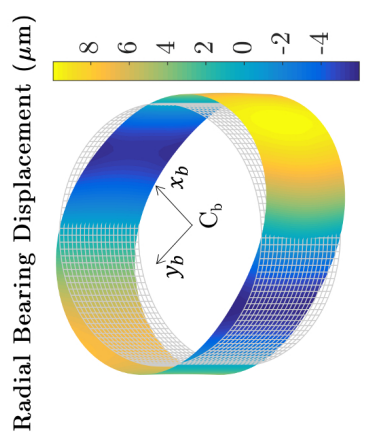

$\frac{1}{0}$
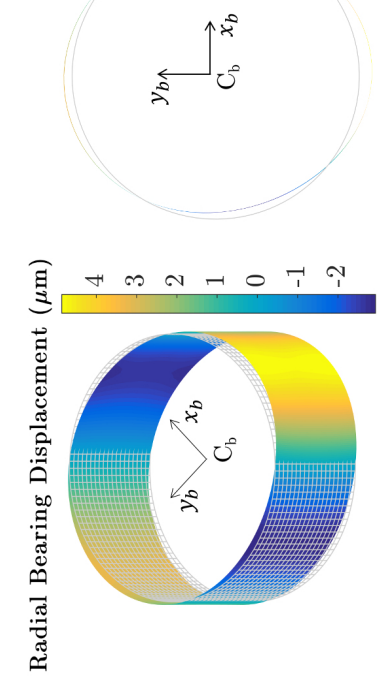

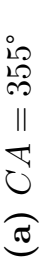

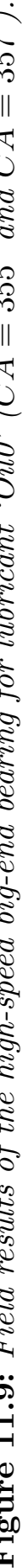




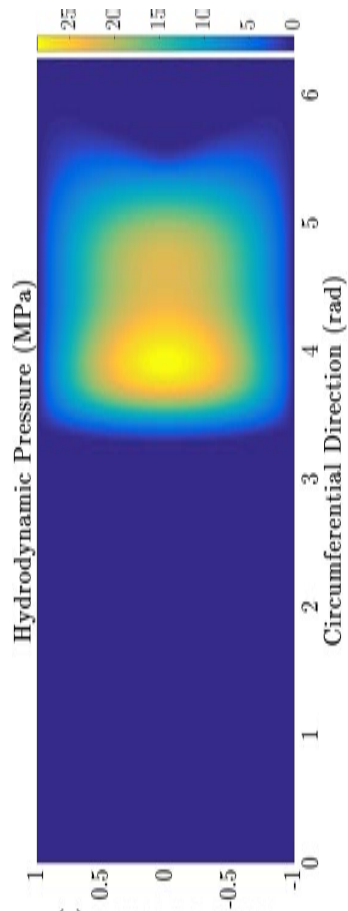

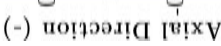

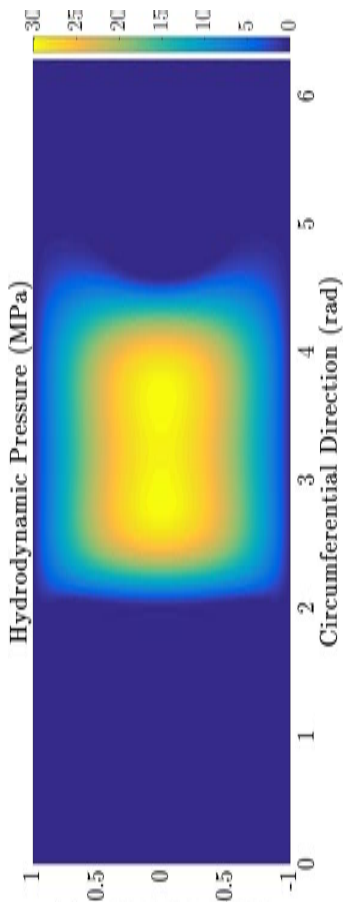

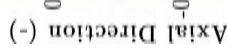

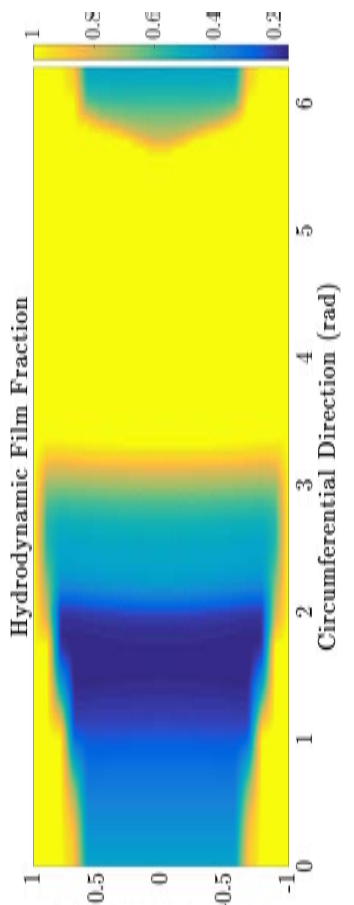

(-) иопрол!ด [в!x

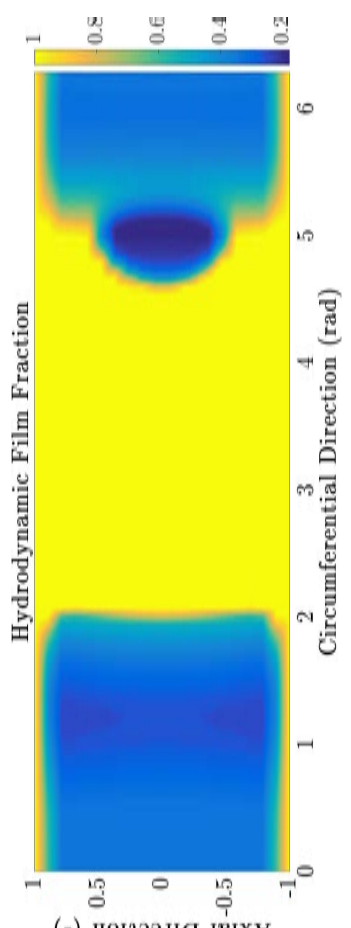

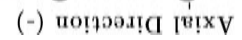

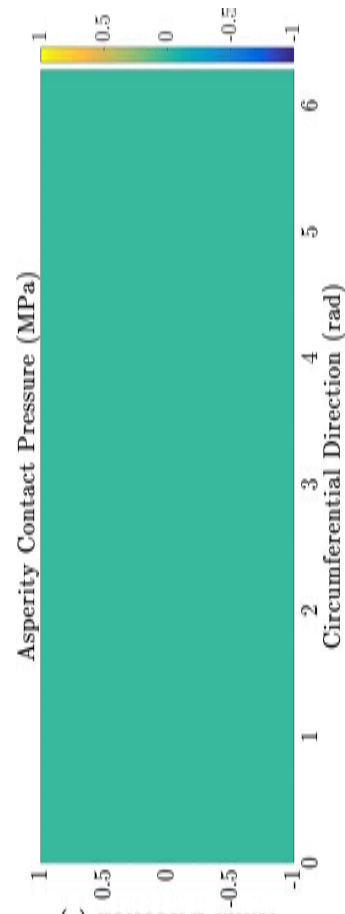

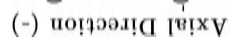

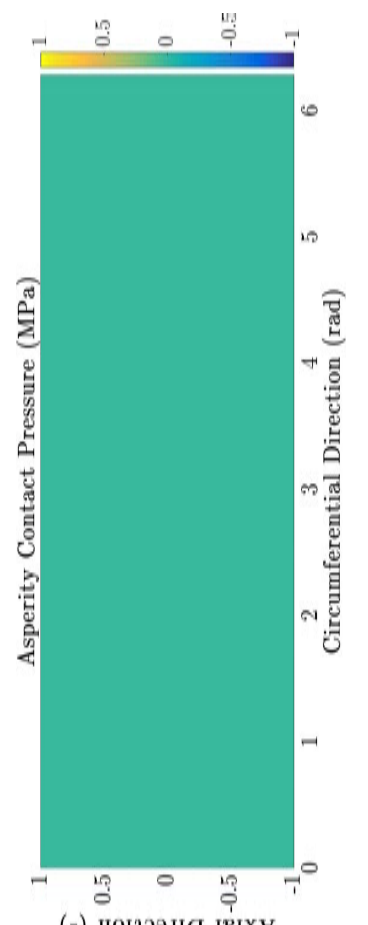

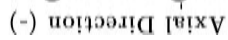

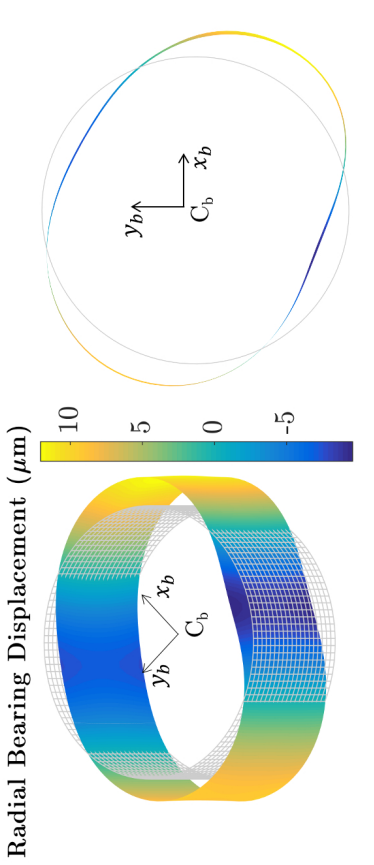

2

ஓ

II $\mid$

ठี

ê

है

0
0
0
0
0

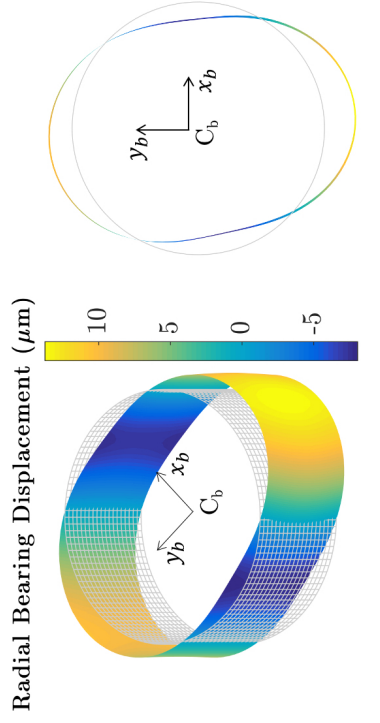

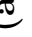




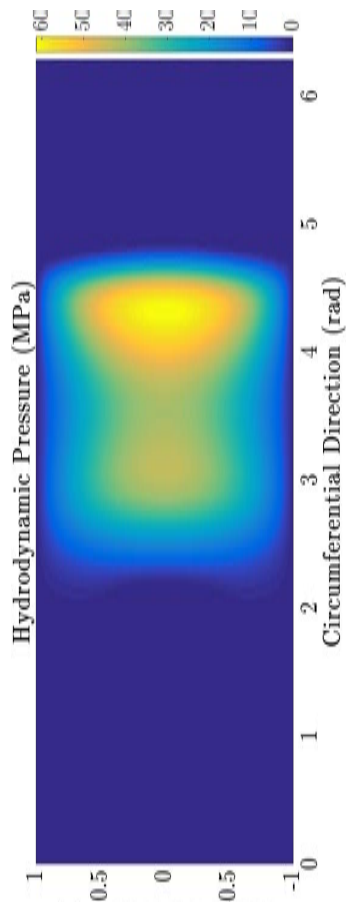

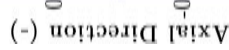

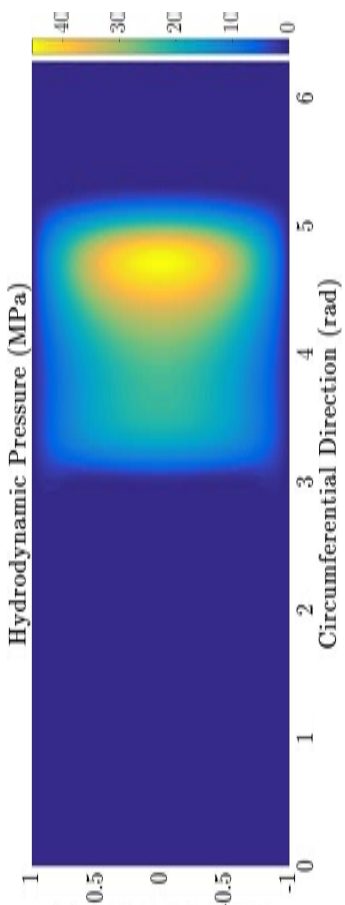

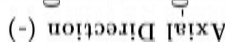

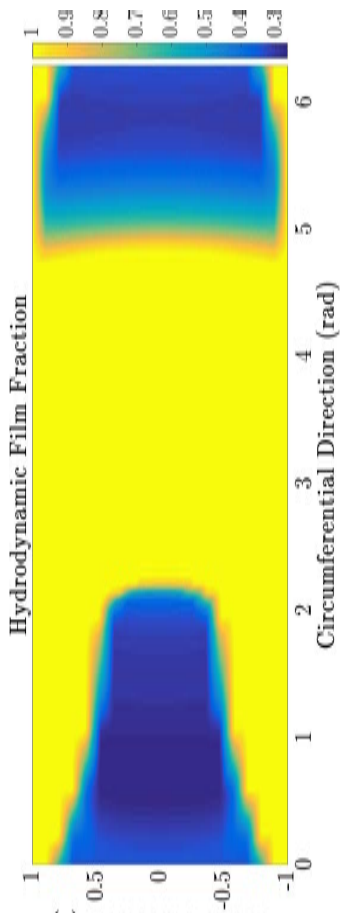

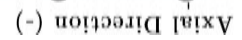

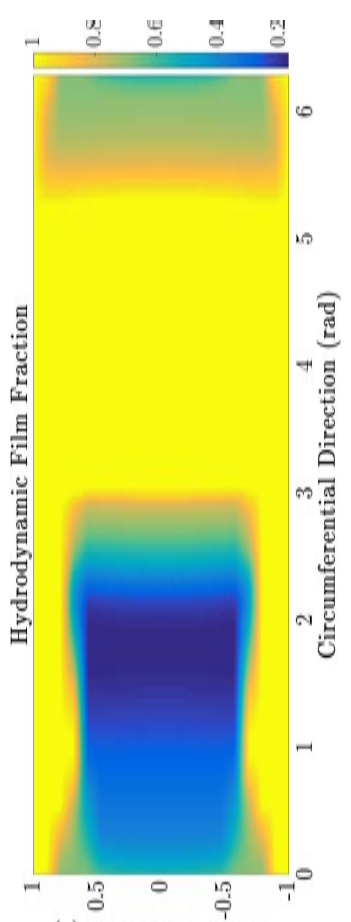

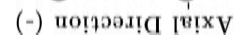

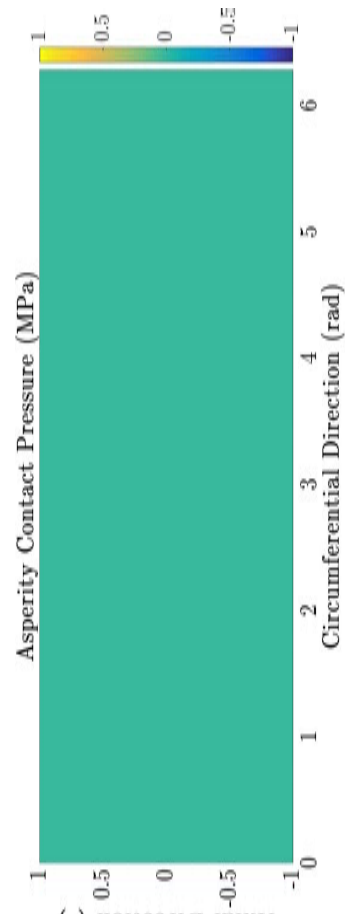

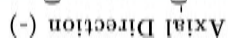

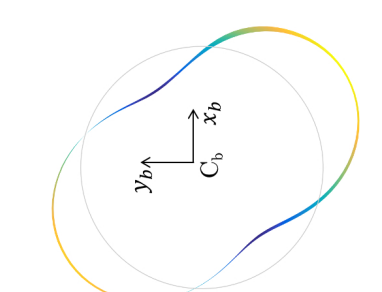

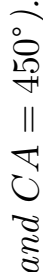

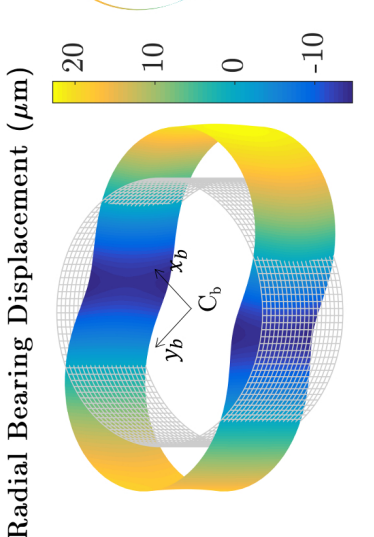

ㅇํㄱ 욱

ठ

อิ
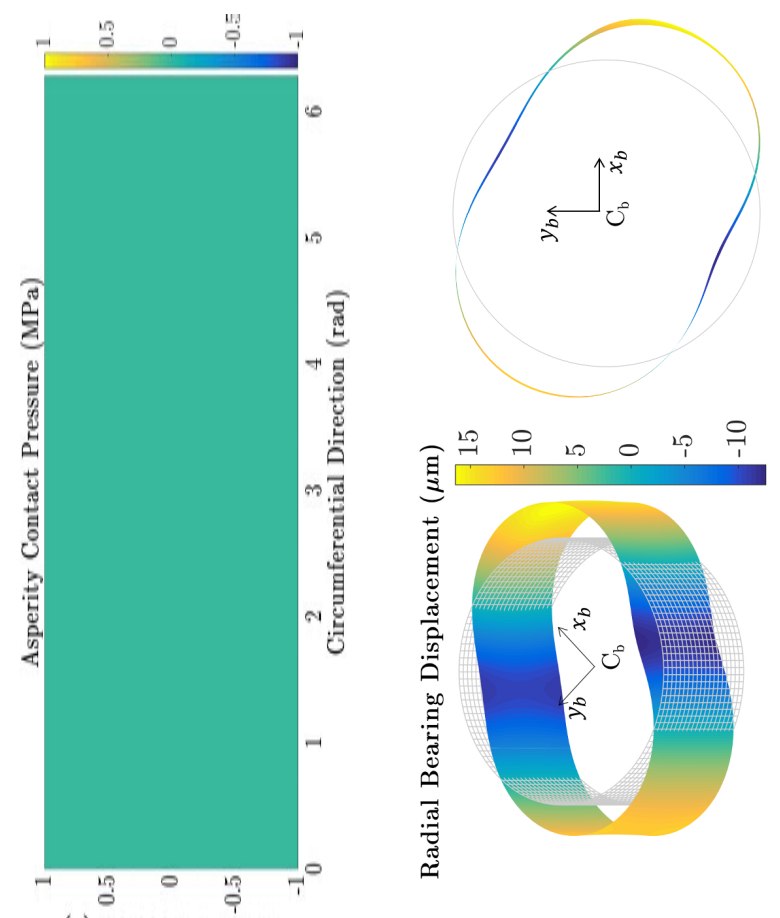

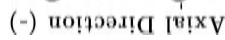

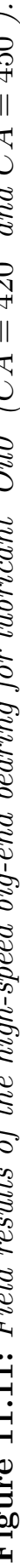




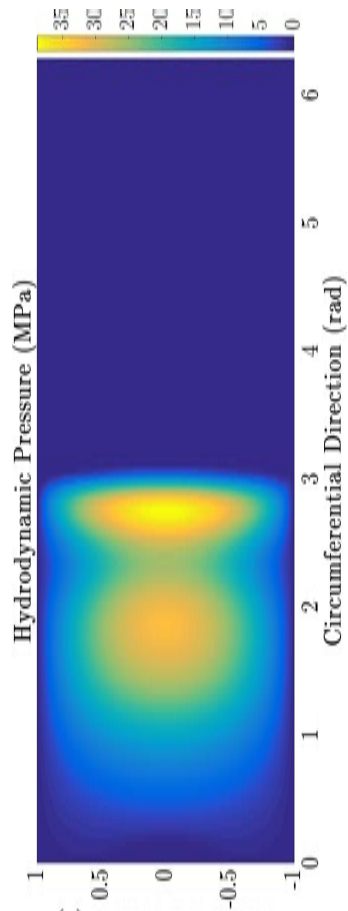

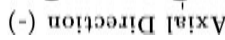

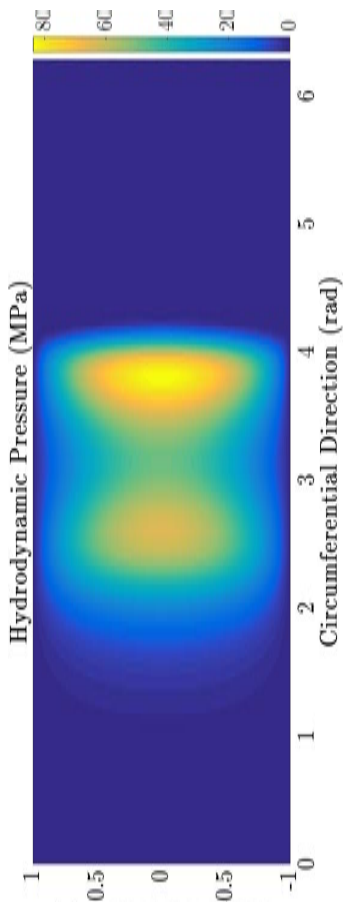

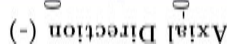

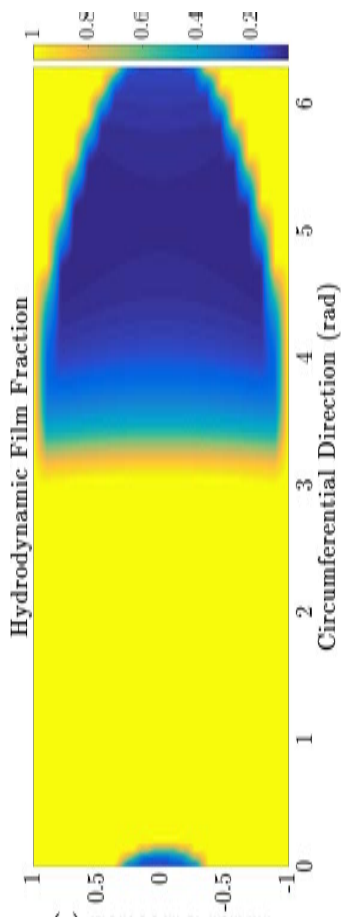

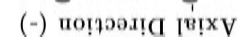

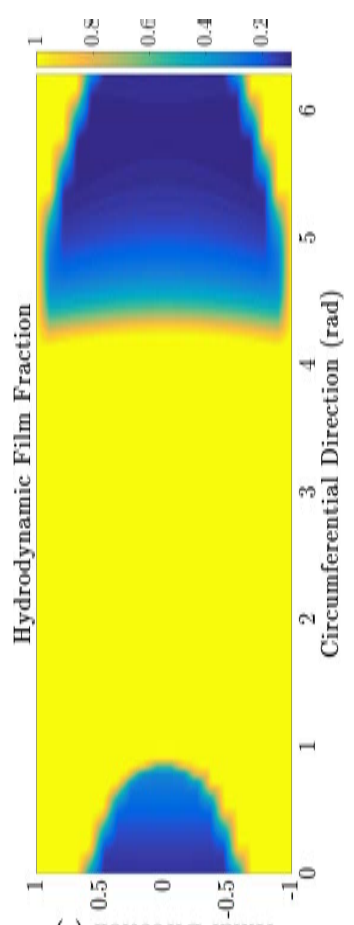

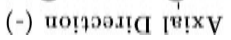

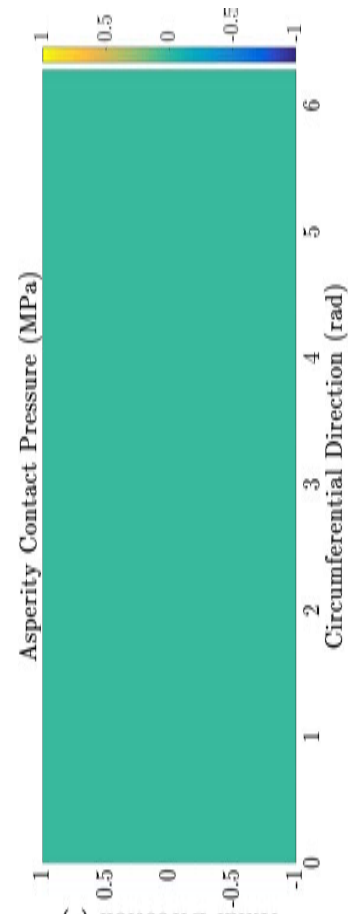

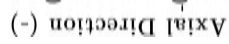

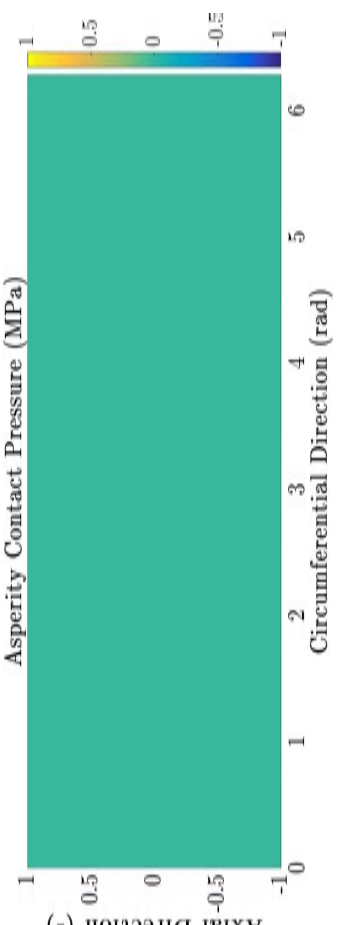

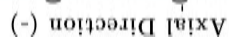

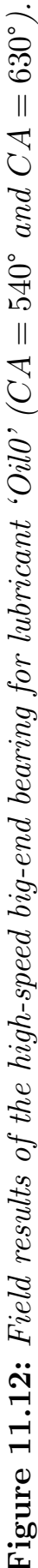
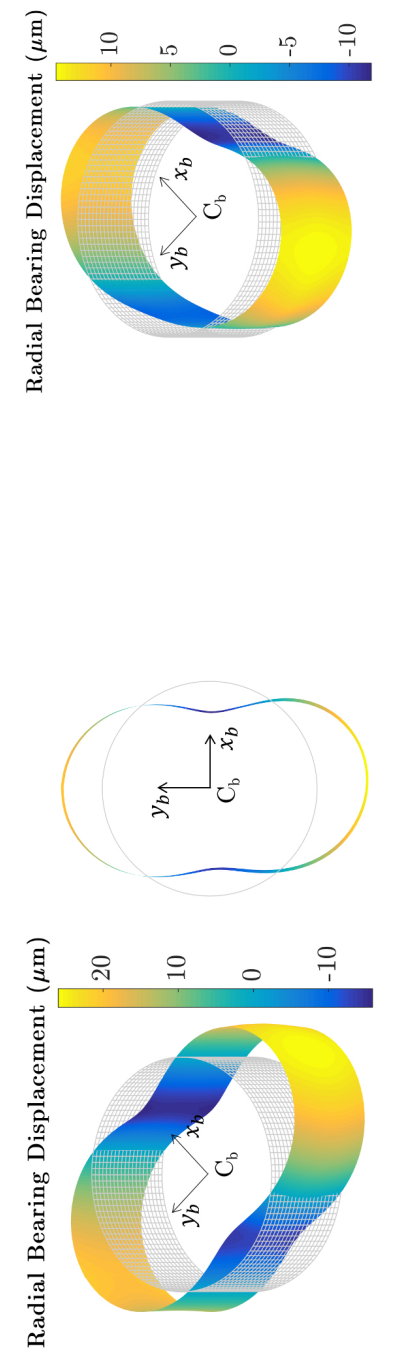

용 


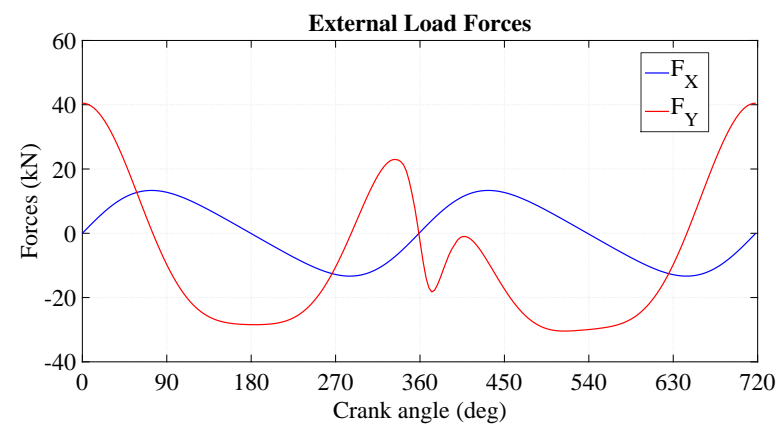

(a) External load forces

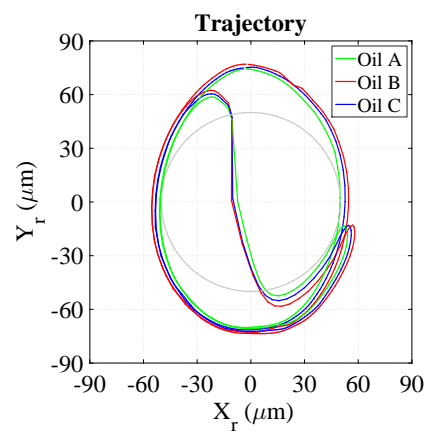

(c) Journal trajectory

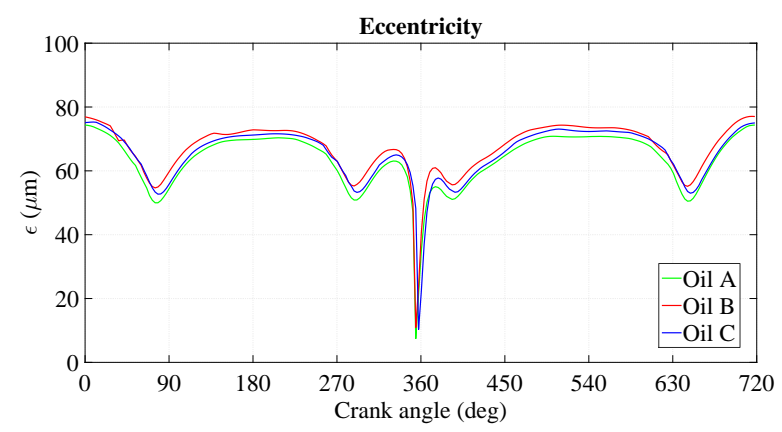

(e) Journal eccentricity

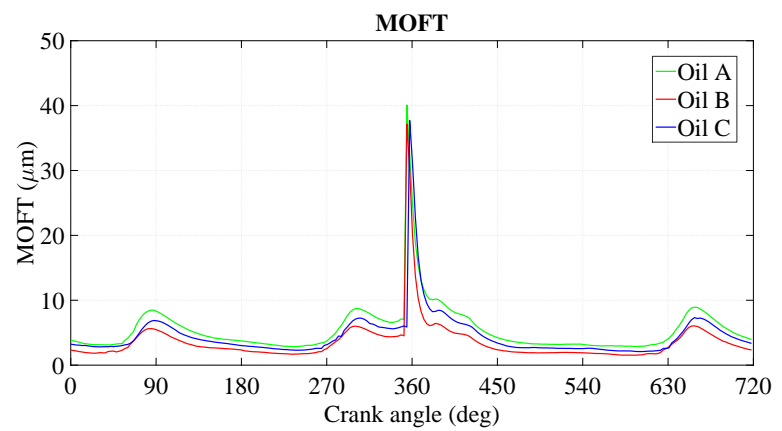

(b) Minimum oil film thickness

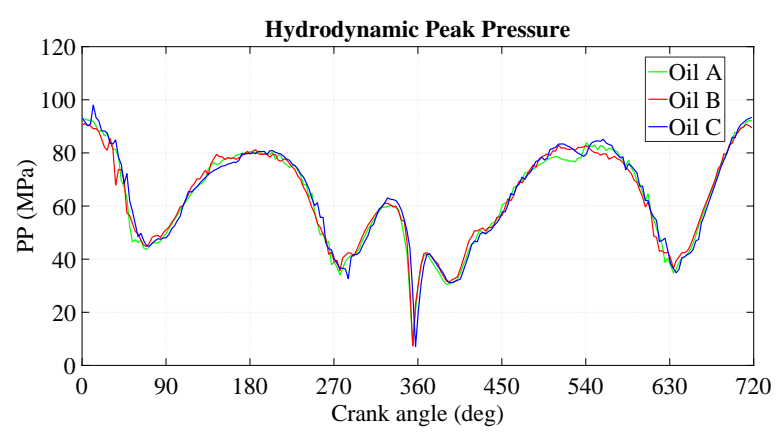

(d) Hydrodynamic peak pressure

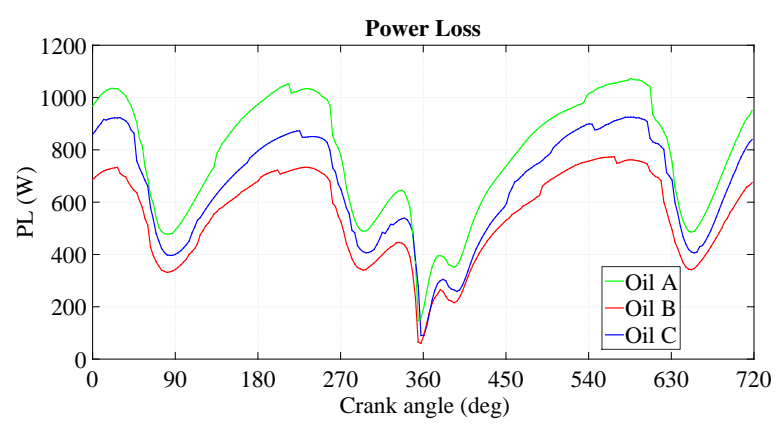

(f) Power loss

Figure 11.13: Scalar results for the dynamically loaded high-speed big-end bearing for the different shear-thinning oils.

\subsection{Simulation results for the shear-thinning oils}

Figure 11.13 outlines the main operational lubrication variables obtained after full EHL simulations for the three shear-thinning oils listed in Table 11.3. Only the flexible bearing case subjected to the same dynamic loading conditions displayed in Fig. 11.2b was considered at this point. As can be seen in Figs. 11.13b-11.13c, the journal movement is hardly affected by the shear-thinning properties of the lubricant. In fact, the journal eccentricity tended to be slightly higher for OilB and progressively lower for OilC and OilA. This is explained by the predominant higher viscosities of OilA in contrast to the reduced values of OilB within the current range of operational shear rate conditions, i.e. $10^{6} \mathrm{~s}^{-1}<\dot{\gamma}<10^{8} \mathrm{~s}^{-1}$ (see Fig. 11.3). Such aspects are also visualized in Fig. 11.13b, where the MOFT is in general subtly superior for OilA and, to the same extent, lower for Oil B. As for the hydrodynamic peak pressures (Fig. 11.13d), no meaningful difference was found among the investigated oils. Regarding the field results, e.g. hydrodynamic pressures, film 
fraction, structural displacements, etc., they were very similar to those calculated for the lubricant Oil0 and illustrated in Figs. 11.6-11.12.

The major influence of the shear-thinning oils on the bearing performance, as expected, was clearly observed for the power losses (Fig. 11.13f). In this case, the less viscous lubricant OilB yielded the lowest overall viscous dissipations, while OilC and OilA successively produced higher frictional losses. Such results indicate, at least from the point of view of conrod bearing applications, the potential benefits of using lubricants with similar shear-thinning properties of OilB for the lubrication of engine components.

\subsection{Performance of the Partitioned Algorithms}

The performance of the coupling algorithms for all the simulation cases undertaken for the present connecting-rod big-end bearing is summarized in Table 11.6. All simulations were carried out in a computer with processor Intel Core i7-3630 CPU 2.40 GHz and 8 GB memory. As can be seen, the PGMA method has shown the best performance, both in terms of the average number of EHL iterations and the computational time for convergence. No convergence has been obtained with the PGMF method within the maximum number of iteration assumed for the problem solution. The values listed in Table 11.6 are the average results per crank angle calculated from their respective values of each time step of the full engine cycle. Notice that all the converged scalar and field results were identical regardless of the coupling method considered.

Table 11.6: Performance of the partitioned algorithms for the high-speed big-end bearing.

\begin{tabular}{|c|c|c|c|}
\hline & Partitioned Method & EHL Iterations ${ }^{*}$ & Time Elapsed* \\
\hline \multirow{3}{*}{ Oil 0} & PGMF & $\mathrm{N} / \mathrm{C}$ & $\mathrm{N} / \mathrm{C}$ \\
\hline & PGMA & $6(5)$ & $13.98 \mathrm{~s}(10.56 \mathrm{~s})$ \\
\hline & IQN-ILS & $7(6)$ & $20.20 \mathrm{~s}(12.66 \mathrm{~s})$ \\
\hline \multirow{3}{*}{ Oil A } & PGMF & $\mathrm{N} / \mathrm{C}$ & $\mathrm{N} / \mathrm{C}$ \\
\hline & PGMA & $6(4)$ & $14.94 \mathrm{~s}(10.96 \mathrm{~s})$ \\
\hline & IQN-ILS & $7(5)$ & $21.35 \mathrm{~s}(14.26 \mathrm{~s})$ \\
\hline \multirow{3}{*}{ Oil B } & PGMF & $\mathrm{N} / \mathrm{C}$ & $\mathrm{N} / \mathrm{C}$ \\
\hline & PGMA & $6(5)$ & $18.45 \mathrm{~s}(13.33 \mathrm{~s})$ \\
\hline & IQN-ILS & $8(6)$ & $24.05 \mathrm{~s}(15.82 \mathrm{~s})$ \\
\hline \multirow{3}{*}{ Oil C } & PGMF & $\mathrm{N} / \mathrm{C}$ & $\mathrm{N} / \mathrm{C}$ \\
\hline & PGMA & $6(5)$ & $15.92 \mathrm{~s}(11.87 \mathrm{~s})$ \\
\hline & IQN-ILS & $8(6)$ & $25.14 \mathrm{~s}(16.89 \mathrm{~s})$ \\
\hline
\end{tabular}

${ }^{*}$ Values between brackets correspond to the statistical medians

$\mathrm{N} / \mathrm{C}$ : not converged 


\section{Chapter 12}

\section{Case III: Effect of Cylinder Liner Wear on the Mixed Lubrication Regime of TLOCRs}

The main purpose of the present application case is the evaluation of the influence of cylinder liner wear on the performance of Twin Land Oil Control Rings (TLOCRs) contacts of internal combustion engines (ICEs). The Oil Control Ring (OCR) is a major contributor to ICE friction losses [138, 204], thus their decrease can directly cause significant reduction of both fuel consumption and $\mathrm{CO}_{2}$ emissions. This investigation has been undertaken by using the full-scale deterministic mixed lubrication modelling described in Section 4.2. In this case, the contact between the OCRs and the cylinder liner topography is assumed parallel and flat, so that the only source of hydrodynamic load carrying capacity is yielded by the lubricant flow through the geometric irregularities of the surface roughness (see Fig. 12.1). Actual 3D roughness measurements taken at the same point of a honed cylinder liner surface before and after a 100h dynamometer engine test are used for the investigation. The ring face is also admitted unchanged since the present evaluations are focused on the bore surface modifications. The results presented in this case study have been published by the author in references $[175,176]$.

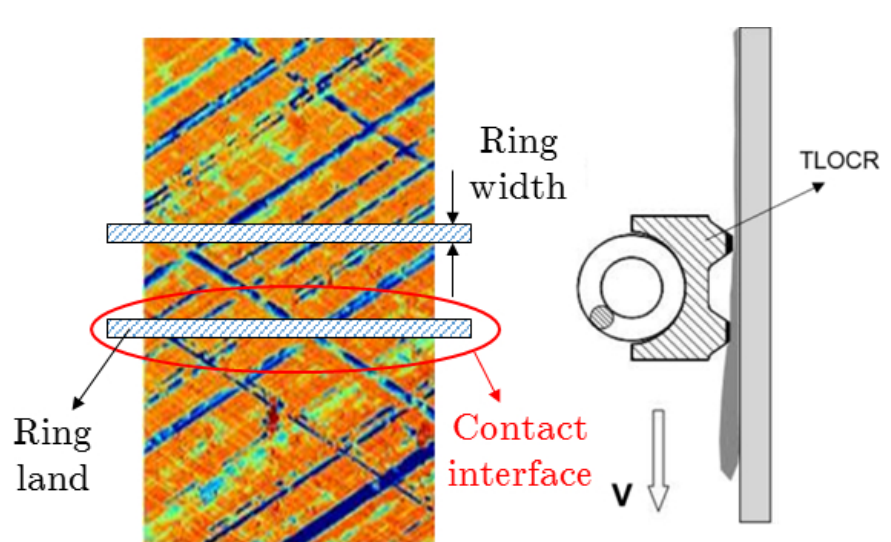

(a) Schematic view showing the contact interface under study.

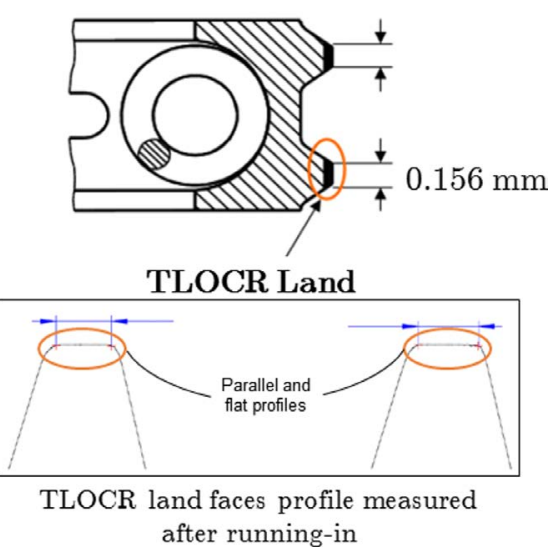

(b) Detailed TLOCR land faces with special attention to the parallel and flat measured profiles after running-in period.

Figure 12.1: Overall illustration of the TLOCR system. Adapted from [51] 


\subsection{Characterization of the Cylinder Liner Topo- graphies}

The cylinder liner topographies of a 12-litre heavy-duty diesel (HDD) engine have been analysed before and after a 100h dynamometer engine test. The engine has a Peak Combustion Pressure (PCP) of 220 bar and uses state-of-the-art piston and piston-ring pack. Top ring and OCR are CrN PVD coated. The engine test consisted initially in a $20 \mathrm{~h}$ break-in period, followed by $10 \mathrm{~h}$ at engine rated power and $70 \mathrm{~h}$ cycle at full load and different engine speeds. The relatively short test duration was chosen in order to preserve most of the original honing. After 100h, only few regions close to the Top Dead Centre (TDC) showed full wear in the initial honing grooves.

The liner topographies were measured in a BMT White Light Interferometer (WLI) at 3 axial and 4 circumferential (angular) positions. A careful fixture reference was used to assure measurements in the same regions before and after the engine test. The set resolution was $1392 \times 1024$ pixels with a grid spacing of $0.892 \mu \mathrm{m}$ in both Cartesian directions, corresponding to an assessed region of $1560 \times 1148 \mu \mathrm{m}$.
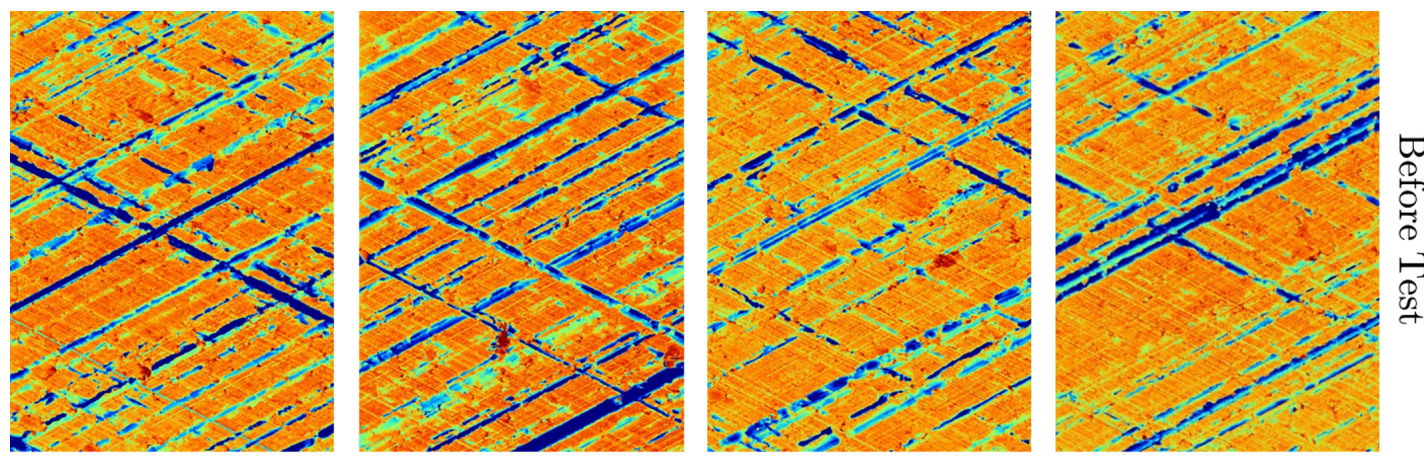

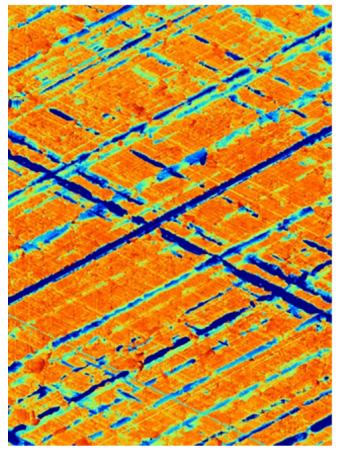

$\operatorname{ATS}\left(0^{\circ}\right)$

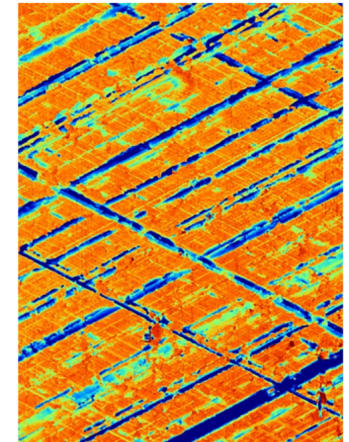

Front $\left(90^{\circ}\right)$

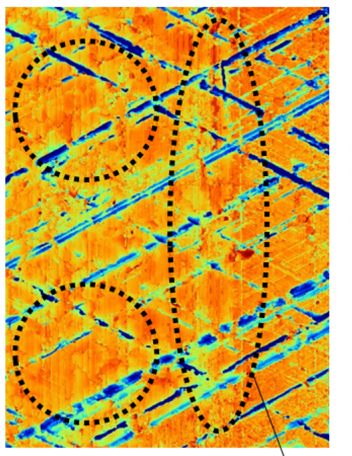

$\operatorname{TS}\left(180^{\circ}\right)$

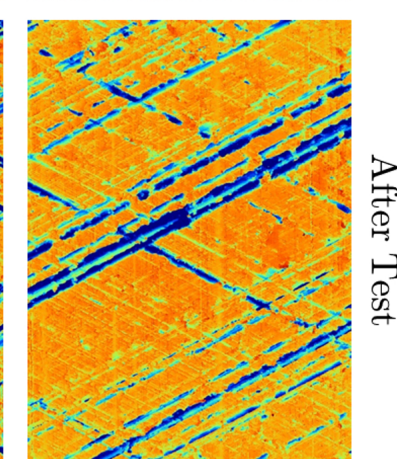

Rear $\left(270^{\circ}\right)$

Axial wear scratches

Figure 12.2: Topography view at $109 \mathrm{~mm}$ from the TDC (approximately in the mid-stroke position).

In order to evaluate in detail the influence of the cylinder liner wear on the mixedlubrication regime of TLOCR contacts, special attention is devoted only to the WLI measurements taken at the mid-Stroke (MS) position, particularly at the Front and Thrust-Side (TS) regions. These surfaces are hereafter designated Front-NEW and Front-WEAR, and analogously TS-NEW and TS-WEAR, where the terms 'NEW' and 'WEAR' denote before (as new) and after the engine test, respectively.

Figure 12.2 shows the roughness topographies measured at $109 \mathrm{~mm}$ from the TDC before and after test; this axial level corresponds approximately to the mid-Stroke (MS) 
position. As observed, the honing is generally well preserved, except for the few scratches visible on the thrust-side (TS) region, hence justifying the use of such portion as the target zone to be analysed by deterministic simulations. Table 12.1 shows the main roughness parameters of the selected topographies for the Front and TS regions, respectively. Notice that the roughness parameters are practically unchanged after the engine test. The Greenwood-Williamson (or contact) parameters calculated using the in-house SUMMITS code [203] are also listed in the table. In this case, only the specimens measured at the TS region showed significant modifications, i.e. the mean asperity height $\left(Z_{S}\right)$ decreased from $0.38 \mu \mathrm{m}$ to $0.32 \mu \mathrm{m}$ and the asperity mean radius $\left(\beta_{S}\right)$ increased from $5.4 \mu \mathrm{m}$ to $11.4 \mu \mathrm{m}$. Such changes are in agreement with the visual inspections highlighted in Fig. 12.2, in which the TS region clearly corresponds to the zone with more severe wear among the evaluated surfaces.

Table 12.1: Roughness and contact parameters for the MS-Front region.

\begin{tabular}{lccccc}
\hline Parameter & $\begin{array}{c}\text { Front } \\
(\text { NEW })\end{array}$ & $\begin{array}{c}\text { Front } \\
(\text { WEAR })\end{array}$ & $\begin{array}{c}\text { TS } \\
(\text { NEW })\end{array}$ & $\begin{array}{c}\text { TS } \\
(\text { WEAR })\end{array}$ & Unit \\
\hline$S p q$ & 0.39 & 0.39 & 0.38 & 0.38 & $\mu \mathrm{m}$ \\
$S s k$ & -2.5 & -2.2 & -3.0 & -3.2 & - \\
$S k u$ & 13.4 & 12.0 & 19.2 & 19.3 & - \\
$S t d$ & 45.1 & 44.8 & 45.3 & 45.1 & $\mathrm{deg}$ \\
\hline$S p k$ & 0.31 & 0.34 & 0.30 & 0.29 & $\mu \mathrm{m}$ \\
$S k$ & 0.71 & 0.64 & 0.61 & 0.57 & $\mu \mathrm{m}$ \\
$S v k$ & 1.90 & 2.01 & 1.70 & 1.60 & $\mu \mathrm{m}$ \\
$S m r 1$ & 6.4 & 5.8 & 8.2 & 7.9 & $\%$ \\
$S m r 2$ & 70.5 & 68.8 & 75.8 & 76.4 & $\%$ \\
\hline$Z_{S}$ & 0.44 & 0.43 & 0.38 & 0.32 & $\mu \mathrm{m}$ \\
$\sigma_{S}$ & 0.26 & 0.29 & 0.23 & 0.23 & $\mu \mathrm{m}$ \\
$\beta_{S}$ & 5.9 & 6.8 & 5.4 & 11.4 & $\mu \mathrm{m}$ \\
$\eta_{S}$ & $5.0 \times 10^{10}$ & $5.0 \times 10^{10}$ & $5.5 \times 10^{10}$ & $4.9 \times 10^{10}$ & $\mathrm{~m}^{-2}$ \\
\hline
\end{tabular}

\subsection{Deterministic Simulations}

In order to carry out the deterministic simulations for the cylinder liner topographies described in Section 12.1, the original measured surfaces were split into smaller narrow slices, each one having $156 \mu \mathrm{m}$ width that corresponds exactly to the dimension of the TLOCR land (see Fig. 12.1). A fixed mesh of $551 \times 59$ grid points was chosen for the discretization of the numerical solutions. In this case, linear interpolation was used for resizing the original topographies to match the number of discrete points established for the numerical mesh. According to this procedure, each measured surface provided 8 slices, as illustrated in Fig. 12.3. Moreover, the TLOCR land was assumed rigid and smooth for all simulation cases, as adopted in [51, 52, 200]. Notice that any micro-EHL effect and rheological variations in the roughness scale were considered in the analyses.

Accordingly, a set of deterministic simulations was performed for every surface slice and for the same fixed values of dimensionless film thickness $(\Lambda)$ raging from 0.05 to 7.00. As for the boundary conditions, the periodic flow condition was imposed on the 


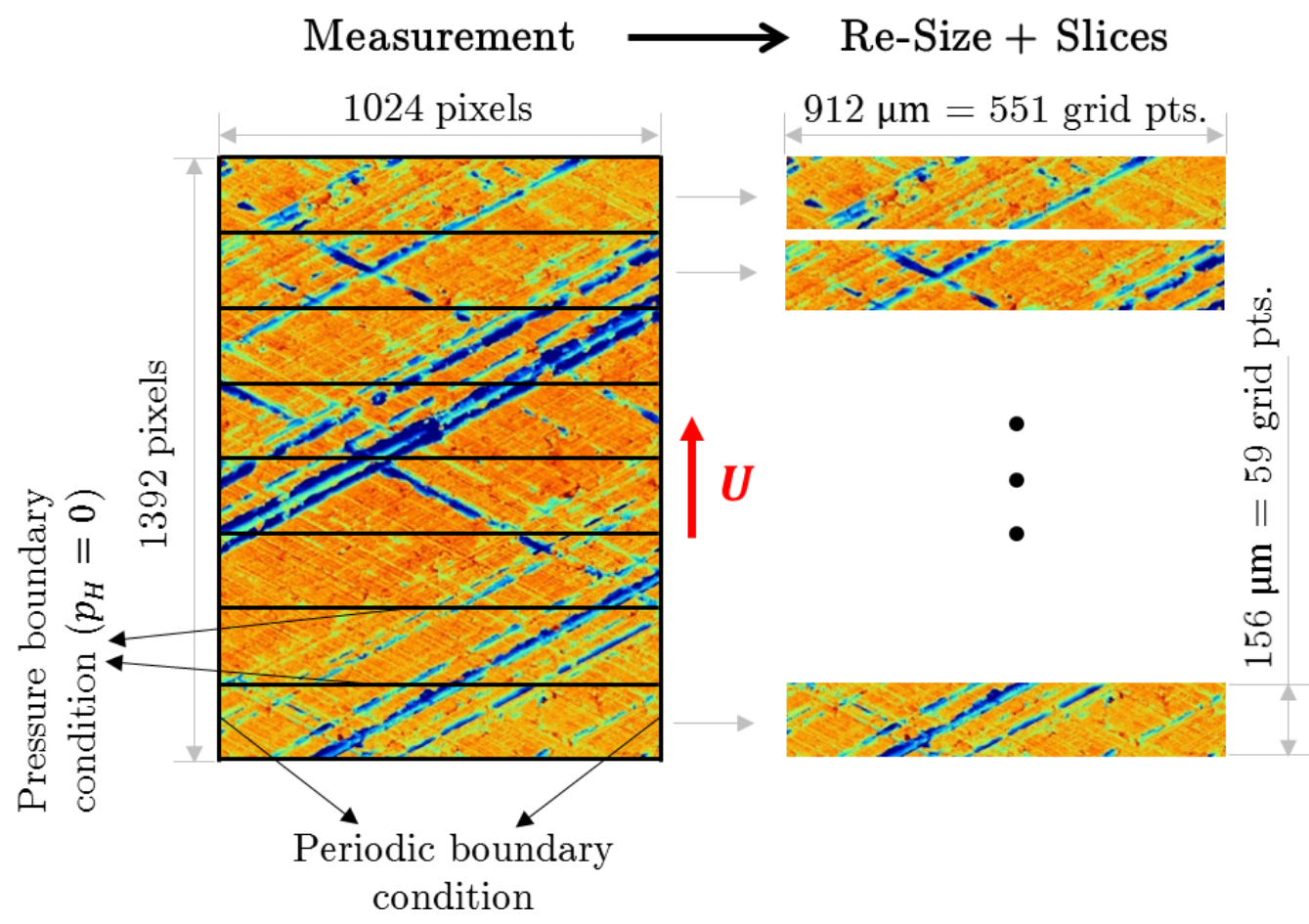

Figure 12.3: Splitting procedure adopted for the generation of the surface slices used in the deterministic simulations.

circumferential bounds of the domain in order to account for the ring periodicity in this axis. Similarly, on the top and bottom boundaries, null relative pressure (equivalent to the absolute atmospheric pressure) was set and no special consideration has been taken into account regarding the amount of lubricant on the contact, i.e. the contact interface is assumed fully-flooded. Furthermore, the following reference values of $U_{0}=3 \mathrm{~m} / \mathrm{s}$ and $\eta_{0}=0.01 \mathrm{Pas}$ were considered for the sliding velocity and lubricant dynamic viscosity, respectively. Such reference quantities were also employed in the deterministic TLOCR model of [51], which further provides correlation formulas to calculate the actual hydrodynamic pressures and shear stresses acting on the TLOCR under different sliding velocities and lubricant viscosities. Such scaling methodology allows for an efficient way of using deterministic solutions in full-cycle engine simulations.

As already stated, although the region of greater concern for wear in cylinder liners is close to the TDC, the present investigation focuses only on the surface changes and their impact on the mixed-lubrication behaviour near the liner MS position. At this position, the piston speed is maximum, so that the larger friction power losses have a significant impact on fuel consumption. Fig. 12.4 depicts an additional view of the TS angular position that highlights the wear scratches and contact area. The red spots zones show roughness heights greater than $0.5 \mu \mathrm{m}$, whereas in the blue regions the heights are located below the surface reference plane. In the same figure, the proportion of real contact area $\left(A_{c} / A_{0}\right)$ is $0.63 \%$ and the void volume derived by the nominal area, i.e. volume ratio of oil on the grooves $\left(V_{\text {oil }} / A_{0}\right)$, is $0.25 \mu \mathrm{m}$.

\subsection{Global (Average) Results}

Figure 12.5 depicts the average hydrodynamic pressures and shear stresses, as well as the average asperity contact pressures calculated for the surface slices measured at the 


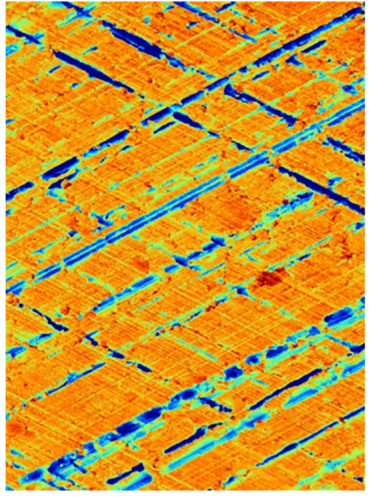

Before test (as new)
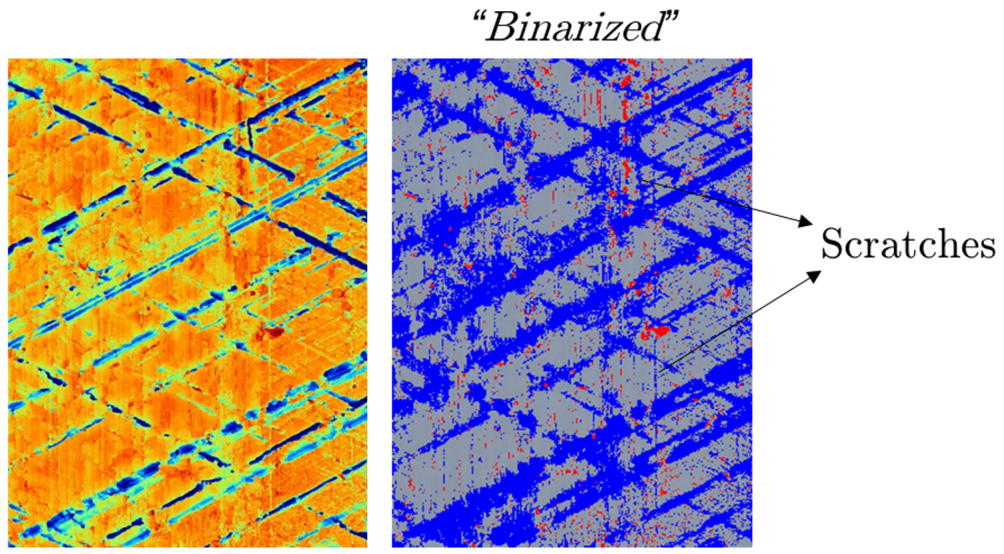

After test

Figure 12.4: Detailed topography view at mid-stroke, thrust-side (TS) position.

front position of the cylinder liner before and after the engine test. The dots represent the average results for each slice, while the curves correspond to the global mean results plotted against the dimensionless separation $(\Lambda)$. As can be observed, the overall changes in the average results were relatively small, which may be explained by the milder superficial wear detected at the liner front position (see Fig. 12.2) that was not significant enough to cause meaningful perturbations on the hydrodynamic and asperity contact effects.

Similarly, Fig. 12.6 illustrates the same set of results for the surface slices measured at the TS position of the cylinder liner before and after the engine test. As noticed, the average hydrodynamic behaviour considerably changed for the after-test (worn) topography, especially in the range of $0.05<\Lambda<3$ which is clearly associated with mixed-lubrication conditions. In particular, the most significant modification was the sharp rise in the hydrodynamic carrying capacity. As for the asperity contact pressures, the magnitude of the values was reduced, essentially due to the wear of the highest asperities.

\subsection{Local Results}

In order to further evaluate the possible reasons behind the significant increase in the hydrodynamic carrying capacity shown in the previous section for the worn topographies, Fig. 12.7 depicts the (local) field results obtained from the deterministic simulations for the TS position surfaces before and after the engine test. Since all the combination pairs of slice/separations presented similar behaviours, only the field results of slice 7 for $\Lambda=1.25$ are considered in the following discussions.

In terms of roughness heights, the overall surface wear can be visualized by comparing the intensity of the colour scale. Additionally, the wear scratches are also clearly observed, especially in the middle-top region of the slice displayed in Fig. 12.7b. Regarding the local-scale lubricant flow effects, the worn surface exhibited a wider region subjected to hydrodynamic pressures ("wetted-regions") and with predominantly higher magnitude values. In this fashion, the average hydrodynamic pressures computed for each case confirm such visual evaluation, i.e. $P_{H}=0.365 \mathrm{MPa}$ for the surface before test and $P_{H}=0.701 \mathrm{MPa}$ for the surface after test. This raise in the fluid pressure may be explained by the reduction in the cavitation area, as can be observed in the dashed circled regions in the figures. For instance, for the original topography (before test) in Fig. 12.7a, approximately $64 \%$ of the lubricated domain is subjected to film rupture (cavitation) with 
no generation of hydrodynamic support. On the other hand, for the after-test surface in Fig. $12.7 \mathrm{~b}$, such proportion is diminished to $49 \%$, which in turn practically doubled the average hydrodynamic pressure.

Physically, the smoothing of the plateau regions caused by the wear-out of the highest asperities, together with the general decrease in the summits curvature, both contributed to facilitate the pressure-driven lubricant flow throughout the inter-grooves zones, hence intensifying the grooves' role in the generation of hydrodynamic pressures, as observed by [37]. Analogously, the widening of regions with pressure-driven flow may be viewed as an inducer of the "building-up" pressure effect verified as the main responsible for providing significant hydrodynamic support to lubrication systems with partial texturing surface $[42,204]$. Finally, the number of summits in contact was reduced for the worn topography due to the same aforementioned removal of the highest roughness protuberances. 
Front Position

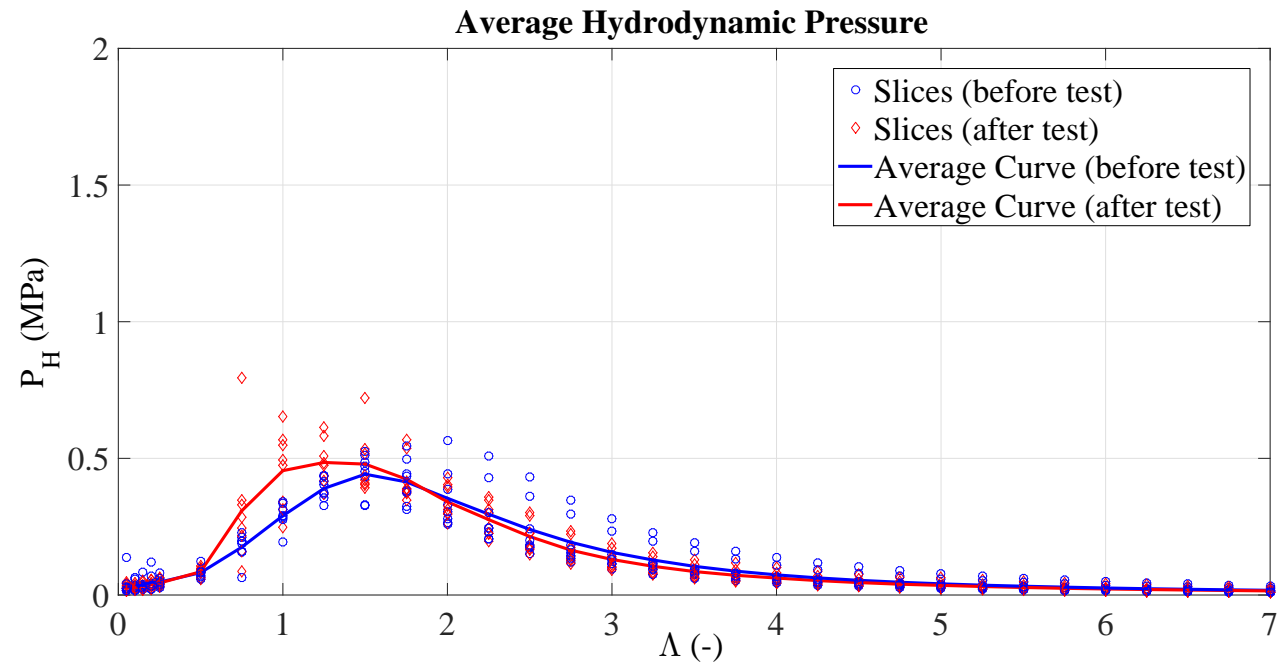

(a) Average Hydrodynamic Pressure

Front Position

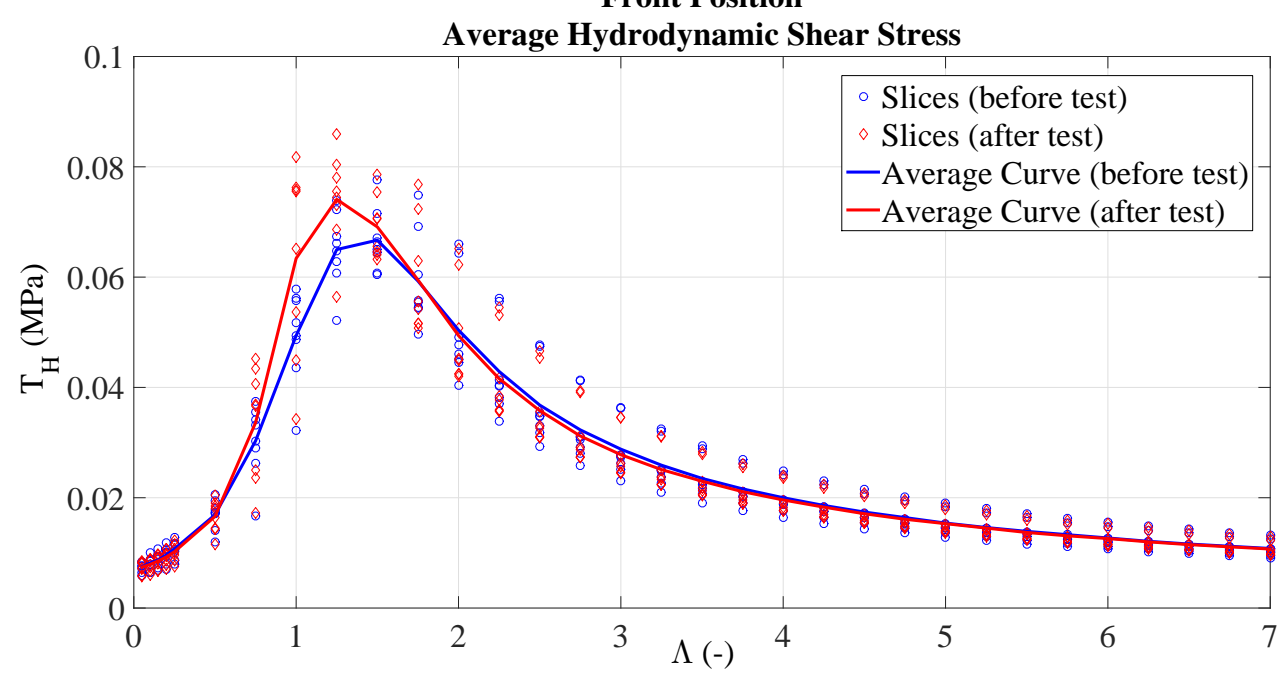

(b) Average Hydrodynamic Shear Stress

Front Position

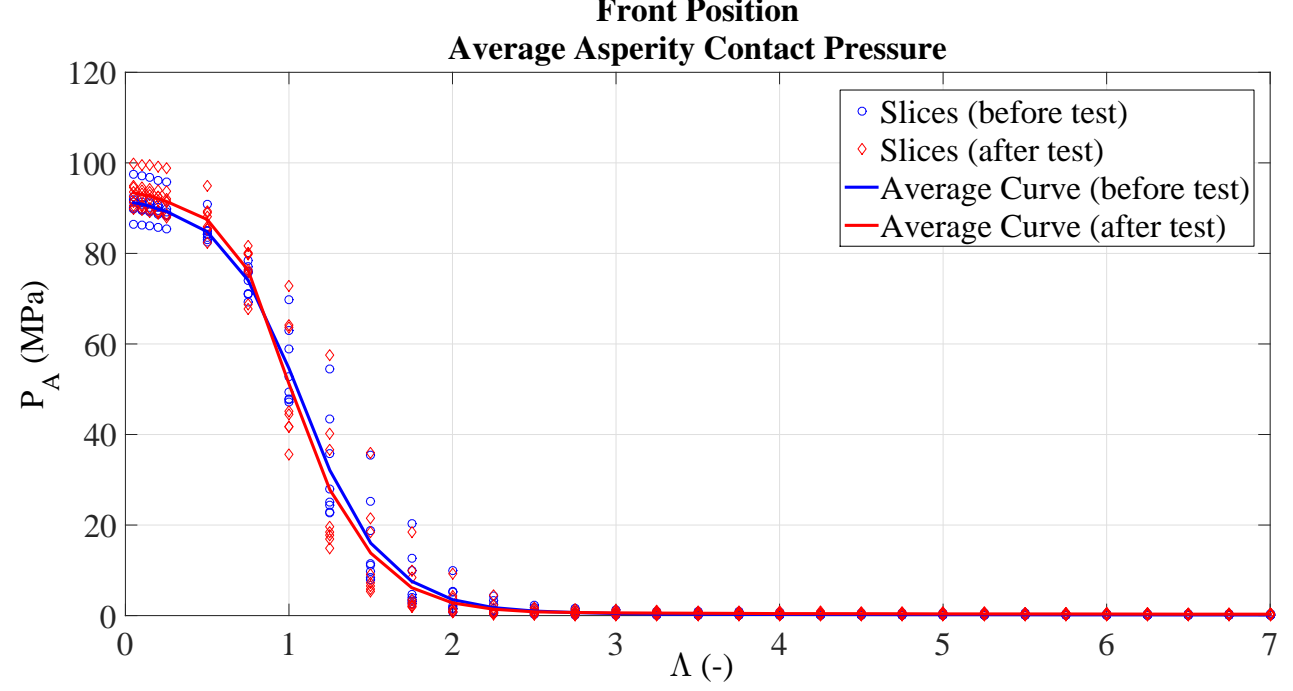

(c) Average Asperity Contact Pressure

Figure 12.5: Average results for the topographies measured at the Front position of the cylinder liner. 
Thrust-Side Position (TS)

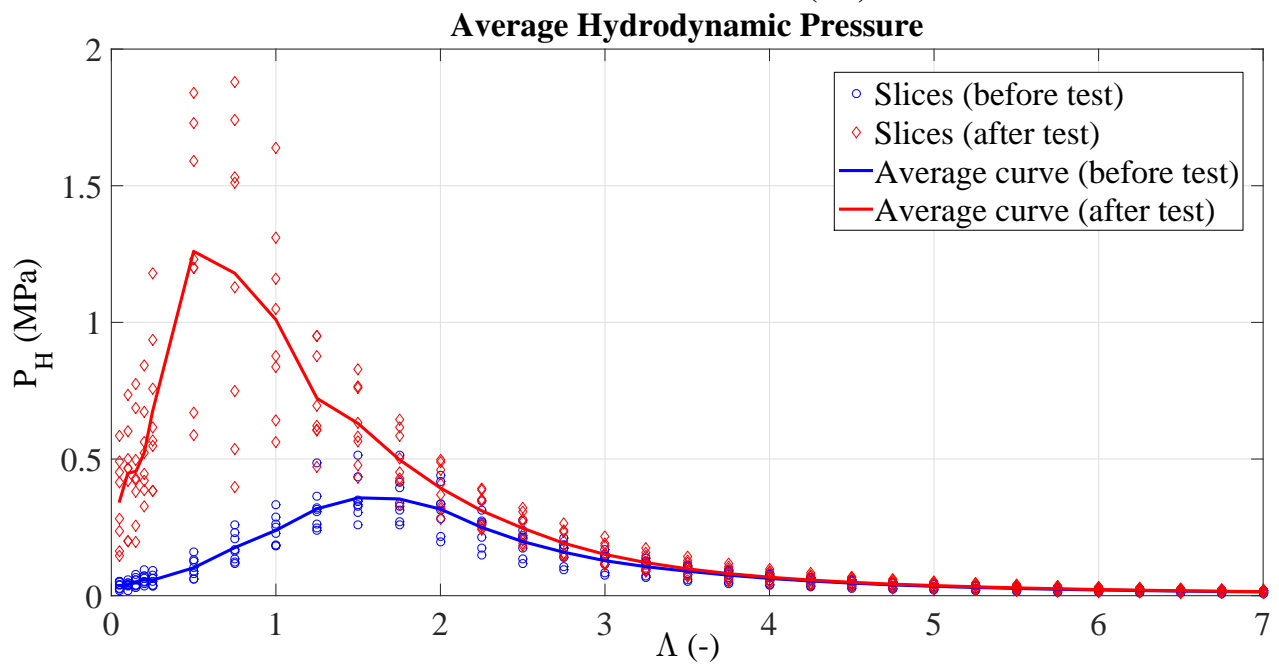

(a) Average Hydrodynamic Pressure

Thrust-Side Position (TS)

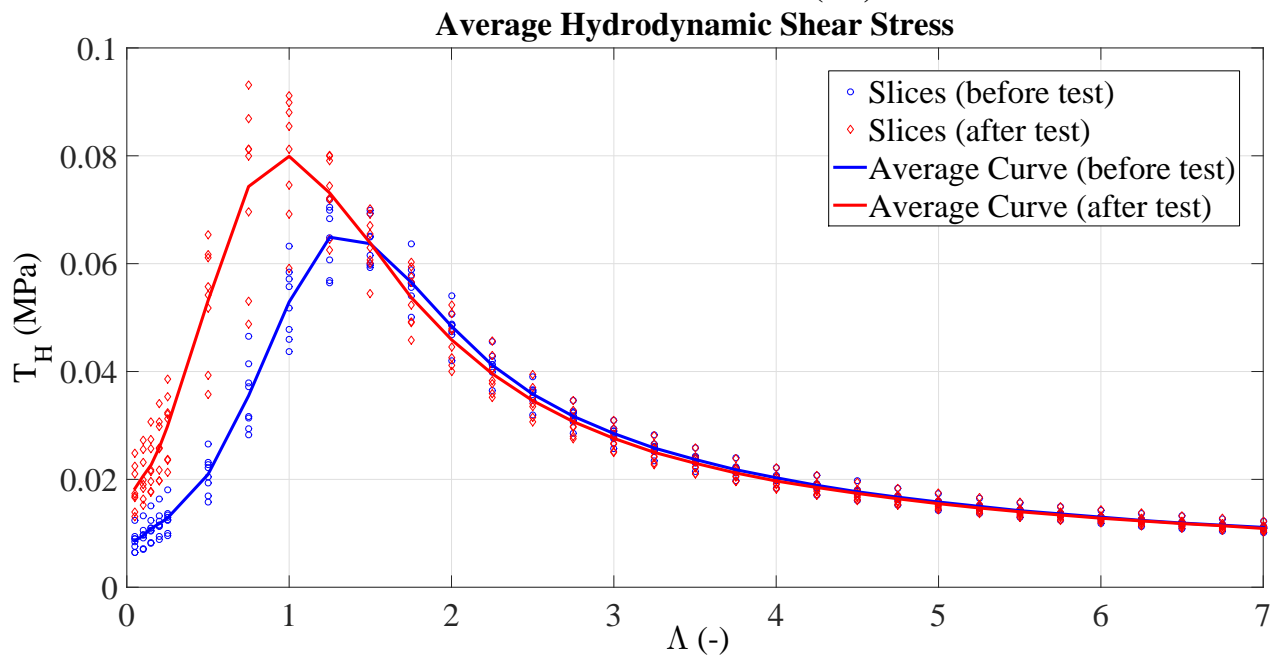

(b) Average Hydrodynamic Shear Stress

Thrust-Side Position (TS)

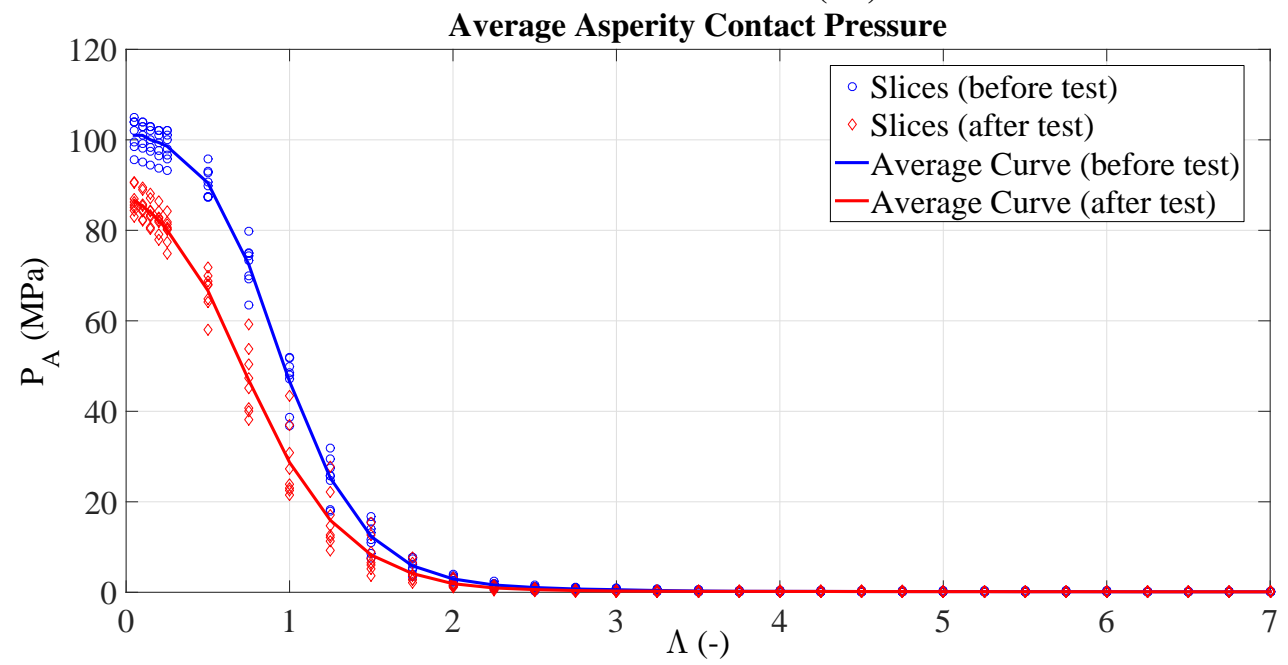

(c) Average Asperity Contact Pressure

Figure 12.6: Average results for the topographies measured at the TS position of the cylinder liner. 

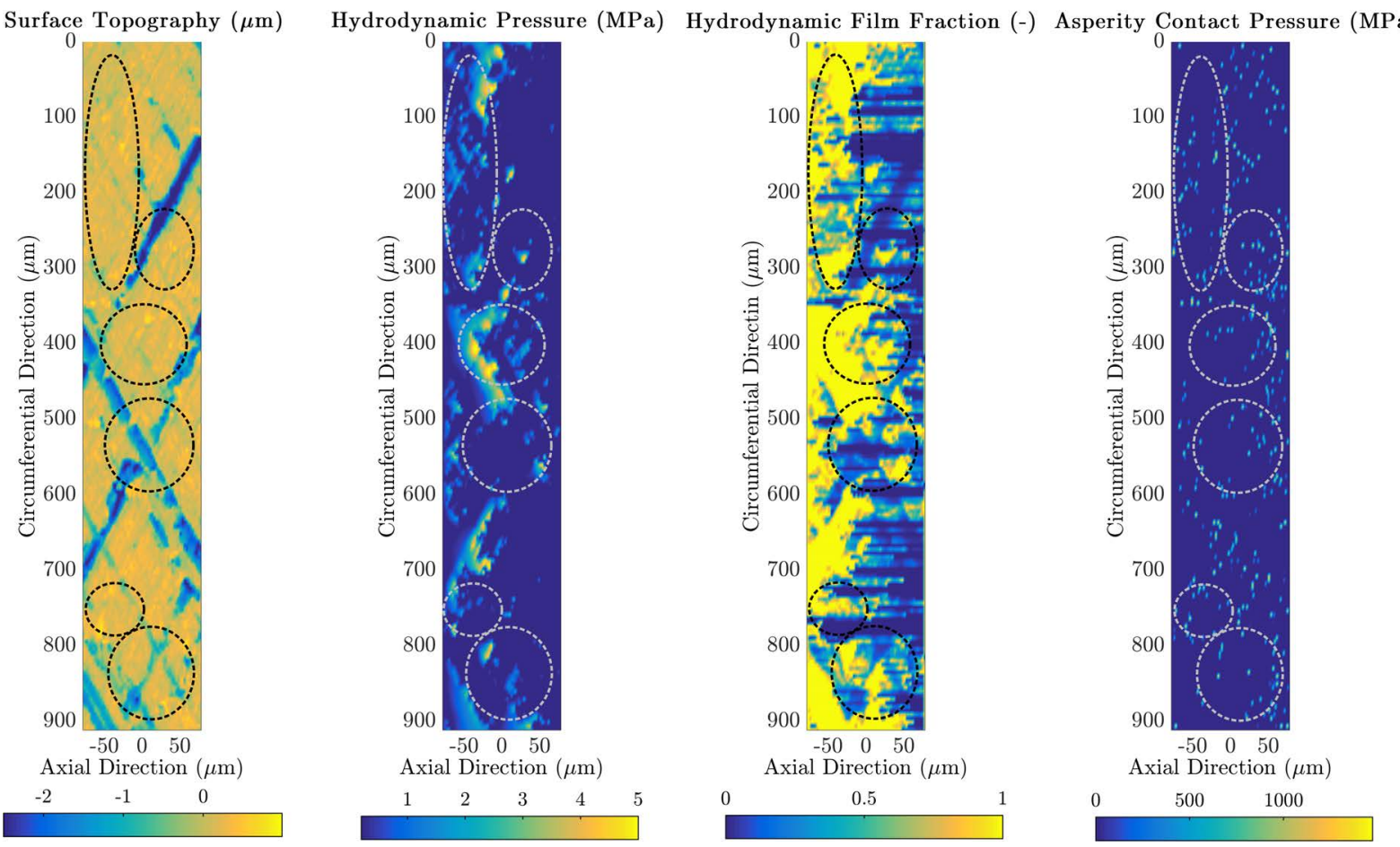

(a) Before engine test.
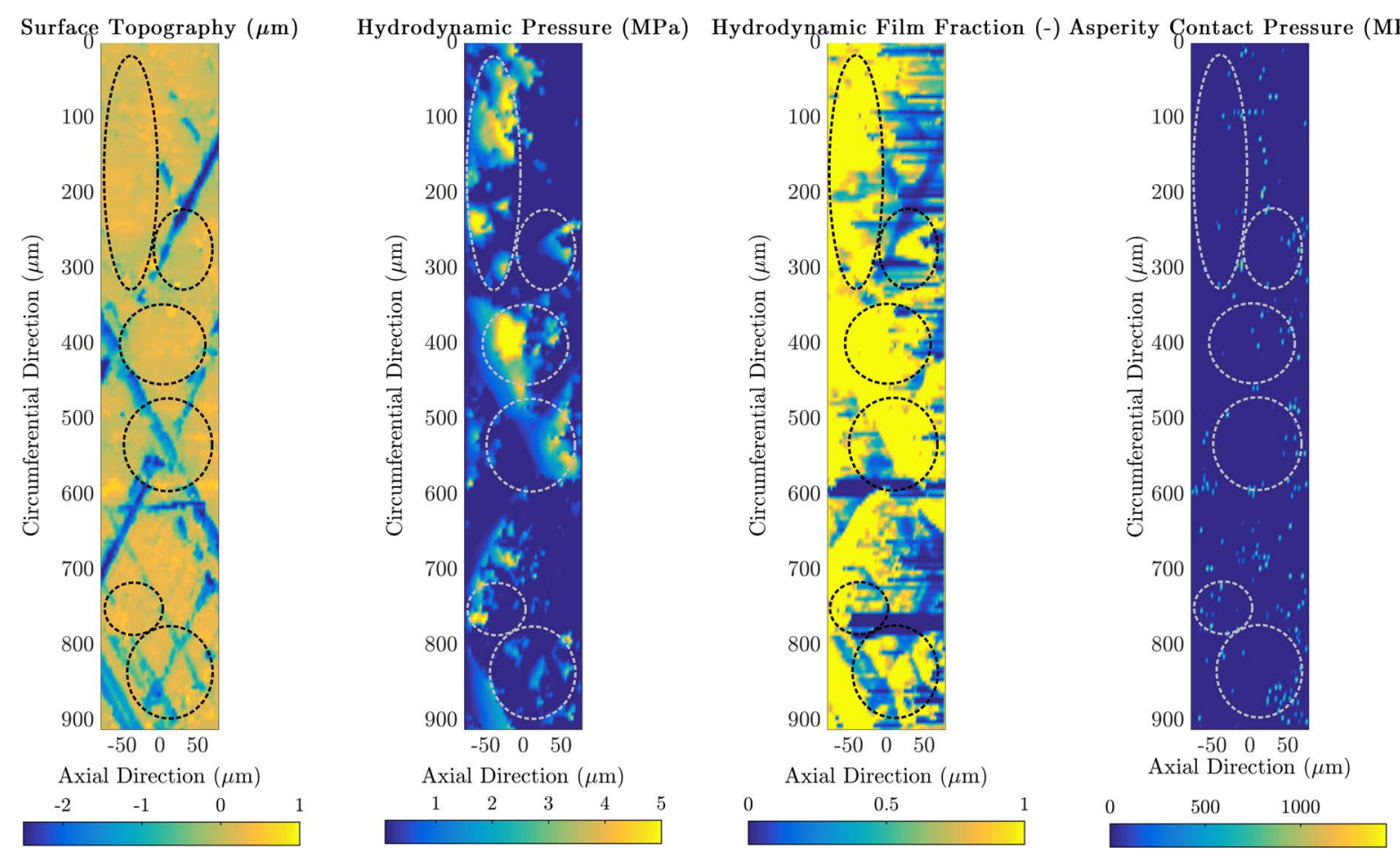

(b) After engine test.

Figure 12.7: Field results for the surface measured at the TS position (slice 7 and $\Lambda=1.25$ ). Dashed circles highlight regions with most significant changes after wear. Sliding velocity from left to right. 


\section{Chapter 13}

\section{Case IV: Textured Sliding Bearing Simulation}

In order to illustrate the robustness, computational efficiency and flexibility of the EbFVM to tackle dense meshes with irregular elements, a set of simulations for a parallel and flat textured sliding bearing have been undertaken for the geometric and operational parameters listed in Table 13.1. Full and partial texturing are considered by varying the number of dimple cells added onto the stationary bearing surface from $10 \times 2$ to $10 \times 10$ cells; the dimples are spherical in shape and cover approximately $20 \%$ of each texture cell (see details of the dimple dimensions in Fig. 13.1). Moreover, the lubricant density and viscosity are assumed constant.
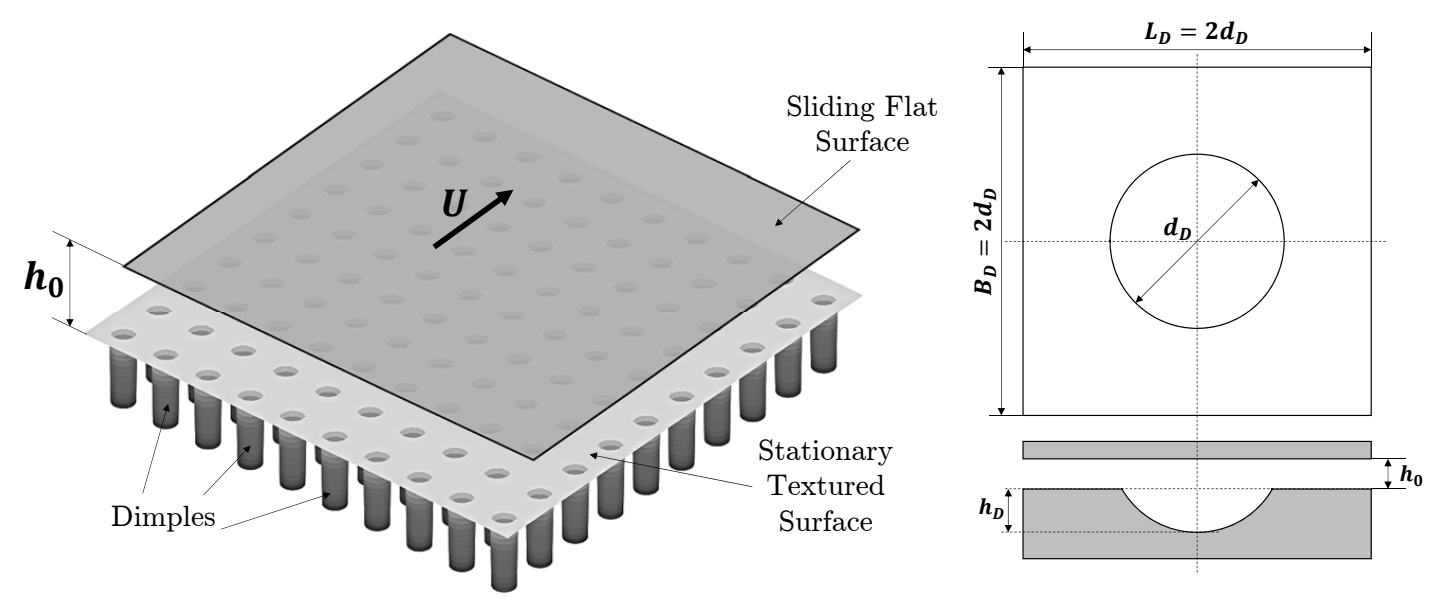

Figure 13.1: Schematic illustration of the textured sliding bearing and the general dimensions of a dimple cell.

The lubricated domains are discretized using both regular and irregular grids; in particular, the use of the latter grid type allows for mesh refinements in the neighbourhoods of the texture features, where the pressure gradients are more pronounced. Such meshing control may well be used to reduce the number of nodes, and hence the computational cost of simulations, needed to discretize the same textured pad when compared to structured grids. Figure 13.2 illustrates both the regular and irregular meshes of 3 representative texture variants with $10 \times 10,10 \times 6$ and $10 \times 2$ dimples cells, as well as the associated hydrodynamic pressure fields calculated; notice the considerable decrease in the number of nodes deployed for the unstructured meshes. 
Table 13.1: Geometric and operational parameters for the textured sliding bearing simulations.

\begin{tabular}{lcc}
\hline Parameter & Value & Unit \\
\hline$a$ (bearing length) & 20 & $\mathrm{~mm}$ \\
$b$ (bearing width) & 20 & $\mathrm{~mm}$ \\
$h_{0}$ & 4.0 & $\mathrm{\mu m}$ \\
$U$ & 10 & $\mathrm{~m} / \mathrm{s}$ \\
$\eta_{0}$ & 10 & $\mathrm{mmPa} \mathrm{s}$ \\
$\rho_{0}$ & 850 & $\mathrm{~kg} / \mathrm{m}^{3}$ \\
$p_{0}$ & 100 & $\mathrm{kPa}$ \\
$p_{c a v}$ & 90 & $\mathrm{kPa}$ \\
$d_{D}$ & 1000 & $\mu \mathrm{m}$ \\
$h_{D}$ & 10 & $\mu \mathrm{m}$ \\
\hline
\end{tabular}
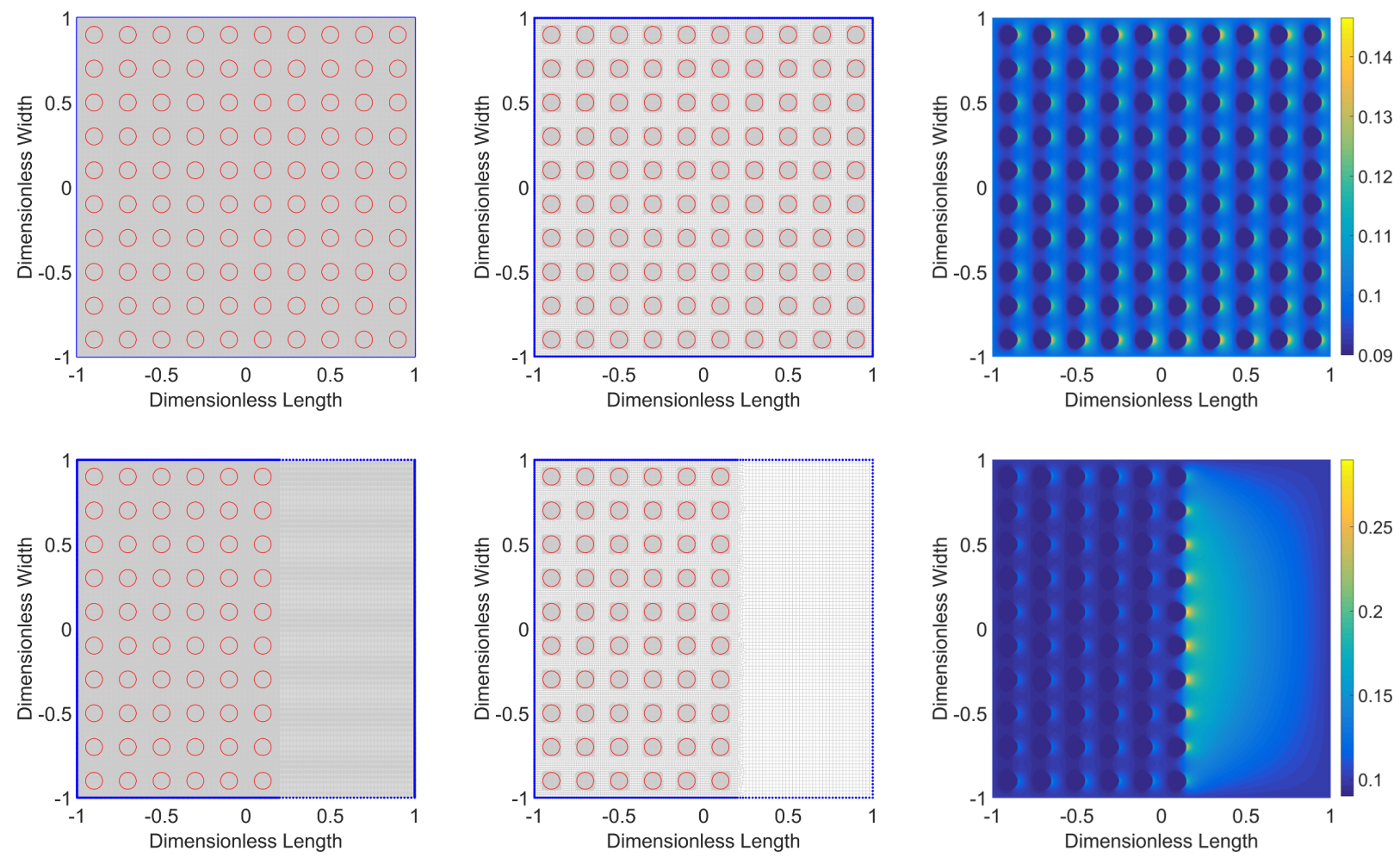

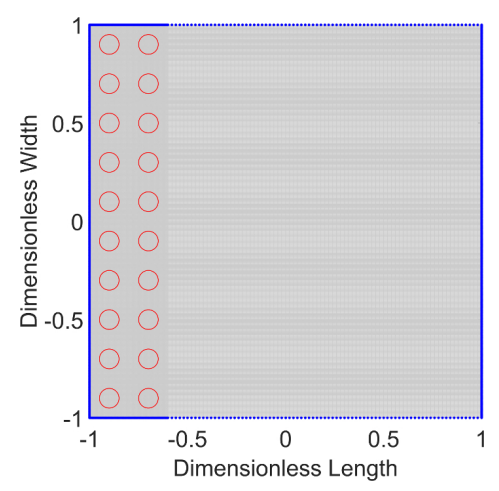

(g) Regular Meshes

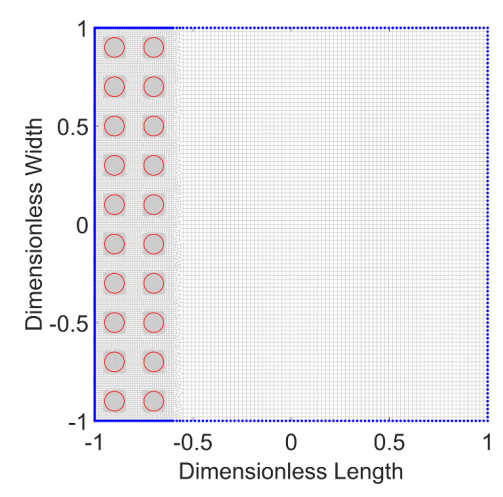

(h) Irregular Meshes

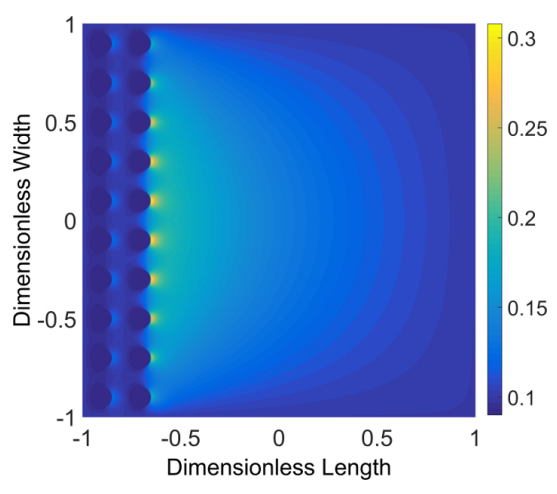

(i) Hydrodynamic Pressures (MPa)

Figure 13.2: Representative texture variants for the sliding bearing simulations and the associated hydrodynamic pressure results. Sliding velocity from left to right. 
The number of outer iterations of the extended SOR algorithm (see Appendix 8), the computational time for convergence and the hydrodynamic load capacity are chosen to compare the results calculated on the regular and irregular meshes. The optimum relaxation factors of the SOR method for pressure $\left(\omega_{p}\right)$ have been found empirically by numerical experimentation. The optimum values for each texture pattern and mesh topology are displayed in Tables 13.2 and 13.3; the relaxation value for the lubricant film fraction (cavitation) is assumed fixed as $\omega_{\theta}=1$. All the simulations were carried out in MATLAB ${ }^{\circledR}$ in a computer with 8 GB RAM and Intel Core i7-3630 CPU $2.40 \mathrm{GHz}$; the algorithm reported in Appendix 8 is implemented as a MATLAB MEX-File that calls a compiled $\mathrm{C}$ function for performance improvement.

Table 13.2: Performance of the EbFVM for the regular meshes

\begin{tabular}{cccccc}
\hline Dimples & DOFs & $\boldsymbol{\omega}_{\boldsymbol{p}}$ & Iterations & Time $(\mathbf{s})$ & $\boldsymbol{W}(\mathbf{N})$ \\
\hline 10x10 & 368,449 & 1.98 & 906 & 11.27 & 38.91 \\
10x8 & 307,142 & 1.98 & 990 & 10.23 & 41.57 \\
10x6 & 245,835 & 1.98 & 1268 & 9.87 & 44.84 \\
10x4 & 183,921 & 1.98 & 1534 & 8.71 & 47.06 \\
10x2 & 122,614 & 1.98 & 1638 & 6.48 & 48.24 \\
\hline
\end{tabular}

Table 13.3: Performance of the EbFVM for the irregular meshes

\begin{tabular}{cccccc}
\hline Dimples & DOFs & $\boldsymbol{\omega}_{\boldsymbol{p}}$ & Iterations & Time $(\mathbf{s})$ & $\boldsymbol{W}(\mathbf{N})$ \\
\hline 10x10 & 140,935 & 1.86 & 954 & 4.58 & 38.91 \\
10x8 & 115,061 & 1.88 & 1572 & 6.18 & 41.6 \\
10x6 & 88,982 & 1.91 & 1867 & 5.74 & 44.91 \\
10x4 & 62,794 & 1.85 & 2579 & 5.05 & 47.15 \\
10x2 & 36,607 & 1.91 & 2092 & 2.14 & 48.35 \\
\hline
\end{tabular}

According to Tables 13.2 and 13.3, the variation in the mesh topology hardly changed the overall load carrying capacity $(W)$ for all texture cases examined, but led to a significant speed-up in the simulation time, yielded essentially by the decrease in the number of degrees of freedom (DOFs) for the irregular grids. Such occurrence demonstrates the enhancements that can be achieved by using the current finite volume method; this is a result of the newly acquired capability of deploying unstructured meshes to simulate lubricated contact, which in turn can contribute either to reducing the number of DOFs in problems with complex geometries or to improving, by mesh refinements, the solution accuracy near regions characterized by special surface features in the contact domain. As for the computational efficiency of the method with dense grids, the current formulation yields faster solutions than those reported in the recent publication [217], in which the computational time needed to simulate similar textured bearings with DOFs varying from 360,000 to 129,600 nodes was shown to be in the range from $47.1 \mathrm{~s}$ to $17.8 \mathrm{~s}$, respectively (no CPU description was provided in [217]). As can be seen from the results summarized in Tables 13.2 and 13.3, for the current formulation with regular meshes, the number of DOFs varies almost within the same range from 368,449 to 122,614 nodes, but the simulation time is significantly lower (ranging from $11.27 \mathrm{~s}$ to $6.48 \mathrm{~s}$ ). 

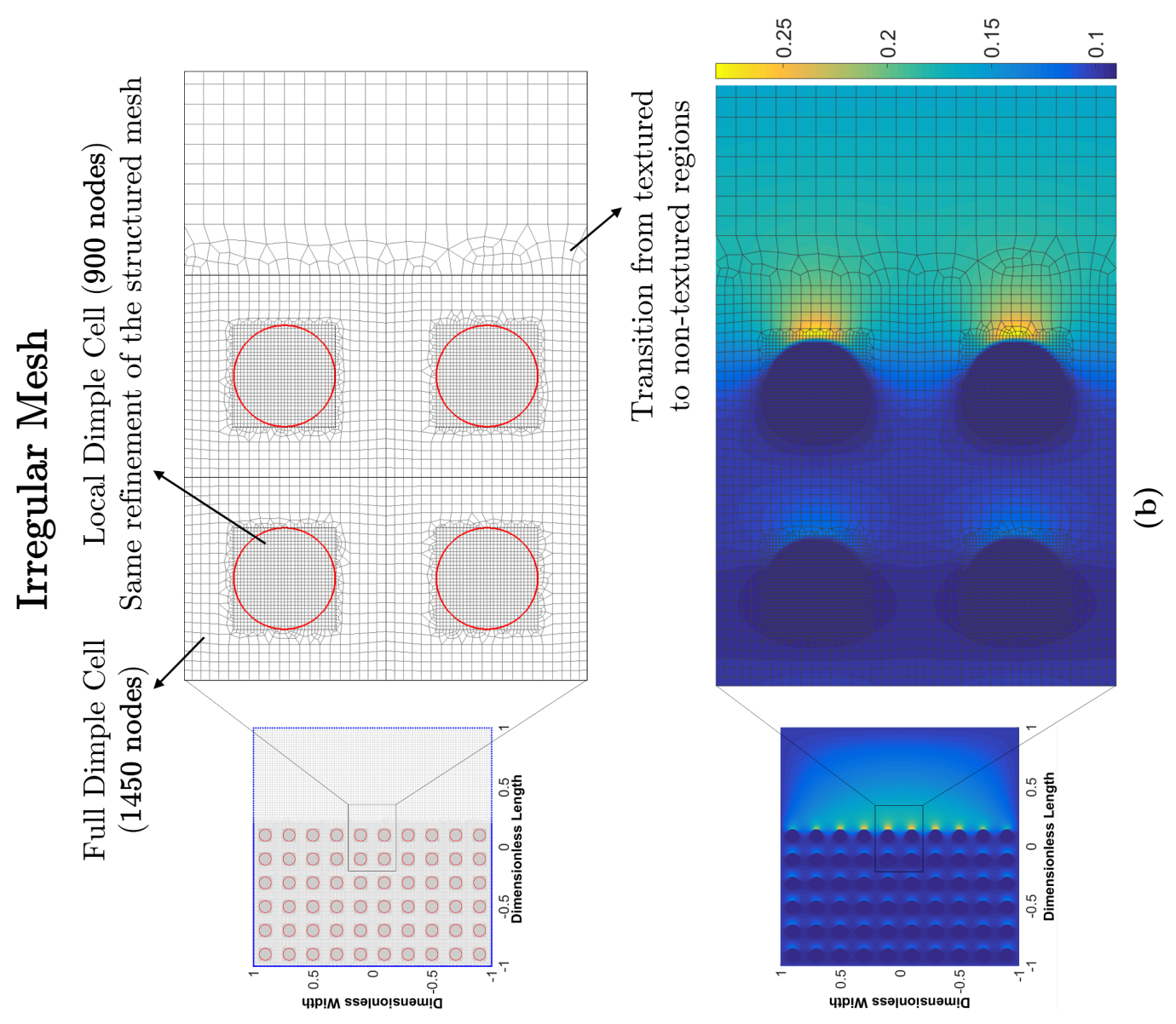

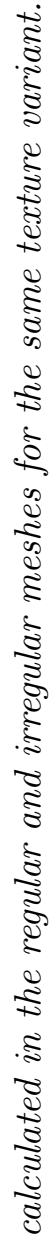
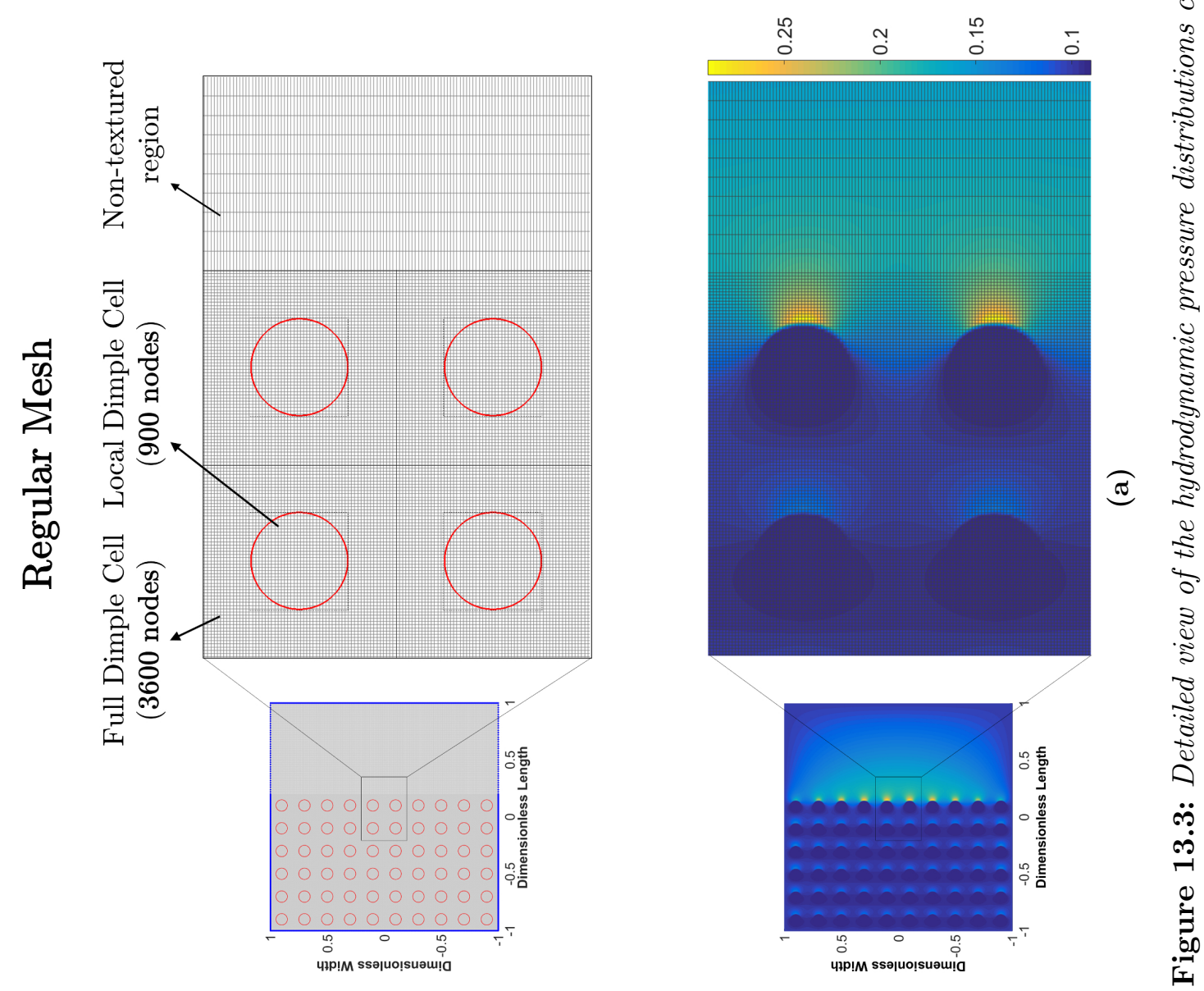
Finally, Fig. 13.3 depicts a detailed view of the hydrodynamic pressure distributions calculated on the regular and irregular meshes for the same texture variant. As expected, no noticeable difference is observed between the pressure fields. Thus, it is possible to conclude that by successfully combining the flexibility of the FEM to deal with irregular geometries and the inherent conservative nature of the FVM, one of the main features of the EbFVM is that it allows the use of irregular meshes to discretize the domain under study. As shown, it confers enormous flexibility to the method and enables to significantly reduce the computational requirements for the solution of lubrication problems in the presence of textured surfaces; hence, the proposed method offers new routes for the improvement and the design optimization of tribological surfaces. The results presented in this case study have been published by the author in references [173, 174]. 


\section{Chapter 14}

\section{Conclusions}

The present thesis research initially proposed the constitution of an extensive computational platform for simulating bearing systems operating under different lubrication regimes and working conditions. This was accomplished for journal and sliding bearing applications through the development of a solid mathematical modelling background based on the full solution of the Isothermal Generalized Equation of the Mechanics of Viscous Thin Films (Reynolds equation), together with the so-called mass-conserving $p-\theta$ Elrod-Adams cavitation model which automatically incorporates the JFO complementary boundary conditions. The bearing models, in particular, account for eventual system angular misalignments, superficial geometric defects (form/waviness deviations, EHL deformations, etc.) or axial movement, as well as the bearing coupling into general multibody dynamic systems (MDS). As for the lubricant rheology, the viscous-pressure, viscous-shear-thinning and density-pressure effects were also considered in the modelling. The soft-EHL conformal contact often present in this sort of bearing components was included in the formulation as well by means of condensation techniques that reduce the full bearing FEM model into an equivalent system retaining only the degrees of freedom of the bearing interface. The effects of the surface roughness under mixed-lubrication situations were addressed by the stochastic Patir and Cheng average flow model and the Greenwood-Williamson and Greenwood-Tripp formulations for asperity contact. A deterministic modelling approach that simultaneously solves the fluid flow problem and the local asperity interactions at the microscopic (roughness) scale was proposed in the same fashion.

Proceeding from the aforementioned computational framework, three major contributions were considered for advanced solutions dedicated to distinct parts of the overall mixed-lubrication modelling previously established. Firstly, a version of the so-called Finite Volume Method Based on Elements (EbFVM), along with a general Successive Over-Relaxation (SOR) algorithm for the iterative calculations, were introduced as a new paradigm in the solution of the modified mass-conserving $p-\theta$ Reynolds lubrication equation on irregular meshes $[173,174]$. This methodology ensures the complete mass conservation of the lubricant flow over the lubricated domain, including the whole cavitation boundaries, regardless of the shape of the grid elements. The new method was successfully validated from comparisons with existing alternative algorithms and full CFD simulations (see Appendix F.5). Test cases were used to demonstrate that the numerical approach proposed is robust and versatile and can incorporate compressibility, piezoviscosity and shear-thinning of the lubricant, while being applicable to any transient hydrodynamic lubrication problem in the presence of cavitation. Furthermore, numerical 
experiments were also undertaken for a textured sliding bearing, showing that the geometric flexibility of the method, which allows mesh refinements concentrated around the texture features, contributes to considerably decreasing the number of nodes required for solving the problem, while keeping the overall results unchanged. At the same extent, the computational efforts for solving the lubrication conditions in the presence of textured surfaces are significantly reduced. In addition, the proposed method affords promising routes for the design optimization of surface texturing.

The second main contribution accomplished by this thesis deals with the analysis of novel robust and stable partitioned techniques, Fixed Point Gauss-Seidel Method (PGMF), Point Gauss-Seidel Method with Aitken Acceleration (PGMA) and Interface Quasi-Newton Method with Inverse Jacobian from Least-Squares approximation (IQNILS), for the dynamic solution of conformal EHL problems [170, 177]. Particularly for the connecting-rod big-end bearings of both heavy-duty diesel and high-speed engines considered for evaluating the performance of the methods, the IQN-ILS scheme was advantageous in terms of computational efforts for statically loaded conditions, whereas the PGMA method showed the best efficiency under dynamic loading situations. The PGMF techniques produced the worst results, presenting no convergence for either loading conditions examined. As for the output results of those simulations, the time variation of several operational parameters were determined, including the journal trajectory and eccentricity, the minimum oil film thickness, peak pressure and power losses. Furthermore, the collapse of cavitation bubbles were also detected under conditions of abrupt changes in the orientation of the bearing external loads. Moreover, the influence of different shear-thinning oils on the performance of the high-speed big-end bearing were investigated as well, clearly showing the significance of the polymeric structure/concentration of the lubricant friction modifiers on the engine power losses. This practical application case illustrates an example of how the solution framework developed herein can be used to support parametric and/or optimization analyses during the engineering design process. The applications of such methods can be certainly extended to other tribological contacts, e.g. line and point contacts, or even to the temperature coupling in thermo-elasto-hydrodynamic (TEHL) calculations. The main advantage of such partitioned approaches is the possibility of using optimized codes to solve hydrodynamic equations and structural equations separately as "black-box" solvers.

Finally, the third major outcome provided by this research concerns the deterministic mixed-lubrication modelling conceived for the full-scale simulation of piston-rings cylinder liner contacts in internal combustion engines [67, 68, 172, 175, 176]. In this case, a solution approach based on solving the fluid flow problem described by Reynolds equation with inter-asperity cavitation concomitantly with the Hertzian asperity contact at the roughness scale was considered. The effect of the cylinder liner wear after a $100 \mathrm{~h}$ dynamometer engine test on the mixed-lubrication behaviour of Twin-Land Oil Control Rings was investigated accordingly. In general, the average hydrodynamic pressures tended to increase whilst the asperity pressures reduced with the evolution of the superficial wear. This may be explained by the fact that the smoothing of the plateau regions caused by the wear-out of the highest asperities, along with the overall decrease in the asperities curvature, contribute to promoting further pressure-driven lubricant flow through the inter-grooves zones, which in turn also highlight the rule of superficial grooves in the generation of hydrodynamic support. This kind of application reinforces the power of deterministic simulations to assess the effects of localized microscopic surface features in the whole tribosystem performance. 


\subsection{Future Works}

The suggestions and recommendations for future researches related to the subjects covered in the current thesis are summarized as follows. The contents are listed rationally according to the order in which the different topics are organized or have been explored throughout the work.

\section{- Bearing Modelling}

- Thermo-hydrodynamic lubrication calculations based on the solution of the Generalized Reynolds equation and the energy equation for the fluid flow. In this case, the lubricant rheology properties, e.g. viscosity and density, can vary across the film thickness.

- Implementation of different bearing systems and configurations, such as multilobe and tilted-pad journal bearings, plane and tilted-pad sliding thrust bearings, etc.

- Calculation of the linear dynamic coefficients of textured or multi-grooved journal bearings.

- Extending the solution framework to include linear and nonlinear rotordynamic analysis.

- Advanced simulations of floating ring bearing assemblies.

- Simulation of wind turbine bearing systems to support the design process of more reliable components.

- Parametric investigations of textured journal and sliding bearings aimed to determine the optimum texturing geometry and pattern distribution for friction reduction in different applications.

- Experimental tests with textured journal bearings for friction reduction and oil shear-thinning analysis.

- Using the Aitken's acceleration adopted in the PGMA partitioned technique for speeding-up the Gauss-Seidel solution with SOR of the $p-\theta$ cavitation algorithm.

\section{- EHL Modelling}

- Full transient EHL solution of journal bearings incorporated into multibody dynamic systems. In this case, the gross system rigid body motion, as well as the structural dynamics, are solved simultaneously in the same solution scheme, i.e. the external load forces action on the bearings are unknown $a$ priori.

- Incorporating the distributed inertia of the bearing structure, which tends to be important in bearing applications with high speed rotations.

- Including thermal distortions caused by the solid temperature gradients in thermo-elastohydrodynamic lubrication analysis.

- Solving more advanced conformal EHL contacts by exploring the "black-box"like approach of the direct coupling methods introduced in this thesis for 
tribological simulations. In this case, more complex contact interactions involving, for instance, nonlinear materials and/or large displacements in the solid problem solution, or even intricate lubricant flows which require the full calculation of the Navier-Stokes equations, could be solved separately in dedicated codes and then coupled into a unique solution using the FSI partitioned techniques analysed in the thesis.

- Proposal of a resolution method for nonlinear contact problems based on the superposition of several (quasi-static) linear approximations of the system configuration. Each quasi-static configuration would be represented by a set of condensate matrices. Possible application: dynamic soft-EHL contacts with rubber material.

- Development of a plug-in with a user interface for EHL calculations in Abaqus ${ }^{\circledR}$ software [62].

- Extension of the analysed partitioned coupling techniques for static and transient solutions of line and point (non-conformal) EHL contacts (simulations of gears and rolling/ball bearings).

\section{- Deterministic Modelling}

- Detailed evaluation of the surface waviness effect on the mixed-lubrication regime; the influence of the method used for separating the waviness and roughness components, i.e. either Gaussian-based filters with a large range of cut-off values or multiscale techniques, can be investigated as well.

- Implementation of both multigrid method and parallel computation to enable the simulation of deterministic problems under dynamic conditions and/or with larger discrete domains.

- Improvements in the overall modelling by including the calculation of the EHL effects, rheological corrections and flash temperature at the microscopic (asperity) scale.

- Incorporating advanced asperity-based models that consider elliptic and/or elasto-plastic/plastic contacts, or even more sophisticated formulations based on the solution of the Boussinesq equation with FFT techniques for rough contacts.

- Developing comprehensive modelling for the boundary lubrication regime at the asperity level, by taking into account effects such as tribofilm formation, interaction of lubricant additives/molecules with the surface topographies (coatings) and their influence on the overall system performance. In-depth knowledge of such complex phenomena could be supported by linking molecular dynamic simulation results to continuum mechanics solutions, e.g. FEM and FVM. 


\section{Appendix A}

\section{Journal Bearing Model}

\section{A.1 Reference Frames and Coordinate Systems Reference Frames}

Fig. A.1 depicts a generic journal bearing coupled to a structure which can move relative to other components in a multibody dynamic system. According to the figure, the following reference frames are defined:

ऽ: Inertial (or Newtonian) reference frame.

$\mathcal{R}$ : Reference frame attached to the structure in which the journal bearing is coupled; in a generic multibody dynamic system, this frame can move with respect to the inertial frame $(\Im)$. On the other hand, in the absence of relative motion, $\mathcal{R}$ coincides with $\Im$, i.e. $\mathcal{R} \equiv \Im$.

$\mathcal{B}$ : Reference frame attached to the mid-plane orthogonal to the bearing axial direction. This frame can experience relative motion with respect to the structure $(\mathcal{R})$ only due to angular misalignments of the bearing, i.e. this frame does not rotate relative to the structure.

$\mathcal{B}_{r}$ : Rotating reference frame attached to the mid-plane orthogonal to the bearing axial direction. This frame is essentially the same as $\mathcal{B}$, except for its possible rotation relative to the structure $(\mathcal{R})$ in cases of unstationary bearings.

$\mathfrak{d}$ : Reference frame attached to the mid-plane orthogonal to the journal axial direction. This frame can experience relative motion with respect to the structure $(\mathcal{R})$ only due to angular misalignments of the journal, i.e. this frame does not rotate relative to the structure.

$\mathcal{J}_{r}$ : Rotating reference frame attached to the mid-plane orthogonal to the journal axial direction. This frame is essentially the same as $\mathcal{J}$, except for its rotation relative to the structure $(\mathcal{R})$ due to the journal speed.

\section{Coordinate Systems}

The definition of coordinate systems coherent to the references frames stated above is important for the general description of the geometric and kinematic features of journal 


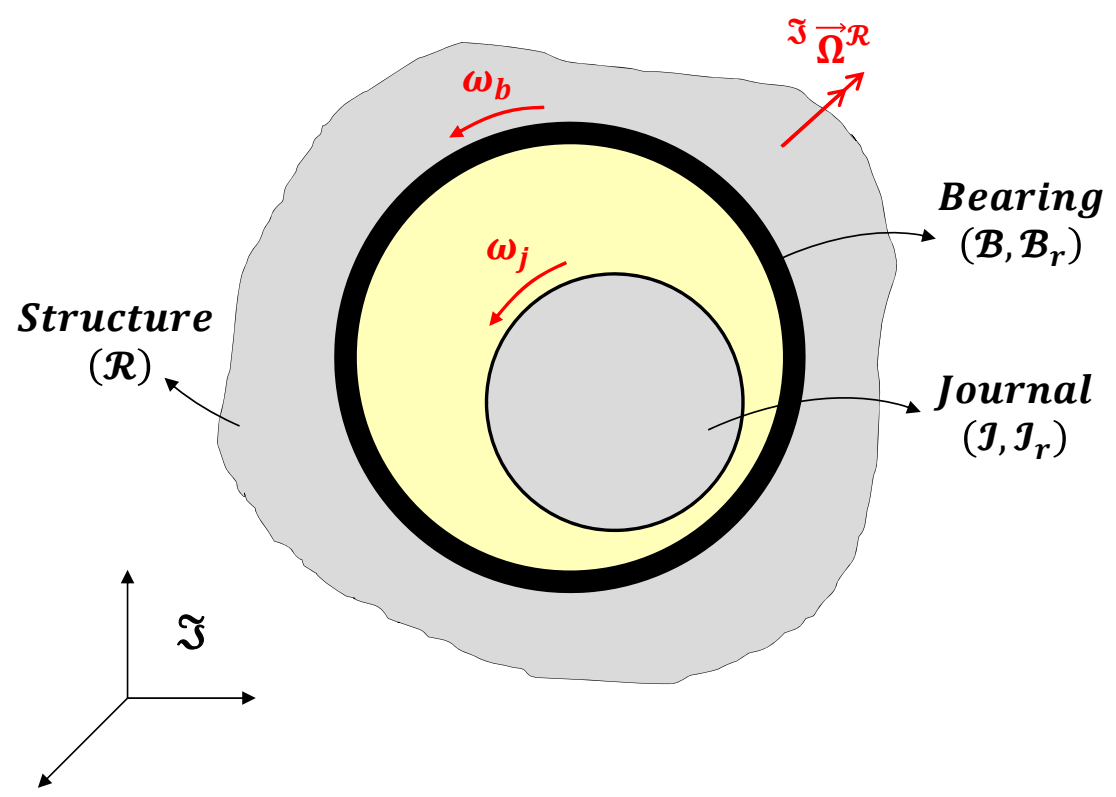

Figure A.1: Reference frames of the journal bearing model.

bearings. The coordinate systems here utilized for the model derivation are listed as follows (see Fig. A.2):

$\boldsymbol{S}_{\mathcal{R}} \rightarrow[O, \overrightarrow{\boldsymbol{I}}, \overrightarrow{\boldsymbol{J}}, \overrightarrow{\boldsymbol{K}}]_{(X, Y, Z)}:$ Coordinate system attached to frame $\mathcal{R}$.

$\mathcal{S}_{\mathcal{B}} \rightarrow\left[C_{b}, \overrightarrow{\boldsymbol{I}}_{\boldsymbol{b}}, \overrightarrow{\boldsymbol{J}}_{\boldsymbol{b}}, \overrightarrow{\boldsymbol{K}}_{\boldsymbol{b}}\right]_{\left(X_{b}, Y_{b}, Z_{b}\right)}:$ Coordinate system attached to frame $\mathcal{B}$ and with origin at the geometric centre of the bearing $\left(C_{b}\right)$. The only relative movements of this system with respect to $\boldsymbol{S}_{\mathcal{R}}$ are due to angular misalignments of the bearing. This reference is also admitted as the principal one from which the relative rigid body motions and all the operational parameters are determined.

$\mathcal{S}_{\mathcal{B}_{r}} \rightarrow\left[C_{b}, \overrightarrow{\boldsymbol{I}}_{\boldsymbol{b r}}, \overrightarrow{\boldsymbol{J}}_{\boldsymbol{b} \boldsymbol{r}}, \overrightarrow{\boldsymbol{K}}_{\boldsymbol{b r}}\right]_{\left(X_{b r}, Y_{b r}, Z_{b r}\right)}:$ Coordinate system attached to frame $\mathcal{B}_{\boldsymbol{r}}$ and with origin at the geometric centre of the bearing $\left(C_{b}\right)$. This coordinate system is essentially the same as $\boldsymbol{S}_{\mathcal{B}}$ plus the bearing rotation around $\overrightarrow{\boldsymbol{K}}_{\boldsymbol{b}}$. Moreover, the complete bearing geometry, including the structural deformations in EHL problems, is described in this system, i.e. the computational mesh used for the numerical solution of Reynolds equation is constructed according to this coordinate system. The use of such general approach to represent the contact interface allows the simulation of non-stationary bearings with complex geometries, e.g. floating ring bearings.

$\boldsymbol{S}_{\mathcal{B}_{l}} \rightarrow\left[P_{b}, \overrightarrow{\boldsymbol{i}}_{\boldsymbol{b l}}, \overrightarrow{\boldsymbol{j}}_{\boldsymbol{b l}}, \overrightarrow{\boldsymbol{k}}_{\boldsymbol{b l}}\right]_{\left(x_{b l}, y_{b l}, z_{b l}\right)}:$ Coordinate system attached to frame $\mathcal{B}_{\boldsymbol{r}}$ and with origin defined locally at every point $P_{b}$ of the bearing surface. This coordinate system defines the local normal, tangential and axial directions at each point around the bearing cylindricity, and used to describe the local fluid film geometry and surfaces kinematic for the Reynolds equation.

$\boldsymbol{S}_{\mathfrak{J}} \rightarrow\left[C_{j}, \overrightarrow{\boldsymbol{I}}_{\boldsymbol{j}}, \overrightarrow{\boldsymbol{J}}_{\boldsymbol{j}}, \overrightarrow{\boldsymbol{K}}_{\boldsymbol{j}}\right]_{\left(X_{j}, Y_{j}, Z_{j}\right)}:$ Coordinate system attached to frame $\boldsymbol{J}$ and with origin at the geometric centre of the journal $\left(C_{j}\right)$. The only relative movements of this system with respect to $\mathcal{S}_{\mathcal{R}}$ are due to angular misalignments of the journal. 


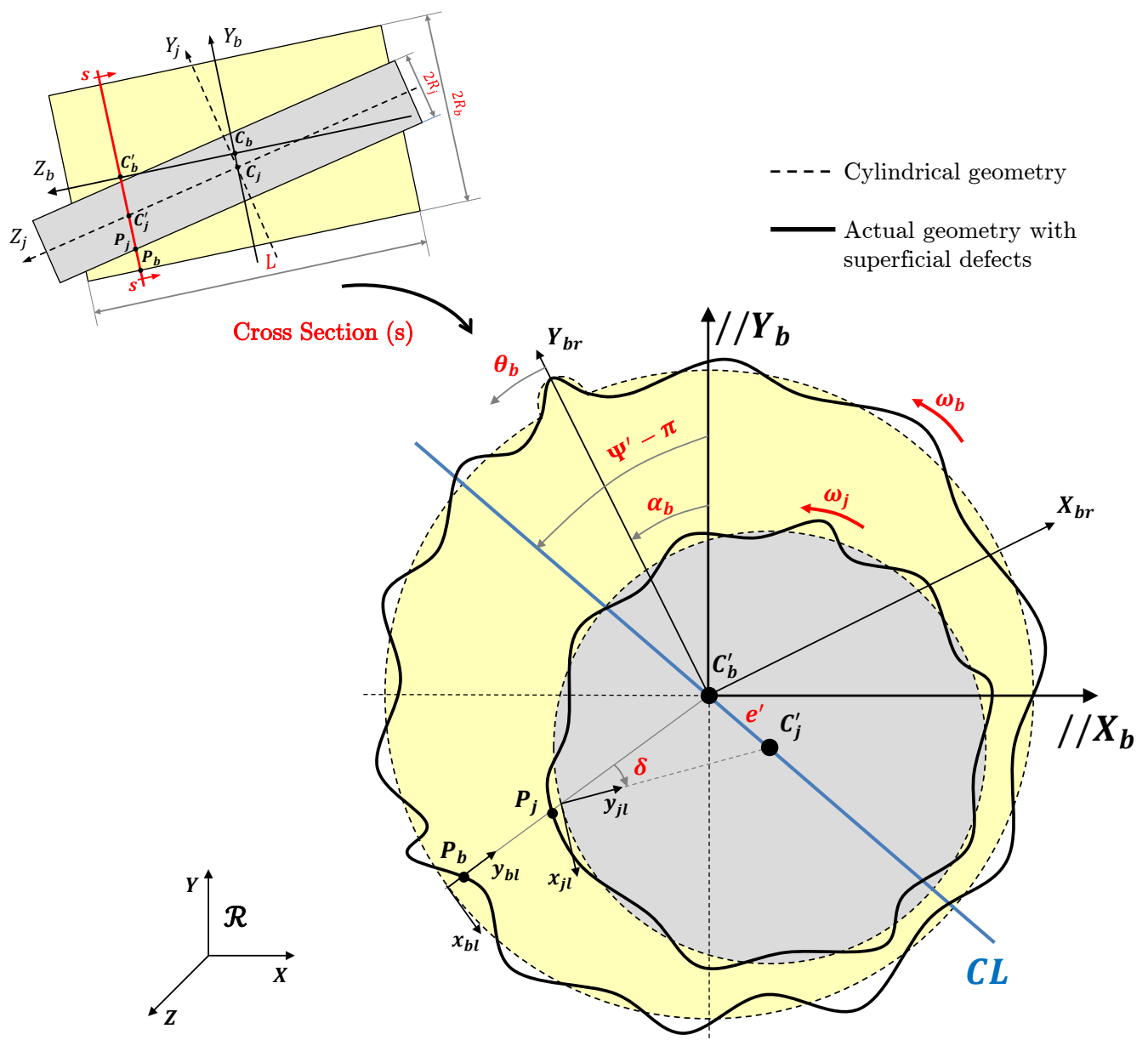

Figure A.2: Main coordinate systems of the journal bearing model.

$\boldsymbol{S}_{\mathfrak{J}_{\boldsymbol{r}}} \rightarrow\left[C_{j}, \overrightarrow{\boldsymbol{I}}_{\boldsymbol{j} \boldsymbol{r}}, \overrightarrow{\boldsymbol{J}}_{\boldsymbol{j} \boldsymbol{r}}, \overrightarrow{\boldsymbol{K}}_{\boldsymbol{j} \boldsymbol{r}}\right]_{\left(X_{j r}, Y_{j r}, Z_{j r}\right)}$ : Coordinate system attached to frame $\mathfrak{J}_{\boldsymbol{r}}$ and with origin at the geometric centre of the journal $\left(C_{j}\right)$. This coordinate system is essentially the same as $\boldsymbol{S}_{\mathfrak{y}}$ plus the journal rotation around $\overrightarrow{\boldsymbol{K}}_{\boldsymbol{j}}$. Moreover, the complete journal geometry, including the structural deformations in EHL problems, is described in this coordinate system.

$\mathcal{S}_{\mathfrak{j}_{l}} \rightarrow\left[C_{j}, \overrightarrow{\boldsymbol{i}}_{\boldsymbol{j} l}, \overrightarrow{\boldsymbol{j}}_{j l}, \overrightarrow{\boldsymbol{k}}_{\boldsymbol{j} l}\right]_{\left(x_{j l}, y_{j l}, z_{j l}\right)}:$ Coordinate system attached to frame $\mathcal{J}_{\boldsymbol{r}}$ and with origin defined locally at every point $P_{j}$ of the journal surface. This coordinate system defines the local normal, tangential and axial directions at each point around the journal cylindricity. Notice that as $\boldsymbol{S}_{\mathcal{B}_{r}}$ is the principal coordinate system from which Reynolds equation is evaluated; whenever (fixed) geometric defects are present on the journal surface, a special procedure has to be used to locally match the instantaneous journal geometry to the actual bearing mesh.

\section{A.2 Transformation Matrices}

In order to correlate vector quantities among the different coordinates systems used for the derivation of the general journal bearing model, the following transformation matrices are defined: 


\section{Transformation Matrix: $\mathcal{S}_{\mathcal{B}, \mathfrak{d}} \rightarrow \mathcal{S}_{\mathcal{R}}$}

In order to correlate the orientations of the unit vectors between $\boldsymbol{S}_{\mathcal{B}}$ and $\boldsymbol{S}_{\mathcal{R}}$, a similar procedure used for determining the so-caller Euler angles in classical mechanics is here adopted. In this case, the following two successive rotations are considered from an auxiliary coordinate system $\boldsymbol{S}_{\mathcal{B}}^{* *}$ defined initially as coincident with $\boldsymbol{S}_{\mathcal{R}}$ : (1) $\boldsymbol{S}_{\mathcal{B}}^{* *}$ is rotated around the $\overrightarrow{\boldsymbol{I}}$ axis by an angle of $A_{b}$, providing intermediate system $\boldsymbol{S}_{\mathcal{B}}^{*}$; and (2) the obtained intermediary system is rotated again by $B_{b}$ around the $\overrightarrow{\boldsymbol{J}}^{*}$ axis, thus establishing the desired coordinate system $\boldsymbol{S}_{\mathcal{B}}$. The relationship between $\boldsymbol{S}_{\mathfrak{J}}$ and $\boldsymbol{S}_{\mathcal{R}}$ is defined analogously by replacing the respective rotation angles with $A_{j}$ and $B_{j}$. Thus, the following transformation matrices are obtained:

$$
\left\{\begin{array}{c}
\overrightarrow{\boldsymbol{I}} \\
\overrightarrow{\boldsymbol{J}} \\
\overrightarrow{\boldsymbol{K}}
\end{array}\right\}=\underbrace{\left[\begin{array}{ccc}
\cos \left(B_{b, j}\right) & 0 & \sin \left(B_{b, j}\right) \\
\sin \left(A_{b, j}\right) \sin \left(B_{b, j}\right) & \cos \left(A_{b, j}\right) & -\cos \left(B_{b, j}\right) \sin \left(A_{b, j}\right) \\
-\cos \left(A_{b, j}\right) \sin \left(B_{b, j}\right) & \sin \left(A_{b, j}\right) & \cos \left(A_{b, j}\right) \cos \left(B_{b, j}\right)
\end{array}\right]}_{\left[\boldsymbol{M}_{\boldsymbol{B}_{\mathcal{B}, \boldsymbol{j}} \rightarrow s_{\mathcal{R}}}\right.}\left\{\begin{array}{c}
\overrightarrow{\boldsymbol{I}}_{\boldsymbol{b}, \boldsymbol{j}} \\
\overrightarrow{\boldsymbol{J}}_{\boldsymbol{b}, \boldsymbol{j}} \\
\overrightarrow{\boldsymbol{K}}_{\boldsymbol{b}, \boldsymbol{j}}
\end{array}\right\}
$$

Angles $A_{b, j}$ and $B_{b, j}$ correspond to the angular misalignments of the bearing/journal relative to reference frame $\mathcal{R}$. Furthermore, as those angles are assumed of small magnitude, i.e. $\cos \left(A_{b, j}\right)=\cos \left(B_{b, j}\right) \approx 1, \sin \left(A_{b, j}\right) \approx A_{b, j}$ and $\sin \left(B_{b, j}\right) \approx B_{b, j}$, Eq. A.1 can be rewritten as:

$$
\left\{\begin{array}{c}
\overrightarrow{\boldsymbol{I}} \\
\overrightarrow{\boldsymbol{J}} \\
\overrightarrow{\boldsymbol{K}}
\end{array}\right\}=\underbrace{\left[\begin{array}{ccc}
1 & 0 & B_{b, j} \\
0 & 1 & -A_{b, j} \\
-B_{b, j} & A_{b, j} & 1
\end{array}\right]}_{[\boldsymbol{M}]_{\mathcal{B}_{\mathcal{B}, \boldsymbol{g}} \rightarrow s_{\mathcal{R}}}}\left\{\begin{array}{c}
\overrightarrow{\boldsymbol{I}}_{\boldsymbol{b}, \boldsymbol{j}} \\
\overrightarrow{\boldsymbol{J}}_{\boldsymbol{b}, \boldsymbol{j}} \\
\overrightarrow{\boldsymbol{K}}_{\boldsymbol{b}, \boldsymbol{j}}
\end{array}\right\}
$$

\section{Transformation Matrix: $\mathcal{S}_{\mathfrak{J}} \rightarrow \mathcal{S}_{\mathcal{B}}$}

The relationship between coordinate systems $\boldsymbol{S}_{\mathfrak{f}}$ and $\boldsymbol{S}_{\mathcal{B}}$ is readily obtained by combining the transformation matrices defined previously in Eq. A.1, i.e. $[\boldsymbol{M}]_{\mathcal{S}_{\mathfrak{g}} \rightarrow \boldsymbol{S}_{\mathcal{B}}}=$ $[\boldsymbol{M}]_{\mathcal{S}_{\mathcal{B}} \rightarrow \mathcal{S}_{\mathcal{R}}}^{-1}[\boldsymbol{M}]_{\mathcal{S}_{\mathfrak{J}} \rightarrow \mathcal{S}_{\mathcal{R}}}$. Thus:

$$
\left\{\begin{array}{c}
\overrightarrow{\boldsymbol{I}}_{\boldsymbol{b}} \\
\overrightarrow{\boldsymbol{J}}_{\boldsymbol{b}} \\
\overrightarrow{\boldsymbol{K}}_{\boldsymbol{b}}
\end{array}\right\}=\underbrace{\left[\begin{array}{rrr}
\cos \left(B_{b}\right) \cos \left(B_{j}\right)+ & -\sin \left(A_{r}\right) \sin \left(B_{b}\right) & -\cos \left(A_{r}\right) \cos \left(B_{j}\right) \sin \left(B_{b}\right)+ \\
\cos \left(A_{r}\right) \sin \left(B_{b}\right) \sin \left(B_{j}\right) & \cos \left(B_{b}\right) \sin \left(B_{j}\right) \\
\sin \left(A_{r}\right) \sin \left(B_{j}\right) & \cos \left(A_{r}\right) & -\cos \left(B_{j}\right) \sin \left(A_{r}\right) \\
\cos \left(B_{j}\right) \sin \left(B_{b}\right)- & \sin \left(A_{r}\right) \cos \left(B_{b}\right) & \cos \left(A_{r}\right) \cos \left(B_{b}\right) \cos \left(B_{j}\right)+ \\
\cos \left(A_{r}\right) \cos \left(B_{b}\right) \sin \left(B_{j}\right) & \sin \left(B_{b}\right) \sin \left(B_{j}\right)
\end{array}\right]}_{\left[\boldsymbol{M}_{\boldsymbol{s}_{\boldsymbol{j}} \rightarrow s_{\mathcal{B}}}\right.}\left\{\begin{array}{c}
\overrightarrow{\boldsymbol{I}}_{\boldsymbol{j}} \\
\overrightarrow{\boldsymbol{J}}_{\boldsymbol{j}} \\
\overrightarrow{\boldsymbol{K}}_{\boldsymbol{j}}
\end{array}\right\}
$$

Considering the same approximations for the (small) misalignment angles, one has:

$$
\left\{\begin{array}{c}
\overrightarrow{\boldsymbol{I}}_{\boldsymbol{b}} \\
\overrightarrow{\boldsymbol{J}}_{\boldsymbol{b}} \\
\overrightarrow{\boldsymbol{K}}_{\boldsymbol{b}}
\end{array}\right\}=\underbrace{\left[\begin{array}{ccc}
1 & 0 & B_{r} \\
0 & 1 & A_{r} \\
-B_{r} & A_{r} & 1
\end{array}\right]}_{[\boldsymbol{M}]_{\boldsymbol{s}_{\mathfrak{j}} \rightarrow \boldsymbol{s}_{\mathcal{B}}}}\left\{\begin{array}{c}
\overrightarrow{\boldsymbol{I}}_{\boldsymbol{j}} \\
\overrightarrow{\boldsymbol{J}}_{\boldsymbol{j}} \\
\overrightarrow{\boldsymbol{K}}_{\boldsymbol{j}}
\end{array}\right\}
$$

where $A_{r}=\left(A_{j}-A_{b}\right)$ and $B_{r}=\left(B_{j}-B_{b}\right)$ are the respective angular misalignments of the journal relative to the bearing. 


\section{Transformation Matrix: $\mathcal{S}_{\mathcal{B}_{r}} \rightarrow \mathcal{S}_{\mathcal{B}}$}

As already stated, $\boldsymbol{S}_{\mathcal{B}_{r}}$ corresponds to the $\boldsymbol{S}_{\mathcal{B}}$ system plus the eventual bearing rotation around $\overrightarrow{\boldsymbol{K}}_{\boldsymbol{b}}$. Thus:

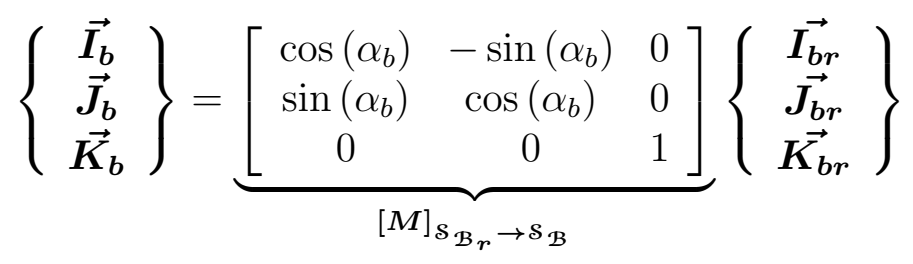

where $\alpha_{b}$ is the angular bearing rotation defined from the $\overrightarrow{\boldsymbol{J}_{\boldsymbol{b}}}$ axis (see Fig. A.2).

\section{Transformation Matrix: $\mathcal{S}_{\mathcal{B}_{l}} \rightarrow \mathcal{S}_{\mathcal{B}_{r}}$}

The relationship between coordinate systems $\boldsymbol{S}_{\mathcal{B}_{r}}$ and $\boldsymbol{S}_{\mathcal{B}_{l}}$ that define the local normal, tangential and axial directions at each point around the bearing cilindricity is given by:

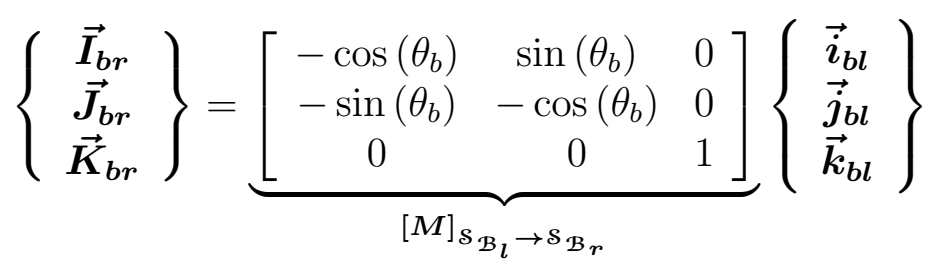

where $\theta_{b}$ is the angular bearing coordinate defined from the $\overrightarrow{\boldsymbol{J}}_{\boldsymbol{b} \boldsymbol{r}}$ axis (see Fig. A.2).

\section{Transformation Matrix: $\mathcal{S}_{\mathfrak{J}_{r}} \rightarrow \mathcal{S}_{\mathfrak{J}}$}

Analogously to the procedure of transformation $\boldsymbol{S}_{\mathcal{B}_{r}} \rightarrow \boldsymbol{S}_{\mathcal{B}}, \boldsymbol{S}_{\mathfrak{J}_{r}}$ corresponds to the $\boldsymbol{S}_{\mathfrak{f}}$ system plus the journal rotation around $\overrightarrow{\boldsymbol{K}}_{\boldsymbol{j}}$. Thus:

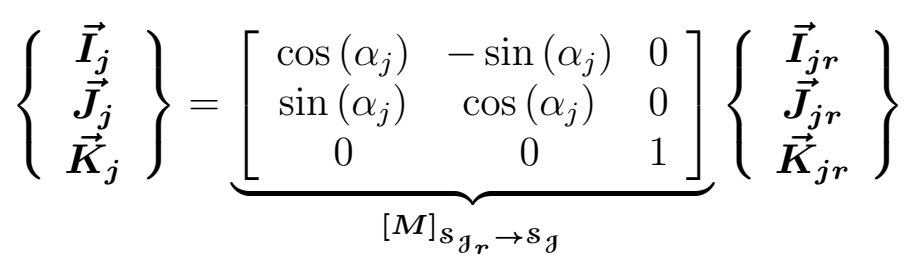

where $\alpha_{j}$ is the angular journal rotation defined from the $\overrightarrow{\boldsymbol{J}}_{\boldsymbol{j}}$ axis.

\section{Transformation Matrix: $\mathcal{S}_{\mathfrak{d}_{l}} \rightarrow \mathcal{S}_{\mathfrak{d}_{r}}$}

Analogously to transformation $\boldsymbol{S}_{\mathcal{B}_{l}} \rightarrow \boldsymbol{S}_{\mathcal{B}_{r}}, \boldsymbol{S}_{\mathfrak{J}_{r}}$, the relationship between coordinate system $\boldsymbol{S}_{\mathfrak{J}_{r}}$ and system $\boldsymbol{S}_{\mathfrak{d}_{l}}$ that define the local normal, tangential and axial directions at each point around the journal cilindricity is given by:

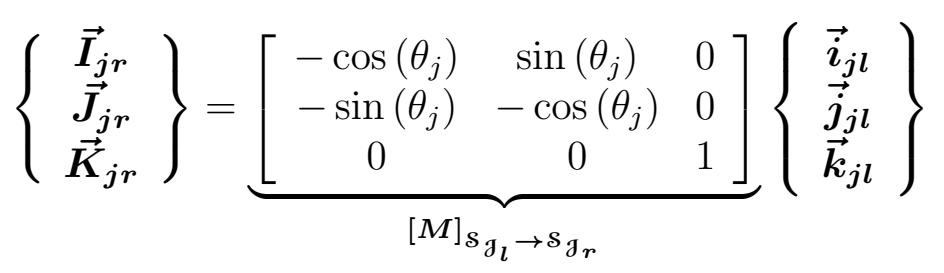

where $\theta_{j}$ is the angular journal coordinate defined from the $\overrightarrow{\boldsymbol{J}}_{\boldsymbol{j} \boldsymbol{r}}$ axis. 


\section{Transformation Matrix: $\mathcal{S}_{\mathfrak{d}_{l}} \rightarrow \mathcal{S}_{\mathcal{B}_{l}}$}

The direct relationship between both local coordinate systems is useful for determining both the geometry of the lubricant film thickness and the kinematic of the lubricated surfaces. Therefore, the following transformation matrices are established:

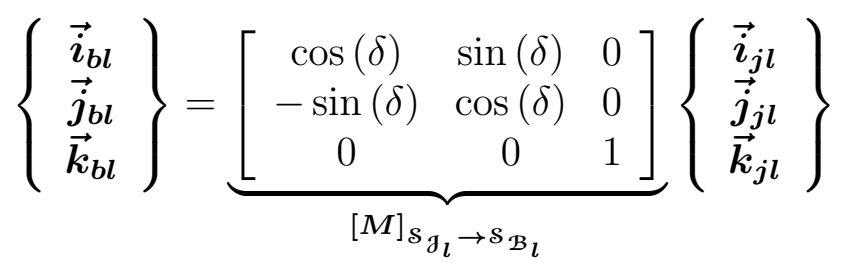

where $\delta$ is the local slope angle defined relative to the journal surface. By assuming the influence of the fluid film curvature as negligible (see Section A.4), Eq. A.9 can be rewritten as:

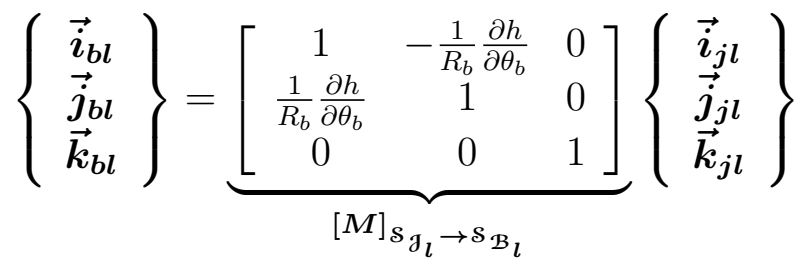

\section{A.3 Dimensionless Parameters}

The dimensionless parameters related to the general journal bearing model are listed as follows for completeness.

\section{Geometric Parameters}

$$
\begin{aligned}
c & =\left(R_{b}-R_{j}\right) & r_{L D} & =\frac{L}{2 R} \\
R & =\frac{\left(R_{b}+R_{j}\right)}{2}=\left(R_{b}-\frac{c}{2}\right) & \varepsilon & =\frac{e}{c}
\end{aligned}
$$

\section{Kinematic Parameters}

$$
\begin{aligned}
& \bar{A}_{b, j, r}=\frac{A_{b, j, r}}{\left(\frac{2 c}{L}\right)} \quad \bar{V}_{1,2}=\frac{V_{1,2}}{c} \quad \dot{\bar{Z}}_{C_{b, j}}=\frac{\dot{Z}_{C_{b, j}}}{\left(\frac{L}{2}\right)} \\
& \dot{\bar{A}}_{b, j, r}=\frac{\dot{A}_{b, j, r}}{\left(\frac{2 c}{L}\right)} \quad \bar{W}_{1,2}=\frac{W_{1,2}}{\left(\frac{L}{2}\right)} \quad \bar{z}_{b, j}=\frac{z_{b, j l}}{\left(\frac{L}{2}\right)} \\
& \bar{B}_{b, j, r}=\frac{B_{b, j, r}}{\left(\frac{2 c}{L}\right)} \quad \dot{\bar{X}}_{b, j, r}=\frac{\dot{X}_{b, j, r}}{c} \quad \bar{\delta}_{b, j, r}^{t, n}=\frac{\delta_{b, j, r}^{t, n}}{c} \\
& \dot{\bar{B}}_{b, j, r}=\frac{\dot{B}_{b, j, r}}{\left(\frac{2 c}{L}\right)} \quad \bar{X}_{b, j, r}=\frac{X_{b, j, r}}{c} \quad \dot{\bar{\delta}}_{b, j, r}^{t, n, a}=\frac{\dot{\delta}_{b, j, r}^{t, n, a}}{c} \\
& \bar{H}_{1,2}=\frac{H_{1,2}}{c} \quad \bar{Y}_{b, j, r}=\frac{Y_{b, j, r}}{c} \quad \theta_{b, j}=\frac{x_{b, j l}}{R} \\
& \bar{h}=\frac{h}{c} \quad \dot{\bar{Y}}_{b, j, r}=\frac{\dot{Y}_{b, j, r}}{c} \quad \bar{\omega}_{b, j}=\frac{\omega_{b, j}}{2 \pi}
\end{aligned}
$$




$$
\bar{U}_{1,2}=\frac{U_{1,2}}{R} \quad \quad \bar{y}_{b, j}=\frac{y_{b, j l}}{c} \quad \Omega=\frac{\left|\omega_{b}+\omega_{j}\right|}{2 \pi}
$$

\section{Hydrodynamic Parameters}

$$
\begin{array}{rlrl}
\bar{p}_{H} & =\frac{p_{H}}{\mu_{0} \Omega\left(\frac{R}{c}\right)^{2}} & \bar{\rho}=\frac{\rho}{\rho_{0}} \\
\bar{q}_{H} & =\frac{q_{H}}{\rho_{0}(\Omega R) c} & \bar{\mu}=\frac{\mu}{\mu_{0}} \\
\bar{\tau}_{H}=\frac{\tau_{H}}{\mu_{0} \Omega\left(\frac{R}{c}\right)} &
\end{array}
$$

\section{Operational Parameters}

$$
\begin{aligned}
\bar{f}_{H} & =\frac{f_{H}}{\left(\frac{c}{R}\right)} & \bar{Q}_{a} & =\frac{Q_{a}}{\rho_{0} \Omega R(c L)} \\
\bar{F}_{H} & =\frac{F_{H}}{\mu_{0} \Omega(2 R L)\left(\frac{R}{c}\right)} & S & =\frac{\mu_{0} \Omega(2 R L)}{\left|\vec{W}_{H}\right|}\left(\frac{R}{c}\right)^{2}=\frac{1}{\left|\bar{W}_{H}\right|} \\
\bar{M}_{H} & =\frac{M_{H}}{\mu_{0} \Omega(2 R L)\left(\frac{R}{c}\right)^{2} L} & \bar{T}_{H} & =\frac{T_{H}}{\mu_{0} \Omega(2 R L)\left(\frac{R}{c}\right) R} \\
\bar{P}_{H} & =\frac{P_{H}}{\mu_{0} \Omega(2 R L)\left(\frac{R}{c}\right) \Omega R} & \bar{W}_{H} & =\frac{W_{H}}{\mu_{0} \Omega(2 R L)\left(\frac{R}{c}\right)^{2}}
\end{aligned}
$$

\section{A.4 Derivation of the Lubricant Film Thickness and Kinematics}

\section{Geometry of the Lubricant Film Thickness}

The geometry of the lubricant film thickness is defined as the absolute normal distance measured between every point of the bearing surface $\left(P_{b}\right)$ and its respective point on the journal $\left(P_{j}\right)$, see Fig. A.3. Mathematically:

$$
H=\left(H_{2}-H_{1}\right)=\left(h+\delta_{b}-\delta_{j}\right)=\left(P_{j}-P_{b}\right) \cdot \vec{j}_{b_{l}}
$$

where $h$ is the lubricant film thickness for a perfectly cylindrical bearing. The point vector term in the above equation can be expressed as:

$$
\left(P_{j}-P_{b}\right)=\left(P_{j}-C_{j}^{\prime}\right)+\left(C_{j}^{\prime}-C_{j}\right)+\left(C_{j}-C_{b}\right)-\left(P_{b}-C_{b}\right)
$$

with

$$
\begin{aligned}
\left(P_{b}-C_{b}\right) & =\left(-R_{b}\right) \overrightarrow{\boldsymbol{j}}_{\boldsymbol{b l}}+\left(z_{b}\right) \overrightarrow{\boldsymbol{k}}_{\boldsymbol{b l}}+\left[\left(\delta_{b}^{t}\right) \overrightarrow{\boldsymbol{i}}_{\boldsymbol{b l}}+\left(-\delta_{b}^{n}\right) \overrightarrow{\boldsymbol{j}}_{\boldsymbol{b l}}+\left(\delta_{b}^{a}\right) \overrightarrow{\boldsymbol{k}}_{\boldsymbol{b l}}\right] \\
\left(P_{j}-C_{j}\right) & =\left(-R_{j}\right) \overrightarrow{\boldsymbol{j}}_{\boldsymbol{j} \boldsymbol{l}}+\left(z_{j}\right) \overrightarrow{\boldsymbol{k}}_{\boldsymbol{j} \boldsymbol{l}}+\left[\left(\delta_{j}^{t}\right) \overrightarrow{\boldsymbol{i}}_{\boldsymbol{j} \boldsymbol{l}}+\left(-\delta_{j}^{n}\right) \overrightarrow{\boldsymbol{j}}_{\boldsymbol{j} \boldsymbol{l}}+\left(\delta_{j}^{a}\right) \overrightarrow{\boldsymbol{k}}_{\boldsymbol{j} \boldsymbol{l}}\right] \\
\left(C_{j}^{\prime}-C_{j}\right) & =\left(z_{j}\right) \overrightarrow{\boldsymbol{K}}_{\boldsymbol{j} \boldsymbol{r}}
\end{aligned}
$$




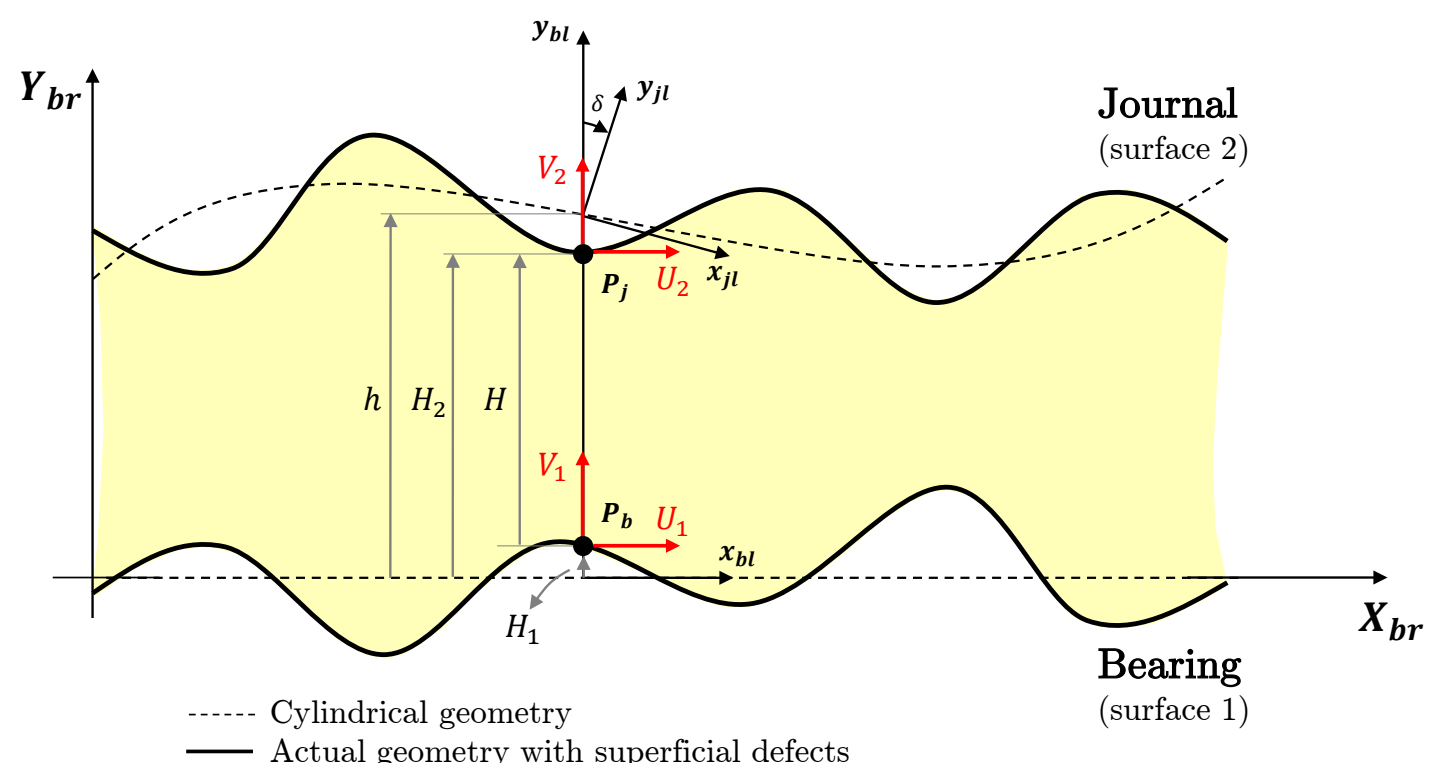

Figure A.3: Illustration of the journal bearing contact interface surrounding the points $P_{b}$ and $P_{j}$.

$$
\left(C_{j}-C_{b}\right)=\left(X_{r}\right) \overrightarrow{\boldsymbol{I}}+\left(Y_{r}\right) \overrightarrow{\boldsymbol{J}}
$$

where

$R_{b, j}$ is the bearing/journal radius [m];

$z_{b, j}$ is the axial bearing/journal coordinate (axial coordinate of $P_{b, j}$ ) $[\mathrm{m}]$;

$\delta_{b, j}^{t, n, a}$ are the tangential, normal and axial components of the combined geometric deviations of the bearing/journal (combined geometric deviations at $P_{b, j}$ ) $[\mathrm{m}]$;

$\left(X_{r}, Y_{r}\right)$ are the rigid body displacements of the journal relative to the bearing in the $\overrightarrow{\boldsymbol{I}}_{\boldsymbol{b}}$ and $\overrightarrow{\boldsymbol{J}}_{\boldsymbol{b}}$ directions, respectively, i.e. $X_{r}=\left(X_{j}-X_{b}\right)$ and $Y_{r}=\left(Y_{j}-Y_{b}\right)$ $[\mathrm{m}]$.

The term $\left(P_{j}-C_{j}^{\prime}\right)$ in Eqs. A.12 is obtained from the projections of the journal cross sections $(s)$. Furthermore, for the later kinematic analysis, it is convenient to represent $\left(P_{b}-C_{b}\right)$ and $\left(P_{j}-C_{j}\right)$ in the rotating coordinate systems $\boldsymbol{S}_{\mathcal{B}_{r}}$ and $\boldsymbol{S}_{\mathfrak{d}_{r}}$, respectively. Accordingly:

$$
\begin{aligned}
\left(P_{b}-C_{b}\right)= & \left\{-\left(R_{b}+\delta_{b}^{n}\right) \sin \left(\theta_{b}\right)-\left(\delta_{b}^{t}\right) \cos \left(\theta_{b}\right)\right\} \overrightarrow{\boldsymbol{I}}_{\boldsymbol{b} \boldsymbol{r}}+ \\
& \left\{\left(R_{b}+\delta_{b}^{n}\right) \cos \left(\theta_{b}\right)-\left(\delta_{b}^{t}\right) \sin \left(\theta_{b}\right)\right\} \overrightarrow{\boldsymbol{J}}_{\boldsymbol{b} \boldsymbol{r}}+ \\
& \left\{\left(z_{b}+\delta_{b}^{a}\right)\right\} \overrightarrow{\boldsymbol{K}}_{\boldsymbol{b} \boldsymbol{r}} \\
\left(P_{j}-C_{j}\right)= & \left\{-\left(R_{j}+\delta_{j}^{n}\right) \sin \left(\theta_{j}\right)-\left(\delta_{j}^{t}\right) \cos \left(\theta_{j}\right)\right\} \overrightarrow{\boldsymbol{I}}_{\boldsymbol{j} \boldsymbol{r}}+ \\
& \left\{\left(R_{j}+\delta_{j}^{n}\right) \cos \left(\theta_{j}\right)-\left(\delta_{j}^{t}\right) \sin \left(\theta_{j}\right)\right\} \overrightarrow{\boldsymbol{J}}_{\boldsymbol{j} \boldsymbol{r}}+ \\
& \left\{-\left[A_{r}\left(R_{j}+\delta_{j}^{n}\right)+B_{r} \delta_{j}^{t}\right] \cos \left(\theta_{j}+\alpha_{j}\right)-\right. \\
& {\left.\left[B_{r}\left(R_{j}+\delta_{j}^{n}\right)-A_{r} \delta_{j}^{t}\right] \sin \left(\theta_{j}+\alpha_{j}\right)+\left(z_{j}+\delta_{j}^{a}\right)\right\} \overrightarrow{\boldsymbol{K}}_{\boldsymbol{j} \boldsymbol{r}} }
\end{aligned}
$$




$$
\begin{aligned}
\left(P_{j}-C_{j}^{\prime}\right)= & \left\{-\left(R_{j}+\delta_{j}^{n}\right) \sin \left(\theta_{j}+\alpha_{j}-\alpha_{b}\right)-\left(\delta_{j}^{t}\right) \cos \left(\theta_{j}+\alpha_{j}-\alpha_{b}\right)\right\} \overrightarrow{\boldsymbol{I}}_{\boldsymbol{b} \boldsymbol{r}}+ \\
& \left\{\left(R_{j}+\delta_{j}^{n}\right) \cos \left(\theta_{j}+\alpha_{j}-\alpha_{b}\right)-\left(\delta_{j}^{t}\right) \sin \left(\theta_{j}+\alpha_{j}-\alpha_{b}\right)\right\} \overrightarrow{\boldsymbol{J}}_{\boldsymbol{b} \boldsymbol{r}}
\end{aligned}
$$

where (see Fig. A.3)

$\left(A_{r}, B_{r}\right)$ are the rigid body angular misalignments of the journal relative to the bearing around the $\overrightarrow{\boldsymbol{I}}_{\boldsymbol{b}}$ and $\overrightarrow{\boldsymbol{J}}_{\boldsymbol{b}}$ directions, respectively, i.e. $A_{r}=\left(A_{j}-A_{b}\right)$ and $B_{r}=\left(B_{j}-B_{b}\right)[\mathrm{rad}]$;

$\theta_{b, j}$ is the angular bearing/journal coordinate defined from the $\overrightarrow{\boldsymbol{J}}_{\boldsymbol{b}_{\boldsymbol{r}}, \boldsymbol{j}_{\boldsymbol{r}}}$ axes [rad];

$\alpha_{b, j}$ is the angular bearing/journal rotation defined from the $\overrightarrow{\boldsymbol{J}}_{\boldsymbol{b}_{\boldsymbol{r}}, \boldsymbol{j}_{\boldsymbol{r}}}$ axes [rad].

The relationship among the angular quantities $\alpha_{b, j}$ and $\theta_{b, j}$ can be written as:

$$
\theta_{j}=\theta_{b}+\left(\alpha_{b}-\alpha_{j}\right)-\delta
$$

where $\delta$ is the local relative slope on the journal surface (see Fig. A.3). By neglecting the lubricant film curvature, i.e. $\delta \ll 1$, the following approximations can be assumed: $\sin (\delta) \approx \delta \approx-\frac{\partial h}{\partial x_{b_{l}}} \approx \frac{1}{R_{b}} \frac{\partial h}{\partial \theta_{b}}$, so that $\left(\theta_{j}+\alpha_{j}\right) \approx\left(\theta_{b}+\alpha_{b}\right)$, where $x_{b_{l}}$ is the local tangential coordinate at $P_{b}$ (see Fig. A.3). Moreover, it is also admitted that the axial coordinates of $C_{b}$ and $C_{j}$ are virtually unchanged during the journal bearing motion, i.e. $z_{j} \equiv z_{b}$. Thus, Eqs. A.12 and A.13 can be expressed as:

$$
\begin{aligned}
\left(P_{j}-C_{j}\right)=\{ & \left.-\left(R_{j}+\delta_{j}^{n}\right) \sin \left(\theta_{j}\right)-\left(\delta_{j}^{t}\right) \cos \left(\theta_{j}\right)\right\} \overrightarrow{\boldsymbol{I}}_{\boldsymbol{j} \boldsymbol{r}}+ \\
& \left\{\left(R_{j}+\delta_{j}^{n}\right) \cos \left(\theta_{j}\right)-\left(\delta_{j}^{t}\right) \sin \left(\theta_{j}\right)\right\} \overrightarrow{\boldsymbol{J}}_{\boldsymbol{j} \boldsymbol{r}}+ \\
& \left\{-\left[A_{r}\left(R_{j}+\delta_{j}^{n}\right)+B_{r} \delta_{j}^{t}\right] \cos \left(\theta_{b}+\alpha_{b}\right)-\right. \\
& {\left[B_{r}\left(R_{j}+\delta_{j}^{n}\right)-A_{r} \delta_{j}^{t}\right] \sin \left(\theta_{b}+\alpha_{b}\right)+} \\
& \left.\left(z_{b}+\delta_{j}^{a}\right)\right\} \overrightarrow{\boldsymbol{K}}_{\boldsymbol{j} \boldsymbol{r}} \\
\left(P_{j}-C_{j}^{\prime}\right)= & \left\{-\left(R_{j}+\delta_{j}^{n}\right) \sin \left(\theta_{b}\right)-\left(\delta_{j}^{t}\right) \cos \left(\theta_{b}\right)\right\} \overrightarrow{\boldsymbol{I}}_{\boldsymbol{b} \boldsymbol{r}} \\
+ & \left\{\left(R_{j}+\delta_{j}^{n}\right) \cos \left(\theta_{b}\right)-\left(\delta_{j}^{t}\right) \sin \left(\theta_{b}\right)\right\} \overrightarrow{\boldsymbol{J}}_{\boldsymbol{b} \boldsymbol{r}}
\end{aligned}
$$

Finally, by combining Eqs. A.15 and Eqs. A.12 and after some coordinate transformations, as well as by disregarding intermediate of magnitude order $\mathcal{O}\left(\frac{c}{R}\right)^{n} \ll 1(n \geq 1)$, the geometry of the lubricant film thickness for the current journal bearing model can be written as follows:

$$
\begin{aligned}
& H_{2}\left(\theta_{b}, z_{b}\right)=\underbrace{c-\left(Y_{r}-A_{r} z_{b}\right) \cos \left(\theta_{b}+\alpha_{b}\right)+\left(X_{r}+B_{r} z_{b}\right) \sin \left(\theta_{b}+\alpha_{b}\right)}_{h}-\delta_{j}^{n}\left(\theta_{b}, z_{b}\right) \\
& H_{1}\left(\theta_{b}, z_{b}\right)=-\delta_{b}^{n}\left(\theta_{b}, z_{b}\right)
\end{aligned}
$$

where $c$ is the nominal radial clearance $c=\left(R_{b}-R_{j}\right)$. The dimensionless version of Eqs. A.16 can be expressed by using the parameters listed in Appendix A.3. Thus:

$$
\begin{aligned}
& \bar{H}_{2}\left(\theta_{b}, \bar{z}_{b}\right)=\underbrace{1-\left(\bar{Y}_{r}-\bar{A}_{r} \bar{z}_{b}\right) \cos \left(\theta_{b}+\alpha_{b}\right)+\left(\bar{X}_{r}+\bar{B}_{r} \bar{z}_{b}\right) \sin \left(\theta_{b}+\alpha_{b}\right)}_{\bar{h}}-\bar{\delta}_{j}^{n}\left(\theta_{b}, \bar{z}_{b}\right) \\
& \bar{H}_{1}\left(\theta_{b}, \bar{z}_{b}\right)=-\bar{\delta}_{b}^{n}\left(\theta_{b}, \bar{z}_{b}\right)
\end{aligned}
$$




\section{Kinematic of the Contact Surfaces}

The absolute velocities of the bearing/journal peripheral points $P_{b, j}$ (see Fig. A.3) relative to the reference frame $\mathcal{R}$ can be defined as:

$$
{ }^{\mathcal{R}} \overrightarrow{\boldsymbol{V}}^{P_{b, j}}={ }^{\mathcal{B}_{r}, \mathcal{J}_{r}} \overrightarrow{\boldsymbol{V}}^{P_{b, j}}+{ }^{\mathcal{R}} \overrightarrow{\boldsymbol{V}}^{C_{b, j}}+{ }^{\mathcal{R}} \overrightarrow{\boldsymbol{\Omega}}^{\mathcal{B}_{r}, \mathcal{J}_{r}} \wedge\left(P_{b, j}-C_{b, j}\right)
$$

with

$$
\begin{aligned}
& { }^{\mathcal{R}} \overrightarrow{\boldsymbol{V}}^{C_{b, j}}=\left(\dot{X}_{C_{b, j}}\right) \overrightarrow{\boldsymbol{I}}+\left(\dot{Y}_{C_{b, j}}\right) \overrightarrow{\boldsymbol{J}}+\left(\dot{Z}_{C_{b, j}}\right) \overrightarrow{\boldsymbol{K}}_{\boldsymbol{b}, \boldsymbol{j}}= \\
& \left\{\left(\dot{X}_{C_{b, j}}\right) \cos \left(\alpha_{b, j}\right)+\left(\dot{Y}_{C_{b, j}}\right) \sin \left(\alpha_{b, j}\right)\right\} \overrightarrow{\boldsymbol{I}}_{\boldsymbol{b} \boldsymbol{r}, \boldsymbol{j} \boldsymbol{r}}+ \\
& \left\{\left(\dot{Y}_{C_{b, j}}\right) \cos \left(\alpha_{b, j}\right)-\left(\dot{X}_{C_{b, j}}\right) \sin \left(\alpha_{b, j}\right)\right\} \overrightarrow{\boldsymbol{J}}_{\boldsymbol{b} \boldsymbol{r}, \boldsymbol{j} \boldsymbol{r}}+ \\
& \left\{\dot{Z}_{C_{b, j}}+B_{b, j} \dot{X}_{C_{b, j}}-A_{b, j} \dot{Y}_{C_{b, j}}\right\} \overrightarrow{\boldsymbol{K}}_{\boldsymbol{b r}, \boldsymbol{j} \boldsymbol{r}} \\
& \mathcal{B}_{\boldsymbol{r}}, \mathfrak{J}_{\boldsymbol{r}} \overrightarrow{\boldsymbol{V}}^{P_{b, j}}=\left(\dot{\delta}_{b, j}^{t}\right) \overrightarrow{\boldsymbol{i}}_{\boldsymbol{b l}, \boldsymbol{j} \boldsymbol{l}}+\left(-\dot{\delta}_{b, j}^{n}\right) \overrightarrow{\boldsymbol{j}}_{\boldsymbol{b l}, \boldsymbol{j} \boldsymbol{l}}+\left(\dot{\delta}_{b, j}^{a}\right) \overrightarrow{\boldsymbol{k}}_{\boldsymbol{b l}, \boldsymbol{j} \boldsymbol{l}}= \\
& \left\{-\left(\dot{\delta}_{b, j}^{t}\right) \cos \left(\theta_{b, j}\right)-\left(\dot{\delta}_{b, j}^{n}\right) \sin \left(\theta_{b, j}\right)\right\} \overrightarrow{\boldsymbol{I}}_{\boldsymbol{b} \boldsymbol{r}, \boldsymbol{j} \boldsymbol{r}}+ \\
& \left\{\left(\dot{\delta}_{b, j}^{n}\right) \cos \left(\theta_{b, j}\right)-\left(\dot{\delta}_{b, j}^{t}\right) \sin \left(\alpha_{b, j}\right)\right\} \overrightarrow{\boldsymbol{J}}_{\boldsymbol{b} \boldsymbol{r}, \boldsymbol{j} \boldsymbol{r}}+ \\
& \left\{\dot{\delta}_{b, j}^{a}\right\} \overrightarrow{\boldsymbol{K}}_{\boldsymbol{b r}, \boldsymbol{j} \boldsymbol{r}} \\
& { }^{\mathcal{R}} \overrightarrow{\boldsymbol{\Omega}}^{\mathcal{B}_{r}, \mathfrak{J}_{r}}=\left\{\dot{A}_{b, j}\right\} \overrightarrow{\boldsymbol{I}}+\left\{\left[\dot{B}_{b, j}\right] \cos \left(A_{b, j}\right)\right\} \overrightarrow{\boldsymbol{J}}+ \\
& \left\{\left[\dot{B}_{b, j}\right] \sin \left(A_{b, j}\right)\right\} \overrightarrow{\boldsymbol{K}}+\left\{\omega_{b, j}\right\} \overrightarrow{\boldsymbol{K}}_{\boldsymbol{b}, \boldsymbol{j}}= \\
& \left\{\left[\dot{A}_{b, j}\right] \cos \left(\alpha_{b, j}\right)+\left[\dot{B}_{b, j}\right] \sin \left(\alpha_{b, j}\right)\right\} \overrightarrow{\boldsymbol{I}}_{\boldsymbol{b} \boldsymbol{r}, \boldsymbol{j} \boldsymbol{r}}+ \\
& \left\{\left[\dot{B}_{b, j}\right] \cos \left(\alpha_{b, j}\right)-\left[\dot{A}_{b, j}\right] \sin \left(\alpha_{b, j}\right)\right\} \overrightarrow{\boldsymbol{J}}_{\boldsymbol{b} \boldsymbol{r}, \boldsymbol{j} \boldsymbol{r}}+ \\
& \left\{\dot{B}_{b, j} \dot{A}_{b, j}+\omega_{b, j}\right\} \overrightarrow{\boldsymbol{K}}_{\boldsymbol{b r}, \boldsymbol{j} \boldsymbol{r}}
\end{aligned}
$$

where the over-dots designate time derivatives and $\dot{Z}_{C_{b, j}}$ are the bearing/journal axial velocities. By substituting Eqs. A.19 and Eqs. A.13 into Eq. A.18 and adopting the dimensionless parameters listed in Appendix A.3, the components of the absolute velocities of $P_{b}$ and $P_{j}$ in the local coordinate system $\boldsymbol{S}_{\mathcal{B}_{l}}$ can be expressed as:

$$
\begin{aligned}
& \bar{U}_{1}=\left\{\left(\frac{c}{R}\right)\left[\left(-\dot{\bar{X}}_{C_{b}}-\dot{\bar{B}}_{b} \bar{z}_{b}\right) \cos \left(\theta_{b}+\alpha_{b}\right)+\left(-\dot{\bar{Y}}_{C_{b}}+\dot{\bar{A}}_{b} \bar{z}_{b}\right) \sin \left(\theta_{b}+\alpha_{b}\right)\right]+\right. \\
&\left.\left(\frac{c}{R}\right)\left[2 \pi \bar{\omega}_{b} \bar{\delta}_{b}^{n}+\dot{\bar{\delta}}_{b}^{t}\right]+\left(2 \pi \bar{\omega}_{b}\right)\right\} \\
& \bar{V}_{1}=\{ {\left[\left(-\dot{\bar{Y}}_{C_{b}}+\dot{\overline{\bar{A}}}_{b} \bar{z}_{b}\right) \cos \left(\theta_{b}+\alpha_{b}\right)+\left(\dot{\bar{X}}_{C_{b}}+\dot{\bar{B}}_{b} \bar{z}_{b}\right) \sin \left(\theta_{b}+\alpha_{b}\right)\right]+} \\
& {\left.\left[2 \pi \bar{\omega}_{b} \bar{\delta}_{b}^{t}-\dot{\bar{\delta}}_{b}^{n}\right]\right\} } \\
& \bar{W}_{1}=\left\{\left(\frac{2 c}{L r_{L D}}\right)\left[\left(\dot{\overline{\bar{A}}}_{b}\right) \cos \left(\theta_{b}+\alpha_{b}\right)+\left(\dot{\bar{B}}_{b}\right) \sin \left(\theta_{b}+\alpha_{b}\right)\right]+\right.
\end{aligned}
$$




$$
\begin{aligned}
& {\left.\left[\left(\frac{2 c}{L}\right) \dot{\bar{\delta}}_{b}^{a}+\dot{\overline{\bar{Z}}}_{C_{b}}\right]\right\} } \\
& \bar{U}_{2}=\left\{\left(\frac{c}{R}\right)\left[\left(-\dot{\bar{X}}_{C_{j}}-\dot{\bar{B}}_{j} \bar{z}_{b}\right) \cos \left(\theta_{b}+\alpha_{b}\right)+\left(-\dot{\bar{Y}}_{C_{j}}+\dot{\bar{A}}_{j} \bar{z}_{b}\right) \sin \left(\theta_{b}+\alpha_{b}\right)\right]+\right. \\
& {\left.\left[\left(\frac{c}{R}\right)\left(2 \pi \bar{\omega}_{j} \bar{\delta}_{j}^{n}+\dot{\bar{\delta}}_{j}^{t}\right)+\left(2 \pi \bar{\omega}_{j}\right)\right]\right\} } \\
& \bar{V}_{2}=\left\{\left[\left(-\dot{\bar{Y}}_{C_{j}}+\dot{\bar{A}}_{j} \bar{z}_{b}\right) \cos \left(\theta_{b}+\alpha_{b}\right)+\left(\dot{\bar{X}}_{C_{j}}+\dot{\bar{B}}_{j} \bar{z}_{b}\right) \sin \left(\theta_{b}+\alpha_{b}\right)\right]+\right. \\
& \bar{W}_{2}=\left\{\left(\frac{2 c}{L r_{L D}}\right)\left[\left(\dot{\overline{\bar{A}}}_{j}+2 \pi \bar{\omega}_{j} \bar{\delta}_{j}^{t}-\dot{\bar{\delta}}_{j}^{n}\right)+\left(2 \pi \bar{\omega}_{j} \frac{\partial \bar{h}}{\partial \theta_{b}}\right)\right]\right\} \\
& {\left.\left[\left(\frac{2 c}{L}\right) \dot{\bar{\delta}}_{j}^{a}+\dot{\overline{\bar{Z}}}_{C_{j}}\right]\right\} }
\end{aligned}
$$

In addition, such components can be further simplified by neglecting the terms of magnitude order $\mathcal{O}\left(\frac{c}{R}\right)^{n} \ll 1, \mathcal{O}\left(\frac{c}{L}\right)^{n} \ll 1, \mathcal{O}\left(\frac{c}{L r_{L D}}\right)^{n} \ll 1(n \geq 1)$. In particular, the terms of order $\mathcal{O}\left(\frac{c}{L r_{L D}}\right)$ are associated with the "axial wedge effect", whose influence on the hydrodynamic pressure calculation is significant only for very short journal bearings [222]. This extreme bearing case will not be considered in the present work. Accordingly:

$$
\begin{aligned}
& \bar{U}_{1}=\left(2 \pi \bar{\omega}_{b}\right) \\
& \bar{V}_{1}=\left\{\left[\left(-\dot{\bar{Y}}_{C_{b}}+\dot{\overline{\bar{A}}}_{b} \bar{z}_{b}\right) \cos \left(\theta_{b}+\alpha_{b}\right)+\left(\dot{\bar{X}}_{C_{b}}+\dot{\bar{B}}_{b} \bar{z}_{b}\right) \sin \left(\theta_{b}+\alpha_{b}\right)\right]+\right. \\
& {\left.\left[2 \pi \bar{\omega}_{b} \bar{\delta}_{b}^{t}-\dot{\bar{\delta}}_{b}^{n}\right]\right\} } \\
& \bar{W}_{1}=\left(\dot{\bar{Z}}_{C_{b}}\right) \\
& \bar{U}_{2}=\left(2 \pi \bar{\omega}_{j}\right) \\
& \bar{V}_{2}=\left\{\left[\left(-\dot{\bar{Y}}_{C_{j}}+\dot{\bar{A}}_{j} \bar{z}_{b}\right) \cos \left(\theta_{b}+\alpha_{b}\right)+\left(\dot{\bar{X}}_{C_{j}}+\dot{\bar{B}}_{j} \bar{z}_{b}\right) \sin \left(\theta_{b}+\alpha_{b}\right)\right]+\right. \\
& {\left.\left[\left(2 \pi \bar{\omega}_{j} \bar{\delta}_{j}^{t}-\dot{\bar{\delta}}_{j}^{n}\right)+\left(2 \pi \bar{\omega}_{j} \frac{\partial \bar{h}_{h}}{\partial \theta_{b}}\right)\right]\right\} } \\
& \bar{W}_{2}=\left(\dot{\bar{Z}}_{C_{j}}\right)
\end{aligned}
$$

\section{A.5 Operational Parameters}

Next, the operational parameters related to the journal bearing model are exposed for completeness. All vector quantities are defined with respect to the bearing coordinate system $\boldsymbol{S}_{\mathcal{B}}$. 
Journal Eccentricity in the Bearing Mid-Plane $\left(\bar{z}_{b}=0\right)$

$$
\varepsilon=\sqrt{\bar{X}_{r}^{2}+\bar{Y}_{r}^{2}}
$$

Attitude Angle in the Bearing Mid-Plane $\left(\bar{z}_{b}=0\right)$

$$
\Psi=\arctan \left(-\frac{\bar{X}_{r}}{\bar{Y}_{r}}\right) \quad \Psi \in[0,2 \pi[
$$

Attack Angle in the Bearing Mid-Plane $\left(\bar{z}_{b}=0\right)$

$$
\varphi=\Psi-\arctan \left(-\frac{\bar{W}_{H_{X}}}{\bar{W}_{H_{Y}}}\right) \quad \varphi \in[0, \pi[
$$

Hydrodynamic Normal Forces

$$
\begin{gathered}
\overrightarrow{\boldsymbol{W}}_{\boldsymbol{H}}=\left[\bar{W}_{H_{X}} \cos \left(\alpha_{b}\right)-\bar{W}_{H_{Y}} \sin \left(\alpha_{b}\right)\right] \overrightarrow{\boldsymbol{I}}_{\boldsymbol{b}}+\left[\bar{W}_{H_{Y}} \cos \left(\alpha_{b}\right)+\bar{W}_{H_{Y}} \sin \left(\alpha_{b}\right)\right] \overrightarrow{\boldsymbol{J}}_{\boldsymbol{b}} \\
\bar{W}_{H_{X}}=\frac{1}{4} \int_{-1}^{1} \int_{0}^{2 \pi}\left[\bar{p}_{H}\left(\theta_{b}, \bar{z}_{b}\right) \sin \left(\theta_{b}\right)\right] d \theta_{b} d \bar{z}_{b} \\
\bar{W}_{H_{Y}}=-\frac{1}{4} \int_{-1}^{1} \int_{0}^{2 \pi}\left[\bar{p}_{H}\left(\theta_{b}, \bar{z}_{b}\right) \cos \left(\theta_{b}\right)\right] d \theta_{b} d \bar{z}_{b} \\
\overrightarrow{\vec{W}}_{\boldsymbol{H}}^{(j)}=\overrightarrow{\boldsymbol{W}}_{\boldsymbol{H}} \quad \overrightarrow{\boldsymbol{W}}_{\boldsymbol{H}}^{(b)}=-\overrightarrow{\boldsymbol{W}}_{\boldsymbol{H}}
\end{gathered}
$$

\section{Asperity Contact Normal Forces}

$$
\begin{aligned}
\overrightarrow{\boldsymbol{W}}_{\boldsymbol{A S P}}=\left[\bar{W}_{A S P_{X}} \cos \left(\alpha_{b}\right)-\bar{W}_{A S P_{Y}} \sin \left(\alpha_{b}\right)\right] \overrightarrow{\boldsymbol{I}}_{\boldsymbol{b}}+\left[\bar{W}_{A S P_{X}} \cos \left(\alpha_{b}\right)+\bar{W}_{A S P_{Y}} \sin \left(\alpha_{b}\right)\right] \overrightarrow{\boldsymbol{J}}_{\boldsymbol{b}} \\
\bar{W}_{A S P_{X}}=\frac{1}{4} \int_{-1}^{1} \int_{0}^{2 \pi}\left[\bar{p}_{A S P}^{G W, G T}\left(\theta_{b}, \bar{z}_{b}\right) \sin \left(\theta_{b}\right)\right] d \theta_{b} d \bar{z}_{b} \\
\bar{W}_{A S P_{Y}}=-\frac{1}{4} \int_{-1}^{1} \int_{0}^{2 \pi}\left[\bar{p}_{A S P}^{(G W, G T)}\left(\theta_{b}, \bar{z}_{b}\right) \cos \left(\theta_{b}\right)\right] d \theta_{b} d \bar{z}_{b} \\
\overrightarrow{\boldsymbol{W}}_{\boldsymbol{A S P}}^{(j)}=\overrightarrow{\boldsymbol{W}}_{\boldsymbol{A S P}} \quad \overrightarrow{\boldsymbol{W}}_{\boldsymbol{A S P}}^{(b)}=-\overrightarrow{\boldsymbol{W}}_{\boldsymbol{A S P}}
\end{aligned}
$$

Moments of the Hydrodynamic Normal Forces

$$
\overrightarrow{\boldsymbol{M}}_{\boldsymbol{H}}^{C_{b}}=\left[\bar{M}_{H_{X}}^{C_{b}} \cos \left(\alpha_{b}\right)-\bar{M}_{H_{Y}}^{C_{b}} \sin \left(\alpha_{b}\right)\right] \overrightarrow{\boldsymbol{I}}_{\boldsymbol{b}}+\left[\bar{M}_{H_{Y}}^{C_{b}} \cos \left(\alpha_{b}\right)+\bar{M}_{H_{X}}^{C_{b}} \sin \left(\alpha_{b}\right)\right] \overrightarrow{\boldsymbol{J}}_{\boldsymbol{b}}
$$




$$
\begin{aligned}
& \bar{M}_{H_{X}}^{C_{b}}=\frac{1}{8} \int_{-1}^{1} \int_{0}^{2 \pi}\left[\bar{p}_{H}\left(\theta_{b}, \bar{z}_{b}\right) \cos \left(\theta_{b}\right) \bar{z}_{b}\right] d \theta_{b} d \bar{z}_{b} \\
& \bar{M}_{H_{Y}}^{C_{b}}=\frac{1}{8} \int_{-1}^{1} \int_{0}^{2 \pi}\left[\bar{p}_{H}\left(\theta_{b}, \bar{z}_{b}\right) \sin \left(\theta_{b}\right) \bar{z}_{b}\right] d \theta_{b} d \bar{z}_{b} \\
& \overrightarrow{\boldsymbol{M}}_{\boldsymbol{H}}^{C_{b}(j)}=\overrightarrow{\boldsymbol{M}}_{\boldsymbol{H}}^{C_{b}} \quad \overrightarrow{\vec{M}}_{\boldsymbol{H}}^{C_{b}(b)}=-\overrightarrow{\vec{M}}_{\boldsymbol{H}}^{C_{b}}
\end{aligned}
$$

\section{Moments of the Asperity Contact Normal Forces}

$$
\begin{gathered}
\overrightarrow{\overrightarrow{\boldsymbol{M}}}_{\boldsymbol{A} \boldsymbol{S P}}^{C_{b}}=\left[\bar{M}_{A S P_{X}}^{C_{b}} \cos \left(\alpha_{b}\right)-\bar{M}_{A S P_{Y}}^{C_{b}} \sin \left(\alpha_{b}\right)\right] \overrightarrow{\boldsymbol{I}}_{\boldsymbol{b}}+\left[\bar{M}_{A S P_{Y}}^{C_{b}} \cos \left(\alpha_{b}\right)+\bar{M}_{A S P_{X}}^{C_{b}} \sin \left(\alpha_{b}\right)\right] \overrightarrow{\boldsymbol{J}}_{\boldsymbol{b}} \\
\bar{M}_{A S P_{X}}^{C_{b}}=\frac{1}{8} \int_{-1}^{1} \int_{0}^{2 \pi}\left[\bar{p}_{A S P}^{(G W, G T)}\left(\theta_{b}, \bar{z}_{b}\right) \cos \left(\theta_{b}\right) \bar{z}_{b}\right] d \theta_{b} d \bar{z}_{b} \\
\bar{M}_{A S P_{Y}}^{C_{b}}=\frac{1}{8} \int_{-1}^{1} \int_{0}^{2 \pi}\left[\bar{p}_{A S P}^{(G W, G T)}\left(\theta_{b}, \bar{z}_{b}\right) \sin \left(\theta_{b}\right) \bar{z}_{b}\right] d \theta_{b} d \bar{z}_{b} \\
\overrightarrow{\boldsymbol{M}}_{\boldsymbol{A S P}}^{C_{b}(j)}=\overrightarrow{\boldsymbol{M}}_{\boldsymbol{A S P}}^{C_{b}} \\
\overrightarrow{\boldsymbol{M}}_{\boldsymbol{A S P}}^{C_{b}(b)}=-\overrightarrow{\boldsymbol{M}}_{\boldsymbol{A S P}}^{C_{b}}
\end{gathered}
$$

Hydrodynamic Friction Forces

$$
\begin{gathered}
\overrightarrow{\overrightarrow{\boldsymbol{F}}}_{\boldsymbol{H}}^{(j, b)}=\left(\bar{F}_{H_{Z}}^{(j, b)}\right) \overrightarrow{\boldsymbol{K}}_{\boldsymbol{b}} \\
\bar{F}_{H_{Z}}^{(j, b)}=\frac{1}{4} \int_{-1}^{1} \int_{0}^{2 \pi}\left[\bar{\tau}_{H_{z_{b} y}}^{(j, b)}\left(\theta_{b}, \bar{z}_{b}\right)\right] d \theta_{b} d \bar{z}_{b}
\end{gathered}
$$

\section{Asperity Contact Friction Forces}

$$
\begin{gathered}
\overrightarrow{\overrightarrow{\boldsymbol{F}}}_{\boldsymbol{A} \boldsymbol{S} \boldsymbol{P}}^{(j, b)}=\left(\bar{F}_{A S P_{Z}}^{(j, b)}\right) \overrightarrow{\boldsymbol{K}}_{\boldsymbol{b}} \\
\bar{F}_{A S P_{Z}}^{(j, b)}=\frac{1}{4} \int_{-1}^{1} \int_{0}^{2 \pi}\left[\bar{\tau}_{A S P_{\bar{z}_{b} y}}^{(j, b)}\left(\theta_{b}, \bar{z}_{b}\right)\right] d \theta_{b} d \bar{z}_{b}
\end{gathered}
$$

Moments of the Hydrodynamic Friction Forces (Torque)

$$
\overrightarrow{\overline{\boldsymbol{T}}}_{\boldsymbol{H}}^{C_{b}(j, b)}=\left(\bar{T}_{H_{Z}}^{C_{b}(j, b)}\right) \overrightarrow{\boldsymbol{K}}_{\boldsymbol{b}}
$$




$$
\bar{T}_{H_{Z}}^{C_{b}(j, b)}=\frac{1}{4} \int_{-1}^{1} \int_{0}^{2 \pi}\left[\bar{\tau}_{H_{\theta_{b}}}^{(j, b)}\left(\theta_{b}, \bar{z}_{b}\right)\right] d \theta_{b} d \bar{z}_{b}
$$

Moments of the Asperity Contact Friction Forces (Torque)

$$
\begin{gathered}
\overrightarrow{\overline{\boldsymbol{T}}}_{\boldsymbol{A S \boldsymbol { P }}}^{C_{b}(j, b)}=\left(\bar{T}_{A S P_{Z}}^{C_{b}(j, b)}\right) \overrightarrow{\boldsymbol{K}}_{\boldsymbol{b}} \\
\bar{T}_{A S P_{Z}}^{C_{b}(j, b)}=\frac{1}{4} \int_{-1}^{1} \int_{0}^{2 \pi}\left[\bar{\tau}_{A S P_{\theta_{b} y}}^{(j, b)}\left(\theta_{b}, \bar{z}_{b}\right)\right] d \theta_{b} d \bar{z}_{b}
\end{gathered}
$$

Hydrodynamic Power Loss

$$
\bar{P}_{H}^{(j, b)}=\left(\frac{2 \pi}{\Omega}\right)\left|\bar{\omega}_{(j, b)} \bar{T}_{H_{Z}}^{C_{b}(j, b)}\right|+\left(\frac{r_{L D}}{\Omega}\right)\left|\dot{\bar{Z}}_{C_{(b, j)}} \bar{F}_{H_{Z}}^{(j, b)}\right|
$$

Asperity Contact Power Loss

$$
\bar{P}_{A S P}^{(j, b)}=\left(\frac{2 \pi}{\Omega}\right)\left|\bar{\omega}_{(j, b)} \bar{T}_{A S P_{Z}}^{C_{b}(j, b)}\right|+\left(\frac{r_{L D}}{\Omega}\right)\left|\dot{\bar{Z}}_{C_{(b, j)}} \bar{F}_{A S P_{Z}}^{(j, b)}\right|
$$

Hydrodynamic Coefficient of Friction

$$
\bar{f}_{H}^{(j, b)}=\frac{\left|\bar{T}_{H_{Z}}^{C_{b}(j, b)}\right|+\left|\bar{F}_{H_{Z}}^{(j, b)}\right|}{\left|\overrightarrow{\boldsymbol{W}}_{\boldsymbol{H}}\right|}
$$

Asperity Contact Coefficient of Friction

$$
\bar{f}_{A S P}^{(j, b)}=\frac{\left|\bar{T}_{A S P_{Z}}^{C_{b}(j, b)}\right|+\left|\bar{F}_{A S P_{Z}}^{(j, b)}\right|}{\left|\overrightarrow{\boldsymbol{W}}_{\boldsymbol{A S P}}\right|}
$$

Side Flow

$$
\bar{Q}_{a}=\left(\frac{1}{2 r_{L D}}\right) \int_{0}^{2 \pi}\left[\bar{q}_{H_{z}}\left(\theta_{b},-1\right)+\bar{q}_{H_{z}}\left(\theta_{b}, 1\right)\right] d \theta_{b}
$$




\section{Appendix B}

\section{Sliding Bearing Model}

\section{B.1 Reference Frames and Coordinate Systems}

\section{Reference Frames}

Fig. B.1 depicts a generic sliding bearing coupled to a structure which can move relative to other components of a multibody dynamic system. According to the figure, the following reference frames are defined:

ऽ: Inertial (or Newtonian) reference frame.

$\mathcal{R}$ : Reference frame attached to the structure to which the sliding bearing is coupled; in a generic multibody dynamic system, this frame can move with respect to the inertial frame $(\Im)$. On the other hand, in the absence of relative motion, $\mathcal{R}$ coincides with $\Im$, i.e. $\mathcal{R} \equiv \Im$.

$\mathcal{B}$ : Reference frame attached to the bearing. This frame can experience relative motion with respect to the structure $(\mathcal{R})$ only due to angular misalignments of the bearing.

$\mathcal{S}$ : Reference frame attached to the slider. This frame can experience relative motion with respect to the structure $(\mathcal{R})$ only due to angular misalignments of the slider.

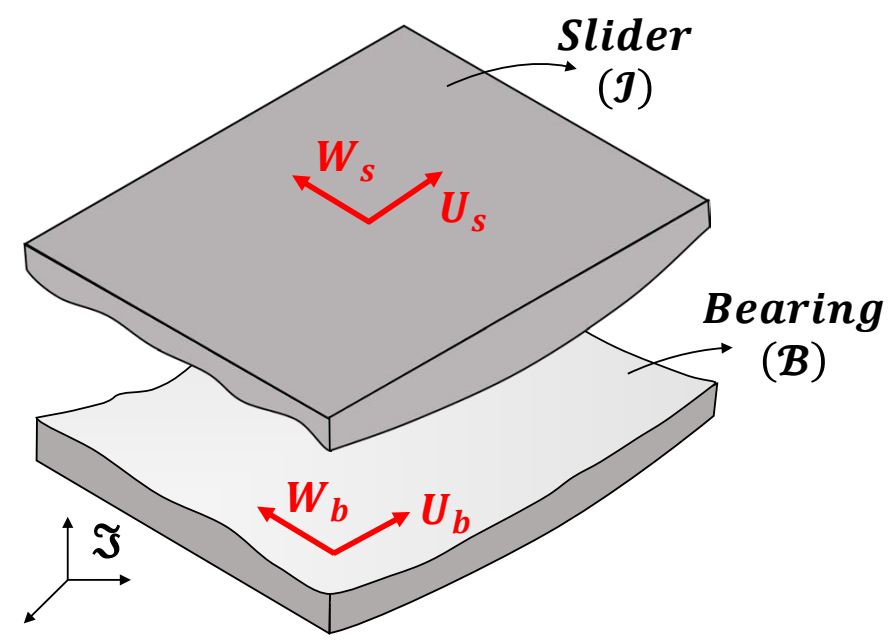

Figure B.1: Reference frames of the sliding bearing model. 

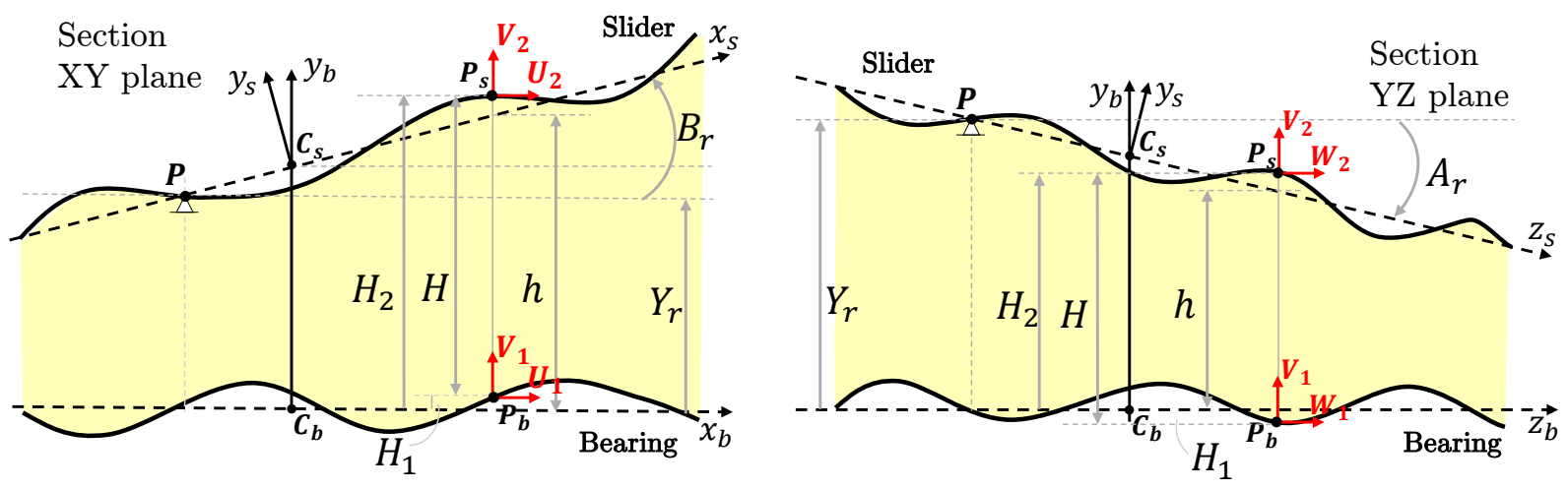

Figure B.2: Main coordinate systems of the sliding bearing model.

\section{Coordinate Systems}

The definition of coordinate systems coherent to the reference frames stated above is important for the general description of the geometric and kinematic features of sliding bearings. The coordinate systems here utilized for the model derivation are listed as follows (see Fig. B.2):

$\mathcal{S}_{\mathcal{R}} \rightarrow[O, \overrightarrow{\boldsymbol{I}}, \overrightarrow{\boldsymbol{J}}, \overrightarrow{\boldsymbol{K}}]_{(X, Y, Z)}:$ Coordinate system attached to frame $\mathcal{R}$.

$\mathcal{S}_{\mathcal{B}} \rightarrow\left[C_{b}, \overrightarrow{\boldsymbol{i}}_{\boldsymbol{b}}, \overrightarrow{\boldsymbol{j}}_{\boldsymbol{b}}, \overrightarrow{\boldsymbol{k}}_{\boldsymbol{b}}\right]_{\left(x_{b}, y_{b}, z_{b}\right)}:$ Coordinate system attached to frame $\mathcal{B}$ and with origin at the geometric centre of the bearing $\left(C_{b}\right)$. The only relative movements of this system with respect to $\mathcal{S}_{\mathcal{R}}$ are due to angular misalignments of the bearing. This reference is also admitted as the principal one from which the system relative rigid body motions and all the operational parameters are determined. Moreover, the complete bearing geometry, including the structural deformations in EHL problems, is described in this system, i.e. the computational mesh used for the numerical solution of Reynolds equation is constructed according to this coordinate system. The local fluid film geometry and surfaces kinematic are expressed in this system as well.

$\boldsymbol{S}_{\mathcal{S}} \rightarrow\left[C_{s}, \overrightarrow{\boldsymbol{i}}_{\boldsymbol{s}}, \overrightarrow{\boldsymbol{j}}_{\boldsymbol{s}}, \overrightarrow{\boldsymbol{k}}_{\boldsymbol{s}}\right]_{\left(x_{s}, y_{s}, z_{s}\right)}:$ Coordinate system attached to frame $\mathcal{S}$ and with origin at the geometric centre of the slider $\left(C_{s}\right)$. The only relative movements of this system with respect to $\mathcal{S}_{\mathcal{R}}$ are due to angular misalignments of the slider. Moreover, the complete slider geometry, including the structural deformations in EHL problems, is described in coordinate system. Notice that as $\boldsymbol{S}_{\mathcal{B}}$ is the principal coordinate system from which Reynolds equation is evaluated, whenever (fixed) geometric defects are present on the slider surface, a special procedure has to be used to locally match the instantaneous slider geometry to the actual bearing mesh.

\section{B.2 Dimensionless Parameters}

As follows, the dimensionless parameters related to the general sliding bearing model are listed for completeness. 
Geometric Parameters

$$
r_{L z x}=\frac{L_{z}}{L_{x}}
$$

\section{Kinematic Parameters}

$$
\begin{array}{rlrl}
\bar{A}_{b, s, r}=\frac{A_{b, s, r}}{\left(\frac{2 h_{0}}{L_{z}}\right)} & \bar{Y}_{b, s, r}=\frac{Y_{b, s, r}}{h_{0}} & \bar{U}_{b, s}=\frac{U_{b, s}}{\left(\frac{L_{x}}{2}\right)} \\
\dot{\bar{A}}_{b, s, r}=\frac{\dot{A}_{b, s, r}}{\left(\frac{2 h_{0}}{L_{z}}\right)} & \dot{\bar{Y}}_{b, s, r}=\frac{\dot{Y}_{b, s, r}}{h_{0}} & \bar{V}_{b, s}=\frac{V_{b, s}}{h_{0}} \\
\bar{B}_{b, s, r}=\frac{B_{b, s, r}}{\left(\frac{2 h b_{0}}{L_{x}}\right)} & \bar{\delta}_{b, s, r}^{n}=\frac{\delta_{b, s, r}^{n}}{h_{0}} & \bar{W}_{b, s}=\frac{W_{b, s}}{\left(\frac{L_{z}}{2}\right)} \\
\dot{\bar{B}}_{b, s, r}=\frac{\dot{B}_{b, s, r}}{\left(\frac{2 h b_{0}}{L_{x}}\right)} & \dot{\bar{\delta}}_{b, s, r}^{n}=\frac{\dot{\delta}_{b, s, r}^{n}}{h_{0}} & \Omega & =\left|\bar{U}_{b}+\bar{U}_{s}\right| \\
\bar{H}_{1,2}=\frac{H_{1,2}}{h_{0}} & \bar{x}_{b, s}=\frac{x_{b, s}}{\left(\frac{L_{x}}{2}\right)} & \bar{y}_{b, s}=\frac{y_{b, s}}{h_{0}} \\
\bar{h} & =\frac{h}{h_{0}} & \bar{z}_{b, s}=\frac{z_{b, s}}{\left(\frac{L_{z}}{2}\right)} &
\end{array}
$$

\section{Hydrodynamic Parameters}

$$
\begin{aligned}
\bar{p}_{H} & =\frac{p_{H}}{\mu_{0} \Omega\left(\frac{L_{x}}{2 h_{0}}\right)^{2}} & \bar{\tau}_{H} & =\frac{\tau_{H}}{\mu_{0} \Omega\left(\frac{L_{x}}{2 h_{0}}\right)} \\
\bar{q}_{H} & =\frac{q_{H}}{\rho_{0} \Omega\left(\frac{L_{x}}{2}\right) h_{0}} & \bar{\rho} & =\frac{\rho}{\rho_{0}} \\
\dot{\bar{\gamma}}_{H} & =\frac{\dot{\gamma}_{H}}{\Omega\left(\frac{L_{x}}{2 h_{0}}\right)} & \bar{\mu} & =\frac{\mu}{\mu_{0}}
\end{aligned}
$$

\section{Operational Parameters}

$$
\begin{aligned}
\bar{f}_{H} & =\frac{f_{H}}{\left(\frac{2 h_{0}}{L_{x}}\right)} & \bar{F}_{H} & =\frac{F_{H}}{\mu_{0} \Omega\left(L_{x} L_{z}\right)\left(\frac{L_{x}}{2 h_{0}}\right)} \\
\bar{P}_{H} & =\frac{P_{H}}{\mu_{0} \Omega\left(L_{x} L_{z}\right)\left(\frac{L_{x}}{2 h_{0}}\right)\left(\frac{L_{x}}{2}\right) \Omega} & S & =\frac{\mu_{0} \Omega\left(L_{x} L_{z}\right)}{\left|\overrightarrow{\boldsymbol{W}}_{H_{Y}}\right|}\left(\frac{L_{x}}{2 h_{0}}\right)^{2}=\frac{1}{\left|\bar{W}_{H_{y_{b}}}\right|} \\
\bar{M}_{H_{H_{x_{b},}, z_{b}}} & =\frac{M_{H_{y_{b}}}}{\mu_{0} \Omega\left(L_{x} L_{z}\right)\left(\frac{L_{x}}{2 h_{0}}\right)^{2}\left(\frac{L_{z, x}}{2}\right)} & \bar{M}_{H_{y_{b}}} & =\frac{L_{x}}{\mu_{0} \Omega\left(L_{x} L_{z}\right)\left(\frac{L_{x}}{2 h_{0}}\right)\left(\frac{L_{z}}{2}\right)}
\end{aligned}
$$




$$
\begin{aligned}
\bar{T}_{H_{x_{b}, z_{b}}} & =\frac{T_{H_{x_{b}, z_{b}}}}{\mu_{0} \Omega\left(L_{x} L_{z}\right)\left(\frac{L_{x}}{2 h_{0}}\right)\left(\frac{L_{z, x}}{2}\right)} & \bar{T}_{H_{y_{b}}} & =\frac{T_{H_{y_{b}}}}{\mu_{0} \Omega\left(L_{x} L_{z}\right)\left(\frac{L_{x}}{2 h_{0}}\right)\left(\frac{L_{z}}{2}\right)} \\
\bar{W}_{H_{x_{b}, z_{b}}} & =\frac{W_{H_{x_{b}, z_{b}}}}{\mu_{0} \Omega\left(L_{x} L_{z}\right)\left(\frac{L_{x}}{2 h_{0}}\right)} & \bar{W}_{H_{y_{b}}} & =\frac{W_{H_{y_{b}}}}{\mu_{0} \Omega\left(L_{x} L_{z}\right)\left(\frac{L_{x}}{2 h_{0}}\right)^{2}}
\end{aligned}
$$

\section{B.3 Operational Parameters}

Next, the operational parameters related to the sliding bearing model are exposed for completeness. All vector quantities are defined with respect to the bearing coordinate system $\boldsymbol{S}_{\mathcal{B}}$.

\section{Hydrodynamic Normal Forces}

$$
\begin{gathered}
\overrightarrow{\boldsymbol{W}}_{\boldsymbol{H}}=\left(\bar{W}_{H_{y_{b}}}\right) \overrightarrow{\boldsymbol{j}}_{\boldsymbol{b}} \\
\bar{W}_{H_{y_{b}}}=\frac{1}{4} \int_{-1}^{1} \int_{-1}^{1}\left[\bar{p}_{H}\left(\bar{x}_{b}, \bar{z}_{b}\right)\right] d \bar{x}_{b} d \bar{z}_{b} \\
\overrightarrow{\overrightarrow{\boldsymbol{W}}}_{\boldsymbol{H}}^{(s)}=\overrightarrow{\overrightarrow{\boldsymbol{W}}}_{\boldsymbol{H}}^{(b)} \quad \overrightarrow{\boldsymbol{W}}_{\boldsymbol{H}}^{(b)}=-\overrightarrow{\boldsymbol{W}}_{\boldsymbol{H}}
\end{gathered}
$$

\section{Asperity Contact Normal Forces}

$$
\begin{gathered}
\overrightarrow{\boldsymbol{W}}_{\boldsymbol{A S P}}=\left(\bar{W}_{A S P_{y_{b}}}\right) \overrightarrow{\boldsymbol{j}}_{\boldsymbol{b}} \\
\bar{W}_{A S P_{y_{b}}}=\frac{1}{4} \int_{-1}^{1} \int_{-1}^{1}\left[\bar{p}_{A S P}\left(\bar{x}_{b}, \bar{z}_{b}\right)\right] d \bar{x}_{b} d \bar{z}_{b} \\
\overrightarrow{\boldsymbol{W}}_{\boldsymbol{A} \boldsymbol{S P}}^{(s)}=\overrightarrow{\boldsymbol{W}}_{\boldsymbol{A S P}}^{(b)} \quad \overrightarrow{\boldsymbol{W}}_{\boldsymbol{A S P}}^{(b)}=-\overrightarrow{\boldsymbol{W}}_{\boldsymbol{A S P}}
\end{gathered}
$$

Moments of the Hydrodynamic Normal Forces

$$
\begin{gathered}
\overrightarrow{\overrightarrow{\boldsymbol{M}}}_{\boldsymbol{H}}^{C_{b}}=\left(\bar{M}_{H_{x_{b}}}^{C_{b}}\right) \overrightarrow{\boldsymbol{i}}_{\boldsymbol{b}}+\left(\bar{M}_{H_{z_{b}}}^{C_{b}}\right) \overrightarrow{\boldsymbol{k}}_{\boldsymbol{b}} \\
\bar{M}_{H_{x_{b}}}^{C_{b}}=-\frac{1}{4} \int_{-1}^{1} \int_{-1}^{1}\left[\bar{p}_{H}\left(\bar{x}_{b}, \bar{z}_{b}\right) \bar{z}_{b}\right] d \bar{x}_{b} d \bar{z}_{b} \\
\bar{M}_{H_{z_{b}}}^{C_{b}}=\frac{1}{4} \int_{-1}^{1} \int_{-1}^{1}\left[\bar{p}_{H}\left(\bar{x}_{b}, \bar{z}_{b}\right) \bar{x}_{b}\right] d \bar{x}_{b} d \bar{z}_{b} \\
\overrightarrow{\boldsymbol{M}}_{\boldsymbol{H}}^{C_{b}(s)}=\overrightarrow{\boldsymbol{M}}_{\boldsymbol{H}}^{C_{b}} \quad \overrightarrow{\vec{M}}_{\boldsymbol{H}}^{C_{b}(b)}=-\overrightarrow{\boldsymbol{M}}_{\boldsymbol{H}}^{C_{b}}
\end{gathered}
$$


Moments of the Asperity Contact Normal Forces

$$
\begin{gathered}
\overrightarrow{\boldsymbol{M}}_{A \boldsymbol{A P}}^{C_{b}}=\left(\bar{M}_{A S P_{x_{b}}}^{C_{b}}\right) \overrightarrow{\boldsymbol{i}}_{\boldsymbol{b}}+\left(\bar{M}_{A S P_{z_{b}}}^{C_{b}}\right) \overrightarrow{\boldsymbol{k}}_{\boldsymbol{b}} \\
\bar{M}_{A S P_{x_{b}}}^{C_{b}}=-\frac{1}{4} \int_{-1}^{1} \int_{-1}^{1}\left[\bar{p}_{A S P}\left(\bar{x}_{b}, \bar{z}_{b}\right) \bar{z}_{b}\right] d \bar{x}_{b} d \bar{z}_{b} \\
\bar{M}_{A S P_{z_{b}}}^{C_{b}}=\frac{1}{4} \int_{-1}^{1} \int_{-1}^{1}\left[\bar{p}_{A S P}\left(\bar{x}_{b}, \bar{z}_{b}\right) \bar{x}_{b}\right] d \bar{x}_{b} d \bar{z}_{b} \\
\overrightarrow{\boldsymbol{M}}_{\boldsymbol{A S P}}^{C_{b}(s)}=\overrightarrow{\boldsymbol{M}}_{\boldsymbol{A S P}}^{C_{b}} \quad \overrightarrow{\vec{M}}_{\boldsymbol{A S P} \boldsymbol{P}}^{C_{b}(b)}=-\overrightarrow{\boldsymbol{M}}_{\boldsymbol{A S P} \boldsymbol{P}}^{C_{b}}
\end{gathered}
$$

Hydrodynamic Friction Forces

$$
\begin{gathered}
\overrightarrow{\boldsymbol{F}}_{\boldsymbol{H}}^{(b, s)}=\left(\bar{F}_{H_{x_{b}}}^{(b, s)}\right) \overrightarrow{\boldsymbol{i}}_{\boldsymbol{b}}+\left(\bar{F}_{H_{z_{b}}}^{(b, s)}\right) \overrightarrow{\boldsymbol{k}}_{\boldsymbol{b}} \\
\bar{F}_{H_{x_{b}, z_{b}}^{(b, s)}}^{(b)}=\frac{1}{4} \int_{-1}^{1} \int_{-1}^{1}\left[\bar{\tau}_{H_{x_{b}}, z_{b}}^{(b, s)}\left(\bar{x}_{b}, \bar{z}_{b}\right)\right] d \bar{x}_{b} d \bar{z}_{b}
\end{gathered}
$$

Asperity Contact Friction Forces

$$
\begin{gathered}
\overrightarrow{\boldsymbol{F}}_{\boldsymbol{A S P}}^{(b, s)}=\left(\bar{F}_{A S P_{x_{b}}}^{(b, s)}\right) \overrightarrow{\boldsymbol{i}}_{\boldsymbol{b}}+\left(\bar{F}_{A S P_{z_{b}}}^{(b, s)}\right) \overrightarrow{\boldsymbol{k}}_{\boldsymbol{b}} \\
\bar{F}_{A S P_{x_{b}, z_{b}}}^{(b, s)}=\frac{1}{4} \int_{-1}^{1} \int_{-1}^{1}\left[\bar{\tau}_{{ }^{\left(b S P x_{b}, z_{b}\right.}}^{(b, s)}\left(\bar{x}_{b}, \bar{z}_{b}\right)\right] d \bar{x}_{b} d \bar{z}_{b}
\end{gathered}
$$

Hydrodynamic Power Loss

$$
\bar{P}_{H}^{(b, s)}=\left(\frac{1}{\Omega}\right)\left|\bar{U}^{(b, s)} \bar{F}_{H_{x_{b}}}^{(b, s)}\right|+\left(\frac{r_{L z x}}{\Omega}\right)\left|\bar{W}^{(b, s)} \bar{F}_{H_{z_{b}}}^{(b, s)}\right|
$$

\section{Asperity Contact Power Loss}

$$
\bar{P}_{A S P}^{(b, s)}=\left(\frac{1}{\Omega}\right)\left|\bar{U}^{(b, s)} \bar{F}_{A S P_{x_{b}}}^{(b, s)}\right|+\left(\frac{r_{L z x}}{\Omega}\right)\left|\bar{W}^{(b, s)} \bar{F}_{A S P_{z_{b}}}^{(b, s)}\right|
$$


Hydrodynamic Coefficient of Friction

$$
\bar{f}_{H}^{(b, s)}=\frac{\left|\overrightarrow{\boldsymbol{F}}_{\boldsymbol{H}}{ }^{(b, s)}\right|}{\left|\bar{W}_{H_{y_{b}}}\right|}
$$

Asperity Contact Coefficient of Friction

$$
\bar{f}_{A S P}^{(b, s)}=\frac{\left|\overrightarrow{\boldsymbol{F}}_{\boldsymbol{A S P}}{ }^{(b, s)}\right|}{\left|\bar{W}_{A S P_{y_{b}}}\right|}
$$




\section{Appendix C}

\section{Conformal EHL Model}

\section{C.1 Calculation of the Area Matrix}

In the FEM, all external loads are converted to equivalent forces distributed in the grid nodes (nodal forces). For EHL applications with FEM model reduction, the hydrodynamic pressures are the only external loads acting on the bearing surfaces. Thus, by considering that those pressures act normally on the contact interface, the nodal forces on every element face of the reduced system can be determined as:

$$
\overrightarrow{f_{r}^{e}}=\oiint_{\Gamma_{e}}\left[\boldsymbol{N}_{e}^{\boldsymbol{T}}\left(p_{H} \overrightarrow{\boldsymbol{n}}_{\boldsymbol{e}}\right)\right] d \Gamma_{e}
$$

where $\vec{f}_{\boldsymbol{r}}^{e}$ and $\overrightarrow{\boldsymbol{n}}_{\boldsymbol{e}}$ are the load and normal unit vectors associated with each face of the reduced model (see Fig. C.1), respectively, and $\boldsymbol{N}_{\boldsymbol{e}}$ is the matrix of interpolation functions (see Appendix F.1). Notice that the integrals in Eq. C.1 are computed over bi-dimensional elements (triangular or quadrangular) and that the nodes which compose the element faces correspond to those retained in the reduced model.

The hydrodynamic pressures on the element faces may be approximated as:

$$
p_{H} \approx \boldsymbol{N}_{e} \overrightarrow{\boldsymbol{p}}_{\boldsymbol{H}}
$$

where $\overrightarrow{\boldsymbol{p}}_{\boldsymbol{H}}$ is the nodal pressure vector of the element face. Substituting Eq. C.2 into Eq. C.1, one obtains:

$$
\vec{f}_{r}^{e}=A^{e} \vec{p}_{H_{e}}
$$

with

$$
\boldsymbol{A}^{\boldsymbol{e}}=\overrightarrow{\boldsymbol{n}}_{\boldsymbol{e}} \oiint_{\Gamma_{e}}\left(\boldsymbol{N}_{e} \boldsymbol{T}^{T} \boldsymbol{N}_{e}\right) d \Gamma_{e}
$$

where $\boldsymbol{A}^{\boldsymbol{e}}$ is the element area matrix that correlates the hydrodynamic pressures and the associated nodal loads acting on each element face. In Eqs. C.3, $\overrightarrow{\boldsymbol{n}}_{\boldsymbol{e}}$ is placed out of the integral once the element faces are always plane in the reduced model.

Finally, after the assembly of every element face that compounds the whole bearing surface, the full vector for the reduced system nodal forces can be expressed as:

$$
\overrightarrow{f_{r}}=A \vec{p}_{H}
$$




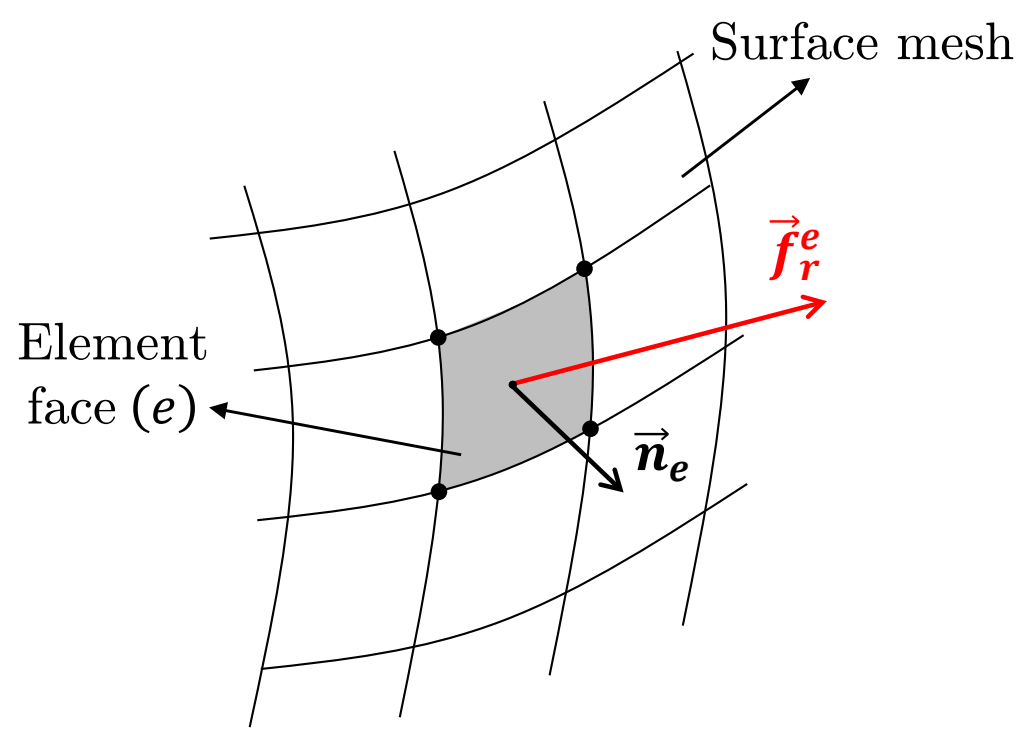

Figure C.1: Illustration of the element faces of the reduced model.

where $\boldsymbol{A}$ is the total area matrix and $\overrightarrow{\boldsymbol{p}}_{\boldsymbol{H}}$ the full nodal vector of the hydrodynamic pressures distributed over the entire bearing surface.

\section{C.2 Substructure Generation into the Abaqus ${ }^{\circledR}$ Soft- ware}

The command lines to be added into the Abaqus ${ }^{\circledR}$ [62] input file for generating the mass, damping and stiffness matrices of the global and reduced FEM models are summarized as follows.

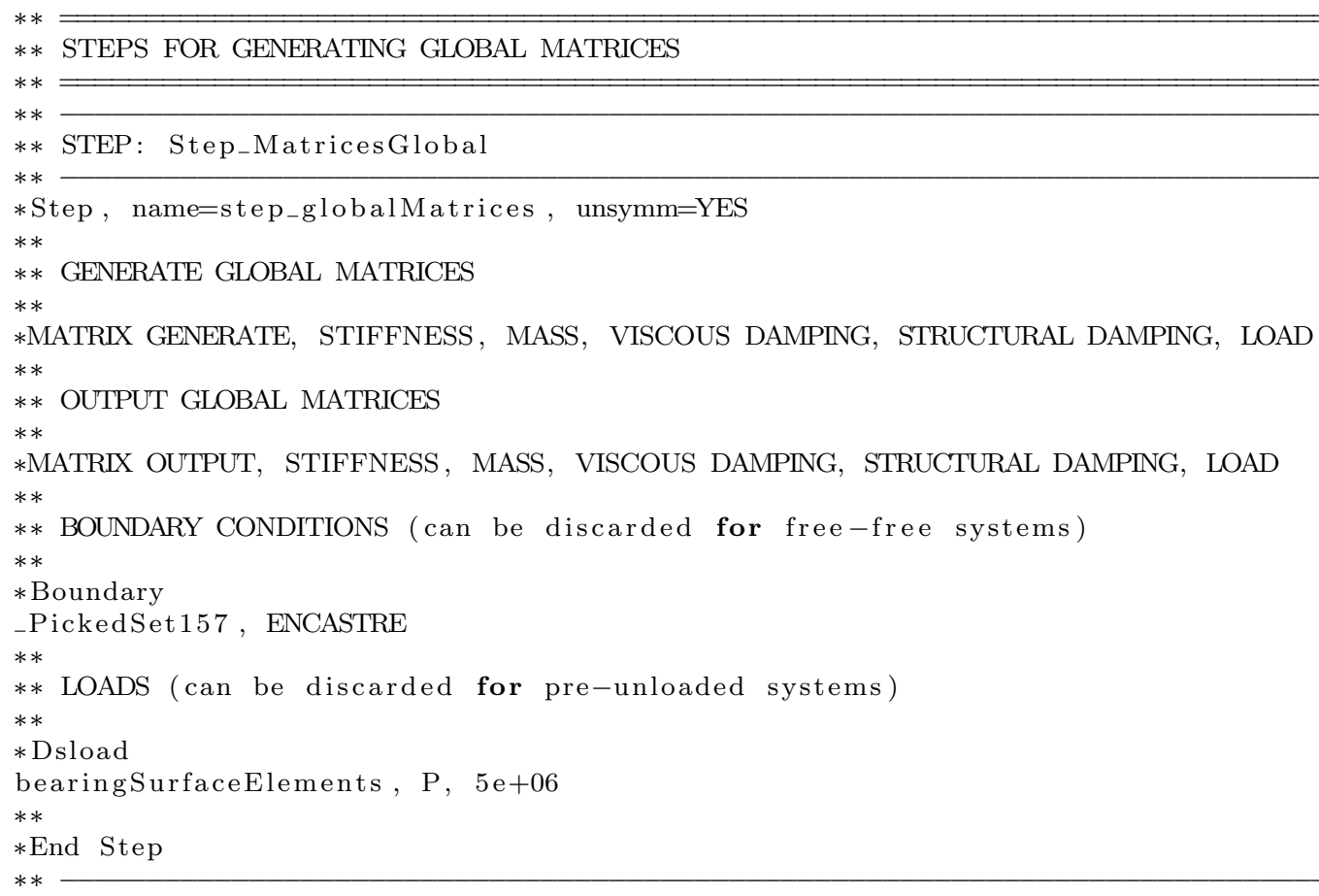




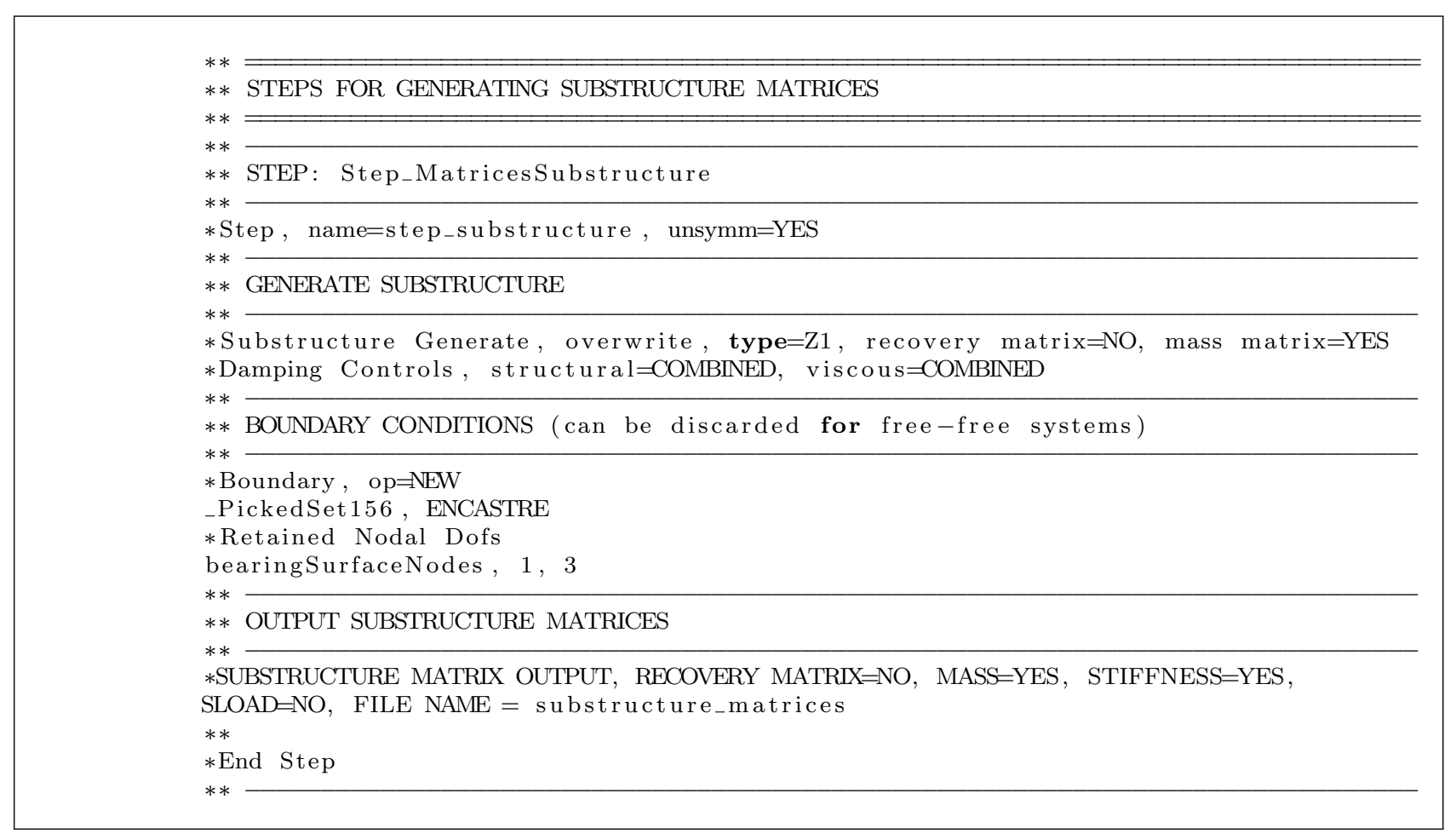

\section{C.3 Dimensionless Parameters}

Next, the dimensionless parameters related to the EHL modelling are listed for completeness.

$$
\ddot{\overrightarrow{\boldsymbol{\delta}}}=\frac{\ddot{\overrightarrow{\boldsymbol{\delta}}}}{c} \quad \dot{\overrightarrow{\boldsymbol{\delta}}}=\frac{\dot{\overrightarrow{\boldsymbol{\delta}}}}{c} \quad \overrightarrow{\overrightarrow{\boldsymbol{\delta}}}=\frac{\overrightarrow{\boldsymbol{\delta}}}{c} \quad \overline{\boldsymbol{T}}_{\boldsymbol{g l}}=\frac{\boldsymbol{T}_{\boldsymbol{g l}}}{c}
$$




\section{Appendix D}

\section{Patir and Cheng's Model}

\section{D.1 Contact and Contact Friction Factors}

Two average quantities, namely contact and contact friction factors, established in the Patir and Cheng's model will be defined, along with their analytical expressions for topographies with Gaussian roughness height distribution.

\section{Contact Factor $\left(\phi_{c}\right)$}

For a generic statistical distribution of the roughness heights, the average gap (separation) $h_{T}^{*}$ that equals to the expected or mean value of $h_{T}$, is defined as follows [164]:

$$
h_{T}^{*}=E\left\{h_{T}\right\}=\int_{-h}^{\infty}[\underbrace{\left(h+\delta_{R}\right)}_{h_{T}} \varphi\left(\delta_{R}\right)] d \delta_{R}
$$

where $\varphi\left(\delta_{R}\right)$ is the probability density function of $\delta_{R}$ and $E\{\}$ the expectancy operator. Under full hydrodynamic lubrication regime, i.e. $\Lambda \gg 3$, no asperity contacts occur, so that $h_{T}^{*} \rightarrow h$. However, whenever partial lubrication takes place, i.e. $\Lambda<3$, the asperity deformations yielded by the direct rough contacts tend to modify the average gap and so $h_{T}^{*} \neq h$. In order to avoid the tedious calculation of Eq. D.1 for every nominal separation, [218] proposed a dimensionless parameter denominated contact factor $\left(\phi_{c}\right)$, which allows a more convenient use of the average Reynolds equation. The contact factor parameter can be expressed as [149, 212]:

$$
\phi_{c}=\frac{h_{T}^{*}}{h} \equiv \frac{1}{\Lambda} \int_{-\Lambda}^{\infty}\left[s \varphi^{*}(s)\right] d s+\int_{-\Lambda}^{\infty}\left[\varphi^{*}(s)\right] d s
$$

where $\Lambda=\frac{h}{\sigma_{R}}$ is the film thickness ratio, $s=\frac{\delta_{R}}{\sigma_{R}}$ the normalized roughness and $\varphi^{*}$ the normalized probability density function of the combined roughness heights. If the probability density function is assumed to follow a Gaussian distribution, one has:

$$
\varphi^{*}(s)=\frac{1}{\sqrt{2 \pi}} \mathrm{e}^{-\frac{s^{2}}{2}}
$$




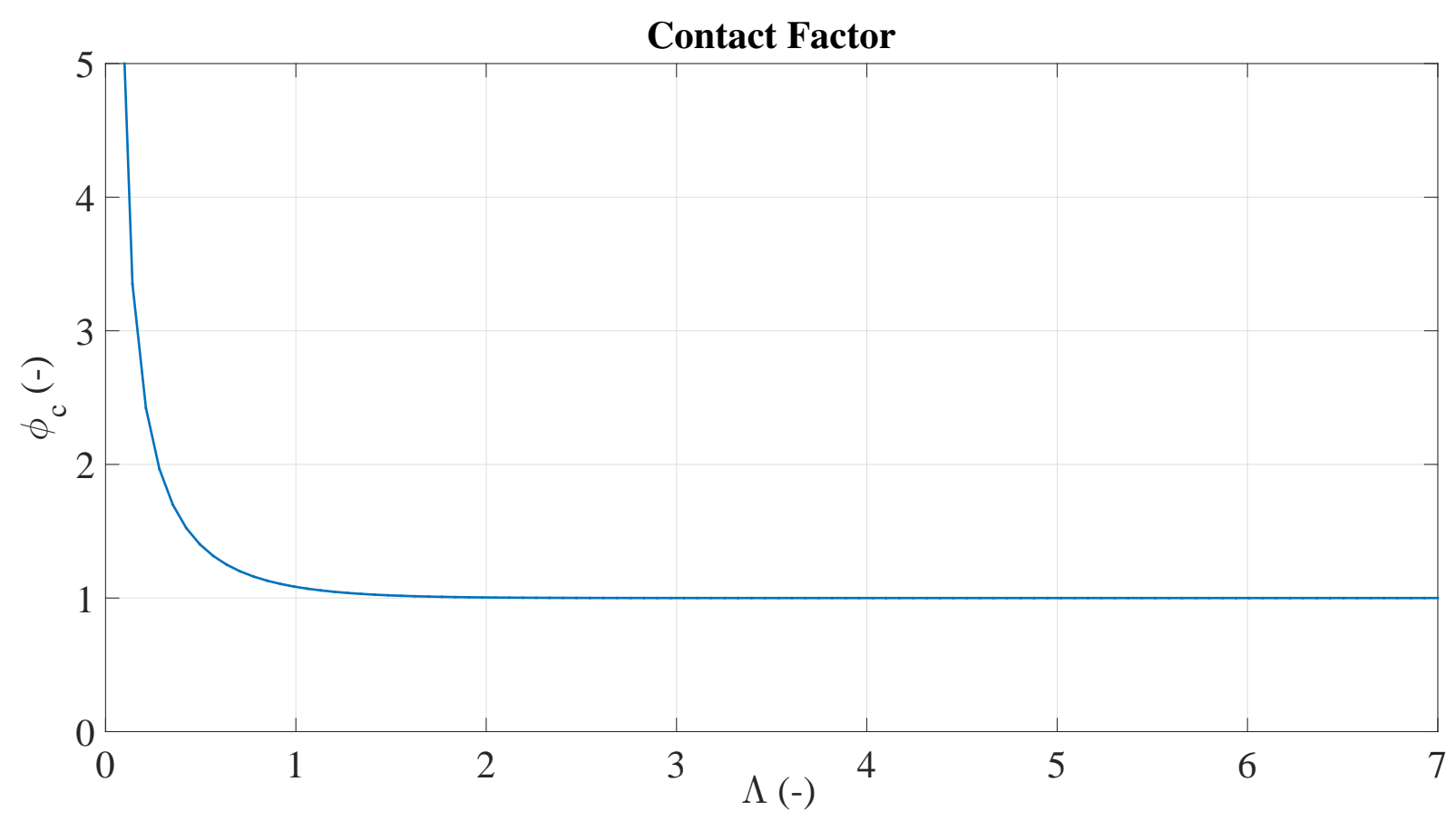

Figure D.1: Contact factor for a Gaussian roughness height distribution.

Thus, the contact factor can be rewritten as:

$$
\phi_{c}=\frac{1}{\sqrt{2 \pi} \Lambda} \int_{-\Lambda}^{\infty}\left[s \mathrm{e}^{-\frac{s^{2}}{2}}\right] d s+\frac{1}{\sqrt{2 \pi}} \int_{-\Lambda}^{\infty}\left[\mathrm{e}^{-\frac{s^{2}}{2}}\right] d s
$$

The analytical solution of the above equation can be obtained in terms of the Gauss error function $\operatorname{erf}()$ as:

$$
\phi_{c}(\Lambda)= \begin{cases}\frac{1}{2}+\frac{1}{2} \operatorname{erf}\left(\frac{\Lambda}{\sqrt{2}}\right)+\frac{\mathrm{e}^{-\frac{\Lambda^{2}}{2}}}{\sqrt{2 \pi} \Lambda}, & \Lambda \leq 3 \\ 1 & , \quad \Lambda>1\end{cases}
$$

Figure D.1 depicts the variation of $\phi_{c}$ with the film thickness ratio.

\section{Contact Friction Factor $\left(\phi_{f}\right)$}

The contact friction factor arises from the average sliding velocities in the shear stress definitions for rough contacts. For a given statistical roughness height distribution, this parameter can be defined as [165]:

$$
\phi_{f}=h E\left\{\frac{1}{h_{T}}\right\} \equiv \Lambda \int_{-\Lambda}^{\infty}\left[\frac{\varphi^{*}(s)}{\Lambda+s}\right] d s
$$

where again $\Lambda$ is the film thickness ratio, s the normalized roughness and $\varphi^{*}$ the normalized probability density function of the combined roughness heights. If the probability density function is assumed to follow a normal distribution (Eq. D.3), the contact factor can be 


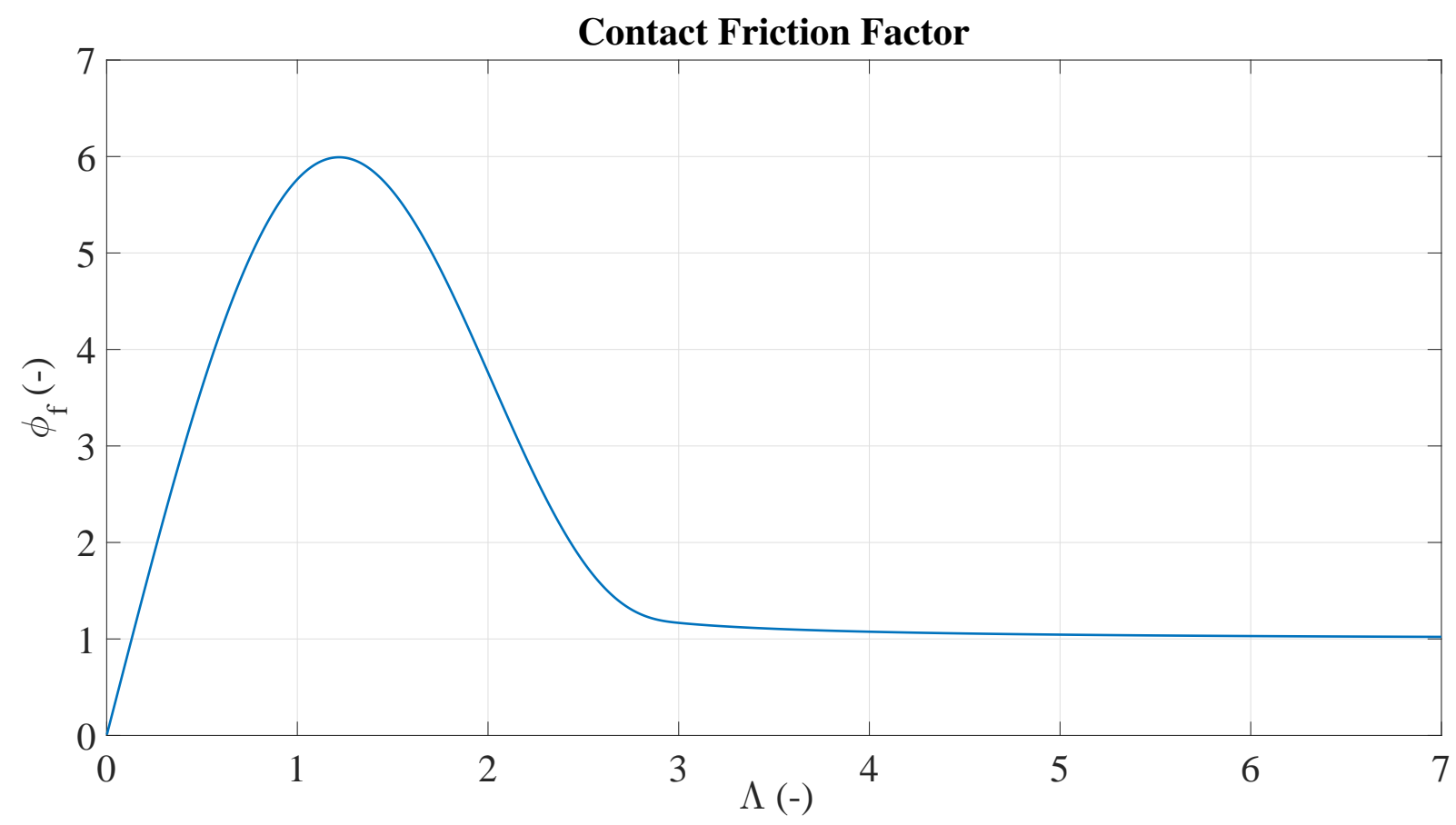

Figure D.2: Contact friction factor for a Gaussian roughness height distribution.

expressed as:

$$
\phi_{f}=\frac{\Lambda}{\sqrt{2 \pi}} \int_{-\Lambda}^{\infty}\left[\frac{\mathrm{e}^{-\frac{s^{2}}{2}}}{\Lambda+s}\right] d s
$$

The numerical solution of the above equation can be curved-fitted to polynomial expressions as:

$$
\phi_{f}(\Lambda)=\left\{\begin{array}{cc}
\frac{35}{32} z\left\{\left(1-z^{2}\right)^{3} \ln \left(\frac{z+1}{10^{9}}\right)+\frac{1}{60}[-55+z(132+z(345+\right. & \\
z(-160+z(-405+z(60+147 z)))))]\} & \\
\frac{35}{32} z\left\{\left(1-z^{2}\right)^{3} \ln \left(\frac{z+1}{z-1}\right)+\frac{z}{15}\left[66+z^{2}\left(30 z^{2}-80\right)\right]\right\} & , \quad \Lambda>3
\end{array}\right.
$$

where $z=\frac{\Lambda}{3}$. Figure D.2 depicts the variation of $\phi_{f}$ with the film thickness ratio.

\section{D.2 Flow Factors}

The pressure, shear and friction flow factors for topographies having Gaussian roughness height distribution were obtained in references $[164,165]$ through numerical simulations carried out at the microscopic scale for a number of small rectangular domains. The results for isotropic and anisotropic surfaces were collected in curves as a function of the film thickness ratio $\Lambda=\frac{h}{\sigma_{R}}$ and the surface pattern parameter $\gamma$. The latter is defined as the ratio of $x$ and $y$ correlation lengths $\gamma=\frac{\lambda_{0.5 x}}{\lambda_{0.5 y}}$, where $x$ is the sliding direction. Thus, $\gamma$ can be interpreted as the aspect ratio of a representative surface asperity characterizing the directional properties of the surface roughness. Figure D.3 illustrates typical contact areas for longitudinally oriented, isotropic and transversally oriented surfaces. 

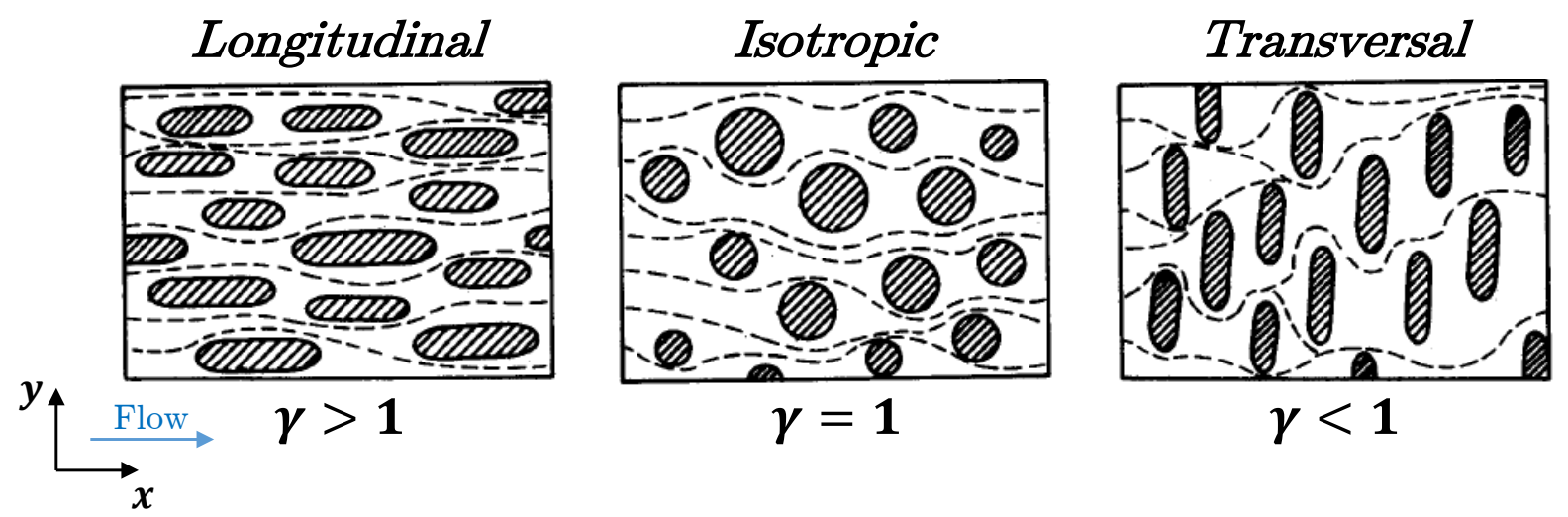

Figure D.3: Typical contact areas for longitudinally orientated, isotropic and transversally orientated surfaces.

Notice that the effect of roughness anisotropy on the lubricant transport is not fully accomplished in the Patir and Cheng's model, and was addressed later by Elrod [76] and Tripp [205]. Furthermore, Patir and Cheng disregarded the influence of the asperity interactions and the inter-asperity cavitation in the flow factors computations. However, for $\Lambda<0.5$ this assumption may not be justified since a large portion of the nominal area is in contact, so that the flow factors values originally computed tend to become less accurate under these conditions.

More recently, variations in the Patir and Cheng's flow factors calculation approach have been proposed, such as: [166] who improved the flow simulation and the generation of "artificial" surface roughness; [134, 143] who studied flow factors for sinusoidal surfaces that can be determined analytically near contact; [121, 213] who used the Patir and Cheng approach with an asperity contact model to extend the formulation for mixedEHD applications; and finally [102] who incorporated the inter-asperity cavitation effect in the flow factors model. Such alternative flow factors results will not be considered in the present thesis.

\section{Pressure Flow Factors $\left(\phi_{p_{x, z}}\right)$}

The pressure flow factors computed in [164] for surfaces with Gaussian roughness height distribution are curve-fitted as:

$$
\phi_{p_{x, z}}(\Lambda, \gamma)= \begin{cases}1-C \mathrm{e}^{r \Lambda} & , \quad \Lambda \leq 1 \\ 1+C H^{-r} & , \quad \Lambda>1\end{cases}
$$

where constants $C$ and $r$ are listed in Table D.1. Figure D.4 depicts the variation of $\phi_{c}$ with $\Lambda$ for different $\gamma$.

\section{Shear Flow Factors $\left(\phi_{s_{x, z}}\right)$}

Unlike the pressure flow factors that are a function of the combined roughness, the shear flow factors depend on the roughness parameters of each surface in contact. In other words, $\phi_{s_{x, z}}$ is not only a function of $\Lambda$ and $\gamma$, but also of $\left(\sigma_{1}^{R}, \sigma_{2}^{R}\right)$ and $\left(\gamma_{1}, \gamma_{2}\right)$ individually. The shear flow factors obtained from numerical simulations in [165] for surfaces with 


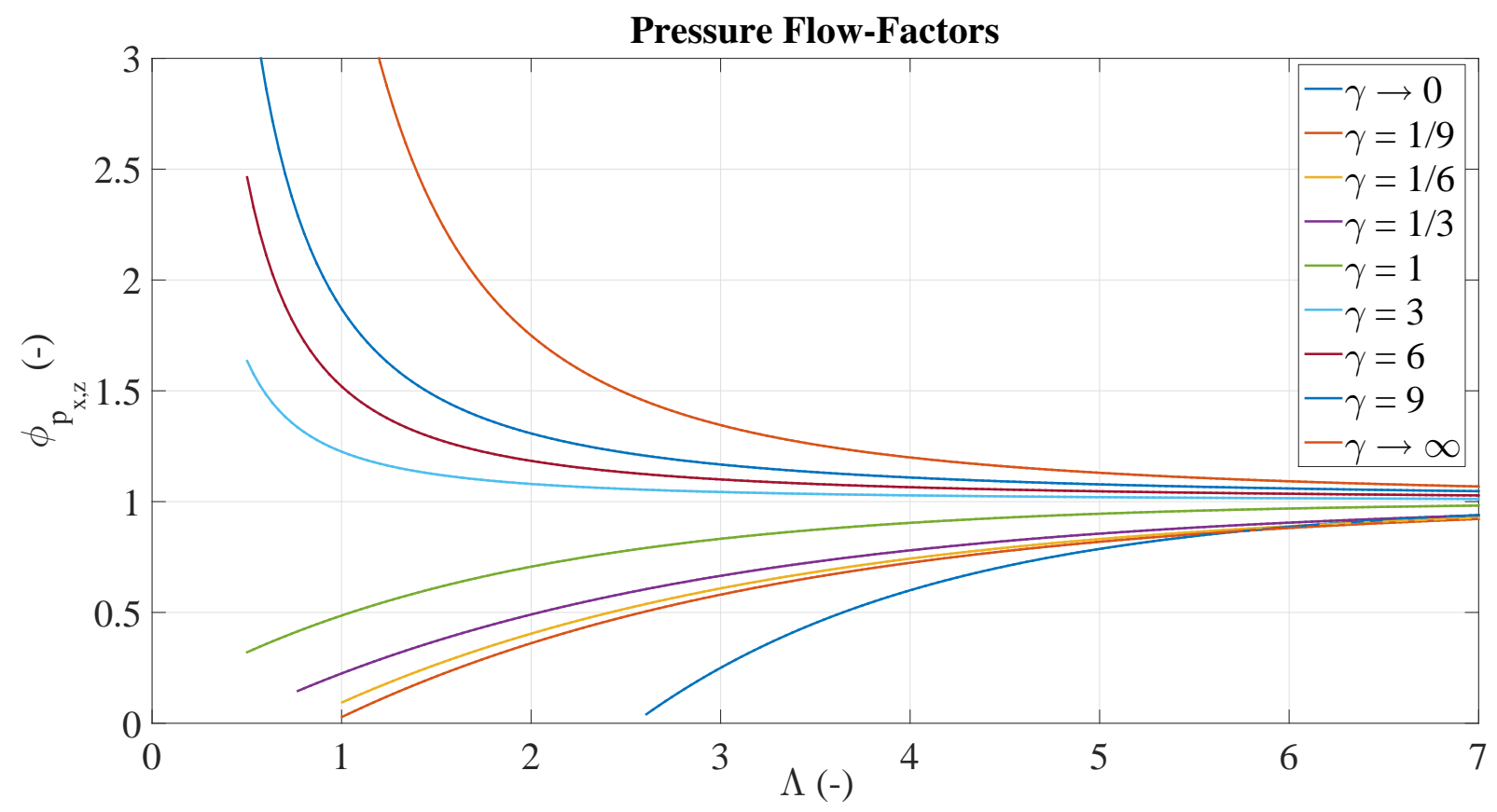

Figure D.4: Pressure flow factors for a Gaussian roughness height distribution.

Table D.1: Curve fitting coefficients of the pressure flow factors for a Gaussian roughness height distribution.

\begin{tabular}{llll}
\hline $\boldsymbol{\gamma}$ & $\boldsymbol{C}$ & $\boldsymbol{r}$ & Range \\
\hline $1 / 9$ & 1.48 & 0.42 & $\mathrm{H}>1$ \\
$1 / 6$ & 1.38 & 0.42 & $\mathrm{H}>1$ \\
$1 / 3$ & 1.18 & 0.42 & $\mathrm{H}>0.75$ \\
1 & 0.90 & 0.56 & $\mathrm{H}>0.5$ \\
3 & 0.225 & 1.50 & $\mathrm{H}>0.5$ \\
6 & 0.520 & 1.50 & $\mathrm{H}>0.5$ \\
9 & 0.870 & 1.50 & $\mathrm{H}>0.5$ \\
\hline
\end{tabular}

Gaussian roughness height distribution are curve-fitted as:

$$
\phi_{s_{x, z}}\left(\Lambda, \sigma_{1}^{R}, \sigma_{2}^{R}, \gamma_{1}, \gamma_{2}\right)=V_{r 1}\left(\sigma_{1}^{R}, \sigma_{R}\right) \Phi_{s_{x, z}}\left(\Lambda, \gamma_{1}\right)-V_{r 2}\left(\sigma_{2}^{R}, \sigma_{R}\right) \Phi_{s_{x, z}}\left(\Lambda, \gamma_{2}\right)
$$

where $V_{r 1}$ and $V_{r 2}$ are the variance ratios given by:

$$
V_{r 1}\left(\sigma_{1}^{R}, \sigma_{R}\right)=\left(\frac{\sigma_{1}^{R}}{\sigma_{R}}\right)^{2} \quad V_{r 2}\left(\sigma_{2}^{R}, \sigma_{R}\right)=\left(\frac{\sigma_{2}^{R}}{\sigma_{R}}\right)^{2}=\left(1-V_{r 1}\right)
$$

$\Phi_{s_{x, z}}$ is a positive function of $\Lambda$ and the surface pattern parameters of a given surface. It can be curve-fitted from simulations results as [165]:

$$
\Phi_{s_{x, z}}\left(\Lambda, \gamma_{1,2}\right)= \begin{cases}A_{1} \Lambda^{\alpha_{1}} \mathrm{e}^{-\alpha_{2} \Lambda+\alpha_{3} \Lambda^{2}} & , \quad \Lambda \leq 5 \\ A_{2} \mathrm{e}^{-0.25 \Lambda} & , \quad \Lambda>5\end{cases}
$$

where the constants $A_{1}, A_{2}, \alpha_{1}, \alpha_{2}, \alpha_{3}$ are listed in Table D.2. Figure D.5 depicts the variation of $\Phi_{s_{x, z}}$ with $\Lambda$ for different $\gamma$. 


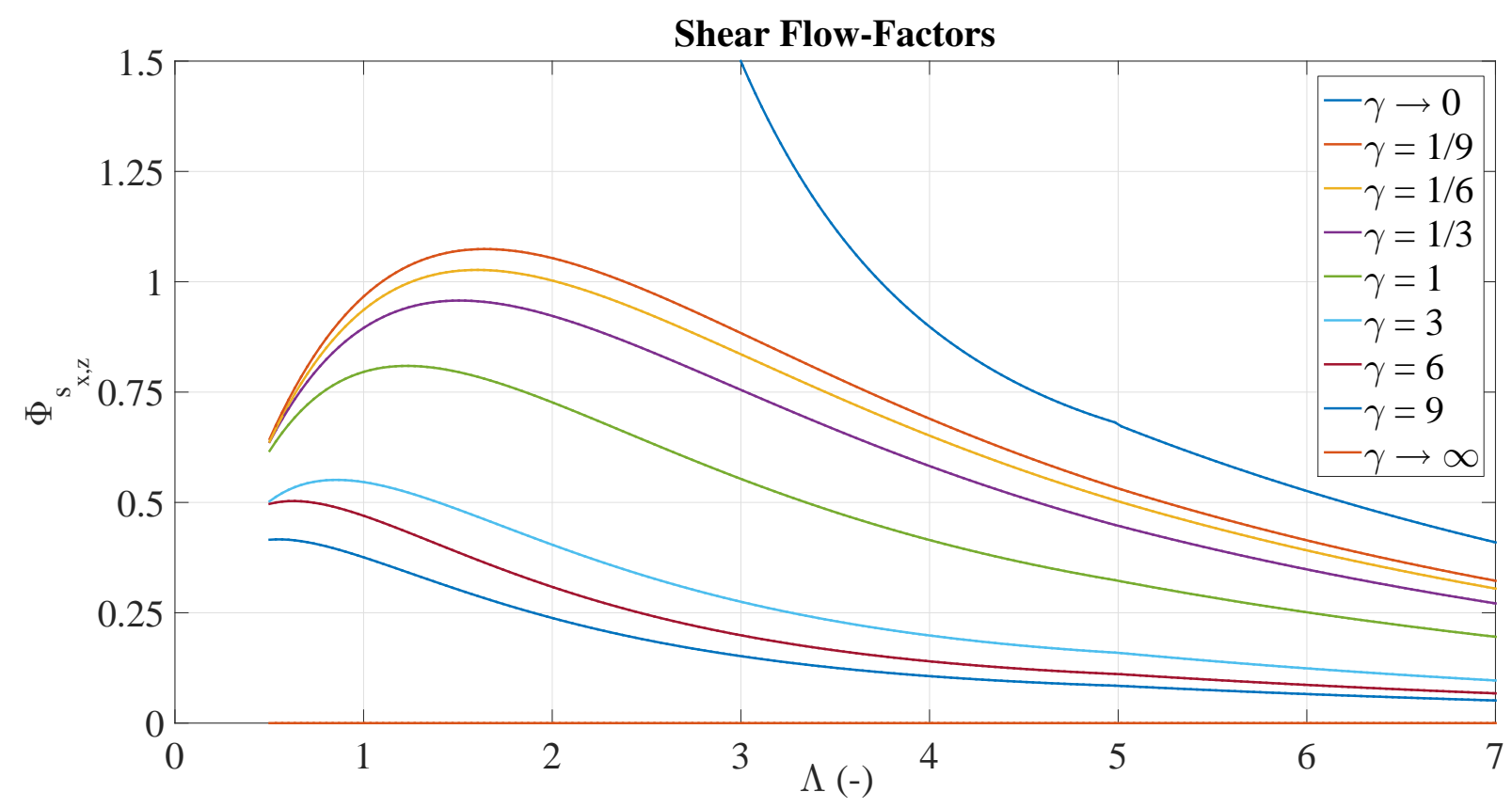

Figure D.5: Shear flow factors for a Gaussian roughness height distribution.

Table D.2: Curve fitting coefficients of the shear flow factors for a Gaussian roughness height distribution.

\begin{tabular}{llllll}
\hline $\boldsymbol{\gamma}$ & $\boldsymbol{A}_{\mathbf{1}}$ & $\boldsymbol{\alpha}_{\mathbf{1}}$ & $\boldsymbol{\alpha}_{\mathbf{2}}$ & $\boldsymbol{\alpha}_{\mathbf{3}}$ & $\boldsymbol{A}_{\mathbf{2}}$ \\
\hline $1 / 9$ & 2.046 & 1.12 & 0.78 & 0.03 & 1.856 \\
$1 / 6$ & 1.962 & 1.08 & 0.77 & 0.03 & 1.754 \\
$1 / 3$ & 1.858 & 1.01 & 0.76 & 0.03 & 1.561 \\
1 & 1.899 & 0.98 & 0.92 & 0.05 & 1.126 \\
3 & 1.560 & 0.85 & 1.13 & 0.08 & 0.556 \\
6 & 1.290 & 0.62 & 1.09 & 0.08 & 0.388 \\
9 & 1.011 & 0.54 & 1.07 & 0.08 & 0.295 \\
\hline
\end{tabular}

\section{Friction Pressure Flow Factors $\left(\phi_{f p_{x, z}}\right)$}

The friction pressure flow factors computed in [165] for surfaces with Gaussian roughness height distribution are curve-fitted as:

$$
\phi_{f p_{x, z}}(\Lambda, \gamma)=1-D e^{s \Lambda}
$$

where constants $D$ an $s$ are listed Table D.3. Figure D.6 depicts the variation of $\phi_{f p_{x, z}}$ with $\Lambda$ for different $\gamma$.

\section{Friction Shear Flow Factors $\left(\phi_{f_{s, z}}\right)$}

Similarly to the shear flow factors, the friction shear flow factors depend on the roughness parameters of each surface in contact. Thus [165]:

$$
\phi_{f s_{x, z}}\left(\Lambda, \sigma_{1}^{R}, \sigma_{2}^{R}, \gamma_{1}, \gamma_{2}\right)=V_{r 1}\left(\sigma_{1}^{R}, \sigma_{R}\right) \Phi_{f s_{x, z}}\left(\Lambda, \gamma_{1}\right)-V_{r 2}\left(\sigma_{2}^{R}, \sigma_{R}\right) \Phi_{f s_{x, z}}\left(\Lambda, \gamma_{2}\right)
$$




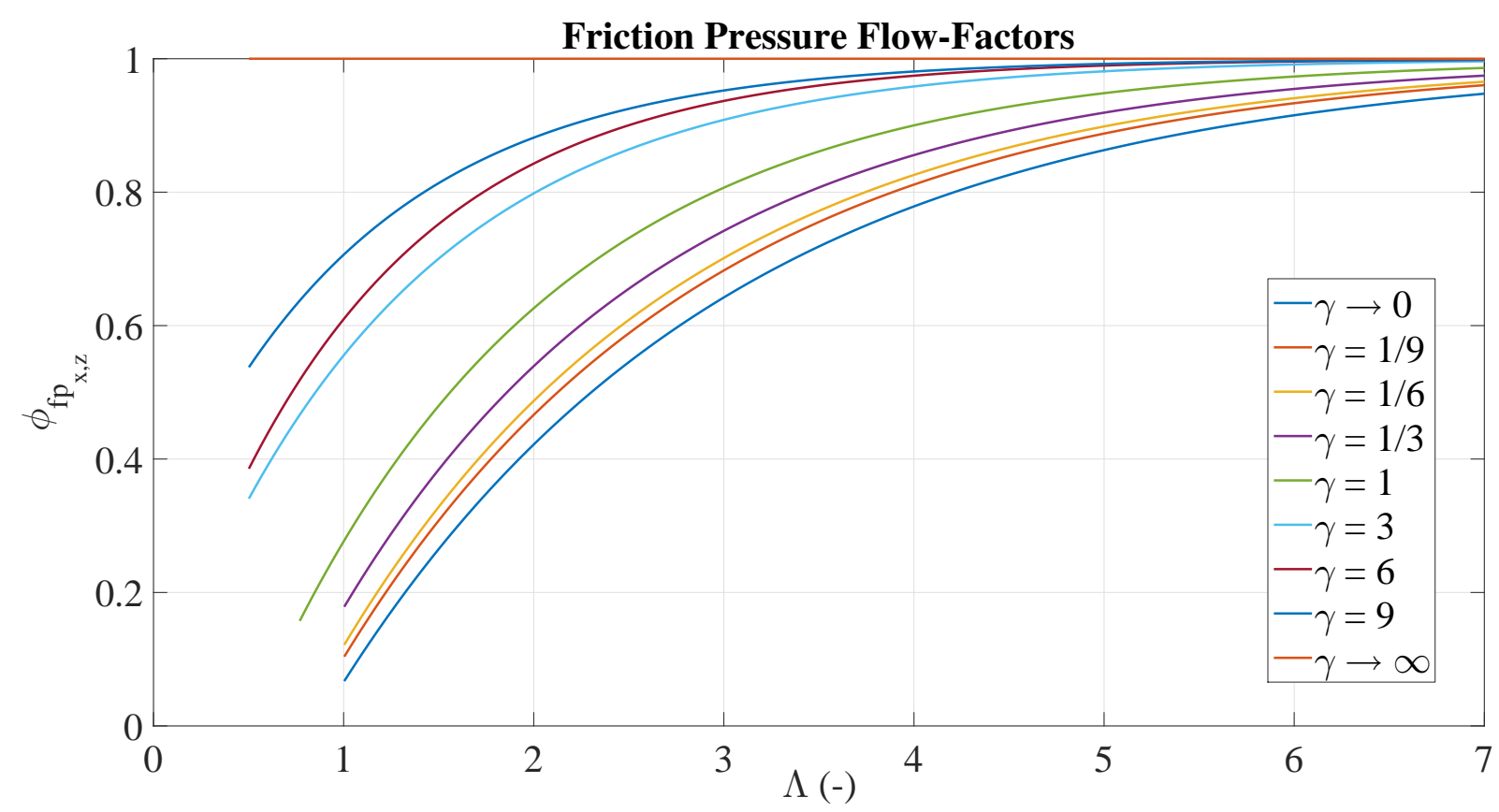

Figure D.6: Friction pressure flow factors for a Gaussian height roughness distribution.

Table D.3: Curve fitting coefficients of the friction pressure flow factors for a Gaussian roughness height distribution.

\begin{tabular}{llll}
\hline $\boldsymbol{\gamma}$ & $\boldsymbol{D}$ & $\boldsymbol{s}$ & Range \\
\hline $1 / 9$ & 1.51 & 0.52 & $\mathrm{H}>1$ \\
$1 / 6$ & 1.51 & 0.54 & $\mathrm{H}>1$ \\
$1 / 3$ & 1.47 & 0.58 & $\mathrm{H}>1$ \\
1 & 1.40 & 0.66 & $\mathrm{H}>0.75$ \\
3 & 0.98 & 0.79 & $\mathrm{H}>0.5$ \\
6 & 0.97 & 0.91 & $\mathrm{H}>0.5$ \\
9 & 0.73 & 0.91 & $\mathrm{H}>0.5$ \\
\hline
\end{tabular}

where $V_{r 1}$ and $V_{r 2}$ are the variance ratios defined in Eq. D.15 and $\Phi_{f s_{x, z}}$ is again a positive function of $\Lambda$ and the surface pattern parameters of a given surface. It can be curve-fitted from simulation results as [165]:

$$
\Phi_{s_{x, z}}\left(\Lambda, \gamma_{1,2}\right)=A_{3} \Lambda^{\alpha_{4}} e^{-\alpha_{5} \Lambda+\alpha_{6} \Lambda^{2}}
$$

where constants $A_{3}, \alpha_{4}, \alpha_{5}, \alpha_{6}$ are listed in Table D.4. Figure D.7 depicts the variation of $\Phi_{s_{x, z}}$ with $\Lambda$ for different $\gamma$. 


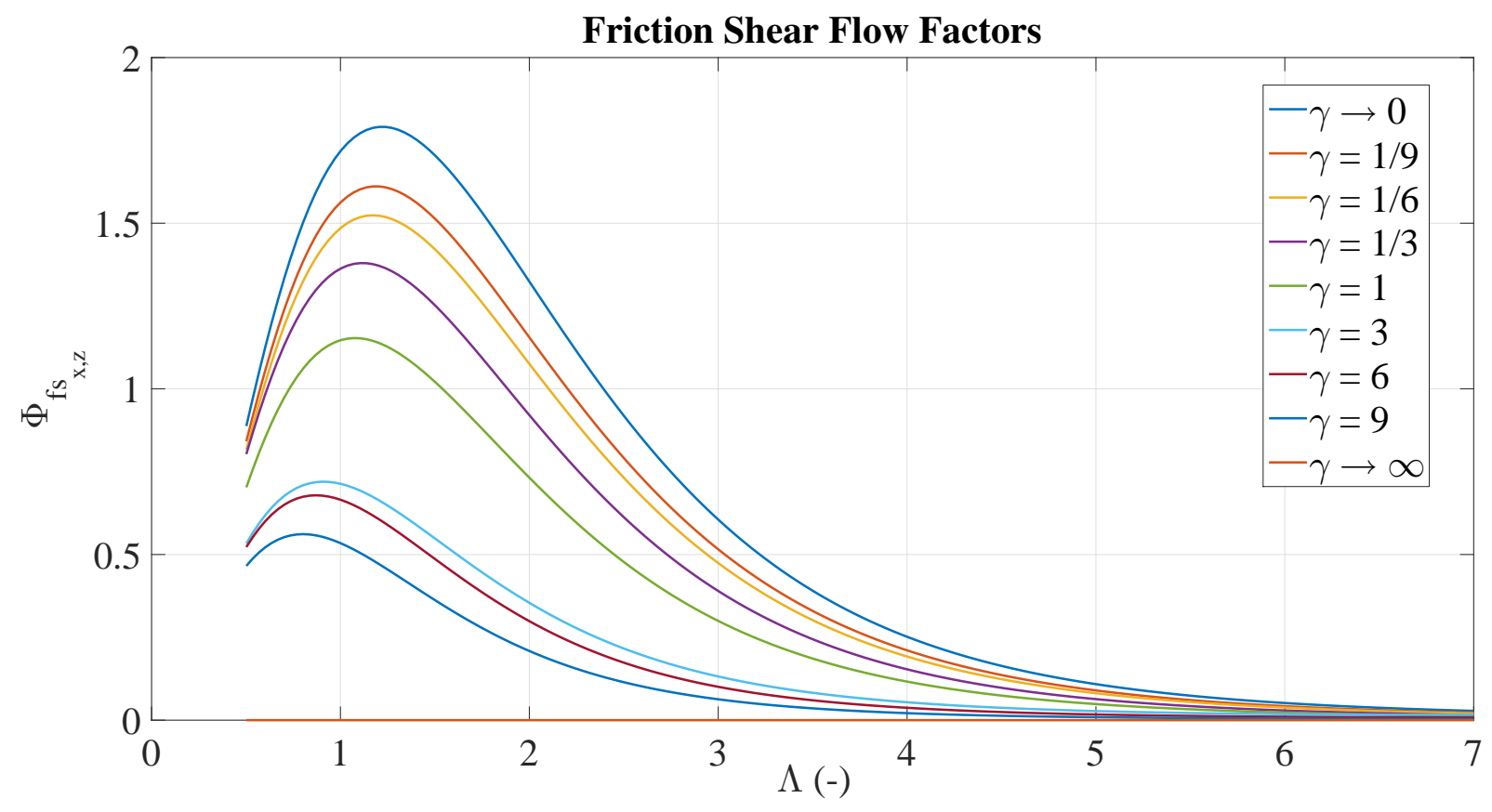

Figure D.7: Friction shear flow factors for a Gaussian roughness height distribution.

Table D.4: Curve fitting coefficients of the friction shear flow factors for a Gaussian roughness height distribution.

\begin{tabular}{lllll}
\hline $\boldsymbol{\gamma}$ & $\boldsymbol{A}_{\mathbf{3}}$ & $\boldsymbol{\alpha}_{\mathbf{4}}$ & $\boldsymbol{\alpha}_{\mathbf{5}}$ & $\boldsymbol{\alpha}_{\mathbf{6}}$ \\
\hline $1 / 9$ & 14.1 & 2.45 & 2.30 & 0.10 \\
$1 / 6$ & 13.4 & 2.42 & 2.30 & 0.10 \\
$1 / 3$ & 12.3 & 2.32 & 2.30 & 0.10 \\
1 & 11.1 & 2.31 & 2.38 & 0.11 \\
3 & 9.8 & 2.25 & 2.80 & 0.18 \\
6 & 10.1 & 2.25 & 2.90 & 0.18 \\
9 & 8.7 & 2.15 & 2.97 & 0.18 \\
\hline
\end{tabular}




\section{Appendix E}

\section{GW/GT Models}

\section{E.1 Dimensionless Parameters}

The dimensionless parameters related to the GW/GT models are listed for completeness.

\section{General Parameters}

$$
\begin{aligned}
& \bar{E}^{*}=\frac{E^{*}}{E_{0}^{*}} \quad \bar{Z}_{S}=\frac{Z_{S}}{Z_{S_{0}}} \quad \bar{\beta}_{S}=\frac{\beta_{S}}{\beta_{S_{0}}} \\
& \bar{Y}=\frac{Y}{Y_{0}} \quad \bar{\sigma}_{S}=\frac{\sigma_{S}}{\sigma_{S_{0}}} \quad \bar{\eta}_{S}=\frac{\eta_{S}}{\eta_{S_{0}}} \\
& \bar{\mu}_{B L}=\frac{\mu_{B L}}{\mu_{B L_{0}}} \\
& \bar{h}_{A S P}=\left(\frac{c}{\sigma_{S_{0}}}\right) \frac{\bar{h}}{\bar{\sigma}_{S}}-\left(\frac{Z_{S_{0}}}{\sigma_{S_{0}}}\right) \frac{\bar{Z}_{S}}{\bar{\sigma}_{S}} \quad \bar{p}_{A S P}^{(G W, G T)}=\frac{p_{A S P}^{(G W, G T)}}{\bar{K}^{(G W, G T)}} \\
& \bar{K}^{(G T)}=\frac{16 \pi \sqrt{2}}{15}\left[E_{0}^{*}\left(\eta_{S_{0}}\right)^{2}\left(\beta_{S_{0}}\right)^{3 / 2}\left(\sigma_{S_{0}}\right)^{5 / 2}\right] \quad \bar{\tau}_{A S P}^{(G W, G T)}=\frac{\tau_{A S P}^{(G W, G T)}}{\mu_{B L_{0}} \bar{K}^{(G W, G T)}} \\
& \bar{K}^{(G W)}=\frac{4}{3}\left[E_{0}^{*}\left(\eta_{S_{0}}\right)\left(\beta_{S_{0}}\right)^{1 / 2}\left(\sigma_{S_{0}}\right)^{3 / 2}\right]
\end{aligned}
$$

\section{Operational Parameters}

$$
\begin{aligned}
\bar{f}_{A S P} & =\frac{f_{A S P}}{\mu_{B L_{0}}} & \bar{P}_{A S P}^{(G W, G T)} & =\frac{P_{A S P}^{(G W, G T)}}{\bar{K}^{(G W, G T)}(2 R L)(\Omega R)} \\
\bar{F}_{A S P}^{(G W, G T)} & =\frac{F_{A S P}^{(G W, G T)}}{\bar{K}^{(G W, G T)}(2 R L)} & \bar{T}_{A S P}^{(G W, G T)} & =\frac{T_{A S P}^{(G W, G T)}}{\bar{K}^{(G W, G T)}(2 R L)(R)} \\
\bar{M}_{A S P}^{(G W, G T)} & =\frac{M_{A S P}^{(G W, G T)}}{\bar{K}^{(G W, G T)}(2 R L) L / 2} & \bar{W}_{A S P}^{(G W, G T)} & =\frac{W_{A S P}^{(G W, G T)}}{\bar{K}^{(G W, G T)}(2 R L)}
\end{aligned}
$$




\section{E.2 Polynomial Fitting of the GW/GT Integrals}

In order to calculate the asperity contact pressures established by the GW/GT models, the integrals defined in Eqs. 4.8 and Eqs. 4.10 have to be evaluated in advance. The values of such integrals depend on the statistical distribution of the asperity heights, as well as on the separation gap between the contact surfaces.

As stated by [97, 202], a Gaussian distribution represents the statistical dispersion of the asperity heights for typical engineering topographies quite well. Figure E.1 shows the example of a surface roughness profile before and after wear. As can be seen, before wear both the roughness heights and the asperity heights located above the reference mean line displayed a near Gaussian distribution. On the other hand, the roughness heights of the worn profile became clearly non-Gaussian, while the asperity ones remained approximately unchanged. In fact, it is worth emphasising that only the statistical behaviour of the asperity heights, i.e. roughness points situated above the topography reference plane, is important for the application of the GW/GT models. The (normalized) probability density function of a Gaussian distribution can be expressed as follows [97]:

$$
\phi^{*}(s)=\frac{1}{\sqrt{2 \pi}} \mathrm{e}^{-\frac{s^{2}}{2}}
$$

Substituting Eq. E.1 into the integral formulas of Eqs. 4.8 and Eqs. 4.10, one obtains:

$$
F_{m}\left(\bar{h}_{A S P}\right)=\frac{1}{\sqrt{2 \pi}} \int_{\bar{h}_{A S P}}^{\infty}\left[\left(s-\bar{h}_{A S P}\right)^{m} \mathrm{e}^{-\frac{s^{2}}{2}}\right] d s
$$

The integrals of Eq. E.2 for $m=0, m=1, m=3 / 2$ and $m=5 / 2$ have no analytical closed-form solution. Thus, the evaluation of such integral functions of $\bar{h}_{A S P}$ is performed numerically and so approximated by polynomial fitting curves as:

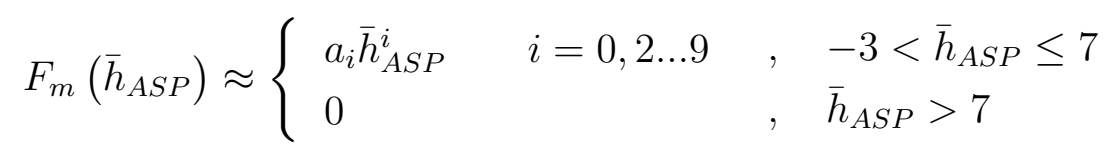

where $a_{i}$ are the coefficients listed in Table E.1. The comparison between the calculated values and the respective fitted-curves of the GW/GT integrals are depicted in Fig. E.2.

Table E.1: Coefficients of the polynomial fitting curves of the $G W / G T$ integrals.

\begin{tabular}{rrrrr}
\hline & \multicolumn{1}{c}{$\boldsymbol{m}=\mathbf{0}$} & \multicolumn{1}{c}{$\boldsymbol{m}=\mathbf{1}$} & \multicolumn{1}{c}{$\boldsymbol{m}=\mathbf{3} \mathbf{2}$} & \multicolumn{1}{c}{$\boldsymbol{m}=\mathbf{5} \mathbf{2}$} \\
\hline$a_{0}$ & $4.983 \times 10^{-1}$ & $4.015 \times 10^{-1}$ & $4.325 \times 10^{-1}$ & $6.16 \times 10^{-1}$ \\
$a_{1}$ & $-3.843 \times 10^{-1}$ & $-4.923 \times 10^{-1}$ & $-6.146 \times 10^{-1}$ & -1.086 \\
$a_{2}$ & $7.063 \times 10^{-3}$ & $1.909 \times 10^{-1}$ & $3.139 \times 10^{-1}$ & $7.72 \times 10^{-1}$ \\
$a_{3}$ & $4.984 \times 10^{-2}$ & $-7.11 \times 10^{-3}$ & $-5.245 \times 10^{-2}$ & $-2.576 \times 10^{-1}$ \\
$a_{4}$ & $-3.69 \times 10^{-3}$ & $-1.116 \times 10^{-2}$ & $-8.35 \times 10^{-3}$ & $3.057 \times 10^{-2}$ \\
$a_{5}$ & $-3.91 \times 10^{-3}$ & $1.59 \times 10^{-3}$ & $3.498 \times 10^{-3}$ & $3.549 \times 10^{-3}$ \\
$a_{6}$ & $5.73 \times 10^{-4}$ & $3.23 \times 10^{-4}$ & $1.54 \times 10^{-5}$ & $-1.05 \times 10^{-3}$ \\
$a_{7}$ & $1.05 \times 10^{-4}$ & $-9.93 \times 10^{-5}$ & $-1.3 \times 10^{-4}$ & $-1.24 \times 10^{-5}$ \\
$a_{8}$ & $-2.71 \times 10^{-5}$ & $8.60 \times 10^{-6}$ & $1.85 \times 10^{-5}$ & $1.84 \times 10^{-5}$ \\
$a_{9}$ & $1.54 \times 10^{-6}$ & $-2.37 \times 10^{-7}$ & $-8.08 \times 10^{-7}$ & $-1.19 \times 10^{-6}$ \\
\hline
\end{tabular}




\section{Before Wear}

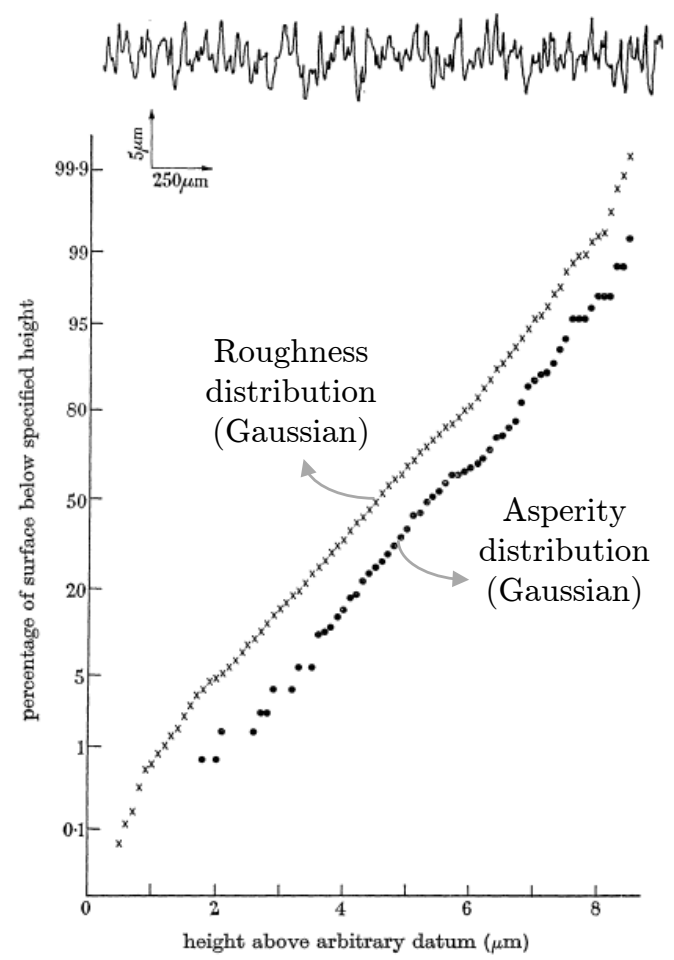

After Wear

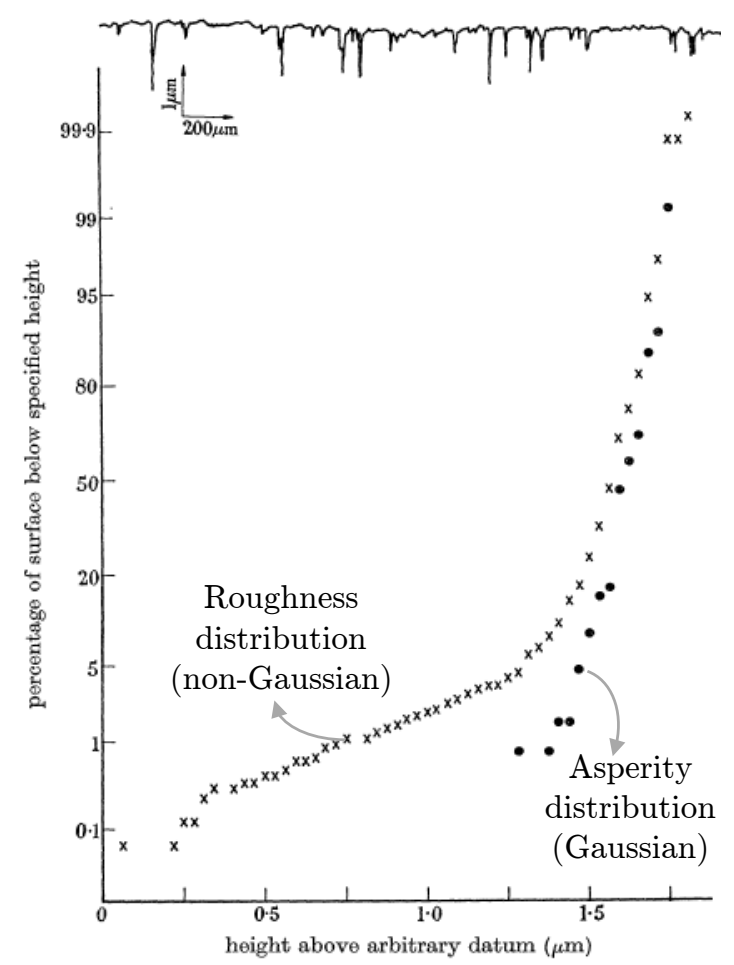

Figure E.1: Cumulative height distribution of a surface roughness profile before and after wear. Adapted from [97].
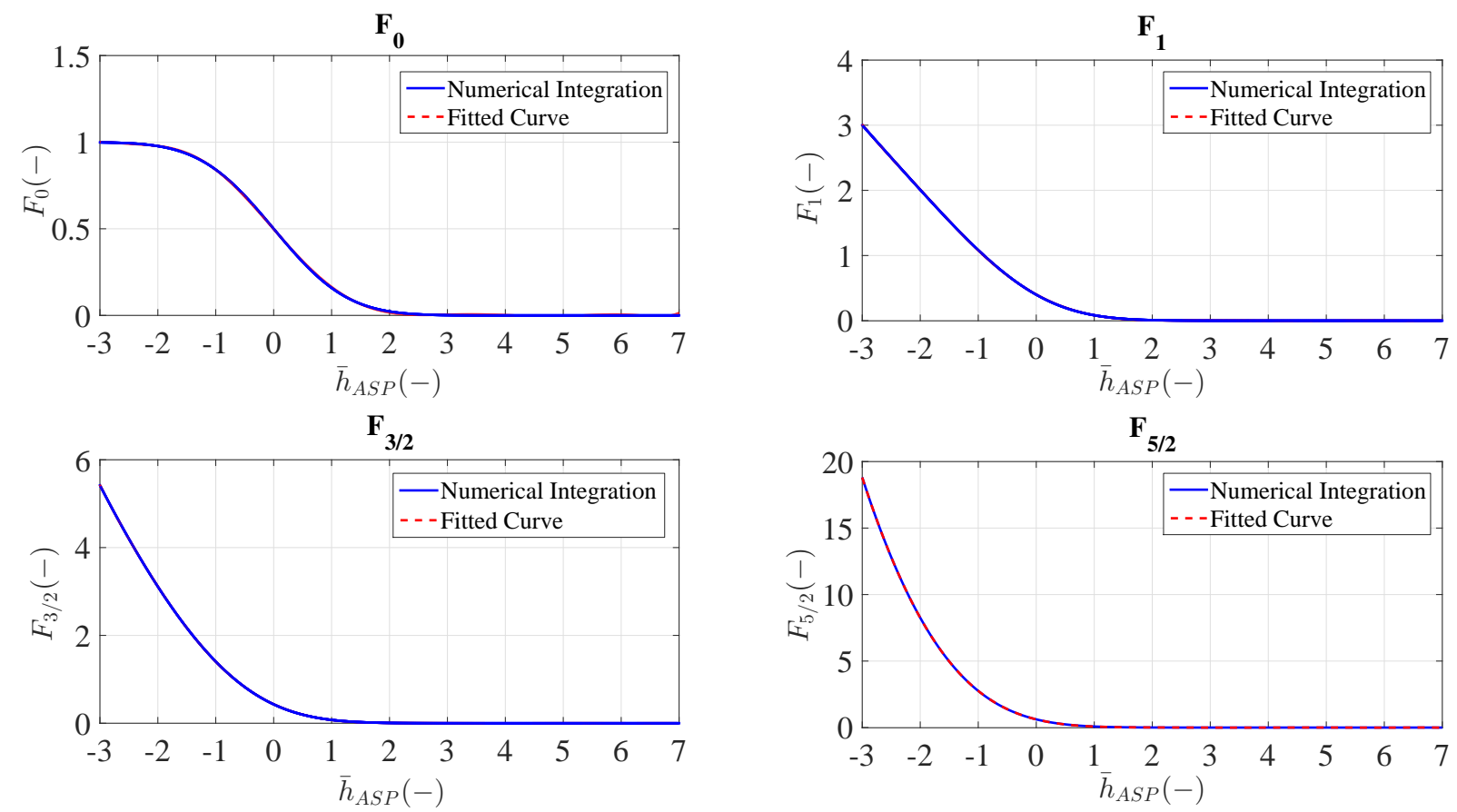

Figure E.2: Curves of the integral functions of the $G W / G T$ models. 


\section{Appendix F}

\section{Numerical Solution of Reynolds Equation}

\section{F.1 Mesh Elements for EbFVM Discretization}

All the geometric and tensor properties of triangular and quadrangular elements are presented for the completeness of the EbFVM derivation. The properties are shown with respect to the standard transformed domain (parametric elements).

\section{Linear Triangular Element}

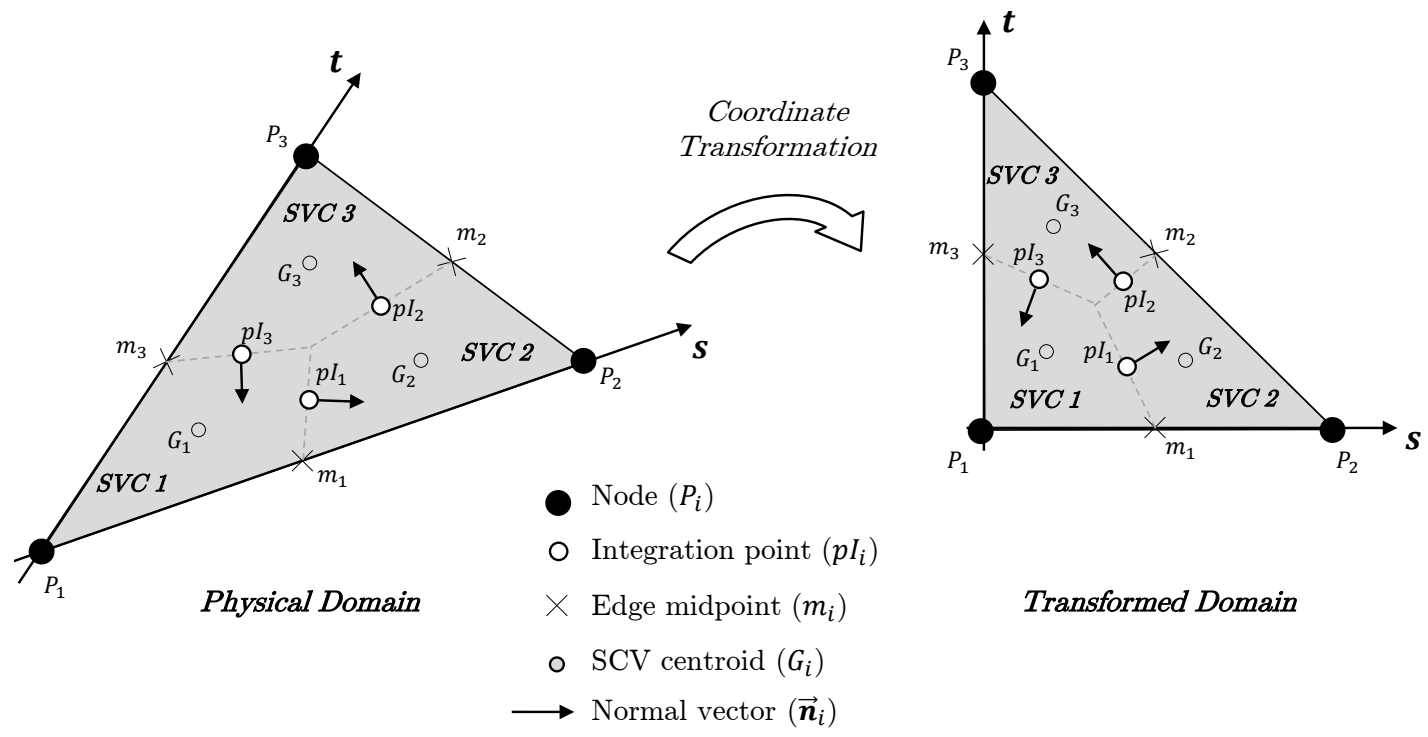

Figure F.1: Coordinate transformation for linear triangular element.

- Coordinates of the element nodes

$$
P_{1}=(0,0) \quad P_{2}=(1,0) \quad P_{3}=(0,1)
$$

- Coordinates of the edges midpoints

$$
m_{1}=\left(\frac{1}{2}, 0\right) \quad m_{2}=\left(\frac{1}{2}, \frac{1}{2}\right) \quad m_{3}=\left(0, \frac{1}{2}\right)
$$


- Coordinates of the integration points

$$
p I_{1}=\left(\frac{5}{12}, \frac{1}{6}\right) \quad p I_{2}=\left(\frac{5}{12}, \frac{5}{12}\right) \quad p I_{3}=\left(\frac{1}{6}, \frac{5}{12}\right)
$$

- Coordinates of the SVC centroids

$$
G_{1}=\left(\frac{5}{24}, \frac{5}{24}\right) \quad G_{2}=\left(\frac{7}{12}, \frac{5}{24}\right) \quad G_{3}=\left(\frac{5}{24}, \frac{7}{12}\right)
$$

- SCV areas

$$
A_{S V C_{1}}=\frac{1}{6} \operatorname{det}\left[\boldsymbol{J}\left(G_{1}\right)\right] \quad A_{S V C_{2}}=\frac{1}{6} \operatorname{det}\left[\boldsymbol{J}\left(G_{2}\right)\right] \quad A_{S V C_{3}}=\frac{1}{6} \operatorname{det}\left[\boldsymbol{J}\left(G_{3}\right)\right]
$$

- Coordinates of the normal vectors (transformed domain)

$$
\overrightarrow{\boldsymbol{n}}_{1}=\left(\frac{1}{6},-\frac{1}{3}\right) \quad \overrightarrow{\boldsymbol{n}}_{2}=\left(\frac{1}{6}, \frac{1}{6}\right) \quad \overrightarrow{\boldsymbol{n}}_{3}=\left(-\frac{1}{3}, \frac{1}{6}\right)
$$

- Coordinates of the normal vectors (physical domain)

$$
\overrightarrow{\boldsymbol{n}}_{1}^{*}=\boldsymbol{R} \boldsymbol{J}\left(p I_{1}\right) \overrightarrow{\boldsymbol{n}}_{1} \quad \overrightarrow{\boldsymbol{n}}_{2}^{*}=\boldsymbol{R} \boldsymbol{J}\left(p I_{2}\right) \overrightarrow{\boldsymbol{n}}_{2} \quad \overrightarrow{\boldsymbol{n}}_{3}^{*}=\boldsymbol{R} \boldsymbol{J}\left(p I_{3}\right) \overrightarrow{\boldsymbol{n}}_{3}
$$

where auxiliary matrix $\boldsymbol{R}$ is defined as:

$$
\boldsymbol{R}=\left[\begin{array}{cc}
0 & -1 \\
1 & 0
\end{array}\right]
$$

- Interpolation and shape functions

$$
N_{1}(s, t)=(1-s-t) \quad N_{2}(s, t)=s \quad N_{3}(s, t)=t
$$

- Matrix for gradients interpolation

$$
\boldsymbol{B}(s, t)=\left[\begin{array}{ll}
\frac{\partial N_{1}(s, t)}{\partial s} & \frac{\partial N_{1}(s, t)}{\partial t} \\
\frac{\partial N_{2}(s, t)}{\partial s} & \frac{\partial N_{2}(s, t)}{\partial t} \\
\frac{\partial N_{3}(s, t)}{\partial s} & \frac{\partial N_{3}(s, t)}{\partial t}
\end{array}\right]=\left[\begin{array}{cc}
-1 & -1 \\
1 & 0 \\
0 & 1
\end{array}\right]
$$

- Jacobian matrix

$$
\boldsymbol{J}(s, t)=\boldsymbol{Z}_{\boldsymbol{e}} \boldsymbol{B}(s, t)
$$

where $\boldsymbol{Z}_{\boldsymbol{e}}$ is the matrix with the global coordinates of the element nodes:

$$
\boldsymbol{Z}_{\boldsymbol{e}}=\left[\begin{array}{lll}
x_{1} & x_{2} & x_{3} \\
y_{1} & y_{2} & y_{3}
\end{array}\right]
$$


- Tensor for gradients interpolation

$$
G_{j k}(s, t) \Rightarrow \boldsymbol{G}(s, t)=\boldsymbol{B}(s, t) \boldsymbol{J}(s, t)^{-1}
$$

- Tensor for diffusion calculation

$$
B_{m j k i s} \Rightarrow\left\{\begin{array}{l}
B_{m j k i 1}=H_{m j k i}\left(p I_{1}\right)-H_{m j k i}\left(p I_{3}\right) \rightarrow S V C_{1} \\
B_{m j k i 2}=H_{m j k i}\left(p I_{2}\right)-H_{m j k i}\left(p I_{1}\right) \rightarrow S V C_{2} \\
B_{m j k i 3}=H_{m j k i}\left(p I_{3}\right)-H_{m j k i}\left(p I_{2}\right) \rightarrow S V C_{3}
\end{array}\right.
$$

where auxiliary tensor $H_{m j k i}$ is defined as:

$$
H_{m j k i}(s, t)=N_{m}(s, t) G_{j k}(s, t) n_{i}^{*}(s, t)
$$

- Matrices for the "flow-weighted" upwind scheme

$$
\begin{aligned}
\boldsymbol{A}^{\boldsymbol{s}} & =\left[\begin{array}{ccc}
0 & \left(1-\alpha_{p I_{1}}\right) & \alpha_{p I_{1}} \\
\alpha_{p I_{2}} & 0 & \left(1-\alpha_{p I_{2}}\right) \\
\left(1-\alpha_{p I_{3}}\right) & \alpha_{p I_{3}} & 0
\end{array}\right] \\
\boldsymbol{B}^{\boldsymbol{s}} & =\left[\begin{array}{ccc}
1 & -\left(1-\alpha_{p I_{1}}\right) \Lambda_{p I_{1}} & -\alpha_{p I_{1}} \Lambda_{p I_{1}} \\
-\alpha_{p I_{2}} \Lambda_{p I_{2}} & 1 & -\left(1-\alpha_{p I_{2}}\right) \Lambda_{p I_{2}} \\
-\left(1-\alpha_{p I_{3}}\right) \Lambda_{p I_{3}} & -\alpha_{p I_{3}} \Lambda_{p I_{3}} & 1
\end{array}\right] \\
\boldsymbol{C}^{\boldsymbol{s}} & =\left[\begin{array}{ccc}
\alpha_{p I_{1}}\left(1-\Lambda_{p I_{1}}\right) & \left(1-\alpha_{p I_{1}}\right)\left(1-\Lambda_{p I_{1}}\right) & 0 \\
0 & \alpha_{p I_{2}}\left(1-\Lambda_{p I_{2}}\right) & \left(1-\alpha_{p I_{2}}\right)\left(1-\Lambda_{p I_{2}}\right) \\
\left(1-\alpha_{p I_{3}}\right)\left(1-\Lambda_{p I_{3}}\right) & 0 & \alpha_{p I_{3}}\left(1-\Lambda_{p I_{3}}\right)
\end{array}\right] \\
\boldsymbol{D}^{\boldsymbol{s}} & =\left[\begin{array}{ccc}
1 & 0 & -1 \\
-1 & 1 & 0 \\
0 & -1 & 1
\end{array}\right]
\end{aligned}
$$

where:

$$
\alpha_{p I_{i}}=\left\{\begin{array}{ll}
1, & \text { if } \quad q_{p I_{i}}>0 \\
0, & \text { if } \quad q_{p I_{i}} \leq 0
\end{array} \quad \overrightarrow{\boldsymbol{\omega}}_{\boldsymbol{p} \boldsymbol{I}}=\frac{\boldsymbol{A}^{\boldsymbol{s}} \overrightarrow{\boldsymbol{q}}_{\boldsymbol{p} \boldsymbol{I}}}{\overrightarrow{\boldsymbol{q}}_{\boldsymbol{p} \boldsymbol{I}}} \quad \overrightarrow{\boldsymbol{\Lambda}}_{\boldsymbol{p} \boldsymbol{I}}=\max \left[\min \left(\overrightarrow{\boldsymbol{\omega}}_{\boldsymbol{p} \boldsymbol{I}}, 1\right), 0\right]\right.
$$

\section{Bilinear Quadrangular Element}

- Coordinates of the element nodes

$$
P_{1}=(-1,-1) \quad P_{2}=(1,-1) \quad P_{3}=(1,1) \quad P_{4}=(-1,1)
$$

- Coordinates of the edges midpoints

$$
m_{1}=(0,-1) \quad m_{2}=(1,0) \quad m_{3}=(0,1) \quad m_{4}=(-1,0)
$$

- Coordinates of the integration points

$$
p I_{1}=\left(0,-\frac{1}{2}\right) \quad p I_{2}=\left(\frac{1}{2}, 0\right) \quad p I_{3}=\left(0, \frac{1}{2}\right) \quad p I_{4}=\left(-\frac{1}{2}, 0\right)
$$



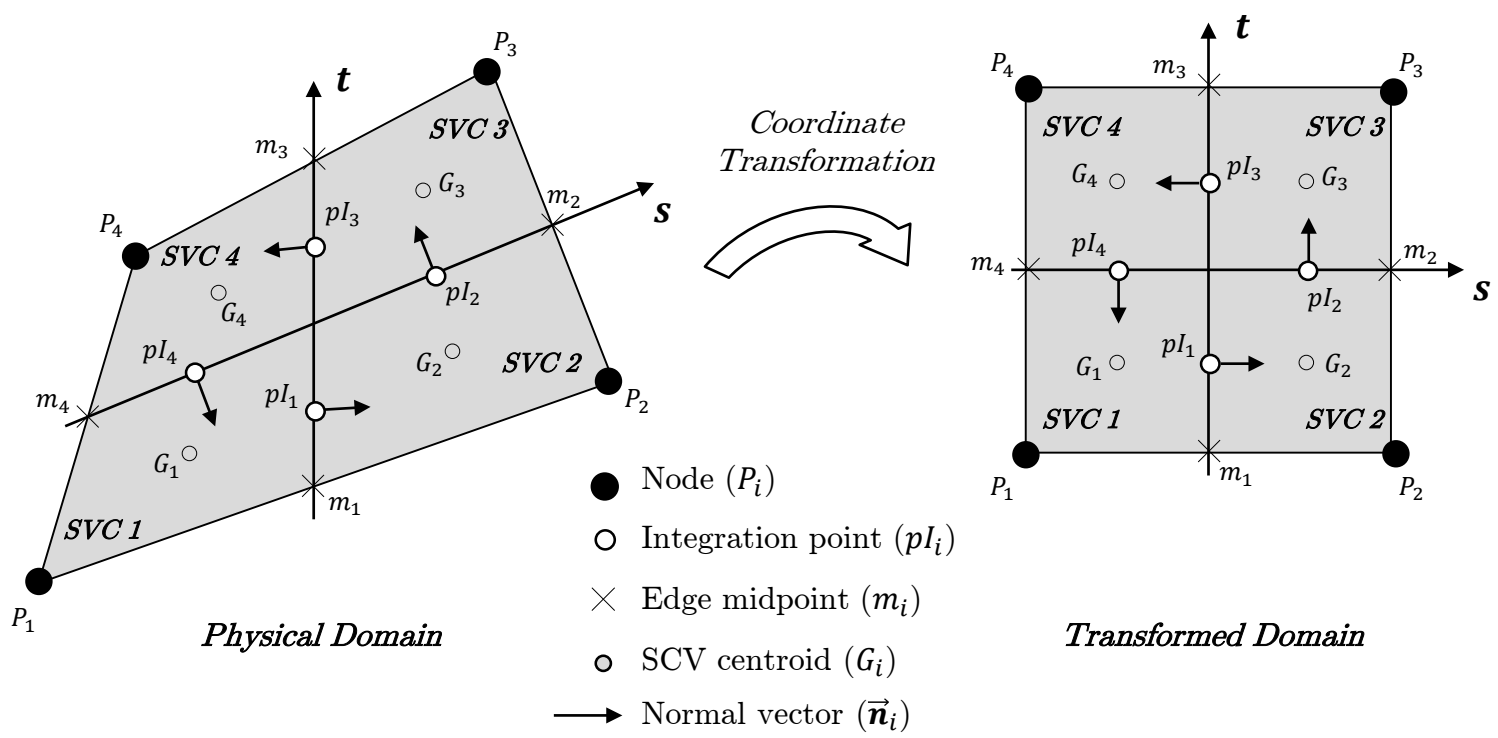

Figure F.2: Coordinate transformation for bilinear quadrangular element.

- Coordinates of the SVC centroids

$$
G_{1}=\left(-\frac{1}{2},-\frac{1}{2}\right) \quad G_{2}=\left(\frac{1}{2},-\frac{1}{2}\right) \quad G_{3}=\left(\frac{1}{2}, \frac{1}{2}\right) \quad G_{4}=\left(-\frac{1}{2}, \frac{1}{2}\right)
$$

- SCV areas

$$
\begin{array}{ll}
A_{S V C_{1}}=\operatorname{det}\left[\boldsymbol{J}\left(G_{1}\right)\right] & A_{S V C_{2}}=\operatorname{det}\left[\boldsymbol{J}\left(G_{2}\right)\right] \\
A_{S V C_{3}}=\operatorname{det}\left[\boldsymbol{J}\left(G_{3}\right)\right] & A_{S V C_{4}}=\operatorname{det}\left[\boldsymbol{J}\left(G_{4}\right)\right]
\end{array}
$$

- Coordinates of the normal vectors (transformed domain)

$$
\overrightarrow{\boldsymbol{n}}_{1}=(0,-1) \quad \overrightarrow{\boldsymbol{n}}_{2}=(1,0) \quad \overrightarrow{\boldsymbol{n}}_{3}=(0,1) \quad \overrightarrow{\boldsymbol{n}}_{4}=(-1,0)
$$

- Coordinates of the normal vectors (physical domain)

$$
\begin{array}{ll}
\overrightarrow{\boldsymbol{n}}_{1}^{*}=\boldsymbol{R} \boldsymbol{J}\left(p I_{1}\right) \overrightarrow{\boldsymbol{n}}_{1} & \overrightarrow{\boldsymbol{n}}_{2}^{*}=\boldsymbol{R} \boldsymbol{J}\left(p I_{2}\right) \overrightarrow{\boldsymbol{n}}_{2} \\
\overrightarrow{\boldsymbol{n}}_{3}^{*}=\boldsymbol{R} \boldsymbol{J}\left(p I_{3}\right) \overrightarrow{\boldsymbol{n}}_{3} & \overrightarrow{\boldsymbol{n}}_{4}^{*}=\boldsymbol{R} \boldsymbol{J}\left(p I_{4}\right) \overrightarrow{\boldsymbol{n}}_{4}
\end{array}
$$

where the auxiliary matrix $\boldsymbol{R}$ is defined as:

$$
\boldsymbol{R}=\left[\begin{array}{cc}
0 & -1 \\
1 & 0
\end{array}\right]
$$

- Interpolation and shape functions

$$
\begin{array}{ll}
N_{1}(s, t)=\frac{1}{4}(1-s)(1-t) & N_{2}(s, t)=\frac{1}{4}(1+s)(1-t) \\
N_{3}(s, t)=\frac{1}{4}(1+s)(1+t) & N_{4}(s, t)=\frac{1}{4}(1-s)(1+t)
\end{array}
$$


- Matrix for gradients interpolation

$$
\boldsymbol{B}(s, t)=\left[\begin{array}{ll}
\frac{\partial N_{1}(s, t)}{\partial s} & \frac{\partial N_{1}(s, t)}{\partial t} \\
\frac{\partial N_{2}(s, t)}{\partial s} & \frac{\partial N_{2}(s, t)}{\partial t} \\
\frac{\partial N_{3}(s, t)}{\partial s} & \frac{\partial N_{3}(s, t)}{\partial t} \\
\frac{\partial N_{4}(s, t)}{\partial s} & \frac{\partial N_{4}(s, t)}{\partial t}
\end{array}\right]=\frac{1}{4}\left[\begin{array}{cc}
(t-1) & (s-1) \\
(1-t) & (-1-t) \\
(1+t) & (1+s) \\
(-1-t) & (1-s)
\end{array}\right]
$$

- Jacobian matrix

$$
\boldsymbol{J}(s, t)=\boldsymbol{Z}_{\boldsymbol{e}} \boldsymbol{B}(s, t)
$$

where $\boldsymbol{Z}_{\boldsymbol{e}}$ is the matrix with the global coordinates of the element nodes:

$$
\boldsymbol{Z}_{\boldsymbol{e}}=\left[\begin{array}{llll}
x_{1} & x_{2} & x_{3} & x_{4} \\
y_{1} & y_{2} & y_{3} & y_{4}
\end{array}\right]
$$

- Tensor for gradients interpolation

$$
G_{j k}(s, t) \Rightarrow \boldsymbol{G}(s, t)=\boldsymbol{B}(s, t) \boldsymbol{J}(s, t)^{-1}
$$

- Tensor for diffusion calculation

$$
B_{m j k i s} \Rightarrow\left\{\begin{array}{l}
B_{m j k i 1}=H_{m j k i}\left(p I_{1}\right)-H_{m j k i}\left(p I_{4}\right) \rightarrow S V C_{1} \\
B_{m j k i 2}=H_{m j k i}\left(p I_{2}\right)-H_{m j k i}\left(p I_{1}\right) \rightarrow S V C_{2} \\
B_{m j k i 3}=H_{m j k i}\left(p I_{3}\right)-H_{m j k i}\left(p I_{2}\right) \rightarrow S V C_{3} \\
B_{m j k i 4}=H_{m j k i}\left(p I_{4}\right)-H_{m j k i}\left(p I_{3}\right) \rightarrow S V C_{4}
\end{array}\right.
$$

where auxiliary tensor $H_{m j k i}$ is defined as:

$$
H_{m j k i}(s, t)=N_{m}(s, t) G_{j k}(s, t) n_{i}^{*}(s, t)
$$

- Matrices for the "flow-weighted" upwind scheme

$$
\begin{aligned}
\boldsymbol{A}^{\boldsymbol{s}} & =\left[\begin{array}{cccc}
0 & \left(1-\alpha_{p I_{1}}\right) & 0 & \alpha_{p I_{1}} \\
\alpha_{p I_{2}} & 0 & \left(1-\alpha_{p I_{2}}\right) & 0 \\
0 & \alpha_{p I_{3}} & 0 & \left(1-\alpha_{p I_{3}}\right) \\
\left(1-\alpha_{p I_{4}}\right) & 0 & \alpha_{p I_{4}} & 0
\end{array}\right] \\
\boldsymbol{B}^{\boldsymbol{s}} & =\left[\begin{array}{cccc}
1 & -\left(1-\alpha_{p I_{1}}\right) \Lambda_{p I_{1}} & 0 & -\alpha_{p I_{1}} \Lambda_{p I_{1}} \\
-\alpha_{p I_{2}} \Lambda_{p I_{2}} & 1 & -\left(1-\alpha_{p I_{2}}\right) \Lambda_{p I_{2}} & 0 \\
0 & -\alpha_{p I_{3}} \Lambda_{p I_{3}} & 1 & -\left(1-\alpha_{p I_{3}}\right) \Lambda_{p I_{3}} \\
-\left(1-\alpha_{p I_{4}}\right) \Lambda_{p I_{4}} & 0 & -\alpha_{p I_{4}} \Lambda_{p I_{4}} & 1
\end{array}\right] \\
\boldsymbol{C}^{\boldsymbol{s}} & =\left[\begin{array}{cccc}
\alpha_{p I_{1}}\left(1-\Lambda_{p I_{1}}\right) & \left(1-\alpha_{p I_{1}}\right)\left(1-\Lambda_{p I_{1}}\right) & 0 & 0 \\
0 & \alpha_{p I_{2}}\left(1-\Lambda_{p I_{2}}\right) & \left(1-\alpha_{p I_{2}}\right)\left(1-\Lambda_{p I_{2}}\right) & 0 \\
0 & 0 & \alpha_{p I_{3}}\left(1-\Lambda_{p I_{3}}\right) & \left(1-\alpha_{p I_{3}}\right)\left(1-\Lambda_{p I_{3}}\right) \\
\left(1-\alpha_{p I_{4}}\right)\left(1-\Lambda_{p I_{4}}\right) & 0 & 0 & \alpha_{p I_{4}}\left(1-\Lambda_{p I_{4}}\right)
\end{array}\right]
\end{aligned}
$$




$$
\boldsymbol{D}^{\boldsymbol{s}}=\left[\begin{array}{cccc}
1 & 0 & 0 & -1 \\
-1 & 1 & 0 & 0 \\
0 & -1 & 1 & 0 \\
0 & 0 & -1 & 1
\end{array}\right]
$$

where:

$$
\alpha_{p I_{i}}=\left\{\begin{array}{ll}
1, & \text { if } \quad q_{p I_{i}}>0 \\
0, & \text { if } \quad q_{p I_{i}} \leq 0
\end{array} \quad \overrightarrow{\boldsymbol{\omega}}_{\boldsymbol{p} \boldsymbol{I}}=\frac{\boldsymbol{A}^{\boldsymbol{s}} \overrightarrow{\boldsymbol{q}}_{\boldsymbol{p} \boldsymbol{I}}}{\overrightarrow{\boldsymbol{q}}_{\boldsymbol{p} \boldsymbol{I}}} \quad \overrightarrow{\boldsymbol{\Lambda}}_{\boldsymbol{p} \boldsymbol{I}}=\max \left[\min \left(\overrightarrow{\boldsymbol{\omega}}_{\boldsymbol{p} \boldsymbol{I}}, 1\right), 0\right]\right.
$$

\section{F.2 Discretization of the Diffusion Term}

According to Eq. 7.6, it is possible to express the diffusive flux across each integration point $(p I)$ of a given element $(e)$ as:

$$
d_{e}^{n}(p I)=\left[\tilde{\boldsymbol{\Gamma}}^{\boldsymbol{p}}(p I) \nabla \tilde{p}_{H}(p I)\right]_{e}^{n} \cdot \overrightarrow{\boldsymbol{n}}^{* e}(p I)
$$

where $p I=\left(s_{p I}, t_{p I}\right)$ denotes the coordinates of the integration points in the transformed domain. In the following deductions, scripts $n$ and $e$ will be suppressed for shortness.

\section{- Approximation of the Diffusivity Matrix at the Integration Points}

Diffusion matrix $\tilde{\boldsymbol{\Gamma}}^{\boldsymbol{p}}(p I)$ may be written in tensor notation as:

$$
\tilde{\boldsymbol{\Gamma}}^{\boldsymbol{p}}(p I) \Rightarrow \tilde{\Gamma}_{i j}^{p}(p I) \quad i, j=1,2
$$

Assuming that each component of the diffusivity matrix is approximated according to the same family of interpolation functions, then:

$$
\tilde{\Gamma}_{i j}^{p}(p I) \approx N_{m}(p I) \tilde{\Gamma}_{i j m}^{p} \quad m=1,2 \ldots, M
$$

where $\tilde{\Gamma}_{i j m}^{p}$ are the nodal values of $\tilde{\Gamma}_{i j}^{p}, M$ the number of nodes of the element and $N_{m}$ the respective interpolation functions (see Appendix F.1).

\section{- Approximation of the Pressure Gradients at the Integration Points}

Pressure gradients $\nabla \tilde{p}_{H}(p I)$ may also be written in tensor notation as:

$$
\nabla \tilde{p}_{H}(p I) \Rightarrow \tilde{g}_{j}(p I)=\frac{\partial \tilde{p}_{H}(p I)}{\partial x_{j}} \quad j=1,2
$$

Assuming the elliptic characteristic of the diffusion term, the approximation of the pressure gradients can be accomplished by adopting linear interpolation based on the shape functions, yielding the following tensor expression:

$$
\tilde{g}_{j}(p I) \approx G_{j k}(p I) \tilde{p}_{H k} \quad k=1,2 \ldots, M
$$

where $\tilde{p}_{H k}$ are the nodal pressures and $G_{j k}$ the interpolation tensor that correlates both pressure gradients approximations and element distortions (see Appendix F.1).

Substituting Eq. F.46 and Eq. F.48 into Eq. F.44, one obtains:

$$
d(p I)=H_{m j k i}(p I)\left(\tilde{\Gamma}_{i j m}^{p} \tilde{p}_{H k}\right) \quad, \quad H_{m j k i}(p I)=N_{m}(p I) G_{j k}(p I) n_{i}^{*}(p I)
$$


Since only geometric features are involved in $H_{m j k i}(p I)$, its value is constant for a fixed grid (non-adaptive mesh) and can hence be calculated once in advance in a pre-processing step of the problem solution (see Appendix F.1).

The total diffusive flux across every $S C V(s)$ that compounds a given element is the sum of the respective fluxes computed for each of the two integration points located on its faces. Mathematically, it can be expressed as:

$$
d_{s}=P_{k s} \tilde{p}_{H k}, \quad P_{k s}=B_{m j k i s} \tilde{\Gamma}_{i j m}^{p} \quad s, k=1,2 \ldots, M
$$

where $P_{k s}$ is defined as the diffusivity matrix and $B_{m j k i s}$ the geometric diffusion tensor of the element (see Appendix F.48). Similarly to the $H_{m j k i}(p I)$ tensor, $B_{m j k i s}$ is also constant for fixed meshes and can thus be calculated only once in a pre-processing procedure.

Finally, Eq. F.50 can be written in matrix notation as:

$$
\overrightarrow{\boldsymbol{d}}_{e}^{n}=\boldsymbol{P}_{e}^{n}\left(\overrightarrow{\tilde{\boldsymbol{p}}}_{\boldsymbol{H}}\right)_{e}^{n}
$$

where the components of vector $\overrightarrow{\boldsymbol{d}}_{e}^{n}$ correspond to the diffusive fluxes through each subcontrol volume, $\left(\overrightarrow{\tilde{\boldsymbol{p}}}_{\boldsymbol{H}}\right)_{e}^{n}$ is the vector of the nodal pressures and $\boldsymbol{P}_{e}^{n}$ is the diffusivity matrix of the element.

\section{F.3 Discretization of the Convective Term}

Analogously to the discretization of the diffusion term, the convective flux of Eq. 7.6 across each integration point $(p I)$ of a given element $(e)$ can be expressed as:

$$
c_{e}^{n}(p I)=[\tilde{q}(p I) \tilde{\theta}(p I)]_{e}^{n}, \quad \tilde{q}(p I)_{e}^{n}=\left[\tilde{\boldsymbol{\Gamma}}^{c}(p I) \overrightarrow{\tilde{\boldsymbol{v}}}(p I)\right]_{e}^{n} \cdot \overrightarrow{\boldsymbol{n}}^{* e}(p I)
$$

where $\tilde{q}(p I)$ is the local convective flux through the integration points $p I=\left(s_{p I}, t_{p I}\right)$. In the following derivations, scripts $n$ and $e$ will be suppressed for shortness.

\section{- Approximation of the Local Convective Flux at the Integration Points}

The local convective flux $\tilde{q}(p I)$ may be written in tensor notation as:

$$
\tilde{q}(p I)=\tilde{C}_{i}(p I) n_{i}^{*}(p I) \quad, \quad \tilde{C}_{i}(p I)=\tilde{\Gamma}_{i j}^{c}(p I) v_{j}(p I) \quad i, j=1,2
$$

Assuming that each component of $\tilde{C}_{i}(p I)$ is approximated according to the same family of interpolation functions, then:

$$
\tilde{C}_{i}(p I) \approx N_{m}(p I) \tilde{C}_{i m} \quad m=1,2 \ldots, M
$$

where $\tilde{C}_{i m}$ are the nodal values of $\tilde{C}_{i}, M$ the number of nodes of the element and $N_{m}$ the respective interpolation functions (see Appendix F.1).

Substituting Eq. F.54 into Eq. F.53, one obtains:

$$
\tilde{q}(p I)=\tilde{Q}_{m i}(p I) \tilde{C}_{i m} \quad, \quad \tilde{C}_{i m}=\left(\tilde{\Gamma}_{i j}^{c} v_{j}\right)_{m} \quad, \quad \tilde{Q}_{m i}(p I)=N_{m}(p I) n_{i}^{*}(p I)
$$

where $\tilde{Q}_{m i}(p I)$ is constant for a fixed grid (non-adaptive mesh) since only geometric features are involved. 


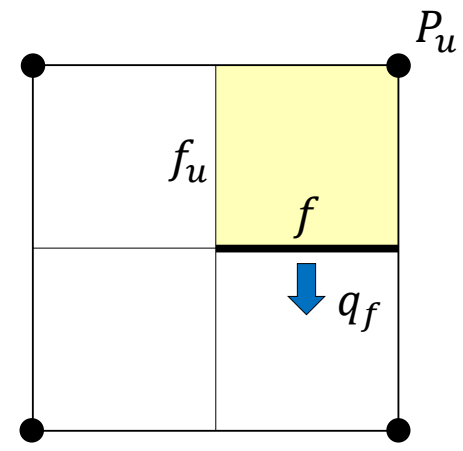

(a)

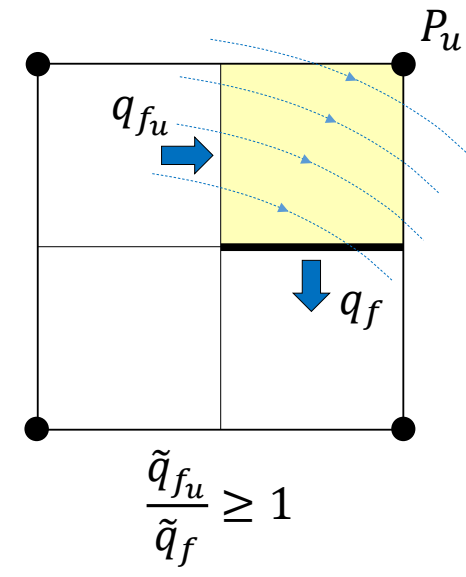

(b)

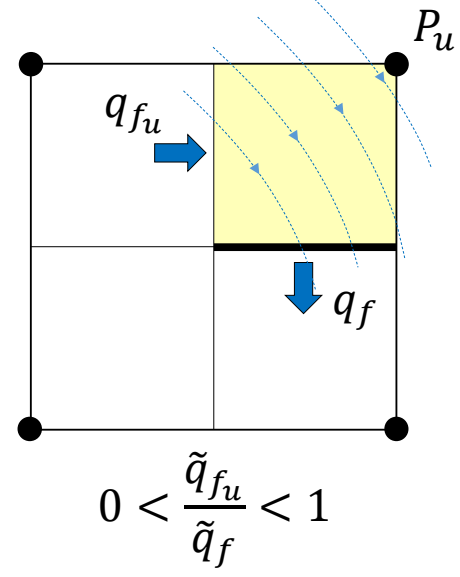

(c)

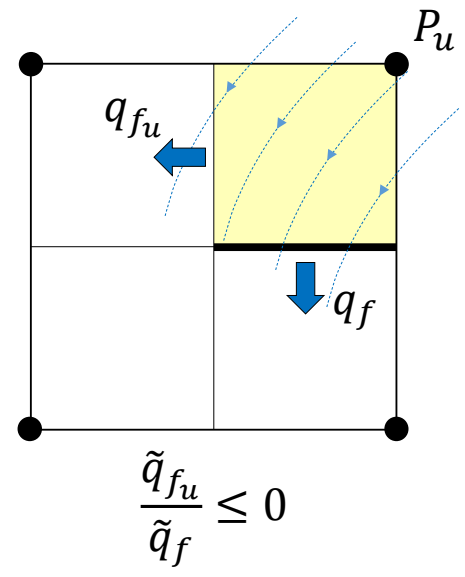

(d)

Figure F.3: Flow entities and illustration of the three typical flow configurations considered in the FWUS for a quadrangular element.

\section{- Approximation of the Film Fraction (Convective Variable) at the Integ- ration Points}

At this point, it is worth highlighting the convective nature of the lubricant film fraction variable $\tilde{\theta}$ present in the hyperbolic term of the modified Reynolds equation. In this sense, the transport of $\tilde{\theta}$ throughout the lubricated contact is strongly associated with the flow velocities induced by surfaces speeds. As well established in computational fluid dynamics (CFD) applications, the numerical discretization of convective terms are highly influenced by the orientation of the fluid flow. Moreover, it is also inappropriate to use linear interpolation functions for approximating such terms, especially because the resulting scheme would retain characteristics of central difference approximations that introduce unrealistic solutions and instabilities in the numerical method $[84,210]$. Therefore, in order to attenuate such drawbacks and to account for the orientation of the fluid flow in the discretization processes, upwindtype interpolations are often employed to cope with hyperbolic-type (convective) equations.

However, when classical upwind formulations are considered in problems with irregular grids, the distorted elements impose complications on the evaluation of the convective flows through the control volumes faces. Furthermore, the customary approaches of using one-dimensional upwind techniques along the grid lines are also prone to cause undesirable and strong dependence of the solutions on the computational grid, the so-called grid orientation (or cross-diffusion) effect [84, 210]. 
In this context, the geometric flexibility of the EbFVM discussed in the present contribution, in which all the computations are performed at the element level on the transformed domain, offers useful advantages to approach the drawbacks mentioned above concerning the compatibility of upwind strategies and irregular meshes. Next, details will be given for one of the most widespread advection scheme devoted for EbFVM solutions, namely Flow Weighted Upwind Scheme (FWUS) [108, 188]. This scheme is based on two important features: (1) the absolute preservation of the positivity of the coefficients of the discretized equations, which contributes to the stability of the numerical solution, and (2) the consideration of the local skewness of the flow, i.e. the local orientation of the fluid flow.

According to the FWUS scheme, the approximated value of the convective scalar variable at a given integration point depends only on the flow configuration in the immediate upstream sub-control volume. As can be seen in Fig. F.3, that sub-control volume is directly related to one node and two element faces, one of them being precisely the face containing the integration point where the convective variable must be determined. Thus, the values of the variable at those three location points can be correlated linearly by means of the following expression:

$$
\tilde{\theta}_{f}=\left(1-\Lambda_{f}\right) \tilde{\theta}_{P_{u}}+\left(\Lambda_{f}\right) \tilde{\theta}_{f_{u}}
$$

where $\Lambda_{f}$ is the interpolation weighting factor that depends on the local flow orientation evaluated in agreement with the local flow ratio parameter $\omega_{f}$, which is defined as the ratio between the convective flux across the upwind face and the flow through the face where the associated integration point is located. Mathematically (see Fig. F.3):

$$
\omega_{f}=\frac{\tilde{q}_{f_{u}}}{\tilde{q}_{f}} \quad, \quad \Lambda_{f}=\max \left[\min \left(\omega_{f}, 1\right), 0\right]
$$

Conforming to Fig. F.3, three typical flow configurations across the referred subcontrol volume can be identified, each one corresponding to a certain range of values of $\omega_{f}$. Accordingly [108, 188]:

- $\boldsymbol{\omega}_{f} \geq 1$ : All the fluid flowing through face $f$ comes from upstream face $f_{u}$, i.e. $\Lambda_{f}=1$ and so $\tilde{\theta}_{f}=\tilde{\theta}_{f_{u}}$. See Fig. F.3 (b).

$-\boldsymbol{\omega}_{\boldsymbol{f}} \leq \mathbf{0}$ : All the fluid flowing thorough both faces comes from inside the subcontrol volume itself, thus carrying only the nodal value $\tilde{\theta}_{P_{u}}$, i.e. $\Lambda_{f}=0$ and so $\tilde{\theta}_{f}=\tilde{\theta}_{P_{u}}$. See Fig. F.3 (d).

$-0<\omega_{f}<1$ : Intermediate situation in which the fluid flowing through face $f$ comes partially from the immediate upstream sub-control volume and partially from the neighbour sub-control volume through face $f_{u}$. In this case, it is possible to claim that $\tilde{\theta}_{f}$ should be proportional to both $\tilde{\theta}_{P_{u}}$ and $\tilde{\theta}_{f_{u}}$, in a measure equivalent to that stated in Eq. F.56. See Fig. F.3 (c).

Considering the local indexation of nodes and faces in an element, Eq. F.56 can be written in a general fashion as [108]:

$$
\tilde{\theta}_{p I_{i}}=\left\{\begin{array}{lll}
\left(1-\Lambda_{p I_{i}}\right) \tilde{\theta}_{P_{i}}+\left(\Lambda_{p I_{i}}\right) \tilde{\theta}_{p I_{i+1}} & \text { if } & \tilde{q}_{p I_{i}}>0 \\
\left(1-\Lambda_{p I_{i}}\right) \tilde{\theta}_{P_{i-1}}+\left(\Lambda_{p I_{i}}\right) \tilde{\theta}_{p I_{i-1}} & \text { if } \quad & \tilde{q}_{p I_{i}} \leq 0
\end{array}\right.
$$


where subscript $i$ belongs to the 4-cycle $\{1,2,3.4\}$ and are associated with each integration point $(p I)$ of the element. Thus, for instance, in a quadrangular element $k+1=1$ when $k=4$, whereas $k-1=4$ when $k=1$. In the above equation, $\Lambda_{p I_{i}}$ is the interpolation weighting factor that depends on the local flow orientation evaluated in agreement with the local flow ratio parameter $\omega_{p I_{i}}$, which in turn is defined as the ratio between the convective flux across the upwind face and the flow thorough the face where the associated integration point is located. Mathematically:

$$
\omega_{p I_{i}}=\left\{\begin{array}{ll}
\frac{\tilde{q}_{p I_{i+1}}}{\tilde{q}_{p I_{i}}} \quad \text { if } \quad \tilde{q}_{p I_{i}}>0 \\
\frac{\tilde{q}_{p I_{i-1}}}{\tilde{q}_{p I_{i}}} \quad \text { if } \quad \tilde{q}_{p I_{i}} \leq 0
\end{array}, \quad \Lambda_{p I_{i}}=\max \left[\min \left(\omega_{p I_{i}}, 1\right), 0\right]\right.
$$

By assembling Eq. F.59 for all integration points of the element, it is possible to define the following matrix expression that correlates $\tilde{\theta}_{i}$ to nodal values $\tilde{\theta}_{P_{i}}$ :

$$
\overrightarrow{\tilde{\boldsymbol{\theta}}}_{p I}=\underbrace{\left(\boldsymbol{B}^{s-1} C^{\boldsymbol{s}}\right)}_{\boldsymbol{U}} \overrightarrow{\tilde{\boldsymbol{\theta}}}=\boldsymbol{U} \overrightarrow{\tilde{\boldsymbol{\theta}}}
$$

where $\boldsymbol{B}^{\boldsymbol{s}}$ and $\boldsymbol{C}^{\boldsymbol{s}}$ are $M \times M$ matrices calculated according to the local convective fluxes $\tilde{q}(p I)$ (see Appendix F.1).

Finally, by substituting Eq. F.55 and Eq. F.60 into Eq. F.52, the total convective flux across every $S C V(s)$ that compounds a given element can be expressed as:

$$
c_{s}=C_{k s} \tilde{\theta}_{k} \quad, \quad \boldsymbol{C}=\boldsymbol{D}[\operatorname{diag}(\overrightarrow{\tilde{\boldsymbol{q}}})] \boldsymbol{U} \quad s, k=1,2 \ldots, M
$$

where $\boldsymbol{D}$ is a $M \times M$ matrix that depends on the element type (see Appendix F.1).

For simplicity, Eq. F.61 can be written in matrix notation as:

$$
\overrightarrow{\boldsymbol{c}}_{e}^{n}=\boldsymbol{C}_{e}^{n}(\overrightarrow{\tilde{\boldsymbol{\theta}}})_{e}^{n}
$$

where the components of vector $\overrightarrow{\boldsymbol{c}}_{e}^{n}$ correspond to the diffusive fluxes through each subcontrol volume, $(\overrightarrow{\tilde{\boldsymbol{\theta}}})_{e}^{n}$ is the vector of the nodal pressures and $\boldsymbol{C}_{e}^{n}$ is the convective matrix of the element.

\section{F.4 Discretization of the Source Terms}

According to Eq. 7.6, it possible to define two source terms associated with each $S C V(s)$ of a given element $(e)$ :

$$
\begin{aligned}
f_{1 e, s}^{n} & =\left[\tilde{\theta}\left(\tilde{S}_{T S}+\tilde{S}_{N S}+\frac{\tilde{S}_{T}}{\Delta t}\right)\right]_{P}^{n} \Delta \mathcal{D}_{\mathcal{H}_{s}^{e}}^{e} \\
f_{2 e, s}^{n} & =-\left[\tilde{\theta}^{n-1}\left(\frac{\tilde{S}_{T}}{\Delta t}\right)^{n}\right]_{P} \Delta \mathcal{D}_{\mathcal{H}_{s}^{e}}^{e} \quad s=1,2 \ldots, M
\end{aligned}
$$


where $\Delta \mathcal{D}_{\mathcal{H}_{s}^{e}}^{e}$ is the physical area of sub-control volume $s$. It is important to notice that both expressions of Eq. F.63 and Eq. F.65 are evaluated explicitly at the element nodes referred to the respective $S C V$. By rearranging the terms of the equations in vector notation, one obtains:

$$
\begin{aligned}
& \left(\overrightarrow{\boldsymbol{f}}_{\mathbf{1}}\right)_{e}^{n}=\left(\overrightarrow{\boldsymbol{F}}_{\mathbf{1}}\right)_{e}^{n} \cdot(\overrightarrow{\tilde{\boldsymbol{\theta}}})_{e}^{n} \quad\left(\overrightarrow{\boldsymbol{F}}_{\mathbf{1}}\right)_{e}^{n} \Rightarrow F_{1 e, s}^{n}=\left[\left(\tilde{S}_{T S}+\tilde{S}_{N S}+\frac{\tilde{S}_{T}}{\Delta t}\right)\right]_{P}^{n} \Delta \mathcal{D}_{\mathcal{H}_{s}^{e}}^{e} \\
& \left(\overrightarrow{\boldsymbol{f}}_{\mathbf{2}}\right)_{e}^{n}=\left(\overrightarrow{\boldsymbol{F}}_{\mathbf{2}}\right)_{e}^{n} \cdot(\overrightarrow{\tilde{\boldsymbol{\theta}}})_{e}^{n-1} \quad\left(\overrightarrow{\boldsymbol{F}}_{\mathbf{2}}\right)_{e}^{n} \Rightarrow F_{2 e, s}^{n}=-\left[\left(\frac{\tilde{S}_{T}}{\Delta t}\right)\right]_{P}^{n} \Delta \mathcal{D}_{\mathcal{H}_{s}^{e}}
\end{aligned}
$$

where the components of $\left(\overrightarrow{\boldsymbol{f}}_{\mathbf{1}}\right)_{e}^{n}$ and $\left(\overrightarrow{\boldsymbol{f}}_{\mathbf{2}}\right)_{e}^{n}$ are the source terms associated with each element node, while $\left(\overrightarrow{\boldsymbol{F}}_{\mathbf{1}}\right)_{e}^{n}$ and $\left(\overrightarrow{\boldsymbol{F}}_{\mathbf{2}}\right)_{e}^{n}$ are auxiliary element vectors. Additionally, $(\overrightarrow{\tilde{\boldsymbol{\theta}}})_{e}^{n}$ and $(\overrightarrow{\tilde{\boldsymbol{\theta}}})_{e}^{n 1}$ are vectors of the nodal lubricant film fraction at time instants $n$ and $(n-1)$, respectively; the operator '.' denotes dot products.

\section{F.5 Validation Cases}

The example cases considered in this section were chosen to test the correctness and accuracy of the proposed formulation and algorithmic implementation by comparison with other solution methodologies published in the recent literature, including both alternative mass-conserving Reynolds solvers and full CFD simulations. Each considered example has been selected to demonstrate the various features considered in the newly developed computational framework.

\section{Single and Double Parabolic Slider with Density-Pressure Cor- rection}

This first example was chosen to evaluate the effectiveness of the proposed EbFVM formulation in comparison with different methodologies for solving lubrication problems involving compressible fluids. To do so, two simulation cases characterized by single and double parabolic sliders similar to those tested in [185] were analysed. However, as proposed in [27], the lubricant film geometry was modified with respect to that used in [185] in order to highlight the effects of the fluid compressibility. The main geometric and operational parameters of each parabolic slider are summarized in Tables F.1 and F.2, as well as the respective comparative results. The results for the single parabolic slider are examined against those calculated using the formulations proposed in [185], the linear complementary problem (LCP) solution in [27] and the incompressible case. Figure F.4 confirms the agreement of the current EbFVM formulation with those obtained with the algorithms proposed by Sahlin et al. [185] and Bertocchi et al. [27], both in terms of hydrodynamic pressures (maximum difference in peak pressure of $0.76 \%$ ) and location of the cavitation boundary separating the pressured and cavitated zones.

Additionally, the double parabolic slider case also proposed by [185] is used to assess the capability of the EbFVM for predicting the fluid film reformation boundary. The results are compared both with respect to a CFD analysis based on the full solution of the Navier-Stokes equations developed using the OpenFOAM code [103], as well as with the same methods employed by Sahlin et al. [185] and Bertocchi et al. [27]. The consideration 
of CFD solutions as a comparative basis is aimed to (1) ensure the validity of Reynold equation for flow problems which satisfy the fundamental lubrication assumptions, and further (2) to demonstrate that in common lubrication applications the intricate flow behaviour of the liquid-vapour/gases mixture within the cavitation zones is in general meaningless for the overall predictions of the system performance. Figure F.5 illustrates an excellent agreement between the results calculated with the current EbFVM scheme compared to those obtained from the CFD simulations and using the methodologies by Sahlin et al. [185] and Bertocchi et al. [27]; the maximum deviation for the hydrodynamic peak pressure is $\leq 0.5 \%$ and the rupture and reformation cavitation boundaries are practically identical.

Table F.1: General simulation parameters for the single parabolic slider case with density-pressure correction.
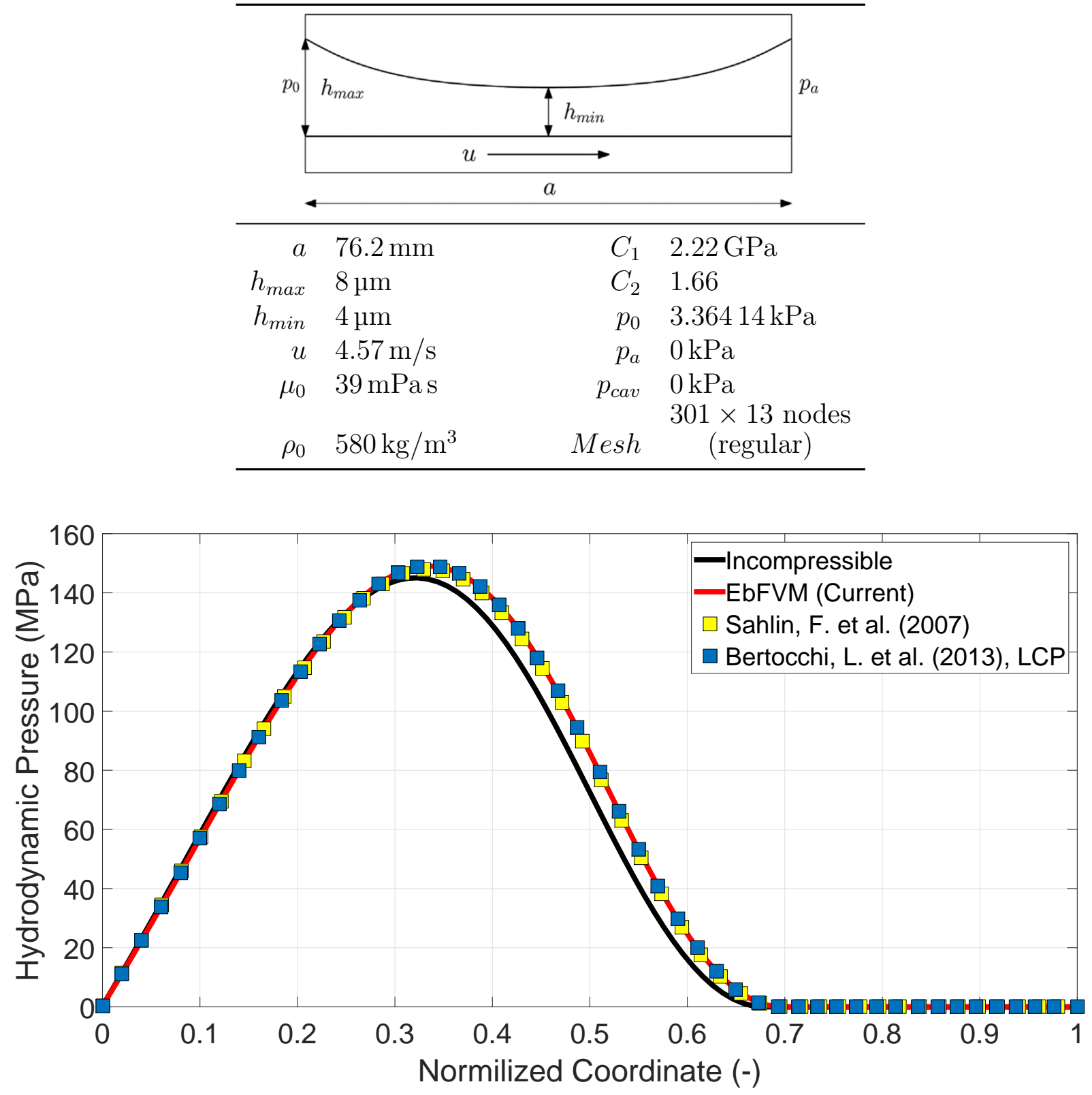

Figure F.4: Comparative results for the single parabolic slider case with densitypressure correction. 
Table F.2: General simulation parameters for the double parabolic slider case with density-pressure correction.
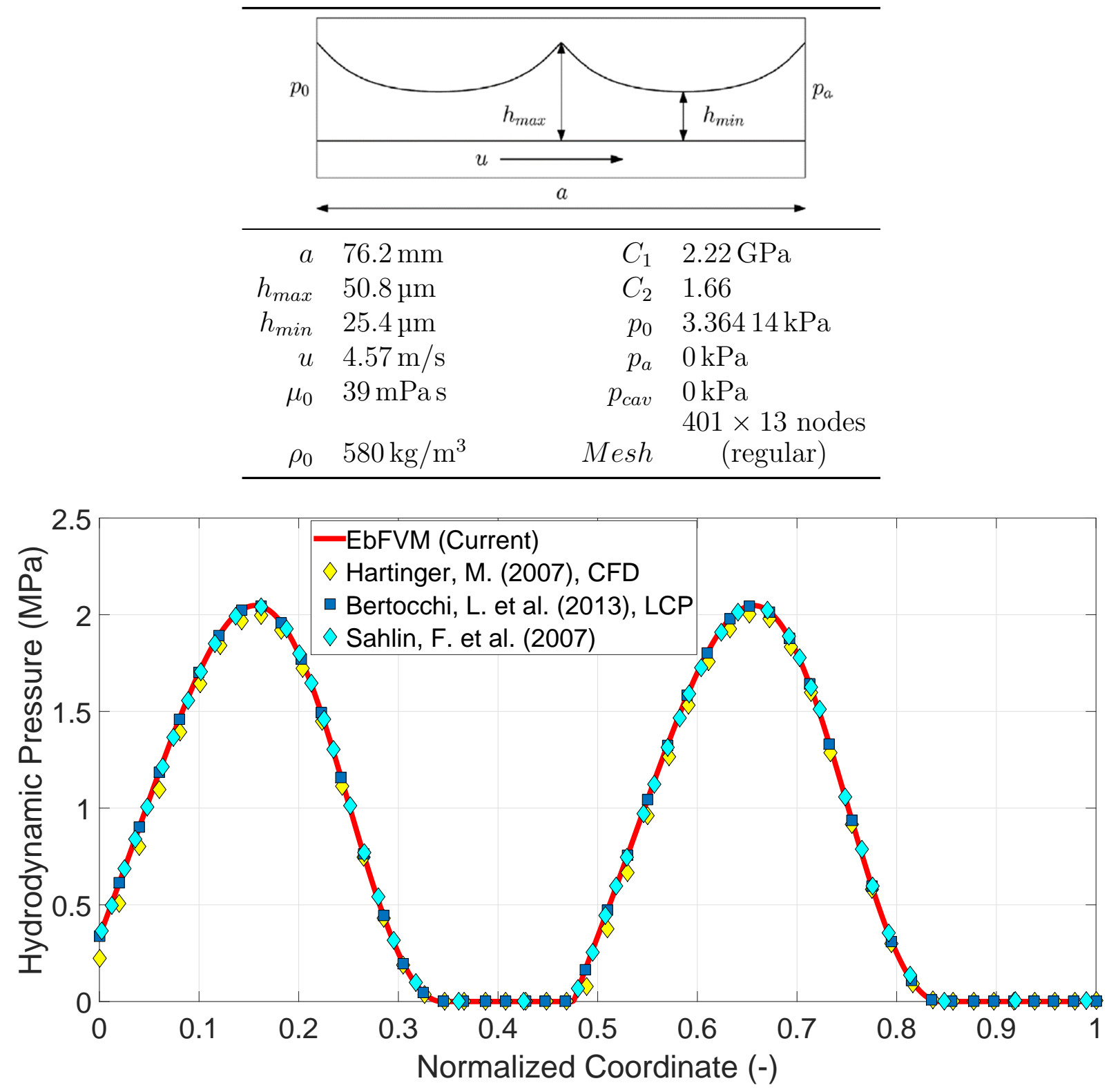

Figure F.5: Comparative results for the double parabolic slider case with densitypressure correction.

\section{Long Journal Bearing with Viscosity-Pressure Correction}

In this second example, the capability of the proposed EbFVM formulation for solving problems with piezoviscous lubricants is tested. Particularly, the simulation case of an infinitely long journal bearing solved in [27] based on the LCP approach for an incompressible piezoviscous lubricant flow is considered. The main problem parameters are listed in Table F.3. Two different operational conditions represented by the fixed eccentricity ratios of $\varepsilon=0.93$ and $\varepsilon=0.95$ are investigated. The Barus equation is adopted for the piezo-viscosity correction. Figures F.6 compare the hydrodynamic pressure profiles along the bearing circumferential directions computed with the current EbFVM formulation against the results from using the LCP method [27] for the two eccentricity ratios 
considered. Again, the results are in good agreement in both cases, with a maximum peak pressure difference of $\leq 0.9 \%$. Excellent agreement is observed for the cavitation boundaries as well.

Table F.3: General simulation parameters for the infinitely long journal bearing case with pressure-viscosity correction.

\begin{tabular}{rlrl}
\hline$R$ & $31.29 \mathrm{~mm}$ & $\rho_{0}$ & $850 \mathrm{~kg} / \mathrm{m}^{3}$ \\
$L$ & $625.8 \mathrm{~mm}$ & $\alpha$ & $11.2 \mathrm{GPa}^{-1}$ \\
$c$ & $40 \mu \mathrm{m}$ & $p_{0}$ & $0 \mathrm{kPa}$ \\
$\omega$ & $250 \mathrm{rad} / \mathrm{s}$ & $p_{\text {cav }}$ & $-100 \mathrm{kPa}$ \\
$\varepsilon$ & 0.93 and 0.95 & Mesh & $601 \times 13$ nodes \\
$\mu_{0}$ & $5.7 \mathrm{mPas}$ & & (regular) \\
\hline
\end{tabular}

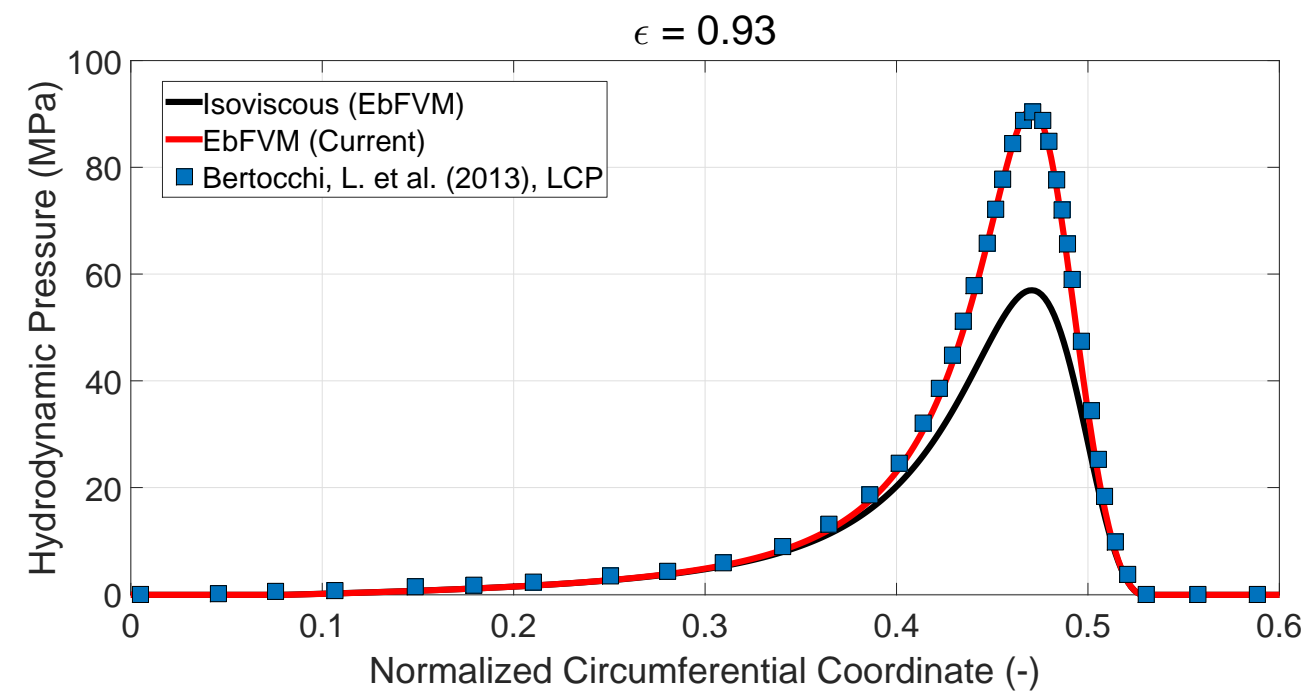

(a)

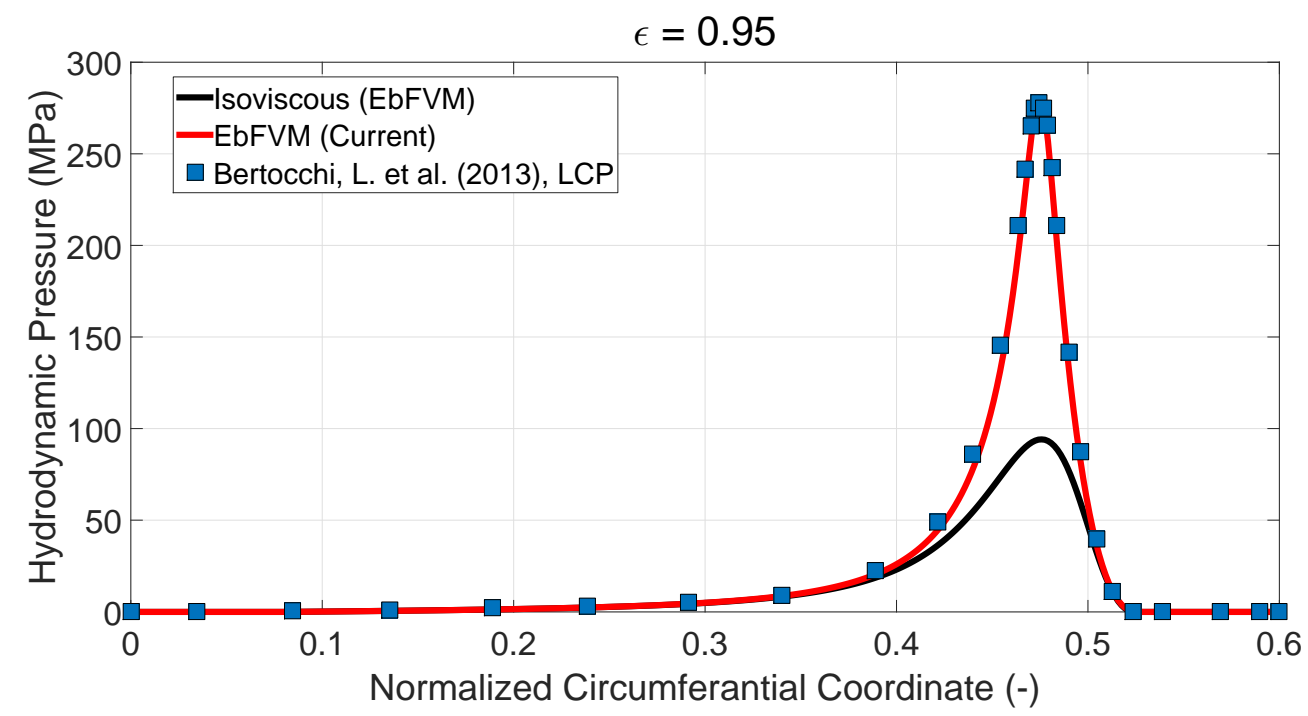

(b)

Figure F.6: Comparative results for the infinitely long journal bearing case with pressure-viscosity correction. 


\section{Pure Squeeze Motion Between Circular Plates}

In this example case, the validation of the proposed EbFVM scheme is assessed for a two-dimensional domain under time dependent conditions. The classical lubrication problem of two circular plates with finite radius and under pure (sinusoidal) squeeze motion is considered. Moreover, any viscosity and density corrections are ignored. The results are compared with those obtained from the analytical solution developed by [162] and the linear complementary method of [27]. The main system parameters are displayed in Table F.4. For the numerical simulation, an irregular grid with 2913 nodes has been used, and 3 oscillating periods with 576 times steps per cycle are adopted for the time solution. Figure F.7 depicts the time variation of the extension of the cavitation zone calculated from the above described methodologies chosen for comparison; notice that the internal "annulus-like" surface plots correspond to the simulated film fraction fields at 3 different times, which illustrate the progressive variation of the cavitation zone (in blue). Once again, the agreement of the results is excellent. Furthermore, the average computational time required for each time step calculation was $0.56 \mathrm{~s}$ for the EbFVM and $150 \mathrm{~s}$ for the LCP case; this highlights the good efficiency of the current proposed finite volume method.

Table F.4: General simulation parameters for the pure squeeze motion case between circular plates.

\begin{tabular}{rlrl}
\hline$r$ & $5 \mathrm{~mm}$ (circular) & $\omega$ & $99.74 \mathrm{rad} / \mathrm{s}$ \\
$h(t)$ & $h_{\min }+h_{a}(1-\cos \omega t)$ & $T$ & $0.063 \mathrm{~s}$ \\
$h_{\min }$ & $9.14 \mathrm{~mm}$ & $\mu_{0}$ & $5 \mathrm{mPas}$ \\
$h_{a}$ & $320.8 \mu \mathrm{m}$ & $\rho_{0}$ & $850 \mathrm{~kg} / \mathrm{m}^{3}$ \\
$t_{\max }$ & $0.1890 \mathrm{~s}(3$ cycles) & $p_{0}$ & $100 \mathrm{kPa}$ \\
& & & 2913 nodes \\
$n_{t}$ & 576 (per cycle) & Mesh & (irregular) \\
\hline
\end{tabular}

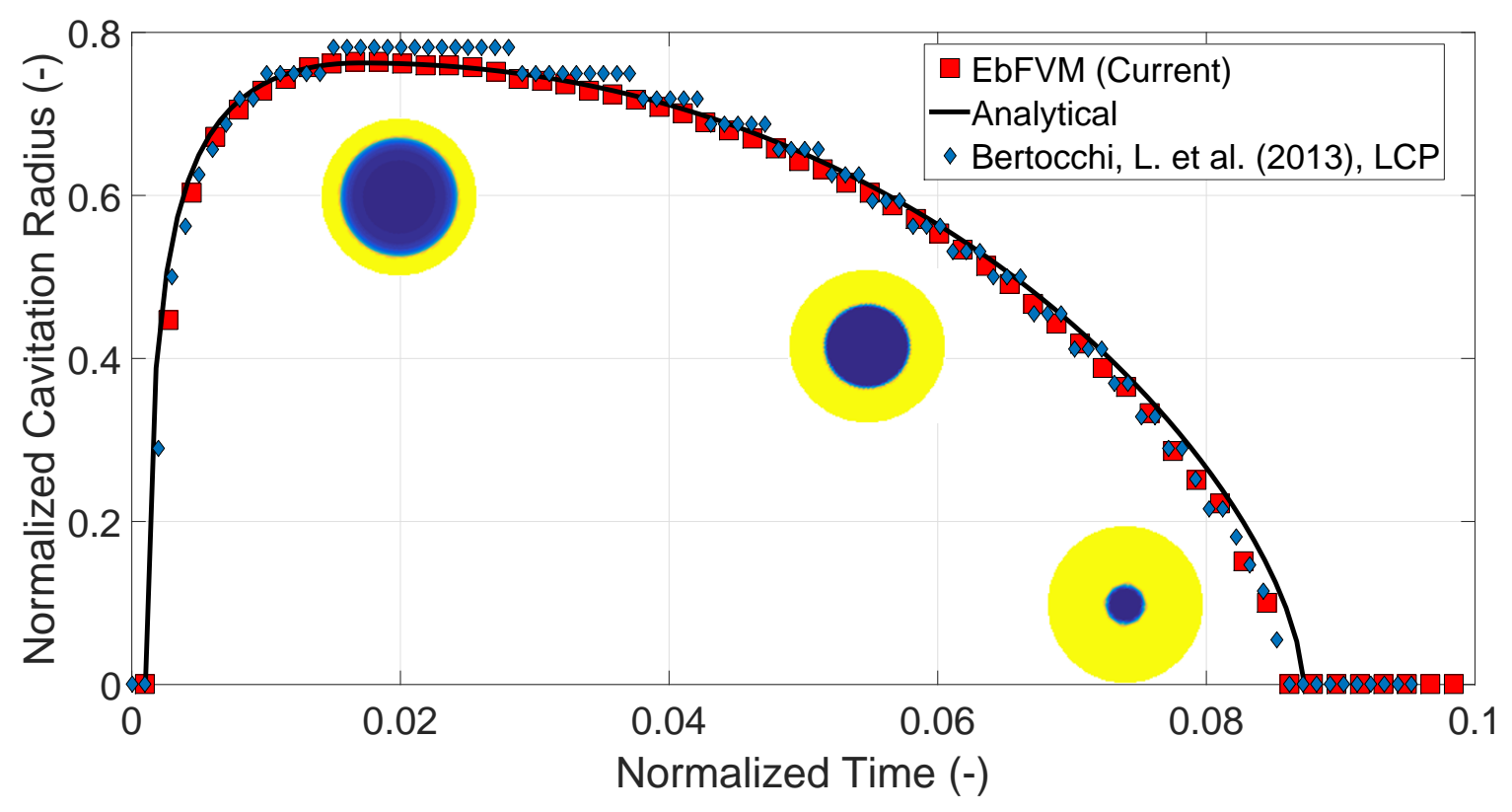

Figure F.7: Comparative results for the pure squeeze motion case between circular plates. 


\section{Sliding Pocket Bearing with Viscosity-Pressure, Viscosity-Shear- Thinning and Density-Pressure Correction}

This second two-dimensional example deals with a sliding pocket bearing lubricated with a compressible, piezoviscous and shear-thinning fluid. Two geometric configurations characterizing a finite and an infinitely long bearing are investigated. All the geometric and operational parameters are listed in Table 5. The figure displayed at the bottom right-hand side of the table compares the pressure profiles along the bearing mid-section $\pi$ calculated with the current EbFVM scheme and with the LCP formulation by Bertocchi et al. [27]. As can be observed, the results computed with the new finite volume scheme match those obtained with LCP technique for both bearing geometries very well, including the analytical solution for the infinitely long bearing case.

Table F.5: General simulation parameters for the sliding bearing case with viscositypressure, viscosity-shear-thinning and density-pressure correction.

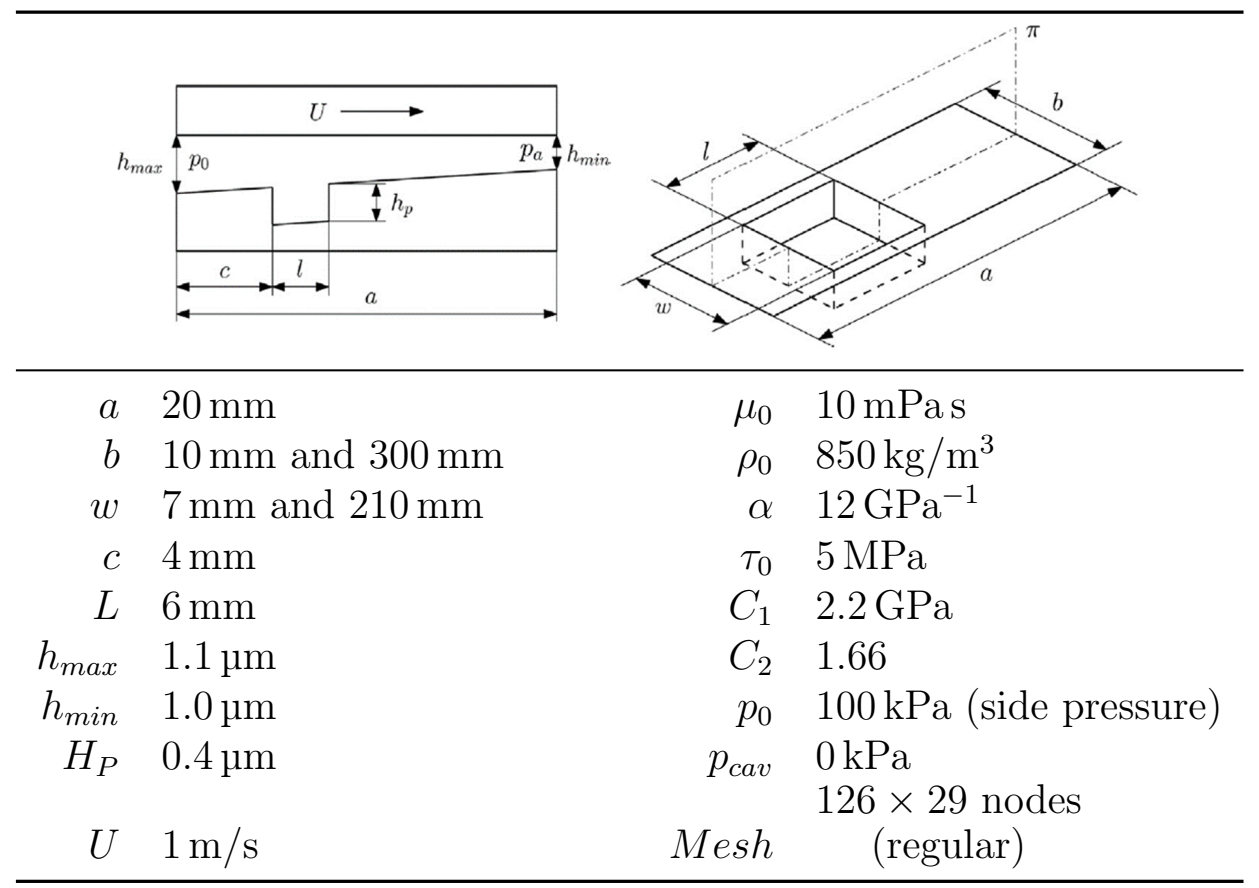

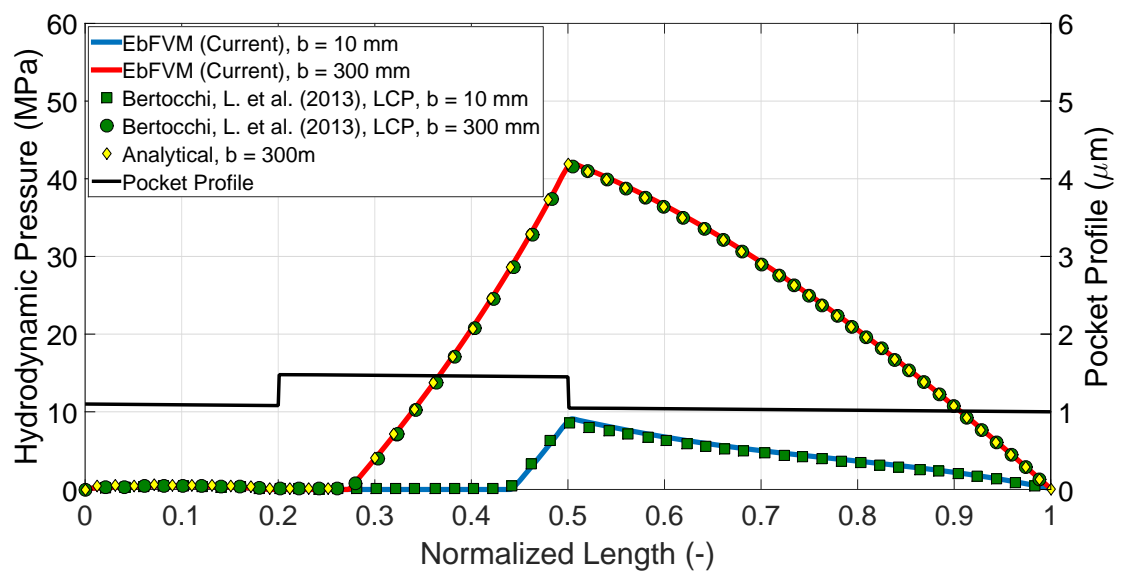

Figure F.8: Comparative results for the sliding bearing case with viscosity-pressure, viscosity-shear-thinning and density-pressure correction. 


\section{Appendix G}

\section{Algorithms}

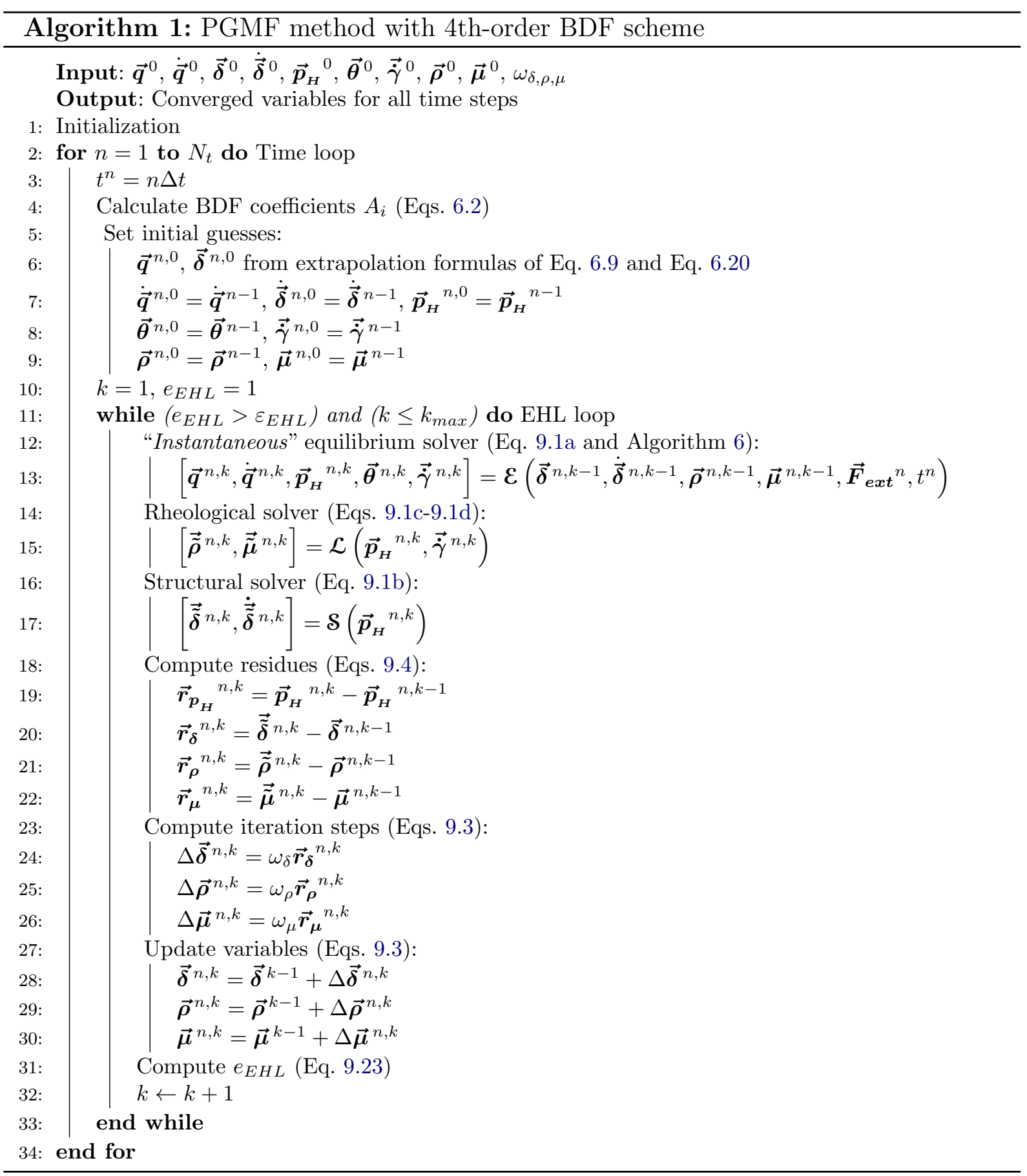




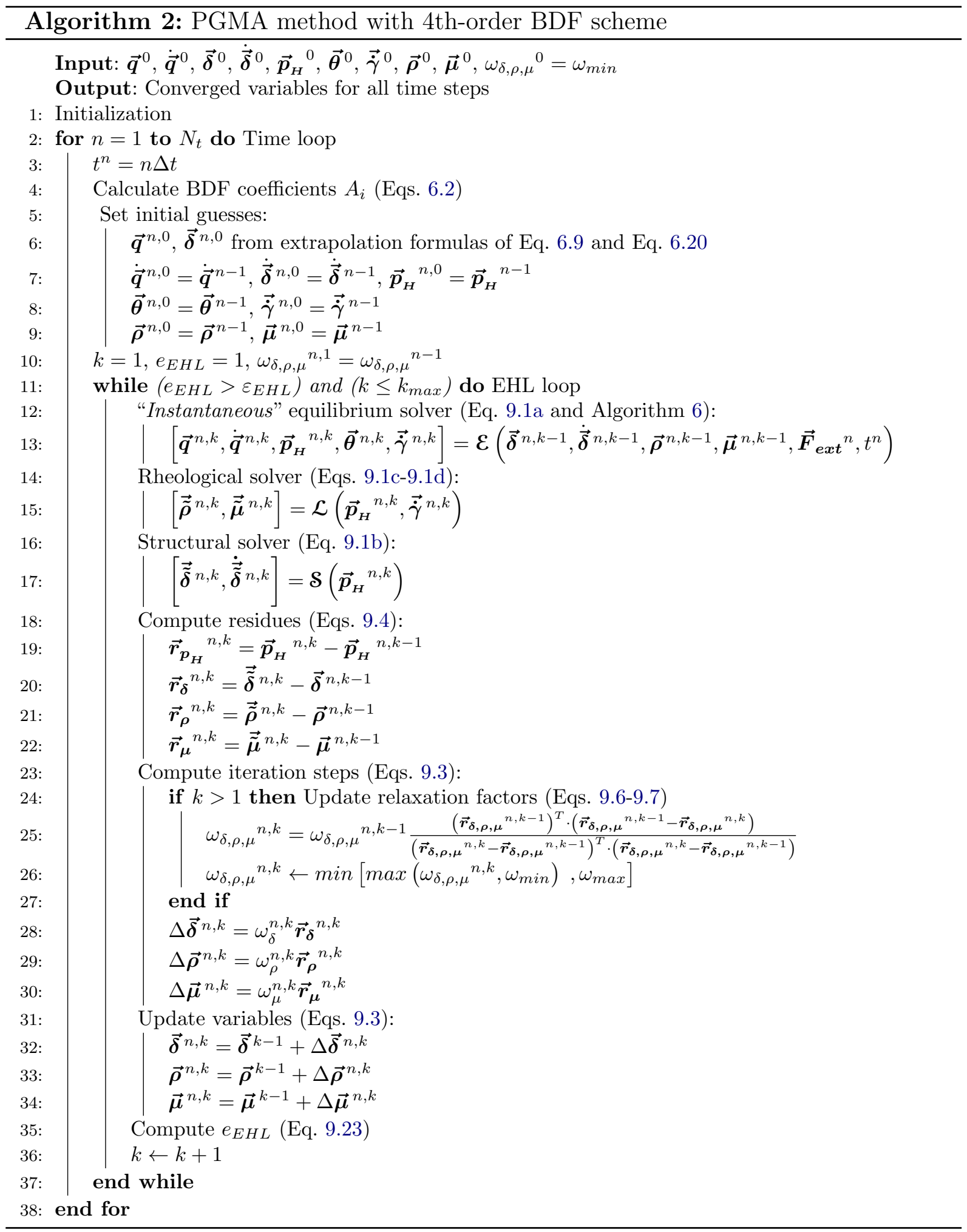




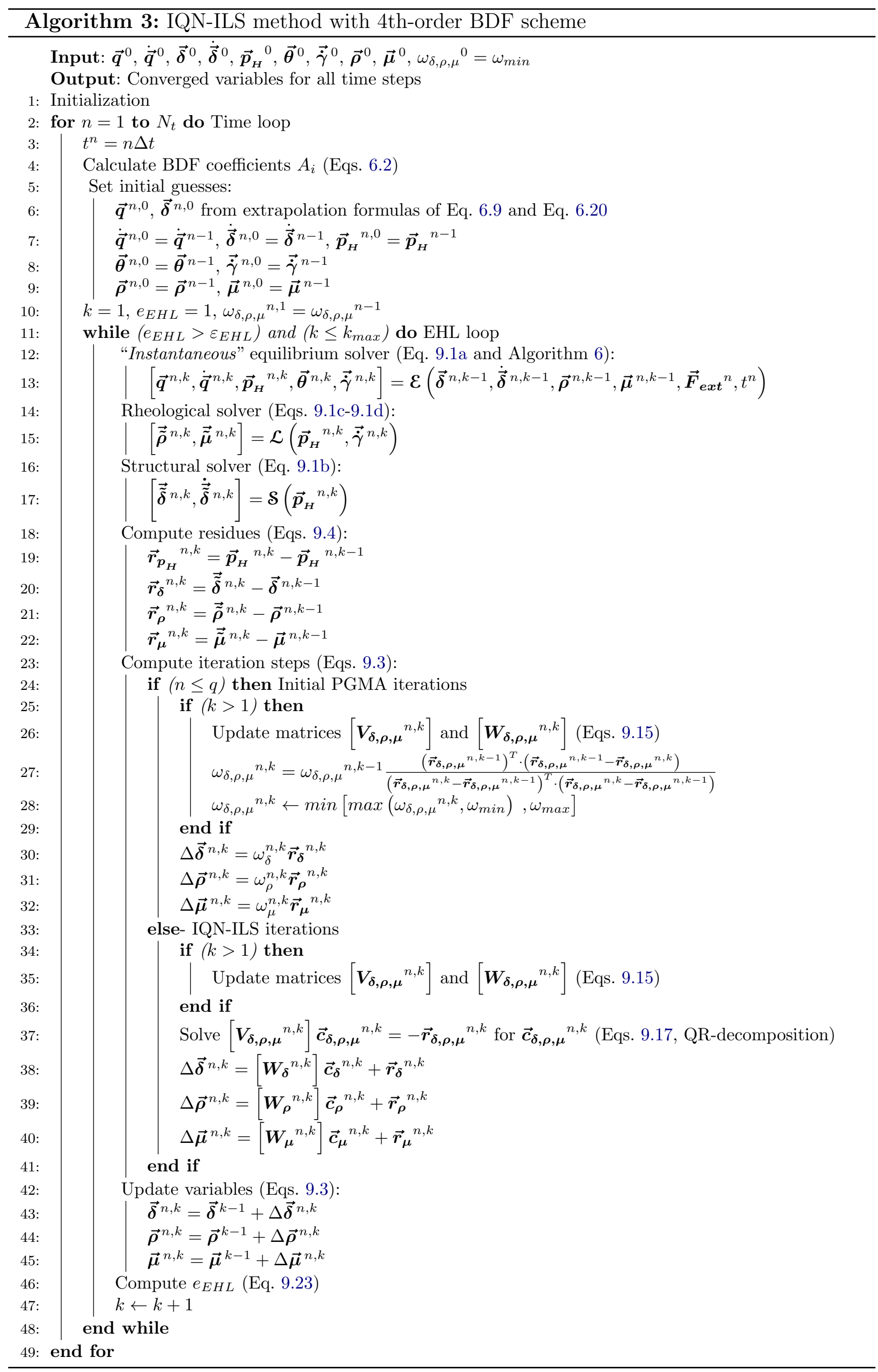


Input: $\overrightarrow{\boldsymbol{q}}^{0}, \dot{\overrightarrow{\boldsymbol{q}}}^{0}, \overrightarrow{\boldsymbol{\delta}}^{0}, \dot{\overrightarrow{\boldsymbol{\delta}}}^{0}, \overrightarrow{\boldsymbol{p}}_{\boldsymbol{H}}{ }^{0}, \overrightarrow{\boldsymbol{\theta}}^{0}, \overrightarrow{\boldsymbol{\gamma}}^{0}, \overrightarrow{\boldsymbol{\rho}}^{0}, \overrightarrow{\boldsymbol{\mu}}^{0}, \omega_{\delta, \rho, \mu}{ }^{0}=\omega_{\min }$

Output: Converged variables for all time steps

1: Initialization

2: for $n=1$ to $N_{t}$ do Time loop

2: $\quad t^{n}=n \Delta t$

Calculate BDF coefficients $A_{i}$ (Eqs. 6.2)

Set initial guesses:

$$
\begin{aligned}
& \overrightarrow{\boldsymbol{q}}^{n, 0}, \overrightarrow{\boldsymbol{\delta}}^{n, 0} \text { from extrapolation formulas of Eq } \\
& \dot{\overrightarrow{\boldsymbol{q}}}^{n, 0}=\dot{\overrightarrow{\boldsymbol{q}}}^{n-1}, \dot{\overrightarrow{\boldsymbol{\delta}}}^{n, 0}=\dot{\overrightarrow{\boldsymbol{\delta}}}^{n-1}, \overrightarrow{\boldsymbol{p}}_{\boldsymbol{H}}{ }^{n, 0}=\overrightarrow{\boldsymbol{p}}_{\boldsymbol{H}}{ }^{n-1} \\
& \overrightarrow{\boldsymbol{\theta}}^{n, 0}=\overrightarrow{\boldsymbol{\theta}}^{n-1}, \overrightarrow{\dot{\gamma}}^{n, 0}=\overrightarrow{\dot{\gamma}}^{n-1} \\
& \overrightarrow{\boldsymbol{\rho}}^{n, 0}=\overrightarrow{\boldsymbol{\rho}}^{n-1}, \overrightarrow{\boldsymbol{\mu}}^{n, 0}=\overrightarrow{\boldsymbol{\mu}}^{n-1} \\
k= & 1, e_{E H L}=1, \omega_{\delta, \rho, \mu}{ }^{n, 1}=\omega_{\delta, \rho, \mu}{ }^{n-1}
\end{aligned}
$$

while $\left(e_{E H L}>\varepsilon_{E H L}\right)$ and $\left(k \leq k_{\max }\right)$ do EHL loop

"Instantaneous" equilibrium solver (Eq. 9.1a and Algorithm 6):

$\mid\left[\overrightarrow{\boldsymbol{q}}^{n, k}, \dot{\overrightarrow{\boldsymbol{q}}}^{n, k}, \overrightarrow{\boldsymbol{p}}_{\boldsymbol{H}}^{n, k}, \overrightarrow{\boldsymbol{\theta}}^{n, k}, \overrightarrow{\dot{\gamma}}^{n, k}\right]=\mathcal{E}\left(\overrightarrow{\boldsymbol{\delta}}^{n, k-1}, \dot{\overrightarrow{\boldsymbol{\delta}}}^{n, k-1}, \overrightarrow{\boldsymbol{\rho}}^{n, k-1}, \overrightarrow{\boldsymbol{\mu}}^{n, k-1}, \overrightarrow{\boldsymbol{F}}_{\boldsymbol{e x t}}^{n}, t^{n}\right)$

Rheological solver (Eqs. 9.1c-9.1d):

$\left[\overrightarrow{\tilde{\boldsymbol{\rho}}}^{n, k}, \overrightarrow{\overrightarrow{\boldsymbol{\mu}}}^{n, k}\right]=\mathcal{L}\left(\overrightarrow{\boldsymbol{p}}_{\boldsymbol{H}}^{n, k}, \overrightarrow{\dot{\gamma}}^{n, k}\right)$

Structural solver (Eq. 9.1b):

$\left[\overrightarrow{\tilde{\boldsymbol{\delta}}}^{n, k}, \dot{\overrightarrow{\tilde{\delta}}}^{n, k}\right]=\mathcal{S}\left(\overrightarrow{\boldsymbol{p}}_{\boldsymbol{H}}^{n, k}\right)$

Compute residues (Eqs. 9.4):

$$
\begin{aligned}
& \overrightarrow{\boldsymbol{r}}_{\boldsymbol{p}_{\boldsymbol{H}}}{ }^{n, k}=\overrightarrow{\boldsymbol{p}}_{\boldsymbol{H}}{ }^{n, k}-\overrightarrow{\boldsymbol{p}}_{\boldsymbol{H}}{ }^{n, k-1} \\
& \overrightarrow{\boldsymbol{r}}_{\boldsymbol{\delta}}{ }^{n, k}=\overrightarrow{\tilde{\boldsymbol{\delta}}}^{n, k}-\overrightarrow{\boldsymbol{\delta}}^{n, k-1} \\
& \overrightarrow{\boldsymbol{r}}_{\boldsymbol{\rho}}{ }^{n, k}=\overrightarrow{\tilde{\boldsymbol{\rho}}}^{n, k}-\overrightarrow{\boldsymbol{\rho}}^{n, k-1} \\
& \overrightarrow{\boldsymbol{r}}_{\boldsymbol{\mu}}{ }^{n, k}=\overrightarrow{\tilde{\boldsymbol{\mu}}}^{n, k}-\overrightarrow{\boldsymbol{\mu}}^{n, k-1}
\end{aligned}
$$

Compute iteration steps (Eqs. 9.3):

if $(n \leq q)$ then Initial PGMA iterations

$$
\begin{aligned}
& \text { if }(k>1) \text { then }
\end{aligned}
$$

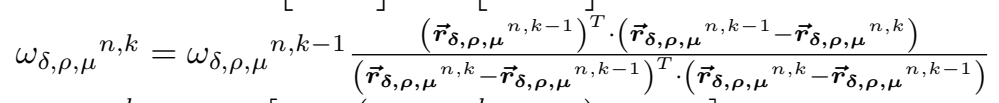

$$
\begin{aligned}
& \omega_{\delta, \rho, \mu}^{n, k} \leftarrow \min \left[\max \left(\omega_{\delta, \rho, \mu}^{n, k}, \omega_{\min }\right), \omega_{\max }\right] \\
& \Delta \overrightarrow{\boldsymbol{\delta}}^{n, k}=\omega_{\delta}^{n, k} \overrightarrow{\boldsymbol{r}}_{\boldsymbol{\delta}}^{n, k} \\
& \Delta \overrightarrow{\boldsymbol{\rho}}^{n, k}=\omega_{\rho}^{n, k} \overrightarrow{\boldsymbol{r}}_{\boldsymbol{\rho}}^{n, k} \\
& \Delta \overrightarrow{\boldsymbol{\mu}}^{n, k}=\omega_{\mu}^{n, k} \overrightarrow{\boldsymbol{r}}_{\boldsymbol{\mu}}{ }^{n, k}
\end{aligned}
$$

\section{end if}

else- IQN-ILS for structural coupling and PGMA for rheological coupling if $(k>1)$ then

$$
\begin{aligned}
& \text { Update matrices }\left[\boldsymbol{V}_{\boldsymbol{\delta}}^{n, k}\right] \text { and }\left[\boldsymbol{W}_{\boldsymbol{\delta}}^{n, k}\right](\text { Eqs. 9.15) } \\
& \omega_{\rho, \mu}^{n, k}=\omega_{\rho, \mu}{ }^{n, k-1} \frac{\left(\overrightarrow{\boldsymbol{r}}_{\rho, \mu}^{n, k-1}\right)^{T} \cdot\left(\overrightarrow{\boldsymbol{r}}_{\rho, \mu}{ }^{n, k-1}-\overrightarrow{\boldsymbol{r}}_{\rho, \mu}{ }^{n, k}\right)}{\left(\overrightarrow{\boldsymbol{r}}_{\rho, \mu}^{n, k}-\overrightarrow{\boldsymbol{r}}_{\rho, \mu}{ }^{n, k-1}\right)^{T} \cdot\left(\overrightarrow{\boldsymbol{r}}_{\rho, \mu}{ }^{n, k}-\overrightarrow{\boldsymbol{r}}_{\rho, \mu}{ }^{n, k-1}\right)} \\
& \omega_{\rho, \mu}^{n, k} \leftarrow \min \left[\max \left(\omega_{\rho, \mu}^{n, k}, \omega_{\min }\right), \omega_{\max }\right]
\end{aligned}
$$

\section{end if}

Solve $\left[\boldsymbol{V}_{\boldsymbol{\delta}}^{n, k}\right] \overrightarrow{\boldsymbol{c}}_{\boldsymbol{\delta}}^{n, k}=-\overrightarrow{\boldsymbol{r}}_{\boldsymbol{\delta}}^{n, k}$ for $\overrightarrow{\boldsymbol{c}}_{\boldsymbol{\delta}}^{n, k}$ (Eqs. 9.17, QR-decomposition)

$\Delta \overrightarrow{\boldsymbol{\delta}}^{n, k}=\left[\boldsymbol{W}_{\boldsymbol{\delta}}^{n, k}\right] \overrightarrow{\boldsymbol{c}}_{\boldsymbol{\delta}}^{n, k}+\overrightarrow{\boldsymbol{r}}_{\boldsymbol{\delta}}^{n, k}$

$\Delta \overrightarrow{\boldsymbol{\rho}}^{n, k}=\omega_{\rho}^{n, k} \overrightarrow{\boldsymbol{r}}_{\boldsymbol{\rho}}^{n, k}$

$\Delta \overrightarrow{\boldsymbol{\mu}}^{n, k}=\omega_{\mu}^{n, k} \overrightarrow{\boldsymbol{r}}_{\boldsymbol{\mu}}{ }^{n, k}$

end if

Update variables (Eqs. 9.3):

$$
\begin{aligned}
& \overrightarrow{\boldsymbol{\delta}}^{n, k}=\overrightarrow{\boldsymbol{\delta}}^{k-1}+\Delta \overrightarrow{\boldsymbol{\delta}}^{n, k} \\
& \overrightarrow{\boldsymbol{\rho}}^{n, k}=\overrightarrow{\boldsymbol{\rho}}^{k-1}+\Delta \overrightarrow{\boldsymbol{\rho}}^{n, k} \\
& \overrightarrow{\boldsymbol{\mu}}^{n, k}=\overrightarrow{\boldsymbol{\mu}}^{k-1}+\Delta \overrightarrow{\boldsymbol{\mu}}^{n, k}
\end{aligned}
$$

Compute $e_{E H L}$ (Eq. 9.23)

$k \leftarrow k+1$ 


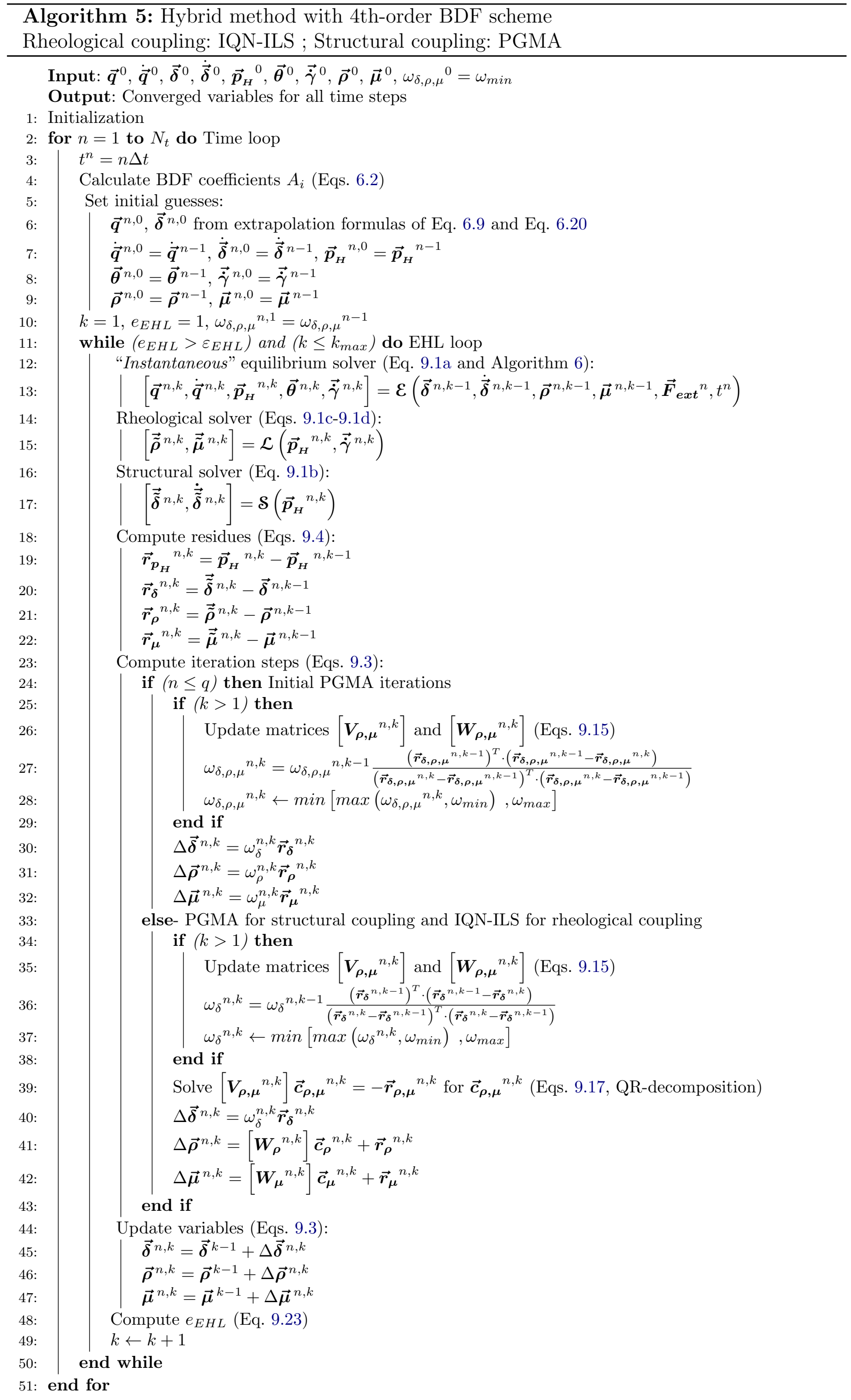



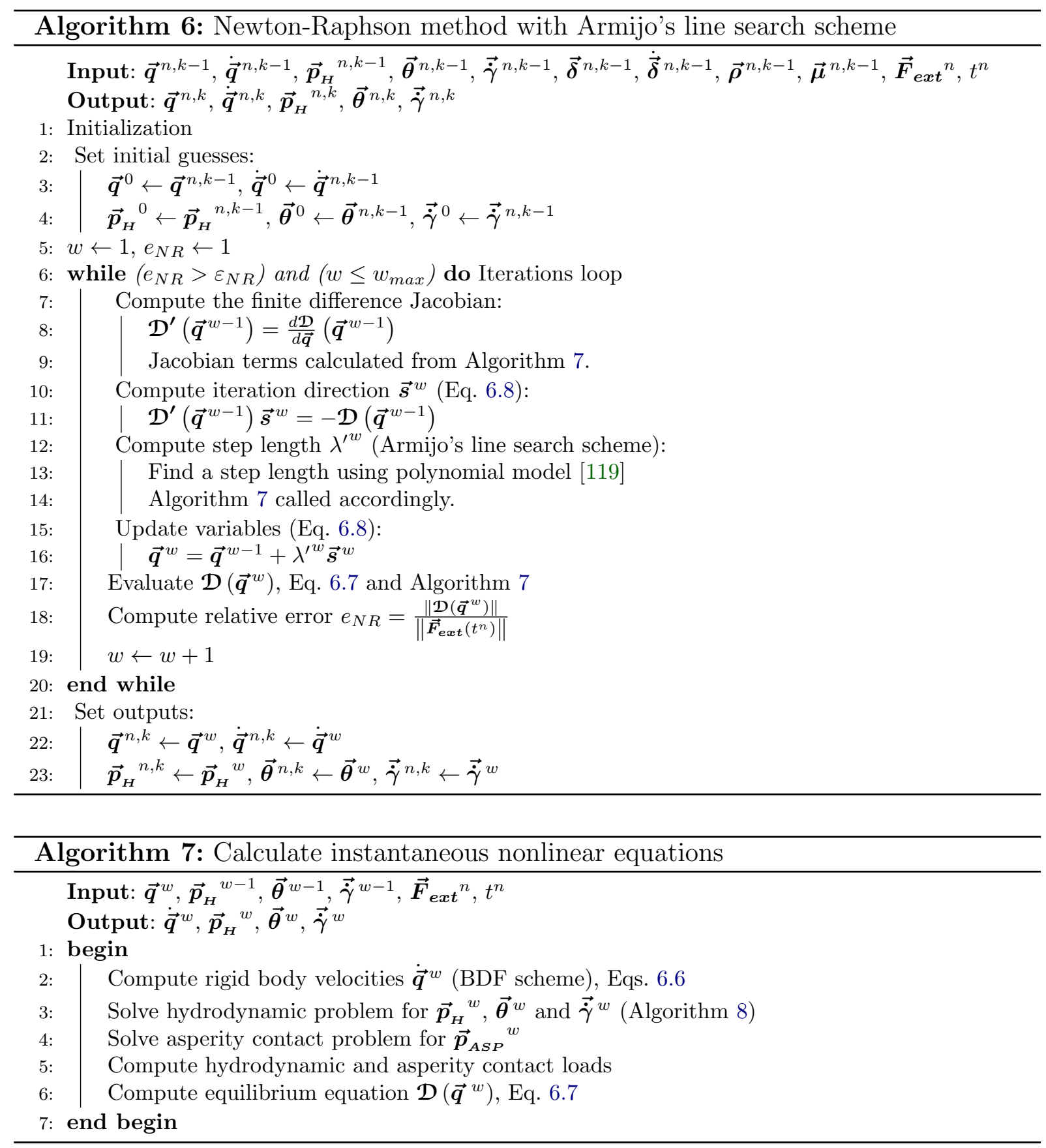


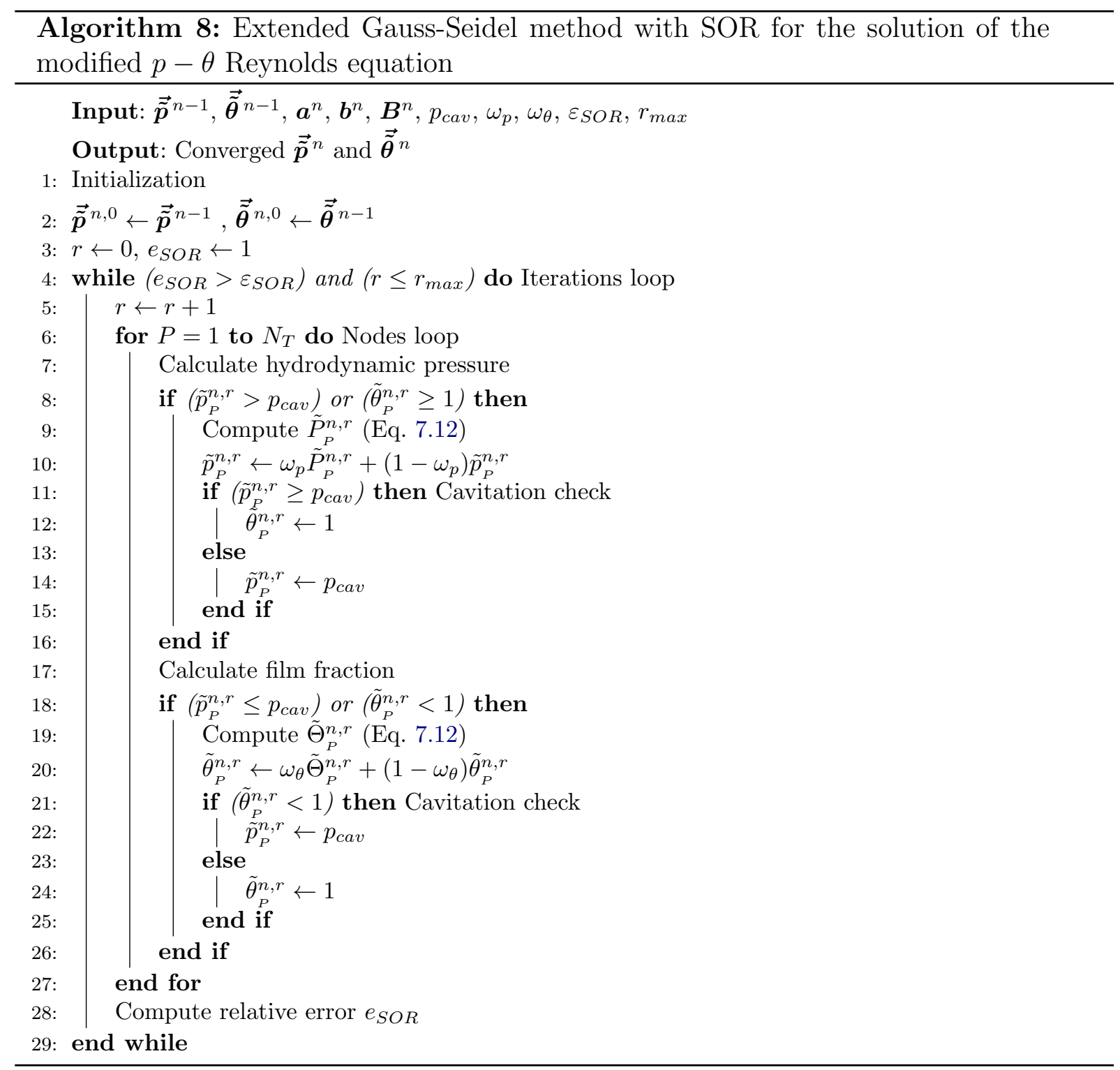




\section{Bibliography}

[1] Adams, G., And Nosonovsky, M. Contact modeling - forces. Tribology International 33, 5-6 (2000), 431-442. 37

[2] Ai, X., Cheng, H. S., And Zheng, L. A transient model for microelastohydrodynamic lubrication with three-dimensional irregularities. Journal of Tribology 115, 1 (1993), 102-110. 47

[3] Aitken, M. B., And McCallion, H. Elastohydrodynamie lubrication of bigend bearings. Part 2: Ratification. Proceedings of the Institution of Mechanical Engineers, Part C: Journal of Mechanical Engineering Science 205, 2 (1991), 107119. 80

[4] Almqvist, A. Homogenization of the Reynolds equation governing hydrodynamic flow in a rotating device. Journal of Tribology 133, 2 (2011), 021705. 44

[5] Almqvist, A., And Dasht, J. The homogenization process of the Reynolds equation describing compressible liquid flow. Tribology International 39, 9 (2006), 994-1002.

[6] Almqvist, A., Essel, E., Persson, L.-E., and Wall, P. Homogenization of the unstationary incompressible Reynolds equation. Tribology International 40, 9 (2007), 1344-1350.

[7] Almqvist, A., Essel, E. K., Fabricius, J., and Wall, P. Reiterated homogenization applied in hydrodynamic lubrication. Proceedings of the Institution of Mechanical Engineers, Part J: Journal of Engineering Tribology 222, 7 (2008), 827-841.

[8] Almqvist, A., Fabricius, J., Spencer, A., And Wall, P. Similarities and differences between the flow factor method by Patir and Cheng and homogenization. Journal of Tribology 133, 3 (2011), 031702.

[9] Almqvist, A., Lukkassen, D., Meidell, A., and Wall, P. New concepts of homogenization applied in rough surface hydrodynamic lubrication. International Journal of Engineering Science 45, 1 (2007), 139-154. 44

[10] Andrés, L. S., And Diaz, S. E. Flow visualization and forces from a squeeze film damper operating with natural air entrainment. Journal of Tribology 125, 2 (2003), 325-333. 12

[11] Arghir, M., Alsayed, A., And Nicolas, D. The finite volume solution of the Reynolds equation of lubrication with film discontinuities. International Journal of Mechanical Sciences 44, 10 (2002), 2119-2132. 2, 59 
[12] Arghir, M., And Frene, J. A triangle based finite volume method for the integration of lubrication's incompressible bulk flow equations. Journal of Tribology 123, 1 (2000), 118-124. 2, 59

[13] Arghir, M., Lez, S. L., And Frene, J. Finite-volume solution of the compressible Reynolds equation: Linear and non-linear analysis of gas bearings. Proceedings of the Institution of Mechanical Engineers, Part J: Journal of Engineering Tribology 220, 7 (2006), 617-627. 2, 59

[14] Ashihara, K., And Hashimoto, H. Theoretical modeling for microgrooved journal bearings under mixed lubrication. Journal of Tribology 132, 4 (2010), 042101. $80,81,102$

[15] Ausas, R. F., Jai, M., And Buscaglia, G. C. A mass-conserving algorithm for dynamical lubrication problems with cavitation. Journal of Tribology 131, 3 (2009), 031702. 2, 59, 64

[16] Ausas, R. F., Jai, M., Ciuperca, I. S., and Buscaglia, G. C. Conservative one-dimensional finite volume discretization of a new cavitation model for pistonring lubrication. Tribology International 57, 0 (2013), 54-66. 2, 59

[17] AVL LIST GMBH. AVL Excite. Graz, Austria, 2015. 37

[18] Balcombe, R., Fowell, M. T., Olver, A. V., Ioannides, S., And Dini, D. A coupled approach for rolling contact fatigue cracks in the hydrodynamic lubrication regime: The importance of fluid/solid interactions. Wear 271, 5-6 (2011), 720-733. 2,59

[19] Bathe, K. Finite Element Procedures. Prentice Hall, 2006. 33

[20] Bayada, G., And Chambat, M. New models in the theory of the hydrodynamic lubrication of rough surfaces. Journal of Tribology 110, 3 (1988), 402-407. 43

[21] Bayada, G., Cid, B., And VÁzquez, C. Two-scale homogenization study of a Reynolds-Rod elastohydrodynamic model. Mathematical Models and Methods in Applied Sciences 13, 2 (2003), 259-293. 44

[22] Bayada, G., And Faure, J. B. A double scale analysis approach of the Reynolds roughness: Comments and application to the journal bearing. Journal of Tribology 111, 2 (1989), 323-330. 43

[23] Bayada, G., Martin, S., And VÁzquez, C. An average flow model of the Reynolds roughness including a mass-flow preserving cavitation model. Journal of Tribology 127, 4 (2005), 793-802. 44

[24] Bayada, G., Martin, S., And VÁzquez, C. Two-scale homogenization of a hydrodynamic Elrod-Adams model. Asymptotic Analysis 44, 1-2 (2005), 75-110. 44

[25] Beheshti, A., And Khonsari, M. Asperity micro-contact models as applied to the deformation of rough line contact. Tribology International 52 (2012), 61-74. 37 
[26] Berthe, D., And Godet, M. A more general form of Reynolds equation - Application to rough surfaces. Wear 27, 3 (1974), 345-357. 8

[27] Bertocchi, L., Dini, D., Giacopini, M., Fowell, M. T., And Baldini, A. Fluid film lubrication in the presence of cavitation: A mass-conserving twodimensional formulation for compressible, piezoviscous and non-Newtonian fluids. Tribology International 67, 0 (2013), 61-71. 2, 58, 177, 178, 179, 181, 182

[28] Birkhoff, G.; HAys, D. Free boundaries in partial lubrication. Journal of Mathematical Physics 42, 2 (1963), 126-138. 16

[29] Boedo, S. Mass conserving cavitation effects in squeeze-film journal bearings subjected to sinusoidal loads. Tribology Transactions 54, 1 (2010), 21-35. 91, 105

[30] Boedo, S., Booker, J., And Wilkie, M. A mass conserving modal analysis for elastohydrodynamic lubrication. In Lubricants and Lubrication: proceedings of the 21th Leeds-Lyon Symposium on Tribology (1995). 2, 58

[31] Boedo, S., ANd Booker, J. F. Surface roughness and structural inertia in a mode-based mass-conserving elastohydrodynamic lubrication model. Journal of Tribology 119, 3 (1997), 449-455. 67

[32] Boedo, S., And Booker, J. F. Finite element analysis of elastic engine bearing lubrication: Application. Revue Européenne des Éléments 10, 6-7 (2001), 725-739. $2,58,80$

[33] Bonneau, D., Fatu, A., And Souchet, D. Internal Combustion Engine Bearings Lubrication in Hydrodynamic Bearings. Numerical methods in engineering series. Wiley, 2014. 79, 102

[34] Bonneau, D., Guines, D., Frêne, J., And Toplosky, J. EHD analysis, including structural inertia effects and a mass-conserving cavitation model. Journal of Tribology 117, 3 (1995), 540-547. 3, 67, 68, 80, 86

[35] Booker, J. F., And Boedo, S. Finite element analysis of elastic engine bearing lubrication: Theory. Revue Européenne des Éléments 10, 6-7 (2001), 705-724. 2, 58

[36] Booker, J. F., Boedo, S., and Bonneau, D. Conformal elastohydrodynamic lubrication analysis for engine bearing design: A brief review. Proceedings of the Institution of Mechanical Engineers, Part C: Journal of Mechanical Engineering Science 224, 12 (2010), 2648-2653. 3, 32, 68

[37] Bouassida, H. Lubricated piston ring cylinder liner contact: Influence of the liner microgeometry. PhD thesis, L'Institut National Des Sciences Appliquees de Lyon (INSA de Lyon), Lyon, France, 2014. 120

[38] Braun, M. J., And Hannon, W. M. Cavitation formation and modelling for fluid film bearings: A review. Proceedings of the Institution of Mechanical Engineers, Part J: Journal of Engineering Tribology 224, 9 (2010), 839-863. 12, 15, 16

[39] Brennen, C. Cavitation and Bubble Dynamics. Cambridge University Press, 2013. 18 
[40] BRewe, D. E. Theoretical modeling of the vapor cavitation in dynamically loaded journal bearings. Journal of Tribology 108, 4 (1986), 628-637. 14, 91, 105

[41] Brewe, D. E., Ball, J. H., and Khonsari, M. M. Current research in cavitating fluid films. Tech. Rep. NASA-TM-103184, E-4924, NAS 1.15:103184, ADA230773, NASA, Army Propulsion Lab.; Cleveland, OH, United States, 1990. 12, $14,91,105$

[42] Brizmer, V., Kligerman, Y., and Etsion, I. A laser surface textured parallel thrust bearing. Tribology Transactions 46, 3 (2003), 397-403. 120

[43] Buscaglia, G., Ciuperca, I., And Jai, M. Homogenization of the transient Reynolds equation. Asymptotic Analysis 32, 2 (2002), 131-152. 43

[44] Buscaglia, G., And Jai, M. Homogenization of the generalized Reynolds equation for ultra-thin gas films and its resolution by FEM. Journal of Tribology 126, 3 (2004), 547-552.

[45] Buscaglia, G. C., And Jai, M. A new numerical scheme for non-uniform homogenized problems: Application to the non-linear Reynolds compressible equation. Mathematical Problems in Engineering 7, 4 (2001), 355-378. 43

[46] Bush, A., Gibson, R., And Thomas, T. The elastic contact of a rough surface. Wear 35, 1 (1975), 87-111. 37

[47] Buzio, R., Boragno, C., Biscarini, F., Buatier De Mongeot, F., And Valbusa, U. The contact mechanics of fractal surfaces. Nature Materials 2, 4 (2003), 233-236. 37

[48] Chang, L., Cusano, C., And Conry, T. F. Effects of lubricant rheology and kinematic conditions on micro-elastohydrodynamic lubrication. Journal of Tribology 111, 2 (1989), 344-351. 47

[49] Chang, L., Webster, M. N., And Jackson, A. A line-contact micro-EHL model with three-dimensional surface topography. Journal of Tribology 116, 1 (1994), 21-28. 47

[50] Chang, W. R., Etsion, I., And Bogy, D. B. An elastic-plastic model for the contact of rough surfaces. Journal of Tribology 109, 2 (1987), 257-263. 37

[51] Chen, H. Modeling the lubrication of the piston ring pack of internal combustion engines using deterministic method. $\mathrm{PhD}$ thesis, Massachusetts Institute of Technology, Cambridge, MA, USA, 2011. x, 3, 48, 115, 117, 118

[52] Chen, H., Li, Y., And Tian, T. A novel approach to model the lubrication and friction between the Twin-Land Oil Control Ring and liner with consideration of micro structure of the liner surface finish in internal combustion engines. $S A E$ Technical Paper (2008), 2008-01-1613. 3, 48, 117

[53] Chow, L. S. H., And Cheng, H. S. The effect of surface roughness on the average film thickness between lubricated rollers. Journal of Tribology 98, 1 (1976), 117-124. 43 
[54] Christensen, H. Stochastic models for hydrodynamic lubrication of rough surfaces. Proceedings of the Institution of Mechanical Engineers 184, 1 (1969), 10131026. 43

[55] Christensen, H. Some aspects of the functional influence of surface roughness in lubrication. Wear 17, 2 (1971), 149-162.

[56] Christensen, H. A theory of mixed lubrication. Proceedings of the Institution of Mechanical Engineers 186, 1 (1972), 421-430. 43

[57] Coyne, J. C., And H. G. Elrod, J. Conditions for the rupture of a lubricating film. Part I: Theoretical model. Journal of Lubrication Technology 92, 3 (1970), 451-456. 16

[58] Coyne, J. C., And H. G. Elrod, J. Conditions for the rupture of a lubricating film. Part II: New boundary conditions for reynolds equation. Journal of Lubrication Technology 93, 1 (1971), 156-167. 16

[59] Craig, R., And Kurdila, A. Fundamentals of Structural Dynamics. Wiley, 2006. 33

[60] Dai, R. X., Dong, Q., And Szeri, A. Z. Approximations in hydrodynamic lubrication. Journal of Tribology 114, 1 (1992), 14-25. 21

[61] Dantzig, J.A., T. I. C. Modeling in Materials Processing. Mechanical Engineering. Cambridge University Press, 2001. 20

[62] Dassault Systemes Simulia Corp. Abaqus/CAE 6.12-1. Providence, RI, USA, 2012. 33, 80, 100, 132, 154

[63] Davies, R., Ed. Cavitation in real liquids: Proceedings of General Motors Corporation Research Laboratories (1964). 12

[64] Degroote, J. Partitioned simulation of fluid-structure interaction. Archives of Computational Methods in Engineering 20, 3 (2013), 185-238. 3, 68, 71, 74

[65] Degroote, J., Bathe, K.-J., And Vierendeels, J. Performance of a new partitioned procedure versus a monolithic procedure in fluid-structure interaction. Comput. Struct. 87, 11-12 (2009), 793-801. 3, 68

[66] Dellis, P., And Arcoumanis, C. Cavitation development in the lubricant film of a reciprocating piston-ring assembly. Proceedings of the Institution of Mechanical Engineers, Part J: Journal of Engineering Tribology 218, 3 (2004), 157-171. 16

[67] Dimkovski, Z., Tomanik, E., And Profito, F. Influence of measurement and filtering type on friction predictions between cylinder liner and oil control ring. Tribology International (2015), In Press (Available Online). 48, 130

[68] Dimkovski, Z., Tomanik, E., And Profito, F. Influence of measurement and filtering type on friction predictions between cylinder liner and oil control ring. In Surface and Interface Mysteries at Different Scales: proceedings of the 42nd LeedsLyon Symposium on Tribology, 7-9 September 2015, Lyon, France (2015). 48, 130 
[69] Dong, S. BDF-like methods for nonlinear dynamic analysis. Journal of Computational Physics 229, 8 (2010), 3019-3045. 54, 55

[70] Dowson, D. A generalized Reynolds equation for fluid-film lubrication. International Journal of Mechanical Sciences 4, 2 (1962), 159-170. 8

[71] Dowson, D. Elastohydrodynamic and micro-elastohydrodynamic lubrication. Wear 190, 2 (1995), 125-138. 31

[72] Dowson, D., Godet, M., And Taylor, C., Eds. Cavitation and related phenomena in lubrication: Proceedings of the 1st Leeds-Lyon Symposium on Tribology, held in the Institute of Tribology, Department of Mechanical Engineering, the University of Leeds, England, September 1974 (1975). 12

[73] Dowson, D., And Taylor, C. Cavitation in bearings. Annual Review of Fluid Mechanics 11 (1979), 35-66. 12, 14

[74] Durany, J., Pereira, J., And Varas, F. A cell-vertex finite volume method for thermohydrodynamic problems in lubrication theory. Computer Methods in Applied Mechanics and Engineering 195, 44-47 (2006), 5949-5961. 2, 58

[75] Durany, J., Pereira, J., And Varas, F. Numerical solution to steady and transient problems in thermohydrodynamic lubrication using a combination of finite element, finite volume and boundary element methods. Finite Elem. Anal. Des. 44, 11 (2008), 686-695. 2, 58

[76] Elrod, H. G. A general theory for laminar lubrication with Reynolds roughness. Journal of Tribology 101, 1 (1979), 8-14. 43, 159

[77] Elrod, H. G. A cavitation and starvation algorithm. Journal of Lubrication Technology 103, 3 (1981), 350-354. 2, 17

[78] Elrod, H. G.; Adams, M. L. A computer program for cavitation and starvation problems. In Cavitation and related phenomena in lubrication: proceedings of the 1st Leeds-Lyon Symposium on Tribology, held in the Institute of Tribology, Department of Mechanical Engineering, the University of Leeds, England, September 1974 (1974). 2, 17

[79] Fantino, B., And Frene, J. Comparison of dynamic behavior of elastic connecting-rod bearing in both petrol and diesel engines. Journal of tribology 10\%, 1 (1985), 87-91. 32

[80] Fantino, B., Godet, M., And Frene, J. Dynamic behaviour of an elastic connecting-rod bearing: Theoretical study. SAE Technical Paper (1983), 830307. 3, 32,68

[81] Farhat, C., Lesoinne, M., and Tallec, P. L. Load and motion transfer algorithms for fluid-structure interaction problems with non-matching discrete interfaces: Momentum and energy conservation, optimal discretization and application to aeroelasticity. Computer Methods in Applied Mechanics and Engineering 15\%, 1-2 (1998), 95-114. 70 
[82] Fatu, A., Hajuam, M., and Bonneau, D. A new model of thermoelastohydrodynamic lubrication in dynamically loaded journal bearings. Journal of Tribology 128, 1 (2006), 85-95. 2, 58, 67

[83] Felippa, C., AND PARK, K. The construction of free-free flexibility matrices for multilevel structural analysis. Computer Methods in Applied Mechanics and Engineering 191, 19-20 (2002), 2139-2168. 35

[84] Ferziger, J., AND Perić, M. Computational Methods for Fluid Dynamics. Springer, 2002. 62, 174

[85] Floberg, L. On hydrodynamic lubrication with special reference to subcavity pressures and number of streamers in cavitation regions. Acta Polytechnica Scandinavica, Mechanical Engineering Series 19 (1965). 16

[86] Floberg, L. Sub-cavity pressures and number of oil streamers in cavitation regions with special reference to the infinite journal bearing. Acta Polytechnica Scandinavica, Mechanical Engineering Series 37 (1968), 1-31.

[87] FloberG, L. On journal bearing lubrication considering the tensile strength of the liquid lubricant. Transactions of the Machine Elements Division, Lund Technical University, Lund, Sweden (1973), 1-26. 2, 14, 16

[88] FloberG, L. Cavitation boundary conditions with regard to the number of streamers and tensile strength of the liquid. In Cavitation and related phenomena in lubrication: proceedings of the 1st Leeds-Lyon Symposium on Tribology, held in the Institute of Tribology, Department of Mechanical Engineering, the University of Leeds, England, September 1974 (1974). 2, 14

[89] Fowell, M., Olver, A. V., Gosman, A. D., Spikes, H. A., And Pegg, I. Entrainment and inlet suction: Two mechanisms of hydrodynamic lubrication in textured bearings. Journal of Tribology 129, 2 (2007), 336-347. 12

[90] Francisco, A., Fatu, A., and Bonneau, D. Using design of experiments to analyze the connecting-rod big-end bearing behavior. Journal of Tribology 131, 1 (2008), 1-13. 3, 68

[91] Frene, J., Desailly, R., And Fantino, B. Hydrodynamics of an elastic connecting-rod bearing: comparison of theoretical and experimental results. In Elastohydrodynamics and related topics: proceedings of the 5th Leeds-Lyon Symposium on Tribology held in the Institute of Tribology, Department of Mechanical Engineering, the University of Leeds, England, September 1978 (1979). 32

[92] Frene, J., Nicolas, D., Degueurce, B., And Berthe, D. Hydrodynamic Lubrication: Bearings and Thrust Bearings. Tribology Series. Elsevier Science, 1997. $8,9,10,11,15$

[93] Giacopini, M., Fowell, M. T., Dini, D., And Strozzi, A. A mass-conserving complementarity formulation to study lubricant films in the presence of cavitation. Journal of Tribology 132, 4 (2010), 041702. 2, 58

[94] Gümbel, L. Das problem der lagerreibung. Mon. Berl. Bezirksverein, V.D.I. 5 (1914), 87-104 and 109-120. 13 
[95] Goenka, P. K., And OH, K. P. An optimum connecting-rod design study - A lubrication viewpoint. Journal of Tribology 108, 3 (1986), 487-493. 3, 67, 68, 86

[96] Greenwood, J. A., And Tripp, J. H. The contact of two nominally flat rough surfaces. Proceedings of the Institution of Mechanical Engineers 185, 1 (1970), 625633. 37,41

[97] Greenwood, J. A., And Williamson, J. B. P. Contact of nominally flat surfaces. Proceedings of the Royal Society of London. Series A, Mathematical and Physical Sciences 295, 1442 (1966), 300-319. 37, 42, 165, 166

[98] Grente, C., Ligier, J., Toplosky, J., and Bonneau, D. The consequence of performance increases of automotive engines on the modelisation of main and connecting-rod bearings. In Tribology Research: From Model Experiment to Industrial Problem A Century of Efforts in Mechanics, Materials Science and PhysicoChemistry Proceedings of the 27th Leeds-Lyon Symposium on Tribology, D. D. G. Dalmaz, A.A. Lubrecht and M. Priest, Eds., vol. 39 of Tribology Series. Elsevier, 2001, pp. 839-850. 3, 67, 68

[99] Gupta, P., And Cook, N. Junction deformation models for asperities in sliding interaction. Wear 20, 1 (1972), 73-87. 37

[100] HajJam, M., And Bonneau, D. A transient finite element cavitation algorithm with application to radial lip seals. Tribology International 40, 8 (2007), 1258-1269. 2,58

[101] Hamrock, B., Schmid, S., And Jacobson, B. Fundamentals of Fluid Film Lubrication. Mechanical Engineering. Taylor \& Francis, 2004. 8, 11, 19, 20, 31, 36, 79, 102

[102] Harp, S. R., And Salant, R. F. An average flow model of rough surface lubrication with inter-asperity cavitation. Journal of Tribology 123, 1 (2000), 134-143. 159

[103] Hartinger, M. CFD modelling of elastohydrodynamic lubrication. PhD thesis, Imperial College London, London, UK, 2007. 177

[104] Hirani, H., Athre, K., And Biswas, S. A simplified mass conserving algorithm for journal bearing under large dynamic loads. International Journal of Rotating Machinery 7, 1 (2001), 41-51. 18

[105] Hopkins, M. R. Viscous flow between rotating cylinders and a sheet moving between them. British Journal of Applied Physics 8, 11 (1957), 442. 16

[106] Hu, Y.-Z., AND ZHU, D. A full numerical solution to the mixed lubrication in point contacts. Journal of Tribology 122, 1 (2000), 1. 47

[107] Hurtado, F. Formulação Tridimensional de Volumes Finitos para Simulação de Reservatórios de Petróleo com Malhas Não-Estruturadas Híbridas. PhD thesis, Universidade Federal de Santa Catarina, Florianópolis, Santa Catarina, Brasil, 2011. In Portuguese. 2, 60, 61 
[108] Hurtado, F. S. V., And Maliska, C. R. A family of positive flow-weighted advection schemes for element-based finite-volume methods. Numerical Heat Transfer, Part B: Fundamentals 62, 2-3 (2012), 113-140. 2, 60, 175

[109] Hutchings, I. Tribology: friction and wear of engineering materials. Edward Arnold, 1992. 42

[110] Irons, B. M., AND TuCK, R. C. A version of the Aitken accelerator for computer iteration. International Journal for Numerical Methods in Engineering 1, 3 (1969), 275-277. 71

[111] Jackson, R. L., And Green, I. A finite element study of elasto-plastic hemispherical contact against a rigid flat. Journal of Tribology 127, 2 (2005), 343-354. 37

[112] Jackson, R. L., And Green, I. A statistical model of elasto-plastic asperity contact between rough surfaces. Tribology International 39, 9 (2006), 906-914. 37

[113] JAI, M. Homogenization and two-scale convergence of the compressible Reynolds lubrication equation modelling the flying characteristics of a rough magnetic head over a rough rigid-disk surface. ESAIM: Mathematical Modelling and Numerical Analysis - Modélisation Mathématique et Analyse Numérique 29, 2 (1995), 199-233. 43

[114] JAI, M., AND Bou-SAID, B. A comparison of homogenization and averaging techniques for the treatment of roughness in slip-flow-modified Reynolds equation. Journal of Tribology 124, 2 (2001), 327-335. 44

[115] Jakobsson, B.; Floberg, L. The finite journal bearing considering vaporization. Transactions of Chalmers University Technology, Guthenburg, Sweden 190 (1957). 2,14

[116] Jocsak, J. The effects of surface finish on piston ring-pack performance in advanced reciprocating engine systems. PhD thesis, Massachusetts Institute of Technology, Cambridge, MA, USA, 2005. 49

[117] Johnson, K. Contact Mechanics. Cambridge University Press, 1987. 42, 48

[118] Kane, M., And Bou-Said, B. Comparison of homogenization and direct techniques for the treatment of roughness in incompressible lubrication. Journal of Tribology 126, 4 (2004), 733-737. 44

[119] Kelley, C. Solving Nonlinear Equations with Newton's Method. Fundamentals of Algorithms. Society for Industrial and Applied Mathematics, 2003. 56, 189

[120] Kim, B.-J., AND KIm, K.-W. Thermo-elastohydrodynamic analysis of connectingrod bearing in internal combustion engine. Journal of Tribology 123, 3 (2001), 444454. 67,80

[121] Kim, T. W., And Cho, Y. J. The flow-factors considering the elastic deformation for the rough surface with a non-Gaussian height distribution. Tribology Transactions 51, 2 (2008), 213-220. 159 
[122] Knoll, G., Lang, J., And RienÄCKer, A. Transient EHD connecting-rod analysis: Full dynamic versus quasi-static deformation. Journal of Tribology 118, 2 (1996), 349-355. 3, 67, 68

[123] Knoll, G., Schönen, R., And Wilhelm, K. Full dynamic analysis of crankshaft and engine block with special respect to elastohydrodynamic bearing coupling. In ASME/ICED 1997 Spring Technical Conference, Fort Collins, CO (1997). 3, 68

[124] Kogut, L., And Etsion, I. Elastic-plastic contact analysis of a sphere and a rigid flat. Journal of Applied Mechanics 69, 5 (2002), 657-662. 37

[125] Kogut, L., And Etsion, I. A finite element based elastic-plastic model for the contact of rough surfaces. Tribology Transactions 46, 3 (2003), 383-390. 37

[126] KüTtLeR, U., AND WALL, W. Fixed-point fluid-structure interaction solvers with dynamic relaxation. Computational Mechanics 43, 1 (2008), 61-72. 71

[127] Kumar, A., Booker, J., And Goenka, P. Dynamically loaded journal bearings: A modal approach to EHL design analysis. In Tribological Design of Machine Elements Proceedings of the 15th Leeds-Lyon Symposium on Tribology held at Bodington Hall, The University of Leeds, M. G. D. Dowson, Cm. Taylor and D. Berthe, Eds., vol. 14 of Tribology Series. Elsevier, 1989, pp. 305-315. 3, 68

[128] Kumar, A., And Booker, J. F. A finite element cavitation algorithm. Journal of Tribology 113, 2 (1991), 276-284. 2, 58

[129] Kumar, A., Goenka, P. K., And Booker, J. F. Modal analysis of elastohydrodynamic lubrication: A connecting rod application. Journal of Tribology 112, 3 (1990), 524-531. 86

[130] Kweh, C. C., Evans, H. P., And Snidle, R. W. Micro-elastohydrodynamic lubrication of an elliptical contact with transverse and three-dimensional sinusoidal roughness. Journal of Tribology 111, 4 (1989), 577-584. 47

[131] Kweh, C. C., Patching, M. J., Evans, H. P., And Snidle, R. W. Simulation of elastohydrodynamic contacts between rough surfaces. Journal of Tribology 114, 3 (1992), 412-419. 47

[132] LaBouff, G. A., And Booker, J. F. Dynamically loaded journal bearings: A finite element treatment for rigid and elastic surfaces. Journal of Tribology 10\%, 4 (1985), 505-513. 3, 32, 67, 68

[133] LEE, S. C., AND REN, N. Behavior of elastic-plastic rough surface contacts as affected by surface topography, load, and material hardness. Tribology Transactions 39, 1 (1996), 67-74. 37

[134] Letalleur, N., Plouraboué, F., and Prat, M. Average flow model of rough surface lubrication: Flow-factors for sinusoidal surfaces. Journal of Tribology 124, 3 (2002), 539-546. 159

[135] LöHner, P. Applied Computational Fluid Dynamics Techniques: An Introduction Based on Finite Element Methods. John Wiley \& Sons, 2008. 62 
[136] LI, Y. Multiphase oil transport at complex micro geometry. PhD thesis, Massachusetts Institute of Technology, Cambridge, MA, USA, 2011. 3, 48

[137] Li, Y., Chen, H., And Tian, T. A deterministic model for lubricant transport within complex geometry under sliding contact and its application in the interaction between the Oil Control Ring and rough liner in internal combustion engines. $S A E$ Technical Paper, 2008-01-1615 (2008).

[138] Liao, K., Liu, Y., Kim, D., Urzua, P., and Tian, T. Practical challenges in determining piston ring friction. Proceedings of the Institution of Mechanical Engineers, Part J: Journal of Engineering Tribology 227, 2 (2012), 112-125. 3, 48, 115

[139] LiU, G., (JANe) WANG, Q., AND Lin, C. A survey of current models for simulating the contact between rough surfaces. Tribology Transactions 42, 3 (1999), 581-591. 37

[140] Liu, W. K., Xiong, S., Guo, Y., Wang, Q. J., Wang, Y., Yang, Q., And Vaidyanathan, K. Finite element method for mixed elastohydrodynamic lubrication of journal-bearing systems. International Journal for Numerical Methods in Engineering 60, 10 (2004), 1759-1790. 3, 68

[141] Lubrecht, A. A., Ten Napel, W. E., And Bosma, R. The influence of longitudinal and transverse roughness on the elastohydrodynamic lubrication of circular contacts. Journal of Tribology 110, 3 (1988), 421-426. 47

[142] Lugt, P., And Morales-Espejel, G. A review of elasto-hydrodynamic lubrication theory. Tribology Transactions 54, 3 (2011), 470-496. 31

[143] Lunde, L., And Tonder, K. Pressure and shear flow in a rough hydrodynamic bearing, flow factor calculation. Journal of Tribology 119, 3 (1997), 549-555. 159

[144] Majumdar, A., And Bhushan, B. Fractal model of elastic-plastic contact between rough surfaces. Journal of Tribology 113, 1 (1991), 1-11. 37

[145] Marx, N., Taylor, I., Morgan, N., And Spikes, H. Influence of polymer temporary shear-thinning on hydrodynamic film formation and friction. In Proceedings of the 2015 STLE Annual Meeting and Exhibition, Dallas, Texas, USA (2015). 101, 102

[146] McCool, J. I. Comparison of models for the contact of rough surfaces. Wear 10\%, 1 (1986), 37-60. 41

[147] McIvor, J. D. C., And Fenner, D. N. Finite element analysis of dynamically loaded flexible journal bearings: A fast Newton-Raphson method. Journal of Tribology 111, 4 (1989), 597-604. 3, 67, 68, 71, 80

[148] Meirovitch, L. Principles and Techniques of Vibrations. Prentice Hall, 1997. 34

[149] Meng, F., Wang, Q. J., Hua, D., And Liu, S. A simple method to calculate contact factor used in average flow model. Journal of Tribology 132, 2 (2010), 024505. 156 
[150] Miao, X., And Huang, X. A complete contact model of a fractal rough surface. Wear 309, 1-2 (2014), 146-151. 37

[151] MoK, D., AND WalL, W. Partitioned analysis schemes for the transient interaction of incompressible flows and nonlinear flexible structures. Trends in computational structural mechanics, Barcelona (2001). 71

[152] Mok, D., Wall, W., AND RAMm, E. Accelerated iterative substructuring schemes for instationary fluid-structure interaction. Computational Fluid and Solid Mechanics (2001), 1325-1328. 71

[153] Moreau, H., Maspeyrot, P., Bonneau, D., and Frene, J. Comparison between experimental film thickness measurements and elastohydrodynamic analysis in a connecting-rod bearing. Proceedings of the Institution of Mechanical Engineers, Part J: Journal of Engineering Tribology 216, 4 (2002), 195-208. 3, 68

[154] Moreau, H., Maspeyrot, P., Chomat-Delalex, A. M., Bonneau, D., And FRENE, J. Dynamic behaviour of elastic engine main bearings: Theory and measurements. Proceedings of the Institution of Mechanical Engineers, Part J: Journal of Engineering Tribology 216, 4 (2002), 179-194. 3, 68

[155] Mori, H., Yabe, H., And Fujita, Y. On the separation boundary conditions for fluid lubrication theories of journal bearing. ASLE Transactions 11 (1968), 196-203. 16

[156] NAYAK, P. Random process model of rough surfaces. Journal of Lubrication Technology 93 (1971), 398-407. 37

[157] Оh, K. P., And Goenka, P. K. The elastohydrodynamic solution of journal bearings under dynamic loading. Journal of Tribology 10\%, 3 (1985), 389-394. 3, $32,67,68,86$

[158] Olson, E., AND Booker, J. Hydrodynamic analysis of journal bearings with structural inertia and elasticity by a modal finite element method. In Elastohydrodynamics - '96 Fundamentals and Applications in Lubrication and Traction: proceedings of the 23rd Leeds-Lyon Symposium on Tribology held in the Institute of Tribology, Department of Mechanical Engineering (1997). 2, 58

[159] Olson, E. G., And Booker, J. F. EHD analysis with distributed structural inertia. Journal of Tribology 123, 3 (2001), 462-468. 67

[160] Olsson, K. Cavitation in dynamically loaded bearing. Transactions of Chalmers University Technology, Guthenburg, Sweden (1965), Report No. 380. 2, 14

[161] Onions, R. A., And Archard, J. F. The contact of surfaces having a random structure. Journal of Physics D: Applied Physics 6, 3 (1973), 289. 37

[162] Optasanu, V., and Bonneau, D. Finite element mass-conserving cavitation algorithm in pure squeeze motion. Validation/Application to a connecting- rod smallend bearing. Journal of Tribology 122, 1 (2000), 162-169. 2, 58, 91, 105, 181 
[163] Ozasa, T., Yamamoto, M., Suzuki, S., and Nozawa, Y. Elastohydrodynamic lubrication model of connecting-rod big-end bearings: Comparison with experiments by diesel engine. SAE Technical Paper (1995), 1995-10-01. 80

[164] Patir, N., And Cheng, H. S. An average flow model for determining effects of three-dimensional roughness on partial hydrodynamic lubrication. Journal of Lubrication Technology 100, 1 (1978), 12-17. 43, 44, 45, 156, 158, 159

[165] Patir, N., And Cheng, H. S. Application of average flow model to lubrication between rough sliding surfaces. Journal of Lubrication Technology 101, 2 (1979), 220-229. 43, 44, 45, 46, 157, 158, 159, 160, 161, 162

[166] Peeken, H. J., Knoll, G., Rienäcker, A., Lang, J., and Schönen, R. On the numerical determination of flow factors. Journal of Tribology 119, 2 (1997), 259-264. 159

[167] Pierre-Eugene, J., Frene, J., Fantino, B., Roussel, G., And Du Parquet, J. Theory and experiments on elastic connecting-rod bearings under steady state conditions. In Tribology of reciprocating engines: proceedings of the 9th Leeds-Lyon Symposium on Tribology held in Bodington Hall, the University of Leeds, England, 7-10 September 1982 (1983). 32

[168] Piffeteau, S., Souchet, D., And Bonneau, D. Influence of thermal and elastic deformations on connecting-rod big-end bearing lubrication under dynamic loading. Journal of Tribology 122, 1 (1999), 181-191. 80

[169] Prat, M., Plouraboué, F., And Letalleur, N. Averaged Reynolds equation for flows between rough surfaces in sliding motion. Transport in Porous Media 48, 3 (2002), 291-313. 43, 44

[170] Profito, F.J.and Dini, D., And Zachariadis, D. Partitioned fluid-structure interaction techniques applied to the conformal ehl solution of dynamically loaded connecting-rod bearings. In Influence of design and materials on journal and thrust bearing performance: proceedings of the 14th EDF/Pprime Workshop, Futuroscope, October 8-9, 2015, Poitiers, France (2015). 69, 99, 130

[171] Profito, F. Modelagem unidimensional do regime misto de lubrificação aplicada a superfícies texturizadas. Master's thesis, Escola Politécnica da Universidade de São Paulo, Setembro 2010. In Portuguese. 10, 36, 66

[172] Profito, F., Cousseau, T., and Tomanik, E. Folded metal effect on lubricant film thickness and friction using a mixed lubrication deterministic model. SAE Technical Paper (2014), 2014-06-0302. 48, 130

[173] Profito, F., Dini, D., And Zachariadis, D. A general finite volume method solution for the reynolds lubrication equation with mass-conserving cavitation model. In 70th STLE Annual Meeting \& Exhibition, 17-21 May 2015, Omni Hotel, Dallas, Texas, USA. 59, 128, 129

[174] Profito, F., Giacopini, M., Zachariadis, D., And Dini, D. A general finite volume method for the solution of the reynolds lubrication equation with a massconserving cavitation model. Tribology Letters 60, 1 (2015). 59, 128, 129 
[175] Profito, F., Tomanik, E., And Zachariadis, D. Effect of cylinder liner wear on the mixed lubrication regime of tlocrs. In Integrated Tribology, proceedings of the 41st Leeds-Lyon Symposium on Tribology, 2-5 September 2014, Leeds, UK (2014). $48,115,130$

[176] Profito, F., Tomanik, E., and Zachariadis, D. Effect of cylinder liner wear on the mixed lubrication regime of TLOCRs. Tribology International 93, 723-732 (2015). 48, 115, 130

[177] Profito, F., And Zachariadis, D. Partitioned fluid-structure methods applied to the solution of elastohydrodynamic conformal contacts. Tribology International 81 (2015), 321-332. 69, 81, 130

[178] Pugliese, G., Tavares, S., Ciulli, E., And Ferreira, L. Rough contacts between actual engineering surfaces. Part II: Contact mechanics. Wear 264, 11-12 (2008), 1116-1128. 37

[179] Pullen, J., And Williamson, J. B. P. On the plastic contact of rough surfaces. Proceedings of the Royal Society of London. Series A, Mathematical and Physical Sciences 327 (1972), 159-173. 37

[180] QIU, Y., And Khonsari, M. M. On the prediction of cavitation in dimples using a mass-conservative algorithm. Journal of Tribology 131, 4 (2009), 041702. 14

[181] Raw, M. J. A New Control Volume-based Finite Element Procedure for the Numerical Solution of the Fluid Flow and Scalar Transport Equations. PhD thesis, University of Waterloo, Waterloo, Ontario, Canada, 1985. 2, 59, 61

[182] Reddy, J. An Introduction to the Finite Element Method. McGraw-Hill Series in Mechanical Engineering. McGraw-Hill, 2006. 61

[183] Reynolds, O. On the theory of lubrication and its application to Mr. Beauchamp Tower's experiments, including an experimental determination of the viscosity of olive oil. Philosophical Transactions of the Royal Society of London 177 (1886), 157-234. 8

[184] Ricardo Limited. Ricardo Software - ENGDYN. Shoreham-by-Sea, UK, 2015. 37

[185] Sahlin, F., Almqvist, A., Larsson, R., And Glavatskin, S. A cavitation algorithm for arbitrary lubricant compressibility. Tribology International 40, 8 (2007), 1294-1300. 177, 178

[186] Sahlin, F., Almqvist, A., Larsson, R., And Glavatskih, S. Rough surface flow factors in full film lubrication based on a homogenization technique. Tribology International 40, 7 (2007), 1025-1034. 44

[187] Sahlin, F., Larsson, R., Almqvist, A., Lugt, P. M., and Marklund, P. A mixed lubrication model incorporating measured surface topography. Part 1: Theory of flow factors. Proceedings of the Institution of Mechanical Engineers, Part J: Journal of Engineering Tribology 224, 4 (2010), 335-351. 44 
[188] Schneider, G. E., AND RAW, M. J. A skewed, positive influence coefficient upwinding procedure for control-volume-based finite element convection-diffusion computation. Numerical Heat Transfer 9, 1 (1986), 1-26. 175

[189] Schneider, G. E., And RaW, M. J. Control volume finite-element method for heat transfer and fluid flow using colocated variables -1 . Computational procedure. Numerical Heat Transfer 11, 4 (1987), 363-390. 2, 59, 61

[190] SpIKes, H. Basics of EHL for practical application. Lubrication Science 27, 1 (2015), 45-67. 31

[191] SpIKes, H., AND JiE, Z. History, origins and prediction of elastohydrodynamic friction. Tribology Letters 56, 1 (2014), 1-25. 19, 20

[192] SpIkes, H. A. Sixty years of EHL. Lubrication Science 18, 4 (2006), 265-291. 31, 49

[193] Stachowiak, G., And Batchelor, A. Engineering Tribology. Elsevier Butterworth-Heinemann, 2005. 19, 41

[194] Stafford, A., Henshell, R., And Dudley, B. Finite element analysis of problems of elastohydrodynamic lubrication. In Elastohydrodynamics and related topics: proceedings of the 5th Leeds-Lyon Symposium on Tribology held in the Institute of Tribology, Department of Mechanical Engineering, the University of Leeds, England, September 1978 (1979). 32, 67

[195] Stefani, F. A., And Rebora, A. U. A nonlinear structure based elastohydrodynamic analysis method for connecting-rod big-end bearings of high performance engines. Journal of Tribology 126, 4 (2004), 664-671. 67, 80

[196] Stieber, W. Das Schwimmlager: Hydrodynamische Theorie des Gleitlagers. VDIVerlag GMbH, 1933. 14

[197] Sun, J., Gui, C.-L., AND WAng, Z.-H. Research on elastohydrodynamic lubrication of a crankshaft bearing with a rough surface considering crankshaft deformation. Proceedings of the Institution of Mechanical Engineers, Part D: Journal of Automobile Engineering 222, 12 (2008), 2403-2414. 80

[198] SwIFT, H. The stability of lubricating films in journal bearings. Proceedings of Institution of Civil Engineers (Lond.) 233 (1932), 267-288. 14

[199] Szeri, A. Fluid Film Lubrication. Cambridge University Press, 2010. 8, 21

[200] Tian, T. Dynamic behaviours of piston rings and their practical impact. Part 1: Ring flutter and ring collapse and their effects on gas flow and oil transport. Proceedings of the Institution of Mechanical Engineers, Part J: Journal of Engineering Tribology 216, 4 (2002), 209-228. 117

[201] Timoshenko, S., And Goodier, J. Theory of Elasticity, 2 ed. McGraw-Hill book Company, 1951. 32

[202] Tomanik, E. Modelamento do Desgaste por Deslizamento em Anéis de Pistão de Motores de Combustão Interna. PhD thesis, Escola Politécnica da Universidade de São Paulo, 2000. In Portuguese. 165 
[203] Tomanik, E. Modelling of the asperity contact area on actual 3D surfaces. SAE Technical Paper (2005), 2005-01-1864. 117

[204] Tomanik, E., Profito, F., And Zachariadis, D. Modelling the hydrodynamic support of cylinder bore and piston rings with laser textured surfaces. Tribology International 59, 0 (2013), 90-96. 115, 120

[205] TRIPP, J. H. Surface roughness effects in hydrodynamic lubrication: The flow factor method. Journal of Tribology 105, 3 (1983), 458-463. 43, 159

[206] Tzeng, S. T., And Saibel, E. Surface roughness effect on slider bearing lubrication. ASLE Transactions 10, 3 (1967), 334-348. 43

[207] Vakis, A. I., And Polycarpou, A. A. An advanced rough surface continuumbased contact and sliding model in the presence of molecularly thin lubricant. Tribology Letters 49, 1 (2013), 227-238. 49

[208] Van Basshuysen, R., And Schäfer, F. Internal Combustion Engine Handbook: Basics, Components, Systems, and Perspectives. Society of Automotive Engineers. SAE International, 2004. 81, 102

[209] Venner, C. H., And ten Napel, W. E. Surface roughness effects in an EHL line contact. Journal of Tribology 114, 3 (1992), 616-622. 47

[210] Versteeg, H., And Malalasekera, W. An Introduction to Computational Fluid Dynamics: The Finite Volume Method. Pearson Education Limited, 2007. 174

[211] Vierendeels, J., Lanoye, L., Degroote, J., And Verdonck, P. Implicit coupling of partitioned fluid-structure interaction problems with reduced order models. Computers \& Structures 85, 11-14 (2007), 970-976. 3, 68

[212] Wang, P., JR., T. G. K., and Vaidyanathan, K. Combined surface roughness pattern and non-Newtonian effects on the performance of dynamically loaded journal bearings. Tribology Transactions 45, 1 (2002), 1-10. 156

[213] Wang, Q. J., Zhu, D., Cheng, H. S., Yu, T., Jiang, X., And Liu, S. Mixed lubrication analyses by a macro-micro approach and a full-scale mixed EHL model. Journal of Tribology 126, 1 (2004), 81-91. 159

[214] Wang, X.-L., Zhang, J.-Y., And Dong, H. Analysis of bearing lubrication under dynamic loading considering micropolar and cavitating effects. Tribology International 44, 9 (2011), 1071 - 1075. 18

[215] Weng, S., Zhu, H.-P., Xia, Y., Ye, L., And Hu, X.-Y. Construction of stiffness and flexibility for substructure-based model updating. Mathematical Problems in Engineering 2013, Article ID 706798 (2013), 14 pages. 35

[216] Whitehouse, D. J., And Archard, J. F. The properties of random surfaces of significance in their contact. Proceedings of the Royal Society of London. A. Mathematical and Physical Sciences 316 (1970), 97-121. 37 
[217] Woloszynski, T., Podsiadlo, P., and Stachowiak, G. Efficient solution to the cavitation problem in hydrodynamic lubrication. Tribology Letters 58, 1 (2015). $2,58,126$

[218] Wu, C., And Zheng, L. An average Reynolds equation for partial film lubrication with a contact factor. Journal of Tribology 111, 1 (1989), 188-191. 156

[219] Xu, G., And Sadeghi, F. Thermal EHL analysis of circular contacts with measured surface roughness. Journal of Tribology 118, 3 (1996), 473-482. 47

[220] Xu, H., AND Smith, E. H. A new approach to the solution of elastohydrodynamic lubrication of crankshaft bearings. Proceedings of the Institution of Mechanical Engineers, Part C: Journal of Mechanical Engineering Science 204, 3 (1990), 187197. $3,68,80$

[221] Zachariadis, D. C. Estudo dos efeitos do desalinhamento angular de mancais hidrodinâmicos nas vibrações de rotores flexíveis. $\mathrm{PhD}$ thesis, Escola Politécnica da Universidade de São Paulo, 2000. In Portuguese. 21

[222] Zachariadis, D. C. Axial wedge effect in tilted hydrodynamic journal bearings. Tribology Transactions 51, 2 (2008), 173-181. 23, 143

[223] Zhang, Y. Boundary lubrication - an important lubrication in the following time. Journal of Molecular Liquids 128, 1-3 (2006), 56-59. 49

[224] Zhao, Y., Maietta, D., And Chang, L. An asperity microcontact model incorporating the transition from elastic deformation to fully plastic flow. Journal of Tribology 122, 1 (1999), 86-93. 37

[225] ZHU, D. On some aspects of numerical solutions of thin-film and mixed elastohydrodynamic lubrication. Proceedings of the Institution of Mechanical Engineers, Part J: Journal of Engineering Tribology 221, 5 (2007), 561-579. 47, 49

[226] Zhu, D., AND Ai, X. Point contact EHL based on optically measured threedimensional rough surfaces. Journal of Tribology 119, 3 (1997), 375-384. 47

[227] ZHU, D., AND Hu, Y. Z. The study of transition from full film elastohydrodynamic to mixed and boundary lubrication. In The Advancing Frontier of Engineering Tribology - Proceedings of the 1999 STLE/ASME H.S. Cheng Tribology Surveillance (Park Ridge, Illinois, USA, 1999), pp. 150-156. 47

[228] ZHU, D., AND Hu, Y.-Z. A computer program package for the prediction of EHL and mixed lubrication characteristics, friction, subsurface stresses and flash temperatures based on measured 3D surface roughness. Tribology Transactions 44 , 3 (2001), 383-390.

[229] ZHu, D., AND Hu, Y.-Z. Effects of rough surface topography and orientation on the characteristics of EHD and mixed lubrication in both circular and elliptical contacts. Tribology Transactions 44, 3 (2001), 391-398.

[230] Zhu, D., AND WANG, Q. J. Elastohydrodynamic lubrication: A gateway to interfacial mechanics - Review and prospect. Journal of Tribology 133, 4 (2011), 041001. 31 
[231] Zhu, D., AND WANG, Q. J. Effect of roughness orientation on the elastohydrodynamic lubrication film thickness. Journal of Tribology 135, 3 (2013), 031501. 47

[232] Zienkiewicz, O., Taylor, R., And Zhu, J. The Finite Element Method: Its Basis And Fundamentals. Elsevier Butterworth-Heinemann, 2005. 33 